\title{
Nickel- and Palladium-Catalyzed Cross- Coupling of Stibines with Organic Halides: Site-Selective Sequential Reactions with Polyhalogenated Arenes
}

Dejiang Zhang, ${ }^{\dagger}$ Ting Tang, ${ }^{\dagger}$ Zhao Zhang, ${ }^{\dagger}$ Liyuan Le,${ }^{\dagger}$ Zhi Xu, ${ }^{\dagger}$ Hao Lu, ${ }^{\dagger}$ Zhou Tong, ${ }^{\dagger}$ Dishu Zeng, ${ }^{\dagger}$ Wai-Yeung Wong, ${ }^{*}{ }^{\$}$ Shuang-Feng Yin, ${ }^{*}, \dagger$ Arash Ghaderi,, Nobuaki Kambe, ${ }^{*} \pitchfork$ Renhua Qiu*,†

'State Key Laboratory of Chemo/Biosensing and Chemometrics, Advanced Catalytic Engineering Research Center of the Ministry of Education, College of Chemistry and Chemical Engineering, Hunan University, Changsha, 410082, P.R. China

*The Institute of Scientific and Industrial Research, Osaka University, 8-1 Mihogaoka, Ibaraki-shi, Osaka 567-0047, Japan

\$Department of Applied Biology and Chemical Technology, The Hong Kong Polytechnic University Hung Hom, Hong Kong, P.R. China

Department of Chemistry, College of Sciences, University of Hormozgan, Bandar Abbas, 7916193145, Iran

\section{Corresponding Author}

*E-mail: renhuaqiu1@hnu.edu.cn (R.Q.)

*E-mail: wai-yeung.wong@polyu.edu.hk (W.Y.W.)

*E-mail: sf_yin@hnu.edu.cn (S. F.)

*E-mail: kambe@chem.eng.osaka-u.ac.jp (N.K.)

\section{Table of Contents}

S0 General Information

S1 Synthesis of Starting Materials

S2 Optimization Details

S2.1 Optimization of Reaction Conditions for Cross-Coupling Reaction of Halostibines with Organic Halides

S2.2 Optimization of Reaction Conditions for Cross-Coupling Reaction of Stibines with Aryl Halides

S2.3 Investigation on the Reactivity of Tri-p-tolylstibane and Stibine 3bf

S2.4 Investigation on the Reaction of Stibine 3ax with 1-Iodo-methylbenzne 2a

S4.1 Mechanism Study on Reductive Cross-Coupling Reaction of Chlorostibines with Aryl Iodides 
S4.1.1 Kinetic Research on Reductive Cross-Coupling Reaction of Chlorostibines with Aryl Iodides

S4.1.2 The Effect of Concentration of Substrates on Reductive Cross-Coupling Reaction of Chlorostibines with Aryl Iodides

S4.1.3 Control Experiments S77

S4.1.4 Proposed Mechanism S86

S4.2 Mechanism Study on Palladium-Catalyzed Cross-Coupling of Sb-aryl Stibines with Aryl Halides

S4.2.1 Kinetic Research on Palladium-Catalyzed Cross-Coupling of Sb-aryl Stibines with Aryl Halides

S4.2.2 The Effect of Concentration of Substrates on Palladium-Catalyzed Cross-Coupling of Sb-aryl Stibines with Aryl Halides S88

S4.2.3 Control Experiments S90

S4.2.4 Proposed Mechanism S96

S5. X-ray Crystallographic Data S96

S6. Reference S99

S7. Copies of NMR Spectrum of All Compounds S100 


\section{S0. General Information}

All reagents were commercially available and used without further purification. The dehydrated solvents were purchased and redistilled before use. All manipulations of air- and moisture-sensitive materials were conducted with a standard Schlenk technique under a nitrogen atmosphere. Glassware was dried in an oven and heated under reduced pressure before use. Column chromatography was undertaken on silica gel (300-400 mesh) using a proper eluent system. Analytical thin layer chromatography (TLC) was performed on Haiyang TLC silica gel GF254 $(0.25 \mathrm{~mm})$ plates. Reactions were monitored by TLC or gas chromatography (GC). Visualization on TLC was achieved by the use of UV light ( $254 \mathrm{~nm})$. Proton, carbon and fluorine nuclear magnetic resonance spectrum $\left({ }^{1} \mathrm{H},{ }^{13} \mathrm{C}\right.$ and ${ }^{19} \mathrm{~F}$ NMR) were recorded on a Bruker-400 (400 MHz for ${ }^{1} \mathrm{H}$ NMR, $101 \mathrm{MHz}$ for ${ }^{13} \mathrm{C}$ NMR and $376 \mathrm{MHz}$ for ${ }^{19} \mathrm{~F}$ NMR spectroscopy) spectrometer with solvent resonance as the internal standard ( ${ }^{1} \mathrm{H} \mathrm{NMR}, \mathrm{CDCl}_{3}$ at $7.26 \mathrm{ppm}$, DMSO- $d_{6}$ at $2.50 \mathrm{ppm}, \mathrm{C}_{6} \mathrm{D}_{6}$ at $7.16 \mathrm{ppm} ;{ }^{13} \mathrm{C} \mathrm{NMR}, \mathrm{CDCl}_{3}$ at $77.16 \mathrm{ppm}$, DMSO- $d_{6}$ at $39.52 \mathrm{ppm}, \mathrm{C}_{6} \mathrm{D}_{6}$ at 128.06 ppm). Chemical shifts are reported in ppm ( $\delta$ ) relative to internal tetramethylsilane (TMS). Data are reported as follows: Chemical shift, multiplicity $(\mathrm{s}=$ singlet, $\mathrm{d}=$ doublet, $\mathrm{t}=$ triplet, $\mathrm{q}=$ quartet, $\mathrm{m}=$ multiplet), Coupling constants $(J)$ are reported in hertz. Melting points were measured using a digital melting point meter WRS-2. Crystal Data were acquired at 296 K on a Rigaku Oxford Diffraction Supernova Dual Source, $\mathrm{Cu}$ at Zero equipped with an Atlas S2 CCD using $\mathrm{Cu} \mathrm{K} \alpha$ radiation. HRMS data were obtained via instrument: Thermo Q Exactive Plus. GC data were obtained on Shimadzu GC-2014.

\section{S1. Synthesis of Starting Materials}<smiles>Cl[Sb]1(Cl)c2ccccc2CN(c2ccccc2)Cc2ccccc21</smiles>

$1 \mathrm{a}$

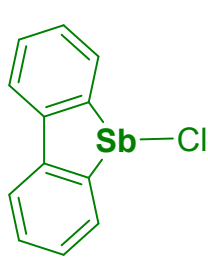

$1 \mathrm{~b}$

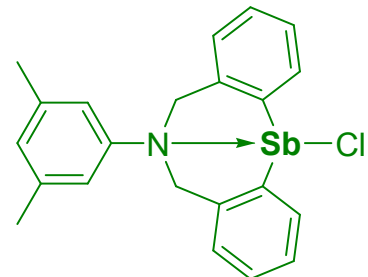

1c<smiles>CC(C)(C)N1Cc2ccccc2[SH](=O)(Cl)c2ccccc21</smiles>

$1 d$<smiles>Fc1ccc(N2Cc3ccccc3S(Cl)(Cl)c3ccccc32)cc1</smiles>

$1 \mathrm{~g}$

Chlorostibines $\mathbf{1 a}, \mathbf{1 b}, \mathbf{1 c}, \mathbf{1 d}$ and $\mathbf{1 g}$ was prepared according to previous report. ${ }^{\mathrm{S} 1}$

12-chloro-6-(4-fluorophenyl)-5,6,7,12-tetrahydrodibenzo[c,f $][1,5]$ azastibocine (1g) 
<smiles>Fc1ccc(N2Cc3ccccc3[Sb]3(Cl)CC2(Cl)c2ccccc23)cc1</smiles>

Chlorostibine $1 \mathrm{~g}$ was obtained in $78 \%$ as a white solid. M.P.: $214.1-215.8{ }^{\circ} \mathrm{C} ;{ }^{1} \mathrm{H}$ NMR $(400 \mathrm{MHz}$, Chloroform- $d$ ) $\delta 8.38$ (d, $J=7.5 \mathrm{~Hz}, 0.5 \mathrm{H}), 8.28(\mathrm{~d}, J=7.5 \mathrm{~Hz}, 1.5 \mathrm{H}), 7.49-7.41(\mathrm{~m}, 2 \mathrm{H}), 7.39-7.32$ (m, 2H), $7.28-7.18(\mathrm{~m}, 4 \mathrm{H}), 7.10-6.97(\mathrm{~m}, 2 \mathrm{H}), 4.65$ (d, $J=14.9 \mathrm{~Hz}, 2 \mathrm{H}), 4.56(\mathrm{~d}, J=14.9 \mathrm{~Hz}, 2 \mathrm{H})$. ${ }^{13} \mathrm{C}$ NMR $\left(101 \mathrm{MHz}, \mathrm{CDCl}_{3}\right) \delta 160.2(\mathrm{~d}, J=244.6 \mathrm{~Hz}), 144.3(\mathrm{~d}, J=3.0 \mathrm{~Hz}), 143.0,142.9,140.6,138.2$, 136.9, 135.4, 129.6, 129.5, 129.5, 129.4, 125.5, 125.5, 121.8, 121.6 (d, $J=8.2 \mathrm{~Hz}), 116.4$ (d, $J=22.3$ $\mathrm{Hz})$, 61.7. ${ }^{19} \mathrm{~F}$ NMR $\left(376 \mathrm{MHz}, \mathrm{CDCl}_{3}\right) \delta-116.65,-116.82$. HRMS-ESI $(\mathrm{m} / \mathrm{z})[\mathrm{M}+\mathrm{H}]^{+}$Calcd for $\mathrm{C}_{20} \mathrm{H}_{17} \mathrm{ClFNSb}^{+}$, 446.0066; Found, 446.0089.

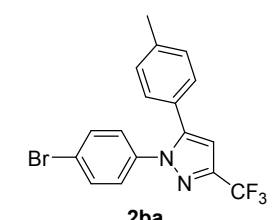

2ba

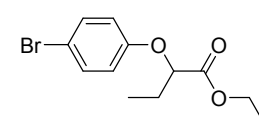

$2 \mathrm{bb}$

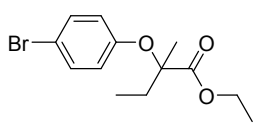

$2 \mathrm{bc}$

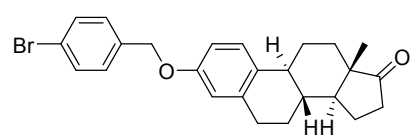

2bd<smiles>CCCN(CCC)S(=O)(=O)c1ccc(OC(=O)c2ccc(I)cc2)cc1</smiles>

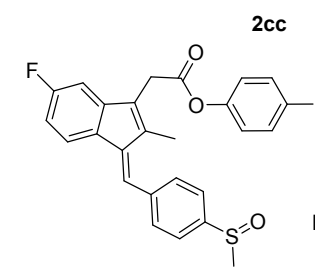

2dc

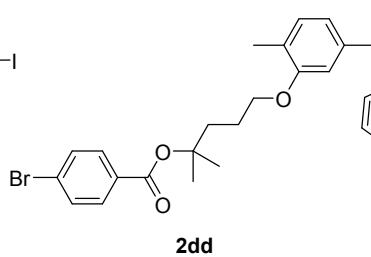

2da

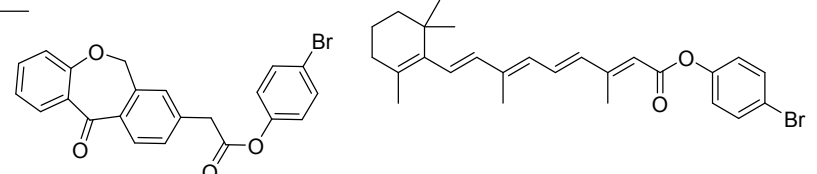

2de

2df

Compound $\mathbf{2 b a}$ was synthesized according to the following procedure. ${ }^{\mathrm{S} 2}$

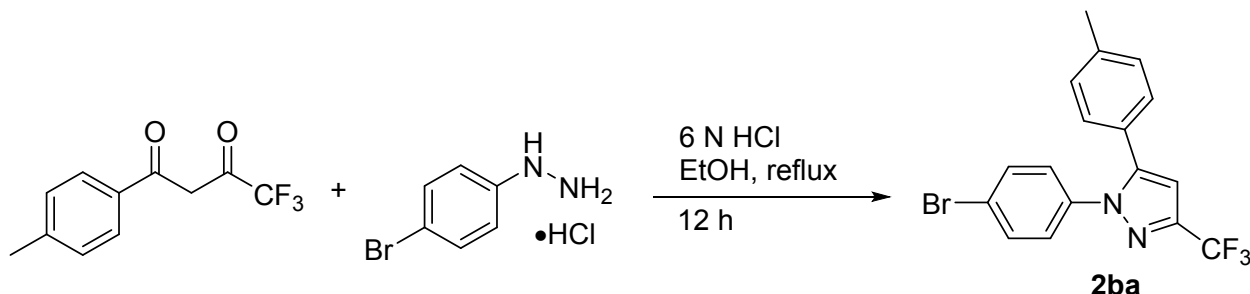

Procedure: A mixture of 4,4,4-trifluoro-1-( $p$-tolyl)butane-1,3-dione (1.19 g, $5.2 \mathrm{mmol})$, (4bromophenyl)hydrazine hydrochloride (1.12 g, $5.0 \mathrm{mmol})$, and $6 \mathrm{~N} \mathrm{HCl}(1.7 \mathrm{~mL}, 10 \mathrm{mmol}, 2.0$ equiv) in ethanol was stirred at $100{ }^{\circ} \mathrm{C}$ for $12 \mathrm{~h}$. After cooling to room temperature, the reaction mixture was diluted with EtOAc $(10 \mathrm{~mL})$, and washed with $10 \mathrm{~mL}$ of saturated $\mathrm{K}_{2} \mathrm{CO}_{3}$ and $10 \mathrm{~mL}$ of brine. The organic layer was dried over anhydrous $\mathrm{Na}_{2} \mathrm{SO}_{4}$ and filtered. The solvent was removed under reduced pressure. The residue was purified by column chromatography on silica gel (eluent: hexane/EtOAc $=$ 
10:1) to afford $2 \mathbf{b a}$ as a white solid (1.71 g, 90\%). ${ }^{1} \mathrm{H}$ NMR (400 MHz, Chloroform- $d$ ) $\delta 7.51-7.44$ (m, 2H), $7.23-7.09(\mathrm{~m}, 6 \mathrm{H}), 6.73(\mathrm{~s}, 1 \mathrm{H}), 2.38(\mathrm{~s}, 3 \mathrm{H}) .{ }^{13} \mathrm{C} \mathrm{NMR}\left(101 \mathrm{MHz}, \mathrm{CDCl}_{3}\right) \delta 144.9,143.5(\mathrm{q}, J=$ $38.3 \mathrm{~Hz}$ ), 139.5, 138.4, 132.2, 129.6, 128.7, 126.9, 126.0, 121.3 (q, $J=269.1 \mathrm{~Hz}), 122.2,105.7,21.3$. ${ }^{19} \mathrm{~F}$ NMR $\left(377 \mathrm{MHz}, \mathrm{CDCl}_{3}\right) \delta-62.16$.

Compounds $\mathbf{2 b b}$ and $\mathbf{2 b c}$ were synthesized according to the following procedure. ${ }^{\mathrm{S} 3}$
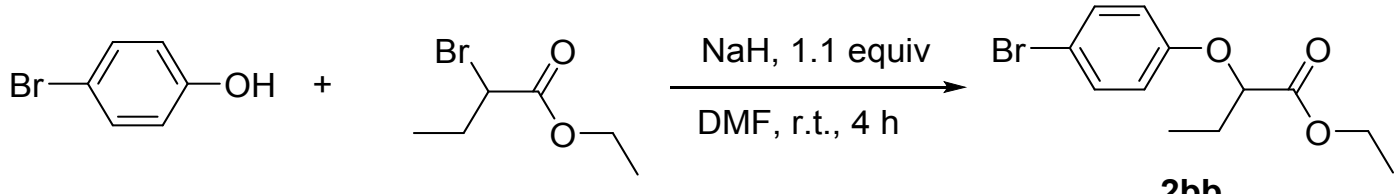

$2 b b$

Procedure: In a $100 \mathrm{~mL}$ round-bottomed flask equipped with a magnetic stir bar, 4-bromophenol (1.0 g, $6.0 \mathrm{mmol})$ in $6.0 \mathrm{~mL}$ of $N, N$-dimethylformamide $(\mathrm{DMF})$ was added dropwise to sodium hydride $(0.26 \mathrm{~g}$, $6.6 \mathrm{mmol}$ ) in $6.0 \mathrm{~mL}$ of DMF. The reaction mixture was stirred at room temperature for 1 hour. Then, ethyl 2-bromobutanoate $(1.15 \mathrm{~g}, 6.0 \mathrm{mmol})$ in $6.0 \mathrm{~mL}$ of DMF was added dropwise, and followed with the addition of sodium iodide $(1.05 \mathrm{~g}, 7.0 \mathrm{mmol})$. The reaction mixture was stirred at $80^{\circ} \mathrm{C}$ for $3 \mathrm{~h}$. After $3 \mathrm{~h}$, the reaction mixture was allowed to cool to room temperature, diluted with $\mathrm{H}_{2} \mathrm{O}(10 \mathrm{~mL})$, and extracted with EtOAc $(3 \times 15 \mathrm{~mL})$. The combined organic layers were washed with $\mathrm{H}_{2} \mathrm{O}(2 \times 10 \mathrm{~mL})$ and filtered. The organic layer was dried over anhydrous $\mathrm{Na}_{2} \mathrm{SO}_{4}$. The solvent was removed under reduced pressure. The residue was purified by silica gel chromatography eluting with hexanes/EtOAc $(\mathrm{v} / \mathrm{v}=1: 1)$ to yield $\mathbf{2 b b}\left(1.41 \mathrm{~g}, 82 \%\right.$ yield) as a colorless oil. ${ }^{1} \mathrm{H}$ NMR (400 MHz, Chloroform- $d$ ) $\delta 7.35$ $(\mathrm{d}, J=8.8 \mathrm{~Hz}, 2 \mathrm{H}), 6.76(\mathrm{~d}, J=8.8 \mathrm{~Hz}, 2 \mathrm{H}), 4.50(\mathrm{t}, J=6.1 \mathrm{~Hz}, 1 \mathrm{H}), 4.20(\mathrm{q}, J=7.1 \mathrm{~Hz}, 2 \mathrm{H}), 2.01-$ $1.93(\mathrm{~m}, 2 \mathrm{H}), 1.23(\mathrm{t}, J=7.1 \mathrm{~Hz}, 3 \mathrm{H}), 1.06(\mathrm{t}, J=7.4 \mathrm{~Hz}, 3 \mathrm{H})$.

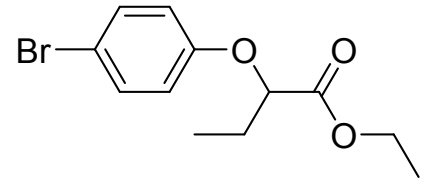

$2 b b$

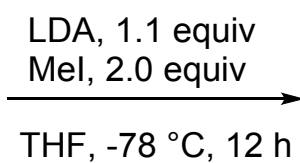

THF, $-78^{\circ} \mathrm{C}, 12 \mathrm{~h}$

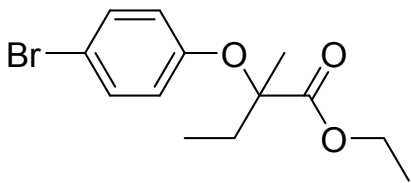

$2 b c$

Procedure: To a solution of lithium diisopropylamide (LDA) at $-78{ }^{\circ} \mathrm{C}$ under a nitrogen atmosphere, ethyl 2-(4-bromophenoxy)butanoate $(1.0 \mathrm{~g}, 3.5 \mathrm{mmol})$ in dry THF $(25 \mathrm{~mL})$ was added dropwise. The reaction mixture was then stirred for $4 \mathrm{~h}$. Methyl iodide $(993.3 \mathrm{mg}, 7.0 \mathrm{mmol})$ in dry THF $(20 \mathrm{~mL})$ was added dropwise. The resulting mixture was allowed to slowly warm to room temperature for $8 \mathrm{~h}$, and monitored by GC. The reaction mixture was quenched with saturated $\mathrm{NH}_{4} \mathrm{Cl}$ solution and extracted with EtOAc $(3 \times 30 \mathrm{~mL})$. The combined organic layer was washed with brine $(2 \times 30 \mathrm{~mL})$, and dried over anhydrous $\mathrm{Na}_{2} \mathrm{SO}_{4}$. The solvent was removed under reduced pressure. The crude material was purified by silica gel chromatography eluting with hexanes/EtOAc $(\mathrm{v} / \mathrm{v}=1: 1)$ to deliver $\mathbf{2 b c}(787.5 \mathrm{mg}, 75 \%$ yield) as a colorless oil. ${ }^{1} \mathrm{H}$ NMR (400 MHz, Chloroform- $d$ ) $\delta 7.35(\mathrm{~d}, J=8.8 \mathrm{~Hz}, 2 \mathrm{H}), 6.76(\mathrm{~d}, J=8.8 \mathrm{~Hz}$, 2H), 4.25 (q, $J=7.1 \mathrm{~Hz}, 2 \mathrm{H}), 2.09-1.89(\mathrm{~m}, 2 \mathrm{H}), 1.51$ (s, 3H), 1.27 (t, $J=7.1 \mathrm{~Hz}, 3 \mathrm{H}), 0.99$ (t, $J=7.5$ $\mathrm{Hz}, 3 \mathrm{H})$. 
Compound $\mathbf{2 b d}$ and $\mathbf{2 c c}$ were synthesized according to the following procedure.
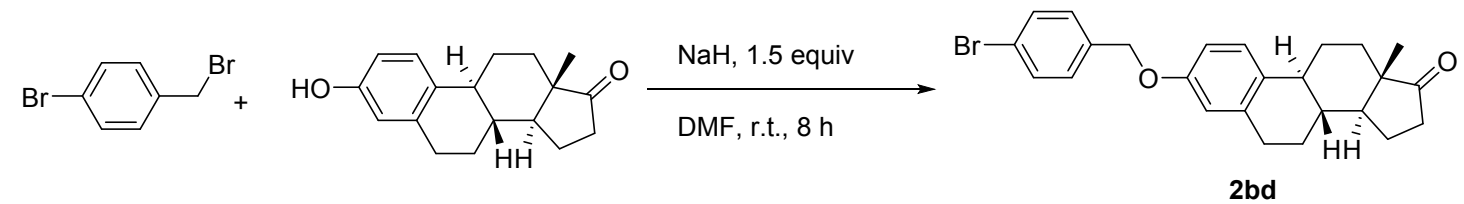

Procedure: A mixture of sodium hydride (300 mg), (8R,9S,13S,14S)-3-hydroxy-13-methyl6,7,8,9,11,12,13,14,15,16-decahydro-17H-cyclopenta[a]phenanthren-17-one (1.35 g, $5.0 \mathrm{mmol})$ and 1bromo-4-(bromomethyl)benzene $(1.25 \mathrm{~g}, 5.0 \mathrm{mmol})$ in DMF was stirred at room temperature for $4 \mathrm{~h}$. The resulting mixture was quenched with $10 \mathrm{~mL}$ of $\mathrm{H}_{2} \mathrm{O}$, and extracted with EtOAc $(3 \times 30 \mathrm{~mL})$. The combined organic layer was dried over anhydrous $\mathrm{Na}_{2} \mathrm{SO}_{4}$. The solvent was removed under reduced pressure. The crude material was purified by silica gel chromatography eluting with hexanes/EtOAc ( $\mathrm{v} / \mathrm{v}=40: 1)$ to yield $2 \mathrm{bd}\left(1.86 \mathrm{~g}, 85 \%\right.$ yield) as a liquid. ${ }^{1} \mathrm{H}$ NMR (400 MHz, Chloroform- $d$ ) $\delta 7.50(\mathrm{~d}, J$ $=8.2 \mathrm{~Hz}, 2 \mathrm{H}), 7.30(\mathrm{~d}, J=8.1 \mathrm{~Hz}, 2 \mathrm{H}), 7.21(\mathrm{~d}, J=8.5 \mathrm{~Hz}, 1 \mathrm{H}), 6.76(\mathrm{~d}, J=8.5 \mathrm{~Hz}, 1 \mathrm{H}), 6.71(\mathrm{~s}, 1 \mathrm{H})$, 4.99 (s, 2H), $2.97-2.78$ (m, 2H), 2.51 (dd, $J=18.8,8.5 \mathrm{~Hz}, 1 \mathrm{H}), 2.39$ (d, $J=9.5 \mathrm{~Hz}, 1 \mathrm{H}), 2.27$ (d, $J=$ $9.3 \mathrm{~Hz}, 1 \mathrm{H}), 2.20-1.91(\mathrm{~m}, 4 \mathrm{H}), 1.72-1.36(\mathrm{~m}, 6 \mathrm{H}), 0.91(\mathrm{~s}, 3 \mathrm{H}) .{ }^{13} \mathrm{C} \mathrm{NMR}\left(101 \mathrm{MHz}, \mathrm{CDCl}_{3}\right) \delta$ 220.9, 156.7, 138.0, 136.5, 132.7, 131.8, 129.1, 126.5, 121.8, 115.1, 112.5, 69.3, 50.5, 48.1, 44.1, 38.5, 36.0, 31.7, 30.0, 26.6, 26.0, 21.7, 14.0. HRMS-ESI (m/z) $[\mathrm{M}+\mathrm{H}]^{+}$Calcd for $\mathrm{C}_{25} \mathrm{H}_{28} \mathrm{BrO}_{2}{ }^{+}, 439.1267$; Found, 439.1259.

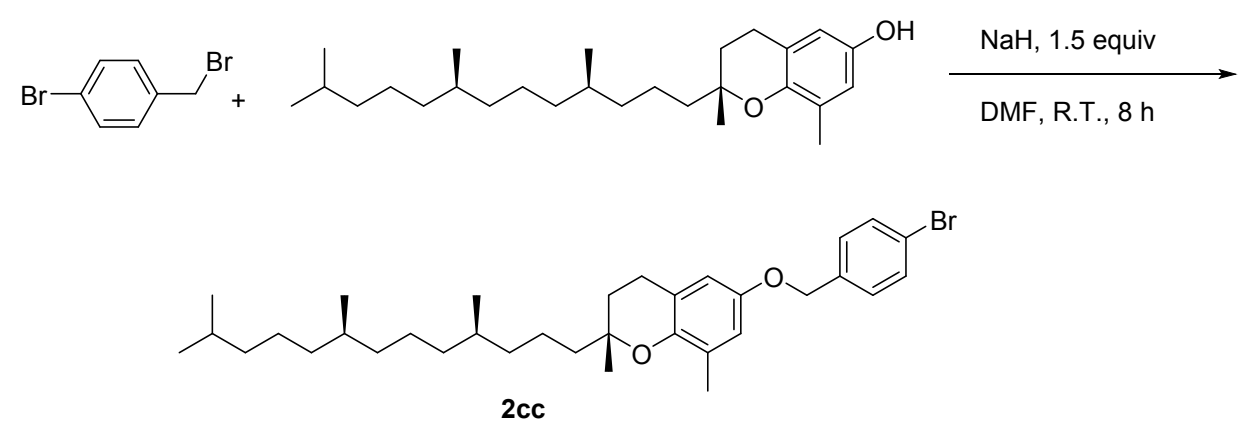

Procedure: A mixture of sodium hydride (300 mg), (R)-2,8-dimethyl-2-((4R,8R)-4,8,12trimethyltridecyl)chroman-6-ol (2.01 g, $5.0 \mathrm{mmol})$, and 1-bromo-4-(bromomethyl)benzene (1.25 g, 5.0 $\mathrm{mmol}$ ) in DMF was stirred at room temperature for $4 \mathrm{~h}$. The resulting mixture was quenched with $10 \mathrm{~mL}$ of $\mathrm{H}_{2} \mathrm{O}$, and extracted with EtOAc $(3 \times 30 \mathrm{~mL})$. The combined organic layer was dried over anhydrous $\mathrm{Na}_{2} \mathrm{SO}_{4}$. The solvent was removed under reduced pressure. The crude material was purified by silica gel chromatography eluting with hexanes/EtOAc ( $\mathrm{v} / \mathrm{v}=40: 1)$ to yield $2 \mathrm{cc}\left(2.45 \mathrm{~g}, 86 \%\right.$ yield) as a lquid. ${ }^{1} \mathrm{H}$ NMR (400 MHz, Chloroform- $d$ ) $\delta 7.55-7.47(\mathrm{~m}, 2 \mathrm{H}), 7.34-7.28(\mathrm{~m}, 2 \mathrm{H}), 6.65(\mathrm{~d}, \mathrm{~J}=3.0 \mathrm{~Hz}, 1 \mathrm{H})$, $6.52(\mathrm{~d}, \mathrm{~J}=3.0 \mathrm{~Hz}, 1 \mathrm{H}), 4.94$ (s, 2H), $2.78-2.69$ (m, 2H), 2.18 (s, 3H), $1.88-1.69$ (m, 2H), $1.64-1.50$ (m, 4H), $1.49-1.35(\mathrm{~m}, 4 \mathrm{H}), 1.29(\mathrm{~d}, \mathrm{~J}=15.3 \mathrm{~Hz}, 9 \mathrm{H}), 1.23-1.13(\mathrm{~m}, 3 \mathrm{H}), 1.12-1.05(\mathrm{~m}, 4 \mathrm{H}), 0.93$ -0.85 (m, 12H). ${ }^{13} \mathrm{C}$ NMR $\left(101 \mathrm{MHz}, \mathrm{CDCl}_{3}\right) \delta 151.2,146.6,136.9,131.7,129.2,127.4,121.7,121.1$, $115.8,112.3,75.8,69.9,40.1,39.5,37.6,37.5,37.4,32.9,32.8,31.5,28.1,25.0,24.6,24.3,22.9,22.8$, 22.7, 21.1, 19.9, 19.8, 16.4. HRMS-EI (m/z) [M] Calcd for $\mathrm{C}_{34} \mathrm{H}_{51} \mathrm{BrO}_{2}$, 570.3072; Found, 570.3076.

Compound $\mathbf{2 d a}, \mathbf{2 d b}, \mathbf{2 d c}, \mathbf{2 d d}, \mathbf{2 d e}$ and $\mathbf{2 d f}$ were synthesized according to the following procedure. ${ }^{\mathrm{S}}$ 


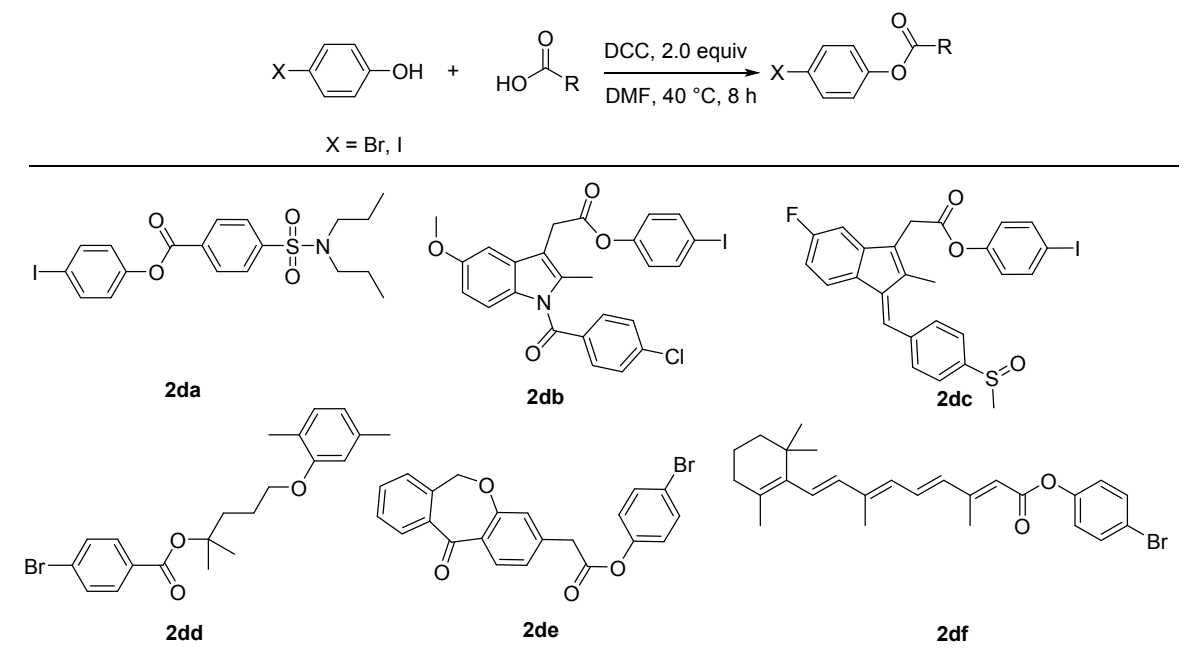

General procedure: A mixture of 4-iodophenol or 4-bromophenol (5.0 mmol), organic acid $(5.0 \mathrm{mmol})$, dicyclohexylcarbodiimide (DCC) $(2.06 \mathrm{~g}, 10 \mathrm{mmol})$ in DMF was stirred at $40{ }^{\circ} \mathrm{C}$ for $8 \mathrm{~h}$. After cooling to room temperature, the reaction mixture was diluted with $20 \mathrm{~mL}$ of $\mathrm{H}_{2} \mathrm{O}$ and extracted with EtOAc (60 $\mathrm{mL}$ ). The solvent was removed under reduced pressure. The residue was purified by silica gel chromatography.

Compound 2da was obtained as a white solid in $80 \%\left(1.95\right.$ g). M.P.: $75.5-76.9^{\circ} \mathrm{C} .{ }^{1} \mathrm{H}$ NMR $(400 \mathrm{MHz}$, Chloroform- $d$ ) $\delta 8.30$ (d, $J=6.6 \mathrm{~Hz}, 2 \mathrm{H}), 7.94(\mathrm{~d}, J=6.6 \mathrm{~Hz}, 2 \mathrm{H}), 7.74$ (d, $J=6.9 \mathrm{~Hz}, 2 \mathrm{H}), 7.01$ (d, $J=$ $6.9 \mathrm{~Hz}, 2 \mathrm{H}), 3.17-3.08(\mathrm{~m}, 4 \mathrm{H}), 1.62-1.49(\mathrm{~m}, 4 \mathrm{H}), 0.88$ (t, $J=7.4 \mathrm{~Hz}, 6 \mathrm{H}) .{ }^{13} \mathrm{C}$ NMR $(101 \mathrm{MHz}$, $\left.\mathrm{CDCl}_{3}\right) \delta 163.5,150.5,145.1,138.7,132.5,130.8,127.2,123.7,90.3,49.9,21.9,11.2$. HRMS-ESI (m/z) $[\mathrm{M}+\mathrm{H}]^{+}$Calcd for $\mathrm{C}_{19} \mathrm{H}_{23} \mathrm{INO}_{4} \mathrm{~S}^{+}, 488.0387$; Found, 488.0394 .

Compound $2 \mathrm{db}$ was obtained as a white solid in $76 \%$ (2.07 g). M.P.: $121.2-123.1{ }^{\circ} \mathrm{C} .{ }^{1} \mathrm{H}$ NMR (400 MHz, Chloroform- $d$ ) $\delta 7.72-7.61(\mathrm{~m}, 4 \mathrm{H}), 7.47$ (d, $J=8.5 \mathrm{~Hz}, 2 \mathrm{H}), 7.04(\mathrm{~s}, 1 \mathrm{H}), 6.93-6.78(\mathrm{~m}, 3 \mathrm{H})$, $6.70(\mathrm{~d}, J=9.0 \mathrm{~Hz}, 1 \mathrm{H}), 3.89$ (s, $2 \mathrm{H}), 3.83(\mathrm{~s}, 3 \mathrm{H}), 2.45$ (s, 3H). ${ }^{13} \mathrm{C}$ NMR $\left(101 \mathrm{MHz}, \mathrm{CDCl}_{3}\right) \delta 169.0$, 168.4, 156.2, 150.6, 139.5, 138.6, 136.4, 133.8, 131.3, 131.0, 130.5, 129.3, 123.7, 115.2, 111.9, 111.8, 101.3, 90.1, 55.8, 30.6, 13.5. HRMS-EI (m/z) [M] Calcd for $\mathrm{C}_{25} \mathrm{H}_{19} \mathrm{ClINO}_{4}$, 559.0047; Found, 559.0041.

Compound $2 \mathbf{d c}$ was obtained as a yellow solid in $86 \%\left(2.13\right.$ g). M.P.: $138.5-140.6{ }^{\circ} \mathrm{C} .{ }^{1} \mathrm{H}$ NMR $(400$ MHz, Chloroform- $d$ ) $\delta 7.78-7.58(\mathrm{~m}, 6 \mathrm{H}), 7.22-7.12(\mathrm{~m}, 2 \mathrm{H}), 7.00-6.90(\mathrm{~m}, 1 \mathrm{H}), 6.84(\mathrm{~d}, J=8.7$ $\mathrm{Hz}, 2 \mathrm{H}), 6.65-6.53(\mathrm{~m}, 1 \mathrm{H}), 3.78(\mathrm{~s}, 2 \mathrm{H}), 2.81(\mathrm{~s}, 3 \mathrm{H}), 2.27$ (s, 3H). ${ }^{13} \mathrm{C} \mathrm{NMR}\left(101 \mathrm{MHz}, \mathrm{CDCl}_{3}\right) \delta$ $168.4,163.5(\mathrm{~d}, J=246.8 \mathrm{~Hz}), 150.6,146.5(\mathrm{~d}, J=8.7 \mathrm{~Hz}), 145.7,141.6,139.6,138.9,138.6,131.0$ (d, $J=2.5 \mathrm{~Hz}), 130.4,129.6(\mathrm{~d}, J=2.8 \mathrm{~Hz}), 128.8(\mathrm{~d}, J=1.9 \mathrm{~Hz}), 124.0,123.9,123.7,111.1$ (d, $J=22.6$ $\mathrm{Hz}), 106.1(\mathrm{~d}, J=24.1 \mathrm{~Hz}), 90.1,44.0,32.0,10.8 .{ }^{19} \mathrm{~F}$ NMR $\left(376 \mathrm{MHz}, \mathrm{CDCl}_{3}\right) \delta-112.58$. HRMS-EI $(\mathrm{m} / \mathrm{z})[\mathrm{M}]$ Calcd for $\mathrm{C}_{26} \mathrm{H}_{20} \mathrm{FIO}_{3} \mathrm{~S}, 558.0162$; Found, 558.0156.

Compound $2 \mathbf{d d}$ was obtained as a liquid in $75 \%(1.51 \mathrm{~g}) .{ }^{1} \mathrm{H}$ NMR $(400 \mathrm{MHz}$, Chloroform- $d) \delta 7.59-$ 7.45 (m, 2H), 7.05 (d, $J=7.5 \mathrm{~Hz}, 1 \mathrm{H}), 6.99-6.92(\mathrm{~m}, 2 \mathrm{H}), 6.72$ (d, $J=7.6 \mathrm{~Hz}, 1 \mathrm{H}), 6.67$ (s, 1H), 4.08 $-3.96(\mathrm{~m}, 2 \mathrm{H}), 2.36(\mathrm{~s}, 3 \mathrm{H}), 2.22(\mathrm{~s}, 3 \mathrm{H}), 1.92(\mathrm{~d}, J=3.6 \mathrm{~Hz}, 4 \mathrm{H}), 1.41$ (d, $J=1.6 \mathrm{~Hz}, 6 \mathrm{H}) .{ }^{13} \mathrm{C}$ NMR 
$\left(101 \mathrm{MHz}, \mathrm{CDCl}_{3}\right) \delta 176.1,156.9,150.1,136.6,132.5,130.5,123.6,123.5,120.9,118.8,112.0,67.7$, 42.6, 37.2, 25.3, 25.2, 21.5, 15.9. HRMS-EI (m/z) [M] Calcd for $\mathrm{C}_{21} \mathrm{H}_{25} \mathrm{BrO}_{3}, 404.0987$; Found, 404.0982.

Compound 2de was obtained as a white solid in $87 \%\left(1.83\right.$ g). M.P.: $151.6-153.2{ }^{\circ} \mathrm{C} .{ }^{1} \mathrm{H}$ NMR $(400$ MHz, Chloroform- $d$ ) $\delta 8.21(\mathrm{~d}, J=2.5 \mathrm{~Hz}, 1 \mathrm{H}), 7.90(\mathrm{~d}, J=7.6 \mathrm{~Hz}, 1 \mathrm{H}), 7.56(\mathrm{t}, J=7.4 \mathrm{~Hz}, 1 \mathrm{H}), 7.52$ -7.44 (m, 4H), 7.36 (d, $J=7.4 \mathrm{~Hz}, 1 \mathrm{H}), 7.07$ (d, $J=8.4 \mathrm{~Hz}, 1 \mathrm{H}), 6.98$ (d, $J=8.4 \mathrm{~Hz}, 2 \mathrm{H}), 5.20(\mathrm{~s}, 2 \mathrm{H})$, 3.87 (s, 2H). ${ }^{13} \mathrm{C}$ NMR (101 MHz, $\left.\mathrm{CDCl}_{3}\right) \delta 190.9,169.6,160.8,149.7,140.5,136.3,135.6,133.0,132.7$, 132.5, 129.6, 129.4, 128.0, 127.0, 125.4, 123.4, 121.4, 119.1, 73.7, 40.3. HRMS-ESI (m/z) $[\mathrm{M}+\mathrm{H}]^{+} \mathrm{Calcd}$ for $\mathrm{C}_{22} \mathrm{H}_{16} \mathrm{BrO}_{4}{ }^{+}$, 423.0226; Found, 423.0225.

Compound 2df was obtained as a yellow solid in $89 \%$ (2.02 g). M.P.: $120.1-120.8^{\circ} \mathrm{C} .{ }^{1} \mathrm{H}$ NMR $(400$ MHz, Chloroform- $d$ ) $\delta 7.49$ (d, $J=8.3 \mathrm{~Hz}, 2 \mathrm{H}), 7.16-6.94(\mathrm{~m}, 3 \mathrm{H}), 6.43-6.30(\mathrm{~m}, 2 \mathrm{H}), 6.23-6.11$ $(\mathrm{m}, 2 \mathrm{H}), 5.97(\mathrm{~s}, 1 \mathrm{H}), 2.41(\mathrm{~s}, 3 \mathrm{H}), 2.03(\mathrm{~s}, 5 \mathrm{H}), 1.73(\mathrm{~s}, 3 \mathrm{H}), 1.66-1.57(\mathrm{~m}, 2 \mathrm{H}), 1.54-1.41(\mathrm{~m}, 2 \mathrm{H})$, 1.05 (s, 6H). ${ }^{13} \mathrm{C}$ NMR (101 MHz, $\left.\mathrm{CDCl}_{3}\right) \delta 165.1,156.2,150.0,140.8,137.8,137.3,134.7,132.5,132.4$, 130.4, 129.5, 129.4, 123.7, 118.6, 116.8, 39.7, 34.4, 33.3, 29.1, 21.9, 19.3, 14.3, 13.1. HRMS-EI (m/z) [M] Calcd for $\mathrm{C}_{26} \mathrm{H}_{31} \mathrm{BrO}_{2}$, 454.1507; Found, 454.1510.

\section{S2. Optimization Details}


S2.1 Optimization of Reaction Conditions for Cross-Coupling Reaction of Halostibines with Organic Halides

Table S1. Optimization of Reaction Conditions for Reductive Cross-Coupling Reaction of 1a with $2 a$

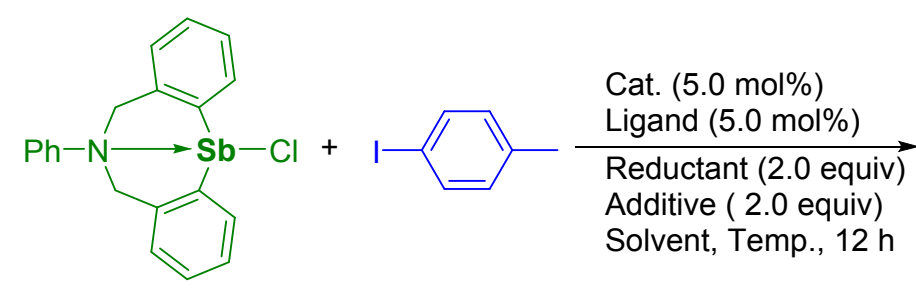

1a, $0.1 \mathrm{mmol}$

2a, $0.1 \mathrm{mmol}$

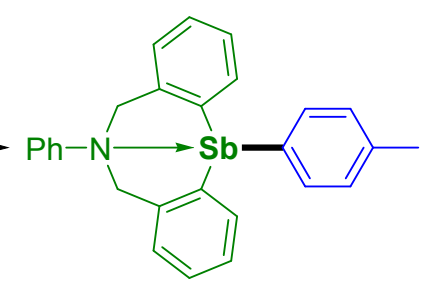

$3 a$

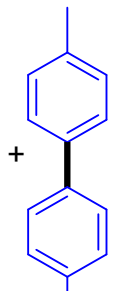

$4 a$

\begin{tabular}{|c|c|c|c|c|c|c|c|c|}
\hline \multirow[t]{2}{*}{ Entry } & \multirow[t]{2}{*}{ Cat. } & \multirow[t]{2}{*}{ Ligand } & \multirow[t]{2}{*}{ Additive } & \multirow[t]{2}{*}{ Solvent } & \multirow{2}{*}{$\begin{array}{l}\text { Reducing } \\
\text { reagent }\end{array}$} & \multirow{2}{*}{$\begin{array}{l}\text { Temp. } \\
\left({ }^{\circ} \mathrm{C}\right)\end{array}$} & \multicolumn{2}{|c|}{ Yield $(\%)^{b}$} \\
\hline & & & & & & & $3 \mathbf{a}$ & $4 \mathbf{a}$ \\
\hline 1 & $\mathrm{PdCl}_{2}$ & L1 & LiI & THF & $\mathrm{Zn}$ & 100 & 35 & 5 \\
\hline 2 & $\mathrm{Pd}(\mathrm{OAc})_{2}$ & L1 & LiI & THF & $\mathrm{Zn}$ & 100 & 42 & 23 \\
\hline 3 & $\mathrm{Pd}\left(\mathrm{PPh}_{3}\right) \mathrm{Cl}_{2}$ & L1 & LiI & THF & $\mathrm{Zn}$ & 100 & 37 & 2 \\
\hline 4 & $\mathrm{Ni}($ dppe $) \mathrm{Cl}_{2}$ & L1 & $\mathrm{LiCl}$ & THF & $\mathrm{Zn}$ & 100 & 57 & 3 \\
\hline 5 & $\mathrm{Ni}($ dppe $) \mathrm{Cl}_{2}$ & $\mathbf{L 1}$ & $\mathrm{LiBr}$ & THF & $\mathrm{Zn}$ & 100 & 79 & 5 \\
\hline 6 & $\mathrm{Ni}($ dppe $) \mathrm{Cl}_{2}$ & $\mathbf{L 1}$ & LiI & THF & $\mathrm{Zn}$ & 100 & 94 & 1 \\
\hline 7 & $\mathrm{NiI}_{2}$ & $\mathbf{L 1}$ & LiI & THF & $\mathrm{Zn}$ & 100 & 38 & 2 \\
\hline 8 & $\mathrm{NiBr}_{2}$ & $\mathbf{L 1}$ & LiI & THF & $\mathrm{Zn}$ & 100 & 59 & 5 \\
\hline 9 & $\mathrm{NiCl}_{2}$ & L1 & LiI & THF & $\mathrm{Zn}$ & 100 & 43 & 3 \\
\hline 10 & $\mathrm{NiF}_{2}$ & $\mathbf{L} 1$ & LiI & THF & $\mathrm{Zn}$ & 100 & 21 & 6 \\
\hline 11 & $\mathrm{Ni}(\text { acac })_{2}$ & $\mathbf{L 1}$ & LiI & THF & $\mathrm{Zn}$ & 100 & 51 & 6 \\
\hline 12 & $\mathrm{Ni}(\operatorname{cod})_{2}$ & L1 & LiI & THF & $\mathrm{Zn}$ & 100 & 25 & 7 \\
\hline 13 & $\mathrm{Ni}(\mathrm{OTf})_{2}$ & $\mathbf{L 1}$ & LiI & THF & $\mathrm{Zn}$ & 100 & 67 & 7 \\
\hline 14 & $\mathrm{Ni}(\mathrm{OAc})_{2}$ & $\mathbf{L 1}$ & LiI & THF & $\mathrm{Zn}$ & 100 & 62 & 4 \\
\hline 15 & $\mathrm{Ni}($ dppe $) \mathrm{Cl}_{2}$ & $\mathbf{L 2}$ & LiI & THF & $\mathrm{Zn}$ & 100 & 51 & 8 \\
\hline 16 & $\mathrm{Ni}($ dppe $) \mathrm{Cl}_{2}$ & $\mathbf{L 3}$ & LiI & THF & $\mathrm{Zn}$ & 100 & 67 & 5 \\
\hline 17 & $\mathrm{Ni}($ dppe $) \mathrm{Cl}_{2}$ & L4 & LiI & THF & $\mathrm{Zn}$ & 100 & 62 & 2 \\
\hline 18 & $\mathrm{Ni}($ dppe $) \mathrm{Cl}_{2}$ & $\mathbf{L 5}$ & LiI & THF & $\mathrm{Zn}$ & 100 & 50 & 4 \\
\hline 19 & $\mathrm{Ni}($ dppe $) \mathrm{Cl}_{2}$ & L6 & LiI & THF & $\mathrm{Zn}$ & 100 & 34 & 3 \\
\hline 20 & $\mathrm{Ni}($ dppe $) \mathrm{Cl}_{2}$ & L7 & LiI & THF & $\mathrm{Zn}$ & 100 & 67 & 1 \\
\hline 21 & $\mathrm{Ni}($ dppe $) \mathrm{Cl}_{2}$ & L8 & LiI & THF & $\mathrm{Zn}$ & 100 & 65 & 2 \\
\hline 22 & $\mathrm{Ni}($ dppe $) \mathrm{Cl}_{2}$ & L1 & LiI & THF & $\mathrm{Mn}$ & 100 & 79 & 3 \\
\hline 23 & $\mathrm{Ni}($ dppe $) \mathrm{Cl}_{2}$ & L1 & LiI & THF & $\mathrm{Zn}$ & 120 & 92 & 3 \\
\hline 24 & $\mathrm{Ni}($ dppe $) \mathrm{Cl}_{2}$ & L1 & LiI & $\mathrm{THF}$ & $\mathrm{Zn}$ & 140 & 85 & 5 \\
\hline 25 & $\mathrm{Ni}($ dppe $) \mathrm{Cl}_{2}$ & L1 & LiI & Dioxane & $\mathrm{Zn}$ & 100 & 47 & 3 \\
\hline
\end{tabular}




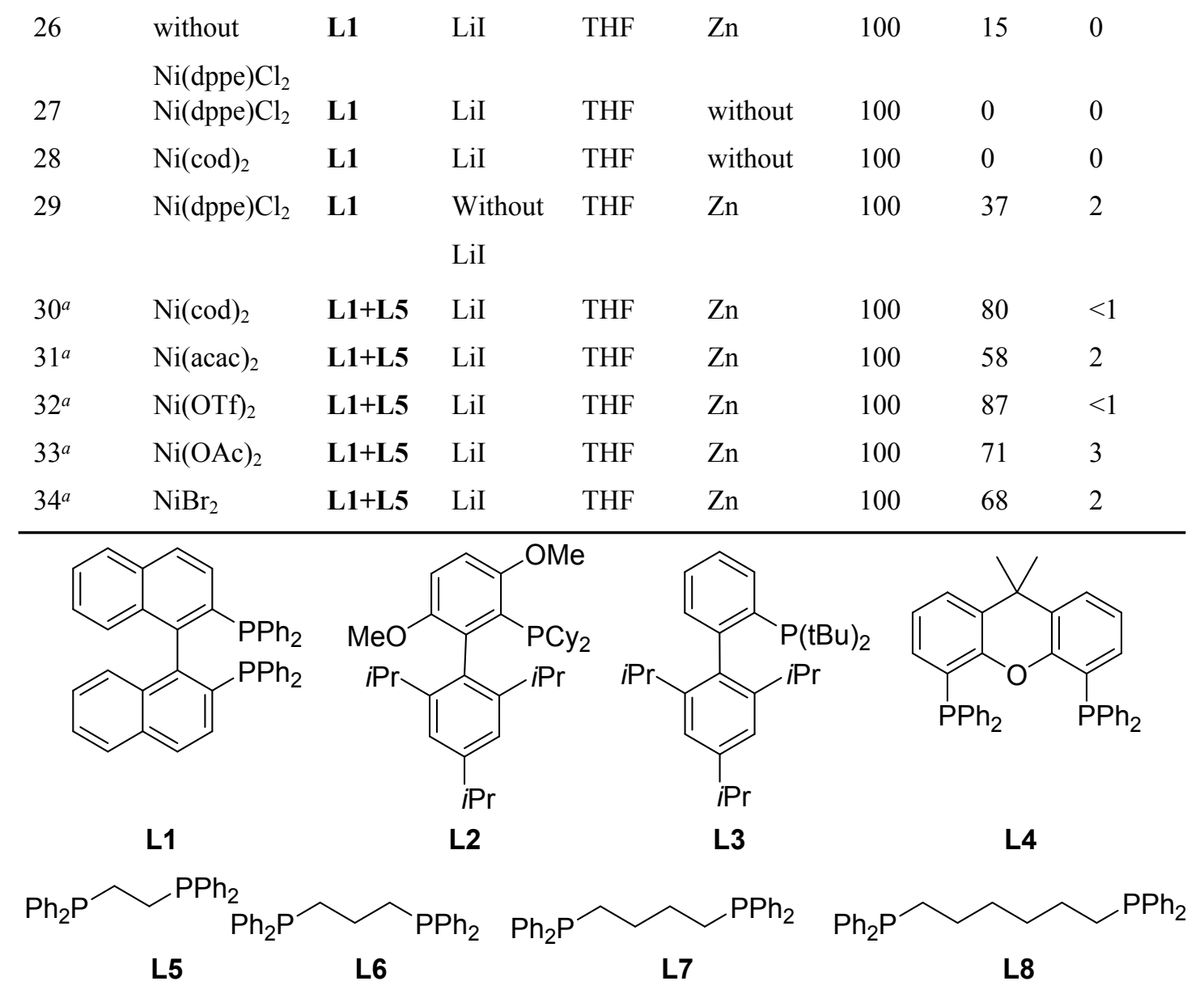

Reaction conditions: 1a $(0.1 \mathrm{mmol}), \mathbf{2 a}(0.1 \mathrm{mmol})$, with or without catalyst $(0.005 \mathrm{mmol}, 5.0 \mathrm{~mol} \%)$, ligand $(0.005 \mathrm{mmol}, 5.0 \mathrm{~mol} \%)$, additive $(0.2 \mathrm{mmol}, 2.0$ equiv), with or without reductant $(0.2 \mathrm{mmol}$, 2.0 equiv), solvent (1.0 mL), at $100-140{ }^{\circ} \mathrm{C}$ for $12 \mathrm{~h} .{ }^{a} \mathbf{L 1}(5.0 \mathrm{~mol} \%), \mathbf{L 5}(5.0 \mathrm{~mol} \%) .{ }^{b}$ Yields were determined by ${ }^{1} \mathrm{H}$ NMR analysis using mesitylene as an internal standard.

Procedure: Under a nitrogen atmosphere, a $10 \mathrm{~mL}$ Schlenk tube equipped with a magnetic stir bar was charged successively with $\mathbf{1 a}(42.7 \mathrm{mg}, 0.10 \mathrm{mmol}), 1$-iodo-4-methylbenzene $\mathbf{2 a}(21.8 \mathrm{mg}, 0.1 \mathrm{mmol})$, catalyst (0.005 mmol, $5.0 \mathrm{~mol} \%)$, ligand $(0.05 \mathrm{mmol}, 5.0 \mathrm{~mol} \%)$, additive ( $0.2 \mathrm{mmol}, 2.0$ equiv), with or without reductant $(0.2 \mathrm{mmol}, 2.0$ equiv), $1.0 \mathrm{~mL}$ of THF and $12 \mathrm{mg}$ mesitylene. The reaction mixture was stirred at $100-140^{\circ} \mathrm{C}$ for $12 \mathrm{~h}$. After the reaction was completed, the resulting mixture was diluted with $1.0 \mathrm{~mL}$ of $\mathrm{CH}_{2} \mathrm{Cl}_{2}$, and filtrated. The residue was washed with $1 \mathrm{~N} \mathrm{HCl}$ solution $(2.0 \mathrm{~mL} \times 3)$ and washed with $2.0 \mathrm{~mL}$ of $\mathrm{H}_{2} \mathrm{O}$. The organic layer was dried over anhydrous $\mathrm{Na}_{2} \mathrm{SO}_{4}$ and concentrated under reduced pressure. The crude product was dissolved in $0.6 \mathrm{~mL}$ of $\mathrm{CDCl}_{3}$, and analyzed by ${ }^{1} \mathrm{H} \mathrm{NMR}$ spectrum using as an internal standard. 


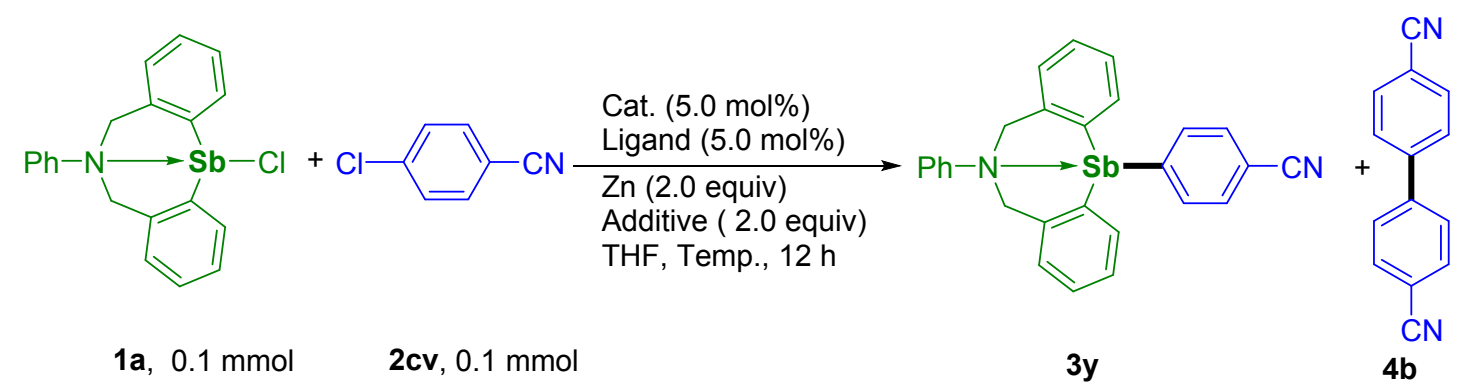

\begin{tabular}{|c|c|c|c|c|c|c|}
\hline \multirow{2}{*}{ Entry } & \multirow{2}{*}[\mathrm{Ni}]{} & \multirow{2}{*}{ Ligand } & \multirow{2}{*}{ Additive } & \multirow{2}{*}{ Temp. $\left({ }^{\circ} \mathrm{C}\right)$} & \multicolumn{2}{|c|}{ Yield (\%) } \\
\hline & & & & & $3 y$ & $4 b$ \\
\hline 1 & $\mathrm{Ni}(\operatorname{cod})_{2}$ & dppe & $\mathrm{LiCl}$ & 120 & 84 & 0 \\
\hline 2 & $\mathrm{Ni}(\operatorname{cod})_{2}$ & dppp & $\mathrm{LiCl}$ & 120 & 67 & 0 \\
\hline 3 & $\mathrm{Ni}(\operatorname{cod})_{2}$ & $\mathrm{dppb}$ & $\mathrm{LiCl}$ & 120 & 59 & 0 \\
\hline 4 & $\mathrm{Ni}($ dppe $) \mathrm{Cl}_{2}$ & BINAP & $\mathrm{LiCl}$ & 120 & 24 & 0 \\
\hline 5 & $\mathrm{Ni}(\mathrm{dppp}) \mathrm{Cl}_{2}$ & BINAP & $\mathrm{LiCl}$ & 120 & 19 & 0 \\
\hline 6 & $\mathrm{NiCl}_{2}$ & BINAP & LiI & 120 & 23 & 0 \\
\hline 7 & $\mathrm{Ni}(\operatorname{cod})_{2}$ & BINAP & $\mathrm{LiCl}$ & 120 & 39 & 0 \\
\hline 8 & $\mathrm{Ni}(\operatorname{cod})_{2}$ & dppe & $\mathrm{LiCl}$ & 140 & 74 & 0 \\
\hline 9 & $\mathrm{Ni}(\operatorname{cod})_{2}$ & dppe & $\mathrm{LiCl}$ & 100 & 58 & 0 \\
\hline
\end{tabular}

Reaction conditions: 1a (0.1 mmol), 4-chlorobenzonitrile 2cv (0.1 mmol), [Ni] (0.005 mmol, $5.0 \mathrm{~mol} \%)$, ligand (0.005 mmol, $5.0 \mathrm{~mol} \%$ ), additive ( $0.2 \mathrm{mmol}, 2.0$ equiv), $\mathrm{Zn}(0.2 \mathrm{mmol}, 2.0$ equiv), THF (1.0 $\mathrm{mL}$ ), at $100-140{ }^{\circ} \mathrm{C}$ for $12 \mathrm{~h} .{ }^{a}$ Yields were determined by ${ }^{1} \mathrm{H}$ NMR using mesitylene as an internal standard.

Procedure: Under a nitrogen atmosphere, a $10 \mathrm{~mL}$ Schlenk tube equipped with a magnetic stir bar was charged successively with $\mathbf{1 a}(42.7 \mathrm{mg}, 0.10 \mathrm{mmol})$, 4-chlorobenzonitrile $\mathbf{2 c v}(13.7 \mathrm{mg}, 0.1 \mathrm{mmol})$, [Ni] (0.005 mmol, $5.0 \mathrm{~mol} \%$ ), ligand (0.05 mmol, $5.0 \mathrm{~mol} \%$ ), additive ( $0.2 \mathrm{mmol}, 2.0$ equiv), $\mathrm{Zn}(13 \mathrm{mg}, 0.2$ mmol, 2.0 equiv), $1.0 \mathrm{~mL}$ of THF and $12 \mathrm{mg}$ mesitylene. The reaction mixture was stirred at $100-140{ }^{\circ} \mathrm{C}$ for $12 \mathrm{~h}$. After the reaction was completed, the resulting mixture was diluted with $1.0 \mathrm{~mL}$ of $\mathrm{CH}_{2} \mathrm{Cl}_{2}$, and filtrated. The residue was washed with $1 \mathrm{~N} \mathrm{HCl}$ solution $(2.0 \mathrm{~mL} \times 3)$ and washed with $2.0 \mathrm{~mL}$ of $\mathrm{H}_{2} \mathrm{O}$. The organic layer was dried over anhydrous $\mathrm{Na}_{2} \mathrm{SO}_{4}$ and concentrated under reduced pressure. The crude product was dissolved in $0.6 \mathrm{~mL}$ of $\mathrm{CDCl}_{3}$, and analyzed by ${ }^{1} \mathrm{H} \mathrm{NMR}$ spectrum using as an internal standard. 


\section{Halides}

Table S3. Exploring the Effect of Palladium Catalysts on Cross-Coupling of Stibine 3a with Aryl Iodide 2b<smiles>Cc1ccc(S2(c3ccccc3)Cc3ccccc3CN(c3ccccc3)C2)cc1</smiles>

3a, $0.11 \mathrm{mmol}$

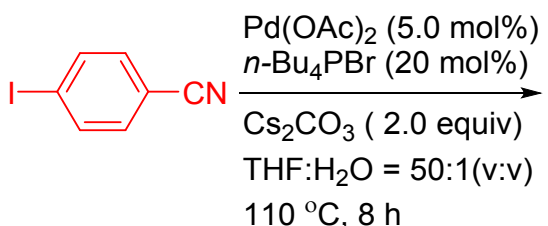

$110^{\circ} \mathrm{C}, 8 \mathrm{~h}$

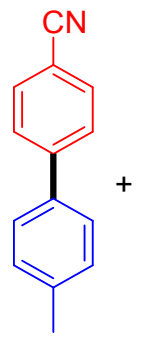

$5 a$

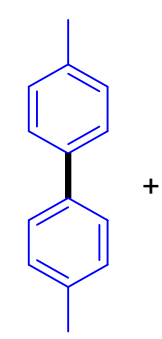

$4 a$<smiles>N#Cc1ccc(-c2ccc(C#N)cc2)cc1</smiles>

4b

\begin{tabular}{|c|c|c|c|c|}
\hline \multirow{2}{*}{ Entry } & \multirow{2}{*}[\mathrm{Pd}]{$(10 \mathrm{~mol} \%)$} & \multicolumn{3}{|c|}{ Yield $(\%)^{a}$} \\
\hline & & $5 a$ & $4 a$ & $4 \mathrm{~b}$ \\
\hline 1 & without [Pd] & 0 & 0 & 0 \\
\hline 2 & $\mathrm{Pd}(\mathrm{OAc})_{2}$ & 94 & 5 & 3 \\
\hline 3 & $\mathrm{Pd}\left(\mathrm{COOCF}_{3}\right)_{2}$ & 70 & 3 & 11 \\
\hline 4 & $\mathrm{Pd}\left(\mathrm{PPh}_{3}\right)_{2} \mathrm{Cl}_{2}$ & 7 & 2 & 25 \\
\hline 5 & $\mathrm{Pd}\left(\mathrm{PCy}_{3}\right)_{2} \mathrm{Cl}_{2}$ & 4 & 3 & 17 \\
\hline 6 & $\operatorname{Pd}(\text { acac })_{2}$ & 77 & 4 & 9 \\
\hline 7 & $\mathrm{PdCl}_{2}$ & 69 & 2 & 12 \\
\hline 8 & $\mathrm{Pd}\left(\mathrm{CH}_{3} \mathrm{CN}\right)_{2} \mathrm{Cl}_{2}$ & 75 & 3 & 10 \\
\hline 9 & $\mathrm{Bn}\left(\mathrm{PPh}_{3}\right)_{2} \mathrm{PdCl}_{2}$ & 41 & 4 & 26 \\
\hline 10 & $\mathrm{Pd}(\mathrm{dba})_{2}$ & 21 & 0 & 19 \\
\hline 11 & $\mathrm{Pd}_{2}(\mathrm{dba})_{3}$ & 26 & 0 & 21 \\
\hline 12 & $\operatorname{Pd}\left(t \mathrm{Bu}_{3}\right)_{2}$ & 2 & 0 & 9 \\
\hline 13 & $\mathrm{Pd}\left(\mathrm{PPh}_{3}\right)_{4}$ & 28 & 0 & 18 \\
\hline 14 & $\mathrm{Pd}\left(\mathrm{PPh}_{3}\right)_{2} \mathrm{Cl}_{2}$, without $\mathrm{Cs}_{2} \mathrm{CO}_{3}, n-\mathrm{Bu}_{4} \mathrm{PBr}$ & 0 & 2 & 3 \\
\hline
\end{tabular}

Reaction conditions: 3a $(0.11 \mathrm{mmol}), \mathbf{2 b}(0.10 \mathrm{mmol}),[\mathrm{Pd}](0.005 \mathrm{mmol}, 5.0 \mathrm{~mol} \%), n-\mathrm{Bu}_{4} \mathrm{PBr}(0.02$ mmol, $20 \mathrm{~mol} \%), \mathrm{Cs}_{2} \mathrm{CO}_{3}\left(0.2 \mathrm{mmol}, 2.0\right.$ equiv), $\mathrm{H}_{2} \mathrm{O}(20 \mu \mathrm{L})$, THF $(1.0 \mathrm{~mL})$, at $110{ }^{\circ} \mathrm{C}$ for $8 \mathrm{~h} .{ }^{a}$ Yields were determined by GC analysis using $n$-dodecane as an internal standard.

Procedure: Under a nitrogen atmosphere, a $10 \mathrm{~mL}$ Schlenk tube equipped with a magnetic stir bar was charged successively with $\mathbf{3 a}(53.1 \mathrm{mg}, 0.11 \mathrm{mmol})$, 4-iodobenzonitrile $\mathbf{2 b}(22.9 \mathrm{mg}, 0.1 \mathrm{mmol}),[\mathrm{Pd}]$ (0.005 mmol, $5.0 \mathrm{~mol} \%$ ), $n$-Bu $\mathrm{BBr}_{4} \mathrm{Pr}\left(6.8 \mathrm{mg}, 0.02 \mathrm{mmol}, 20 \mathrm{~mol} \%\right.$ ), $\mathrm{Cs}_{2} \mathrm{CO}_{3}$ (65 mg, $0.2 \mathrm{mmol}, 2.0$ equiv), $1.0 \mathrm{~mL}$ of THF, $20 \mu \mathrm{L}$ of $\mathrm{H}_{2} \mathrm{O}$ and $30 \mu \mathrm{L}$ of $n$-dodecane. The reaction mixture was stirred at 110 ${ }^{\circ} \mathrm{C}$ for $8 \mathrm{~h}$. Yields of products were determined by GC analysis using $n$-dodecane as an internal standard. 


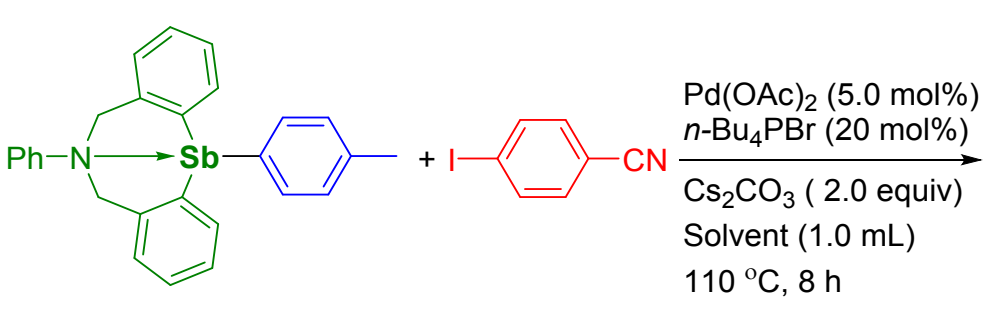

3a, $0.11 \mathrm{mmol}$

2b, $0.1 \mathrm{mmol}$

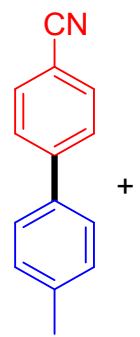

$5 a$

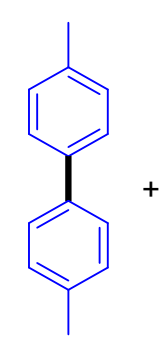

4a<smiles>N#Cc1ccc(-c2ccc(C#N)cc2)cc1</smiles>

4b

\begin{tabular}{lllll}
\hline Entry & Solvent & \multicolumn{3}{c}{ Yield (\%) $^{a}$} \\
\cline { 3 - 5 } & & $\mathbf{5 a}$ & $\mathbf{4 a}$ & $\mathbf{4 b}$ \\
\hline 1 & $\mathrm{ClCH}_{2} \mathrm{CH}_{2} \mathrm{Cl}$ & 25 & 3 & 5 \\
2 & $\mathrm{CH}_{2} \mathrm{Cl}_{2}$ & 26 & $<1$ & 6 \\
3 & $\mathrm{DMSO}$ & 0 & 2 & 2 \\
4 & $\mathrm{H}_{2} \mathrm{O}$ & 2 & 2 & 5 \\
5 & $\mathrm{THF}$ & 32 & 10 & 8 \\
6 & Toluene & 45 & 8 & 6 \\
7 & tert-butyl methyl ether & 8 & 11 & 11 \\
8 & Dioxane & 26 & 9
\end{tabular}

Reaction conditions: 3a $(0.11 \mathrm{mmol}), \mathbf{2 b}(0.10 \mathrm{mmol}), \mathrm{Pd}(\mathrm{OAc})_{2}(0.005 \mathrm{mmol}, 5.0 \mathrm{~mol} \%), n-\mathrm{Bu}_{4} \mathrm{PBr}$ (0.02 mmol, $20 \mathrm{~mol} \%), \mathrm{Cs}_{2} \mathrm{CO}_{3}\left(0.2 \mathrm{mmol}, 2.0\right.$ equiv), solvent $(1.0 \mathrm{~mL})$, at $110^{\circ} \mathrm{C}$ for $8 \mathrm{~h} .{ }^{a}$ Yields were determined by GC analysis $n$-dodecane as an internal standard.

Procedure: Under a nitrogen atmosphere, a $10 \mathrm{~mL}$ Schlenk tube equipped with a magnetic stir bar was charged successively with $\mathbf{3 a}(53.1 \mathrm{mg}, 0.11 \mathrm{mmol})$, 4-iodobenzonitrile $\mathbf{2 b}(22.9 \mathrm{mg}, 0.1 \mathrm{mmol})$, $\mathrm{Pd}(\mathrm{OAc})_{2}(1.1 \mathrm{mg}, 0.005 \mathrm{mmol}, 5.0 \mathrm{~mol} \%), n-\mathrm{Bu}_{4} \mathrm{PBr}(6.8 \mathrm{mg}, 0.02 \mathrm{mmol}, 20 \mathrm{~mol} \%), \mathrm{Cs}_{2} \mathrm{CO}_{3}(65 \mathrm{mg}$, $0.2 \mathrm{mmol}, 2.0$ equiv), $1.0 \mathrm{~mL}$ of solvent and $30 \mu \mathrm{L}$ of $n$-dodecane. The reaction mixture was stirred at $110{ }^{\circ} \mathrm{C}$ for $8 \mathrm{~h}$. Yields of products were determined by GC analysis using $n$-dodecane as an internal standard 


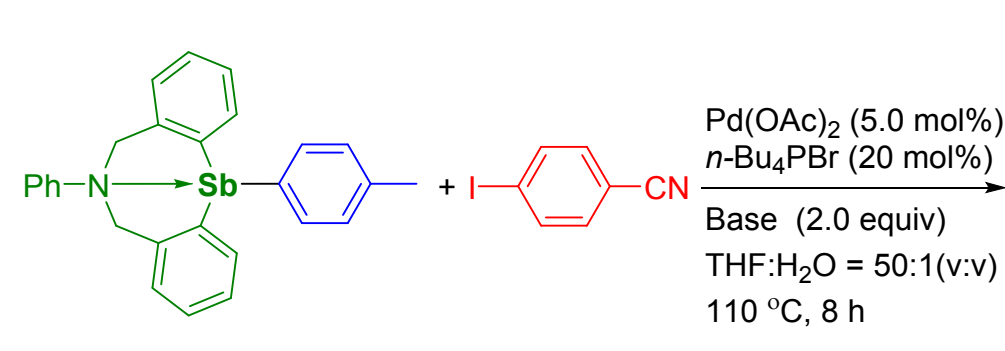

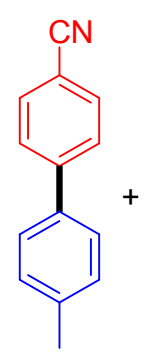

$5 a$

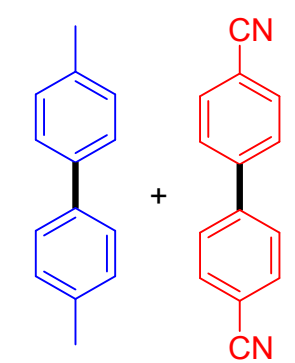

4a

4b

\begin{tabular}{lllll}
\hline \multirow{2}{*}{ Entry } & \multirow{2}{*}{ Base (2.0 equiv) } & \multicolumn{3}{c}{ Yield (\%) $^{a}$} \\
\cline { 3 - 5 } & & $\mathbf{5 a}$ & $\mathbf{4 a}$ & $\mathbf{4 b}$ \\
\hline 1 & $\mathrm{Cs}_{2} \mathrm{CO}_{3}$ & 72 & 7 & 3 \\
2 & $\mathrm{CsF}$ & 8 & 13 \\
3 & $t \mathrm{BuOK}$ & 25 & 12 & 32 \\
4 & $t \mathrm{BuONa}$ & 59 & 13 & 15 \\
5 & $\mathrm{Na}_{2} \mathrm{CO}_{3}$ & 67 & 10 & 10 \\
6 & $\mathrm{NaHCO}_{3}$ & 42 & 8 & 6 \\
7 & $\mathrm{~K}_{2} \mathrm{CO}_{3}$ & 31 & 14 & 21 \\
8 & $\mathrm{CH}_{3} \mathrm{COOK}_{9}$ & 62 & 10 & 19 \\
9 & $\mathrm{CH}_{3} \mathrm{ONa}$ & 54 & $<1$ & 13 \\
10 & without $\mathrm{Cs}_{2} \mathrm{CO}_{3}$ & 12 & $<1$ \\
\hline
\end{tabular}

Reaction conditions: 3a $(0.11 \mathrm{mmol}), \mathbf{2 b}(0.10 \mathrm{mmol}), \mathrm{Pd}(\mathrm{OAc})_{2}(0.005 \mathrm{mmol}, 5.0 \mathrm{~mol} \%), n-\mathrm{Bu} 4 \mathrm{PBr}$ (0.02 mmol, $20 \mathrm{~mol} \%), \mathrm{Cs}_{2} \mathrm{CO}_{3}\left(0.2 \mathrm{mmol}, 2.0\right.$ equiv), THF $(1.0 \mathrm{~mL}), \mathrm{H}_{2} \mathrm{O}(20 \mu \mathrm{L})$, at $110{ }^{\circ} \mathrm{C}$ for $8 \mathrm{~h}$. ${ }^{a}$ Yields were determined by GC analysis using $n$-dodecane as an internal standard.

Procedure: Under a nitrogen atmosphere, a $10 \mathrm{~mL}$ Schlenk tube equipped with a magnetic stir bar was charged successively with $\mathbf{3 a}(53.1 \mathrm{mg}, 0.11 \mathrm{mmol})$, 4-iodobenzonitrile $\mathbf{2 b}(22.9 \mathrm{mg}, 0.1 \mathrm{mmol})$, $\mathrm{Pd}(\mathrm{OAc})_{2}(1.1 \mathrm{mg}, 0.005 \mathrm{mmol}, 5.0 \mathrm{~mol} \%), n-\mathrm{Bu}_{4} \mathrm{PBr}(6.8 \mathrm{mg}, 0.02 \mathrm{mmol}, 20 \mathrm{~mol} \%)$, base (0.2 mmol, 2.0 equiv), $1.0 \mathrm{~mL}$ of THF, $20 \mu \mathrm{L}$ of $\mathrm{H}_{2} \mathrm{O}$ and $30 \mu \mathrm{L}$ of $n$-dodecane. The reaction mixture was stirred at $110{ }^{\circ} \mathrm{C}$ for $8 \mathrm{~h}$. The resulting mixture was diluted with $\mathrm{CH}_{2} \mathrm{Cl}_{2}$. Yields of products were determined by GC analysis using $n$-dodecane as an internal standard. 
<smiles>Cc1ccc([Sb]2(c3ccccc3)CN3Cc4ccccc4C2(c2ccccc2)c2ccccc23)cc1</smiles>

3a, $0.11 \mathrm{mmol}$

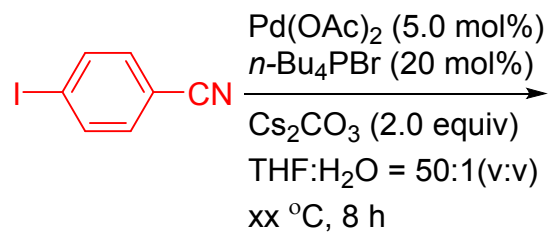

2b, $0.1 \mathrm{mmol}$

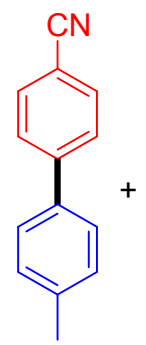

$5 a$

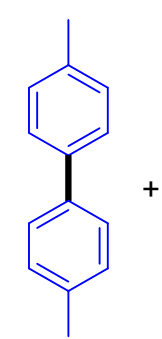

$4 a$<smiles>N#Cc1ccc(-c2ccc(C#N)cc2)cc1</smiles>

4b

\begin{tabular}{lllll}
\hline \multirow{2}{*}{ Entry } & \multirow{2}{*}{ Temp. $\left({ }^{\circ} \mathrm{C}\right)$} & \multicolumn{3}{c}{${\text { Yield }(\%)^{a}}^{a}$} \\
\cline { 3 - 5 } & & $\mathbf{5 a}$ & $\mathbf{4 a}$ & $\mathbf{4 b}$ \\
\hline 1 & 20 & 3 & 1 & 0 \\
2 & 40 & 12 & 2 & 0 \\
3 & 60 & 23 & 3 & 2 \\
4 & 80 & 37 & 1 & 4 \\
5 & 100 & 84 & 4 & 3 \\
6 & 110 & 94 & 5 & 3 \\
7 & 120 & 85 & 9 & 7 \\
8 & 130 & 80 & 10 & 8 \\
9 & 140 & 76 & 16 & 11 \\
\hline
\end{tabular}

Reaction conditions: 3a $(0.11 \mathrm{mmol}), \mathbf{2 b}(0.10 \mathrm{mmol}), \mathrm{Pd}(\mathrm{OAc})_{2}(0.005 \mathrm{mmol}, 5.0 \mathrm{~mol} \%), n-\mathrm{Bu}_{4} \mathrm{PBr}$ (0.02 mmol, $20 \mathrm{~mol} \%), \mathrm{Cs}_{2} \mathrm{CO}_{3}\left(0.2 \mathrm{mmol}, 2.0\right.$ equiv), THF $(1.0 \mathrm{~mL}), \mathrm{H}_{2} \mathrm{O}(20 \mu \mathrm{L})$, at $20-140{ }^{\circ} \mathrm{C}$ for 8 h. ${ }^{a}$ Yields were determined by GC analysis using $n$-dodecane as an internal standard.

Procedure: Under a nitrogen atmosphere, a $10 \mathrm{~mL}$ Schlenk tube equipped with a magnetic stir bar was charged successively with $\mathbf{3 a}(53.1 \mathrm{mg}, 0.11 \mathrm{mmol})$, 4-iodobenzonitrile $\mathbf{2 b}(22.9 \mathrm{mg}, 0.1 \mathrm{mmol})$, $\mathrm{Pd}(\mathrm{OAc})_{2}(1.1 \mathrm{mg}, 0.005 \mathrm{mmol}, 5.0 \mathrm{~mol} \%), n-\mathrm{Bu} 4 \mathrm{PBr}(6.8 \mathrm{mg}, 0.02 \mathrm{mmol}, 20 \mathrm{~mol} \%), \mathrm{Cs}_{2} \mathrm{CO}_{3}(65 \mathrm{mg}$, $0.2 \mathrm{mmol}, 2.0$ equiv), $1.0 \mathrm{~mL}$ of THF, $20 \mu \mathrm{L}$ of $\mathrm{H}_{2} \mathrm{O}$ and $30 \mu \mathrm{L}$ of $n$-dodecane. The reaction mixture was stirred at $20-140{ }^{\circ} \mathrm{C}$ for $8 \mathrm{~h}$. Yields of products were determined by GC analysis using $n$-dodecane as an internal standard. 
Table S7. Exploring the Effect of the Amount of Water on Cross-Coupling of Stibine 3a with Aryl Iodide 2b<smiles>Cc1ccc(S(=O)(=O)c2ccccc2CN2Cc3ccccc3S2)cc1</smiles>

3a, $0.11 \mathrm{mmol}$

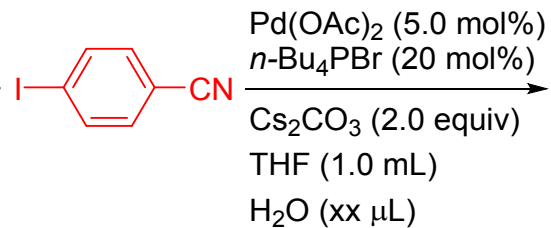

$110^{\circ} \mathrm{C}, 8 \mathrm{~h}$

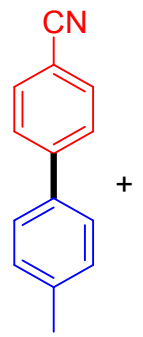

$5 a$

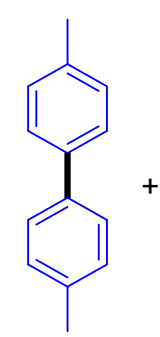

$4 a$<smiles>N#Cc1ccc(-c2ccc(C#N)cc2)cc1</smiles>

4b

\begin{tabular}{lllll}
\hline \multirow{2}{*}{ Entry } & \multirow{2}{*}{$\mathrm{H}_{2} \mathrm{O}(\mu \mathrm{L})$} & $\mathbf{5 a}$ & $\mathbf{4 a}$ & $\mathbf{4}$ Yield $(\%)^{a}$ \\
\cline { 3 - 5 } & & 32 & 2 & 8 \\
2 & 0 & 34 & 2 & 15 \\
3 & 5 & 59 & 3 & 12 \\
4 & 10 & 77 & 2 & 13 \\
5 & 15 & 94 & 5 & 3 \\
6 & 20 & 86 & 2 & 3 \\
7 & 30 & 84 & 2 & 4 \\
8 & 40 & 73 & 3 & 12 \\
9 & 50 & 70 & 4 & 11 \\
10 & 100 & 69 & 4 & 9 \\
11 & 200 & 60 & 4 & 12 \\
12 & 250 & 55 & 4 & 12 \\
13 & 500 & 47 & 5 & 13 \\
\hline
\end{tabular}

Reaction conditions: 3a $(0.11 \mathrm{mmol}), \mathbf{2 b}(0.10 \mathrm{mmol}), \mathrm{Pd}(\mathrm{OAc})_{2}(0.005 \mathrm{mmol}, 5.0 \mathrm{~mol} \%), n-\mathrm{Bu} 4 \mathrm{PBr}$ (0.02 mmol, $20 \mathrm{~mol} \%), \mathrm{Cs}_{2} \mathrm{CO}_{3}\left(0.2 \mathrm{mmol}, 2.0\right.$ equiv), THF $(1.0 \mathrm{~mL})$, without or with $\mathrm{H}_{2} \mathrm{O}(5-500 \mu \mathrm{L})$ at $110{ }^{\circ} \mathrm{C}$ for $8 \mathrm{~h} .{ }^{a}$ Yields were determined by GC analysis using $n$-dodecane as an internal standard.

Procedure: Under a nitrogen atmosphere, a $10 \mathrm{~mL}$ Schlenk tube equipped with a magnetic stir bar was charged successively with 3a $(53.1 \mathrm{mg}, 0.11 \mathrm{mmol})$, 4-iodobenzonitrile $\mathbf{2 b}(22.9 \mathrm{mg}, 0.1 \mathrm{mmol})$, $\mathrm{Pd}(\mathrm{OAc})_{2}(1.1 \mathrm{mg}, 0.005 \mathrm{mmol}, 5.0 \mathrm{~mol} \%), n-\mathrm{Bu} 4 \mathrm{PBr}(6.8 \mathrm{mg}, 0.02 \mathrm{mmol}, 20 \mathrm{~mol} \%), \mathrm{Cs}_{2} \mathrm{CO}_{3}(65 \mathrm{mg}$, $0.2 \mathrm{mmol}, 2.0$ equiv), $1.0 \mathrm{~mL}$ of THF, $5-500 \mu \mathrm{L}$ of $\mathrm{H}_{2} \mathrm{O}$ and $30 \mu \mathrm{L}$ of $n$-dodecane. The reaction mixture was stirred at $110^{\circ} \mathrm{C}$ for $8 \mathrm{~h}$. Yields of products were determined by GC analysis using $n$-dodecane as an internal standard. Experimental results in Table S7 showed that this cross-coupling process could be accelerated by the suitable amount of water, which may be due to the increase of the solubility of $\mathrm{Cs}_{2} \mathrm{CO}_{3}$ in the system in the presence of water. 
Table S8. Exploring the Effect of the Amount of $\boldsymbol{n}$-Bu ${ }_{4} \mathrm{PBr}$ on Cross-Coupling of Stibine 3a with Aryl Iodide 2b

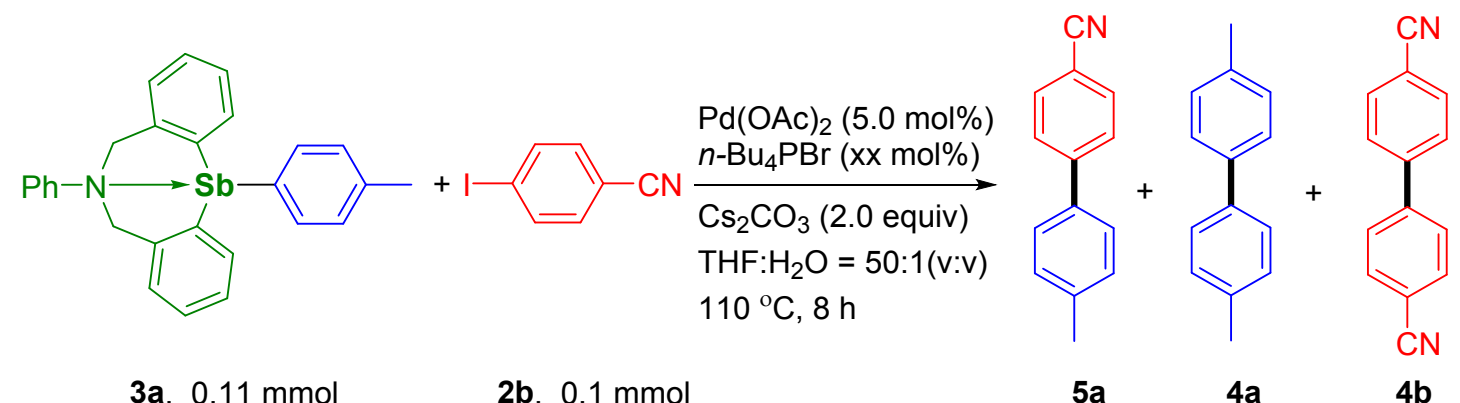

\begin{tabular}{lllll}
\hline \multirow{2}{*}{ Entry } & $n-\mathrm{Bu}_{4} \mathrm{PBr}(\mathrm{mol} \%)$ & \multicolumn{3}{c}{${\text { Yield }(\%)^{a}}^{a}$} \\
\cline { 3 - 5 } & & $\mathbf{5 a}$ & $\mathbf{4 a}$ & $\mathbf{4 b}$ \\
\hline 1 & 0 & 19 & 2 & 10 \\
2 & 1 & 33 & 11 & 16 \\
3 & 5 & 43 & 10 & 13 \\
4 & 10 & 52 & 10 & 19 \\
5 & 15 & 67 & 11 & 21 \\
6 & 20 & 94 & 5 & 3 \\
7 & 40 & 83 & 3 & 7 \\
8 & 60 & 81 & 3 & 6 \\
9 & 80 & 73 & 4 & 9 \\
10 & 100 & 58 & 4 & 11 \\
11 & 200 & 51 & 4 & 12 \\
\hline
\end{tabular}

Reaction conditions: $\mathbf{3 a}(0.11 \mathrm{mmol}), \mathbf{2 b}(0.10 \mathrm{mmol}), \mathrm{Pd}(\mathrm{OAc})_{2}(0.005 \mathrm{mmol}, 5.0 \mathrm{~mol} \%)$, without or with $n-\mathrm{Bu}_{4} \mathrm{PBr}(0-0.2 \mathrm{mmol}, 0-200 \mathrm{~mol} \%), \mathrm{Cs}_{2} \mathrm{CO}_{3}$ (0.2 mmol, 2.0 equiv), THF (1.0 mL), $\mathrm{H}_{2} \mathrm{O}$ (20 $\mu \mathrm{L})$ at $110{ }^{\circ} \mathrm{C}$ for 8 h. ${ }^{a}$ Yields were determined by GC analysis using $n$-dodecane as an internal standard.

Procedure: Under a nitrogen atmosphere, a $10 \mathrm{~mL}$ Schlenk tube equipped with a magnetic stir bar was charged successively with $\mathbf{3 a}(53.1 \mathrm{mg}, 0.11 \mathrm{mmol})$, 4-iodobenzonitrile $\mathbf{2 b}(22.9 \mathrm{mg}, 0.1 \mathrm{mmol})$, $\mathrm{Pd}(\mathrm{OAc})_{2}(1.1 \mathrm{mg}, 0.005 \mathrm{mmol}, 5.0 \mathrm{~mol} \%), n-\mathrm{Bu}_{4} \mathrm{PBr}(0-67.8 \mathrm{mg}, 0-0.2 \mathrm{mmol}), \mathrm{Cs}_{2} \mathrm{CO}_{3}(65 \mathrm{mg}, 0.2$ mmol, 2.0 equiv), $1.0 \mathrm{~mL}$ of THF, $20 \mu \mathrm{L}$ of $\mathrm{H}_{2} \mathrm{O}$ and $30 \mu \mathrm{L}$ of $n$-dodecane. The reaction mixture was stirred at $110{ }^{\circ} \mathrm{C}$ for $8 \mathrm{~h}$. Yields of products were determined by GC analysis using $n$-dodecane as an internal standard. 


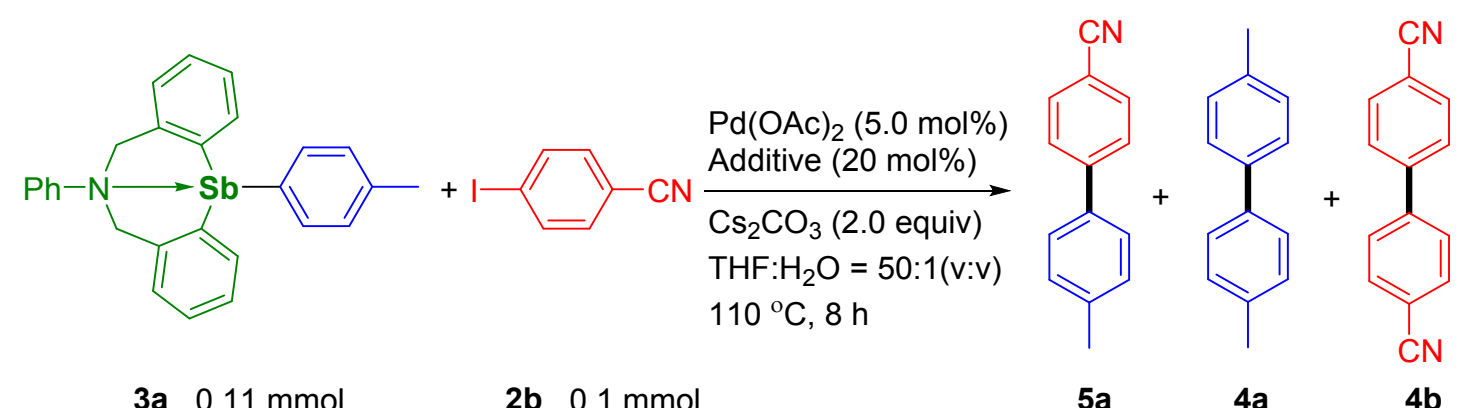

\begin{tabular}{lllll}
\hline \multirow{2}{*}{ Entry } & \multirow{2}{*}{ Additive $(20$ mol\%) } & \multicolumn{3}{c}{${\text { Yield }(\%)^{a}}^{a}$} \\
\cline { 3 - 5 } & & $\mathbf{5 a}$ & 2 & $\mathbf{4 b}$ \\
\hline 1 & without additive & 19 & 3 & 10 \\
2 & $\mathrm{KI}$ & 57 & 3 & 17 \\
3 & $\mathrm{KBr}$ & 67 & 2 & 15 \\
4 & $\mathrm{KCl}$ & 68 & 3 & 13 \\
5 & $\mathrm{NaI}$ & 56 & 3 & 16 \\
6 & $\mathrm{NaBr}$ & 53 & 4 & 7 \\
7 & $\mathrm{NaCl}$ & 50 & 3 & 14 \\
8 & $\mathrm{LiBr}$ & 57 & 4 & 11 \\
9 & $\mathrm{LiCl}$ & 54 & 3 & 13 \\
10 & $n-\mathrm{Bu}{ }_{4} \mathrm{NBr}$ & 81 & 2 & 10 \\
11 & $n-\mathrm{Et}_{4} \mathrm{NBr}$ & 71 & 5 & 11 \\
12 & $n-\mathrm{Bu} 4 \mathrm{PBr}$ & 94 & 3 \\
\hline
\end{tabular}

Reaction conditions: 3a $(0.11 \mathrm{mmol}), \mathbf{2 b}(0.10 \mathrm{mmol}), \mathrm{Pd}(\mathrm{OAc})_{2}(0.005 \mathrm{mmol}, 5.0 \mathrm{~mol} \%)$, without or with an additive ( $0.02 \mathrm{mmol}, 20 \mathrm{~mol} \%), \mathrm{Cs}_{2} \mathrm{CO}_{3}\left(0.20 \mathrm{mmol}, 2.0\right.$ equiv), THF $(1.0 \mathrm{~mL}), \mathrm{H}_{2} \mathrm{O}(20 \mu \mathrm{L})$ at $110^{\circ} \mathrm{C}$ for $8 \mathrm{~h} .{ }^{a}$ Yields were determined by GC analysis uisng $n$-dodecane as an internal standard.

Procedure: Under a nitrogen atmosphere, a $10 \mathrm{~mL}$ Schlenk tube equipped with a magnetic stir bar was charged successively with $\mathbf{3 a}(53.1 \mathrm{mg}, 0.11 \mathrm{mmol})$, 4-iodobenzonitrile $\mathbf{2 b}(22.9 \mathrm{mg}, 0.10 \mathrm{mmol})$, $\mathrm{Pd}(\mathrm{OAc})_{2}(1.1 \mathrm{mg}, 0.005 \mathrm{mmol}, 5.0 \mathrm{~mol} \%)$, without or with an additive $(0.02 \mathrm{mmol}, 20 \mathrm{~mol} \%), \mathrm{Cs}_{2} \mathrm{CO}_{3}$ ( $65 \mathrm{mg}, 0.2 \mathrm{mmol}, 2.0$ equiv), $1.0 \mathrm{~mL}$ of THF, $20 \mu \mathrm{L}$ of $\mathrm{H}_{2} \mathrm{O}$ and 30 of $\mu \mathrm{L}$ of $n$-dodecane. The reaction mixture was stirred at $110^{\circ} \mathrm{C}$ for $8 \mathrm{~h}$. Yields of products were determined by GC analysis using $n$ dodecane as an internal standard.

Table S10. Exploring the Effect of the Ligand on Cross-Coupling of Stibine 3a with Aryl Iodide 2b 

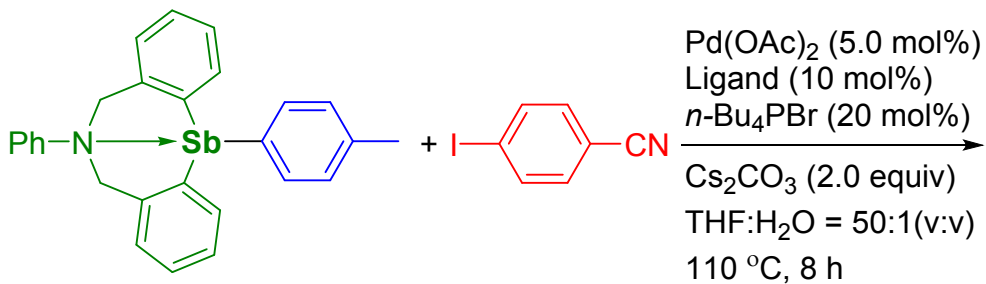

3a, $0.11 \mathrm{mmol}$

2b, $0.1 \mathrm{mmol}$

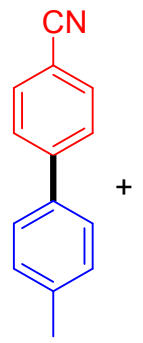

$5 a$

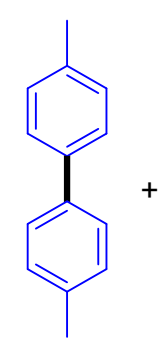

$4 a$<smiles>N#Cc1ccc(-c2ccc(C#N)cc2)cc1</smiles>

4b

\begin{tabular}{|c|c|c|c|c|}
\hline \multirow{2}{*}{ Entry } & \multirow{2}{*}{ Ligand (10 mol\%) } & \multicolumn{3}{|c|}{ Yield $(\%)^{a}$} \\
\hline & & $5 a$ & $4 a$ & $4 \mathbf{b}$ \\
\hline 1 & without ligand & 94 & 5 & 3 \\
\hline 2 & bpy & 10 & 3 & 10 \\
\hline 3 & dtbbpy & 45 & 3 & 15 \\
\hline 4 & $\mathrm{PPh}_{3}$ & 57 & 3 & 11 \\
\hline 5 & $\mathrm{PCy}_{3}$ & 7 & 3 & 2 \\
\hline 6 & ${ }^{t} \mathrm{Bu}_{3} \mathrm{P}$ & 5 & 4 & 2 \\
\hline 7 & $n-\mathrm{Bu}_{3} \mathrm{P}$ & 4 & 4 & 3 \\
\hline 8 & BINAP & 5 & 3 & 6 \\
\hline 9 & Xantphos & 36 & 4 & 9 \\
\hline 10 & Brettphos & 31 & 4 & 8 \\
\hline
\end{tabular}

Reaction conditions: 3a $(0.11 \mathrm{mmol}), \mathbf{2 b}(0.10 \mathrm{mmol}), \mathrm{Pd}(\mathrm{OAc})_{2}(0.005 \mathrm{mmol}, 5.0 \mathrm{~mol} \%), n-\mathrm{Bu}_{4} \mathrm{PBr}$ (0.02 mmol, $20 \mathrm{~mol} \%)$, ligand ( $0.01 \mathrm{mmol}, 10 \mathrm{~mol} \%), \mathrm{Cs}_{2} \mathrm{CO}_{3}(0.2 \mathrm{mmol}, 2.0$ equiv), THF (1.0 mL), $\mathrm{H}_{2} \mathrm{O}(20 \mu \mathrm{L})$ at $110^{\circ} \mathrm{C}$ for $8 \mathrm{~h} .{ }^{a}$ Yields were determined by $\mathrm{GC}$ analysis using $n$-dodecane as an internal standard.

Procedure: Under a nitrogen atmosphere, a $10 \mathrm{~mL}$ Schlenk tube equipped with a magnetic stir bar was charged successively with $\mathbf{3 a}(53.1 \mathrm{mg}, 0.11 \mathrm{mmol})$, 4-iodobenzonitrile $\mathbf{2 b}(22.9 \mathrm{mg}, 0.1 \mathrm{mmol})$, $\mathrm{Pd}(\mathrm{OAc})_{2}(1.1 \mathrm{mg}, 0.005 \mathrm{mmol}, 5.0 \mathrm{~mol} \%), n-\mathrm{Bu}_{4} \mathrm{PBr}(6.8 \mathrm{mg}, 0.02 \mathrm{mmol})$, ligand $(0.01 \mathrm{mmol}, 10$ mol\%), $\mathrm{Cs}_{2} \mathrm{CO}_{3}$ (65 mg, $0.2 \mathrm{mmol}, 2.0$ equiv), $1.0 \mathrm{~mL}$ of THF, $20 \mu \mathrm{L}$ of $\mathrm{H}_{2} \mathrm{O}$ and $30 \mu \mathrm{L}$ of $n$-dodecane. The reaction mixture was stirred at $110^{\circ} \mathrm{C}$ for $8 \mathrm{~h}$. Yields of products were determined by GC analysis using $n$-dodecane as an internal standard.

Table S11. Exploring the Effect of the Amount of $\mathrm{PPh}_{3}$ on Cross-Coupling of Stibine 3a with Aryl Iodide 2b 


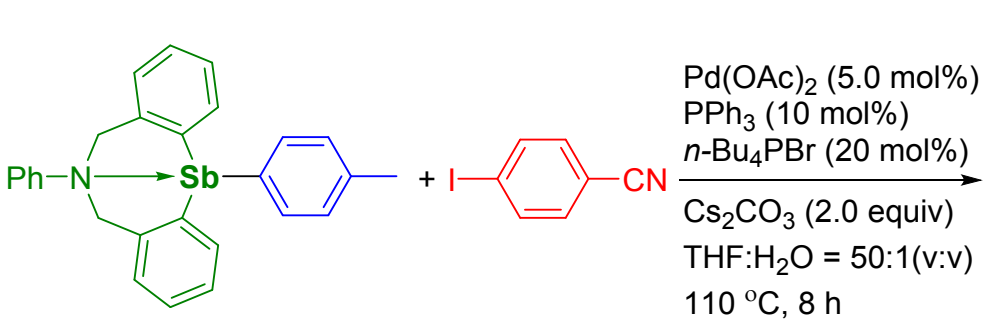

3a, $0.11 \mathrm{mmol}$

2b, $0.1 \mathrm{mmol}$

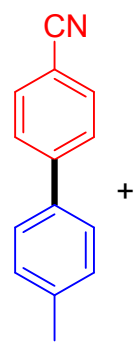

$5 a$

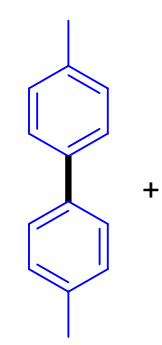

$4 a$<smiles>N#Cc1ccc(-c2ccc(C#N)cc2)cc1</smiles>

4b

\begin{tabular}{llccc}
\hline \multirow{2}{*}{ Entry } & $\mathrm{PPh}_{3}(\mathrm{~mol} \%)$ & $\mathbf{5 a}$ & $\mathbf{4 a}$ & $\mathbf{4 b}$ \\
\cline { 2 - 5 } & & 94 & 5 & 3 \\
\hline 1 & 0 & 65 & 4 & 10 \\
2 & 5 & 57 & 3 & 11 \\
3 & 10 & 55 & 4 & 11 \\
4 & 20 & 37 & 3 & 10 \\
5 & 40 & 21 & 2 & 5 \\
6 & 60 & 17 & 2 & 4 \\
7 & 80 & 10 & 2 & 3 \\
8 & 100 & 5 & 1 & 2 \\
9 & 150 & 2 & 1 & 1 \\
10 & 200 & $<1$ & 1 & $<1$ \\
11 & 400 & 2 & \multicolumn{3}{c}{${ }^{a}$} & \\
\hline
\end{tabular}

Reaction conditions: 3a $(0.11 \mathrm{mmol}), \mathbf{2 b}(0.10 \mathrm{mmol}), \mathrm{Pd}(\mathrm{OAc})_{2}(0.005 \mathrm{mmol}, 5.0 \mathrm{~mol} \%), n-\mathrm{Bu}_{4} \mathrm{PBr}$ (0.02 mmol, 20 mol\%), without or with $\mathrm{PPh}_{3}(0-0.4 \mathrm{mmol}, 0$ - $400 \mathrm{~mol} \%), \mathrm{Cs}_{2} \mathrm{CO}_{3}(0.2 \mathrm{mmol}, 2.0$ equiv), THF (1.0 mL), $\mathrm{H}_{2} \mathrm{O}(20 \mu \mathrm{L})$ at $110{ }^{\circ} \mathrm{C}$ for $8 \mathrm{~h} .{ }^{a}$ Yields were determined by $\mathrm{GC}$ analysis using $n$ dodecane as an internal standard.

Procedure: Under a nitrogen atmosphere, a $10 \mathrm{~mL}$ Schlenk tube equipped with a magnetic stir bar was charged successively with $\mathbf{3 a}(53.1 \mathrm{mg}, 0.11 \mathrm{mmol})$, 4-iodobenzonitrile $\mathbf{2 b}(22.9 \mathrm{mg}, 0.1 \mathrm{mmol})$, $\mathrm{Pd}(\mathrm{OAc})_{2}(1.1 \mathrm{mg}, 0.005 \mathrm{mmol}, 5.0 \mathrm{~mol} \%), n-\mathrm{Bu}_{4} \mathrm{PBr}$ (6.8 mg, $\left.0.02 \mathrm{mmol}\right), \mathrm{PPh}_{3}(0-104.8 \mathrm{mg}, 0-0.4$ mmol), $\mathrm{Cs}_{2} \mathrm{CO}_{3}$ ( $65 \mathrm{mg}, 0.2 \mathrm{mmol}, 2.0$ equiv), $1.0 \mathrm{~mL}$ of THF, $20 \mu \mathrm{L}$ of $\mathrm{H}_{2} \mathrm{O}$ and $30 \mu \mathrm{L}$ of $n$-dodecane. The reaction mixture was stirred at $110^{\circ} \mathrm{C}$ for $8 \mathrm{~h}$. Yields of products were determined by GC analysis using $n$-dodecane as an internal standard.

\section{S2.3. Investigation on the Reactivity of Tri-p-tolylstibane and Stibine 3bf}




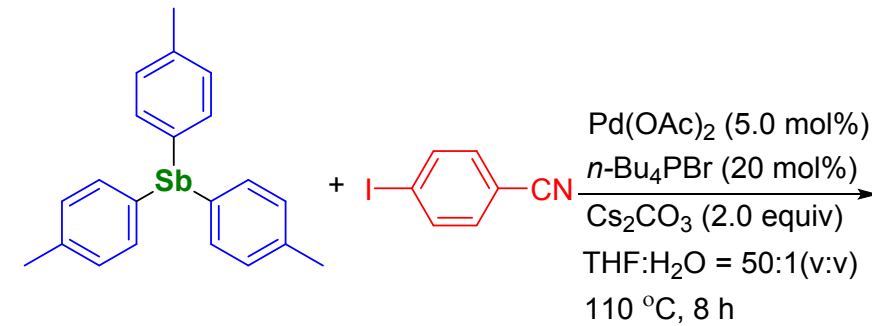

$0.1 \mathrm{mmol}$

2b, $0.3 \mathrm{mmol}$

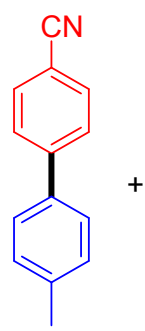

5a, $36 \%$

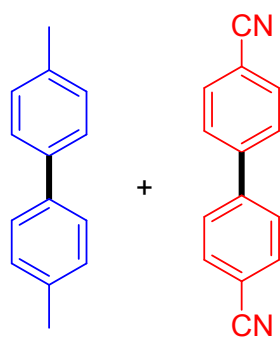

$4 a, 7 \%$

4b, $6 \%$

Procedure: Under a nitrogen atmosphere, a $10 \mathrm{~mL}$ Schlenk tube equipped with a magnetic stir bar was charged successively with tri-p-tolylstibane ( $39.4 \mathrm{mg}, 0.1 \mathrm{mmol}$ ), 4-iodobenzonitrile $\mathbf{2 b}$ ( $69 \mathrm{mg}, 0.3$ mmol), $\mathrm{Pd}(\mathrm{OAc})_{2}(1.1 \mathrm{mg}, 0.005 \mathrm{mmol}, 5.0 \mathrm{~mol} \%), n$-Bu ${ }_{4} \mathrm{PBr}$ (6.8 mg, $\left.0.02 \mathrm{mmol}, 20 \mathrm{~mol} \%\right), \mathrm{Cs}_{2} \mathrm{CO}_{3}$ (65 mg, $0.2 \mathrm{mmol}, 2.0$ equiv), $1.0 \mathrm{~mL}$ of THF, $20 \mu \mathrm{L}$ of $\mathrm{H}_{2} \mathrm{O}$ and $30 \mu \mathrm{L}$ of n-dodecane. The reaction mixture was stirred at $110^{\circ} \mathrm{C}$ for $8 \mathrm{~h}$. The resulting mixture was diluted with $\mathrm{CH}_{2} \mathrm{Cl}_{2}$. Yields of products were determined by $\mathrm{GC}$ analysis using $n$-dodecane as an internal standard. The cross-coupling product 5a was detected in 36\% yield. The homo-coupling products $4 \mathbf{a}$ and $\mathbf{4 b}$ were detected in $7 \%$ yield and $6 \%$ yield, respectively. Experimental results showed that the reactivity of tri-p-tolylstibane was poor.

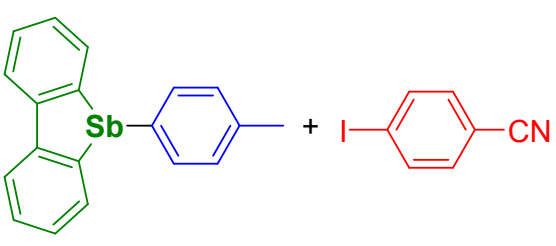

3bf, $0.1 \mathrm{mmol}$
$\mathrm{Pd}(\mathrm{OAc})_{2}(5.0 \mathrm{~mol} \%)$

$\underset{\mathrm{Cs}_{2} \mathrm{CO}_{3} \text { (2.0 equiv) }}{n-\mathrm{Bu}_{4} \mathrm{PBr}(20 \text { mol\%) }}$

THF: $\mathrm{H}_{2} \mathrm{O}=50: 1(\mathrm{v}: \mathrm{v})$

$110^{\circ} \mathrm{C}, 8 \mathrm{~h}$<smiles>Cc1ccc(-c2ccc(C#N)cc2)cc1</smiles>

$5 a, 66 \%$

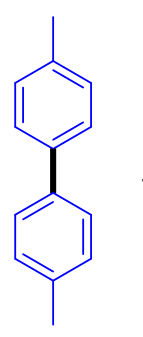

$4 a, 11 \%$<smiles>N#Cc1ccc(-c2ccc(C#N)cc2)cc1</smiles>

$4 b, 1 \%$

Procedure: Under a nitrogen atmosphere, a $10 \mathrm{~mL}$ Schlenk tube equipped with a magnetic stir bar was charged successively with $\mathbf{3 b f}(36.4 \mathrm{mg}, 0.1 \mathrm{mmol})$, 4-iodobenzonitrile $\mathbf{2 b}$ (22.9 mg, $0.1 \mathrm{mmol})$, $\mathrm{Pd}(\mathrm{OAc})_{2}(1.1 \mathrm{mg}, 0.005 \mathrm{mmol}, 5.0 \mathrm{~mol} \%), n-\mathrm{Bu}_{4} \mathrm{PBr}(6.8 \mathrm{mg}, 0.02 \mathrm{mmol}, 20 \mathrm{~mol} \%), \mathrm{Cs}_{2} \mathrm{CO}_{3}(65 \mathrm{mg}$, $0.2 \mathrm{mmol}, 2.0$ equiv), $1.0 \mathrm{~mL}$ of THF, $20 \mu \mathrm{L}$ of $\mathrm{H}_{2} \mathrm{O}$ and $30 \mu \mathrm{L}$ of $n$-dodecane. The reaction mixture was stirred at $110{ }^{\circ} \mathrm{C}$ for $8 \mathrm{~h}$. The resulting mixture was diluted with $\mathrm{CH}_{2} \mathrm{Cl}_{2}$. Yields of products were determined by GC analysis using $n$-dodecane as an internal standard. The cross-coupling product 5a was detected in $66 \%$ yield. The homo-coupling products $4 \mathbf{a}$ and $\mathbf{4 b}$ were detected in $11 \%$ yield and $1 \%$ yield, respectively.

The above experimental results showed that the reactivity order is $\mathbf{3 a}>\mathbf{3 b f}>$ tri- $p$-tolylstibane.

S2.4 Investigation on the Reaction of Stibine 3az with 1-Iodo-methylbenzne 2a

Table S12. Investigation on the Reaction of 3az with 2 a under the Reported Reaction Conditions ${ }^{\mathrm{S} 2}$ 


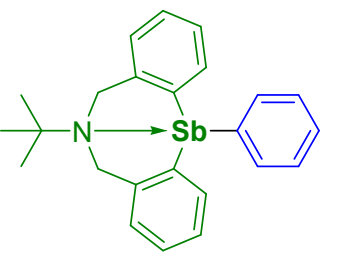

3az, $0.1 \mathrm{mmol}$

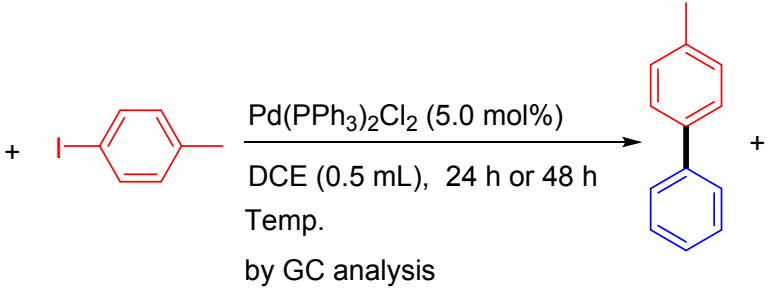

2a, $0.15 \mathrm{mmol}$

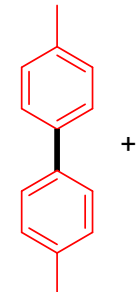

$4 a$

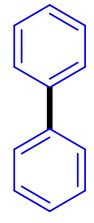

49

\begin{tabular}{lllccc}
\hline Entry & Time (h) & Temp. $\left({ }^{\circ} \mathrm{C}\right)$ & \multicolumn{3}{c}{${\text { Yield }(\%)^{a}}^{a}$} \\
\cline { 3 - 6 } & & & $\mathbf{5 e}$ & $\mathbf{4 a}$ & $\mathbf{4 g}$ \\
\hline $1^{\mathrm{S} 2}$ & 24 & 80 & 18.3 & 1.8 & 1.8 \\
$2^{\mathrm{S} 2}$ & 24 & 80 & 17.9 & 2.1 & 0.6 \\
$3^{\mathrm{S} 2}$ & 24 & 80 & 18.6 & 0.5 & 3.5 \\
Average $^{\mathrm{S} 2}$ & & & 18.3 & 1.5 & 1.9 \\
4 & 48 & 80 & 33.2 & 3.1 & 2.5 \\
5 & 24 & 80 & 18.4 & 1.1 & 2.5 \\
6 & 24 & 90 & 34.4 & 1.2 & 3.1 \\
7 & 24 & 100 & 45.2 & 1.8 & 2.6 \\
8 & 24 & 120 & 59.3 & 2.6 & 3.7 \\
\hline
\end{tabular}

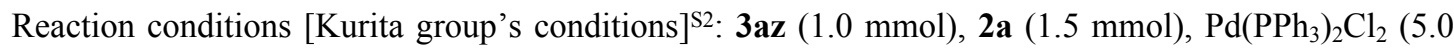
mol\%) and $5.0 \mathrm{~mL}$ of $\mathrm{ClCH}_{2} \mathrm{CH}_{2} \mathrm{Cl}$ at $80-120{ }^{\circ} \mathrm{C}$ for $24 \mathrm{~h}$ or $48 \mathrm{~h}$. ${ }^{a}$ Yields were determined by GC analysis using $n$-dodecane as an internal standard.

Procedure: Under nitrogen, a Schlenk tube of $10 \mathrm{~mL}$ equipped with a magnetic stir bar was charged successively with $\mathbf{3 a z}(44.9 \mathrm{mg}, 0.1 \mathrm{mmol})$, 1-iodo-4-methylbenzene 2a $(32.7 \mathrm{mg}, 0.15 \mathrm{mmol})$, $\mathrm{Pd}\left(\mathrm{PPh}_{3}\right)_{2} \mathrm{Cl}_{2}$ (3.5 mg, $0.005 \mathrm{mmol}, 5.0 \mathrm{~mol} \%$ ), $0.5 \mathrm{~mL}$ of $\mathrm{ClCH}_{2} \mathrm{CH}_{2} \mathrm{Cl}$ (DCE) and $30 \mu \mathrm{L}$ of $n$-dodecane. The reaction mixture was stirred at $80-120^{\circ} \mathrm{C}$ for $24 \mathrm{~h}$ or $48 \mathrm{~h}$. Yields of products were determined by GC analysis using $n$-dodecane as an internal standard. Experimental results were shown in Table S12. The average yield of $\mathbf{5 e}$ was $18.3 \%$, which was very different from the $68 \%$ yield reported in the literature. ${ }^{\mathrm{S} 2}$ This result puzzled us. Then, we tried to explore the effect of temperature on the reaction. When the temperature reached $120{ }^{\circ} \mathrm{C}, \mathbf{5 e}$ was obtained with $59.3 \%$ yield, which was close to the value $(68 \%)$.

Table S13. Monitoring the Reaction Progress under the Reported Reaction Conditions ${ }^{\mathrm{S} 2}$ 


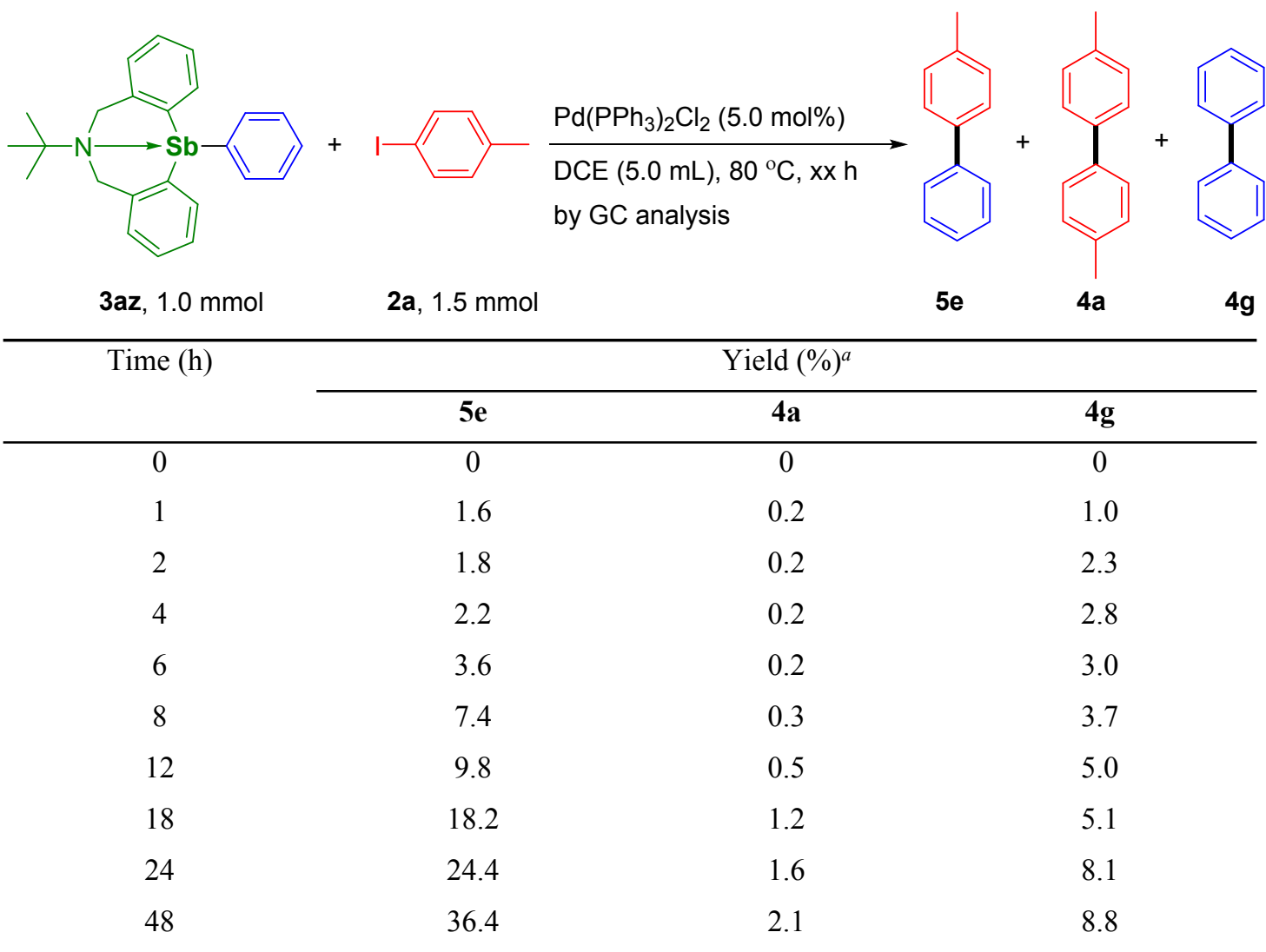

Reaction conditions: 3az $(1.0 \mathrm{mmol}), 2 \mathrm{a}(1.5 \mathrm{mmol}), \mathrm{Pd}\left(\mathrm{PPh}_{3}\right)_{2} \mathrm{Cl}_{2}(5.0 \mathrm{~mol} \%)$ and $5.0 \mathrm{~mL}$ of $\mathrm{ClCH}_{2} \mathrm{CH}_{2} \mathrm{Cl}$ at $80^{\circ} \mathrm{C}$ for $0-48 \mathrm{~h}$. Yields of $5 \mathbf{e}$ and $4 \mathbf{a}$ were based on $1.0 \mathrm{mmol}$ scale. Yields of $4 \mathrm{~g}$ were based on 1.5 mmol scale. ${ }^{a}$ Yields were determined by GC analysis using $n$-dodecane as an internal standard.

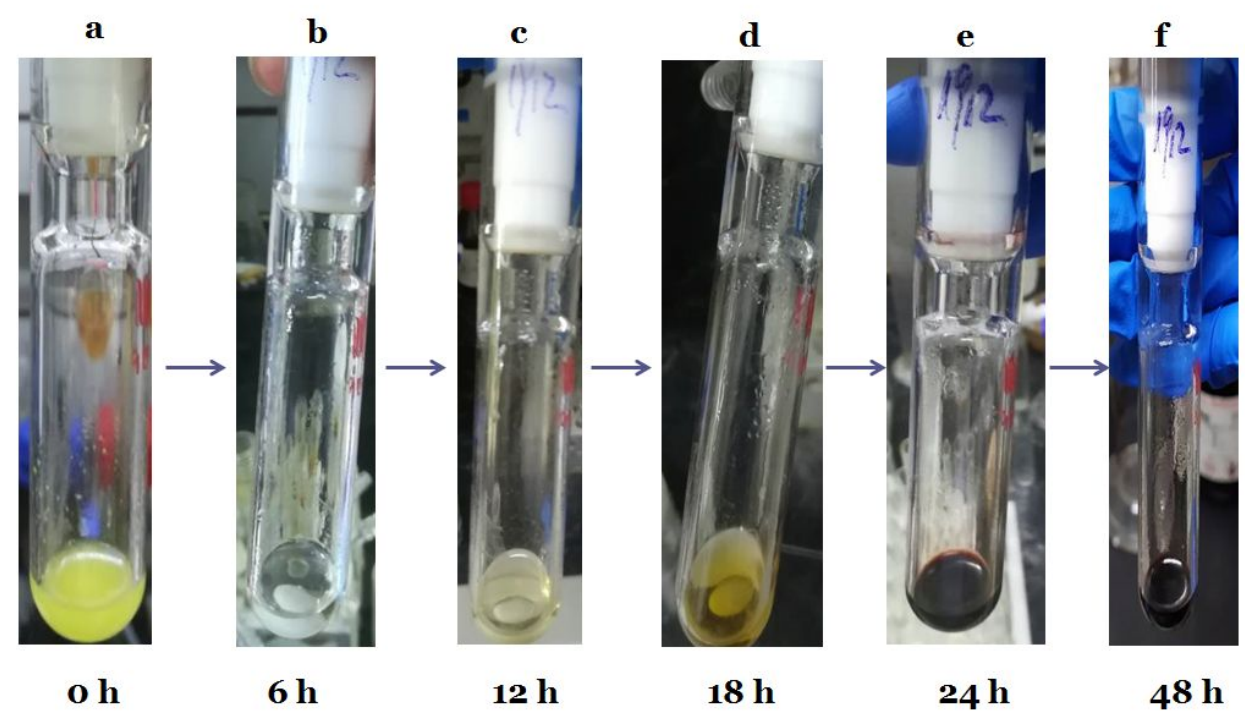

Figure S1. The color change of the reaction solution in different time

Procedure: Under a nitrogen atmosphere, a $10 \mathrm{~mL}$ Schlenk tube equipped with a magnetic stir bar was charged successively with $\mathbf{3 a z}$ (449 mg, $1.0 \mathrm{mmol}$ ), 1-iodo-4-methylbenzene $\mathbf{2 a}(327 \mathrm{mg}, 1.5 \mathrm{mmol})$, 
$\mathrm{Pd}\left(\mathrm{PPh}_{3}\right)_{2} \mathrm{Cl}_{2}(35 \mathrm{mg}, 0.05 \mathrm{mmol}, 5.0 \mathrm{~mol} \%), 5.0 \mathrm{~mL}$ of $\mathrm{ClCH}_{2} \mathrm{CH}_{2} \mathrm{Cl}$ and $30 \mu \mathrm{L}$ of $n$-dodecane. The reaction mixture was stirred at $80^{\circ} \mathrm{C}$ for $0-48 \mathrm{~h}$. Reaction progress was monitored by GC analysis. As shown in Figure S1, the difference of the color of the solution at different time periods was obvious, the solution changed from yellow to colorless, and then from colorless to brownish red. The yields of products were determined by GC analysis. Experimental results were shown in Table S13. Crosscoupling product $5 \mathbf{e}$ was formed in $24.4 \%$ yield when the reaction was stirred at $80{ }^{\circ} \mathrm{C}$ for $24 \mathrm{~h}$. The reaction mixture continued to be stirred at $80{ }^{\circ} \mathrm{C}$ for $24 \mathrm{~h}$, the yield of $5 \mathbf{e}$ reached $36.4 \%$. These results suggested reaction time $(24 \mathrm{~h})$ was not optimal in the literature. ${ }^{\mathrm{S} 2}$

\section{S3 Experimental Details}

\section{S3.1 Synthesis of Triarylstibines and Analytical Data}

\section{General Procedure A for Synthesis of Triarylstibines via Reductive Cross-Coupling of Chlorostibines with Organic Halides}

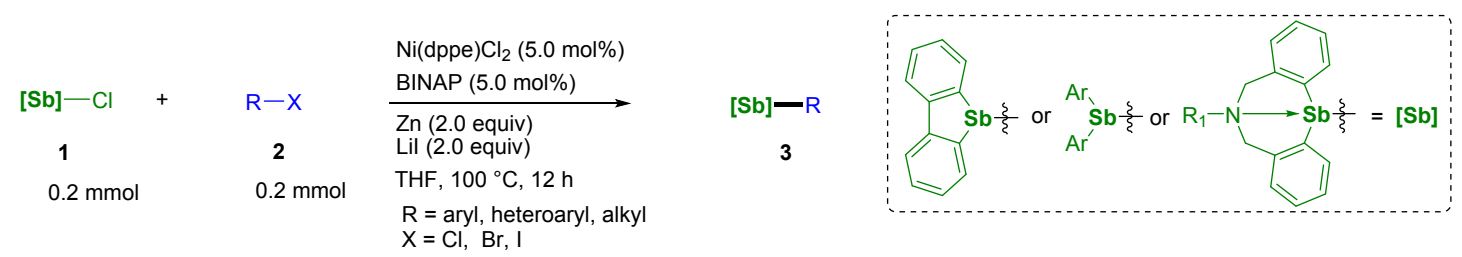

Under a nitrogen atmosphere, a $10 \mathrm{~mL}$ Schlenk tube equipped with a magnetic stir bar was charged successively with chlorostibine $(0.2 \mathrm{mmol})$, organic halide $(0.2 \mathrm{mmol}), \mathrm{Ni}(\mathrm{dppe}) \mathrm{Cl}_{2}(5.3 \mathrm{mg}, 0.01 \mathrm{mmol})$, LiI (53.5 mg, $0.4 \mathrm{mmol})$, BINAP (6.2 mg, $0.01 \mathrm{mmol})$, zinc powder $(26 \mathrm{mg}, 0.4 \mathrm{mmol})$, and $2.0 \mathrm{~mL}$ of THF. The reaction mixture was stirred at $100^{\circ} \mathrm{C}$ for $12 \mathrm{~h}$. After the reaction was completed, the resulting mixture was diluted with $3.0 \mathrm{~mL}$ of $\mathrm{CH}_{2} \mathrm{Cl}_{2}$, filtrated. The residue was washed with $1 \mathrm{~N} \mathrm{HCl}$ solution (5.0 $\mathrm{mL} \times 3$ ) and washed with $5.0 \mathrm{~mL}$ of $\mathrm{H}_{2} \mathrm{O}$. The organic layer was dried over anhydrous $\mathrm{Na}_{2} \mathrm{SO}_{4}$ and concentrated under reduced pressure. Purification of the crude product by flash chromatography on silica gel using the mixed solvent system of petroleum ether (PE) and ethyl acetate (EA) afforded the desired products.

Scheme S1. Substrate Scope for the Cross-Electrophile Reaction of Chlorostibines with Organic Halides 


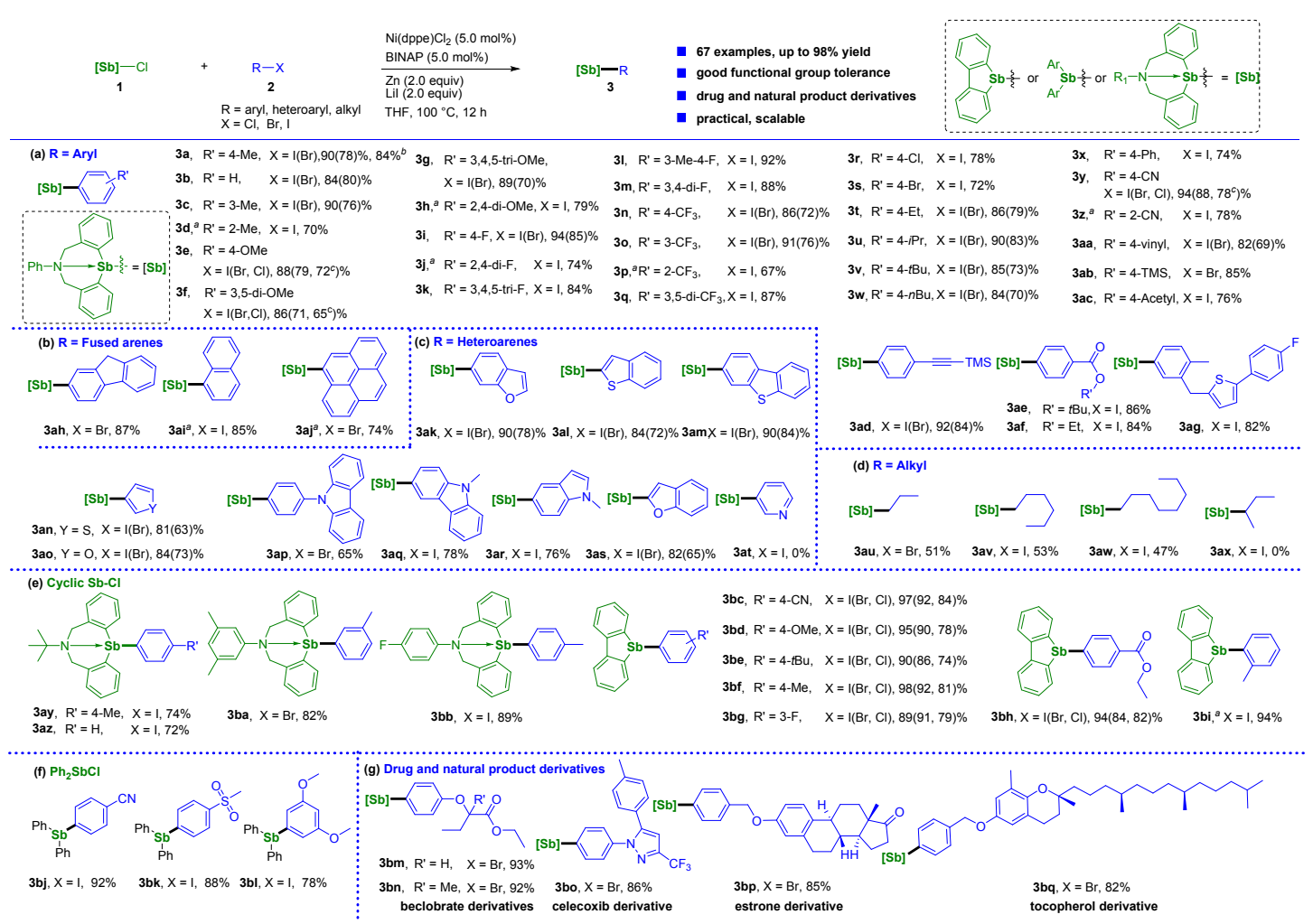

Stibines 3a-e, 3i, 3l, 3n-y, 3aa-c, 3ae, 3ai, 3aj, 3al-p, 3as, 3ay, 3az, 3ba, 3bd, 3bf, 3bg, 3bh, 3bj, 3bk and $\mathbf{3 b l}$ were reported. $\mathrm{s} 1$

6-phenyl-12-(p-tolyl)-5,6,7,12-tetrahydrodibenzo $[c, f][1,5]$ azastibocine (3a)<smiles>Cc1ccc([Sb]2(c3ccccc3)CN(c3ccccc3)Cc3ccccc32)cc1</smiles>

1-Iodo-4-methylbenzene $\mathbf{2 a}(43.6 \mathrm{mg}, 0.20 \mathrm{mmol})$ was used, the desired product $\mathbf{3 a}$ was obtained in $90 \%$ yield; 1-bromo-4-methylbenzene 2 al $(34.0 \mathrm{mg}, 0.2 \mathrm{mmol}$ ) was used, the desired product 3a was obtained in $78 \%$ yield $(75.3 \mathrm{mg}) .{ }^{1} \mathrm{H}$ NMR $\left(400 \mathrm{MHz}, \mathrm{CDCl}_{3}\right) \delta 7.40(\mathrm{~d}, J=7.2 \mathrm{~Hz}, 2 \mathrm{H}), 7.12-7.03(\mathrm{~m}, 10 \mathrm{H})$, 6.99-6.91 (m, 4H), 6.77 (t, $J=7.2 \mathrm{~Hz}, 1 \mathrm{H}), 4.56$ (d, $J=15.1 \mathrm{~Hz}, 2 \mathrm{H}), 4.29$ (d, J=15.1 Hz, 2H), 2.27 (s, 3H). ${ }^{13} \mathrm{C} \mathrm{NMR}\left(101 \mathrm{MHz}, \mathrm{CDCl}_{3}\right) \delta 148.6,144.4,139.4,139.0,138.5,136.6,135.8,129.6,129.1,128.4$, $128.0,126.9,121.3,117.5,57.5,21.7$.

Gram-scale experiment: Under a nitrogen atmosphere, a Schlenk tube of $100 \mathrm{~mL}$ equipped with a magnetic stir bar was charged successively with chlorostibine 1a (5.34 g, 12.5 mmol), 1-iodo-4methylbenzene $2 \mathrm{a}(2.72 \mathrm{~g}, 12.5 \mathrm{mmol}), \mathrm{Ni}(\mathrm{dppe}) \mathrm{Cl}_{2}$ (331.2 mg, $\left.5.0 \mathrm{~mol} \%\right)$, zinc powder (1.62 g, 2.0 equiv), BINAP (375 mg, $5.0 \mathrm{~mol} \%$ ), LiI (3.34 g, 2.0 equiv) and THF (40 mL). The reaction mixture was stirred at $100{ }^{\circ} \mathrm{C}$ for $48 \mathrm{~h}$. After the reaction was completed, the resulting mixture was diluted with 30 $\mathrm{mL}$ of $\mathrm{CH}_{2} \mathrm{Cl}_{2}$, filtrated. The residue was washed with $1 \mathrm{~N} \mathrm{HCl}$ solution $(125.0 \mathrm{~mL} \times 3)$ and washed with $125.0 \mathrm{~mL}$ of $\mathrm{H}_{2} \mathrm{O}$. The organic layer was dried over anhydrous $\mathrm{Na}_{2} \mathrm{SO}_{4}$ and concentrated under reduced 
pressure. The crude product was purified by column chromatography on silica gel and eluted with the mixture solvent of PE:EA (80:1(v:v)) to afford 3a as a white solid in $84 \%$ yield (5.0 g)

\section{6,12-diphenyl-5,6,7,12-tetrahydrodibenzo $[c, f][1,5]$ azastibocine $(3 \mathrm{~b})$}<smiles></smiles>

Iodobenzene $2 \mathbf{c}$ ( $40.8 \mathrm{mg}, 0.20 \mathrm{mmol}$ ) was used, the desired product $\mathbf{3 b}$ was obtained as a white solid in $84 \%$ yield $(78.8 \mathrm{mg}$ ); bromobenzene $2 \mathbf{d}(31.2 \mathrm{mg}, 0.2 \mathrm{mmol})$ was used, the desired product $\mathbf{3 b}$ was obtained in $80 \%$ yield $(75.0 \mathrm{mg}) .{ }^{1} \mathrm{H}$ NMR $\left(400 \mathrm{MHz}, \mathrm{CDCl}_{3}\right) \delta 7.74-7.56(\mathrm{~m}, 2 \mathrm{H}), 7.45-7.35(\mathrm{~m}, 3 \mathrm{H})$, 7.26-7.17 (m, 8H), 7.10 (d, $J=7.6 \mathrm{~Hz}, 4 \mathrm{H}), 6.92(\mathrm{t}, J=7.4 \mathrm{~Hz}, 1 \mathrm{H}), 4.70$ (d, $J=15.1 \mathrm{~Hz}, 2 \mathrm{H}), 4.44$ (d, $J=15.1 \mathrm{~Hz}, 2 \mathrm{H}) .{ }^{13} \mathrm{C} \mathrm{NMR}\left(101 \mathrm{MHz}, \mathrm{CDCl}_{3}\right) \delta 148.5,144.4,143.4,139.0,136.6,135.7,129.1,128.7$, 128.7, 128.4, 128.1, 126.9, 121.4, 117.6, 57.5.

\section{6-phenyl-12-(m-tolyl)-5,6,7,12-tetrahydrodibenzo[c,f $][1,5]$ azastibocine (3c)}<smiles></smiles>

1-Iodo-3-methylbenzene $2 \mathbf{e}(43.6 \mathrm{mg}, 0.20 \mathrm{mmol}$ ) was used, the desired product $3 \mathbf{c}$ was obtained as a white solid in $90 \%$ yield $(86.9 \mathrm{mg}$ ); 1-bromo-3-methylbenzene $\mathbf{2 f}$ ( $34.0 \mathrm{mg}, 0.2 \mathrm{mmol}$ ) was used, the desired product $3 \mathbf{c}$ was obtained in $76 \%$ yield $(73.4 \mathrm{mg}) .{ }^{1} \mathrm{H}$ NMR $\left(400 \mathrm{MHz}, \mathrm{CDCl}_{3}\right) \delta 7.70(\mathrm{~s}, 1 \mathrm{H})$, $7.64(\mathrm{~d}, J=7.1 \mathrm{~Hz}, 1 \mathrm{H}), 7.48(\mathrm{t}, J=7.3 \mathrm{~Hz}, 1 \mathrm{H}), 7.45-7.33(\mathrm{~m}, 9 \mathrm{H}), 7.30-7.23(\mathrm{~m}, 4 \mathrm{H}), 7.08(\mathrm{t}, J=$ $7.2 \mathrm{~Hz}, 1 \mathrm{H}), 4.85(\mathrm{~d}, J=15.1 \mathrm{~Hz}, 2 \mathrm{H}), 4.59$ (d, $J=15.1 \mathrm{~Hz}, 2 \mathrm{H}), 2.53(\mathrm{~s}, 3 \mathrm{H}) .{ }^{13} \mathrm{C}$ NMR $(101 \mathrm{MHz}$, $\left.\mathrm{CDCl}_{3}\right) \delta 148.5,144.3,143.0,139.6,138.0,136.6,135.9,135.7,129.5,129.0,128.5,128.3,128.0,126.9$, $121.3,117.5,57.5,21.6$.

6-phenyl-12-(o-tolyl)-5,6,7,12-tetrahydrodibenzo[c,f $][1,5]$ azastibocine (3d)<smiles>Cc1ccccc1Sc1ccccc1CN1Cc2ccccc2Sc2ccccc21</smiles>

1-Iodo-2-methylbenzene $2 \mathbf{g}$ ( $43.6 \mathrm{mg}, 0.20 \mathrm{mmol}$ ) was used, the desired product $\mathbf{3 d}$ was obtained as a white solid in 70\% yield $(67.6 \mathrm{mg}) .{ }^{1} \mathrm{H}$ NMR $\left(400 \mathrm{MHz}, \mathrm{CDCl}_{3}\right) \delta 7.57(\mathrm{~d}, J=6.5 \mathrm{~Hz}, 1 \mathrm{H}), 7.41-7.25$ 
(m, 8H), 7.22 (d, $J=7.1 \mathrm{~Hz}, 3 \mathrm{H}), 7.19-7.09$ (m, 4H), 6.97 (t, $J=6.9 \mathrm{~Hz}, 1 \mathrm{H}), 4.74(\mathrm{~d}, J=15.1 \mathrm{~Hz}$, $2 \mathrm{H}), 4.52(\mathrm{~d}, J=15.1 \mathrm{~Hz}, 2 \mathrm{H}), 2.51(\mathrm{~s}, 3 \mathrm{H}) .{ }^{13} \mathrm{C} \mathrm{NMR}\left(101 \mathrm{MHz}, \mathrm{CDCl}_{3}\right) \delta 148.6,146.0,144.5,143.6$, $140.3,136.8,135.7,129.4,129.0,128.9,128.3,128.0,127.0,126.2,121.4,118.0,57.7,25.3$.

12-(4-methoxyphenyl)-6-phenyl-5,6,7,12-tetrahydrodibenzo[c, $f][1,5]$ azastibocine $(3 \mathrm{e})$

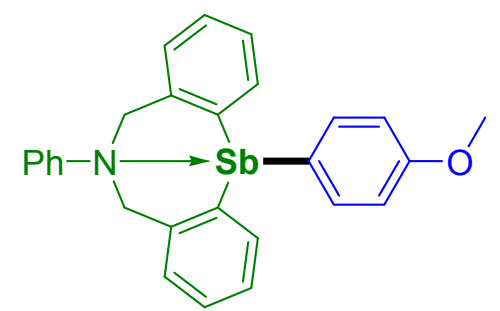

1-Iodo-4-methoxybenzene $\mathbf{2 h}(46.8 \mathrm{mg}, 0.20 \mathrm{mmol})$ was used, the desired product $\mathbf{3 e}$ was obtained as a white solid in $88 \%$ yield $(87.8 \mathrm{mg})$; 1-bromo-4-methoxybenzene $2 \mathbf{i}(37.2 \mathrm{mg}, 0.2 \mathrm{mmol})$ was used, the desired product $3 \mathbf{e}$ was obtained in $79 \%$ yield $(78.8 \mathrm{mg}$ ); 1-chloro-4-methoxybenzene $2 \mathbf{c w}$ ( $28.4 \mathrm{mg}, 0.2$ mmol) was used, the combination of $\mathrm{Ni}(\mathrm{cod})_{2}(2.8 \mathrm{mg}, 5.0 \mathrm{~mol} \%)$, dppe (4.0 mg, 5.0\%), $\mathrm{LiCl}(16.8 \mathrm{mg}$, 2.0 equiv) and zinc (26 mg, 2.0 equiv) gave the desired product $3 \mathrm{e}$ in $72 \%$ yield $(71.8 \mathrm{mg}) .{ }^{1} \mathrm{H} \mathrm{NMR}$ $\left(400 \mathrm{MHz}, \mathrm{CDCl}_{3}\right) \delta 7.66(\mathrm{~d}, J=7.5 \mathrm{~Hz}, 2 \mathrm{H}), 7.36-7.26(\mathrm{~m}, 8 \mathrm{H}), 7.24-7.15(\mathrm{~m}, 4 \mathrm{H}), 7.07(\mathrm{~d}, J=7.6$ $\mathrm{Hz}, 2 \mathrm{H}), 7.01(\mathrm{t}, J=7.2 \mathrm{~Hz}, 1 \mathrm{H}), 4.79$ (d, $J=15.1 \mathrm{~Hz}, 2 \mathrm{H}), 4.53$ (d, $J=15.1 \mathrm{~Hz}, 2 \mathrm{H}), 3.94(\mathrm{~s}, 3 \mathrm{H}) .{ }^{13} \mathrm{C}$ NMR $\left(101 \mathrm{MHz}, \mathrm{CDCl}_{3}\right) \delta 160.3,148.6,144.3,140.2,136.6,135.9,133.4,129.1,128.3,128.0,126.9$, $121.3,117.5,114.6,57.5,55.2$.

12-(3,5-dimethoxyphenyl)-6-phenyl-5,6,7,12-tetrahydrodibenzo $[c, f][1,5]$ azastibocine (3f)<smiles>COc1cc(OC)cc([Sb]2(c3ccccc3)CN3Cc4ccccc4C2(C)c2ccccc23)c1</smiles>

1-Iodo-3,5-dimethoxybenzene $\mathbf{2} \mathbf{j}$ (52.8 $\mathrm{mg}, 0.20 \mathrm{mmol}$ ) was used, the desired product $\mathbf{3 f}$ was obtained as a white solid in $86 \%$ yield $(90.9 \mathrm{mg})$; 1-bromo-3,5-dimethoxybenzene $2 \mathbf{k}$ (43.2 $\mathrm{mg}, 0.2 \mathrm{mmol})$ was used, the desired product $\mathbf{3 f}$ was obtained in 71\% yield (75.1 mg); 1-chloro-3,5-dimethoxybenzene 2dc (34.4 mg, $0.2 \mathrm{mmol})$ was used, the combination of $\mathrm{Ni}(\mathrm{cod})_{2}(2.8 \mathrm{mg}, 5.0 \mathrm{~mol} \%)$, dppe $(4.0 \mathrm{mg}, 5.0 \%)$, $\mathrm{LiCl}$ (16.8 mg, 2.0 equiv) and zinc (26 mg, 2.0 equiv) gave the desired product $3 \mathrm{e}$ in $65 \%$ yield (68.8 mg). Melting point (M.P.): $181.2-182.9^{\circ} \mathrm{C} .{ }^{1} \mathrm{H}$ NMR $\left(400 \mathrm{MHz}, \mathrm{CDCl}_{3}\right) \delta 7.29$ (d, J= $\left.7.4 \mathrm{~Hz}, 2 \mathrm{H}\right)$, 7.26-7.17 (m, 8H), 7.15-7.05 (m, 4H), $6.91(\mathrm{t}, J=7.2 \mathrm{~Hz}, 1 \mathrm{H}), 6.84(\mathrm{~s}, 2 \mathrm{H}), 6.51(\mathrm{~s}, 1 \mathrm{H}), 4.69(\mathrm{~d}, J=$ $15.2 \mathrm{~Hz}, 2 \mathrm{H}), 4.43(\mathrm{~d}, J=15.2 \mathrm{~Hz}, 2 \mathrm{H}), 3.74(\mathrm{~s}, 6 \mathrm{H}) .{ }^{13} \mathrm{C} \mathrm{NMR}\left(101 \mathrm{MHz}, \mathrm{CDCl}_{3}\right) \delta 160.7,148.4,145.5$, 144.3, 136.8, 135.6, 129.1, 128.4, 128.0, 126.9, 121.5, 117.7, 115.9, 101.5, 57.6, 55.5. HRMS-ESI (m/z) $[\mathrm{M}+\mathrm{H}]^{+}$Calcd for $\mathrm{C}_{28} \mathrm{H}_{27} \mathrm{NO}_{2} \mathrm{Sb}^{+}, 530.1074$; Found, 530.1077.

6-phenyl-12-(3,4,5-trimethoxyphenyl)-5,6,7,12-tetrahydrodibenzo[c,f][1,5] azastibocine $(3 \mathrm{~g})$ 
<smiles></smiles>

5-Iodo-1,2,3-trimethoxybenzene $2 \mathbf{l}$ ( $58.8 \mathrm{mg}, 0.20 \mathrm{mmol}$ ) was used, the desired product $\mathbf{3 g}$ was obtained as a white solid in $89 \%$ yield ( $99.5 \mathrm{mg})$; 5-bromo-1,2,3-trimethoxybenzene $\mathbf{2 m}(49.2 \mathrm{mg}, 0.2 \mathrm{mmol})$ was used, the desired product $3 \mathrm{~g}$ was obtained in $70 \%$ yield $(78.3 \mathrm{mg})$. M.P.: $170.2-171.4{ }^{\circ} \mathrm{C} .{ }^{1} \mathrm{H}$ NMR $(400$ $\left.\mathrm{MHz}, \mathrm{CDCl}_{3}\right) \delta 7.45(\mathrm{~d}, J=6.3 \mathrm{~Hz}, 2 \mathrm{H}), 7.40-7.31(\mathrm{~m}, 6 \mathrm{H}), 7.30-7.19(\mathrm{~m}, 4 \mathrm{H}), 7.03(\mathrm{~s}, 3 \mathrm{H}), 4.82(\mathrm{~d}$, $J=15.0 \mathrm{~Hz}, 2 \mathrm{H}), 4.56(\mathrm{~d}, J=15.1 \mathrm{~Hz}, 2 \mathrm{H}), 4.08$ (s, 3H), 3.91 (s, 6H). ${ }^{13} \mathrm{C} \mathrm{NMR}\left(101 \mathrm{MHz}, \mathrm{CDCl}_{3}\right) \delta$ 153.3, 148.3, 144.2, 138.5, 137.6, 136.6, 135.6, 128.9, 128.4, 127.9, 126.8, 121.4, 117.5, 115.1, 60.9, 57.4, 56.2. HRMS-ESI (m/z) $[\mathrm{M}+\mathrm{H}]^{+}$Calcd for $\mathrm{C}_{29} \mathrm{H}_{29} \mathrm{NO}_{3} \mathrm{Sb}^{+}$, 560.1180; Found, 560.1182 .

12-(2,4-dimethoxyphenyl)-6-phenyl-5,6,7,12-tetrahydrodibenzo $[c, f][1,5]$ azastibocine (3h)<smiles></smiles>

1-Iodo-2,4-dimethoxybenzene $\mathbf{2 n}(52.8 \mathrm{mg}, 0.20 \mathrm{mmol})$ was used, the desired product $\mathbf{3 h}$ as a white solid in $79 \%$ yield $(83.6 \mathrm{mg})$. M.P.: $42.3-44.7^{\circ} \mathrm{C} .{ }^{1} \mathrm{H}$ NMR $\left(400 \mathrm{MHz}, \mathrm{CDCl}_{3}\right) \delta 7.40(\mathrm{~d}, J=8.1 \mathrm{~Hz}, 1 \mathrm{H})$, $7.34-7.22(\mathrm{~m}, 8 \mathrm{H}), 7.19-7.12(\mathrm{~m}, 4 \mathrm{H}), 6.96(\mathrm{t}, J=7.1 \mathrm{~Hz}, 1 \mathrm{H}), 6.63(\mathrm{~d}, J=8.1 \mathrm{~Hz}, 1 \mathrm{H}), 6.58(\mathrm{~s}, 1 \mathrm{H})$, $4.74(\mathrm{~d}, J=15.0 \mathrm{~Hz}, 2 \mathrm{H}), 4.50$ (d, $J=15.0 \mathrm{~Hz}, 2 \mathrm{H}), 3.93$ (s, 3H), 3.77 (s, 3H). ${ }^{13} \mathrm{C}$ NMR $(101 \mathrm{MHz}$, $\left.\mathrm{CDCl}_{3}\right) \delta$ 166.0, 162.1, 148.7, 144.7, 140.8, 136.7, 136.2, 129.0, 128.1, 127.8, 126.8, 121.9, 121.2, 117.7, 106.4, 97.6, 57.5, 55.6, 55.4. HRMS-ESI $(\mathrm{m} / \mathrm{z})[\mathrm{M}+\mathrm{H}]^{+}$Calcd for $\mathrm{C}_{28} \mathrm{H}_{27} \mathrm{NO}_{2} \mathrm{Sb}^{+}, 530.1074$; Found, 530.1082 .

12-(4-fluorophenyl)-6-phenyl-5,6,7,12-tetrahydrodibenzo $[c, f][1,5]$ azastibocine (3i)<smiles></smiles>

1-Fluoro-4-iodobenzene $2 \mathbf{0}(44.4 \mathrm{mg}, 0.20 \mathrm{mmol})$ was used, the desired product $3 \mathbf{i}$ was obtained as a white solid in 94\% yield (91.6 mg); 1-bromo-4-fluorobenzene $\mathbf{2 p}$ (34.8 $\mathrm{mg}, 0.2 \mathrm{mmol}$ ) was used, the desired product $3 \mathbf{i}$ was obtained in $85 \%$ yield $(82.8 \mathrm{mg}) .{ }^{1} \mathrm{H}$ NMR $\left(400 \mathrm{MHz}, \mathrm{CDCl}_{3}\right) \delta 7.75(\mathrm{t}, J=6.9$ $\mathrm{Hz}, 2 \mathrm{H}), 7.40-7.28(\mathrm{~m}, 8 \mathrm{H}), 7.27-7.18(\mathrm{~s}, 6 \mathrm{H}), 7.05(\mathrm{t}, J=7.2 \mathrm{~Hz}, 1 \mathrm{H}), 4.82(\mathrm{~d}, J=15.2 \mathrm{~Hz}, 2 \mathrm{H})$, $4.56(\mathrm{~d}, J=15.2 \mathrm{~Hz}, 2 \mathrm{H}) .{ }^{13} \mathrm{C} \mathrm{NMR}\left(101 \mathrm{MHz}, \mathrm{CDCl}_{3}\right) \delta 163.6(\mathrm{~d}, J=246.1 \mathrm{~Hz}), 148.5,144.3,140.7$ 
$(\mathrm{d}, J=7.0 \mathrm{~Hz}), 138.4(\mathrm{~d}, J=3.9 \mathrm{~Hz}), 136.4,135.6,129.1,128.5,128.1,126.9,121.6,117.7,116.0$ (d, $J$ $=19.4 \mathrm{~Hz}), 57.6 .{ }^{19} \mathrm{~F}$ NMR $\left(376 \mathrm{MHz}, \mathrm{CDCl}_{3}\right) \delta-112.47$.

\section{2-(2,4-difluorophenyl)-6-phenyl-5,6,7,12-tetrahydrodibenzo $[c, f][1,5]$ azastibocine $(3 \mathbf{j})$}<smiles>Fc1ccc([Sb]2(c3ccccc3)CN3Cc4ccccc4CN(c4ccccc4)[SH]32)c(F)c1</smiles>

2,4-Difluoro-1-iodobenzene $\mathbf{2 q}$ ( $48.0 \mathrm{mg}, 0.20 \mathrm{mmol}$ ) was used, the desired product $\mathbf{3} \mathbf{j}$ was obtained as a white solid in $74 \%$ yield $(74.7 \mathrm{mg})$. M.P.: $169.7-171.9^{\circ} \mathrm{C} .{ }^{1} \mathrm{H}$ NMR $\left(400 \mathrm{MHz}, \mathrm{CDCl}_{3}\right) \delta 7.46(\mathrm{q}, J$ $=7.4 \mathrm{~Hz}, 1 \mathrm{H}), 7.29-7.19(\mathrm{~m}, 6 \mathrm{H}), 7.18-7.08(\mathrm{~m}, 6 \mathrm{H}), 7.00-6.88(\mathrm{~m}, 2 \mathrm{H}), 6.87-6.79(\mathrm{~m}, 1 \mathrm{H}), 4.69$ $(\mathrm{d}, J=15.2 \mathrm{~Hz}, 2 \mathrm{H}), 4.45(\mathrm{~d}, J=15.2 \mathrm{~Hz}, 2 \mathrm{H}) .{ }^{13} \mathrm{C} \mathrm{NMR}\left(101 \mathrm{MHz}, \mathrm{CDCl}_{3}\right) \delta 167.9(\mathrm{dd}, J=11.4 \mathrm{~Hz}$, $238.9 \mathrm{~Hz}$ ), 164.4 (dd, $J=11.9 \mathrm{~Hz}, 247.6 \mathrm{~Hz}), 148.2,144.2,141.3$ (dd, $J=13.5,8.8 \mathrm{~Hz}), 136.4,134.8$, 129.1, 128.6, 128.2, 127.0, 124.3 (dd, $J=35.3,3.9 \mathrm{~Hz}), 122.0,118.3,112.5(\mathrm{dd}, J=19.6,3.6 \mathrm{~Hz}), 103.3$ $(\mathrm{dd}, J=31.6,23.7 \mathrm{~Hz}), 57.8 .{ }^{19} \mathrm{~F}$ NMR $\left(376 \mathrm{MHz}, \mathrm{CDCl}_{3}\right) \delta-90.93(\mathrm{~d}, J=9.8 \mathrm{~Hz}),-109.41$ (d, $J=10.5$ Hz). HRMS-ESI (m/z) [M+H] $]^{+}$Calcd for $\mathrm{C}_{26} \mathrm{H}_{21} \mathrm{~F}_{2} \mathrm{NSb}^{+}$, 506.0675; Found, 506.0682.

6-phenyl-12-(3,4,5-trifluorophenyl)-5,6,7,12-tetrahydrodibenzo[c,f][1,5]azastibocine (3k)<smiles>Fc1cc([Sb]2c3ccccc3CN(c3ccccc3)Cc3ccccc32)cc(F)c1F</smiles>

1,2,3-Trifluoro-5-iodobenzene $2 \mathbf{r}(51.6 \mathrm{mg}, 0.20 \mathrm{mmol})$ was used, the desired product $3 \mathbf{k}$ was obtained as a white solid in $84 \%$ yield $(87.8 \mathrm{mg})$. M.P.: $201.3-204.1^{\circ} \mathrm{C} .{ }^{1} \mathrm{H}$ NMR $\left(400 \mathrm{MHz}, \mathrm{CDCl}_{3}\right) \delta 7.41-$ 7.30 (m, 8H), $7.28-7.17$ (m, 6H), 7.06 (t, $J=7.0 \mathrm{~Hz}, 1 \mathrm{H}), 4.78$ (d, $J=15.2 \mathrm{~Hz}, 2 \mathrm{H}), 4.53$ (d, $J=15.2$ $\mathrm{Hz}, 2 \mathrm{H}) .{ }^{13} \mathrm{C}$ NMR $\left(101 \mathrm{MHz}, \mathrm{CDCl}_{3}\right) \delta 151.5(\mathrm{dd}, J=256.3,9.8 \mathrm{~Hz}), 148.1,144.3,140.2(\mathrm{dt}, J=266.0$, $15.3 \mathrm{~Hz}$ ), 139.7, 139.6, 136.3, 135.1, 129.2, 128.9, 128.4, 127.1, 121.9, 121.8 (dd, $J=13.3,4.3 \mathrm{~Hz}$ ), 57.8. ${ }^{19} \mathrm{~F}$ NMR $\left(376 \mathrm{MHz}, \mathrm{CDCl}_{3}\right) \delta-134.76(\mathrm{dd}, J=20.9,7.2 \mathrm{~Hz}),-159.85--160.55(\mathrm{~m})$. HRMS-ESI $(\mathrm{m} / \mathrm{z})[\mathrm{M}+\mathrm{H}]^{+}$Calcd for $\mathrm{C}_{26} \mathrm{H}_{20} \mathrm{~F}_{3} \mathrm{NSb}^{+}, 524.0581$; Found, 524.0591 .

12-(4-fluoro-3-methylphenyl)-6-phenyl-5,6,7,12-tetrahydrodibenzo[c,f][1,5]azastibocine (31) 
<smiles>Cc1cc([Sb]2(c3ccccc3)CN3Cc4ccccc4C2(C)c2ccccc23)ccc1F</smiles>

1-Fluoro-4-iodo-2-methylbenzene $2 \mathrm{~s}(47.2 \mathrm{mg}, 0.20 \mathrm{mmol})$ was used, the desired product $3 \mathrm{l}$ was obtained as a white solid in $92 \%$ yield $(92.1 \mathrm{mg}) .{ }^{1} \mathrm{H} \mathrm{NMR}\left(400 \mathrm{MHz}, \mathrm{CDCl}_{3}\right) \delta 7.55(\mathrm{~d}, J=8.4 \mathrm{~Hz}, 1 \mathrm{H})$, 7.49 (t, $J=6.7 \mathrm{~Hz}, 1 \mathrm{H}), 7.36-7.24(\mathrm{~m}, 8 \mathrm{H}), 7.22-7.09$ (m, 5H), $7.0(\mathrm{t}, J=7.2 \mathrm{~Hz}, 1 \mathrm{H}), 4.77$ (d, $J=$ $15.2 \mathrm{~Hz}, 2 \mathrm{H}), 4.51$ (d, $J=15.2 \mathrm{~Hz}, 2 \mathrm{H}), 2.37(\mathrm{~s}, 3 \mathrm{H}) .{ }^{13} \mathrm{C} \mathrm{NMR}\left(101 \mathrm{MHz}, \mathrm{CDCl}_{3}\right) \delta 162.2$ (d, $J=244.7$ Hz), 148.5, 144.3, 142.1 (d, $J=4.6 \mathrm{~Hz}), 138.0$ (d, $J=7.2 \mathrm{~Hz}), 137.9$ (d, $J=4.6 \mathrm{~Hz}), 136.5,135.6,129.1$, 128.5, 128.1, 126.9, 125.2 (d, $J=15.8 \mathrm{~Hz}), 121.5,117.6,115.6$ (d, $J=20.6 \mathrm{~Hz}), 57.5,14.7$ (d, $J=3.8$ $\mathrm{Hz}) .{ }^{19} \mathrm{~F}$ NMR $\left(376 \mathrm{MHz}, \mathrm{CDCl}_{3}\right) \delta-117.13$.

\section{2-(3,4-difluorophenyl)-6-phenyl-5,6,7,12-tetrahydrodibenzo[c,ff][1,5]azastibocine (3m)}<smiles></smiles>

1,2-Difluoro-4-iodobenzene $2 \mathbf{t}$ ( $48.0 \mathrm{mg}, 0.20 \mathrm{mmol}$ ) was used, the desired product $\mathbf{3 m}$ was obtained as a white solid in $88 \%$ yield $(88.9 \mathrm{mg})$. M.P.: $162.1-164.5^{\circ} \mathrm{C} .{ }^{1} \mathrm{H}$ NMR $(400 \mathrm{MHz}, \mathrm{CDCl} 3) \delta 7.58(\mathrm{t}, \mathrm{J}$ $=8.9 \mathrm{~Hz}, 1 \mathrm{H}), 7.53-7.46(\mathrm{~m}, 1 \mathrm{H}), 7.42-7.30(\mathrm{~m}, 7 \mathrm{H}), 7.29-7.19(\mathrm{~m}, 6 \mathrm{H}), 7.07(\mathrm{t}, \mathrm{J}=7.1 \mathrm{~Hz}, 1 \mathrm{H})$, $4.81(\mathrm{~d}, \mathrm{~J}=15.2 \mathrm{~Hz}, 2 \mathrm{H}), 4.56(\mathrm{~d}, \mathrm{~J}=15.2 \mathrm{~Hz}, 2 \mathrm{H}) .{ }^{13} \mathrm{C} \mathrm{NMR}(101 \mathrm{MHz}, \mathrm{CDCl} 3) \delta 151.0(\mathrm{dd}, \mathrm{J}=246.0$, $9.1 \mathrm{~Hz}), 150.8$ (dd, J = 252.0, $10.7 \mathrm{~Hz}), 148.3,144.3,140.2(\mathrm{~d}, \mathrm{~J}=5.0 \mathrm{~Hz}), 136.3,135.2$ - 134.9 (m), 135.0, 129.1, 128.7, 128.2, 127.0, 126.8, 121.9, 117.9, 117.8 (d, J = 15.7 Hz), 57.7. ${ }^{19} \mathrm{~F}$ NMR (376 MHz, CDCl3) $\delta-137.62--137.80(\mathrm{~m}),-138.09--138.25(\mathrm{~m})$. HRMS-ESI $(\mathrm{m} / \mathrm{z})[\mathrm{M}+\mathrm{H}]+$ Calcd for $\mathrm{C} 26 \mathrm{H} 21 \mathrm{~F} 2 \mathrm{NSb}+$, 506.0675; Found, 506.0683.

\section{6-phenyl-12-(4-(trifluoromethyl)phenyl)-5,6,7,12-tetrahydrodibenzo[c,ff][1,5]azastibocine (3n)}<smiles>FC(F)(F)c1ccc([Sb]2(c3ccccc3)c3ccccc3CN(c3ccccc3)Cc3ccccc32)cc1</smiles>

1-Iodo-4-(trifluoromethyl)benzene $\mathbf{2 u}(54.4 \mathrm{mg}, 0.20 \mathrm{mmol})$ was used, the desired product $\mathbf{3 n}$ was obtained as a white solid in $86 \%$ yield ( $92.3 \mathrm{mg}$ ); 1-bromo-4-(trifluoromethyl)benzene $2 \mathbf{v}$ ( $44.8 \mathrm{mg}, 0.2$ mmol) was used, the desired product $\mathbf{3 n}$ was obtained in $72 \%$ yield $(77.3 \mathrm{mg}) .{ }^{1} \mathrm{H}$ NMR (400 MHz, 
$\left.\mathrm{CDCl}_{3}\right) \delta 7.89(\mathrm{~d}, J=7.7 \mathrm{~Hz}, 2 \mathrm{H}), 7.74(\mathrm{~d}, J=7.7 \mathrm{~Hz}, 2 \mathrm{H}), 7.43-7.28(\mathrm{~m}, 6 \mathrm{H}), 7.25-7.16(\mathrm{~m}, 6 \mathrm{H})$, $7.04(\mathrm{t}, J=7.2 \mathrm{~Hz}, 1 \mathrm{H}), 4.80(\mathrm{~d}, J=15.2 \mathrm{~Hz}, 2 \mathrm{H}), 4.54(\mathrm{~d}, J=15.2 \mathrm{~Hz}, 2 \mathrm{H}) .{ }^{13} \mathrm{C} \mathrm{NMR}(101 \mathrm{MHz}$, $\left.\mathrm{CDCl}_{3}\right) \delta 149.3,148.3,144.3,139.3,136.5,135.2,130.6(\mathrm{q}, J=32.0 \mathrm{~Hz}), 129.1,128.7,128.3,127.1$, $125.1(\mathrm{q}, J=3.7 \mathrm{~Hz}), 124.6(\mathrm{q}, J=272.2 \mathrm{~Hz}), 121.8,117.9,57.7 .{ }^{19} \mathrm{~F} \mathrm{NMR}\left(376 \mathrm{MHz}, \mathrm{CDCl}_{3}\right) \delta-62.42$.

6-phenyl-12-(3-(trifluoromethyl)phenyl)-5,6,7,12-tetrahydrodibenzo $[c, f][1,5]$ azastibocine (3o)<smiles></smiles>

1-Iodo-3-(trifluoromethyl)benzene $2 \mathbf{w}(54.4 \mathrm{mg}, 0.20 \mathrm{mmol})$ was used, the desired product 30 was obtained as a white solid in 91\% yield $(97.7 \mathrm{mg}$ ); 1-bromo-3-(trifluoromethyl)benzene $2 \mathbf{x}$ (44.8 mg, 0.2 mmol) was used, the desired product 30 was obtained in $76 \%$ yield $(81.6 \mathrm{mg}) .{ }^{1} \mathrm{H} \mathrm{NMR}(400 \mathrm{MHz}$, $\left.\mathrm{CDCl}_{3}\right) \delta 7.96(\mathrm{~s}, 1 \mathrm{H}), 7.83(\mathrm{~d}, J=7.3 \mathrm{~Hz}, 1 \mathrm{H}), 7.67(\mathrm{~d}, J=7.7 \mathrm{~Hz}, 1 \mathrm{H}), 7.50(\mathrm{t}, J=7.5 \mathrm{~Hz}, 1 \mathrm{H}), 7.31-$ $7.18(\mathrm{~m}, 6 \mathrm{H}), 7.17-7.04(\mathrm{~m}, 6 \mathrm{H}), 6.94(\mathrm{t}, J=7.2 \mathrm{~Hz}, 1 \mathrm{H}), 4.71(\mathrm{~d}, J=15.2 \mathrm{~Hz}, 2 \mathrm{H}), 4.45(\mathrm{~d}, J=15.2$ $\mathrm{Hz}, 2 \mathrm{H}) .{ }^{13} \mathrm{C} \mathrm{NMR}\left(101 \mathrm{MHz}, \mathrm{CDCl}_{3}\right) \delta 148.3,145.2,144.3,142.3,136.4,135.4$ (q, $\left.J=3.6 \mathrm{~Hz}\right), 135.2$, $130.6(\mathrm{q}, J=31.6 \mathrm{~Hz}), 129.2,128.7,128.6,128.3,127.05,125.5$ (q, $J=3.7 \mathrm{~Hz}), 124.5$ (d, $J=272.8 \mathrm{~Hz})$, 121.9, 117.9, 57.7. ${ }^{19} \mathrm{~F}$ NMR $\left(376 \mathrm{MHz}, \mathrm{CDCl}_{3}\right) \delta-62.29$.

6-phenyl-12-(2-(trifluoromethyl)phenyl)-5,6,7,12-tetrahydrodibenzo $[c, f][1,5]$ azastibocine $(3 p)$<smiles>FC(F)(F)c1ccccc1Sc1ccccc1CN1Cc2ccccc2S(c2ccccc2)(c2ccccc2)C1</smiles>

1-Iodo-2-(trifluoromethyl)benzene $2 \mathbf{y}(54.4 \mathrm{mg}, 0.20 \mathrm{mmol})$ was used, the desired product $\mathbf{3 p}$ was obtained as a white solid in $67 \%$ yield $(71.9 \mathrm{mg}) .{ }^{1} \mathrm{H}$ NMR $\left(400 \mathrm{MHz}, \mathrm{CDCl}_{3}\right) \delta 7.77(\mathrm{~d}, J=7.8 \mathrm{~Hz}, 1 \mathrm{H})$, 7.69 (d, $J=7.4 \mathrm{~Hz}, 1 \mathrm{H}), 7.44(\mathrm{dt}, J=27.5,7.4 \mathrm{~Hz}, 2 \mathrm{H}), 7.26-7.19(\mathrm{~m}, 6 \mathrm{H}), 7.15(\mathrm{~d}, J=8.1 \mathrm{~Hz}, 2 \mathrm{H})$, $7.12-7.03(\mathrm{~m}, 2 \mathrm{H}), 7.02-6.90(\mathrm{~m}, 3 \mathrm{H}), 4.68(\mathrm{~d}, J=15.2 \mathrm{~Hz}, 2 \mathrm{H}), 4.48(\mathrm{~d}, J=15.2 \mathrm{~Hz}, 2 \mathrm{H}) .{ }^{13} \mathrm{C}$ NMR $\left(101 \mathrm{MHz}, \mathrm{CDCl}_{3}\right) \delta 148.2,145.3,144.7,142.2,138.1$ (q, $\left.J=29.6 \mathrm{~Hz}\right), 137.1,136.9$ (q, $\left.J=2.4 \mathrm{~Hz}\right)$, $131.5,129.1,128.5,128.3,128.1,126.9,125.0$, (q, $J=5.5 \mathrm{~Hz}) 124.9$ (q, $J=273.9 \mathrm{~Hz}), 122.1,118.6$, 58.0. ${ }^{19} \mathrm{~F}$ NMR $\left(376 \mathrm{MHz}, \mathrm{CDCl}_{3}\right) \delta-56.83$.

12-(3,5-bis(trifluoromethyl)phenyl)-6-phenyl-5,6,7,12-tetrahydrodibenzo $[c, f][1,5]$ azastibocine $(3 q)$ 
<smiles>FC(F)(F)c1cc(C(F)(F)F)cc([Sb]2(c3ccccc3)CN(c3ccccc3)Cc3ccccc32)c1</smiles>

1-Iodo-3,5-bis(trifluoromethyl)benzene $\mathbf{2 z}(68.0 \mathrm{mg}, 0.20 \mathrm{mmol}$ ) was used, the desired product $\mathbf{3 q}$ was obtained as a white solid in $87 \%$ yield $(105.3 \mathrm{mg}) .{ }^{1} \mathrm{H}$ NMR $\left(400 \mathrm{MHz}, \mathrm{CDCl}_{3}\right) \delta 8.12(\mathrm{~s}, 2 \mathrm{H}), 7.92(\mathrm{~s}$, 1H), $7.32-7.23$ (m, 6H), $7.19-7.09$ (m, 4H), $7.02-6.93$ (m, 3H), 4.73 (d, $J=15.3 \mathrm{~Hz}, 2 \mathrm{H}), 4.48$ (d, $J$ $=15.3 \mathrm{~Hz}, 2 \mathrm{H}) \cdot{ }^{13} \mathrm{C} \mathrm{NMR}\left(101 \mathrm{MHz}, \mathrm{CDCl}_{3}\right) \delta 148.1,147.9,144.3,138.9,136.1,134.7,131.1(\mathrm{q}, J=$ $32.2 \mathrm{~Hz}), 129.2,129.0,128.5,127.2,123.8$ (q, $J=271.6 \mathrm{~Hz}), 122.6$ (q, $J=3.8 \mathrm{~Hz}), 122.5,118.3$, 57.9. ${ }^{19} \mathrm{~F}$ NMR $\left(376 \mathrm{MHz}, \mathrm{CDCl}_{3}\right) \delta-62.60$.

\section{2-(4-chlorophenyl)-6-phenyl-5,6,7,12-tetrahydrodibenzo $[c, f][1,5]$ azastibocine (3r)}<smiles></smiles>

1-Chloro-4-iodobenzene $\mathbf{2 a a}(47.6 \mathrm{mg}, 0.20 \mathrm{mmol})$ was used, the desired product $\mathbf{3 r}$ was obtained as a white solid in $78 \%$ yield $(78.5 \mathrm{mg}) .{ }^{1} \mathrm{H}$ NMR $\left(400 \mathrm{MHz}, \mathrm{CDCl}_{3}\right) \delta 7.62(\mathrm{~d}, J=7.6 \mathrm{~Hz}, 2 \mathrm{H}), 7.42(\mathrm{~d}, J=$ $7.7 \mathrm{~Hz}, 2 \mathrm{H}), 7.32-7.23(\mathrm{~m}, 6 \mathrm{H}), 7.22-7.09(\mathrm{~m}, 6 \mathrm{H}), 6.98$ (t, $J=7.2 \mathrm{~Hz}, 1 \mathrm{H}), 4.74(\mathrm{~d}, J=15.2 \mathrm{~Hz}, 2 \mathrm{H})$, $4.48(\mathrm{~d}, J=15.2 \mathrm{~Hz}, 2 \mathrm{H}) .{ }^{13} \mathrm{C} \mathrm{NMR}\left(101 \mathrm{MHz}, \mathrm{CDCl}_{3}\right) \delta 148.4,144.3,141.8,140.3,136.5,135.4,135.1$, $129.1,129.0,128.6,128.2,127.0,121.7,117.8,57.6$.

\section{2-(4-bromophenyl)-6-phenyl-5,6,7,12-tetrahydrodibenzo[c,fl[1,5] azastibocine (3s)}<smiles></smiles>

1-Bromo-4-iodobenzene $\mathbf{2 a b}(56.4 \mathrm{mg}, 0.20 \mathrm{mmol})$ was used, the desired product $3 \mathrm{~s}$ as a white solid in $72 \%$ yield $(78.8 \mathrm{mg}) .{ }^{1} \mathrm{H}$ NMR $\left(400 \mathrm{MHz}, \mathrm{CDCl}_{3}\right) \delta 7.58-7.46(\mathrm{~m}, 4 \mathrm{H}), 7.27-7.18(\mathrm{~m}, 6 \mathrm{H}), 7.17-$ $7.06(\mathrm{~m}, 6 \mathrm{H}), 6.93(\mathrm{t}, J=7.2 \mathrm{~Hz}, 1 \mathrm{H}), 4.69(\mathrm{~d}, J=15.2 \mathrm{~Hz}, 2 \mathrm{H}), 4.43$ (d, $J=15.2 \mathrm{~Hz}, 2 \mathrm{H}) .{ }^{13} \mathrm{C}$ NMR $\left(101 \mathrm{MHz}, \mathrm{CDCl}_{3}\right) \delta 148.4,144.3,142.4,140.6,136.5,135.4,131.8,129.1,128.6,128.2,127.0,123.6$, 121.7, 117.8, 57.6. 
<smiles></smiles>

1-Ethyl-4-iodobenzene 2 ac (46.4 $\mathrm{mg}, 0.20 \mathrm{mmol}$ ) was used, the desired product 3t was obtained as a white solid in $86 \%$ yield ( $85.5 \mathrm{mg}$ ); 1-bromo-4-ethylbenzene 2 ad (36.8 $\mathrm{mg}, 0.2 \mathrm{mmol}$ ) was used, the desired product $3 \mathrm{t}$ was obtained in $79 \%$ yield $(78.5 \mathrm{mg}) .{ }^{1} \mathrm{H}$ NMR $\left(400 \mathrm{MHz}, \mathrm{CDCl}_{3}\right) \delta 7.56(\mathrm{~d}, \mathrm{~J}=7.4$ $\mathrm{Hz}, 2 \mathrm{H}), 7.29-7.16(\mathrm{~m}, 10 \mathrm{H}), 7.13-7.04(\mathrm{~m}, 4 \mathrm{H}), 6.90$ (t, J = 7.2 Hz, 1H), 4.68 (d, J = 15.1 Hz, 2H), $4.42(\mathrm{~d}, \mathrm{~J}=15.1 \mathrm{~Hz}, 2 \mathrm{H}), 2.71(\mathrm{q}, \mathrm{J}=7.6 \mathrm{~Hz}, 2 \mathrm{H}), 1.29$ (t, J = 7.6 Hz, 3H). ${ }^{13} \mathrm{C}$ NMR (101 MHz, CDCl3) $\delta 148.6,144.7,144.3,139.6,139.0,136.6,135.8,129.1,128.4,128.3,128.0,126.9,121.2,117.5,57.5$, 29.0, 15.5.

\section{2-(4-isopropylphenyl)-6-phenyl-5,6,7,12-tetrahydrodibenzo $[c, f][1,5]$ azastibocine (3u)}<smiles>CC(C)c1ccc(S(=O)(=O)c2ccccc2CN2Cc3ccccc3P2c2ccccc2)cc1</smiles>

1-Iodo-4-isopropylbenzene $\mathbf{2 a e}(49.2 \mathrm{mg}, 0.20 \mathrm{mmol})$ was used, the desired product $\mathbf{3} \mathbf{u}$ was obtained as a white solid in 90\% yield (92.0 mg); 1-bromo-4-isopropylbenzene 2af (39.6 mg, $0.2 \mathrm{mmol}$ ) was used, the desired product $3 \mathbf{u}$ was obtained in $83 \%$ yield $(84.8 \mathrm{mg}) .{ }^{1} \mathrm{H}$ NMR $\left(400 \mathrm{MHz}, \mathrm{CDCl}_{3}\right) \delta 7.67(\mathrm{~d}, J=$ $7.7 \mathrm{~Hz}, 2 \mathrm{H}), 7.37(\mathrm{~d}, J=7.7 \mathrm{~Hz}, 2 \mathrm{H}), 7.35-7.26(\mathrm{~m}, 8 \mathrm{H}), 7.24-7.15(\mathrm{~m}, 4 \mathrm{H}), 7.01(\mathrm{t}, J=7.3 \mathrm{~Hz}, 1 \mathrm{H})$, $4.79(\mathrm{~d}, J=15.1 \mathrm{~Hz}, 2 \mathrm{H}), 4.52(\mathrm{~d}, J=15.1 \mathrm{~Hz}, 2 \mathrm{H}), 3.12-2.97(\mathrm{~m}, 1 \mathrm{H}), 1.41(\mathrm{~d}, J=6.9 \mathrm{~Hz}, 6 \mathrm{H}) .{ }^{13} \mathrm{C}$ NMR $\left(101 \mathrm{MHz}, \mathrm{CDCl}_{3}\right) \delta 149.3,148.6,144.3,139.6,138.9,136.7,135.7,129.1,128.3,128.0,127.0$, $126.9,121.2,117.4,57.5,34.2,24.1$.

\section{2-(4-(tert-butyl)phenyl)-6-phenyl-5,6,7,12-tetrahydrodibenzo $[c, f][1,5]$ azastibocine $(3 \mathrm{v})$}

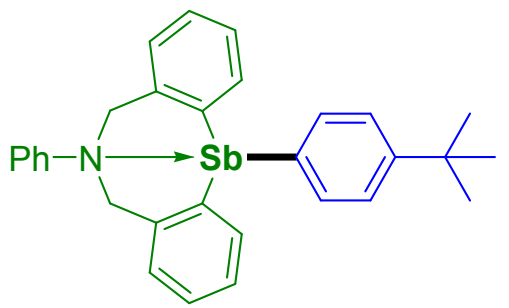

1-(Tert-butyl)-4-iodobenzene $2 \mathbf{a g}$ ( $52.0 \mathrm{mg}, 0.20 \mathrm{mmol})$ was used, the desired product $\mathbf{3 v}$ was obtained as a white solid in $85 \%$ yield $(89.2 \mathrm{mg})$; 1-bromo-4-(tert-butyl)benzene 2 ah (42.4 $\mathrm{mg}, 0.2 \mathrm{mmol})$ was used, the desired product $3 \mathbf{v}$ was obtained in $73 \%$ yield $(76.7 \mathrm{mg}) .{ }^{1} \mathrm{H}$ NMR $\left(400 \mathrm{MHz}, \mathrm{CDCl}_{3}\right) \delta 7.57$ $(\mathrm{d}, J=7.4 \mathrm{~Hz}, 2 \mathrm{H}), 7.42(\mathrm{~d}, J=7.4 \mathrm{~Hz}, 2 \mathrm{H}), 7.29-7.15(\mathrm{~m}, 8 \mathrm{H}), 7.14-7.03(\mathrm{~m}, 4 \mathrm{H}), 6.90(\mathrm{t}, J=7.0$ $\mathrm{Hz}, 1 \mathrm{H}), 4.68(\mathrm{~d}, J=15.1 \mathrm{~Hz}, 2 \mathrm{H}), 4.42(\mathrm{~d}, J=15.1 \mathrm{~Hz}, 2 \mathrm{H}), 1.37(\mathrm{~s}, 9 \mathrm{H}) .{ }^{13} \mathrm{C} \mathrm{NMR}\left(101 \mathrm{MHz}, \mathrm{CDCl}_{3}\right)$ 
$\delta 151.6,148.6,144.3,139.2,138.7,136.7,135.7,129.1,128.3,128.0,126.9,125.8,121.2,117.4,57.5$, 34.9, 31.5.

12-(4-butylphenyl)-6-phenyl-5,6,7,12-tetrahydrodibenzo[c, $f$ ][1,5] azastibocine (3w)

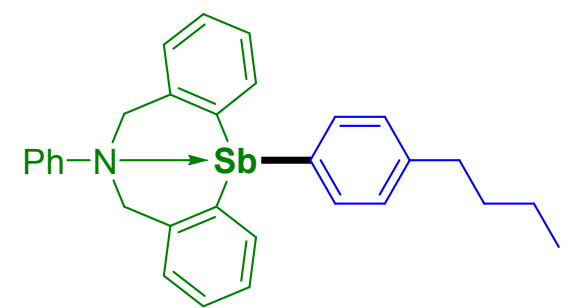

1-Butyl-4-iodobenzene $2 \mathbf{a i}(52.0 \mathrm{mg}, 0.20 \mathrm{mmol}$ ) was used, the desired product $\mathbf{3} \mathbf{w}$ was obtained as a white solid in $84 \%$ yield ( $88.2 \mathrm{mg}$ ); 1-butyl-4-bromobenzene 2 aj $(42.4 \mathrm{mg}, 0.20 \mathrm{mmol}$ ) was used, the product $3 \mathbf{w}$ was obtained in $70 \%$ yield $(73.5 \mathrm{mg}) .{ }^{1} \mathrm{H}$ NMR $\left(400 \mathrm{MHz}, \mathrm{CDCl}_{3}\right) \delta 7.65(\mathrm{~d}, J=7.3 \mathrm{~Hz}$, 2H), $7.37-7.25$ (m, 10H), $7.22-7.14(\mathrm{~m}, 4 \mathrm{H}), 7.00(\mathrm{t}, J=7.2 \mathrm{~Hz}, 1 \mathrm{H}), 4.78$ (d, $J=15.1 \mathrm{~Hz}, 2 \mathrm{H}), 4.52$ (d, $J=15.1 \mathrm{~Hz}, 2 \mathrm{H}), 2.76$ (t, $J=7.8 \mathrm{~Hz}, 2 \mathrm{H}), 1.84-1.68(\mathrm{~m}, 2 \mathrm{H}), 1.56-1.45$ (m, 2H), 1.06 (t, $J=7.3$ $\mathrm{Hz}, 3 \mathrm{H}) .{ }^{13} \mathrm{C} \mathrm{NMR}\left(101 \mathrm{MHz}, \mathrm{CDCl}_{3}\right) \delta 148.6,144.4,143.5,139.5,138.9,136.7,135.8,129.1,129.0$, $128.4,128.1,126.9,121.3,117.5,57.5,35.9,33.8,22.8,14.2$.

\section{2-([1,1'-biphenyl]-4-yl)-6-phenyl-5,6,7,12-tetrahydrodibenzo $[c, f][1,5]$ azastibocine (3x)}<smiles>c1ccc(-c2ccc([Sb]3(c4ccccc4)Cc4ccccc4CN(c4ccccc4)C3)cc2)cc1</smiles>

4-Iodo-1,1'-biphenyl $\mathbf{2 a k}(56.2 \mathrm{mg}, 0.20 \mathrm{mmol}$ ) was used, the desired product $3 \mathbf{x}$ was obtained as a white solid in $74 \%$ yield $(80.7 \mathrm{mg}) .{ }^{1} \mathrm{H}$ NMR $\left(400 \mathrm{MHz}, \mathrm{CDCl}_{3}\right) \delta 7.91(\mathrm{~d}, J=7.4 \mathrm{~Hz}, 2 \mathrm{H}), 7.83(\mathrm{t}, J=7.8 \mathrm{~Hz}$, 4H), 7.60 (t, $J=7.5 \mathrm{~Hz}, 2 \mathrm{H}), 7.53-7.33(\mathrm{~m}, 9 \mathrm{H}), 7.30-7.24(\mathrm{~m}, 4 \mathrm{H}), 7.08(\mathrm{t}, J=7.2 \mathrm{~Hz}, 1 \mathrm{H}), 4.85$ (d, $J=15.2 \mathrm{~Hz}, 2 \mathrm{H}), 4.59$ (d, $J=15.2 \mathrm{~Hz}, 2 \mathrm{H}) .{ }^{13} \mathrm{C} \mathrm{NMR}\left(101 \mathrm{MHz}, \mathrm{CDCl}_{3}\right) \delta 148.5,144.3,142.2,141.2$, 141.1, 139.4, 136.6, 135.6, 129.1, 128. 9, 128.4, 128.1, 127.5, 127.3, 127.2, 126.9, 121.4, 117.6, 57.5.

\section{4-(6-phenyl-6,7-dihydrodibenzo[c,f][1,5]azastibocin-12(5H)-yl)benzonitrile (3y)}<smiles></smiles>

4-Iodobenzonitrile $\mathbf{2 b}$ ( $45.8 \mathrm{mg}, 0.20 \mathrm{mmol}$ ) was used, the desired product $\mathbf{3 y}$ was obtained as a white solid in 94\% yield (92.8 mg); 4-bromobenzonitrile $2 \mathrm{am}$ (36.2 $\mathrm{mg}, 0.20 \mathrm{mmol})$ was used, the desired product $3 \mathbf{y}$ was obtained in $88 \%$ yield $(86.9 \mathrm{mg})$; 4-chlorobenzonitrile $2 \mathbf{c v}(27.4 \mathrm{mg}, 0.20 \mathrm{mmol}$ ) was 
used, the product $3 \mathbf{y}$ was obtained in $78 \%$ yield $(77.1 \mathrm{mg}) .{ }^{1} \mathrm{H}$ NMR $\left(400 \mathrm{MHz}, \mathrm{CDCl}_{3}\right) \delta 7.77(\mathrm{~d}, J=$ $6.7 \mathrm{~Hz}, 2 \mathrm{H}), 7.62$ (d, $J=6.8 \mathrm{~Hz}, 2 \mathrm{H}), 7.31-7.18(\mathrm{~m}, 6 \mathrm{H}), 7.11(\mathrm{~d}, J=5.8 \mathrm{~Hz}, 4 \mathrm{H}), 7.03(\mathrm{~d}, J=7.4 \mathrm{~Hz}$, 2H), $6.95(\mathrm{t}, J=7.2 \mathrm{~Hz}, 1 \mathrm{H}), 4.69$ (d, $J=15.2 \mathrm{~Hz}, 2 \mathrm{H}), 4.44(\mathrm{~d}, J=15.2 \mathrm{~Hz}, 2 \mathrm{H}) .{ }^{13} \mathrm{C}$ NMR $(101 \mathrm{MHz}$, $\left.\mathrm{CDCl}_{3}\right) \delta$ 152.0, 148.1, 144.3, 139.5, 136.3, 135.0, 131.5, 129.1, 128.7, 128.3, 127.1, 122.0, 119.3, 118.0, $112.2,57.7$.

\section{2-(6-phenyl-6,7-dihydrodibenzo[ $[c, f][1,5]$ azastibocin-12(5H)-yl)benzonitrile (3z)}

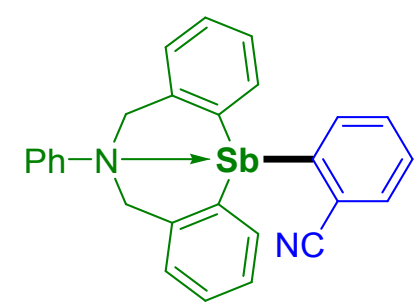

2-Iodobenzonitrile $2 \mathbf{a n}$ ( $45.8 \mathrm{mg}, 0.20 \mathrm{mmol}$ ) was used, the desired product $\mathbf{3 z}$ was obtained as a white solid in $78 \%$ yield $(78.5 \mathrm{mg}) .{ }^{1} \mathrm{H}$ NMR $(400 \mathrm{MHz}$, Chloroform- $d$ ) $\delta 7.80(\mathrm{dd}, J=19.4,7.3 \mathrm{~Hz}, 2 \mathrm{H}), 7.63$ - 7.52 (m, 2H), $7.36-7.28(\mathrm{~m}, 6 \mathrm{H}), 7.22(\mathrm{~d}, J=8.1 \mathrm{~Hz}, 2 \mathrm{H}), 7.19-7.13(\mathrm{~m}, 2 \mathrm{H}), 7.08$ (d, $J=7.4 \mathrm{~Hz}$, 2H), $7.03(\mathrm{t}, J=7.2 \mathrm{~Hz}, 1 \mathrm{H}), 4.77$ (d, $J=15.2 \mathrm{~Hz}, 2 \mathrm{H}), 4.54$ (d, $J=15.2 \mathrm{~Hz}, 2 \mathrm{H}) .{ }^{13} \mathrm{C}$ NMR $(101 \mathrm{MHz}$, $\left.\mathrm{CDCl}_{3}\right) \delta 150.6,148.1,144.4,140.2,136.4,135.5,133.5,132.2,129.1,129.0,128.7,128.2,127.1,122.4$, 122.3, 119.9, 118.7, 58.0. HRMS-ESI (m/z) $[\mathrm{M}+\mathrm{H}]^{+}$Calcd for $\mathrm{C}_{27} \mathrm{H}_{22} \mathrm{~N}_{2} \mathrm{Sb}^{+}, 495.0816$, Found, 495.0812.

6-phenyl-12-(4-vinylphenyl)-5,6,7,12-tetrahydrodibenzo[c,f][1,5]azastibocine (3aa)

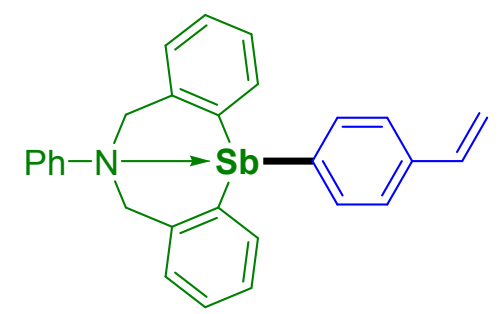

1-Iodo-4-vinylbenzene $\mathbf{2 a o}(46.2 \mathrm{mg}, 0.20 \mathrm{mmol})$ was used, the desired product 3aa was obtained as a white solid in $82 \%$ yield ( $81.2 \mathrm{mg}$ ); 1-bromo-4-vinylbenzene $2 \mathrm{ap}$ (36.4 $\mathrm{mg}, 0.20 \mathrm{mmol}$ ) was used, the desired product 3aa was obtained in $69 \%$ yield $(68.3 \mathrm{mg}){ }^{1} \mathrm{H}$ NMR $(400 \mathrm{MHz}$, Chloroform- $d$ ) $\delta 7.63(\mathrm{~d}$, $J=7.5 \mathrm{~Hz}, 2 \mathrm{H}), 7.45$ (d, $J=7.5 \mathrm{~Hz}, 2 \mathrm{H}), 7.27-7.18(\mathrm{~m}, 8 \mathrm{H}), 7.15-7.06(\mathrm{~m}, 4 \mathrm{H}), 6.92$ (t, $J=7.3 \mathrm{~Hz}$, 1H), $6.76(\mathrm{dd}, J=17.6,10.9 \mathrm{~Hz}, 1 \mathrm{H}), 5.84(\mathrm{~d}, J=17.6 \mathrm{~Hz}, 1 \mathrm{H}), 5.29$ (d, $J=10.9 \mathrm{~Hz}, 1 \mathrm{H}), 4.70(\mathrm{~d}, J=$ $15.2 \mathrm{~Hz}, 2 \mathrm{H}), 4.44(\mathrm{~d}, J=15.2 \mathrm{~Hz}, 2 \mathrm{H}) .{ }^{13} \mathrm{C} \mathrm{NMR}\left(101 \mathrm{MHz}, \mathrm{CDCl}_{3}\right) \delta 148.5,144.3,143.3,139.2$, 137.7, 137.1, 136.6, 135.6, 129.1, 128.4, 128.1, 126.9, 126.5, 121.4, 117.6, 114.3, 57.5.

6-phenyl-12-(4-(trimethylsilyl)phenyl)-5,6,7,12-tetrahydrodibenzo $[c, f][1,5]$ azastibocine (3ab) 


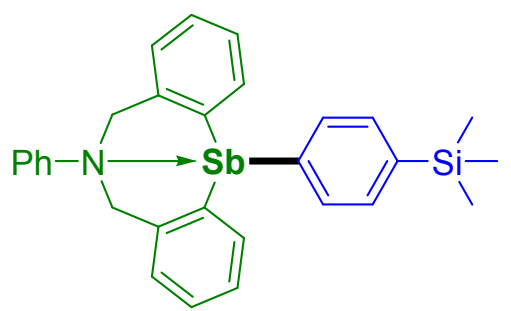

(4-Bromophenyl)trimethylsilane 2 as $(45.6 \mathrm{mg}, 0.20 \mathrm{mmol})$ was used, the desired product 3 ab was obtained as a white solid in $85 \%$ yield $(92.0 \mathrm{mg}) .{ }^{1} \mathrm{H}$ NMR $\left(400 \mathrm{MHz}, \mathrm{CDCl}_{3}\right) \delta 7.64(\mathrm{~d}, J=7.7 \mathrm{~Hz}, 2 \mathrm{H})$, $7.55(\mathrm{~d}, J=7.7 \mathrm{~Hz}, 2 \mathrm{H}), 7.27-7.19(\mathrm{~m}, 8 \mathrm{H}), 7.13-7.08(\mathrm{~m}, 4 \mathrm{H}), 6.92(\mathrm{t}, J=7.3 \mathrm{~Hz}, 1 \mathrm{H}), 4.71(\mathrm{~d}, J=$ $15.1 \mathrm{~Hz}, 2 \mathrm{H}), 4.44(\mathrm{~d}, J=15.1 \mathrm{~Hz}, 2 \mathrm{H}), 0.32(\mathrm{~s}, 9 \mathrm{H}) .{ }^{13} \mathrm{C} \mathrm{NMR}\left(101 \mathrm{MHz}, \mathrm{CDCl}_{3}\right) \delta 148.6,144.4,143.9$, $140.8,138.2,136.7,135.7,133.5,129.1,128.4,128.1,126.9,121.4,117.6,57.6,-0.9$.

\section{1-(4-(6-phenyl-6,7-dihydrodibenzo[c,f][1,5] azastibocin-12(5H)-yl)phenyl)ethan-1-one (3ac)}<smiles></smiles>

1-(4-Iodophenyl)ethan-1-one $\mathbf{2 a v}(49.2 \mathrm{mg}, 0.20 \mathrm{mmol})$ was used, the desired product 3ac was obtained as a white solid in $76 \%$ yield $(77.6 \mathrm{mg}) .{ }^{1} \mathrm{H} \mathrm{NMR}\left(400 \mathrm{MHz}, \mathrm{CDCl}_{3}\right) \delta 8.04(\mathrm{~d}, J=7.6 \mathrm{~Hz}, 2 \mathrm{H}), 7.87(\mathrm{~d}$, $J=7.7 \mathrm{~Hz}, 2 \mathrm{H}), 7.36-7.27(\mathrm{~m}, 6 \mathrm{H}), 7.23-7.13(\mathrm{~m}, 6 \mathrm{H}), 7.02(\mathrm{t}, J=7.2 \mathrm{~Hz}, 1 \mathrm{H}), 4.78(\mathrm{~d}, J=15.2 \mathrm{~Hz}$, 2H), 4.52 (d, $J=15.2 \mathrm{~Hz}, 2 \mathrm{H}), 2.72$ (s, 3H). ${ }^{13} \mathrm{C} \mathrm{NMR}\left(101 \mathrm{MHz}, \mathrm{CDCl}_{3}\right) \delta 198.8,151.6,148.3,144.3$, $139.2,137.1,136.4,135.2,129.1,128.6,128.2,127.9,127.0,121.7,117.8,57.6,26.8$.

6-phenyl-12-(4-((trimethylsilyl)ethynyl)phenyl)-5,6,7,12-tetrahydrodibenzo $[c, f][1,5]$ azastibocine (3ad)<smiles></smiles>

((4-Iodophenyl)ethynyl)trimethylsilane $2 \mathbf{a q}(60.0 \mathrm{mg}, 0.20 \mathrm{mmol})$ was used, the desired product $\mathbf{3 a d}$ was obtained as a white solid in 92\% yield (103.9 mg); ((4-bromophenyl)ethynyl)trimethylsilane 2ar $(50.4 \mathrm{mg}, 0.20 \mathrm{mmol})$ was used, the product 3ad was obtained in 84\% yield $(94.9 \mathrm{mg})$. M.P.: 195.1 $198.2{ }^{\circ} \mathrm{C} .{ }^{1} \mathrm{H}$ NMR $\left(400 \mathrm{MHz}, \mathrm{CDCl}_{3}\right) \delta 7.71(\mathrm{~d}, J=7.7 \mathrm{~Hz}, 2 \mathrm{H}), 7.61(\mathrm{~d}, J=7.7 \mathrm{~Hz}, 2 \mathrm{H}), 7.36-7.28$ (m, 6H), $7.24(\mathrm{~d}, J=7.2 \mathrm{~Hz}, 2 \mathrm{H}), 7.19$ (d, $J=7.8 \mathrm{~Hz}, 4 \mathrm{H}), 7.02(\mathrm{t}, J=7.3 \mathrm{~Hz}, 1 \mathrm{H}), 4.78(\mathrm{~d}, J=15.2 \mathrm{~Hz}$, $2 \mathrm{H}), 4.52$ (d, $J=15.2 \mathrm{~Hz}, 2 \mathrm{H}), 0.38(\mathrm{~s}, 9 \mathrm{H}) .{ }^{13} \mathrm{C} \mathrm{NMR}\left(101 \mathrm{MHz}, \mathrm{CDCl}_{3}\right) \delta 148.4,145.0,144.3,138.8$, 136.6, 135.4, 131.9, 129.1, 128.5, 128.1, 126.9, 123.1, 121.6, 117.7, 105.4, 95.0, 57.6, 0.2. HRMS-ESI $(\mathrm{m} / \mathrm{z})[\mathrm{M}+\mathrm{H}]^{+}$Calcd for $\mathrm{C}_{31} \mathrm{H}_{31} \mathrm{NSbSi}^{+}, 566.1258$, Found, 566.1265. 


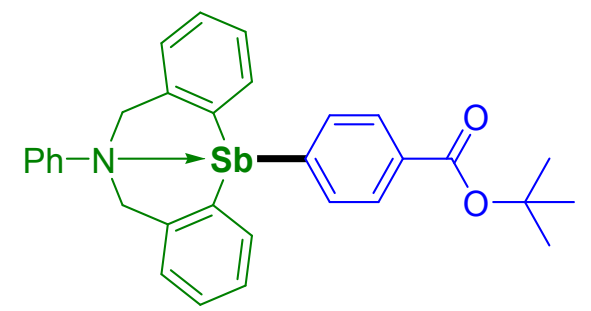

Tert-butyl 4-iodobenzoate $\mathbf{2 a u}(60.8 \mathrm{mg}, 0.20 \mathrm{mmol})$ was used, the desired product 3ae was obtained as a white solid in 86\% yield (97.8 mg). ${ }^{1} \mathrm{H}$ NMR $\left(400 \mathrm{MHz}, \mathrm{CDCl}_{3}\right) \delta 8.04(\mathrm{~d}, J=7.8 \mathrm{~Hz}, 2 \mathrm{H}), 7.78(\mathrm{~d}, J$ $=7.8 \mathrm{~Hz}, 2 \mathrm{H}), 7.32-7.23(\mathrm{~m}, 6 \mathrm{H}), 7.20-7.08(\mathrm{~m}, 6 \mathrm{H}), 6.98(\mathrm{t}, J=7.2 \mathrm{~Hz}, 1 \mathrm{H}), 4.75(\mathrm{~d}, J=15.2 \mathrm{~Hz}$, 2H), 4.49 (d, $J=15.2 \mathrm{~Hz}, 2 \mathrm{H}), 1.66$ (s, 9H). ${ }^{13} \mathrm{C} \mathrm{NMR}\left(101 \mathrm{MHz}, \mathrm{CDCl}_{3}\right) \delta 166.3,150.2,148.4,144.3$, $138.9,136.6,135.3,132.2,129.2,129.1,128.6,128.2,127.0,121.7,117.8,81.1,57.6,28.4$.

\section{Ethyl 4-(6-phenyl-6,7-dihydrodibenzo[c,f][1,5] azastibocin-12(5H)-yl)benzoate (3af)}<smiles></smiles>

Ethyl 4-iodobenzoate 2at (55.2 $\mathrm{mg}, 0.20 \mathrm{mmol})$ was used, the desired product 3af was obtained as a white solid in $84 \%$ yield $(90.9 \mathrm{mg})$ M.P.: $180.3-182.1{ }^{\circ} \mathrm{C} .{ }^{1} \mathrm{H}$ NMR $\left(400 \mathrm{MHz}, \mathrm{CDCl}_{3}\right) \delta 8.16(\mathrm{~d}, J=$ $7.6 \mathrm{~Hz}, 2 \mathrm{H}), 7.86(\mathrm{~d}, J=7.6 \mathrm{~Hz}, 2 \mathrm{H}), 7.36-7.28(\mathrm{~m}, 6 \mathrm{H}), 7.24-7.15(\mathrm{~m}, 6 \mathrm{H}), 7.03(\mathrm{t}, J=7.2 \mathrm{~Hz}, 1 \mathrm{H})$, $4.80(\mathrm{~d}, J=15.2 \mathrm{~Hz}, 2 \mathrm{H}), 4.57-4.47(\mathrm{~m}, 4 \mathrm{H}), 1.51(\mathrm{t}, J=7.1 \mathrm{~Hz}, 3 \mathrm{H}) .{ }^{13} \mathrm{C} \mathrm{NMR}\left(101 \mathrm{MHz}, \mathrm{CDCl}_{3}\right) \delta$ 167.1, 150.9, 148.4, 144.3, 139.0, 136.5, 135.3, 130.6, 129.2, 129.1, 128.6, 128.2, 127.0, 121.7, 117.8, 61.1, 57.6, 14.5. HRMS-ESI (m/z) [M+Na] $]^{+}$Calcd for $\mathrm{C}_{29} \mathrm{H}_{26} \mathrm{NNaO}_{2} \mathrm{Sb}^{+}$, 564.0894, Found, 564.0886.

\section{2-(3-((5-(4-fluorophenyl)thiophen-2-yl)methyl)-4-methylphenyl)-6-phenyl-5,6,7,12-tetrahydrodi} benzo $[c, f][1,5]$ azastibocine (3ag)<smiles></smiles>

2-(4-Fluorophenyl)-5-(5-iodo-2-methylbenzyl)thiophene 2ay (81.6 mg, $0.20 \mathrm{mmol}$ ) was used, the desired product $3 \mathbf{a g}$ was obtained as a white solid in $82 \%$ yield $(110.4 \mathrm{mg})$. M.P.: $180.3-183.4{ }^{\circ} \mathrm{C} .{ }^{1} \mathrm{H}$ NMR (400 MHz, $\left.\mathrm{CDCl}_{3}\right) \delta 7.59(\mathrm{~s}, 1 \mathrm{H}), 7.57-7.51(\mathrm{~m}, 3 \mathrm{H}), 7.35-7.27(\mathrm{~m}, 9 \mathrm{H}), 7.18-7.12(\mathrm{~m}, 4 \mathrm{H})$, $7.11-7.05(\mathrm{~m}, 3 \mathrm{H}), 6.99(\mathrm{t}, J=7.2 \mathrm{~Hz}, 1 \mathrm{H}), 6.75(\mathrm{~d}, J=3.5 \mathrm{~Hz}, 1 \mathrm{H}), 4.78(\mathrm{~d}, J=15.2 \mathrm{~Hz}, 2 \mathrm{H}), 4.51(\mathrm{~d}$, $J=15.2 \mathrm{~Hz}, 2 \mathrm{H}), 4.19$ (s, 2H), 2.46 (s, 3H). ${ }^{13} \mathrm{C} \mathrm{NMR}\left(101 \mathrm{MHz}, \mathrm{CDCl}_{3}\right) \delta 162.2(\mathrm{~d}, J=246.5 \mathrm{~Hz})$, 148.6, 144.4, 144.1, 141.5, 140.6, 140.1, 138.7, 137.8, 137.0, 136.7, 135.8, 131.1(d, J=3.4 Hz), 130.9, 
129.1, 128.4, 128.1, $127.2(\mathrm{~d}, J=7.9 \mathrm{~Hz}), 126.9,125.9,122.7,121.3,117.5,115.8(\mathrm{~d}, J=21.7 \mathrm{~Hz}), 57.5$, 34.3, 19.6. ${ }^{19} \mathrm{~F}$ NMR $\left(376 \mathrm{MHz}, \mathrm{CDCl}_{3}\right) \delta$-115.27. HRMS-ESI $(\mathrm{m} / \mathrm{z})[\mathrm{M}+\mathrm{H}]^{+}$Calcd for $\mathrm{C}_{38} \mathrm{H}_{32} \mathrm{FNSSb}^{+}$, 674.1272, Found, 674.1269.

12-(9H-fluoren-2-yl)-6-phenyl-5,6,7,12-tetrahydrodibenzo[c,f][1,5] azastibocine (3ah)<smiles>c1ccc([Sb]2(c3ccccc3)CN3Cc4ccccc4C2c2ccccc23)cc1</smiles>

2-Bromo-9H-fluorene 2 be $(48.8 \mathrm{mg}, 0.20 \mathrm{mmol})$ was used, the desired product 3ah was obtained as a white solid in $87 \%$ yield $(96.9 \mathrm{mg})$. M.P.: $235.6-237.2^{\circ} \mathrm{C} .{ }^{1} \mathrm{H}$ NMR $\left(400 \mathrm{MHz}, \mathrm{CDCl}_{3}\right) \delta 7.90-7.78$ (m, 3H), $7.67(\mathrm{~d}, J=7.4 \mathrm{~Hz}, 1 \mathrm{H}), 7.56(\mathrm{~d}, J=7.4 \mathrm{~Hz}, 1 \mathrm{H}), 7.39$ (t, $J=7.4 \mathrm{~Hz}, 1 \mathrm{H}), 7.32$ (t, $J=7.3 \mathrm{~Hz}$, 1H), $7.28-7.20(\mathrm{~m}, 8 \mathrm{H}), 7.15-7.06(\mathrm{~m}, 4 \mathrm{H}), 6.93(\mathrm{t}, J=7.3 \mathrm{~Hz}, 1 \mathrm{H}), 4.72(\mathrm{~d}, J=15.1 \mathrm{~Hz}, 2 \mathrm{H}), 4.46$ $(\mathrm{d}, J=15.1 \mathrm{~Hz}, 2 \mathrm{H}), 3.92(\mathrm{~s}, 2 \mathrm{H}) .{ }^{13} \mathrm{C} \mathrm{NMR}\left(101 \mathrm{MHz}, \mathrm{CDCl}_{3}\right) \delta 148.6,144.4,143.6,143.5,142.3$, 141.9, 141.8, 137.3, 136.7, 135.9, 135.5, 129.1, 128.4, 128.1, 127.1, 127.0, 126.9, 125.3, 121.4, 120.2, 120.1, 117.6, 57.6, 37.0. HRMS-ESI (m/z) $[\mathrm{M}+\mathrm{H}]^{+}$Calcd for $\mathrm{C}_{33} \mathrm{H}_{27} \mathrm{NSb}^{+}$, 558.1176, Found, 558.1183.

12-(naphthalen-1-yl)-6-phenyl-5,6,7,12-tetrahydrodibenzo[ $c, f][1,5]$ azastibocine (3ai)

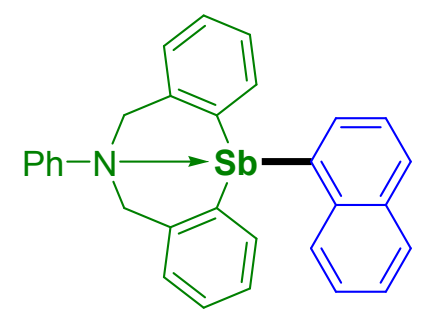

1-Iodonaphthalene $\mathbf{2 b g}$ (50.8 $\mathrm{mg}, 0.20 \mathrm{mmol}$ ) was used, the desired product 3ai was obtained as a white solid in $85 \%$ yield $(88.2 \mathrm{mg})$; 1-bromonaphthalene $2 \mathbf{b h}(41.2 \mathrm{mg}, 0.20 \mathrm{mmol})$ was used, the desired product 3ai was obtained in $82 \%$ yield $(85.1 \mathrm{mg}) .{ }^{1} \mathrm{H}$ NMR $\left(400 \mathrm{MHz}, \mathrm{CDCl}_{3}\right) \delta 7.89$ (dd, $J=8.2 \mathrm{~Hz}$, $17.3 \mathrm{~Hz}, 3 \mathrm{H}), 7.58-7.31$ (m, 3H), $7.30-7.19$ (m, 9H), 7.09 (s, 2H), $6.94(\mathrm{t}, J=7.1 \mathrm{~Hz}, 3 \mathrm{H}), 4.75$ (d, $J$ $=15.2 \mathrm{~Hz}, 2 \mathrm{H}), 4.54(\mathrm{~d}, J=15.2 \mathrm{~Hz}, 2 \mathrm{H}) .{ }^{13} \mathrm{C} \mathrm{NMR}\left(101 \mathrm{MHz}, \mathrm{CDCl}_{3}\right) \delta 148.5,144.2,139.7,137.3$, 135.3, 133.9, 129.1, 129.0, 128.4, 128.0, 126.9, 126.1, 125.8, 125.6, 121.6, 118.2, 57.9.

6-phenyl-12-(pyren-4-yl)-5,6,7,12-tetrahydrodibenzo[c,f][1,5]azastibocine (3aj)<smiles>C1=Cc2cccc3c2C=CC1=C1C(=C3)[Sb](c2ccccc2)c2ccccc2CN1c1ccccc1</smiles> 
4-Bromopyrene $2 \mathbf{b f}(56.2 \mathrm{mg}, 0.20 \mathrm{mmol}$ ) was used, the desired product 3aj was obtained as a white solid in 74\% yield (87.8 mg). ${ }^{1} \mathrm{H}$ NMR $(400 \mathrm{MHz}$, Chloroform- $d$ ) $\delta 9.18-8.55(\mathrm{~m}, 1 \mathrm{H}), 8.47-8.25(\mathrm{~m}$, 1H), $8.23-8.08(\mathrm{~m}, 5 \mathrm{H}), 8.03-7.96(\mathrm{~m}, 1 \mathrm{H}), 7.36-7.16(\mathrm{~m}, 9 \mathrm{H}), 7.06(\mathrm{~d}, J=7.5 \mathrm{~Hz}, 2 \mathrm{H}), 7.04-6.69$ $(\mathrm{m}, 3 \mathrm{H}), 4.80(\mathrm{~d}, J=15.2 \mathrm{~Hz}, 2 \mathrm{H}), 4.59$ (d, $J=15.4 \mathrm{~Hz}, 2 \mathrm{H}) .{ }^{13} \mathrm{C} \mathrm{NMR}\left(101 \mathrm{MHz}, \mathrm{CDCl}_{3}\right) \delta 148.5$, $138.6,137.3$, 135.5, 131.5, 131.2, 129.1, 128.5, 128.1, 128.0, 127.8, 127.0, 125.9, 125.2, 125.1, 125.0, $121.7,118.2,57.9$

\section{2-(2,3-dihydrobenzofuran-6-yl)-6-phenyl-5,6,7,12-tetrahydrodibenzo $[c, f][1,5]$ azastibocine (3ak)}<smiles></smiles>

6-Iodo-2,3-dihydrobenzofuran $\mathbf{2} \mathbf{b m}(49.2 \mathrm{mg}, 0.20 \mathrm{mmol})$ was used, the desired product 3 ak was obtained as a white solid in 90\% yield (92.0 mg); 6-bromo-2,3-dihydrobenzofuran 2 bn (39.6 mg, 0.20 mmol) was used, the desired product 3ak was obtained in $78 \%$ yield (79.7 mg). M.P.: $190.0-192.1^{\circ} \mathrm{C}$. ${ }^{1} \mathrm{H}$ NMR $\left(400 \mathrm{MHz}, \mathrm{CDCl}_{3}\right) \delta 7.54(\mathrm{~s}, 1 \mathrm{H}), 7.46(\mathrm{~d}, J=7.8 \mathrm{~Hz}, 1 \mathrm{H}), 7.35-7.26(\mathrm{~m}, 8 \mathrm{H}), 7.22-7.11$ (m, 4H), $7.04-6.89(\mathrm{~m}, 2 \mathrm{H}), 4.77$ (d, $J=15.1 \mathrm{~Hz}, 2 \mathrm{H}), 4.67(\mathrm{t}, J=8.6 \mathrm{~Hz}, 2 \mathrm{H}), 4.51$ (d, $J=15.1 \mathrm{~Hz}$, $2 \mathrm{H}), 3.29(\mathrm{t}, J=8.7 \mathrm{~Hz}, 2 \mathrm{H}) .{ }^{13} \mathrm{C} \mathrm{NMR}\left(101 \mathrm{MHz}, \mathrm{CDCl}_{3}\right) \delta 160.9,148.6,144.4,138.9,136.6,136.1$, 135.3, 133.1, 129.1, 128.3, 128.0, 127.7, 126.9, 121.2, 117.5, 110.1, 71.2, 57.5, 29.7. HRMS-ESI (m/z) $[\mathrm{M}+\mathrm{H}]^{+}$Calcd for $\mathrm{C}_{28} \mathrm{H}_{25} \mathrm{NOSb}^{+}, 512.0969$, Found, 512.0972.

\section{2-(benzo[b]thiophen-2-yl)-6-phenyl-5,6,7,12-tetrahydrodibenzo[c,f][1,5] azastibocine (3al)}<smiles>c1ccc(N2Cc3ccccc3S(c3ccccc3)(c3ccccc3)C2)cc1</smiles>

2-Iodobenzo[b]thiophene $\mathbf{2} \mathbf{b q}(52.0 \mathrm{mg}, 0.20 \mathrm{mmol})$ was used, the desired product $\mathbf{3 a l}$ was obtained as a white solid in $84 \%$ yield $(88.2 \mathrm{mg})$; 2-bromobenzo[ $b]$ thiophene $2 \mathrm{br}$ (42.4 $\mathrm{mg}, 0.20 \mathrm{mmol}$ ) was used, the desired product 3 al was obtained in $72 \%$ yield $(75.6 \mathrm{mg}) .{ }^{1} \mathrm{H}$ NMR $\left(400 \mathrm{MHz}, \mathrm{CDCl}_{3}\right) \delta 7.98(\mathrm{~d}, J=$ $7.6 \mathrm{~Hz}, 1 \mathrm{H}), 7.92$ (d, J=7.5 Hz, 1H), $7.75(\mathrm{~s}, 1 \mathrm{H}), 7.54$ (d, J=7.5 Hz, 2H), $7.46-7.36$ (m, 2H), 7.35 $7.25(\mathrm{~m}, 6 \mathrm{H}), 7.20$ (d, $J=7.3 \mathrm{~Hz}, 4 \mathrm{H}), 7.03(\mathrm{t}, J=7.3 \mathrm{~Hz}, 1 \mathrm{H}), 4.77$ (d, $J=15.2 \mathrm{~Hz}, 2 \mathrm{H}), 4.52(\mathrm{~d}, J=$ $15.2 \mathrm{~Hz}, 2 \mathrm{H}) .{ }^{13} \mathrm{C} \mathrm{NMR}\left(101 \mathrm{MHz}, \mathrm{CDCl}_{3}\right) \delta 148.3,145.6,143.8,142.8,141.4,136.9,136.0,135.1$, $129.2,128.8,128.3,126.8,124.0,123.9,123.3,122.3,118.3,58.0$.

12-(dibenzo $[b, d]$ thiophen-3-yl)-6-phenyl-5,6,7,12-tetrahydrodibenzo $[c, f][1,5]$ azastibocine (3am) 
<smiles>c1ccc(Nc2ccccc2[Sb]2(c3ccccc3)CN3Cc4ccccc4[Sn]32)cc1</smiles>

3-Iododibenzo[ $[b, d]$ thiophene $\mathbf{2 b s}(62.0 \mathrm{mg}, 0.20 \mathrm{mmol})$ was used, the desired product $\mathbf{3 a m}$ was obtained as a white solid in $90 \%$ yield (103.5 mg); 3-bromodibenzo[b,d] thiophene $2 \mathbf{b t}(52.4 \mathrm{mg}, 0.20 \mathrm{mmol})$ was used, the desired product 3am was obtained in $84 \%$ yield $(96.6 \mathrm{mg})$. M.P.: $219.4-221.6{ }^{\circ} \mathrm{C} .{ }^{1} \mathrm{H}$ NMR $\left(400 \mathrm{MHz}, \mathrm{CDCl}_{3}\right) \delta 8.50(\mathrm{~s}, 1 \mathrm{H}), 8.08(\mathrm{~d}, J=7.4 \mathrm{~Hz}, 1 \mathrm{H}), 7.92-7.80(\mathrm{~m}, 2 \mathrm{H}), 7.70(\mathrm{~d}, J=7.9 \mathrm{~Hz}, 1 \mathrm{H})$, $7.46-7.36$ (m, 2H), $7.26-7.19(\mathrm{~m}, 8 \mathrm{H}), 7.13(\mathrm{~d}, J=8.1 \mathrm{~Hz}, 2 \mathrm{H}), 7.10-7.02(\mathrm{~m}, 2 \mathrm{H}), 6.94(\mathrm{t}, J=7.3$ $\mathrm{Hz}, 1 \mathrm{H}), 4.73(\mathrm{~d}, J=15.2 \mathrm{~Hz}, 2 \mathrm{H}), 4.47(\mathrm{~d}, J=15.2 \mathrm{~Hz}, 2 \mathrm{H}) .{ }^{13} \mathrm{C}$ NMR $\left(101 \mathrm{MHz}, \mathrm{CDCl}_{3}\right) \delta 148.5$, 144.4, 140.2, 139.3, 138.7, 136.8, 136.7, 136.0, 135.7, 135.6, 132.3, 129.1, 128.5, 128.2, 127.0, 126.8, 124.6, 123.1, 122.9, 121.8, 121.6, 117.8, 57.7. HRMS-ESI $(\mathrm{m} / \mathrm{z})[\mathrm{M}+\mathrm{H}]^{+}$Calcd for $\mathrm{C}_{32} \mathrm{H}_{25} \mathrm{NSSb}^{+}$, 576.0740, Found, 576.0741 .

\section{6-phenyl-12-(thiophen-3-yl)-5,6,7,12-tetrahydrodibenzo $[c, f][1,5]$ azastibocine (3an)}<smiles></smiles>

3-Iodothiophene $2 \mathbf{b o}$ ( $42.0 \mathrm{mg}, 0.20 \mathrm{mmol}$ ) was used, the desired product 3an was obtained as a white solid in $81 \%$ yield ( $77.0 \mathrm{mg}$ ); 3-bromothiophene $2 \mathbf{b p}$ ( $32.4 \mathrm{mg}, 0.20 \mathrm{mmol}$ ) was used, the desired product 3an was obtained in $63 \%$ yield $(59.9 \mathrm{mg}) .{ }^{1} \mathrm{H}$ NMR $\left(400 \mathrm{MHz}, \mathrm{CDCl}_{3}\right) \delta 7.73(\mathrm{~s}, 1 \mathrm{H}), 7.67-7.58(\mathrm{~m}$, 1H), $7.41-7.31(\mathrm{~m}, 9 \mathrm{H}), 7.25(\mathrm{t}, J=8.1 \mathrm{~Hz}, 4 \mathrm{H}), 7.07(\mathrm{t}, J=7.2 \mathrm{~Hz}, 1 \mathrm{H}), 4.84(\mathrm{~d}, J=15.2 \mathrm{~Hz}, 2 \mathrm{H})$, $4.57(\mathrm{~d}, J=15.2 \mathrm{~Hz}, 2 \mathrm{H}) .{ }^{13} \mathrm{C} \mathrm{NMR}\left(101 \mathrm{MHz}, \mathrm{CDCl}_{3}\right) \delta 148.4,144.0,139.8,136.5,135.4,135.1,134.9$, $129.1,128.4,128.1,126.8,126.1,121.5,117.6,57.5$.

12-(furan-3-yl)-6-phenyl-5,6,7,12-tetrahydrodibenzo[c,fl[1,5]azastibocine (3ao)<smiles></smiles>

3-Iodofuran $2 \mathbf{b i}$ ( $38.8 \mathrm{mg}, 0.20 \mathrm{mmol}$ ) was used, the desired product 3ao was obtained as a white solid in $84 \%$ yield ( $77.1 \mathrm{mg}$ ); 3-bromofuran $\mathbf{2 b j}$ ( $29.2 \mathrm{mg}, 0.20 \mathrm{mmol}$ ) was used, the desired product 3ao was obtained in $73 \%$ yield $(67.0 \mathrm{mg}) .{ }^{1} \mathrm{H}$ NMR $\left(400 \mathrm{MHz}, \mathrm{CDCl}_{3}\right) \delta 7.66(\mathrm{t}, J=1.5 \mathrm{~Hz}, 1 \mathrm{H}), 7.55-7.50(\mathrm{~m}$, 1H), $7.42-7.35(\mathrm{~m}, 2 \mathrm{H}), 7.28-7.19(\mathrm{~m}, 6 \mathrm{H}), 7.18-7.12(\mathrm{~m}, 2 \mathrm{H}), 7.12-7.07(\mathrm{~m}, 2 \mathrm{H}), 6.98-6.90(\mathrm{~m}$, 
$1 \mathrm{H}), 6.45-6.38(\mathrm{~m}, 1 \mathrm{H}), 4.71(\mathrm{~d}, J=15.2 \mathrm{~Hz}, 2 \mathrm{H}), 4.43(\mathrm{~d}, J=15.2 \mathrm{~Hz}, 2 \mathrm{H}) .{ }^{13} \mathrm{C}$ NMR $(101 \mathrm{MHz}$, $\left.\mathrm{CDCl}_{3}\right) \delta 150.6,148.6,143.9,143.5,136.5,134.5,129.2,128.5,128.1,126.8,121.6,117.6,115.9$, 57.6.

12-(4-(9H-carbazol-9-yl)phenyl)-6-phenyl-5,6,7,12-tetrahydrodibenzo[ $\left.c_{2} f\right][1,5]$ azastibocine (3ap)<smiles>c1ccc(N2Cc3ccccc3Sc3ccccc32)cc1</smiles>

9-(4-Bromophenyl)-9H-carbazole $2 \mathbf{a z}(64.2 \mathrm{mg}, 0.20 \mathrm{mmol})$ was used, the desired product 3ap was obtained as a white solid in $65 \%$ yield $(82.4 \mathrm{mg}) .{ }^{1} \mathrm{H} \mathrm{NMR}\left(400 \mathrm{MHz}, \mathrm{CDCl}_{3}\right) \delta 8.16(\mathrm{~d}, J=7.7 \mathrm{~Hz}, 2 \mathrm{H})$, 7.89 (d, $J=7.6 \mathrm{~Hz}, 2 \mathrm{H}), 7.59$ (dd, $J=7.9 \mathrm{~Hz}, 21.6 \mathrm{~Hz}, 4 \mathrm{H}), 7.45$ (t, $J=7.7 \mathrm{~Hz}, 2 \mathrm{H}), 7.33-7.23$ (m, $10 \mathrm{H}), 7.20$ (t, $J=7.3 \mathrm{~Hz}, 2 \mathrm{H}), 7.14$ (d, $J=8.2 \mathrm{~Hz}, 2 \mathrm{H}), 6.96$ (t, $J=7.3 \mathrm{~Hz}, 1 \mathrm{H}), 4.74$ (d, $J=15.2 \mathrm{~Hz}$, 2H), $4.48(\mathrm{~d}, J=15.2 \mathrm{~Hz}, 2 \mathrm{H}) .{ }^{13} \mathrm{C} \mathrm{NMR}\left(101 \mathrm{MHz}, \mathrm{CDCl}_{3}\right) \delta 148.5,144.4,142.8,140.9,140.4,138.2$, 136.6, 135.6, 129.2, 128.6, 128.3, 127.1, 127.0, 126.1, 123.6, 121.7, 120.5, 120.1, 117.8, 110.1, 57.7.

12-(9-methyl-9H-carbazol-3-yl)-6-phenyl-5,6,7,12-tetrahydrodibenzo $[c, f][1,5]$ azastibocine (3aq)<smiles></smiles>

3-Iodo-9-methyl-9H-carbazole 2aw $(61.4 \mathrm{mg}, 0.20 \mathrm{mmol})$ was used, the desired product 3aq was obtained as a white solid in $78 \%$ yield $(89.2 \mathrm{mg})$. M.P.: $248.7-251.3{ }^{\circ} \mathrm{C} .{ }^{1} \mathrm{H}$ NMR $(400 \mathrm{MHz}$, Chloroform- $d$ ) $\delta 8.35$ (s, 1H), 7.97 (d, $J=7.7 \mathrm{~Hz}, 1 \mathrm{H}), 7.64(\mathrm{~d}, J=8.0 \mathrm{~Hz}, 1 \mathrm{H}), 7.43-7.31(\mathrm{~m}, 3 \mathrm{H})$, $7.20-7.11(\mathrm{~m}, 10 \mathrm{H}), 7.05(\mathrm{~d}, J=8.2 \mathrm{~Hz}, 2 \mathrm{H}), 7.01-6.93(\mathrm{~m}, 2 \mathrm{H}), 6.84(\mathrm{t}, J=7.3 \mathrm{~Hz}, 1 \mathrm{H}), 4.66(\mathrm{~d}, J$ $=15.2 \mathrm{~Hz}, 2 \mathrm{H}), 4.40(\mathrm{~d}, J=15.2 \mathrm{~Hz}, 2 \mathrm{H}), 3.79(\mathrm{~s}, 3 \mathrm{H}) .{ }^{13} \mathrm{C} \mathrm{NMR}\left(101 \mathrm{MHz}, \mathrm{CDCl}_{3}\right) \delta 148.7,144.4$, 141.6, 141.0, 136.8, 136.3, 136.0, 131.3, 131.1, 129.1, 128.3, 128.0, 126.9, 125.8, 123.7, 122.8, 121.3, 120.5, 119.2, 117.5, 109.2, 108.6, 57.6, 29.2. HRMS-ESI $(\mathrm{m} / \mathrm{z})[\mathrm{M}+\mathrm{H}]^{+}$Calcd for $\mathrm{C}_{33} \mathrm{H}_{28} \mathrm{~N}_{2} \mathrm{Sb}^{+}$, 573.1285, Found, 573.1271.

\section{2-(1-methyl-1 $H$-indol-5-yl)-6-phenyl-5,6,7,12-tetrahydrodibenzo[c, $f][1,5]$ azastibocine (3ar)}<smiles>Cn1ccc2cc(S(=O)(=O)c3ccccc3CN3Cc4ccccc43)ccc21</smiles> 
5-Iodo-1-methyl-1H-indole $2 \mathbf{a x}(51.4 \mathrm{mg}, 0.20 \mathrm{mmol})$ was used, the desired product $\mathbf{3 a r}$ was obtained as a white solid in $76 \%$ yield (79.3 mg). M.P.: $194.5-197.1{ }^{\circ} \mathrm{C} .{ }^{1} \mathrm{H}$ NMR $\left(400 \mathrm{MHz}, \mathrm{CDCl}_{3}\right) \delta 8.04(\mathrm{~s}$, 1H), $7.55-7.43(\mathrm{~m}, 2 \mathrm{H}), 7.34-7.26(\mathrm{~m}, 8 \mathrm{H}), 7.17(\mathrm{~d}, J=8.0 \mathrm{~Hz}, 2 \mathrm{H}), 7.15-7.09(\mathrm{~m}, 3 \mathrm{H}), 6.98(\mathrm{t}, J=$ $7.3 \mathrm{~Hz}, 1 \mathrm{H}), 6.56$ (d, $J=3.0 \mathrm{~Hz}, 1 \mathrm{H}), 4.79$ (d, $J=15.1 \mathrm{~Hz}, 2 \mathrm{H}), 4.53$ (d, $J=15.1 \mathrm{~Hz}, 2 \mathrm{H}), 3.89$ (s, 3H). ${ }^{13} \mathrm{C}$ NMR $\left(101 \mathrm{MHz}, \mathrm{CDCl}_{3}\right) \delta 148.8,144.4,137.2,136.9,136.4,132.3,131.6,131.5,129.4,129.1$, 128.7, 128.2, 127.9, 126.8, 121.1, 117.4, 110.0, 101.1, 57.5, 33.0. HRMS-ESI (m/z) $[\mathrm{M}+\mathrm{H}]^{+}$Calcd for $\mathrm{C}_{29} \mathrm{H}_{26} \mathrm{~N}_{2} \mathrm{Sb}^{+}, 523.1129$, Found, 523.1118.

\section{2-(benzofuran-2-yl)-6-phenyl-5,6,7,12-tetrahydrodibenzo[c, $f][1,5]$ azastibocine (3as)}<smiles></smiles>

2-Iodobenzofuran $\mathbf{2 b k}$ ( $48.8 \mathrm{mg}, 0.20 \mathrm{mmol}$ ) was used, the desired product 3as was obtained as a white solid in $82 \%$ yield $(77.1 \mathrm{mg})$; 2-bromobenzofuran $2 \mathbf{b l}(39.2 \mathrm{mg}, 0.20 \mathrm{mmol})$ was used, the desired product 3as was obtained in $65 \%$ yield $(67.0 \mathrm{mg}) .{ }^{1} \mathrm{H}$ NMR $\left(400 \mathrm{MHz}, \mathrm{CDCl}_{3}\right) \delta 7.69(\mathrm{~s}, 1 \mathrm{H}), 7.60(\mathrm{~d}, J$ $=8.2 \mathrm{~Hz}, 1 \mathrm{H}), 7.32-7.19(\mathrm{~m}, 9 \mathrm{H}), 7.17-7.09(\mathrm{~m}, 2 \mathrm{H}), 7.08(\mathrm{~d}, J=6.5 \mathrm{~Hz}, 1 \mathrm{H}), 7.04-6.91(\mathrm{~m}, 4 \mathrm{H})$, $4.74(\mathrm{~d}, J=15.2 \mathrm{~Hz}, 2 \mathrm{H}), 4.50(\mathrm{~d}, J=15.2 \mathrm{~Hz}, 2 \mathrm{H}) .{ }^{13} \mathrm{C} \mathrm{NMR}\left(101 \mathrm{MHz}, \mathrm{CDCl}_{3}\right) \delta 156.2,153.1,148.5$, 143.8, 136.9, 133.0, 131.1, 129.2, 128.6, 128.1, 126.9, 124.3, 124.1, 122.3, 121.8, 117.9, 114.9, 111.4, 57.8 .

6-phenyl-12-propyl-5,6,7,12-tetrahydrodibenzo[c, $f][1,5]$ azastibocine (3au)<smiles>CCC[Sb]c1ccccc1CN1Cc2ccccc2S(C)(c2ccccc2)C1</smiles>

1-Iodopropane $2 \operatorname{cr}(34.0 \mathrm{mg}, 0.20 \mathrm{mmol})$ was used, the desired product 3au was obtained as a white solid in 51\% yield (44.3 mg). M.P.: $137.2-138.4{ }^{\circ} \mathrm{C} .{ }^{1} \mathrm{H}$ NMR (400 MHz, $\left.\mathrm{CDCl}_{3}\right) \delta 7.55-7.45(\mathrm{~m}, 2 \mathrm{H})$, $7.27-7.13(\mathrm{~m}, 8 \mathrm{H}), 6.98(\mathrm{~d}, J=8.3 \mathrm{~Hz}, 2 \mathrm{H}), 6.87(\mathrm{t}, J=7.3 \mathrm{~Hz}, 1 \mathrm{H}), 4.64$ (d, $J=14.9 \mathrm{~Hz}, 2 \mathrm{H}), 4.31$ (d, $J=14.9 \mathrm{~Hz}, 2 \mathrm{H}), 1.90-1.68(\mathrm{~m}, 4 \mathrm{H}), 1.15(\mathrm{t}, J=6.6 \mathrm{~Hz}, 3 \mathrm{H}) .{ }^{13} \mathrm{C} \mathrm{NMR}\left(101 \mathrm{MHz}, \mathrm{CDCl}_{3}\right) \delta 149.0$, 144.2, 134.9, 134.1, 129.0, 128.2, 128.1, 127.0, 120.4, 116.2, 56.9, 21.8, 21.2, 18.5. HRMS-ESI (m/z) $[\mathrm{M}+\mathrm{Na}]^{+}$Calcd for $\mathrm{C}_{23} \mathrm{H}_{24} \mathrm{NNaSb}^{+}, 458.0839$, Found, 458.0844 .

12-hexyl-6-phenyl-5,6,7,12-tetrahydrodibenzo $[c, f][1,5]$ azastibocine (3av) 


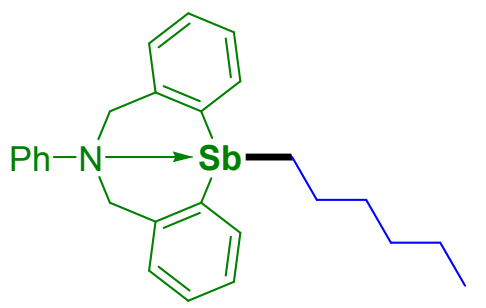

1-Iodopentane $2 \mathbf{c s}$ (39.6 $\mathrm{mg}, 0.20 \mathrm{mmol}$ ) was used, the desired product 3av was obtained as a white solid in 53\% yield (50.5 mg). Mp: 90.1-91.3 ${ }^{\circ} \mathrm{C} .{ }^{1} \mathrm{H}$ NMR (400 MHz, $\left.\mathrm{CDCl}_{3}\right) \delta 7.74-7.65(\mathrm{~m}, 2 \mathrm{H}), 7.44-$ $7.34(\mathrm{~m}, 6 \mathrm{H}), 7.33-7.28(\mathrm{~m}, 2 \mathrm{H}), 7.15(\mathrm{~d}, J=8.6 \mathrm{~Hz}, 2 \mathrm{H}), 7.04(\mathrm{t}, J=7.3 \mathrm{~Hz}, 1 \mathrm{H}), 4.80(\mathrm{~d}, J=14.9 \mathrm{~Hz}$, 2H), $4.46(\mathrm{~d}, J=14.9 \mathrm{~Hz}, 2 \mathrm{H}), 2.09-1.86(\mathrm{~m}, 4 \mathrm{H}), 1.77-1.65(\mathrm{~m}, 2 \mathrm{H}), 1.60-1.49(\mathrm{~m}, 4 \mathrm{H}), 1.10(\mathrm{t}, J$ $=7.0 \mathrm{~Hz}, 3 \mathrm{H}) .{ }^{13} \mathrm{C} \mathrm{NMR}\left(100 \mathrm{MHz}, \mathrm{CDCl}_{3}\right) \delta 148.8,144.0,134.8,133.9,128.9,128.0,127.9,126.8$, 120.3, 116.0, 56.7, 33.4, 31.5, 27.6, 22.6, 19.0, 14.1. HRMS-ESI $(\mathrm{m} / \mathrm{z})[\mathrm{M}+\mathrm{Na}]^{+}$Calcd for $\mathrm{C}_{26} \mathrm{H}_{30} \mathrm{NNaSb}^{+}, 500.1309$, Found, 500.1306.

\section{2-octyl-6-phenyl-5,6,7,12-tetrahydrodibenzo[c, $f][1,5]$ azastibocine (3aw)}

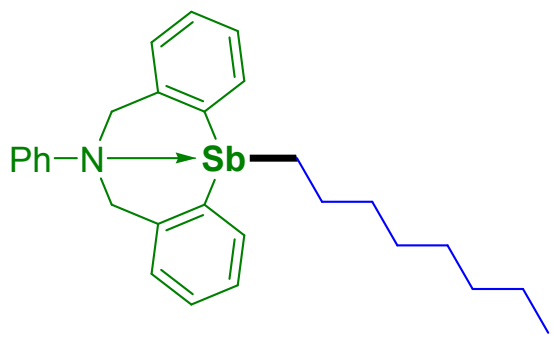

1-Iodooctane 2ct (48.0 mg, $0.20 \mathrm{mmol}$ ) was used, the desired product 3aw was obtained as a white solid in 47\% yield (47.4 mg). M.P.: 85.6 - 87.1 ${ }^{\circ} \mathrm{C} .{ }^{1} \mathrm{H}$ NMR (400 MHz, $\left.\mathrm{CDCl}_{3}\right) \delta 7.55-7.45(\mathrm{~m}, 2 \mathrm{H}), 7.25$ $-7.17(\mathrm{~m}, 6 \mathrm{H}), 7.17-7.11(\mathrm{~m}, 2 \mathrm{H}), 6.97$ (d, $J=8.1 \mathrm{~Hz}, 2 \mathrm{H}), 6.86(\mathrm{t}, J=7.4 \mathrm{~Hz}, 1 \mathrm{H}), 4.63(\mathrm{~d}, J=14.9$ $\mathrm{Hz}, 2 \mathrm{H}), 4.29$ (d, J=14.9 Hz, 2H), $1.86-1.68$ (m, 4H), $1.55-1.47$ (m, 2H), $1.43-1.16(\mathrm{~m}, 8 \mathrm{H}), 0.92$ $-0.84(\mathrm{~m}, 3 \mathrm{H}) .{ }^{13} \mathrm{C}$ NMR $\left(101 \mathrm{MHz}, \mathrm{CDCl}_{3}\right) \delta 149.0,144.2,135.0,134.1,129.0,128.2,128.1,127.0$, 120.4, 116.2, 56.9, 33.9, 32.1, 29.5, 29.4, 27.7, 22.8, 19.2, 14.3. HRMS-ESI (m/z) $[\mathrm{M}+\mathrm{Na}]^{+}$Calcd for $\mathrm{C}_{28} \mathrm{H}_{34} \mathrm{NNaSb}^{+}, 528.1622$, Found, 528.1619.

\section{6-(tert-butyl)-12-(p-tolyl)-5,6,7,12-tetrahydrodibenzo[c,f][1,5]azastibocine (3ay)}

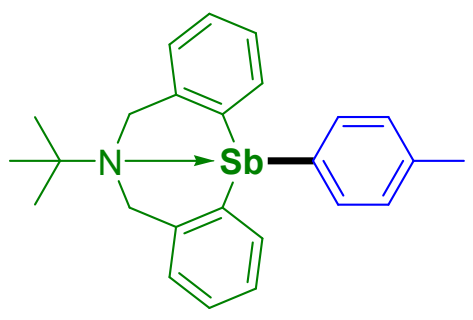

1-Iodo-4-methylbenzene $\mathbf{2 a}(43.6 \mathrm{mg}, 0.20 \mathrm{mmol})$ was used, the desired product 3ay was obtained as a white solid in $74 \%$ yield $(68.5 \mathrm{mg}) .{ }^{1} \mathrm{H}$ NMR $\left(400 \mathrm{MHz}, \mathrm{CDCl}_{3}\right) \delta 7.56(\mathrm{~d}, J=7.6 \mathrm{~Hz}, 2 \mathrm{H}), 7.25-7.18$ (m, 4H), $7.14(\mathrm{t}, J=7.3 \mathrm{~Hz}, 2 \mathrm{H}), 7.06-6.99(\mathrm{~m}, 4 \mathrm{H}), 4.16(\mathrm{~d}, J=15.3 \mathrm{~Hz}, 2 \mathrm{H}), 3.85(\mathrm{~d}, J=15.3 \mathrm{~Hz}$, 
2H), $2.42(\mathrm{~s}, 3 \mathrm{H}), 1.23(\mathrm{~s}, 9 \mathrm{H}) .{ }^{13} \mathrm{C} \mathrm{NMR}\left(101 \mathrm{MHz}, \mathrm{CDCl}_{3}\right) \delta 146.9,142.6,139.0,137.9,136.7,136.4$, $129.4,128.0,127.2,126.3,57.7,54.9,27.1,21.7$.

6-(tert-butyl)-12-phenyl-5,6,7,12-tetrahydrodibenzo[ $c, f][1,5]$ azastibocine (3az)<smiles>CC(C)(C)N1Cc2ccccc2[Sb](c2ccccc2)c2ccccc2C1</smiles>

Iodobenzene $\mathbf{2 c}$ ( $40.8 \mathrm{mg}, 0.20 \mathrm{mmol}$ ) was used, the desired product $3 \mathbf{a z}$ was obtained as a white solid in $72 \%$ yield $(64.6 \mathrm{mg}) .{ }^{1} \mathrm{H}$ NMR $\left(400 \mathrm{MHz}, \mathrm{CDCl}_{3}\right) \delta 7.83-7.74(\mathrm{~m}, 2 \mathrm{H}), 7.54-7.46(\mathrm{~m}, 3 \mathrm{H}), 7.32-$ $7.22(\mathrm{~m}, 4 \mathrm{H}), 7.18-7.08(\mathrm{~m}, 4 \mathrm{H}), 4.27(\mathrm{~d}, J=15.3 \mathrm{~Hz}, 2 \mathrm{H}), 3.96(\mathrm{~d}, J=15.3 \mathrm{~Hz}, 2 \mathrm{H}), 1.34(\mathrm{~s}, 9 \mathrm{H}) .{ }^{13} \mathrm{C}$ NMR $\left(101 \mathrm{MHz}, \mathrm{CDCl}_{3}\right) \delta 146.9,146.6,139.0,136.6,136.4,128.5,128.2,128.0,127.2,126.3,57.7$, 54.9, 27.0.

6-(3,5-dimethylphenyl)-12-(m-tolyl)-5,6,7,12-tetrahydrodibenzo[c,fl[1,5]azastibocine (3ba)<smiles>Cc1cc(C)cc(N2Cc3ccccc3[Sb](c3ccccc3)(c3cccc(C)c3)C2)c1</smiles>

1-Bromo-3-methylbenzene $2 \mathbf{e}$ (34.0 $\mathrm{mg}, 0.20 \mathrm{mmol})$ was used, the desired product $\mathbf{3 b a}$ was obtained as a white solid in $82 \%$ yield $(83.8 \mathrm{mg}) .{ }^{1} \mathrm{H}$ NMR $\left(400 \mathrm{MHz}, \mathrm{CDCl}_{3}\right) \delta 7.51(\mathrm{~s}, 1 \mathrm{H}), 7.45(\mathrm{~d}, J=7.2 \mathrm{~Hz}$, $1 \mathrm{H}), 7.30(\mathrm{t}, J=7.4 \mathrm{~Hz}, 1 \mathrm{H}), 7.25-7.16(\mathrm{~m}, 7 \mathrm{H}), 7.08(\mathrm{t}, J=7.1 \mathrm{~Hz}, 2 \mathrm{H}), 6.73(\mathrm{~s}, 2 \mathrm{H}), 6.58(\mathrm{~s}, 1 \mathrm{H})$, $4.67(\mathrm{~d}, J=15.1 \mathrm{~Hz}, 2 \mathrm{H}), 4.39$ (d, $J=15.1 \mathrm{~Hz}, 2 \mathrm{H}), 2.36(\mathrm{~s}, 3 \mathrm{H}), 2.22(\mathrm{~s}, 6 \mathrm{H}) .{ }^{13} \mathrm{C}$ NMR $(101 \mathrm{MHz}$, $\mathrm{CDCl}_{3}$ ) $\delta 148.7,144.6,143.4,139.7,138.5,138.0,136.7,135.9,135.8,129.4,128.5,128.3,128.0,126.8$, $123.4,115.8,57.7,21.9,21.7$.

6-(4-fluorophenyl)-12-(p-tolyl)-5,6,7,12-tetrahydrodibenzo[ $c, f][1,5]$ azastibocine (3bb)

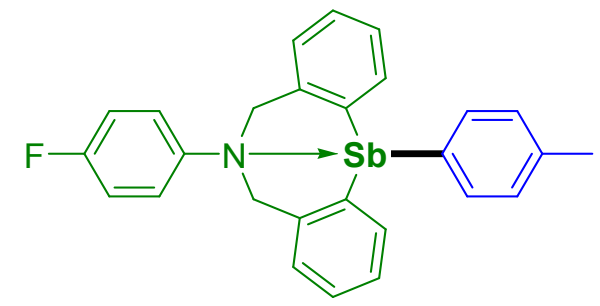

1-Iodo-4-methylbenzene $(21.8 \mathrm{mg}, 0.10 \mathrm{mmol})$ was used, the desired product $\mathbf{3 b b}$ was obtained as a liquid in $89 \%$ yield (44.6 mg). ${ }^{1} \mathrm{H}$ NMR (400 MHz, Chloroform- $d$ ) $\delta 7.64(\mathrm{~d}, J=7.3 \mathrm{~Hz}, 2 \mathrm{H}), 7.35-$ $7.24(\mathrm{~m}, 8 \mathrm{H}), 7.22-7.10(\mathrm{~m}, 4 \mathrm{H}), 7.01(\mathrm{~d}, J=8.4 \mathrm{~Hz}, 2 \mathrm{H}), 4.67$ (d, $J=15.1 \mathrm{~Hz}, 2 \mathrm{H}), 4.51$ (d, $J=15.1$ $\mathrm{Hz}, 2 \mathrm{H}), 2.51$ (s, 3H). ${ }^{13} \mathrm{C} \mathrm{NMR}\left(101 \mathrm{MHz}, \mathrm{CDCl}_{3}\right) \delta 157.9(\mathrm{~d}, J=239.0 \mathrm{~Hz}), 144.8(\mathrm{~d}, J=2.5 \mathrm{~Hz})$, 
144.1, 139.3, 138.9, 138.5, 136.6, 135.8, 129.6, 128.4, 128.1, 126.9, 119.0 (d, $J=7.5 \mathrm{~Hz}), 115.6$ (d, $J=$ $21.9 \mathrm{~Hz}), 57.9$, 21.6. HRMS-ESI (m/z) $[\mathrm{M}+\mathrm{H}]^{+}$Calcd for $\mathrm{C}_{27} \mathrm{H}_{24} \mathrm{FNSb}^{+}, 502.0925$, Found, 502.0912.

4-(5H-dibenzo $[b, d]$ stibol-5-yl)benzonitrile (3bc)

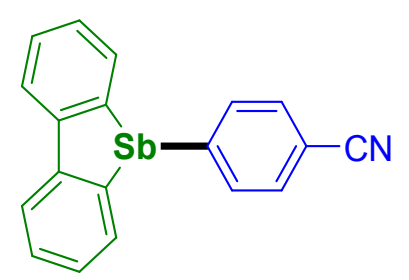

4-Iodobenzonitrile $\mathbf{2 b}$ ( $45.8 \mathrm{mg}, 0.20 \mathrm{mmol}$ ) was used, the desired product $\mathbf{3 b c}$ was obtained as a white solid in $97 \%$ yield $(72.7 \mathrm{mg}$ ); 4-bromobenzonitrile $2 \mathrm{am}(36.2 \mathrm{mg}, 0.20 \mathrm{mmol})$ was used, the desired product 3bc was obtained in 92\% yield $(69.0 \mathrm{mg})$; 4-chlorobenzonitrile $2 \mathbf{c v}(27.4 \mathrm{mg}, 0.20 \mathrm{mmol})$ was used, the desired product $3 \mathbf{b c}$ was obtained in $84 \%$ yield $(63.0 \mathrm{mg})$. M.P.: $155.6-157.4{ }^{\circ} \mathrm{C} .{ }^{1} \mathrm{H}$ NMR $\left(400 \mathrm{MHz}, \mathrm{CDCl}_{3}\right) \delta 7.94(\mathrm{~d}, J=7.8 \mathrm{~Hz}, 2 \mathrm{H}), 7.72(\mathrm{~d}, J=7.2 \mathrm{~Hz}, 2 \mathrm{H}), 7.51-7.45(\mathrm{~m}, 2 \mathrm{H}), 7.45-7.36$ (m, 4H), 7.33 (t, $J=7.3 \mathrm{~Hz}, 2 \mathrm{H}) .{ }^{13} \mathrm{C}$ NMR $\left(101 \mathrm{MHz}, \mathrm{CDCl}_{3}\right) \delta 150.4,145.7,144.1,135.7,135.3$, 131.7, 129.5, 128.3, 123.8, 118.9, 112.1. HRMS-ESI (m/z) [M+H] ${ }^{+}$Calcd for $\mathrm{C}_{19} \mathrm{H}_{13} \mathrm{NSb}^{+}, 376.0081$; Found, 376.0079 .

\section{5-(4-methoxyphenyl)-5H-dibenzo $[b, d]$ stibole (3bd)}

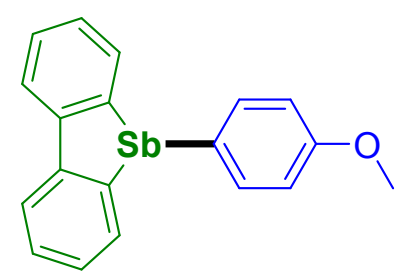

1-Iodo-4-methoxybenzene $\mathbf{2 h}$ ( $46.8 \mathrm{mg}, 0.20 \mathrm{mmol}$ ) was used, the desired product $\mathbf{3 b d}$ was obtained as a white solid in 95\% yield (72.2 mg); 1-bromo-4-methoxybenzene $2 \mathbf{i}$ (37.2 $\mathrm{mg}, 0.20 \mathrm{mmol}$ ) was used, the desired product 3 bd was obtained in $90 \%$ yield (68.4 mg); 1-chloro-4-methoxybenzene $2 \mathbf{c w}$ (28.4 $\mathrm{mg}, 0.20 \mathrm{mmol}$ ) was used, the desired product 3 bd was obtained in $78 \%$ yield $\left(59.3 \mathrm{mg}\right.$ ). ${ }^{1} \mathrm{H}$ NMR (400 $\left.\mathrm{MHz}, \mathrm{CDCl}_{3}\right) \delta 7.89$ (d, $\left.J=7.8 \mathrm{~Hz}, 2 \mathrm{H}\right), 7.66(\mathrm{~d}, J=7.2 \mathrm{~Hz}, 2 \mathrm{H}), 7.39$ (t, $\left.J=7.3 \mathrm{~Hz}, 2 \mathrm{H}\right), 7.28-7.14$ (m, 4H), $6.66(\mathrm{~d}, J=8.4 \mathrm{~Hz}, 2 \mathrm{H}), 3.61$ (s, 3H). ${ }^{13} \mathrm{C} \mathrm{NMR}\left(101 \mathrm{MHz}, \mathrm{CDCl}_{3}\right) \delta 160.2,150.2,145.5,136.7$, $135.4,128.9,128.3,127.9,123.5,114.7,55.0$.

\section{5-(4-(tert-butyl)phenyl)-5H-dibenzo[b,d] stibole (3be)}

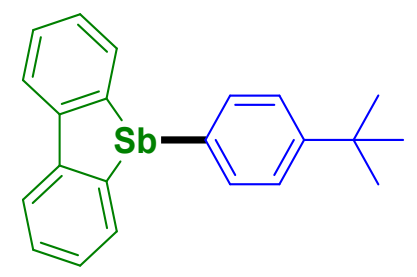

1-(Tert-butyl)-4-iodobenzene 2ag ( $52.0 \mathrm{mg}, 0.20 \mathrm{mmol}$ ) was used, the desired product 3be was obtained as a white solid in $90 \%$ yield (73.1 mg); 1-bromo-4-(tert-butyl)benzene 2ah (42.4 mg, $0.20 \mathrm{mmol})$ was 
used, the desired product $3 \mathbf{b e}$ was obtained in $86 \%$ yield (69.8 $\mathrm{mg}$ ); 1-chloro-4-(tert-butyl)benzene $\mathbf{2 e x}$ (33.6 mg, $0.20 \mathrm{mmol}$ ) was used, the desired product 3be was obtained in $74 \%$ yield $(60.1 \mathrm{mg})$. M.P.: $99.4-101.2^{\circ} \mathrm{C} .{ }^{1} \mathrm{H}$ NMR $\left(400 \mathrm{MHz}, \mathrm{CDCl}_{3}\right) \delta 8.00(\mathrm{~d}, J=7.9 \mathrm{~Hz}, 2 \mathrm{H}), 7.78(\mathrm{~d}, J=7.2 \mathrm{~Hz}, 2 \mathrm{H}), 7.50$ (t, $J=7.6 \mathrm{~Hz}, 2 \mathrm{H}), 7.38-7.30(\mathrm{~m}, 4 \mathrm{H}), 7.26$ (d, $J=8.1 \mathrm{~Hz}, 2 \mathrm{H}), 1.28(\mathrm{~s}, 9 \mathrm{H}) .{ }^{13} \mathrm{C}$ NMR $(101 \mathrm{MHz}$, $\left.\mathrm{CDCl}_{3}\right) \delta 151.6,150.4,145.3,135.5,135.2,134.5,128.9,127.9,126.0,123.5,34.7,31.3$. HRMS-ESI $(\mathrm{m} / \mathrm{z})[\mathrm{M}+\mathrm{H}]^{+}$Calcd for $\mathrm{C}_{22} \mathrm{H}_{22} \mathrm{Sb}^{+}$, 407.0754; Found, 407.0757.

\section{5-(p-tolyl)-5H-dibenzo[b,d] stibole (3bf)}

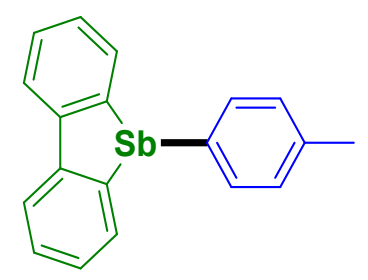

1-Iodo-4-methylbenzene $\mathbf{2 a}$ ( $43.6 \mathrm{mg}, 0.20 \mathrm{mmol}$ ) was used, the desired product $\mathbf{3 b f}$ was obtained as a white solid in $98 \%$ yield $(71.3 \mathrm{mg}$ ); 1-bromo-4-methylbenzene $2 \mathrm{al}(34.0 \mathrm{mg}, 0.20 \mathrm{mmol}$ ) was used, the desired product $\mathbf{3 b f}$ was obtained in $92 \%$ yield (67.0 mg); 1-chloro-4-methylbenzene $2 \mathbf{c y}$ (25.2 mg, 0.20 mmol) was used, the desired product $3 \mathbf{b f}$ was obtained in $81 \%$ yield $(58.9 \mathrm{mg}) .{ }^{1} \mathrm{H}$ NMR (400 MHz, $\left.\mathrm{CDCl}_{3}\right) \delta 7.93(\mathrm{~d}, J=7.9 \mathrm{~Hz}, 2 \mathrm{H}), 7.70(\mathrm{~d}, J=7.2 \mathrm{~Hz}, 2 \mathrm{H}), 7.43(\mathrm{t}, J=7.6 \mathrm{~Hz}, 2 \mathrm{H}), 7.31-7.24(\mathrm{~m}, 2 \mathrm{H})$, $7.21(\mathrm{~d}, J=7.6 \mathrm{~Hz}, 2 \mathrm{H}), 6.98(\mathrm{~d}, J=7.6 \mathrm{~Hz}, 2 \mathrm{H}), 2.22(\mathrm{~s}, 3 \mathrm{H}) .{ }^{13} \mathrm{C} \mathrm{NMR}\left(101 \mathrm{MHz}, \mathrm{CDCl}_{3}\right) \delta 150.3$, $145.3,138.6,135.4,134.4,129.7,128.9,128.0,123.5,21.4$.

\section{5-(3-fluorophenyl)-5H-dibenzo $[b, d]$ stibole (3bg)}

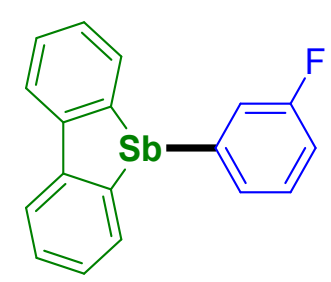

1-Fluoro-3-iodobenzene $\mathbf{2 b u}$ ( $44.4 \mathrm{mg}, 0.20 \mathrm{mmol}$ ) was used, the desired product $\mathbf{3 b g}$ was obtained as a white solid in $89 \%$ yield ( $65.5 \mathrm{mg}$ ); 1-fluoro-3-bromobenzene $\mathbf{2 b v}(34.8 \mathrm{mg}, 0.20 \mathrm{mmol}$ ) was used, the desired product $\mathbf{3 b g}$ was obtained in 91\% yield (66.9 mg); 1-chloro-3-fluorobenzene $2 \mathbf{c u}$ (26.2 $\mathrm{mg}, 0.20$ $\mathrm{mmol}$ ), the desired product $3 \mathrm{bg}$ was obtained in $79 \%$ yield $(58.1 \mathrm{mg}) .{ }^{1} \mathrm{H} \mathrm{NMR}\left(400 \mathrm{MHz}, \mathrm{CDCl}_{3}\right) \delta$ $8.00(\mathrm{~d}, J=7.8 \mathrm{~Hz}, 2 \mathrm{H}), 7.78(\mathrm{~d}, J=7.2 \mathrm{~Hz}, 2 \mathrm{H}), 7.52(\mathrm{t}, J=7.6 \mathrm{~Hz}, 2 \mathrm{H}), 7.37$ (t, $J=7.3 \mathrm{~Hz}, 2 \mathrm{H}), 7.25$ $-7.17(\mathrm{~m}, 2 \mathrm{H}), 7.04(\mathrm{dd}, J=2.5 \mathrm{~Hz}, 8.1 \mathrm{~Hz}, 1 \mathrm{H}), 6.98-6.89(\mathrm{~m}, 1 \mathrm{H}) .{ }^{13} \mathrm{C} \mathrm{NMR}\left(101 \mathrm{MHz}, \mathrm{CDCl}_{3}\right) \delta$ $162.9(\mathrm{~d}, J=251.0 \mathrm{~Hz}), 150.4,144.7,140.6(\mathrm{~d}, J=2.7 \mathrm{~Hz}), 135.4,131.0(\mathrm{~d}, J=3.0 \mathrm{~Hz}), 130.2$ (d, $J=$ $6.7 \mathrm{~Hz}), 129.3,128.2,123.7,121.6(\mathrm{~d}, J=19.3 \mathrm{~Hz}), 115.7$ (d, $J=21.2 \mathrm{~Hz}) .{ }^{19} \mathrm{~F}$ NMR $\left(376 \mathrm{MHz}, \mathrm{CDCl}_{3}\right)$ $\delta-112.41$.

Ethyl 4-(5H-dibenzo $[b, d]$ stibol-5-yl)benzoate (3bh) 


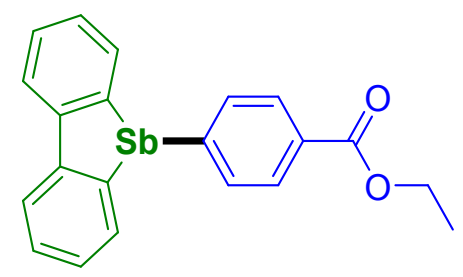

Ethyl 4-iodobenzoate 2at (55.2 $\mathrm{mg}, 0.20 \mathrm{mmol}$ ) was used, the desired product $\mathbf{3 b h}$ was obtained as a white solid in $94 \%$ yield (79.3 mg); ethyl 4-bromobenzoate $2 \mathbf{b w}$ (45.6 $\mathrm{mg}, 0.20 \mathrm{mmol}$ ) was used, the desired product $\mathbf{3 b h}$ was obtained in $84 \%$ yield $(70.9 \mathrm{mg}$ ); ethyl 4-chlorobenzoate $\mathbf{2 c z}(36.8 \mathrm{mg}, 0.20$ mmol) was used, the desired product $3 \mathrm{bh}$ was obtained in $82 \%$ yield $(69.2 \mathrm{mg}) .{ }^{1} \mathrm{H} \mathrm{NMR} \mathrm{(400} \mathrm{MHz,}$ $\left.\mathrm{CDCl}_{3}\right) \delta 7.94(\mathrm{~d}, J=7.8 \mathrm{~Hz}, 2 \mathrm{H}), 7.82(\mathrm{~d}, J=8.1 \mathrm{~Hz}, 2 \mathrm{H}), 7.73(\mathrm{~d}, J=7.2 \mathrm{~Hz}, 2 \mathrm{H}), 7.51-7.39$ (m, $4 \mathrm{H}), 7.31(\mathrm{t}, J=7.3 \mathrm{~Hz}, 2 \mathrm{H}), 4.31$ (q, $J=7.1 \mathrm{~Hz}, 2 \mathrm{H}), 1.32(\mathrm{t}, J=7.1 \mathrm{~Hz}, 3 \mathrm{H}) .{ }^{13} \mathrm{C} \mathrm{NMR}(101 \mathrm{MHz}$, $\left.\mathrm{CDCl}_{3}\right) \delta 166.6,150.4,145.0,144.6,135.3,135.2,130.5,129.4,129.2,128.1,123.7,61.0,14.4$.

5-(o-tolyl)-5H-dibenzo $[b, d]$ stibole (3bi)

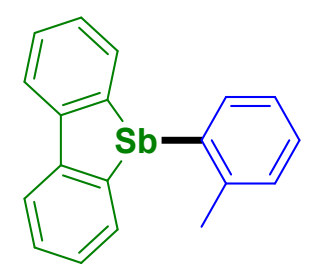

1-Iodo-2-methylbenzene $(21.8 \mathrm{mg}, 0.10 \mathrm{mmol})$ was used, the desired product $3 \mathbf{b i}$ was obtained as a white solid in 94\% yield (34.2 mg). M.P.: $220.3-221.9{ }^{\circ} \mathrm{C} .{ }^{1} \mathrm{H}$ NMR (400 MHz, Chloroform- $d$ ) $\delta 7.97(\mathrm{~d}, J=$ $7.7 \mathrm{~Hz}, 2 \mathrm{H}), 7.81(\mathrm{~d}, J=7.2 \mathrm{~Hz}, 2 \mathrm{H}), 7.49$ (t, $J=7.6 \mathrm{~Hz}, 2 \mathrm{H}), 7.35(\mathrm{t}, J=7.3 \mathrm{~Hz}, 2 \mathrm{H}), 7.23-7.11(\mathrm{~m}$, 2H), $7.04(\mathrm{~d}, J=7.9 \mathrm{~Hz}, 1 \mathrm{H}), 6.91(\mathrm{t}, J=7.4 \mathrm{~Hz}, 1 \mathrm{H}), 2.77(\mathrm{~s}, 3 \mathrm{H}) .{ }^{13} \mathrm{C} \mathrm{NMR}\left(101 \mathrm{MHz}, \mathrm{CDCl}_{3}\right) \delta 150.3$, 144.6, 143.5, 138.6, 135.8, 134.6, 129.5, 128.9, 128.8, 127.8, 126.4, 123.7, 25.2. HRMS-ESI (m/z) $[\mathrm{M}+\mathrm{H}]^{+}$Calcd for $\mathrm{C}_{19} \mathrm{H}_{16} \mathrm{Sb}^{+}, 365.0285$, Found, 365.0273 .

\section{4-(diphenylstibanyl)benzonitrile (3bj)}

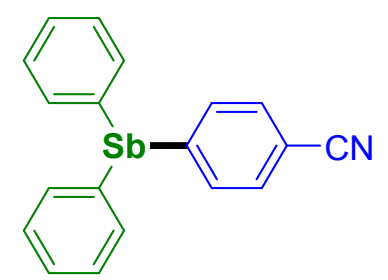

$\mathrm{Ph}_{2} \mathrm{SbCl}^{\mathrm{S} 1}$ (62.0 mg, $\left.0.2 \mathrm{mmol}\right)$ in situ generated from $\mathrm{SbPh}_{3}(34.1 \mathrm{mg}, 0.13 \mathrm{mmol})$ and $\mathrm{SbCl}_{3}(13.7 \mathrm{mg}$, $0.06 \mathrm{mmol})$ and 4-iodobenzonitrile $(45.8 \mathrm{mg}, 0.20 \mathrm{mmol})$ were used, the desired product 3bj was obtained as a white solid in 92\% yield (69.4 mg). ${ }^{1} \mathrm{H}$ NMR (400 MHz, Chloroform- $\left.d\right) \delta 7.57(\mathrm{~d}, J=1.4$ $\mathrm{Hz}, 4 \mathrm{H}), 7.46-7.42$ (m, 4H), $7.40-7.36$ (m, 6H).

\section{(4-(methylsulfonyl)phenyl)diphenylstibane (3bk)}




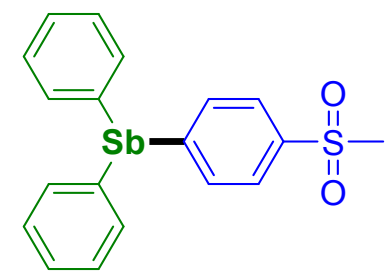

$\mathrm{Ph}_{2} \mathrm{SbCl}^{\mathrm{S} 1}$ (62.0 mg, $\left.0.2 \mathrm{mmol}\right)$ in situ generated from $\mathrm{SbPh}_{3}(34.1 \mathrm{mg}, 0.13 \mathrm{mmol})$ and $\mathrm{SbCl}_{3}(13.7 \mathrm{mg}$, $0.06 \mathrm{mmol})$ and 1-iodo-4-(methylsulfonyl)benzene $(56.3 \mathrm{mg}, 0.20 \mathrm{mmol})$ were used, the desired product 3bk was obtained as a white solid in $88 \%$ yield $(75.7 \mathrm{mg}) .{ }^{1} \mathrm{H}$ NMR $(400 \mathrm{MHz}$, Chloroform- $d$ ) $\delta 7.84$ $(\mathrm{d}, J=8.3 \mathrm{~Hz}, 2 \mathrm{H}), 7.64(\mathrm{~d}, J=8.3 \mathrm{~Hz}, 2 \mathrm{H}), 7.46-7.39(\mathrm{~m}, 4 \mathrm{H}), 7.38-7.31(\mathrm{~m}, 6 \mathrm{H}), 3.03(\mathrm{~s}, 3 \mathrm{H})$.

\section{(3,5-dimethoxyphenyl)diphenylstibane (3bl)}

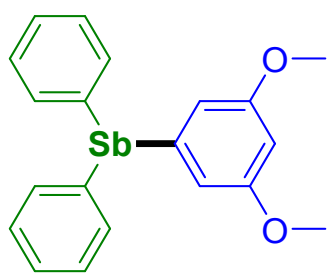

$\mathrm{Ph}_{2} \mathrm{SbCl}^{\mathrm{S} 1}$ (62.0 mg, $\left.0.2 \mathrm{mmol}\right)$ in situ generated from $\mathrm{SbPh}_{3}(34.1 \mathrm{mg}, 0.13 \mathrm{mmol})$ and $\mathrm{SbCl}_{3}(13.7 \mathrm{mg}$, $0.06 \mathrm{mmol})$ and 1-iodo-3,5-dimethoxybenzene $(52.8 \mathrm{mg}, 0.20 \mathrm{mmol})$ were used, the desired product $3 \mathbf{b l}$ was obtained as a white solid in $78 \%$ yield $(64.3 \mathrm{mg}) .{ }^{1} \mathrm{H}$ NMR $(400 \mathrm{MHz}$, Chloroform- $d$ ) $\delta 7.49-7.39$ $(\mathrm{m}, 4 \mathrm{H}), 7.36-7.29(\mathrm{~m}, 6 \mathrm{H}), 6.59(\mathrm{~d}, J=2.3 \mathrm{~Hz}, 2 \mathrm{H}), 6.42(\mathrm{t}, J=2.3 \mathrm{~Hz}, 1 \mathrm{H}), 3.70(\mathrm{~s}, 6 \mathrm{H})$.

\section{Ethyl 2-(4-(6-phenyl-6,7-dihydrodibenzo[c,f][1,5]azastibocin-12(5H)-yl)phenoxy)butanoate (3bm)}<smiles>CCOC(=O)C(CC)Oc1ccc(S2(c3ccccc3)CN3Cc4ccccc4C2(c2ccccc2)c2ccccc23)cc1</smiles>

Ethyl 2-(4-bromophenoxy)butanoate $\mathbf{2 b b}(57.2 \mathrm{mg}, 0.20 \mathrm{mmol})$ was used, the desired product $\mathbf{3 b m}$ was obtained as a liquid in 93\% yield (111.4 mg). ${ }^{1} \mathrm{H}$ NMR (400 MHz, $\left.\mathrm{CDCl}_{3}\right) \delta 7.53(\mathrm{~d}, J=7.8 \mathrm{~Hz}, 2 \mathrm{H})$, $7.26-7.17(\mathrm{~m}, 8 \mathrm{H}), 7.12-7.06(\mathrm{~m}, 4 \mathrm{H}), 6.96-6.89(\mathrm{~m}, 3 \mathrm{H}), 4.70(\mathrm{~d}, J=15.1 \mathrm{~Hz}, 2 \mathrm{H}), 4.63(\mathrm{t}, J=6.2$ $\mathrm{Hz}, 1 \mathrm{H}), 4.43(\mathrm{~d}, J=15.1 \mathrm{~Hz}, 2 \mathrm{H}), 4.31-4.20(\mathrm{~m}, 2 \mathrm{H}), 2.07-1.97(\mathrm{~m}, 2 \mathrm{H}), 1.26$ (t, J= 7.1 Hz, 3H), $1.12(\mathrm{t}, J=7.5 \mathrm{~Hz}, 3 \mathrm{H}) .{ }^{13} \mathrm{C} \mathrm{NMR}\left(101 \mathrm{MHz}, \mathrm{CDCl}_{3}\right) \delta 171.9,158.7,148.6,144.3,144.3,140.3,136.7$, 136.6, 135.8, 135.8, 134.5, 129.1, 128.4, 128.1, 128.0, 126.9, 121.3, 117.5, 115.6, 61.3, 57.5, 57.4, 26.4, 14.3, 9.9. HRMS-ESI (m/z) $[\mathrm{M}+\mathrm{H}]^{+}$Calcd for $\mathrm{C}_{32} \mathrm{H}_{33} \mathrm{NO}_{3} \mathrm{Sb}^{+}, 600.1493$, Found, 600.1503 .

Ethyl 2-methyl-2-(4-(6-phenyl-6,7-dihydrodibenzo[c,f][1,5]azastibocin-12(5H)-yl)phenoxy)butane ate (3bn) 


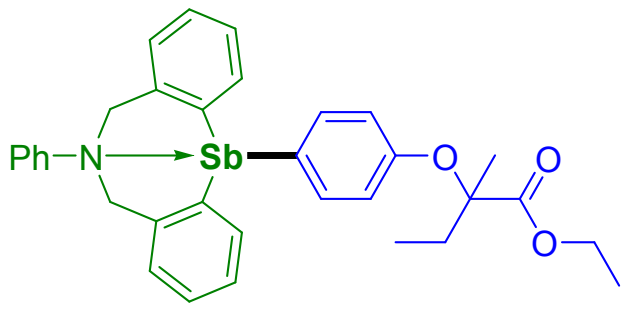

Ethyl 2-(4-bromophenoxy)-2-methylbutanoate $\mathbf{2 b c}(60.0 \mathrm{mg}, 0.20 \mathrm{mmol})$ was used, the desired product 3bn was obtained as a liquid in $92 \%$ yield $(112.8 \mathrm{mg}) .{ }^{1} \mathrm{H}$ NMR $\left(400 \mathrm{MHz}, \mathrm{CDCl}_{3}\right) \delta 7.63(\mathrm{~d}, J=7.9$ $\mathrm{Hz}, 2 \mathrm{H}), 7.36-7.27$ (m, 8H), 7.20 (d, J=7.9 Hz, 4H), $7.06-6.99$ (m, 3H), 4.79 (d, J=15.2 Hz, 2H), $4.52(\mathrm{~d}, J=15.2 \mathrm{~Hz}, 2 \mathrm{H}), 4.44-4.30(\mathrm{~m}, 2 \mathrm{H}), 2.27-2.04(\mathrm{~m}, 2 \mathrm{H}), 1.72(\mathrm{~s}, 3 \mathrm{H}), 1.36(\mathrm{t}, J=7.1 \mathrm{~Hz}$, $3 \mathrm{H}), 1.15(\mathrm{t}, J=7.5 \mathrm{~Hz}, 3 \mathrm{H}) .{ }^{13} \mathrm{C} \mathrm{NMR}\left(101 \mathrm{MHz}, \mathrm{CDCl}_{3}\right) \delta 174.0,156.3,148.5,144.3,139.8,136.5$, 135.8, 134.8, 129.0, 128.3, 127.9, 126.8, 121.3, 119.1, 117.5, 81.8, 61.3, 57.4, 33.0, 20.9, 14.2, 8.0. HRMS-ESI (m/z) [M+H] $]^{+}$Calcd for $\mathrm{C}_{33} \mathrm{H}_{35} \mathrm{NO}_{3} \mathrm{Sb}^{+}, 614.1650$, Found, 614.1660.

6-phenyl-12-(4-(5-(p-tolyl)-3-(trifluoromethyl)-1 H-pyrazol-1-yl)phenyl)-5,6,7,12-tetrahydrodi benzo $[c, f][1,5]$ azastibocine $(3 \mathrm{bo})$<smiles></smiles>

1-(4-Bromophenyl)-5-(p-tolyl)-3-(trifluoromethyl)-1H-pyrazole $2 \mathbf{b a}(76.2 \mathrm{mg}, 0.20 \mathrm{mmol})$ was used, the desired product $3 \mathbf{b o}$ was obtained as a white solid in $86 \%$ yield $(119.2 \mathrm{mg})$. M.P.: $210.7-211.0^{\circ} \mathrm{C}$. ${ }^{1} \mathrm{H}$ NMR $\left(400 \mathrm{MHz}, \mathrm{CDCl}_{3}\right) \delta 7.78(\mathrm{~d}, J=7.5 \mathrm{~Hz}, 2 \mathrm{H}), 7.47(\mathrm{~d}, J=7.5 \mathrm{~Hz}, 2 \mathrm{H}), 7.43-7.30(\mathrm{~m}, 7 \mathrm{H})$, $7.29-7.19$ (m, 9H), $7.06(\mathrm{t}, J=7.2 \mathrm{~Hz}, 1 \mathrm{H}), 6.87$ (s, 1H), $4.82(\mathrm{~d}, J=15.2 \mathrm{~Hz}, 2 \mathrm{H}), 4.56(\mathrm{~d}, J=15.2$ $\mathrm{Hz}, 2 \mathrm{H}), 2.49$ (s, 3H). ${ }^{13} \mathrm{C}$ NMR (101 MHz, $\left.\mathrm{CDCl}_{3}\right) \delta 148.4,145.0,144.7,144.4,143.3$ (q, J=38.2 Hz), $139.7,139.2$, 136.5, 135.4, 129.5, 129.1, 128.8, 128.6, 128.2, 127.0, 126.5, 125.6, 121.7, 121.5 (d, $J=$ 268.9 Hz), 117.9, 105.4, 105.3, 57.7, 21.4. ${ }^{19} \mathrm{~F}$ NMR (376 MHz, $\left.\mathrm{CDCl}_{3}\right) \delta-62.05 . \mathrm{HRMS}-\mathrm{ESI}(\mathrm{m} / \mathrm{z})$ $[\mathrm{M}+\mathrm{Na}]^{+}$Calcd for $\mathrm{C}_{37} \mathrm{H}_{29} \mathrm{~F}_{3} \mathrm{~N}_{3} \mathrm{NaSb}^{+}, 716.1244$, Found, 716.1251.

$(8 S, 9 S, 13 S, 14 S)-13-m e t h y l-3-((4-(6-p h e n y l-6,7-d i h y d r o d i b e n z o[c, f][1,5]$ azastibocin-12(5H)yl)ben zyl)oxy)-6,7,8,9,11,12,13,14,15,16-decahydro-17H-cyclopenta[a]phenanthren-17-one (3bp)<smiles>C[C@]12CCC3c4ccc(OCc5ccc([Sb]6c7ccccc7CN(c7ccccc7)Cc7ccccc76)cc5)cc4CC[C@H]3[C@H]1CCC2=O</smiles> 

cyclopenta[a]phenanthren-17-one $2 \mathbf{b d}(87.6 \mathrm{mg}, 0.20 \mathrm{mmol})$ was used, the desired product $3 \mathbf{b p}$ was obtained as a white solid in $85 \%$ yield $(127.7 \mathrm{mg})$. M.P.: $217.0-219.3{ }^{\circ} \mathrm{C} .{ }^{1} \mathrm{H} \mathrm{NMR}\left(400 \mathrm{MHz}, \mathrm{CDCl}_{3}\right)$ $\delta 7.72(\mathrm{~d}, J=7.7 \mathrm{~Hz}, 2 \mathrm{H}), 7.52(\mathrm{~d}, J=7.6 \mathrm{~Hz}, 2 \mathrm{H}), 7.31-7.23(\mathrm{~m}, 9 \mathrm{H}), 7.18-7.10(\mathrm{~m}, 4 \mathrm{H}), 6.97(\mathrm{t}, J$ $=7.3 \mathrm{~Hz}, 1 \mathrm{H}), 6.89(\mathrm{dd}, J=2.8 \mathrm{~Hz}, 8.6 \mathrm{~Hz}, 1 \mathrm{H}), 6.83(\mathrm{~d}, J=2.7 \mathrm{~Hz}, 1 \mathrm{H}), 5.13(\mathrm{~s}, 2 \mathrm{H}), 4.75(\mathrm{~d}, J=15.2$ Hz, 2H), 4.49 (d, $J=15.2 \mathrm{~Hz}, 2 \mathrm{H}), 2.99-2.92$ (m, 2H), 2.55 (dd, $J=8.6 \mathrm{~Hz}, 18.8 \mathrm{~Hz}, 1 \mathrm{H}), 2.49-2.42$ $(\mathrm{m}, 1 \mathrm{H}), 2.35-2.27(\mathrm{~m}, 1 \mathrm{H}), 2.24-2.14(\mathrm{~m}, 1 \mathrm{H}), 2.13-1.97(\mathrm{~m}, 3 \mathrm{H}), 1.68-1.50(\mathrm{~m}, 6 \mathrm{H}), 0.95(\mathrm{~s}$, 3H). ${ }^{13} \mathrm{C} \mathrm{NMR}\left(101 \mathrm{MHz}, \mathrm{CDCl}_{3}\right) \delta 221.1,157.1,148.5,144.4,143.1,139.2,138.0,137.6,136.6,135.6$, $132.5,129.1,128.4,128.1,127.8,126.9,126.5,121.5,117.6,115.1,112.5,70.3,57.6,50.5,48.2$, 44.1, 38.5, 36.0, 31.7, 29.8, 26.7, 26.0, 21.7, 14.0. HRMS-ESI $(\mathrm{m} / \mathrm{z})[\mathrm{M}+\mathrm{H}]^{+}$Calcd for $\mathrm{C}_{45} \mathrm{H}_{45} \mathrm{NO}_{2} \mathrm{Sb}^{+}$, 752.2483, Found, 752.2473 .

12-(4-((((R)-2,8-dimethyl-2-((4R,8R)-4,8,12-trimethyltridecyl)chroman-6-yl)oxy)methyl) phenyl)6-phenyl-5,6,7,12-tetrahydrodibenzo $[c, f][1,5]$ azastibocine (3bq)

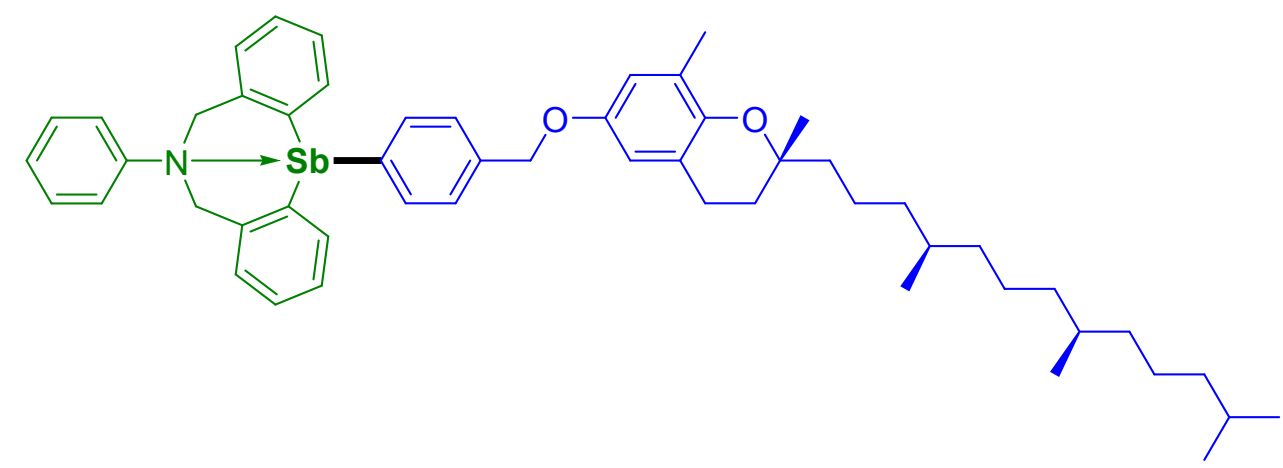

(R)-6-((4-bromobenzyl)oxy)-2,8-dimethyl-2-((4R,8R)-4,8,12-trimethyl tridecyl)chromane 2 da (114.0 $\mathrm{mg}, 0.20 \mathrm{mmol}$ ) was used, the desired product $\mathbf{3 b q}$ as a liquid in $82 \%$ yield $(144.8 \mathrm{mg}) .{ }^{1} \mathrm{H} \mathrm{NMR}$ (400 $\left.\mathrm{MHz}, \mathrm{CDCl}_{3}\right) \delta 7.82-7.67(\mathrm{~m}, 2 \mathrm{H}), 7.61-7.49(\mathrm{~m}, 2 \mathrm{H}), 7.34-7.25(\mathrm{~m}, 8 \mathrm{H}), 7.21-7.14(\mathrm{~m}, 4 \mathrm{H}), 6.99$ $(\mathrm{t}, J=7.3 \mathrm{~Hz}, 1 \mathrm{H}), 6.78(\mathrm{~s}, 1 \mathrm{H}), 6.65(\mathrm{~s}, 1 \mathrm{H}), 5.09(\mathrm{~s}, 2 \mathrm{H}), 4.77(\mathrm{~d}, J=15.2 \mathrm{~Hz}, 2 \mathrm{H}), 4.51(\mathrm{~d}, J=15.2$ $\mathrm{Hz}, 2 \mathrm{H}), 2.80$ (t, $J=7.1 \mathrm{~Hz}, 2 \mathrm{H}), 2.24$ (d, $J=2.6 \mathrm{~Hz}, 3 \mathrm{H}), 1.92-1.75(\mathrm{~m}, 2 \mathrm{H}), 1.71-1.54(\mathrm{~m}, 4 \mathrm{H}), 1.52$ $-1.40(\mathrm{~m}, 4 \mathrm{H}), 1.39-1.27(\mathrm{~m}, 10 \mathrm{H}), 1.23-1.10(\mathrm{~m}, 6 \mathrm{H}), 0.96-0.89(\mathrm{~m}, 12 \mathrm{H}) .{ }^{13} \mathrm{C} \mathrm{NMR}(101 \mathrm{MHz}$, $\left.\mathrm{CDCl}_{3}\right) \delta 151.6,148.5,146.6,144.4,142.8,139.2,138.1,136.7,135.6,129.1,128.4,128.1,127.9,127.4$, 126.9, 121.4, 121.1, 117.6, 115.9, 112.4, 75.7, 70.9, 57.5, 40.2, 39.5, 37.6, 37.6, 37.4, 32.9, 32.8, 31.5, 28.1, 24.9, 24.6, 24.3, 22.9, 22.8, 22.7, 21.1, 19.9, 19.8, 16.4. HRMS-ESI (m/z) $[\mathrm{M}+\mathrm{H}]^{+}$Calcd for $\mathrm{C}_{54} \mathrm{H}_{69} \mathrm{NO}_{2} \mathrm{Sb}^{+}, 884.4361$, Found, 884.4368.

\section{S3.2 Synthesis of Biaryls}

\section{General Procedure B for Synthesis of Biaryls via Cross-Coupling of Stibines with Aryl Halides}


<smiles>S[Sb](c1ccccc1)(c1ccccc1)c1ccccc1CN1Cc2ccccc21</smiles>

3

$0.22 \mathrm{mmol}$

$$
A r^{\prime}-X
$$

$\mathrm{X}=\mathrm{I}$ or $\mathrm{Br}$

2

$0.2 \mathrm{mmol}$
$\mathrm{Pd}(\mathrm{OAc})_{2}(5.0 \mathrm{~mol} \%)$

$n-\mathrm{Bu}_{4} \mathrm{PBr}(20 \mathrm{~mol} \%)$

$\mathrm{Cs}_{2} \mathrm{CO}_{3}$ ( 2.0 equiv)

THF: $\mathrm{H}_{2} \mathrm{O}=50: 1(\mathrm{v}: \mathrm{v}$ )

$110^{\circ} \mathrm{C}, 8 \mathrm{~h}$

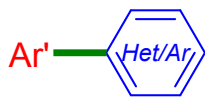

5

Under a nitrogen atmosphere, a $10 \mathrm{~mL}$ Schlenk tube equipped with a magnetic stir bar was charged successively with stibine $(0.22 \mathrm{mmol})$, aryl halide $(0.20 \mathrm{mmol}), \mathrm{Pd}(\mathrm{OAc})_{2}(0.01 \mathrm{mmol}), n-\mathrm{Bu}_{4} \mathrm{PBr}(0.04$ $\mathrm{mmol}), \mathrm{Cs}_{2} \mathrm{CO}_{3}(0.40 \mathrm{mmol}), 2.0 \mathrm{~mL}$ of THF and $40 \mu \mathrm{L}$ of $\mathrm{H}_{2} \mathrm{O}$. The reaction mixture was stirred at 110 ${ }^{\circ} \mathrm{C}$ for $8 \mathrm{~h}$. After the reaction was completed, the resulting mixture was diluted with of $\mathrm{CH}_{2} \mathrm{Cl}_{2}$ and concentrated under reduced pressure. The crude product was purified by flash chromatography on silica gel using petroleum ether (PE) or the mixed solvent system of petroleum ether (PE) and ethyl acetate (EA) to afford the desired products.

Scheme S2. Substrate Scope for the Cross-Coupling of Stibines with Aryl Halides

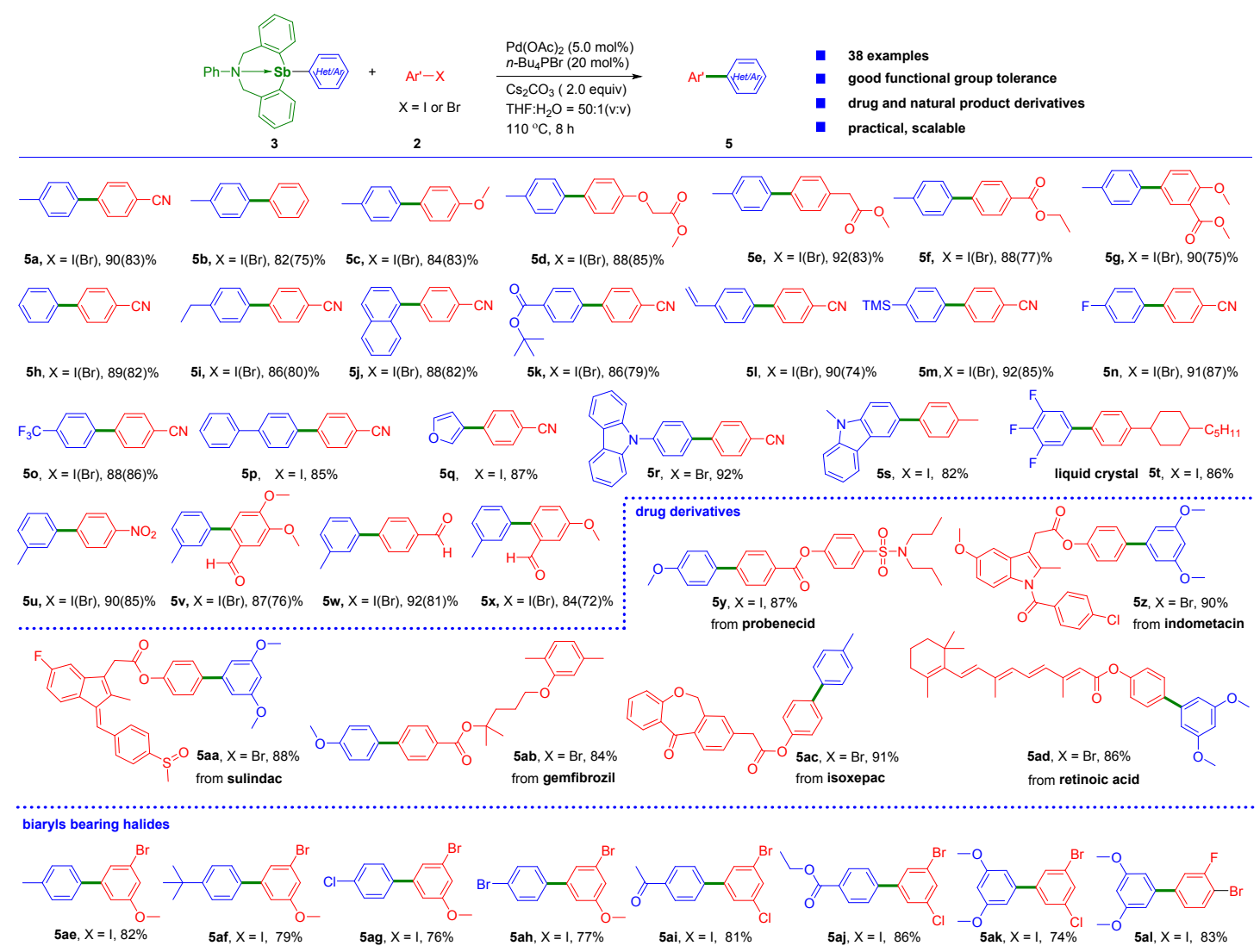

Biaryls $5 \mathbf{5 a}-\mathbf{c}, \mathbf{5 f}, \mathbf{5 j}-\mathbf{k}, \mathbf{5 l}, \mathbf{5 m}, \mathbf{5 u - x}$ and $\mathbf{5 s}$ were reported. ${ }^{\mathrm{S} 1}{ }_{\mathbf{5 e}},{ }^{\mathrm{S} 5} \mathbf{5 g},{ }^{\mathrm{S} 6} \mathbf{5 n}-\mathbf{0},{ }^{\mathrm{S} 7} \mathbf{5 h}-\mathbf{i}, \mathbf{5 r},{ }^{\mathrm{S} 8} \mathbf{5 t},{ }^{\mathrm{S} 9} \mathbf{5 p},{ }^{\mathrm{S} 10}$ and $\mathbf{5} \mathbf{q}^{\mathrm{S} 11}$ were reported.

4'-Methyl-[1,1'-biphenyl]-4-carbonitrile (5a) 


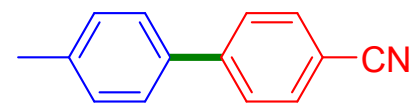

Stibine 3a (106.3 mg, $0.22 \mathrm{mmol}$ ) and 4-iodobenzonitrile $\mathbf{2 b}$ (45.8 $\mathrm{mg}, 0.20 \mathrm{mmol}$ ) were used, the desired product 5a was obtained as as a white solid in 90\% yield (34.7 mg); 4-bromobenzonitrile 2 am was used, the desired product 5a was obtained in $83 \%$ yield $(32.0 \mathrm{mg}) .{ }^{1} \mathrm{H}$ NMR $\left(400 \mathrm{MHz}, \mathrm{CDCl}_{3}\right) \delta 7.68(\mathrm{q}, J=$ $8.4 \mathrm{~Hz}, 4 \mathrm{H}), 7.49$ (d, $J=8.0 \mathrm{~Hz}, 2 \mathrm{H}), 7.29$ (d, $J=7.9 \mathrm{~Hz}, 2 \mathrm{H}), 2.41(\mathrm{~s}, 3 \mathrm{H}) .{ }^{13} \mathrm{C} \mathrm{NMR}\left(101 \mathrm{MHz}, \mathrm{CDCl}_{3}\right)$ $\delta 145.7,138.9,136.4,132.7,130.0,127.6,127.2,119.1,110.7,21.3$.

\section{4-Methyl-1,1'-biphenyl (5b)}

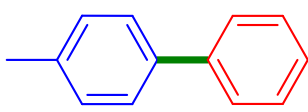

Stibine 3a (106.3 mg, $0.22 \mathrm{mmol})$ and iodobenzene $2 \mathbf{c}(40.8 \mathrm{mg}, 0.20 \mathrm{mmol})$ were used, the desired product $\mathbf{5 b}$ was obtained as as a white solid in $82 \%$ yield $(27.6 \mathrm{mg})$; bromobenzene $\mathbf{2 d}(31.4 \mathrm{mg}, 0.20$ mmol) was used, the desired product $\mathbf{5 b}$ was obtained in $75 \%$ yield $(25.2 \mathrm{mg}) .{ }^{1} \mathrm{H} \mathrm{NMR}(400 \mathrm{MHz}$, $\left.\mathrm{CDCl}_{3}\right) \delta 7.63(\mathrm{~d}, J=7.8 \mathrm{~Hz}, 2 \mathrm{H}), 7.54(\mathrm{~d}, J=7.9 \mathrm{~Hz}, 2 \mathrm{H}), 7.47(\mathrm{t}, J=7.6 \mathrm{~Hz}, 2 \mathrm{H}), 7.37$ (t, $J=7.3 \mathrm{~Hz}$, 1H), 7.30 (d, $J=7.9 \mathrm{~Hz}, 2 \mathrm{H}), 2.44$ (s, 3H). ${ }^{13} \mathrm{C} \mathrm{NMR}\left(101 \mathrm{MHz}, \mathrm{CDCl}_{3}\right) \delta 141.3,138.5,137.1,129.6$, $128.8,127.1,127.1,21.2$.

\section{4-Methoxy-4'-methyl-1,1'-biphenyl (5c)}

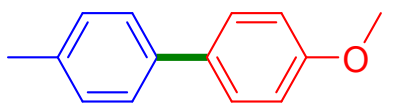

Stibine 3a (106.3 mg, $0.22 \mathrm{mmol})$ and 1-iodo-4-methoxybenzene $2 \mathbf{h}(46.8 \mathrm{mg}, 0.20 \mathrm{mmol})$ were used, the desired product $\mathbf{5 c}$ was obtained as as a white solid in $84 \%$ yield $(33.3 \mathrm{mg})$; 1-bromo-4methoxybenzene $2 \mathbf{i}$ (37.2 $\mathrm{mg}, 0.20 \mathrm{mmol}$ ) was used, the desired product $\mathbf{5 c}$ was obtained in $83 \%$ yield (32.9 mg). ${ }^{1} \mathrm{H}$ NMR (400 MHz, $\left.\mathrm{CDCl}_{3}\right) \delta 7.56(\mathrm{~d}, J=8.3 \mathrm{~Hz}, 2 \mathrm{H}), 7.50(\mathrm{~d}, J=7.7 \mathrm{~Hz}, 2 \mathrm{H}), 7.28(\mathrm{~d}, J=$ $7.7 \mathrm{~Hz}, 2 \mathrm{H}), 7.02(\mathrm{~d}, J=8.3 \mathrm{~Hz}, 2 \mathrm{H}), 3.89(\mathrm{~s}, 3 \mathrm{H}), 2.43(\mathrm{~s}, 3 \mathrm{H}) .{ }^{13} \mathrm{C} \mathrm{NMR}\left(101 \mathrm{MHz}, \mathrm{CDCl}_{3}\right) \delta 159.0$, $138.1,136.5,133.9,129.6,128.1,126.7,114.3,55.5,21.2$.

\section{Methyl 2-((4'-methyl-[1,1'-biphenyl]-4-yl)oxy)acetate (5d)}

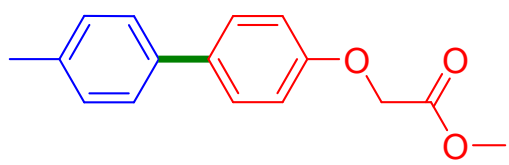

Stibine 3a (106.3 mg, $0.22 \mathrm{mmol}$ ) and methyl 2-(4-iodophenoxy)acetate 2 co were used, the desired product 5d was obtained as a white solid in $88 \%$ yield $(45.1 \mathrm{mg})$; methyl 2-(4-bromophenoxy)acetate $\mathbf{2 c p}(48.8 \mathrm{mg}, 0.20 \mathrm{mmol}$ ) was used, the desired product $\mathbf{5 d}$ was obtained in $85 \%$ yield (43.5 mg). M.P.: 120.5 - $123.1^{\circ} \mathrm{C} .{ }^{1} \mathrm{H}$ NMR $(400 \mathrm{MHz}$, Chloroform- $d) \delta 7.54(\mathrm{~d}, J=8.3 \mathrm{~Hz}, 2 \mathrm{H}), 7.47$ (d, $J=7.7 \mathrm{~Hz}$, 2H), $7.26(\mathrm{~d}, J=7.7 \mathrm{~Hz}, 2 \mathrm{H}), 7.00(\mathrm{~d}, J=8.3 \mathrm{~Hz}, 2 \mathrm{H}), 4.71(\mathrm{~s}, 2 \mathrm{H}), 3.86(\mathrm{~s}, 3 \mathrm{H}), 2.42(\mathrm{~s}, 3 \mathrm{H}) .{ }^{13} \mathrm{C}$ NMR $\left(101 \mathrm{MHz}, \mathrm{CDCl}_{3}\right) \delta 169.5,157.2,137.8,136.7,135.0,129.6,128.2,126.7,115.0,65.5,52.4,21.2$. HRMS-EI (m/z) [M] Calcd for $\mathrm{C}_{16} \mathrm{H}_{16} \mathrm{O}_{3}, 256.1099$; Found, 256.1096. 


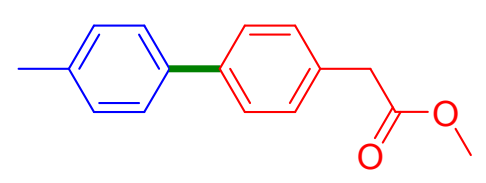

Stibine 3a (106.3 mg, $0.22 \mathrm{mmol})$ and methyl 2-(4-iodophenyl)acetate $2 \mathrm{~cm}(55.2 \mathrm{mg}, 0.20 \mathrm{mmol})$ were used, the desired product $\mathbf{5 e}$ was obtained as a white solid in $92 \%$ yield $(44.2 \mathrm{mg})$; methyl 2-(4bromophenyl)acetate $2 \mathbf{c n}(45.6 \mathrm{mg}, 0.20 \mathrm{mmol})$ was used, the desired product $5 \mathbf{e}$ was obtained in $83 \%$ yield (39.8 mg). M.P.: $33.4-34.0{ }^{\circ} \mathrm{C} .{ }^{1} \mathrm{H}$ NMR $\left(400 \mathrm{MHz}, \mathrm{CDCl}_{3}\right) \delta 7.53(\mathrm{~d}, J=8.1 \mathrm{~Hz}, 2 \mathrm{H}), 7.46(\mathrm{~d}, J$ $=8.1 \mathrm{~Hz}, 2 \mathrm{H}), 7.32(\mathrm{~d}, J=8.1 \mathrm{~Hz}, 2 \mathrm{H}), 7.22(\mathrm{~d}, J=7.9 \mathrm{~Hz}, 2 \mathrm{H}), 3.69$ (s, 3H), $3.64(\mathrm{~s}, 2 \mathrm{H}), 2.37$ (s, 3H). ${ }^{13} \mathrm{C}$ NMR $\left(101 \mathrm{MHz}, \mathrm{CDCl}_{3}\right) \delta 172.1,140.1,138.0,137.1,132.8,129.7,129.6,127.2,127.0,52.2,40.9$, 21.2. HRMS-EI (m/z) [M] Calcd for $\mathrm{C}_{16} \mathrm{H}_{16} \mathrm{O}_{2}, 240.1150$; Found, 240.1149.

\section{Ethyl 4'-methyl-[1,1'-biphenyl]-4-carboxylate (5f)}

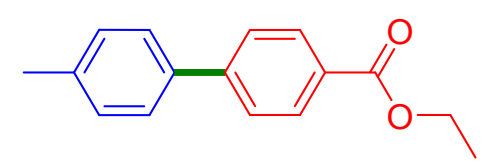

Stibine 3a (106.3 mg, $0.22 \mathrm{mmol})$ and ethyl 4-iodobenzoate 2at $(55.2 \mathrm{mg}, 0.20 \mathrm{mmol})$ were used, the desired product $\mathbf{5 f}$ was obtained as as a white solid in $88 \%$ yield $(42.2 \mathrm{mg}$ ); ethyl 4-bromobenzoate $\mathbf{2 b w}$ ( $45.6 \mathrm{mg}, 0.20 \mathrm{mmol}$ ) was used, the desired product $\mathbf{5 f}$ was obtained in $77 \%$ yield $(37.0 \mathrm{mg})$. ${ }^{1} \mathrm{H}$ NMR $\left(400 \mathrm{MHz}, \mathrm{CDCl}_{3}\right) \delta 8.14(\mathrm{~d}, J=8.3 \mathrm{~Hz}, 2 \mathrm{H}), 7.69(\mathrm{~d}, J=7.1 \mathrm{~Hz}, 2 \mathrm{H}), 7.57(\mathrm{~d}, J=7.7 \mathrm{~Hz}, 2 \mathrm{H}), 7.32(\mathrm{~d}$, $J=8.3 \mathrm{~Hz}, 2 \mathrm{H}), 4.44$ (q, $J=7.0 \mathrm{~Hz}, 2 \mathrm{H}), 2.45$ (s, 3H), 1.46 (t, $J=7.1 \mathrm{~Hz}, 3 \mathrm{H}) .{ }^{13} \mathrm{C}$ NMR $(101 \mathrm{MHz}$, $\left.\mathrm{CDCl}_{3}\right) \delta 166.7,145.6,138.2,137.3,130.2,129.8,129.1,127.2,126.9,61.1,21.3,14.5$.

\section{Methyl 4-methoxy-4'-methyl-[1,1'-biphenyl]-3-carboxylate (5g)}

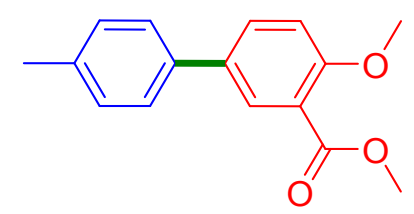

Stibine 3a (106.3 mg, $0.22 \mathrm{mmol})$ and methyl 5-iodo-2-methoxybenzoate 2ck (58.4 mg, $0.20 \mathrm{mmol})$ were used, the desired product $5 \mathrm{~g}$ was obtained as a white solid in $90 \%$ yield $(46.1 \mathrm{mg})$; methyl 5-bromo2-methoxybenzoate $\mathbf{2 c l}(48.8 \mathrm{mg}, 0.20 \mathrm{mmol}$ ) was used, the desired product $\mathbf{5 g}$ was obtained in $75 \%$ yield (38.4 mg). M.P.: $63.0-64.1^{\circ} \mathrm{C} .{ }^{1} \mathrm{H}$ NMR $\left(400 \mathrm{MHz}, \mathrm{CDCl}_{3}\right) \delta 8.08(\mathrm{~s}, 1 \mathrm{H}), 7.73(\mathrm{~d}, J=8.7 \mathrm{~Hz}$, $1 \mathrm{H}), 7.51$ (d, $J=7.9 \mathrm{~Hz}, 2 \mathrm{H}), 7.29$ (d, $J=7.8 \mathrm{~Hz}, 2 \mathrm{H}), 7.09$ (d, $J=8.6 \mathrm{~Hz}, 1 \mathrm{H}), 3.99$ (s, 3H), 3.97 (s, 3H), 2.44 (s, 3H). ${ }^{13} \mathrm{C}$ NMR (101 MHz, $\mathrm{CDCl}_{3}$ ) $\delta 166.8,158.5,137.0,136.9,133.4,131.8,130.2,129.7$, 126.7, 120.4, 112.6, 56.3, 52.2, 21.2. HRMS-EI (m/z) [M] Calcd for $\mathrm{C}_{16} \mathrm{H}_{16} \mathrm{O}_{3}, 256.1099$; Found, 256.1099 .

\section{[1,1'-Biphenyl]-4-carbonitrile (5h)}




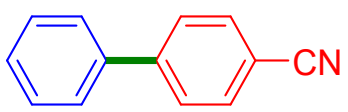

Stibine 3b (103.2 mg, $0.22 \mathrm{mmol}$ ) and 4-iodobenzonitrile $\mathbf{2 b}$ (45.8 mg, $0.20 \mathrm{mmol}$ ) were used, the desired product $\mathbf{5 h}$ was obtained as a white solid in $89 \%$ yield $(31.9 \mathrm{mg})$; 4-bromobenzonitrile $\mathbf{2} \mathbf{a m}(36.2 \mathrm{mg}$, $0.20 \mathrm{mmol}$ ) was used, the desired product $\mathbf{5 h}$ was obtained in $82 \%$ yield $(29.4 \mathrm{mg})$. M.P.: $85.6-86.1^{\circ} \mathrm{C}$. ${ }^{1} \mathrm{H}$ NMR (400 MHz, $\left.\mathrm{CDCl}_{3}\right) \delta 7.71$ (q, $\left.J=7.8 \mathrm{~Hz}, 4 \mathrm{H}\right), 7.59(\mathrm{~d}, J=7.1 \mathrm{~Hz}, 2 \mathrm{H}), 7.49(\mathrm{t}, J=7.1 \mathrm{~Hz}, 2 \mathrm{H})$, $7.46-7.38(\mathrm{~m}, 1 \mathrm{H}) .{ }^{13} \mathrm{C} \mathrm{NMR}\left(101 \mathrm{MHz}, \mathrm{CDCl}_{3}\right) \delta 145.8,139.3,132.7,129.2,128.8,127.8,127.3$, 119.0, 111.0. HRMS-EI (m/z) [M] Calcd for $\mathrm{C}_{13} \mathrm{H}_{9} \mathrm{~N}, 179.0735$; Found, 179.0732.

4'-Ethyl-[1,1'-biphenyl]-4-carbonitrile (5i)

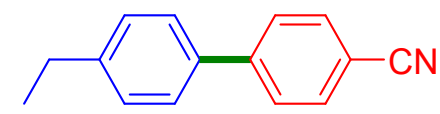

Stibine $3 \mathbf{t}$ (109.3 mg, $0.22 \mathrm{mmol}$ ) and 4-iodobenzonitrile $\mathbf{2 b}$ (45.8 mg, $0.20 \mathrm{mmol}$ ) were used, the desired product 5i was obtained as a white solid in $86 \%$ yield $(35.6 \mathrm{mg})$; 4-bromobenzonitrile $2 \mathrm{am}(36.2 \mathrm{mg}$, $0.20 \mathrm{mmol}$ ) was used, the desired product $5 \mathbf{i}$ was obtained in $80 \%$ yield $(33.1 \mathrm{mg}) .{ }^{1} \mathrm{H} \mathrm{NMR}(400 \mathrm{MHz}$, Chloroform- $d$ ) $\delta 7.69$ (q, $J=8.1 \mathrm{~Hz}, 4 \mathrm{H}), 7.53(\mathrm{~d}, J=7.8 \mathrm{~Hz}, 2 \mathrm{H}), 7.33(\mathrm{~d}, J=7.7 \mathrm{~Hz}, 2 \mathrm{H}), 2.72(\mathrm{q}, J=$ $7.5 \mathrm{~Hz}, 2 \mathrm{H}), 1.29$ (t, $J=7.6 \mathrm{~Hz}, 3 \mathrm{H}) .{ }^{13} \mathrm{C} \mathrm{NMR}\left(101 \mathrm{MHz}, \mathrm{CDCl}_{3}\right) \delta 145.7,145.2,136.6,132.7,128.8$, $127.6,127.3,119.1,110.7,28.7,15.6$.

\section{4-(Naphthalen-1-yl)benzonitrile (5j)}

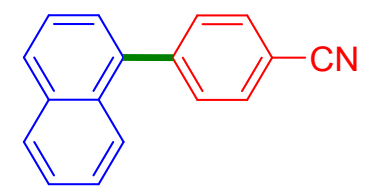

Stibine 3ai (114.2 mg, $0.22 \mathrm{mmol}$ ) and 4-iodobenzonitrile $2 \mathbf{b}(45.8 \mathrm{mg}, 0.20 \mathrm{mmol})$ were used, the desired product $\mathbf{5 j}$ was obtained as a white solid in $88 \%$ yield $(40.3 \mathrm{mg})$; 4-bromobenzonitrile 2 am $(36.2$ $\mathrm{mg}, 0.20 \mathrm{mmol}$ ) was used, the desired product $\mathbf{5 j}$ was obtained in $82 \%$ yield $(37.6 \mathrm{mg})$. ${ }^{1} \mathrm{H}$ NMR (400 $\left.\mathrm{MHz} \mathrm{CDCl}_{3}\right) \delta 7.95(\mathrm{t}, J=7.6 \mathrm{~Hz}, 2 \mathrm{H}), 7.85-7.71(\mathrm{~m}, 3 \mathrm{H}), 7.64-7.53(\mathrm{~m}, 4 \mathrm{H}), 7.49(\mathrm{t}, J=7.5 \mathrm{~Hz}$, 1H), $7.41(\mathrm{~d}, J=7.0 \mathrm{~Hz}, 1 \mathrm{H}) .{ }^{13} \mathrm{C} \mathrm{NMR}\left(101 \mathrm{MHz}, \mathrm{CDCl}_{3}\right) \delta 145.6,138.2,133.8,132.1,131.0,130.8$, $128.8,128.6,127.1,126.7,126.2,125.4,125.2,118.9,111.2$.

\section{Tert-butyl 4'-cyano-[1,1'-biphenyl]-4-carboxylate (5k)}

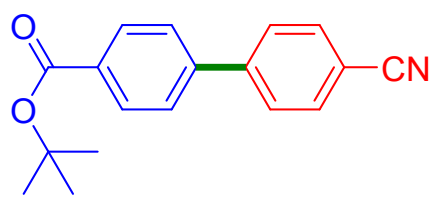

Stibine 3ae (125.2 $\mathrm{mg}, 0.22 \mathrm{mmol})$ and 4-iodobenzonitrile $\mathbf{2 b}(45.8 \mathrm{mg}, 0.20 \mathrm{mmol})$ were used, the desired product $\mathbf{5 k}$ was obtained as a white solid in $86 \%$ yield $(48.0 \mathrm{mg})$; 4-bromobenzonitrile 2 am $(36.2$ $\mathrm{mg}, 0.20 \mathrm{mmol}$ ) was used, the desired product 5k was obtained in 79\% yield (44.1 mg). ${ }^{1} \mathrm{H}$ NMR (400 $\left.\mathrm{MHz} \mathrm{CDCl}_{3}\right) \delta 8.08(\mathrm{~d}, J=8.1 \mathrm{~Hz}, 2 \mathrm{H}), 7.72(\mathrm{q}, J=8.2 \mathrm{~Hz}, 4 \mathrm{H}), 7.62(\mathrm{~d}, J=8.1 \mathrm{~Hz}, 2 \mathrm{H}), 1.61(\mathrm{~s}, 9 \mathrm{H})$. 
${ }^{13} \mathrm{C}$ NMR $\left(101 \mathrm{MHz}, \mathrm{CDCl}_{3}\right) \delta 165.3,144.7,143.0,132.8,132.2,130.3,128.0,127.1,118.8,111.8,81.5$, 28.3 .

4'-Vinyl-[1,1'-biphenyl]-4-carbonitrile (5l)

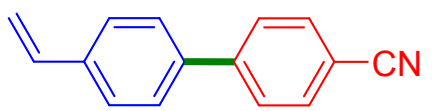

Stibine 3aa (108.9 $\mathrm{mg}, 0.22 \mathrm{mmol})$ and 4-iodobenzonitrile $\mathbf{2 b}(45.8 \mathrm{mg}, 0.20 \mathrm{mmol})$ were used, the desired product $\mathbf{5} \mathbf{l}$ was obtained as a white solid in $90 \%$ yield $(36.9 \mathrm{mg})$; 4-bromobenzonitrile $\mathbf{2 a m}(36.2$ $\mathrm{mg}, 0.20 \mathrm{mmol}$ ) was used, the desired product $5 \mathrm{l}$ was obtained in $74 \%$ yield $(30.3 \mathrm{mg}) .{ }^{1} \mathrm{H}$ NMR (400 MHz, $\left.\mathrm{CDCl}_{3}\right) \delta 7.75-7.65(\mathrm{~m}, 4 \mathrm{H}), 7.61-7.49(\mathrm{~m}, 4 \mathrm{H}), 6.77$ (dd, $\left.J=10.9 \mathrm{~Hz}, 17.6 \mathrm{~Hz}, 1 \mathrm{H}\right), 5.84(\mathrm{~d}$, $J=17.6 \mathrm{~Hz}, 1 \mathrm{H}), 5.34$ (d, $J=10.8 \mathrm{~Hz}, 1 \mathrm{H}) .{ }^{13} \mathrm{C} \mathrm{NMR}\left(101 \mathrm{MHz}, \mathrm{CDCl}_{3}\right) \delta 145.2,138.4,138.0,136.0$, $132.6,127.5,127.4,126.9,118.9,115.0,110.9$.

4'-(Trimethylsilyl)-[1,1'-biphenyl]-4-carbonitrile (5m)

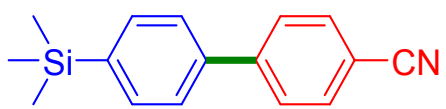

Stibine 3ac (119.0 mg, $0.22 \mathrm{mmol})$ and 4-iodobenzonitrile $\mathbf{2 b}(45.8 \mathrm{mg}, 0.20 \mathrm{mmol})$ were used, the desired product $\mathbf{5 m}$ was obtained as a white solid in $92 \%$ yield $(46.2 \mathrm{mg})$; 4-bromobenzonitrile 2am ( $36.2 \mathrm{mg}, 0.20 \mathrm{mmol}$ ) was used, the desired product $5 \mathrm{~m}$ was obtained in $85 \%$ yield $\left(42.7 \mathrm{mg}\right.$ ). ${ }^{1} \mathrm{H}$ NMR $\left(400 \mathrm{MHz}, \mathrm{CDCl}_{3}\right) \delta 7.72$ (q, $\left.J=8.4 \mathrm{~Hz}, 4 \mathrm{H}\right), 7.65$ (d, $\left.J=7.9 \mathrm{~Hz}, 2 \mathrm{H}\right), 7.59$ (d, $\left.J=8.2 \mathrm{~Hz}, 2 \mathrm{H}\right), 0.32$ (s, 9H). ${ }^{13} \mathrm{C}$ NMR (101 MHz, $\left.\mathrm{CDCl}_{3}\right) \delta 145.8,141.4,139.6,134.2,132.7,127.8,126.6,119.1,111.1,-1.0$.

4'-Fluoro-[1,1'-biphenyl]-4-carbonitrile (5n)

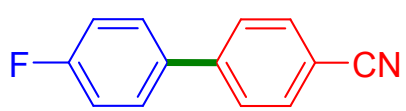

Stibine $3 \mathbf{i}$ (107.1 $\mathrm{mg}, 0.22 \mathrm{mmol}$ ) and 4-iodobenzonitrile $\mathbf{2 b}$ ( $45.8 \mathrm{mg}, 0.20 \mathrm{mmol})$ were used, the desired product $\mathbf{5 n}$ was obtained as a white solid in $91 \%$ yield $(35.9 \mathrm{mg})$; 4-bromobenzonitrile $2 \mathbf{a m}(36.2 \mathrm{mg}$, $0.20 \mathrm{mmol}$ ) was used, the desired product $\mathbf{5 n}$ was obtained in $87 \%$ yield $(34.3 \mathrm{mg})$. M.P.: $108.3-110.1$ ${ }^{\circ} \mathrm{C} .{ }^{1} \mathrm{H}$ NMR $\left(400 \mathrm{MHz}, \mathrm{CDCl}_{3}\right) \delta 7.74(\mathrm{~d}, J=7.8 \mathrm{~Hz}, 2 \mathrm{H}), 7.66(\mathrm{~d}, J=7.8 \mathrm{~Hz}, 2 \mathrm{H}), 7.62-7.54(\mathrm{~m}$, 2H), $7.19(\mathrm{t}, J=8.1 \mathrm{~Hz}, 2 \mathrm{H}) .{ }^{13} \mathrm{C} \mathrm{NMR}\left(101 \mathrm{MHz}, \mathrm{CDCl}_{3}\right) \delta 163.3(\mathrm{~d}, J=248.9 \mathrm{~Hz}), 144.7,135.4(\mathrm{~d}, J$ $=3.3 \mathrm{~Hz}), 132.8,129.1(\mathrm{~d}, J=8.3 \mathrm{~Hz}), 127.7,118.9,116.2(\mathrm{~d}, J=21.7 \mathrm{~Hz}), 111.1 .{ }^{19} \mathrm{~F}$ NMR $(376 \mathrm{MHz}$, $\left.\mathrm{CDCl}_{3}\right) \delta$-113.16. HRMS-EI (m/z) [M] Calcd for $\mathrm{C}_{13} \mathrm{H}_{8} \mathrm{FN}$, 197.0641; Found, 197.0647.

4'-(Trifluoromethyl)-[1,1'-biphenyl]-4-carbonitrile (5o)

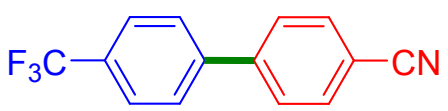

Stibine 3n (118.1 mg, $0.22 \mathrm{mmol}$ ) and 4-iodobenzonitrile $2 \mathbf{b}$ ( $45.8 \mathrm{mg}, 0.20 \mathrm{mmol})$ were used, the desired product $5 \mathbf{0}$ was obtained as a white solid in $88 \%$ yield $(43.5 \mathrm{mg}$ ); 4-bromobenzonitrile $2 \mathbf{a m}(36.2 \mathrm{mg}$, 
$0.20 \mathrm{mmol}$ ) was used, the desired product 50 was obtained in $86 \%$ yield $(42.5 \mathrm{mg})$. M.P.: $126.8-$ 127.6 ${ }^{\circ} \mathrm{C} .{ }^{1} \mathrm{H}$ NMR $\left(400 \mathrm{MHz}, \mathrm{CDCl}_{3}\right) \delta 7.78(\mathrm{t}, J=7.8 \mathrm{~Hz}, 4 \mathrm{H}), 7.72(\mathrm{~d}, J=7.9 \mathrm{~Hz}, 4 \mathrm{H}) .{ }^{13} \mathrm{C}$ NMR $\left(101 \mathrm{MHz}, \mathrm{CDCl}_{3}\right) \delta 144.2,142.8,132.9,130.7$ (q, $\left.J=32.7 \mathrm{~Hz}\right), 128.0,127.7,126.1$ (q, $J=3.7 \mathrm{~Hz}$ ), $124.1(\mathrm{q}, J=272.2 \mathrm{~Hz}), 118.7,112.1 .{ }^{19} \mathrm{~F}$ NMR $\left(376 \mathrm{MHz}, \mathrm{CDCl}_{3}\right) \delta-62.60$. HRMS-EI $(\mathrm{m} / \mathrm{z})[\mathrm{M}] \mathrm{Calcd}$ for $\mathrm{C}_{14} \mathrm{H}_{8} \mathrm{~F}_{3} \mathrm{~N}, 247.0609$; Found, 247.0610.

\section{$\left[1,1^{\prime}: 4^{\prime}, 1^{\prime \prime}-\right.$ Terphenyl]-4-carbonitrile (5p)}

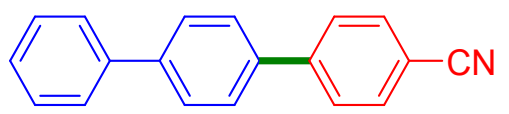

Stibine $3 \mathbf{x}$ (119.9 $\mathrm{mg}, 0.22 \mathrm{mmol})$ and 4-iodobenzonitrile $\mathbf{2 b}$ (45.8 $\mathrm{mg}, 0.20 \mathrm{mmol})$ were used, the desired product $5 \mathbf{p}$ was obtained as a white solid in $85 \%$ yield $(43.4 \mathrm{mg})$. M.P.: $177.0-179.3^{\circ} \mathrm{C} .{ }^{1} \mathrm{H}$ NMR $(400$ $\left.\mathrm{MHz}, \mathrm{CDCl}_{3}\right) \delta 7.73(\mathrm{~d}, J=9.6 \mathrm{~Hz}, 6 \mathrm{H}), 7.70-7.63$ (m, 4H), 7.49 (t, $\left.J=7.4 \mathrm{~Hz}, 2 \mathrm{H}\right), 7.40$ (t, $J=7.2$ $\mathrm{Hz}, 1 \mathrm{H}) .{ }^{13} \mathrm{C}$ NMR (101 MHz, $\left.\mathrm{CDCl}_{3}\right) \delta 145.2,141.6,140.3,138.0,132.7,129.0,127.9,127.9,127.7$, 127.6, 127.2, 119.1, 111.0. HRMS-ESI (m/z) $[\mathrm{M}+\mathrm{H}]^{+}$Calcd for $\mathrm{C}_{19} \mathrm{H}_{14} \mathrm{~N}^{+}$, 256.1121; Found, 256.1129 .

\section{4-(Furan-3-yl)benzonitrile (5q)}

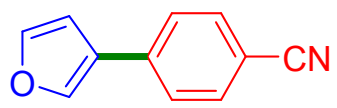

Stibine 3as (101.0 mg, $0.22 \mathrm{mmol})$ and 4-iodobenzonitrile $2 \mathbf{b}(45.8 \mathrm{mg}, 0.20 \mathrm{mmol})$ were used, the desired product $\mathbf{5 q}$ was obtained as a white solid in $87 \%$ yield $(29.4 \mathrm{mg})$. M.P.: $55.6-58.2{ }^{\circ} \mathrm{C}$. ${ }^{1} \mathrm{H}$ NMR $\left(400 \mathrm{MHz}, \mathrm{CDCl}_{3}\right) \delta 7.82(\mathrm{t}, J=1.2 \mathrm{~Hz}, 1 \mathrm{H}), 7.67-7.65(\mathrm{~m}, 1 \mathrm{H}), 7.64-7.62(\mathrm{~m}, 1 \mathrm{H}), 7.58-7.56(\mathrm{~m}$, $1 \mathrm{H}), 7.56-7.54(\mathrm{~m}, 1 \mathrm{H}), 7.52(\mathrm{t}, J=1.7 \mathrm{~Hz}, 1 \mathrm{H}), 6.72-6.70(\mathrm{~m}, 1 \mathrm{H}) .{ }^{13} \mathrm{C} \mathrm{NMR}\left(101 \mathrm{MHz}, \mathrm{CDCl}_{3}\right) \delta$ 144.5, 140.0, 137.2, 132.8, 126.3, 125.2, 119.0, 110.4, 108.5. HRMS-EI $(\mathrm{m} / \mathrm{z})[\mathrm{M}+\mathrm{Na}]^{+}$Calcd for $\mathrm{C}_{11} \mathrm{H}_{7} \mathrm{NNaO}^{+}$, 192.0420; Found, 192.0420 .

4'-(9H-carbazol-9-yl)-[1,1'-biphenyl]-4-carbonitrile (5r)

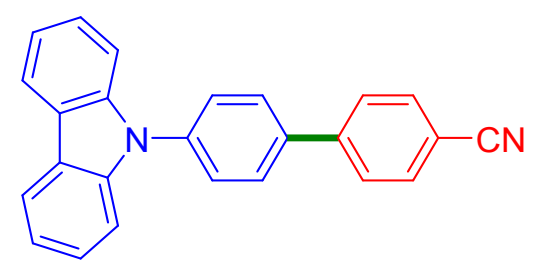

Stibine 3ak (139.5 mg, $0.22 \mathrm{mmol}$ ) and 4-bromobenzonitrile $2 \mathbf{a m}(36.2 \mathrm{mg}, 0.20 \mathrm{mmol})$ were used, the desired product $\mathbf{5 r}$ was obtained as a white solid in $92 \%$ yield $(63.3 \mathrm{mg})$. M.P.: $251.8-252.2{ }^{\circ} \mathrm{C} .{ }^{1} \mathrm{H}$ $\operatorname{NMR}\left(400 \mathrm{MHz}, \mathrm{CDCl}_{3}\right) \delta 8.17(\mathrm{~d}, J=7.7 \mathrm{~Hz}, 2 \mathrm{H}), 7.87-7.75(\mathrm{~m}, 6 \mathrm{H}), 7.70(\mathrm{~d}, J=8.3 \mathrm{~Hz}, 2 \mathrm{H}), 7.52$ $-7.40(\mathrm{~m}, 4 \mathrm{H}), 7.33(\mathrm{t}, J=7.3 \mathrm{~Hz}, 2 \mathrm{H}) .{ }^{13} \mathrm{C} \mathrm{NMR}\left(101 \mathrm{MHz}, \mathrm{CDCl}_{3}\right) \delta 144.8,140.8,138.4,138.2,132.9$, 128.8, 127.8, 127.7, 126.2, 123.7, 120.6, 120.4, 119.0, 111.5, 109.9. HRMS-EI (m/z) [M] Calcd for $\mathrm{C}_{25} \mathrm{H}_{16} \mathrm{~N}_{2}, 344.1313$; Found, 344.1317 .

9-Methyl-3-(p-tolyl)-9H-carbazole (5s) 


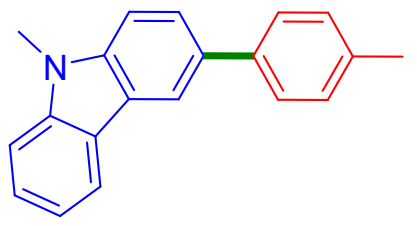

Stibine 3al (125.8 mg, $0.22 \mathrm{mmol})$ and 1-iodo-4-methylbenzene 2a (43.6 mg, $0.20 \mathrm{mmol})$ were used, the desired product $\mathbf{5 s}$ was obtained as a white solid in $82 \%$ yield $(44.4 \mathrm{mg}) .{ }^{1} \mathrm{H} \mathrm{NMR}\left(400 \mathrm{MHz}, \mathrm{CDCl}_{3}\right) \delta$ $8.32(\mathrm{~s}, 1 \mathrm{H}), 8.17(\mathrm{~d}, J=7.7 \mathrm{~Hz}, 1 \mathrm{H}), 7.79-7.70(\mathrm{~m}, 1 \mathrm{H}), 7.65(\mathrm{~d}, J=8.0 \mathrm{~Hz}, 2 \mathrm{H}), 7.55-7.40(\mathrm{~m}, 3 \mathrm{H})$, $7.32(\mathrm{~d}, J=7.8 \mathrm{~Hz}, 2 \mathrm{H}), 7.28(\mathrm{~d}, J=1.9 \mathrm{~Hz}, 1 \mathrm{H}), 3.90(\mathrm{~s}, 3 \mathrm{H}), 2.45(\mathrm{~s}, 3 \mathrm{H}) .{ }^{13} \mathrm{C} \mathrm{NMR}\left(101 \mathrm{MHz}, \mathrm{CDCl}_{3}\right)$ $\delta 141.6,140.5,139.4,136.2,132.5,129.6,127.3,126.0,125.3,123.4,123.1,120.5,119.1,118.7,108.7$, $108.7,29.3,21.2$.

\section{3,4,5-Trifluoro-4'-(4-pentylcyclohexyl)-1,1'-biphenyl (5t)}

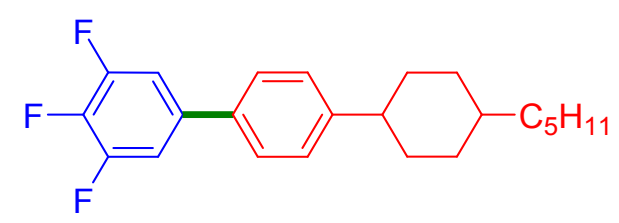

Stibine 3k (115.1 mg, $0.22 \mathrm{mmol}$ ) and 1-iodo-4-(4-pentylcyclohexyl)benzene 2cq (71.2 mg, $0.20 \mathrm{mmol})$ were used, the desired product $\mathbf{5 t}$ was obtained as a liquid in $86 \%$ yield $(61.9 \mathrm{mg}) .{ }^{1} \mathrm{H} \mathrm{NMR}(400 \mathrm{MHz}$, $\left.\mathrm{CDCl}_{3}\right) \delta 7.46(\mathrm{~d}, J=7.9 \mathrm{~Hz}, 2 \mathrm{H}), 7.33(\mathrm{~d}, J=7.9 \mathrm{~Hz}, 2 \mathrm{H}), 7.25-7.16(\mathrm{~m}, 2 \mathrm{H}), 2.55(\mathrm{t}, J=12.1 \mathrm{~Hz}$, $1 \mathrm{H}), 1.95(\mathrm{t}, J=9.4 \mathrm{~Hz}, 4 \mathrm{H}), 1.58-1.45(\mathrm{~m}, 2 \mathrm{H}), 1.41-1.24(\mathrm{~m}, 9 \mathrm{H}), 1.17-1.05(\mathrm{~m}, 2 \mathrm{H}), 0.95(\mathrm{t}, J=$ $6.7 \mathrm{~Hz}, 3 \mathrm{H}) .{ }^{13} \mathrm{C} \mathrm{NMR}\left(101 \mathrm{MHz}, \mathrm{CDCl}_{3}\right) \delta 152.6(\mathrm{dd}, J=9.9,4.4 \mathrm{~Hz}), 150.2(\mathrm{dd}, J=10.0,4.2 \mathrm{~Hz})$, 148.4, 140.3 (t, $J=15.3 \mathrm{~Hz}), 137.8$ (t, $J=15.6 \mathrm{~Hz}), 137.3$ (dd, $J=7.8,3.2 \mathrm{~Hz}), 135.7,127.6,126.7$, $110.8(\mathrm{dd}, J=15.8,5.7 \mathrm{~Hz}), 44.3,37.4,37.3,34.3,33.6,32.3,26.7,22.8,14.2 .{ }^{19} \mathrm{~F}$ NMR $(376 \mathrm{MHz}$, $\left.\mathrm{CDCl}_{3}\right) \delta-134.41(\mathrm{~d}, J=20.9 \mathrm{~Hz}),-163.26(\mathrm{t}, J=20.9 \mathrm{~Hz})$. HRMS-EI (m/z) [M] Calcd for $\mathrm{C}_{23} \mathrm{H}_{27} \mathrm{~F}_{3}$, 360.2065; Found, 360.2063.

\section{3-Methyl-4'-nitro-1,1'-biphenyl (5u)}

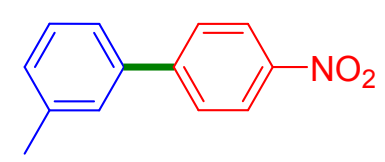

Stibine 3c (106.3 mg, $0.22 \mathrm{mmol}$ ) and 1-iodo-4-nitrobenzene $\mathbf{2 b z}$ (49.8 $\mathrm{mg}, 0.20 \mathrm{mmol}$ ) were used, the desired product $\mathbf{5 u}$ was obtained as as a white solid in 90\% yield (38.3 mg); 1-bromo-4-nitrobenzene $\mathbf{2 c a}$ (40.2 mg, $0.20 \mathrm{mmol}$ ) was used, the desired product $\mathbf{5 u}$ was obtained in $85 \%$ yield $(36.2 \mathrm{mg}) .{ }^{1} \mathrm{H}$ NMR $\left(400 \mathrm{MHz}, \mathrm{CDCl}_{3}\right) \delta 8.31(\mathrm{~d}, J=8.8 \mathrm{~Hz}, 2 \mathrm{H}), 7.75(\mathrm{~d}, J=8.8 \mathrm{~Hz}, 2 \mathrm{H}), 7.48-7.38(\mathrm{~m}, 3 \mathrm{H}), 7.29(\mathrm{~d}, J=$ $6.0 \mathrm{~Hz}, 1 \mathrm{H}), 2.47$ (s, 3H). ${ }^{13} \mathrm{C} \mathrm{NMR}\left(101 \mathrm{MHz}, \mathrm{CDCl}_{3}\right) \delta 147.9,147.1,139.0,138.9,129.8,129.2,128.2$, $127.9,124.6,124.2,21.7$.

4,5-Dimethoxy-3'-methyl-[1,1'-biphenyl]-2-carbaldehyde (5v) 


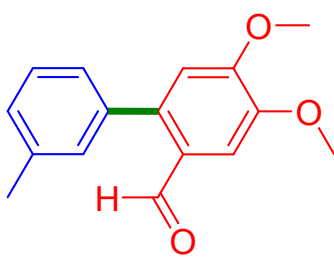

Stibine 3c (106.3 mg, $0.22 \mathrm{mmol})$ and 2-iodo-4,5-dimethoxybenzaldehyde 2ce (58.4 mg, $0.20 \mathrm{mmol})$ were used, the desired product $\mathbf{5} \mathbf{v}$ was obtained as a white solid in $87 \%$ yield $(44.5 \mathrm{mg})$; 2-bromo-4,5dimethoxybenzaldehyde $2 \mathbf{c f}$ ( $48.8 \mathrm{mg}, 0.20 \mathrm{mmol}$ ) was used, the desired product $\mathbf{5 v}$ was obtained in $76 \%$ yield (38.9 mg). ${ }^{1} \mathrm{H}$ NMR (400 MHz, Chloroform- $d$ ) $\delta 9.81(\mathrm{~s}, 1 \mathrm{H}), 7.52(\mathrm{~s}, 1 \mathrm{H}), 7.32(\mathrm{t}, J=7.4 \mathrm{~Hz}, 1 \mathrm{H})$, $7.22(\mathrm{~d}, J=7.5 \mathrm{~Hz}, 1 \mathrm{H}), 7.16(\mathrm{~d}, J=10.5 \mathrm{~Hz}, 2 \mathrm{H}), 6.84(\mathrm{~s}, 1 \mathrm{H}), 3.96(\mathrm{~s}, 6 \mathrm{H}), 2.41(\mathrm{~s}, 3 \mathrm{H}) .{ }^{13} \mathrm{C}$ NMR $\left(101 \mathrm{MHz}, \mathrm{CDCl}_{3}\right) \delta 191.2,153.4,148.7,141.7,138.1,137.6,131.0,128.8,128.3,127.4,127.0,112.6$, $108.6,56.3,56.1,21.5$.

\section{3'-Methyl-[1,1'-biphenyl]-4-carbaldehyde (5w)}

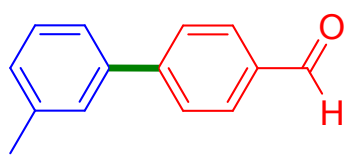

Stibine 3c (106.3 mg, $0.22 \mathrm{mmol})$ and 4-iodobenzaldehyde $2 \mathrm{cg}$ (46.4 mg, $0.20 \mathrm{mmol})$ were used, the desired product $\mathbf{5 w}$ was obtained as a white solid in $92 \%$ yield $(36.1 \mathrm{mg})$; 4-bromobenzaldehyde $\mathbf{2} \mathbf{c h}$ (36.8 $\mathrm{mg}, 0.20 \mathrm{mmol})$ was used, the desired product $\mathbf{5 w}$ was obtained in $81 \%$ yield $(31.8 \mathrm{mg}) .{ }^{1} \mathrm{H} \mathrm{NMR}$ $\left(400 \mathrm{MHz}, \mathrm{CDCl}_{3}\right) \delta 10.10(\mathrm{~s}, 1 \mathrm{H}), 7.99(\mathrm{~d}, J=8.2 \mathrm{~Hz}, 2 \mathrm{H}), 7.80(\mathrm{~d}, J=8.2 \mathrm{~Hz}, 2 \mathrm{H}), 7.49(\mathrm{~d}, J=8.1$ $\mathrm{Hz}, 2 \mathrm{H}), 7.42$ (t, $J=7.5 \mathrm{~Hz}, 1 \mathrm{H}), 7.32-7.26$ (m, 1H), 2.49 (s, 3H). ${ }^{13} \mathrm{C} \mathrm{NMR}\left(101 \mathrm{MHz}, \mathrm{CDCl}_{3}\right) \delta 192.1$, $147.5,139.9,138.8,135.3,130.4,129.4,129.0,128.3,127.8,124.6,21.7$.

\section{4-Methoxy-3'-methyl-[1,1'-biphenyl]-2-carbaldehyde (5x)}

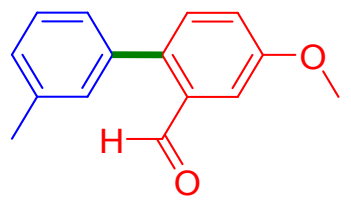

Stibine 3c (106.3 mg, $0.22 \mathrm{mmol})$ and 2-iodo-5-methoxybenzaldehyde 2ci (52.4 mg, $0.20 \mathrm{mmol})$ were used, the desired product $\mathbf{5} \mathbf{x}$ as a white solid in $84 \%$ yield $(38.0 \mathrm{mg})$; 2-bromo-5-methoxybenzaldehyde 2cj (42.8 mg, $0.20 \mathrm{mmol}$ ) was used, the desired product $\mathbf{5 x}$ was obtained in $72 \%$ yield $(32.5 \mathrm{mg}) .{ }^{1} \mathrm{H}$ NMR (400 MHz, Chloroform-d) $\delta 9.99$ (s, 1H), 7.55 (d, J=2.4 Hz, 1H), $7.44-7.35$ (m, 2H), 7.29 $7.16(\mathrm{~m}, 4 \mathrm{H}), 3.95$ (s, 3H), 2.46 (s, 3H). ${ }^{13} \mathrm{C} \mathrm{NMR}\left(101 \mathrm{MHz}, \mathrm{CDCl}_{3}\right) \delta 192.6,159.2,139.5,138.2,137.5$, 134.6, 132.2, 131.1, 128.7, 128.4, 127.5, 121.5, 109.9, 55.7, 21.6.

4-(N,N-dipropylsulfamoyl)phenyl 4'-methoxy-[1,1'-biphenyl]-4-carboxylate (5y) 


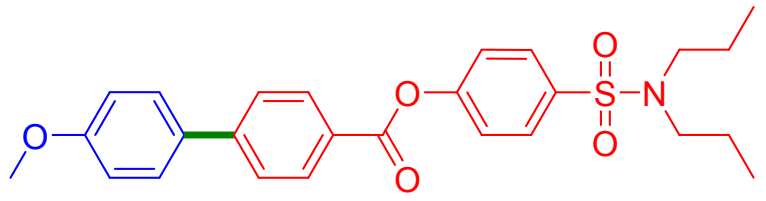

Stibine 3e (109.8 mg, $0.22 \mathrm{mmol})$ and 4-(N,N-dipropylsulfamoyl)phenyl 4-iodobenzoate 2da (97.4 mg, $0.20 \mathrm{mmol})$ were used, the desired product $\mathbf{5 y}$ as a white solid in $87 \%$ yield $(81.3 \mathrm{mg})$. M.P.: 124.7 $126.5^{\circ} \mathrm{C} .{ }^{1} \mathrm{H}$ NMR (400 MHz, Chloroform- $d$ ) $\delta 8.37$ (d, $\left.J=8.4 \mathrm{~Hz}, 2 \mathrm{H}\right), 7.99(\mathrm{~d}, J=8.2 \mathrm{~Hz}, 2 \mathrm{H}), 7.60$ (dd, $J=32.6,8.5 \mathrm{~Hz}, 4 \mathrm{H}), 7.30$ (d, $J=8.3 \mathrm{~Hz}, 2 \mathrm{H}), 7.02$ (d, $J=8.5 \mathrm{~Hz}, 2 \mathrm{H}), 3.89$ (s, 3H), $3.42-2.87$ $(\mathrm{m}, 4 \mathrm{H}), 1.66-1.55(\mathrm{~m}, 4 \mathrm{H}), 0.93(\mathrm{t}, J=7.4 \mathrm{~Hz}, 6 \mathrm{H}) .{ }^{13} \mathrm{C} \mathrm{NMR}\left(101 \mathrm{MHz}, \mathrm{CDCl}_{3}\right) \delta 164.1,159.4$, 149.7, 145.0, 139.1, 133.0, 132.8, 130.9, 128.3, 128.0, 127.3, 121.8, 114.4, 55.5, 50.1, 22.1, 11.3. HRMSESI (m/z) $[\mathrm{M}+\mathrm{H}]^{+}$Calcd for $\mathrm{C}_{26} \mathrm{H}_{30} \mathrm{NO}_{5} \mathrm{~S}^{+}, 468.1839$; Found, 468.1842.

3',5'-Dimethoxy-[1,1'-biphenyl]-4-yl-2-(1-(4-chlorobenzoyl)-5-methoxy-2-methyl-1H-indol-3yl)acetate (5z)

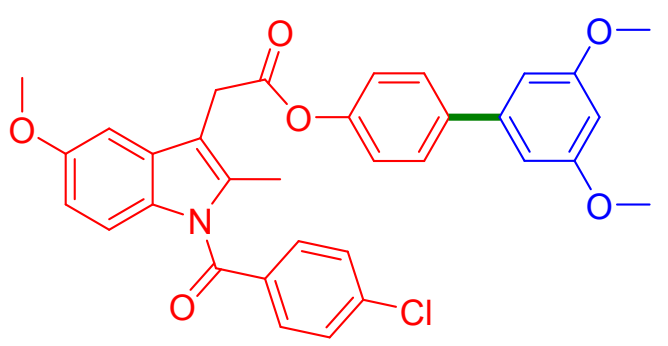

Stibine 3f (116.4 mg, $0.22 \mathrm{mmol})$ and 4-iodophenyl-2-(1-(4-chlorobenzoyl)-5-methoxy-2-methyl-1Hindol-3-yl)acetate $\mathbf{2 d b}(109.0 \mathrm{mg}, 0.20 \mathrm{mmol})$ were used, the desired product $\mathbf{5 z}$ as a white solid in $90 \%$ yield (102.4 mg). M.P.: $112.2-123.4{ }^{\circ} \mathrm{C} .{ }^{1} \mathrm{H}$ NMR (400 MHz, Chloroform- $d$ ) $\delta 7.69(\mathrm{~d}, J=8.6 \mathrm{~Hz}, 2 \mathrm{H})$, $7.56(\mathrm{~d}, J=8.8 \mathrm{~Hz}, 2 \mathrm{H}), 7.48(\mathrm{~d}, J=8.6 \mathrm{~Hz}, 2 \mathrm{H}), 7.19-7.07(\mathrm{~m}, 3 \mathrm{H}), 6.93(\mathrm{~d}, J=8.9 \mathrm{~Hz}, 1 \mathrm{H}), 6.76-$ $6.64(\mathrm{~m}, 3 \mathrm{H}), 6.51-6.43(\mathrm{~m}, 1 \mathrm{H}), 3.94(\mathrm{~s}, 2 \mathrm{H}), 3.86(\mathrm{~s}, 3 \mathrm{H}), 3.84(\mathrm{~s}, 6 \mathrm{H}), 2.48(\mathrm{~s}, 3 \mathrm{H}) .{ }^{13} \mathrm{C} \mathrm{NMR}(101$ $\left.\mathrm{MHz}_{2} \mathrm{CDCl}_{3}\right) \delta 169.4,168.4,161.1,156.2,150.3,142.5,139.4,139.1,136.3,133.9,131.3,130.9,130.6$, 129.2, 128.2, 121.7, 115.1, 112.1, 111.9, 105.5, 101.3, 99.4, 55.8, 55.5, 30.6, 13.5. HRMS-ESI (m/z) $[\mathrm{M}+\mathrm{H}]^{+}$Calcd for $\mathrm{C}_{33} \mathrm{H}_{29} \mathrm{ClNO}_{6}{ }^{+}, 570.1678$; Found, 570.1683 .

3',5'-Dimethoxy-[1,1'-biphenyl]-4-yl (E)-2-(5-fluoro-2-methyl-1-(4-(methylsulfinyl)benzylidene)1H-inden-3-yl)acetate (5aa)<smiles>COc1cc(OC)cc(-c2ccc(OC(=O)CC3=C(C)/C(=C\c4ccc(S(C)=O)cc4)c4ccc(F)cc43)cc2)c1</smiles> 
Stibine 3f (116.4 mg, $0.22 \mathrm{mmol})$ and 4-iodophenyl (E)-2-(5-fluoro-2-methyl-1-(4(methylsulfinyl)benzylidene)-1H-inden-3-yl)acetate $2 \mathbf{d c}(99.2 \mathrm{mg}, 0.20 \mathrm{mmol}$ ) were used, the desired product 5aa was obtained as a white solid in $88 \%$ yield $(99.9 \mathrm{mg})$. M.P.: $138.5-140.6{ }^{\circ} \mathrm{C} .{ }^{1} \mathrm{H}$ NMR (400 MHz, Chloroform- $d$ ) $\delta 7.71$ (q, $J=8.2 \mathrm{~Hz}, 4 \mathrm{H}$ ), 7.55 (d, $J=8.6 \mathrm{~Hz}, 2 \mathrm{H}), 7.23-7.16$ (m, 2H), 7.13 $(\mathrm{d}, J=8.6 \mathrm{~Hz}, 2 \mathrm{H}), 7.01(\mathrm{dd}, J=8.9,2.4 \mathrm{~Hz}, 1 \mathrm{H}), 6.67(\mathrm{~d}, J=2.2 \mathrm{~Hz}, 2 \mathrm{H}), 6.60$ (td, $J=8.8,2.4 \mathrm{~Hz}$, $1 \mathrm{H}), 6.46(\mathrm{t}, J=2.2 \mathrm{~Hz}, 1 \mathrm{H}), 3.83(\mathrm{~s}, 8 \mathrm{H}), 2.81(\mathrm{~s}, 3 \mathrm{H}), 2.29(\mathrm{~s}, 3 \mathrm{H}) .{ }^{13} \mathrm{C} \mathrm{NMR}\left(101 \mathrm{MHz}, \mathrm{CDCl}_{3}\right) \delta$ $168.8,163.5$ (d, $J=246.7 \mathrm{~Hz}), 161.2,150.4,146.7,145.7,142.6,141.7,139.7,139.2,138.8,131.3$ (d, $J$ $=2.4 \mathrm{~Hz}), 130.4,129.7(\mathrm{~d}, J=3.0 \mathrm{~Hz}), 128.6(\mathrm{~d}, J=1.4 \mathrm{~Hz}), 128.3,124.0,123.9,121.7,111.1(\mathrm{~d}, J=$ $22.2 \mathrm{~Hz}), 106.2(\mathrm{~d}, J=23.9 \mathrm{~Hz}), 105.6,99.5,55.6,44.0,32.1,10.8 .{ }^{19} \mathrm{~F} \mathrm{NMR}\left(376 \mathrm{MHz}, \mathrm{CDCl}_{3}\right) \delta$ 112.67. HRMS-EI (m/z) [M] Calcd for $\mathrm{C}_{34} \mathrm{H}_{29} \mathrm{FO}_{5} \mathrm{~S}, 568.1720$; Found, 568.1726.

5-(2,5-Dimethylphenoxy)-2-methylpentan-2-yl 4'-methoxy-[1,1'-biphenyl]-4-carboxylate (5ab)

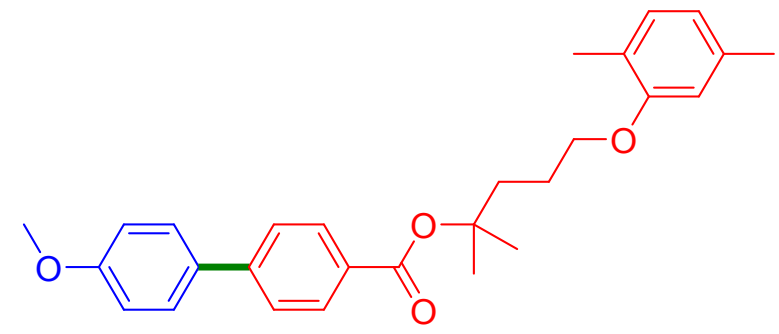

Stibine 3e (109.8 mg, $0.22 \mathrm{mmol})$ and 5-(2,5-dimethylphenoxy)-2-methylpentan-2-yl 4-bromobenzoate 2dd $(80.8 \mathrm{mg}, 0.20 \mathrm{mmol})$ were used, the desired product $\mathbf{5 a b}$ was obtained as a liquid in $84 \%$ yield (72.6 mg). ${ }^{1} \mathrm{H}$ NMR (400 MHz, Chloroform- $d$ ) $\delta 7.56(\mathrm{dd}, J=13.4,8.3 \mathrm{~Hz}, 4 \mathrm{H}), 7.14(\mathrm{~d}, J=8.3 \mathrm{~Hz}$, 2H), $7.05(\mathrm{dd}, J=20.0,7.9 \mathrm{~Hz}, 3 \mathrm{H}), 6.79-6.66(\mathrm{~m}, 2 \mathrm{H}), 4.09-4.02(\mathrm{~m}, 2 \mathrm{H}), 3.88(\mathrm{~s}, 3 \mathrm{H}), 2.37$ (s, 3H), $2.26(\mathrm{~s}, 3 \mathrm{H}), 1.96(\mathrm{~d}, J=3.0 \mathrm{~Hz}, 3 \mathrm{H}), 1.45(\mathrm{~s}, 6 \mathrm{H}) .{ }^{13} \mathrm{C} \mathrm{NMR}\left(101 \mathrm{MHz}, \mathrm{CDCl}_{3}\right) \delta 176.5,159.2,156.9$, $150.1,138.5,136.6,133.1,130.5,128.2,127.7,123.7,121.8,120.8,114.3,112.0,67.8,55.4,42.5,37.3$, 25.4, 25.3, 21.5, 15.9. HRMS-ESI (m/z) [M+H] $]^{+}$Calcd for $\mathrm{C}_{28} \mathrm{H}_{33} \mathrm{O}_{4}{ }^{+}, 433.2373$; Found, 433.2374.

4'-Methyl-[1,1'-biphenyl]-4-yl 2-(11-oxo-6,11-dihydrodibenzo[b,e]oxepin-3-yl)acetate (5ac)

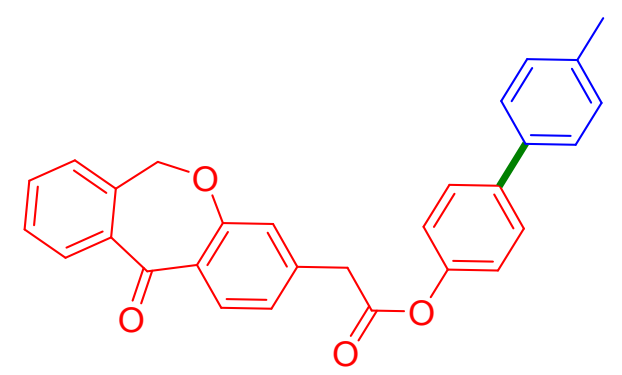

Stibine 3a (106.3 mg, $0.22 \mathrm{mmol})$ and 4-bromophenyl 2-(11-oxo-6,11-dihydrodibenzo[b,e]oxepin-3yl)acetate $2 \mathrm{de}(84.4 \mathrm{mg}, 0.20 \mathrm{mmol})$ were used, the desired product $5 \mathbf{a c}$ was obtained as a white solid in 91\% yield (78.9 mg). M.P.: $115.5-117.8^{\circ} \mathrm{C} .{ }^{1} \mathrm{H}$ NMR $(400 \mathrm{MHz}$, Chloroform- $d$ ) $\delta 8.28(\mathrm{~d}, J=2.4 \mathrm{~Hz}$, 1H), 7.95 (dd, $J=7.7,1.4 \mathrm{~Hz}, 1 \mathrm{H}), 7.63-7.55$ (m, 4H), $7.54-7.46$ (m, 3H), 7.40 (dd, $J=7.4,1.3 \mathrm{~Hz}$, 1H), $7.29-7.25(\mathrm{~m}, 2 \mathrm{H}), 7.21-7.15(\mathrm{~m}, 2 \mathrm{H}), 7.12(\mathrm{~d}, J=8.4 \mathrm{~Hz}, 1 \mathrm{H}), 5.24(\mathrm{~s}, 2 \mathrm{H}), 3.95(\mathrm{~s}, 2 \mathrm{H}), 2.42$ 
(s, 3H). ${ }^{13} \mathrm{C}$ NMR $\left(101 \mathrm{MHz}, \mathrm{CDCl}_{3}\right) \delta 190.9,170.0,160.7,150.0,140.5,139.1,137.5,137.3,136.4$, 135.6, 132.9, 132.7, 129.6, 129.4, 128.0, 127.9, 127.3, 127.0, 125.3, 121.8, 121.4, 73.7, 40.4, 21.2. HRMS-ESI (m/z) $[\mathrm{M}+\mathrm{H}]^{+}$Calcd for $\mathrm{C}_{29} \mathrm{H}_{23} \mathrm{O}_{4}{ }^{+}, 435.1591$; Found, 435.1590.

3',5'-Dimethoxy-[1,1'-biphenyl]-4-yl $(2 E, 4 E, 6 E, 8 E)$-3,7-dimethyl-9-(2,6,6-trimethylcyclohex-1-en1-yl)nona-2,4,6,8-tetraenoate (5ad)<smiles>COc1cc(OC)cc(-c2ccc(OC(=O)/C=C(C)/C=C/C=C(C)/C=C/C3=C(C)CCCC3(C)C)cc2)c1</smiles>

Stibine 3f (116.4 mg, $0.22 \mathrm{mmol})$ and 4-bromophenyl (2E,4E,6E,8E)-3,7-dimethyl-9-(2,6,6trimethylcyclohex-1-en-1-yl)nona-2,4,6,8-tetraenoate $2 \mathbf{d f}$ ( $90.8 \mathrm{mg}, 0.20 \mathrm{mmol}$ ) were used, the desired product 5 ad was obtained as a yellow liquid in $86 \%$ yield $(88.1 \mathrm{mg}) .{ }^{1} \mathrm{H}$ NMR $(400 \mathrm{MHz}$, Chloroformd) $\delta 7.58(\mathrm{~d}, J=8.4 \mathrm{~Hz}, 2 \mathrm{H}), 7.19(\mathrm{~d}, J=8.6 \mathrm{~Hz}, 2 \mathrm{H}), 7.10(\mathrm{dd}, J=15.1,11.4 \mathrm{~Hz}, 1 \mathrm{H}), 6.72(\mathrm{~d}, J=2.3$ $\mathrm{Hz}, 2 \mathrm{H}), 6.47$ (t, $J=2.2 \mathrm{~Hz}, 1 \mathrm{H}), 6.36(\mathrm{dd}, J=25.0,15.5 \mathrm{~Hz}, 2 \mathrm{H}), 6.19$ (dd, $J=13.8,9.2 \mathrm{~Hz}, 2 \mathrm{H}), 6.02$ (s, 1H), $3.85(\mathrm{~s}, 6 \mathrm{H}), 2.56-2.28(\mathrm{~m}, 3 \mathrm{H}), 2.07-2.00(\mathrm{~m}, 5 \mathrm{H}), 1.74(\mathrm{~s}, 3 \mathrm{H}), 1.67-1.58(\mathrm{~m}, 2 \mathrm{H}), 1.52-$ $1.45(\mathrm{~m}, 2 \mathrm{H}), 1.05(\mathrm{~s}, 6 \mathrm{H}) .{ }^{13} \mathrm{C}$ NMR $\left(101 \mathrm{MHz}, \mathrm{CDCl}_{3}\right) \delta 165.6,161.2,155.8,150.5,142.9,140.6$, 138.7, 137.8, 137.3, 134.8, 132.2, 130.4, 129.5, 129.3, 128.2, 122.1, 117.2, 105.6, 99.4, 55.5, 39.7, 34.4, 33.3, 29.1, 21.9, 19.3, 14.2, 13.1. HRMS-ESI (m/z) $[\mathrm{M}+\mathrm{H}]^{+}$Calcd for $\mathrm{C}_{34} \mathrm{H}_{41} \mathrm{O}_{4}{ }^{+}, 513.2999$; Found, 513.2993.

3-Bromo-5-methoxy-4'-methyl-1,1'-biphenyl (5ae)

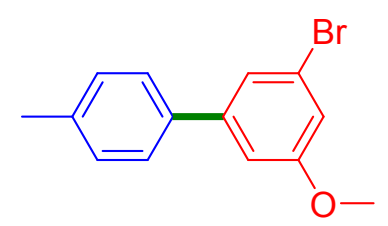

Stibine 3a (106.3 mg, $0.22 \mathrm{mmol}$ ) and 1-bromo-3-iodo-5-methoxybenzene 2dg (62.4 mg, $0.20 \mathrm{mmol})$ were used, the desired product 5ae was obtained as a liquid in $82 \%$ yield $(45.2 \mathrm{mg}) .{ }^{1} \mathrm{H} \mathrm{NMR}(400 \mathrm{MHz}$, Chloroform- $d$ ) $\delta 7.47(\mathrm{~d}, J=8.3 \mathrm{~Hz}, 2 \mathrm{H}), 7.36-7.30(\mathrm{~m}, 1 \mathrm{H}), 7.27(\mathrm{~d}, J=7.4 \mathrm{~Hz}, 2 \mathrm{H}), 7.09-6.99$ (m, 2H), 3.86 (s, 3H), 2.42 (s, 3H). $\left.{ }^{13} \mathrm{C} \mathrm{NMR} \mathrm{(101} \mathrm{MHz,} \mathrm{CDCl}_{3}\right) \delta 160.7,144.2,138.0,136.9,129.7,127.1$, 123.2, 122.7, 115.6, 112.1, 55.7, 21.2. HRMS-EI (m/z) [M] Calcd for $\mathrm{C}_{14} \mathrm{H}_{13} \mathrm{BrO}, 276.0150$; Found, 276.0151 .

3-Bromo-4'-(tert-butyl)-5-methoxy-1,1'-biphenyl (5af) 


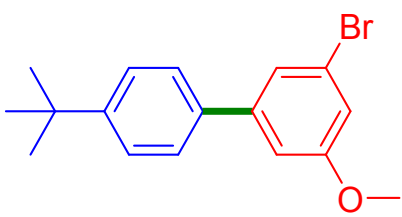

Stibine 3v (115.5 mg, $0.22 \mathrm{mmol}$ ) and 1-bromo-3-iodo-5-methoxybenzene 2dg (62.4 mg, $0.20 \mathrm{mmol})$ were used, the desired product 5af was obtained as a liquid in 79\% yield (50.2 mg). ${ }^{1} \mathrm{H} \mathrm{NMR} \mathrm{(400} \mathrm{MHz,}$ Chloroform- $d$ ) $\delta 7.58-7.46(\mathrm{~m}, 4 \mathrm{H}), 7.43-7.32(\mathrm{~m}, 1 \mathrm{H}), 7.16-6.98(\mathrm{~m}, 2 \mathrm{H}), 3.86(\mathrm{~s}, 3 \mathrm{H}), 1.40(\mathrm{~s}$, 9H). ${ }^{13} \mathrm{C} \mathrm{NMR}\left(101 \mathrm{MHz}, \mathrm{CDCl}_{3}\right) \delta 160.7,151.2,144.1,136.9,126.9,125.9,123.2,122.7,115.6,112.1$, 55.6, 34.7, 31.4. HRMS-EI (m/z) [M] Calcd for $\mathrm{C}_{17} \mathrm{H}_{19} \mathrm{BrO}, 318.0619$; Found, 318.0622 .

\section{3-Bromo-4'-chloro-5-methoxy-1,1'-biphenyl (5ag)}

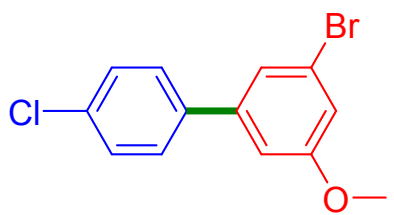

Stibine 3r (110.7 mg, $0.22 \mathrm{mmol})$ and 1-bromo-3-iodo-5-methoxybenzene 2dg (62.4 mg, $0.20 \mathrm{mmol})$ were used, the desired product $\mathbf{5} \mathbf{a g}$ was obtained as a white solid in 76\% yield (44.9 mg). M.P.: 70.5 $70.9{ }^{\circ} \mathrm{C} .{ }^{1} \mathrm{H}$ NMR $(400 \mathrm{MHz}$, Chloroform- $d) \delta 7.53-7.38(\mathrm{~m}, 4 \mathrm{H}), 7.32-7.27(\mathrm{~m}, 1 \mathrm{H}), 7.04(\mathrm{~d}, J=$ $24.5 \mathrm{~Hz}, 2 \mathrm{H}), 3.86(\mathrm{~s}, 3 \mathrm{H}) .{ }^{13} \mathrm{C} \mathrm{NMR}\left(101 \mathrm{MHz}, \mathrm{CDCl}_{3}\right) \delta 160.8,143.0,138.3,134.3,129.2,128.5$, 123.4, 122.7, 116.1, 112.3, 55.8. HRMS-EI (m/z) [M] Calcd for $\mathrm{C}_{13} \mathrm{H}_{10} \mathrm{BrClO}, 295.9604$; Found, 295.9608 .

3,4'-Dibromo-5-methoxy-1,1'-biphenyl (5ah)

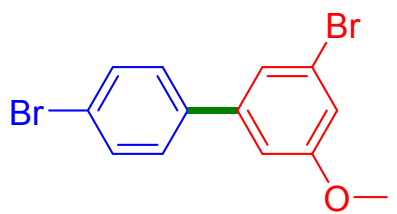

Stibine 3s (120.3 mg, $0.22 \mathrm{mmol})$ and 1-bromo-3-iodo-5-methoxybenzene $2 \mathrm{dg}$ (62.4 $\mathrm{mg}, 0.20 \mathrm{mmol})$ were used, the desired product $\mathbf{5 a h}$ was obtained as a white solid in 77\% yield (52.3 mg). M.P.: 67.6 $69.3{ }^{\circ} \mathrm{C} .{ }^{1} \mathrm{H}$ NMR $(400 \mathrm{MHz}$, Chloroform- $d) \delta 7.58(\mathrm{~d}, J=8.1 \mathrm{~Hz}, 2 \mathrm{H}), 7.42(\mathrm{~d}, J=8.1 \mathrm{~Hz}, 2 \mathrm{H}), 7.30$ (s, 1H), $7.05(\mathrm{~d}, J=26.2 \mathrm{~Hz}, 2 \mathrm{H}), 3.87(\mathrm{~s}, 3 \mathrm{H}) .{ }^{13} \mathrm{C} \mathrm{NMR}\left(101 \mathrm{MHz}, \mathrm{CDCl}_{3}\right) \delta 160.8,143.0,138.8$, 132.1, 128.8, 123.4, 122.6, 122.5, 116.1, 112.2, 55.8. HRMS-EI (m/z) [M] Calcd for $\mathrm{C}_{13} \mathrm{H}_{10} \mathrm{Br}_{2} \mathrm{O}$, 339.9098; Found, 339.9095.

1-(3'-Bromo-5'-chloro-[1,1'-biphenyl]-4-yl)ethan-1-one (5ai) 


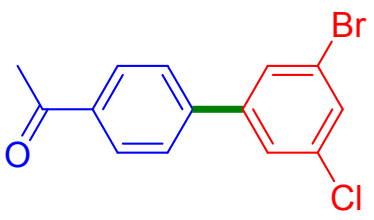

Stibine 3af (112.4 mg, $0.22 \mathrm{mmol})$ and 1-bromo-3-chloro-5-iodobenzene $2 \mathrm{di}$ (63.4 mg, $0.20 \mathrm{mmol})$ were used, the desired product 5 ai was obtained as a white solid in $81 \%$ yield $(49.9 \mathrm{mg})$. M.P.: $97.2-98.7^{\circ} \mathrm{C}$. ${ }^{1} \mathrm{H}$ NMR (400 MHz, Chloroform- $d$ ) $\delta 8.03(\mathrm{~d}, J=8.0 \mathrm{~Hz}, 2 \mathrm{H}), 7.65-7.58(\mathrm{~m}, 3 \mathrm{H}), 7.57-7.46(\mathrm{~m}, 2 \mathrm{H})$, 2.64 (s, 3H). ${ }^{13} \mathrm{C}$ NMR $\left(101 \mathrm{MHz}, \mathrm{CDCl}_{3}\right) \delta$ 197.6, 143.2, 142.9, 136.9, 135.8, 130.9, 129.2, 128.8, 127.4, 126.3, 123.4, 26.8. HRMS-EI (m/z) [M] Calcd for $\mathrm{C}_{14} \mathrm{H}_{10} \mathrm{BrClO}, 307.9604$; Found, 307.9600 .

\section{Ethyl 3'-bromo-5'-chloro-[1,1'-biphenyl]-4-carboxylate (5aj)}<smiles>CCOC(=O)c1ccc(-c2cc(Cl)cc(Br)c2)cc1</smiles>

Stibine 3ad (119.0 mg, $0.22 \mathrm{mmol}$ ) and 1-bromo-3-chloro-5-iodobenzene 2di (63.4 mg, 0.20 mmol) were used, the desired product $\mathbf{5 a j}$ was obtained as a white solid in $86 \%$ yield $(58.1 \mathrm{mg})$. M.P.: $95.8-96.3{ }^{\circ} \mathrm{C}$. ${ }^{1} \mathrm{H}$ NMR (400 MHz, Chloroform- $d$ ) $\delta 8.17-8.00(\mathrm{~m}, 2 \mathrm{H}), 7.68-7.41(\mathrm{~m}, 5 \mathrm{H}), 4.40(\mathrm{q}, J=7.1 \mathrm{~Hz}, 2 \mathrm{H})$, $1.41(\mathrm{t}, J=7.1 \mathrm{~Hz}, 3 \mathrm{H}) .{ }^{13} \mathrm{C} \mathrm{NMR}\left(101 \mathrm{MHz}, \mathrm{CDCl}_{3}\right) \delta 166.2,143.3,142.6,135.7,130.7,130.5,130.3$, 128.7, 127.1, 126.3, 123.3, 61.3, 14.4. HRMS-EI (m/z) [M] Calcd for $\mathrm{C}_{15} \mathrm{H}_{12} \mathrm{BrClO}_{2}$, 337.9709; Found, 337.9712 .

3-Bromo-5-chloro-3',5'-dimethoxy-1,1'-biphenyl (5ak)<smiles>COc1cc(OC)cc(-c2cc(Cl)cc(Br)c2)c1</smiles>

Stibine 3f (116.4 mg, $0.22 \mathrm{mmol}$ ) and 1-bromo-3-chloro-5-iodobenzene 2di (63.4 mg, $0.20 \mathrm{mmol})$ were used, the desired product 5 ak was obtained as a white solid in $74 \%$ yield (48.2 mg). M.P.: $82.1-84.1^{\circ} \mathrm{C}$. ${ }^{1} \mathrm{H}$ NMR (400 MHz, Chloroform- $d$ ) $\delta 7.61-7.55(\mathrm{~m}, 1 \mathrm{H}), 7.51-7.43(\mathrm{~m}, 2 \mathrm{H}), 6.64(\mathrm{~d}, J=2.3 \mathrm{~Hz}, 2 \mathrm{H})$, $6.50(\mathrm{t}, J=2.2 \mathrm{~Hz}, 1 \mathrm{H}), 3.85(\mathrm{~s}, 6 \mathrm{H}) .{ }^{13} \mathrm{C} \mathrm{NMR}\left(101 \mathrm{MHz}, \mathrm{CDCl}_{3}\right) \delta 161.3,144.5,140.6,135.4,130.2$, 128.6, 126.2, 123.0, 105.4, 100.4, 55.56. HRMS-EI (m/z) [M] Calcd for $\mathrm{C}_{14} \mathrm{H}_{12} \mathrm{BrClO}_{2}$, 325.9709; Found, 325.9707 .

\section{4-Bromo-3-fluoro-3',5'-dimethoxy-1,1'-biphenyl (5al)}




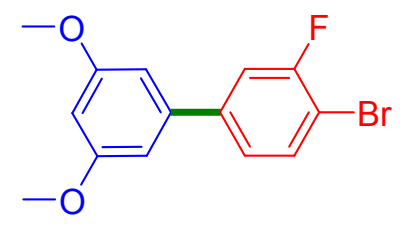

Stibine 3f (116.4 mg, $0.22 \mathrm{mmol})$ and 1-bromo-2-fluoro-4-iodobenzene $2 \mathbf{d h}(60.0 \mathrm{mg}, 0.20 \mathrm{mmol})$ were used, the desired product 5 al was obtained as a white solid in $83 \%$ yield $(51.5 \mathrm{mg})$. M.P.: $74.6-75.3^{\circ} \mathrm{C}$. ${ }^{1} \mathrm{H}$ NMR (400 MHz, Chloroform- $d$ ) $\delta 7.57$ (t, $\left.J=7.7 \mathrm{~Hz}, 1 \mathrm{H}\right), 7.37-7.29(\mathrm{~m}, 1 \mathrm{H}), 7.25-7.20(\mathrm{~m}, 1 \mathrm{H})$, $6.67(\mathrm{~d}, J=2.3 \mathrm{~Hz}, 2 \mathrm{H}), 6.50(\mathrm{t}, J=2.3 \mathrm{~Hz}, 1 \mathrm{H}), 3.85(\mathrm{~s}, 6 \mathrm{H}) .{ }^{13} \mathrm{C} \mathrm{NMR}\left(101 \mathrm{MHz}, \mathrm{CDCl}_{3}\right) \delta 161.4$, $159.4(\mathrm{~d}, J=247.1 \mathrm{~Hz}), 142.8$ (d, $J=7.1 \mathrm{~Hz}), 141.2$ (d, $J=2.1 \mathrm{~Hz}), 133.8,124.0(\mathrm{~d}, J=3.3 \mathrm{~Hz}), 115.3$ $(\mathrm{d}, J=22.9 \mathrm{~Hz}), 108.2(\mathrm{~d}, J=21.1 \mathrm{~Hz}), 105.4,100.1,55.6 .{ }^{19} \mathrm{~F}$ NMR $\left(376 \mathrm{MHz}, \mathrm{CDCl}_{3}\right) \delta-107.21$. HRMS-EI (m/z) [M] Calcd for $\mathrm{C}_{14} \mathrm{H}_{12} \mathrm{BrFO}_{2}, 310.0005$; Found, 310.0002.

\section{Gram-Scale Synthesis of Drug Difunisal ${ }^{\mathrm{S} 12}$}

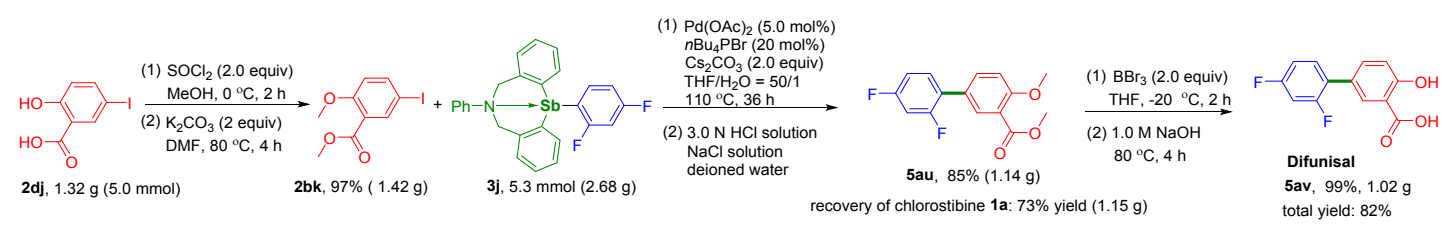

Procedure (a): Under a nitrogen atmoshpere, a $100 \mathrm{~mL}$ Schlenk tube equipped with a magnetic stir bar was charged successively with 2-hydroxy-5-iodobenzoic acid $\mathbf{2 d j}$ (1.32 g, $5.0 \mathrm{mmol})$, methanol (20 mL) and $\mathrm{SOCl}_{2}(1.19 \mathrm{~g}, 10.0 \mathrm{mmol})$. The reaction mixture was stirred at $0{ }^{\circ} \mathrm{C}$ for $2 \mathrm{~h}$. After the reaction was completed, the resulting mixture was qunenched with $\mathrm{H}_{2} \mathrm{O}$ and extracted with EA. The organic layer was concentrated under reduced pressure. The crude product was directly used without purification. The crude product reacted with $\mathrm{MeI}$ in the presence of $\mathrm{K}_{2} \mathrm{CO}_{3}$. Methyl 5-iodo-2-methoxybenzoate 2dk was obtained in 97\% yield (1.42 g). ${ }^{1} \mathrm{H}$ NMR (400 MHz, $\left.\mathrm{CDCl}_{3}\right) \delta 10.71(\mathrm{~s}, 1 \mathrm{H}), 8.12(\mathrm{~s}, 1 \mathrm{H}), 7.69$ (d, $J=$ $8.8 \mathrm{~Hz}, 1 \mathrm{H}), 6.77(\mathrm{~d}, J=8.8 \mathrm{~Hz}, 1 \mathrm{H}), 3.95$ (s, 3H). ${ }^{13} \mathrm{C} \mathrm{NMR}\left(101 \mathrm{MHz}, \mathrm{CDCl}_{3}\right) \delta 169.3,161.2,144.1$, $138.3,120.0,114.5,80.1,52.6$.

Procedure (b): Under a nitrogen atmoshpere, a $100 \mathrm{~mL}$ Schlenk tube equipped with a magnetic stir bar was charged successively with stibine $\mathbf{3 j}$ (2.68 g, $5.3 \mathrm{mmol}$ ), methyl 5-iodo-2-methoxybenzoate $\mathbf{2 d k}$ (1.42 g, $4.85 \mathrm{mmol}), \mathrm{Pd}(\mathrm{OAc})_{2}(54.6 \mathrm{mg}, 0.24 \mathrm{mmol}), n-\mathrm{Bu}_{4} \mathrm{PBr}(329.1 \mathrm{mg}, 0.97 \mathrm{mmol}), \mathrm{Cs}_{2} \mathrm{CO}_{3}(3.15$ g, $9.7 \mathrm{mmol}$ ), $40 \mathrm{~mL}$ of $\mathrm{THF}$ and $0.8 \mathrm{~mL}$ of $\mathrm{H}_{2} \mathrm{O}$. The reaction mixture was stirred at $110^{\circ} \mathrm{C}$ for $36 \mathrm{~h}$. After the reaction was completed, the resulting mixture was quenched with $10 \mathrm{~mL}$ of deionized water, and extracted with $\mathrm{CH}_{2} \mathrm{Cl}_{2}(10 \mathrm{~mL}$ x 3). Subsequently, the organic layer was washed with $3.0 \mathrm{M}(\mathrm{mol} / \mathrm{L})$ $\mathrm{HCl}$ solution $(50 \mathrm{~mL} \times 3)$, and then successively washed with $50 \mathrm{~mL}$ of saturated $\mathrm{NaCl}$ solution and 50 $\mathrm{mL}$ of deionized water. The organic layer was dried over anhydrous $\mathrm{Na}_{2} \mathrm{SO}_{4}$, concentrated under reduced pressure. The crude product was purified by column chromatography on silica gel and firstly eluted with the mixed solvent of PE:EA (20:1(v: v)) to provide the cross-coupling product 5au, and continued to elute with the mixed solvent of $\mathrm{CH}_{2} \mathrm{Cl}_{2}: \mathrm{CH}_{3} \mathrm{OH}(5: 1(\mathrm{v}: \mathrm{v}))$ to obtain chlorostibine 1a. 5au was afforded in $85 \%$ yield, and $73 \%$ of chlorostibine 1a was recycled. Melting point of 5au: $70.1-72.3{ }^{\circ} \mathrm{C} .{ }^{1} \mathrm{H}$ NMR 
$\left(400 \mathrm{MHz}, \mathrm{CDCl}_{3}\right) \delta 7.92(\mathrm{~s}, 1 \mathrm{H}), 7.57(\mathrm{~d}, J=8.7 \mathrm{~Hz}, 1 \mathrm{H}), 7.39-7.28(\mathrm{~m}, 1 \mathrm{H}), 7.01(\mathrm{~d}, J=8.7 \mathrm{~Hz}, 1 \mathrm{H})$, $6.94-6.81(\mathrm{~m}, 2 \mathrm{H}), 3.90(\mathrm{~s}, 3 \mathrm{H}), 3.88(\mathrm{~s}, 3 \mathrm{H}) .{ }^{13} \mathrm{C} \mathrm{NMR}\left(101 \mathrm{MHz}, \mathrm{CDCl}_{3}\right) \delta 166.3,162.1(\mathrm{dd}, J=$ 247.5, $11.9 \mathrm{~Hz}), 160.7$ (dd, $J=248.5,11.7 \mathrm{~Hz}), 158.6,133.8$ (d, $J=3.5 \mathrm{~Hz}), 131.9$ (d, $J=2.6 \mathrm{~Hz}), 131.0$ (dd, $J=9.5,4.8 \mathrm{~Hz}), 126.8,123.8(\mathrm{dd}, J=13.5,3.8 \mathrm{~Hz}), 120.0,112.1,111.6(\mathrm{dd}, J=21.1,3.8 \mathrm{~Hz}), 104.3$ (dd, $J=26.7,25.2 \mathrm{~Hz}), 56.0,52.0 .{ }^{19} \mathrm{~F}$ NMR (376 MHz, $\left.\mathrm{CDCl}_{3}\right) \delta-111.35$ (d, $J=7.4 \mathrm{~Hz}$ ), -113.74 (d, $J$ $=7.4 \mathrm{~Hz})$. HRMS-ESI (m/z) $[\mathrm{M}+\mathrm{Na}]^{+}$Calcd for $\mathrm{C}_{15} \mathrm{H}_{12} \mathrm{~F}_{2} \mathrm{NaO}_{3}{ }^{+}, 301.0647$; Found, 301.0655 .

Procedure (c): To a solution of methyl 2',4'-difluoro-4-methoxy-[1,1'-biphenyl]-3-carboxylate 5au (1.14 $\mathrm{g}, 4.1 \mathrm{mmol}$ ) in $20 \mathrm{~mL}$ THF, $\mathrm{BBr}_{3}$ (2.0 equiv, $1 \mathrm{M}$ solution in THF) was added at $-20^{\circ} \mathrm{C}$ under a nitrogen atmoshpere. The reaction mixture was stirred at $-20{ }^{\circ} \mathrm{C}$ for $2 \mathrm{~h}$. Then, it was quenched with $\mathrm{H}_{2} \mathrm{O}$, extracted with $\mathrm{CH}_{2} \mathrm{Cl}_{2}$, washed with brine and concentrated under reduced pressure. The residue was dissolve in $20 \mathrm{~mL} \mathrm{MeOH}$ and $20 \mathrm{~mL} 1.0 \mathrm{M} \mathrm{NaOH}$ and refluxed for $4 \mathrm{~h}$. After the reaction was completed, the mixture was concentrated under reduced pressure and then acidified by diluted $\mathrm{HCl}$ and extracted by $\mathrm{CH}_{2} \mathrm{Cl}_{2}$ and dried by $\mathrm{Na}_{2} \mathrm{SO}_{4} .1 .04 \mathrm{~g}$ white solid $(99 \%)$ was obtained after the solvent has been removed. Melting point of 5av: $>300{ }^{\circ} \mathrm{C} .{ }^{1} \mathrm{H}$ NMR $\left(400 \mathrm{MHz}\right.$, DMSO- $\left.d_{6}\right) \delta 7.96(\mathrm{~s}, 1 \mathrm{H}), 7.67-7.04(\mathrm{~m}, 4 \mathrm{H}), 6.88$ (s, $1 \mathrm{H}) .{ }^{13} \mathrm{C}$ NMR $\left(101 \mathrm{MHz}, \mathrm{DMSO}-d_{6}\right) \delta 171.8,162.3,160.0(\mathrm{dd}, J=39.7,12.2 \mathrm{~Hz}), 157.7(\mathrm{~d}, J=$ $12.1 \mathrm{~Hz}), 132.8(\mathrm{~d}, J=2.7 \mathrm{~Hz}), 131.3(\mathrm{dd}, J=9.5,5.0 \mathrm{~Hz}), 130.4(\mathrm{~d}, J=3.1 \mathrm{~Hz}), 125.0(\mathrm{dd}, J=13.5$, $3.7 \mathrm{~Hz}), 122.8,118.7,116.6,111.8(\mathrm{dd}, J=21.0,3.5 \mathrm{~Hz}), 104.9-103.8(\mathrm{~m}) .{ }^{19} \mathrm{~F} \mathrm{NMR}\left(376 \mathrm{MHz}, \mathrm{CDCl}_{3}\right)$ $\delta-112.12--112.36(\mathrm{~m}),-113.50--113.80(\mathrm{~m})$. HRMS-ESI $(\mathrm{m} / \mathrm{z})[\mathrm{M}+\mathrm{H}]^{+}$Calcd for $\mathrm{C}_{13} \mathrm{H}_{9} \mathrm{~F}_{2} \mathrm{O}_{3}{ }^{+}$, 251.0514; Found, 251.0515.

\section{Gram-Scale Synthesis of Drug Fenbufen ${ }^{\mathrm{S} 13}$}

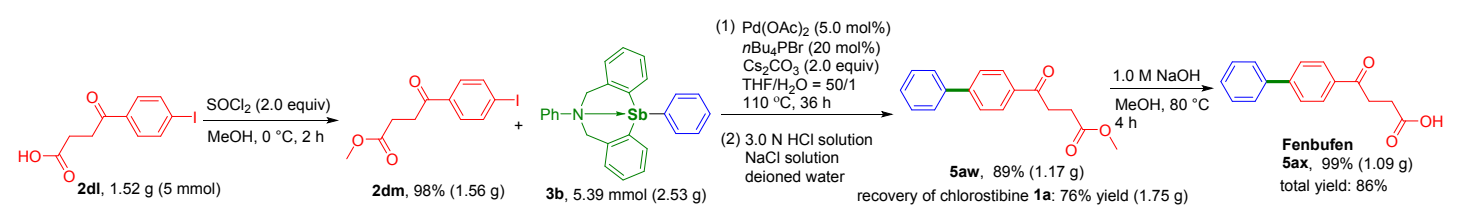

Procedure (a): Under a nitrogen atmoshpere, a $100 \mathrm{~mL}$ Schlenk tube equipped with a magnetic stir bar was charged successively with 4-(4-iodophenyl)-4-oxobutanoic acid $2 \mathbf{d l}$ (1.52 g, $5.0 \mathrm{mmol}$ ), methanol $(20 \mathrm{~mL})$ and $\mathrm{SOCl}_{2}(1.19 \mathrm{~g}, 10.0 \mathrm{mmol})$. The reaction mixture was stirred at $0{ }^{\circ} \mathrm{C}$ for $2 \mathrm{~h}$. After the reaction was completed, the resulting mixture was qunenched with $\mathrm{H}_{2} \mathrm{O}$ and extracted with EA. The organic layer was concentrated under reduced pressure. Methyl 4-(4-iodophenyl)-4-oxobutanoate $\mathbf{2 d m}$ was obtained in $98 \%$ yield $(1.56 \mathrm{~g}) .{ }^{1} \mathrm{H}$ NMR $\left(400 \mathrm{MHz}, \mathrm{CDCl}_{3}\right) \delta 7.80(\mathrm{~d}, J=8.1 \mathrm{~Hz}, 2 \mathrm{H}), 7.56(\mathrm{~d}, J=$ $8.2 \mathrm{~Hz}, 2 \mathrm{H}), 3.66$ (s, 3H), 3.24 (t, $J=6.5 \mathrm{~Hz}, 2 \mathrm{H}), 2.72(\mathrm{t}, J=6.5 \mathrm{~Hz}, 2 \mathrm{H}) .{ }^{13} \mathrm{C} \mathrm{NMR}\left(101 \mathrm{MHz}, \mathrm{CDCl}_{3}\right.$ ) $\delta 197.0,173.2,135.2,131.9,129.6,128.4,51.9,33.3,27.9$.

Procedure (b): Under a nitrogen atmoshpere, a $100 \mathrm{~mL}$ Schlenk tube equipped with a magnetic stir bar was charged successively with stibine $\mathbf{3 b}$ ( $2.53 \mathrm{~g}, 5.39 \mathrm{mmol})$, methyl 4-(4-iodophenyl)-4-oxobutanoate $2 \mathbf{d m}(1.56 \mathrm{~g}, 4.9 \mathrm{mmol}), \mathrm{Pd}(\mathrm{OAc})_{2}(54.6 \mathrm{mg}, 0.24 \mathrm{mmol}), n-\mathrm{Bu}_{4} \mathrm{PBr}(329.1 \mathrm{mg}, 0.97 \mathrm{mmol}), \mathrm{Cs}_{2} \mathrm{CO}_{3}$ ( $3.15 \mathrm{~g}, 9.7 \mathrm{mmol}$ ), $40 \mathrm{~mL}$ of THF and $0.8 \mathrm{~mL}$ of $\mathrm{H}_{2} \mathrm{O}$. The reaction mixture was stirred at $110{ }^{\circ} \mathrm{C}$ for $36 \mathrm{~h}$. After the reaction was completed, the resulting mixture was quenched with $10 \mathrm{~mL}$ of deionized 
water, and extracted with $\mathrm{CH}_{2} \mathrm{Cl}_{2}(10 \mathrm{~mL} \times 3)$. Subsequently, the organic layer was washed with $3.0 \mathrm{M}$ $(\mathrm{mol} / \mathrm{L}) \mathrm{HCl}$ solution $(50 \mathrm{~mL} \times 3)$, and then successively washed with $50 \mathrm{~mL}$ of saturated $\mathrm{NaCl}$ solution and $50 \mathrm{~mL}$ of deionized water. The organic layer was dried over anhydrous $\mathrm{Na}_{2} \mathrm{SO}_{4}$, and concentrated under reduced pressure. The crude product was purified by column chromatography on silica gel and firstly eluted with the mixed solvent of PE:EA (20:1(v: v)) to obtain cross-coupling product 5aw, and continued to elute with the mixed solvent of $\mathrm{CH}_{2} \mathrm{Cl}_{2}: \mathrm{CH}_{3} \mathrm{OH}(5: 1(\mathrm{v}: \mathrm{v}))$ to obtain chlorostibine 1a. 5aw was afforded in $89 \%$ yield, and $76 \%$ of chlorostibine 1a was recycled. Melting point of 5aw: $99.5-100.6$ ${ }^{\circ} \mathrm{C} .{ }^{1} \mathrm{H}$ NMR (400 MHz, Chloroform- $d$ ) $\delta 8.04$ (d, $\left.J=8.1 \mathrm{~Hz}, 2 \mathrm{H}\right), 7.67$ (d, $\left.J=8.0 \mathrm{~Hz}, 2 \mathrm{H}\right), 7.61$ (d, $J=$ $7.3 \mathrm{~Hz}, 2 \mathrm{H}), 7.45$ (t, $J=7.5 \mathrm{~Hz}, 2 \mathrm{H}), 7.38$ (t, $J=7.3 \mathrm{~Hz}, 1 \mathrm{H}), 3.70$ (s, 3H), 3.33 (t, $J=6.6 \mathrm{~Hz}, 2 \mathrm{H}), 2.78$ $(\mathrm{t}, J=6.6 \mathrm{~Hz}, 2 \mathrm{H}) .{ }^{13} \mathrm{C} \mathrm{NMR}\left(101 \mathrm{MHz}, \mathrm{CDCl}_{3}\right) \delta 197.6,173.4,145.8,139.8,135.2,129.0,128.6,128.3$, 127.3, 127.2, 51.9, 33.4, 28.1. HRMS-EI (m/z) [M] Calcd for $\mathrm{C}_{17} \mathrm{H}_{16} \mathrm{O}_{3}, 268.1099$; Found, 268.1098.

Note: Reaction between $\mathbf{2 d m}$ and $\mathbf{3 b}$ resulted in the formation of the possible mixture of $\mathrm{Sb}-\mathrm{I}(\mathbf{1 i})$, $\mathrm{Sb}-\operatorname{Br}(\mathbf{1} \mathbf{h}), \mathrm{Sb}-\mathrm{OAc}(\mathbf{1 j})$, and the desired product 5aw. After the reaction was completed, the reaction mixture was subjected to test by HRMS analysis, and the result was shown in the Figure $\mathrm{S} 2$. There's still a little bit of starting material $\mathbf{3 b}$ left, and $\mathrm{Sb}-\mathrm{I}(\mathbf{1 i}), \mathrm{Sb}-\mathrm{Br}(\mathbf{1} \mathbf{h})$ andSb-OAc(1j) were detected.
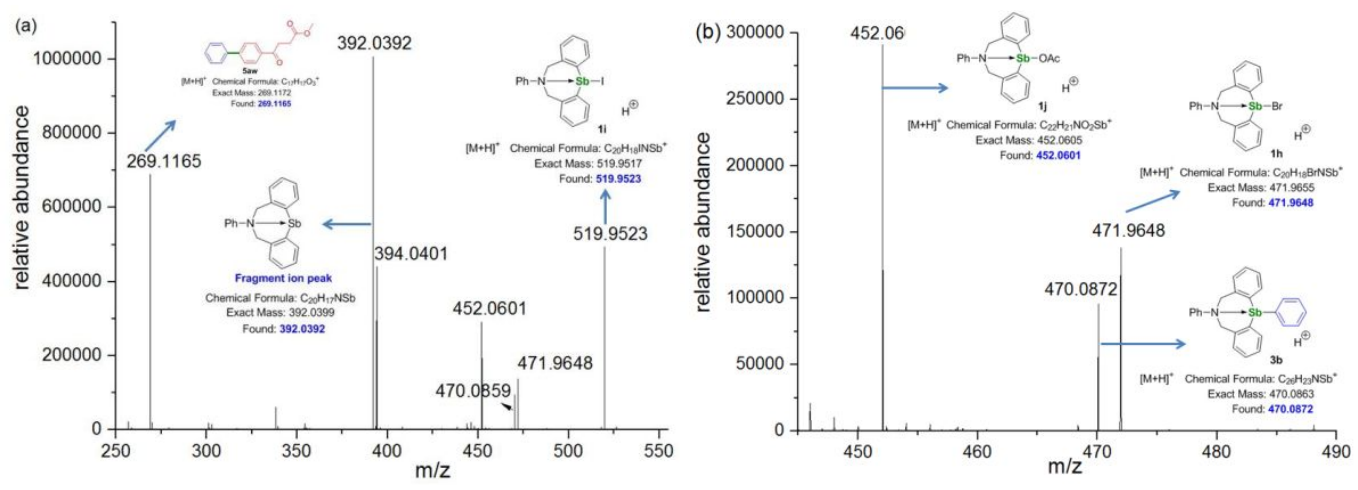

Figure S2 HRMS data of reaction mixture

We speculated that the reaction might occur in the equation 1 . The reaction mixture was treated with $3.0 \mathrm{~mol} / \mathrm{L} \mathrm{HCl}$ solution and stirred at $40{ }^{\circ} \mathrm{C}$ for $4 \mathrm{~h}$. Next, saturated $\mathrm{NaCl}$ solution was added. The organic phase was dried with anhydrous $\mathrm{Na}_{2} \mathrm{SO}_{4}$, and concentrated under reduced. The crude product was purified by column chromatography. The obtained compound was determined by HRMS analysis. The outcome in Figure S3 showed the recycled product is chlorostibine 1a instead of other stibines.<smiles>[X][Se]1(c2ccccc2CN2Cc3ccccc3C2)Cc2ccccc21</smiles>

$X=\mathrm{I}, \mathrm{Br}, \mathrm{OAc}$

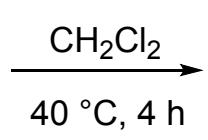<smiles>Cl[Sb]1(Cl)c2ccccc2CN(c2ccccc2)Cc2ccccc21</smiles>

$1 \mathbf{a}$ 


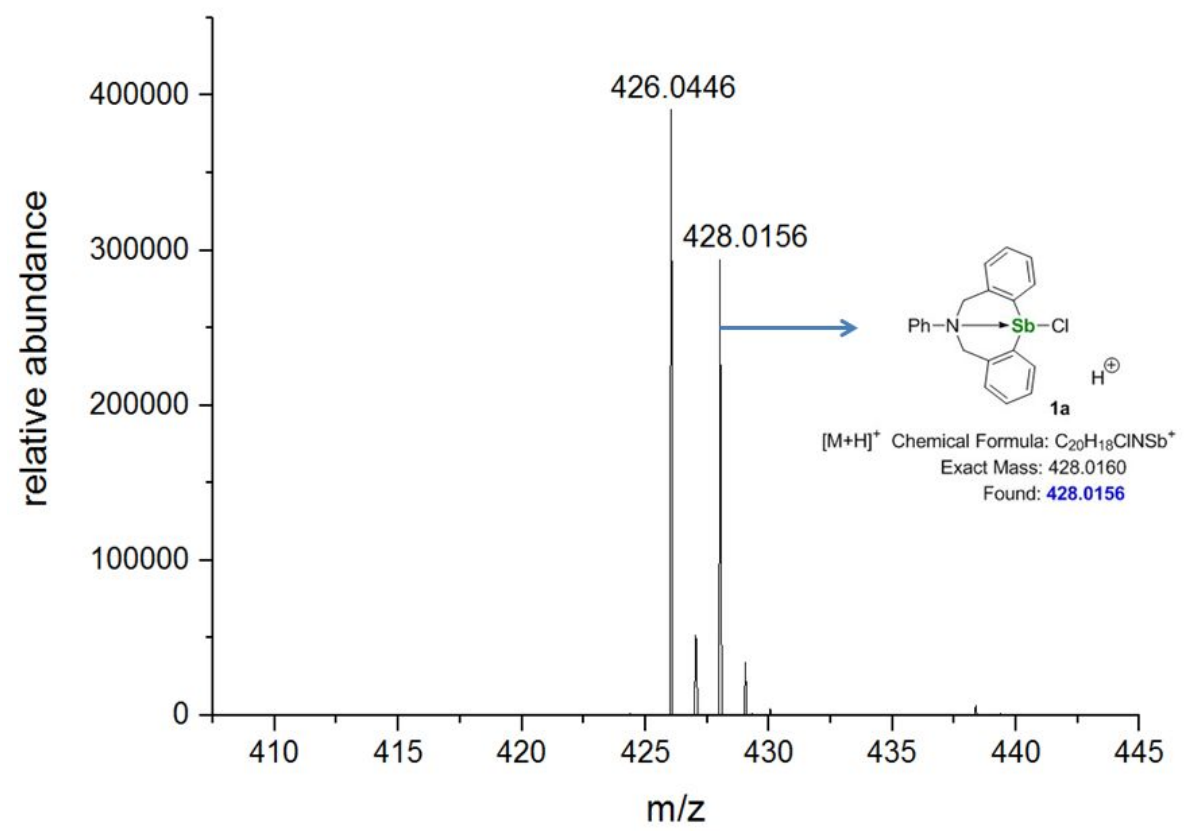

Figure. S3 HRMS data of recycled compound

Procedure (c): To a solution of methyl 4-([1,1'-biphenyl]-4-yl)-4-oxobutanoate 5aw (1.17 g, $4.36 \mathrm{mmol})$ in $20 \mathrm{~mL}$ of $\mathrm{MeOH}, 20 \mathrm{~mL}$ of $1.0 \mathrm{M} \mathrm{NaOH}$ was added. The reaction mixture was refluxed for $4 \mathrm{~h}$. After the reaction was completed, the resulting mixture was concentrated under reduced pressure and then acidified by diluted $\mathrm{HCl}$ and extracted with $\mathrm{CH}_{2} \mathrm{Cl}_{2}$ and dried with $\mathrm{Na}_{2} \mathrm{SO}_{4} .1 .09 \mathrm{~g}$ white solid $\mathbf{5 a x}(99 \%)$ was obtained. M.P.: $184.5-187.2{ }^{\circ} \mathrm{C} .{ }^{1} \mathrm{H}$ NMR $\left(400 \mathrm{MHz}, \mathrm{DMSO}-d_{6}\right) \delta 12.20(\mathrm{~s}, 1 \mathrm{H}), 8.06(\mathrm{~d}, J=8.1$ $\mathrm{Hz}, 2 \mathrm{H}), 7.82$ (d, $J=8.1 \mathrm{~Hz}, 2 \mathrm{H}), 7.74(\mathrm{~d}, J=7.5 \mathrm{~Hz}, 2 \mathrm{H}), 7.50(\mathrm{t}, J=7.4 \mathrm{~Hz}, 2 \mathrm{H}), 7.43(\mathrm{t}, J=7.1 \mathrm{~Hz}$, 1H), 3.28 (t, $J=5.9 \mathrm{~Hz}, 2 \mathrm{H}), 2.61(\mathrm{t}, J=5.9 \mathrm{~Hz}, 2 \mathrm{H}) .{ }^{13} \mathrm{C}$ NMR (101 MHz, DMSO- $\left.d_{6}\right) \delta 198.0,173.8$, 144.5, 138.9, 135.3, 129.1, 128.6, 128.4, 127.0, 126.9, 33.1, 27.9. HRMS-EI (m/z) [M] Calcd for $\mathrm{C}_{16} \mathrm{H}_{14} \mathrm{O}_{3}, 254.0943$; Found, 254.0942.

\section{Late-Stage Modification of Drug Fenofibrate}

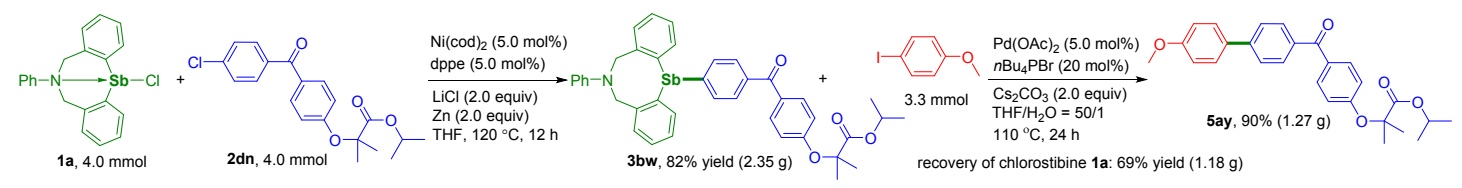

Procedure (a): Under a nitrogen atmosphere, a $100 \mathrm{~mL}$ Schlenk tube equipped with a magnetic stir bar was charged successively with chlorostibine 1a (1.71 g, $4.0 \mathrm{mmol})$, isopropyl 2-(4-(4chlorobenzoyl)phenoxy)-2-methylpropanoate $2 \mathbf{d n}(1.44 \mathrm{~g}, 4.0 \mathrm{mmol}), \mathrm{Ni}(\mathrm{cod})_{2}(55.0 \mathrm{mg}, 5.0 \mathrm{~mol} \%)$, zinc powder (520 mg, 2.0 equiv), dppe ( $80 \mathrm{mg}, 5.0 \mathrm{~mol} \%$ ), LiCl (336 mg, 2.0 equiv) and THF (30 mL). The reaction mixture was stirred at $120{ }^{\circ} \mathrm{C}$ for $12 \mathrm{~h}$. After the reaction was completed, the resulting mixture was diluted with $3.0 \mathrm{~mL}$ of $\mathrm{CH}_{2} \mathrm{Cl}_{2}$, and filtrated. The residue was washed with $3 \mathrm{~N} \mathrm{HCl}$ solution ( $30 \mathrm{~mL} \times 3$ ) and washed with $30 \mathrm{~mL}$ of $\mathrm{H}_{2} \mathrm{O}$. The organic layer was dried over anhydrous $\mathrm{Na}_{2} \mathrm{SO}_{4}$, and concentrated under reduced pressure. The crude product was purified by column chromatography on silica gel and eluted with the mixed solvent of PE:EA (20:1(v:v)) to afford $\mathbf{3 b w}$ as a white solid in $82 \%$ yield (2.35 g). M.P.: 157.8 - 159.3 ${ }^{\circ} \mathrm{C} .{ }^{1} \mathrm{H}$ NMR (400 MHz, Chloroform-d) $\delta 7.92(\mathrm{~d}, J=8.4 \mathrm{~Hz}, 2 \mathrm{H})$, 
$7.89-7.81(\mathrm{~m}, 4 \mathrm{H}), 7.36-7.28(\mathrm{~m}, 6 \mathrm{H}), 7.26(\mathrm{~d}, J=7.3 \mathrm{~Hz}, 2 \mathrm{H}), 7.22-7.16(\mathrm{~m}, 4 \mathrm{H}), 7.05-6.94(\mathrm{~m}$, $3 \mathrm{H}), 5.22-5.11(\mathrm{~m}, 1 \mathrm{H}), 4.78(\mathrm{~d}, J=15.2 \mathrm{~Hz}, 2 \mathrm{H}), 4.52(\mathrm{~d}, J=15.2 \mathrm{~Hz}, 2 \mathrm{H}), 1.74(\mathrm{~s}, 6 \mathrm{H}), 1.28(\mathrm{~d}, J=$ $6.2 \mathrm{~Hz}, 6 \mathrm{H}) .{ }^{13} \mathrm{C} \mathrm{NMR}\left(101 \mathrm{MHz}, \mathrm{CDCl}_{3}\right) \delta$ 196.0, 173.3, 159.7, 149.8, 148.3, 144.3, 138.8, 138.2, 136.6, 135.3, 132.2, 130.7, 129.5, 129.1, 128.6, 128.2, 127.0, 121.7, 117.8, 117.3, 79.5, 69.4, 57.6, 25.5, 21.6. HRMS-ESI (m/z) $[\mathrm{M}+\mathrm{H}]^{+}$Calcd for $\mathrm{C}_{40} \mathrm{H}_{39} \mathrm{NO}_{4} \mathrm{Sb}^{+}, 718.1912$; Found, 718.1917.

Procedure (b): Under a nitrogen atmoshpere, a $100 \mathrm{~mL}$ Schlenk tube equipped with a magnetic stir bar was charged successively with stibine $\mathbf{3 b w}$ ( $2.35 \mathrm{~g}, 3.28 \mathrm{mmol}$ ), 1-iodo-4-methoxybenzene $\mathbf{2 h}(772.2$ $\mathrm{mg}, 3.3 \mathrm{mmol}), \mathrm{Pd}(\mathrm{OAc})_{2}(88.0 \mathrm{mg}, 0.01 \mathrm{mmol}), n-\mathrm{Bu}_{4} \mathrm{PBr}(224.4 \mathrm{mg}, 0.66 \mathrm{mmol}), \mathrm{Cs}_{2} \mathrm{CO}_{3}(2.14 \mathrm{~g}, 6.6$ mmol), $20 \mathrm{~mL}$ of THF and $400 \mu \mathrm{L}$ of $\mathrm{H}_{2} \mathrm{O}$. The reaction mixture was stirred at $110^{\circ} \mathrm{C}$ for $24 \mathrm{~h}$. After the reaction was completed, the resulting mixture was diluted with $\mathrm{CH}_{2} \mathrm{Cl}_{2}$ and concentrated under reduced pressure. The crude product was purified by column chromatography on silica gel and eluted with the mixted solvent of PE/EA(20/1 (v:v)) to afford 5ay as a white solid in 90\% yield (1.27 g). The residue was continued to elute with the mixed solvent of $\mathrm{CH}_{2} \mathrm{Cl}_{2}: \mathrm{CH}_{3} \mathrm{OH}(5: 1(\mathrm{v}: \mathrm{v}))$ to obtain chlorostibine 1a (1.18 g). M.P.: $127.9-129.2{ }^{\circ} \mathrm{C} .{ }^{1} \mathrm{H}$ NMR $(400 \mathrm{MHz}$, Chloroform- $d$ ) $\delta 7.81$ (t, $J=9.0$ $\mathrm{Hz}, 4 \mathrm{H}), 7.62(\mathrm{dd}, J=23.0,8.0 \mathrm{~Hz}, 4 \mathrm{H}), 7.00(\mathrm{~d}, J=8.2 \mathrm{~Hz}, 2 \mathrm{H}), 6.88(\mathrm{~d}, J=8.3 \mathrm{~Hz}, 2 \mathrm{H}), 5.18-5.02$ (m, 1H), 3.85 (s, 3H), 1.67 (s, 6H), $1.21(\mathrm{~d}, J=6.2 \mathrm{~Hz}, 6 \mathrm{H}) .{ }^{13} \mathrm{C} \mathrm{NMR}\left(101 \mathrm{MHz}, \mathrm{CDCl}_{3}\right) \delta$ 195.2, 173.3, 159.9, 159.6, 144.5, 136.2, 132.5, 132.0, 131.0, 130.6, 128.4, 126.4, 117.3, 114.5, 79.4, 69.4, 55.5, 25.5, 21.6. HRMS-ESI (m/z) $[\mathrm{M}+\mathrm{H}]^{+}$Calcd for $\mathrm{C}_{27} \mathrm{H}_{29} \mathrm{O}_{5}{ }^{+}$, 433.2010; Found, 433.2010.

\section{Scheme S3. Selective Sequential Reactions of Stibines with Polyhalogenated Arenes}




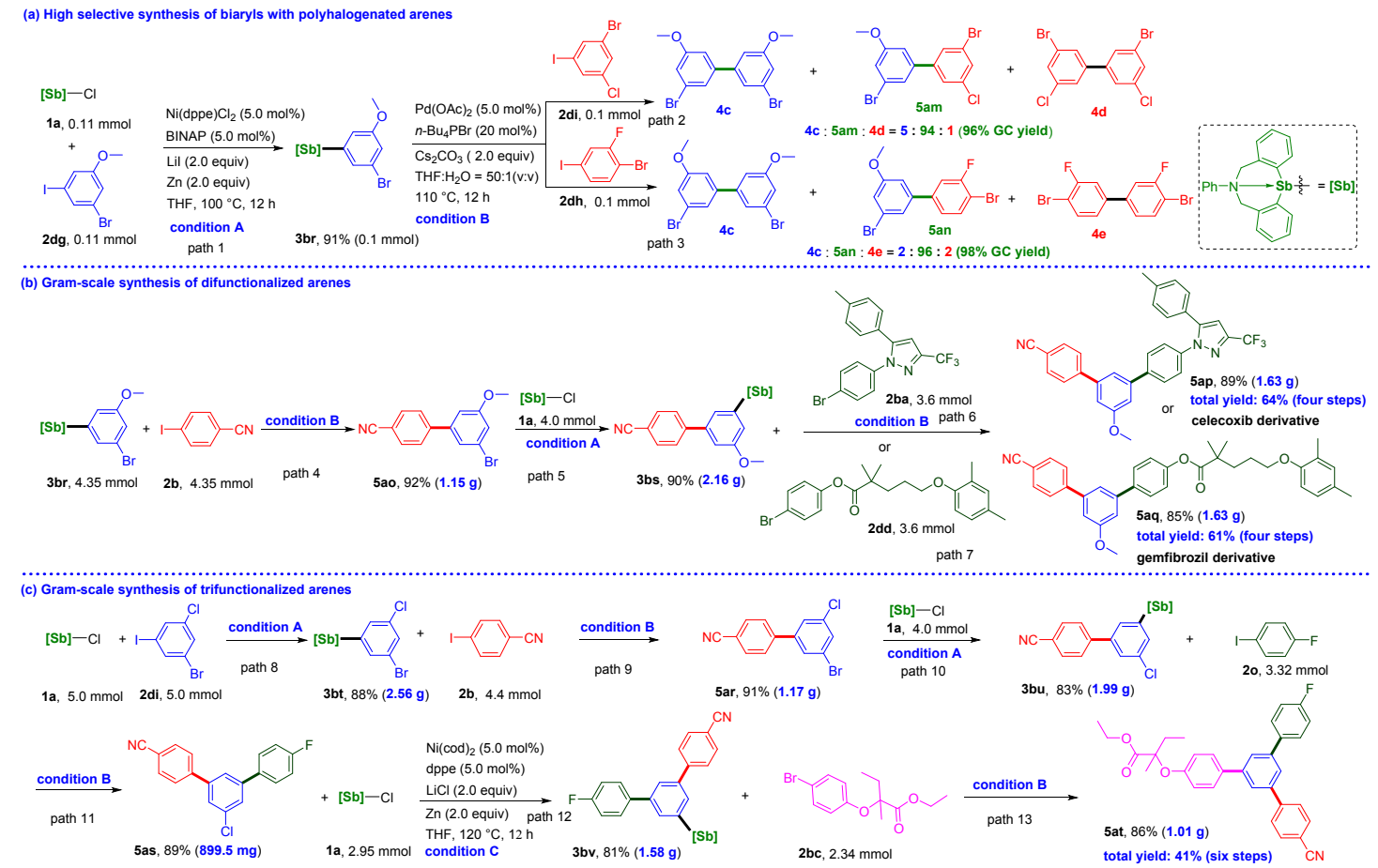

Procedure for path 1: Under a nitrogen atmosphere, a $10 \mathrm{~mL}$ Schlenk tube equipped with a magnetic stir bar was charged successively with chlorostibine 1a $(47.0 \mathrm{mg}, 0.11 \mathrm{mmol}), 1$-bromo-3-iodo-5methoxybenzene $2 \mathrm{dg}(34.3 \mathrm{mg}, 0.11 \mathrm{mmol}), \mathrm{Ni}(\mathrm{dppe}) \mathrm{Cl}_{2}(2.9 \mathrm{mg}, 5.0 \mathrm{~mol} \%)$, BINAP (3.3 mg, 5.0 mol\%), zinc powder (14.3 mg, 2.0 equiv), LiI (29.5, 2.0 equiv) and THF (1.0 mL). The reaction mixture was stirred at $100{ }^{\circ} \mathrm{C}$ for $12 \mathrm{~h}$. After the reaction was completed, the resulting mixture was diluted with $2.0 \mathrm{~mL}$ of $\mathrm{CH}_{2} \mathrm{Cl}_{2}$, and filtrated. The residue was washed with $1.0 \mathrm{~N} \mathrm{HCl}$ solution $(2.0 \mathrm{~mL} \times 3)$, and washed with $2.0 \mathrm{~mL}$ of $\mathrm{H}_{2} \mathrm{O}$. The organic layer was dried over anhydrous $\mathrm{Na}_{2} \mathrm{SO}_{4}$ and concentrated under reduced pressure. The crude product was purified by column chromatography on silica gel and eluted with the mixed solvent of PE:EA (80:1(v:v)) to afford 3 br as a white solid in 91\% yield (57.7 mg). M.P.: 171.2 - $172.3{ }^{\circ} \mathrm{C} .{ }^{1} \mathrm{H}$ NMR $(400 \mathrm{MHz}$, Chloroform- $d$ ) $\delta 7.47(\mathrm{~s}, 1 \mathrm{H}), 7.37-7.27$ (m, 8H), $7.26-7.17$ (m, 6H), $7.02(\mathrm{t}, J=7.3 \mathrm{~Hz}, 1 \mathrm{H}), 4.77(\mathrm{~d}, J=15.2 \mathrm{~Hz}, 2 \mathrm{H}), 4.52(\mathrm{~d}, J=15.1 \mathrm{~Hz}, 2 \mathrm{H}), 3.83(\mathrm{~s}, 3 \mathrm{H}) .{ }^{13} \mathrm{C}$ NMR $\left(101 \mathrm{MHz}, \mathrm{CDCl}_{3}\right) \delta 160.3,148.3,147.3,144.2,136.5,135.4,133.1,129.1,128.6,128.2,127.0$, 123.5, 122.6, 121.8, 118.0, 117.7, 57.7, 55.6. HRMS-ESI $(\mathrm{m} / \mathrm{z})[\mathrm{M}+\mathrm{H}]^{+}$Calcd for $\mathrm{C}_{27} \mathrm{H}_{24} \mathrm{BrNOSb}^{+}$, 578.0074; Found, 578.0081.

Procedure for path 2: Under a nitrogen atmoshpere, a $10 \mathrm{~mL}$ Schlenk tube equipped with a magnetic stir bar was charged successively with stibine 3 br $(57.7 \mathrm{mg}, 0.1 \mathrm{mmol})$, 1-bromo-3-chloro-5iodobenzene 2di (31.6 mg, $0.1 \mathrm{mmol}), \mathrm{Pd}(\mathrm{OAc})_{2}(1.1 \mathrm{mg}, 0.005 \mathrm{mmol}), n-\mathrm{Bu} 4 \mathrm{PBr}(6.8 \mathrm{mg}, 0.02 \mathrm{mmol})$, $\mathrm{Cs}_{2} \mathrm{CO}_{3}(65 \mathrm{mg}, 0.20 \mathrm{mmol}), 1.0 \mathrm{~mL}$ of THF and $20 \mu \mathrm{L}$ of $\mathrm{H}_{2} \mathrm{O}$. The reaction mixture was stirred at 110 ${ }^{\circ} \mathrm{C}$ for $12 \mathrm{~h}$. After the reaction was completed, the resulting mixture was diluted with $\mathrm{CH}_{2} \mathrm{Cl}_{2}$, and $30 \mu \mathrm{L}$ of $n$-dodecane was added. The yields of cross-coupling product 5am and homo-coupling products $\mathbf{4 c}$ and 4d were determinded by GC analysis. 4c: 5am: 4d = 5:94:1 (96\% yield). In addition, the mixture was purified by column chromatography on silica gel and eluted with the mixed solvent of PE/EA(40/1 (v:v)) to provide the pure product $\mathbf{5} \mathbf{a m}$ as a white solid in $90 \%$ yield. M. P.: $99.7-100.5{ }^{\circ} \mathrm{C} .{ }^{1} \mathrm{H}$ NMR (400 MHz, Chloroform-d) $\delta 7.53$ (d, $J=19.4$ Hz, 2H), 7.44 (s, 1H), 7.24 (s, 1H), 7.07 (s, 1H), 6.95 (s, 1H), 
$3.85(\mathrm{~s}, 3 \mathrm{H}) .{ }^{13} \mathrm{C} \mathrm{NMR}\left(101 \mathrm{MHz}, \mathrm{CDCl}_{3}\right) \delta 160.8,143.0,141.3,135.6,130.7,128.6,126.2,123.6,123.3$, 122.7, 117.0, 112.2, 55.8. HRMS-ESI (m/z) [M] Calcd for $\mathrm{C}_{13} \mathrm{H}_{9} \mathrm{Br}_{2} \mathrm{ClO}$, 373.8709; Found, 373.8705.

Procedure for path 3: Under a nitrogen atmoshpere, a $10 \mathrm{~mL}$ Schlenk tube equipped with a magnetic stir bar was charged successively with stibine $3 \mathbf{b r}(57.7 \mathrm{mg}, 0.1 \mathrm{mmol})$, 1-bromo-2-fluoro-4-iodobenzene $2 \mathrm{dh}$ (30.0 mg, $0.1 \mathrm{mmol}), \mathrm{Pd}(\mathrm{OAc})_{2}$ (1.1 mg, $\left.0.005 \mathrm{mmol}\right), n-\mathrm{Bu}_{4} \mathrm{PBr}$ (6.8 mg, $\left.0.02 \mathrm{mmol}\right), \mathrm{Cs}_{2} \mathrm{CO}_{3}(65$ $\mathrm{mg}, 0.20 \mathrm{mmol}$ ), $1.0 \mathrm{~mL}$ of THF and $20 \mu \mathrm{L}$ of $\mathrm{H}_{2} \mathrm{O}$. The reaction mixture was stirred at $110^{\circ} \mathrm{C}$ for $12 \mathrm{~h}$. After the reaction was completed, the resulting mixture was diluted with $\mathrm{CH}_{2} \mathrm{Cl}_{2}$, and $30 \mu \mathrm{L}$ of $n$ dodecane was added. The yields of the cross-coupling product 5an and homo-coupling products $\mathbf{4 c}$ as well as $\mathbf{4 e}$ were determinded by GC analysis. $4 \mathbf{c}: \mathbf{5 a n}: \mathbf{4 e}=2: 96: 2$ (98\% yield). In addition, the mixture was purified by column chromatography on silica gel and eluted with the mixed solvent of PE/EA(40/1 (v:v)) to provide the product 5 an as a white solid in 94\% yield. M. P.: $78.5-80.3{ }^{\circ} \mathrm{C}$. ${ }^{1} \mathrm{H}$ NMR $(400$ MHz, Chloroform- $d$ ) $\delta 7.62(\mathrm{t}, J=7.8 \mathrm{~Hz}, 1 \mathrm{H}), 7.34-7.27(\mathrm{~m}, 2 \mathrm{H}), 7.22(\mathrm{~d}, J=8.3 \mathrm{~Hz}, 1 \mathrm{H}), 7.09$ (s, 1H), $7.00(\mathrm{~s}, 1 \mathrm{H}), 3.88(\mathrm{~s}, 3 \mathrm{H}) .{ }^{13} \mathrm{C} \mathrm{NMR}\left(101 \mathrm{MHz}, \mathrm{CDCl}_{3}\right) \delta 160.8,159.4$ (d, J=247.8 Hz), 141.9 (d, $J=2.0 \mathrm{~Hz}), 141.2$ (d, $J=7.1 \mathrm{~Hz}), 134.0,123.9$ (d, $J=3.4 \mathrm{~Hz}), 123.6,122.5,116.6,115.2(\mathrm{~d}, J=23.2$ $\mathrm{Hz}), 112.2,108.8(\mathrm{~d}, J=20.9 \mathrm{~Hz}), 55.8 .{ }^{19} \mathrm{~F}$ NMR (377 MHz, $\left.\mathrm{CDCl}_{3}\right) \delta-106.70 . \mathrm{HRMS}-\mathrm{EI}(\mathrm{m} / \mathrm{z})[\mathrm{M}]$ Calcd for $\mathrm{C}_{13} \mathrm{H}_{9} \mathrm{Br}_{2} \mathrm{FO}, 357.9004$; Found, 357.8999 .

Procedure for path 4: Under a nitrogen atmoshpere, a $100 \mathrm{~mL}$ Schlenk tube equipped with a magnetic stir bar was charged successively with stibine $3 \mathbf{b r}$ (2.51 g, $4.35 \mathrm{mmol})$, 4-iodobenzonitrile $2 \mathbf{b}$ (996.1 mg, $4.35 \mathrm{mmol}), \mathrm{Pd}(\mathrm{OAc})_{2}(48.9 \mathrm{mg}, 0.217 \mathrm{mmol}), n-\mathrm{Bu}_{4} \mathrm{PBr}(295.8 \mathrm{mg}, 0.87 \mathrm{mmol}), \mathrm{Cs}_{2} \mathrm{CO}_{3}(2.827 \mathrm{~g}, 8.70$ mmol), $40 \mathrm{~mL}$ of THF and $800 \mu \mathrm{L}$ of $\mathrm{H}_{2} \mathrm{O}$. The reaction mixture was stirred at $110{ }^{\circ} \mathrm{C}$ for $12 \mathrm{~h}$. After the reaction was completed, the resulting mixture was diluted with $\mathrm{CH}_{2} \mathrm{Cl}_{2}$ and concentrated under reduced pressure. The crude product was purified by column chromatography on silica gel and eluted with the mixed solvent of PE/EA(20/1 (v:v)) to afford the desired product 5ao as a white solid in $92 \%$ yield (1.15 g). M. P.: $125.4-127.1{ }^{\circ} \mathrm{C} ;{ }^{1} \mathrm{H}$ NMR (400 MHz, Chloroform- $d$ ) $\delta 7.78-7.70(\mathrm{~m}, 2 \mathrm{H}), 7.69$ $-7.60(\mathrm{~m}, 2 \mathrm{H}), 7.35-7.29(\mathrm{~m}, 1 \mathrm{H}), 7.08(\mathrm{~d}, J=32.1 \mathrm{~Hz}, 2 \mathrm{H}), 3.87(\mathrm{~s}, 3 \mathrm{H}) .{ }^{13} \mathrm{C} \mathrm{NMR}\left(101 \mathrm{MHz}, \mathrm{CDCl}_{3}\right)$ $\delta 160.8,144.2,142.1,132.7,127.9,123.6,122.8,118.7,117.0,112.5,111.8,55.8$. HRMS-EI (m/z) [M] Calcd for $\mathrm{C}_{14} \mathrm{H}_{10} \mathrm{BrNO}, 286.9946$; Found, 286.9942.

Procedure for path 5: Under a nitrogen atmosphere, a $100 \mathrm{~mL}$ Schlenk tube equipped with a magnetic stir bar was charged successively with chlorostibine $1 \mathrm{a}$ (1.71 g, $4.0 \mathrm{mmol})$, 3'-bromo-5'-methoxy-[1,1'biphenyl]-4-carbonitrile $5 \mathbf{a o}(1.15 \mathrm{~g}, 4.0 \mathrm{mmol})$, Ni(dppe)Cl 2 (105.6 mg, $5.0 \mathrm{~mol} \%$ ), BINAP (124.5 mg, $5.0 \mathrm{~mol} \%)$, zinc powder (520 mg, 2.0 equiv), LiI (1.07 g, 2.0 equiv) and THF (40 mL). The reaction mixture was stirred at $100{ }^{\circ} \mathrm{C}$ for $12 \mathrm{~h}$. After the reaction was completed, the resulting mixture was diluted with $30 \mathrm{~mL}$ of $\mathrm{CH}_{2} \mathrm{Cl}_{2}$, and filtrated. The residue was washed with $1.0 \mathrm{~N} \mathrm{HCl}$ solution $(50 \mathrm{~mL} \mathrm{x}$ 3) and washed with $50 \mathrm{~mL}$ of $\mathrm{H}_{2} \mathrm{O}$. The organic layer was dried over anhydrous $\mathrm{Na}_{2} \mathrm{SO}_{4}$ and concentrated under reduced pressure. The crude product was purified by column chromatography on silica gel and eluted with the mixed solvent of PE:EA (20:1(v:v)) to afford 3bs as a white solid in 90\% yield (2.16 g). M.P.: 197.8 - $199.2{ }^{\circ} \mathrm{C} .{ }^{1} \mathrm{H}$ NMR (400 MHz, Chloroform- $d$ ) $\delta 7.66(\mathrm{~s}, 4 \mathrm{H}), 7.48(\mathrm{~s}, 1 \mathrm{H}), 7.29-7.21(\mathrm{~m}$, 9H), $7.17-7.09$ (m, 5H), 6.94 (t, $J=7.3 \mathrm{~Hz}, 1 \mathrm{H}), 4.73$ (d, J=15.2 Hz, 2H), 4.46 (d, J=15.2 Hz, 2H), $3.83(\mathrm{~s}, 3 \mathrm{H}) .{ }^{13} \mathrm{C} \mathrm{NMR}\left(101 \mathrm{MHz}, \mathrm{CDCl}_{3}\right) \delta 160.0,148.4,146.4,145.7,144.4,140.5,136.6,135.5,132.6$, 
130.0, 129.1, 128.6, 128.1, 127.9, 127.0, 123.7, 121.7, 119.1, 117.8, 113.9, 111.0, 57.7, 55.6. HRMSESI (m/z) $[\mathrm{M}+\mathrm{H}]^{+}$Calcd for $\mathrm{C}_{34} \mathrm{H}_{28} \mathrm{~N}_{2} \mathrm{OSb}^{+}, 601.1234$; Found, 601.1238.

Procedure for path 6: Under a nitrogen atmoshpere, a $100 \mathrm{~mL}$ Schlenk tube equipped with a magnetic stir bar was charged successively with stibine $3 \mathrm{bs}$ ( $2.16 \mathrm{~g}, 3.6 \mathrm{mmol})$, 1-(4-bromophenyl)-5-( $p$-tolyl)-3(trifluoromethyl)-1 $H$-pyrazole $2 \mathbf{b a}(1.37 \mathrm{~g}, 3.6 \mathrm{mmol}), \mathrm{Pd}(\mathrm{OAc})_{2}(40.5 \mathrm{mg}, 0.18 \mathrm{mmol}), n-\mathrm{Bu}_{4} \mathrm{PBr}$ (244.3 mg, $0.72 \mathrm{mmol}), \mathrm{Cs}_{2} \mathrm{CO}_{3}(2.34 \mathrm{~g}, 7.2 \mathrm{mmol}), 40 \mathrm{~mL}$ of THF and $800 \mu \mathrm{L}$ of $\mathrm{H}_{2} \mathrm{O}$. The reaction mixture was stirred at $110{ }^{\circ} \mathrm{C}$ for $12 \mathrm{~h}$. After the reaction was completed, the resulting mixture was diluted with $\mathrm{CH}_{2} \mathrm{Cl}_{2}$ and concentrated under reduced pressure. The crude product was purified by column chromatography on silica gel and eluted with the mixed solvent of PE/EA(20/1 (v:v)) to afford the desired product 5ap as a white solid in $89 \%$ yield $(1.63 \mathrm{~g})$. M. P.: $109.1-110.4{ }^{\circ} \mathrm{C} .{ }^{1} \mathrm{H}$ NMR $(400 \mathrm{MHz}$, Chloroform- $d$ ) $\delta 7.76-7.67(\mathrm{~s}, 4 \mathrm{H}), 7.61(\mathrm{~d}, J=8.2 \mathrm{~Hz}, 2 \mathrm{H}), 7.45-7.32(\mathrm{~m}, 3 \mathrm{H}), 7.22-7.07(\mathrm{~m}, 6 \mathrm{H})$, $6.75(\mathrm{~s}, 1 \mathrm{H}), 3.93(\mathrm{~s}, 3 \mathrm{H}), 2.37$ (s, 3H). ${ }^{13} \mathrm{C}$ NMR $\left(101 \mathrm{MHz}, \mathrm{CDCl}_{3}\right) \delta 160.6,145.3,144.9,143.3$ (q, $J$ $=38.2 \mathrm{~Hz}), 142.1,141.2,140.5,139.3,138.9,132.6,129.5,128.7,127.9,127.8,126.2,125.7,121.4(\mathrm{q}$, $J=269.1 \mathrm{~Hz}), 118.8,118.6,112.9,112.3,111.3,105.6,55.6,21.3 .{ }^{19} \mathrm{~F}$ NMR $\left(376 \mathrm{MHz}, \mathrm{CDCl}_{3}\right) \delta-$ 62.01. HRMS-EI (m/z) [M] Calcd for $\mathrm{C}_{31} \mathrm{H}_{22} \mathrm{~F}_{3} \mathrm{~N}_{3} \mathrm{O}$, 509.1715; Found, 509.1711.

Procedure for path 7: Under a nitrogen atmoshpere, a $100 \mathrm{~mL}$ Schlenk tube equipped with a magnetic stir bar was charged successively with stibine 3 bs $(2.16 \mathrm{~g}$, $3.6 \mathrm{mmol})$, 4-bromophenyl 5-(2,4dimethylphenoxy)-2,2-dimethylpentanoate 2dd (1.45 g, $3.6 \mathrm{mmol}), \mathrm{Pd}(\mathrm{OAc})_{2}(40.5 \mathrm{mg}, 0.18 \mathrm{mmol}), n$ $\mathrm{Bu}_{4} \mathrm{PBr}(67.8 \mathrm{mg}, 0.72 \mathrm{mmol}), \mathrm{Cs}_{2} \mathrm{CO}_{3}(2.34 \mathrm{~g}, 7.2 \mathrm{mmol}), 40 \mathrm{~mL}$ of THF and $800 \mu \mathrm{L}$ of $\mathrm{H}_{2} \mathrm{O}$. The reaction mixture was stirred at $110^{\circ} \mathrm{C}$ for $12 \mathrm{~h}$. After the reaction was completed, the resulting mixture was diluted with $\mathrm{CH}_{2} \mathrm{Cl}_{2}$ and concentrated under reduced pressure. The crude product was purified by column chromatography on silica gel and eluted with PE/EA(20/1 (v:v)) to afford the desired product 5aq as a liquid in $85 \%$ yield $(1.63 \mathrm{~g}) .{ }^{1} \mathrm{H}$ NMR $(400 \mathrm{MHz}$, Chloroform- $d$ ) $\delta 7.78-7.70(\mathrm{~m}, 4 \mathrm{H}), 7.65-$ $7.58(\mathrm{~m}, 2 \mathrm{H}), 7.35(\mathrm{t}, J=1.6 \mathrm{~Hz}, 1 \mathrm{H}), 7.18-7.12(\mathrm{~m}, 3 \mathrm{H}), 7.11-7.07(\mathrm{~m}, 1 \mathrm{H}), 7.03(\mathrm{~d}, J=7.3 \mathrm{~Hz}, 1 \mathrm{H})$, $6.73-6.62(\mathrm{~m}, 2 \mathrm{H}), 4.06-3.98(\mathrm{~m}, 2 \mathrm{H}), 3.94(\mathrm{~s}, 3 \mathrm{H}), 2.33(\mathrm{~s}, 3 \mathrm{H}), 2.21$ (s, 3H), $1.97-1.87$ (m, 4H), 1.42 (s, 6H). ${ }^{13} \mathrm{C}$ NMR $\left(101 \mathrm{MHz}, \mathrm{CDCl}_{3}\right) \delta 176.5,160.6,157.0,151.0,145.6,142.9,141.2,138.3,136.6$, 132.7, 130.5, 128.3, 128.0, 123.7, 122.1, 120.9, 119.0, 118.9, 113.0, 112.0, 112.0, 111.3, 67.8, 55.6, 42.6, 37.3, 25.4, 25.3, 21.5, 15.9. HRMS-ESI $(\mathrm{m} / \mathrm{z})[\mathrm{M}+\mathrm{H}]^{+}$Calcd for $\mathrm{C}_{35} \mathrm{H}_{36} \mathrm{NO}_{4}{ }^{+}, 534.2639$; Found, 534.2640 .

Procedure for path 8: Under a nitrogen atmosphere, a $100 \mathrm{~mL}$ Schlenk tube equipped with a magnetic stir bar was charged successively with chlorostibine 1a $(2.14 \mathrm{~g}, 5.0 \mathrm{mmol})$, 1-bromo-3-chloro-5iodobenzene 1di (1.58 g, $5.0 \mathrm{mmol}$ ), Ni(dppe)Cl 2 (132.5 mg, $5.0 \mathrm{~mol} \%$ ), BINAP (150 mg, $5.0 \mathrm{~mol} \%$ ), zinc powder ( $650 \mathrm{mg}, 2.0$ equiv), LiI (1.34 g, 2.0 equiv) and THF $(40 \mathrm{~mL})$. The reaction mixture was stirred at $100{ }^{\circ} \mathrm{C}$ for $12 \mathrm{~h}$. After the reaction was completed, the resulting mixture was diluted with 15 $\mathrm{mL}$ of $\mathrm{CH}_{2} \mathrm{Cl}_{2}$, filtrated. The residue was washed with $1.0 \mathrm{~N} \mathrm{HCl}$ solution $(50 \mathrm{~mL} \times 3)$ and washed with $50 \mathrm{~mL}$ of $\mathrm{H}_{2} \mathrm{O}$. The organic layer was dried over anhydrous $\mathrm{Na}_{2} \mathrm{SO}_{4}$ and concentrated under reduced pressure. The crude product was purified by column chromatography on silica gel and eluted with the mixed solvent of PE:EA (80:1(v:v)) to afford the desired product $3 \mathbf{b t}$ as a white solid in $88 \%$ yield $(2.56$ g). M.P.: $204.4-205.4{ }^{\circ} \mathrm{C} .{ }^{1} \mathrm{H}$ NMR (400 MHz, Chloroform- $d$ ) $\delta 7.74$ (s, 1H), 7.63 (s, 2H), $7.37-7.27$ (m, 6H), $7.26-7.16(\mathrm{~m}, 6 \mathrm{H}), 7.03(\mathrm{t}, J=7.3 \mathrm{~Hz}, 1 \mathrm{H}), 4.76(\mathrm{~d}, J=15.1 \mathrm{~Hz}, 2 \mathrm{H}), 4.51(\mathrm{~d}, J=15.1 \mathrm{~Hz}$, 
2H). ${ }^{13} \mathrm{C}$ NMR (101 MHz, $\left.\mathrm{CDCl}_{3}\right) \delta$ 148.8, 148.2, 144.2, 139.2, 136.8, 136.3, 135.4, 135.1, 131.4, 129.2, 128.8, 128.5, 127.0, 123.5, 122.2, 118.2, 57.8. HRMS-ESI (m/z) $[\mathrm{M}+\mathrm{H}]^{+}$Calcd for $\mathrm{C}_{26} \mathrm{H}_{20} \mathrm{BrClNNaSb}^{+}$, 603.9398; Found, 603.9397.

Procedure for path 9: Under a nitrogen atmoshpere, a $100 \mathrm{~mL}$ Schlenk tube equipped with a magnetic stir bar was charged successively with stibine $\mathbf{3 b t}$ ( $2.56 \mathrm{~g}, 4.4 \mathrm{mmol}$ ), 4-iodobenzonitrile $\mathbf{2 b}$ ( $1.00 \mathrm{~g}, 4.4$ mmol), $\mathrm{Pd}(\mathrm{OAc})_{2}$ (49.5 mg, $\left.0.22 \mathrm{mmol}\right), n-\mathrm{Bu}_{4} \mathrm{PBr}(298.6 \mathrm{mg}, 0.88 \mathrm{mmol}), \mathrm{Cs}_{2} \mathrm{CO}_{3}(2.86 \mathrm{~g}, 8.8 \mathrm{mmol}$ ), $40 \mathrm{~mL}$ of THF and $800 \mu \mathrm{L}$ of $\mathrm{H}_{2} \mathrm{O}$. The reaction mixture was stirred at $110^{\circ} \mathrm{C}$ for $12 \mathrm{~h}$. After the reaction was completed, the resulting mixture was diluted with $\mathrm{CH}_{2} \mathrm{Cl}_{2}$ and concentrated under reduced pressure. The crude product was purified by column chromatography on silica gel and eluted with the mixed solvent of PE/EA(40/1 (v:v)) to afford the desired product 5 ar as a white solid in $91 \%$ yield $(1.17 \mathrm{~g})$. M.P.: $115.3-117.6^{\circ} \mathrm{C} .{ }^{1} \mathrm{H}$ NMR (400 MHz, Chloroform- $d$ ) $\delta 7.79-7.71(\mathrm{~m}, 2 \mathrm{H}), 7.68-7.46(\mathrm{~m}, 5 \mathrm{H})$. ${ }^{13} \mathrm{C}$ NMR (101 MHz, $\left.\mathrm{CDCl}_{3}\right) \delta 142.9,142.5,136.0,133.0,131.4,128.8,127.9,126.4,123.6,118.6$, 112.4. HRMS-EI (m/z) [M] Calcd for $\mathrm{C}_{13} \mathrm{H}_{7} \mathrm{BrClN}$, 290.9450; Found, 290.9445.

Procedure for path 10: Under a nitrogen atmosphere, a $100 \mathrm{~mL}$ Schlenk tube equipped with a magnetic stir bar was charged successively with chlorostibine 1a (1.71 g, $4.0 \mathrm{mmol}), 3$ '-bromo-5'-chloro-[1,1'biphenyl]-4-carbonitrile $5 \mathrm{ar}(1.17 \mathrm{~g}, 4.0 \mathrm{mmol})$, Ni(dppe)Cl 2 (105.6 mg, $5.0 \mathrm{~mol} \%$ ), BINAP (124.4 mg, $5.0 \mathrm{~mol} \%)$, zinc powder (520 mg, 2.0 equiv), LiI (1.07 g, 2.0 equiv) and THF (40 mL). The reaction mixture was stirred at $100{ }^{\circ} \mathrm{C}$ for $12 \mathrm{~h}$. After the reaction was completed, the resulting mixture was diluted with $15 \mathrm{~mL}$ of $\mathrm{CH}_{2} \mathrm{Cl}_{2}$, filtrated. The residue was washed with $1 \mathrm{~N} \mathrm{HCl}$ solution $(50 \mathrm{~mL} \times 3)$ and washed with $50 \mathrm{~mL}$ of $\mathrm{H}_{2} \mathrm{O}$. The organic layer was dried over anhydrous $\mathrm{Na}_{2} \mathrm{SO}_{4}$ and concentrated under reduced pressure. The crude product was purified by column chromatography on silica gel and eluted with the mixed solvent of PE:EA (40:1(v:v)) to afford the desired product $3 \mathbf{b u}$ as a white solid in $83 \%$ yield (1.99 g). M.P.: $196.8-198.1^{\circ} \mathrm{C} .{ }^{1} \mathrm{H}$ NMR (400 MHz, Chloroform- $d$ ) $\delta 7.81-7.64(\mathrm{~m}, 7 \mathrm{H}), 7.36$ $-7.26(\mathrm{~m}, 6 \mathrm{H}), 7.25-7.15(\mathrm{~m}, 6 \mathrm{H}), 7.01(\mathrm{t}, J=7.3 \mathrm{~Hz}, 1 \mathrm{H}), 4.77$ (d, $J=15.2 \mathrm{~Hz}, 2 \mathrm{H}), 4.51$ (d, $J=15.2$ $\mathrm{Hz}, 2 \mathrm{H}) .{ }^{13} \mathrm{C}$ NMR (101 MHz, $\left.\mathrm{CDCl}_{3}\right) \delta 148.2,147.7,144.5,144.4,140.9,138.4,136.4,135.6,135.5$, 135.2, 132.8, 129.2, 128.8, 128.3, 127.9, 127.6, 127.1, 122.0, 118.9, 118.1, 111.5, 57.8. HRMS-ESI (m/z) $[\mathrm{M}+\mathrm{H}]^{+}$Calcd for $\mathrm{C}_{33} \mathrm{H}_{25} \mathrm{ClN}_{2} \mathrm{Sb}^{+}, 605.0739$; Found, 605.0739.

Procedure for path 11: Under a nitrogen atmoshpere, a $100 \mathrm{~mL}$ Schlenk tube equipped with a magnetic stir bar was charged successively with stibine $\mathbf{3 b u}$ (1.99 g, $3.32 \mathrm{mmol})$, 1-fluoro-4-iodobenzene $2 \mathrm{o}$ (737.0 mg, $3.32 \mathrm{mmol}$ ), $\mathrm{Pd}(\mathrm{OAc})_{2}(37.3 \mathrm{mg}, 0.166 \mathrm{mmol}), n-\mathrm{Bu} 4 \mathrm{PBr}(225.3 \mathrm{mg}, 0.664 \mathrm{mmol}), \mathrm{Cs}_{2} \mathrm{CO}_{3}$ (2.16 g, $6.64 \mathrm{mmol}$ ), $40 \mathrm{~mL}$ of THF and $800 \mu \mathrm{L}$ of $\mathrm{H}_{2} \mathrm{O}$. The reaction mixture was stirred at $110^{\circ} \mathrm{C}$ for $12 \mathrm{~h}$. After the reaction was completed, the resulting mixture was diluted with $\mathrm{CH}_{2} \mathrm{Cl}_{2}$ and concentrated under reduced pressure. The crude product was purified by column chromatography on silica gel and eluted with the mixed solvent of PE/EA(40/1 (v:v)) to afford the desired product 5as as a white solid in $89 \%$ yield (899.5 mg). M.P.: $144.6-146.3{ }^{\circ} \mathrm{C} .{ }^{1} \mathrm{H}$ NMR (400 MHz, Chloroform- $d$ ) $\delta 7.78-7.68$ (m, $4 \mathrm{H}), 7.56(\mathrm{t}, J=11.8 \mathrm{~Hz}, 5 \mathrm{H}), 7.16(\mathrm{t}, J=8.5 \mathrm{~Hz}, 2 \mathrm{H}) .{ }^{13} \mathrm{C} \mathrm{NMR}\left(101 \mathrm{MHz}, \mathrm{CDCl}_{3}\right) \delta 163.0(\mathrm{~d}, J=248.3$ Hz), 144.1, 142.9, 141.5, 135.5, 135.5 (d, $J=3.4 \mathrm{~Hz}), 132.8,128.9$ (d, $J=8.1 \mathrm{~Hz}), 127.9,127.9,127.2$, 126.1, 124.2, 118.6, $116.0(\mathrm{~d}, J=21.6 \mathrm{~Hz}), 111.8 .{ }^{19} \mathrm{~F} \mathrm{NMR}\left(376 \mathrm{MHz}, \mathrm{CDCl}_{3}\right) \delta-113.80$. HRMS-EI $(\mathrm{m} / \mathrm{z})[\mathrm{M}]$ Calcd for $\mathrm{C}_{19} \mathrm{H}_{11} \mathrm{ClFN}, 307.0564$; Found, 307.0562. 
Procedure for path 12: Under a nitrogen atmoshpere, a $100 \mathrm{~mL}$ Schlenk tube equipped with a magnetic stir bar was charged successively with stibine 1a (1.26 g, $2.95 \mathrm{mmol})$, 5'-chloro-4"-fluoro-[1,1':3',1"terphenyl]-4-carbonitrile 5as (905.6 mg, $2.95 \mathrm{mmol}), \mathrm{Ni}(\mathrm{cod})_{2}(40.4 \mathrm{mg}, 0.147 \mathrm{mmol})$, dppe (62.0 mg, $0.147 \mathrm{mmol}), \mathrm{LiCl}(247.8 \mathrm{mg}, 5.9 \mathrm{mmol}), 40 \mathrm{~mL}$ of THF. The reaction mixture was stirred at $110^{\circ} \mathrm{C}$ for $12 \mathrm{~h}$. After the reaction was completed, the resulting mixture was diluted with $\mathrm{CH}_{2} \mathrm{Cl}_{2}$. The residue was washed with $1 \mathrm{~N} \mathrm{HCl}$ solution $(50 \mathrm{~mL}$ x 3$)$ and washed with $50 \mathrm{~mL}$ of $\mathrm{H}_{2} \mathrm{O}$. The organic layer was dried over anhydrous $\mathrm{Na}_{2} \mathrm{SO}_{4}$ and concentrated under reduced pressure. The crude product was purified by column chromatography on silica gel and eluted with the mixed solvent of PE:EA (40:1(v:v)) to afford the desired product $\mathbf{3 b v}$ as a white solid in $81 \%$ yield $(1.58 \mathrm{~g})$. M.P.: $244.8-246.0{ }^{\circ} \mathrm{C}$. ${ }^{1} \mathrm{H}$ NMR $(400$ $\mathrm{MHz}$, Chloroform- $d$ ) $\delta 7.89(\mathrm{~d}, J=16.9 \mathrm{~Hz}, 2 \mathrm{H}), 7.81-.7 .76(\mathrm{~m}, 1 \mathrm{H}), 7.75-7.67(\mathrm{~m}, 4 \mathrm{H}), 7.62-7.54$ (m, 2H), $7.32-7.21(\mathrm{~m}, 8 \mathrm{H}), 7.17-7.08(\mathrm{~m}, 6 \mathrm{H}), 6.96(\mathrm{t}, J=7.3 \mathrm{~Hz}, 1 \mathrm{H}), 4.75(\mathrm{~d}, J=15.2 \mathrm{~Hz}, 2 \mathrm{H})$, $4.48(\mathrm{~d}, J=15.3 \mathrm{~Hz}, 2 \mathrm{H}) .{ }^{13} \mathrm{C} \mathrm{NMR}\left(101 \mathrm{MHz}, \mathrm{CDCl}_{3}\right) \delta 162.7(\mathrm{~d}, J=247.0 \mathrm{~Hz}), 148.3,145.8(\mathrm{~d}, J=$ $5.2 \mathrm{~Hz}), 144.5,141.0,139.7,137.9,137.0$ (d, $J=3.1 \mathrm{~Hz}), 136.6,136.5,135.4,132.7,129.2,129.0,128.9$, 128.7, 128.2, 128.0, 127.1, 126.4, 121.8, 119.1, 117.9, 115.9 (d, $J=21.5 \mathrm{~Hz}), 111.1,57.7 .{ }^{19} \mathrm{~F} \mathrm{NMR}(376$ $\left.\mathrm{MHz}, \mathrm{CDCl}_{3}\right) \delta-115.05$. HRMS-ESI (m/z) $[\mathrm{M}+\mathrm{H}]^{+}$Calcd for $\mathrm{C}_{39} \mathrm{H}_{29} \mathrm{FN}_{2} \mathrm{Sb}^{+}, 665.1347$; Found, 665.1355. Procedure for path 13: Under a nitrogen atmoshpere, a $100 \mathrm{~mL}$ Schlenk tube equipped with a magnetic stir bar was charged successively with stibine $3 \mathbf{b v}$ (1.56 g, $2.36 \mathrm{mmol}$ ), ethyl 2-(4-bromophenoxy)-2methylbutanoate $2 \mathbf{b c}(702.0 \mathrm{mg}, 2.34 \mathrm{mmol}), \mathrm{Pd}(\mathrm{OAc})_{2}(26.3 \mathrm{mg}, 0.117 \mathrm{mmol}), n-\mathrm{Bu}_{4} \mathrm{PBr}(158.8 \mathrm{mg}$, $0.468 \mathrm{mmol}), \mathrm{Cs}_{2} \mathrm{CO}_{3}(1.52 \mathrm{~g}, 4.68 \mathrm{mmol}), 40 \mathrm{~mL}$ of THF and $800 \mu \mathrm{L}$ of $\mathrm{H}_{2} \mathrm{O}$. The reaction mixture was stirred at $110{ }^{\circ} \mathrm{C}$ for $12 \mathrm{~h}$. After the reaction was completed, the resulting mixture was diluted with $\mathrm{CH}_{2} \mathrm{Cl}_{2}$ and concentrated under reduced pressure. The crude product was purified by column chromatography on silica gel and eluted with PE/EA(40/1 (v:v)) to afford the desired product 5at as a liquid in $86 \%$ yield $(1.01 \mathrm{mg}) .{ }^{1} \mathrm{H}$ NMR (400 MHz, Chloroform- $d$ ) $\delta 7.80-7.69(\mathrm{~m}, 6 \mathrm{H}), 7.66-7.53$ $(\mathrm{m}, 5 \mathrm{H}), 7.17(\mathrm{t}, J=7.7 \mathrm{~Hz}, 2 \mathrm{H}), 6.97$ (d, $J=8.8 \mathrm{~Hz}, 2 \mathrm{H}), 4.28$ (q, $J=7.1 \mathrm{~Hz}, 2 \mathrm{H}), 2.15-1.94(\mathrm{~m}, 2 \mathrm{H})$, $1.57(\mathrm{~s}, 3 \mathrm{H}), 1.29$ (t, $J=7.1 \mathrm{~Hz}, 3 \mathrm{H}), 1.02$ (t, $J=7.4 \mathrm{~Hz}, 3 \mathrm{H}) .{ }^{13} \mathrm{C} \mathrm{NMR}\left(101 \mathrm{MHz}, \mathrm{CDCl}_{3}\right) \delta 173.9$, 162.7 (d, $J=247.2 \mathrm{~Hz}), 155.6,145.6,142.3,141.7,140.4,136.8$ (d, $J=3.4 \mathrm{~Hz}), 134.1,132.7,128.9$ (d, $J=8.1 \mathrm{~Hz}), 128.0,127.9,125.9,124.7,124.5,119.3,118.9,115.8$ (d, $J=21.5 \mathrm{~Hz}), 111.3,82.1,61.4$, 32.6, 20.8, 14.2 , 7.9. ${ }^{19} \mathrm{~F}$ NMR (376 MHz, $\left.\mathrm{CDCl}_{3}\right) \delta$-114.79. HRMS-EI (m/z) [M] Calcd for $\mathrm{C}_{32} \mathrm{H}_{28} \mathrm{FNO}_{3}$, 493.2053; Found, 493.2057.

\section{S4. Mechanism Study}




\section{S4.1.1 Kinetic Research on Reductive Cross-Coupling Reaction of Chlorostibines with Aryl Iodides}

Table S14. Time-Course Monitoring Results<smiles>[O-]c1ccccc1CN1Cc2ccccc2[Se]Cc2ccccc21</smiles>

1a, $0.1 \mathrm{mmol}$<smiles>Cc1ccc(I)cc1</smiles>

2a, $0.1 \mathrm{mmol}$
$\mathrm{Ni}($ dppe $) \mathrm{Cl}_{2}(5 \mathrm{~mol} \%)$ BINAP (5 mol \%)

Zn (2.2 equiv)

Lil ( 2 equiv)

$\operatorname{THF}(1 \mathrm{~mL}), 100^{\circ} \mathrm{C}, 0-12 \mathrm{~h}$

by ${ }^{1} \mathrm{H}$ NMR analysis

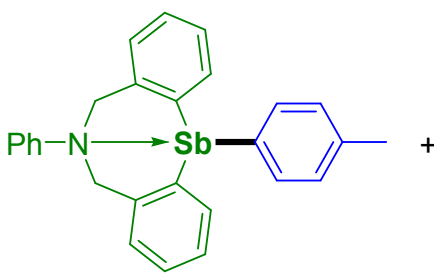

$3 a$

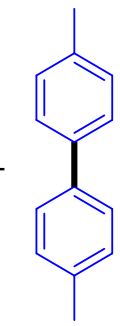

$4 a$

\begin{tabular}{llllllllll}
\hline Time (h) & 0 & 0.5 & 1 & 2 & 4 & 6 & 8 & 10 & 12 \\
\hline Yield of 3a (\%) & 0 & 25 & 40 & 55 & 72 & 82 & 90 & 93 & 94 \\
Yield of $\mathbf{4 a}(\%)^{a}$ & 0 & 0 & 0 & 0 & 0.5 & 1 & 2 & 2 & 3 \\
Surplus of 2a $(\%)^{a}$ & 100 & 75 & 60 & 45 & 27 & 16 & 6 & 2 & 0
\end{tabular}

Reaction conditions: 1a $(0.10 \mathrm{mmol}), \mathbf{2 a}(0.10 \mathrm{mmol}), \mathrm{Ni}(\mathrm{dppe}) \mathrm{Cl}_{2}(0.005 \mathrm{mmol}), \mathrm{BINAP}(0.005 \mathrm{mmol})$, Zn $(0.20 \mathrm{mmol})$, LiI $(0.20 \mathrm{mmol})$, THF $(1.0 \mathrm{~mL})$ at $100{ }^{\circ} \mathrm{C}$ for $0-12 \mathrm{~h} .{ }^{a}$ Yields were determined by ${ }^{1} \mathrm{H}$ NMR analysis using mesitylene as an internal standard.

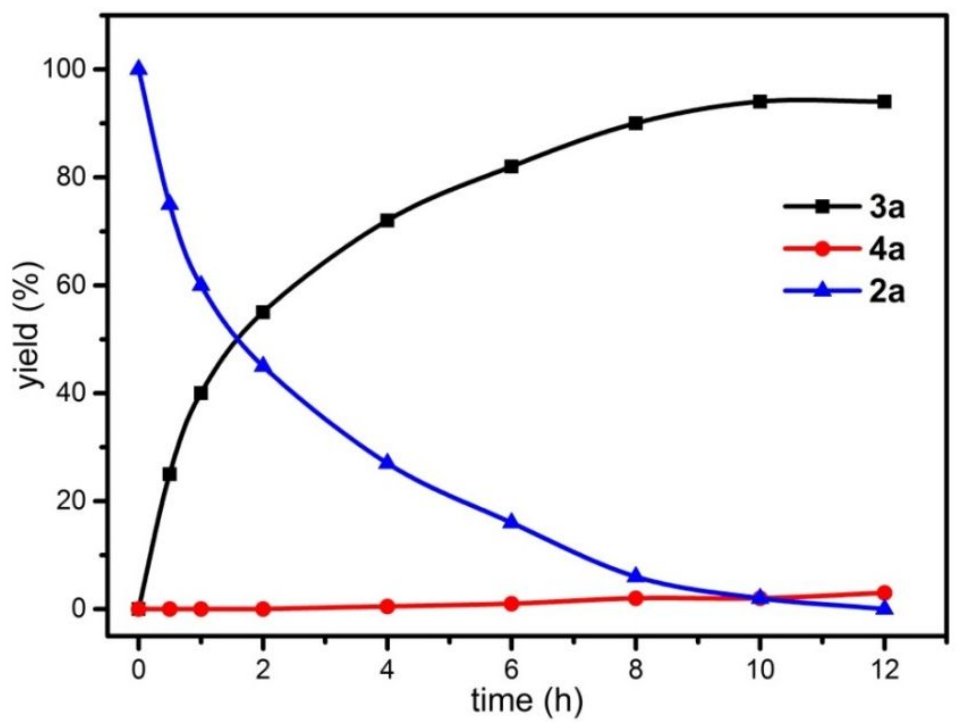

Figure S4. Time-course plots of reductive cross-coupling of chlorostibine 1a with 1-iodo-4methylbenzene 2a

Procedure: Under a nitrogen atmosphere, a $10 \mathrm{~mL}$ Schlenk tube equipped with a magnetic stir bar was charged successively with chlorostibine $1 \mathbf{a}(42.7 \mathrm{mg}, 0.1 \mathrm{mmol}), 1$-iodo-4-methylbenzene $2 \mathrm{a}(21.8 \mathrm{mg}$, $0.1 \mathrm{mmol}), \mathrm{Ni}(\mathrm{dppe}) \mathrm{Cl}_{2}(3.5 \mathrm{mg}, 0.005 \mathrm{mmol}, 5.0 \mathrm{~mol} \%$ ), 1, 1'-binaphthyl-2, 2'-diphemyl phosphine (BINAP) (3.1 mg, $0.005 \mathrm{mmol}, 5.0 \mathrm{~mol} \%$ ), LiI (26.8 mg, $0.2 \mathrm{mmol}, 2.0$ equiv), zinc powder (13.0 mg, 
$0.2 \mathrm{mmol}, 2.0$ equiv) and $1.0 \mathrm{~mL}$ of THF. The reaction mixture was stirred at $100{ }^{\circ} \mathrm{C}$ for $0-12 \mathrm{~h}$. The resulting mixture was diluted with $2.0 \mathrm{~mL}$ of $\mathrm{CH}_{2} \mathrm{Cl}_{2}$, filtrated, washed with $1.0 \mathrm{~N} \mathrm{HCl}$ solution $(3.0 \mathrm{~mL}$ x 3), dried over anhydrous $\mathrm{Na}_{2} \mathrm{SO}_{4}$. The solvent was removed by rotary evaporation under reduced pressure, and then $0.6 \mathrm{~mL}$ of $\mathrm{CDCl}_{3}$ and $12.0 \mathrm{mg}$ of mesitylene were added. The yields of products were determined by ${ }^{1} \mathrm{H}$ NMR analysis.

S4.1.2 The Effect of Concentration of Substrates on Reductive Cross-Coupling Reaction of Chlorostibines with Aryl Iodides

Table S15. Investigation on the Effect of Concentration of Substrates on Reductive Cross-Coupling of Chlorostibine 1a with 1-Iodo-4-Methylbenzene 2a<smiles>Cl[Sn]1(Cl)c2ccccc2CN(c2ccccc2)Cc2ccccc21</smiles>

1a, $0.1 \mathrm{mmol}$<smiles>Cc1ccc(I)cc1</smiles>

2a, $0.1 \mathrm{mmol}$
$\mathrm{Ni}($ dppe $) \mathrm{Cl}_{2}$ (5 mol \%) BINAP (5 mol \%)

$\mathrm{Zn}$ (2.2 equiv) Lil ( 2 equiv) THF (1 mL), $100^{\circ} \mathrm{C}, 20 \mathrm{~min}$ by ${ }^{1} \mathrm{H}$ NMR analysis

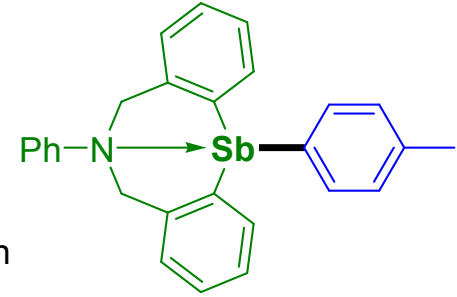

$3 a$

\begin{tabular}{llll}
\hline Entry & $\mathbf{1 a}(\mathrm{mmol})$ & $\mathbf{2 a}(\mathrm{mmol})$ & ${\text { Yield of } \mathbf{3 a}(\%)^{a}}^{a}$ \\
\hline 1 & 0.1 & 0.1 & 12 \\
2 & 0.2 & 0.1 & 33 \\
3 & 0.3 & 0.1 & 47 \\
4 & 0.1 & 0.2 & 13 \\
5 & 0.1 & 0.3 & 14 \\
\hline
\end{tabular}

Note: When changing the amount of 1a (from $0.1 \mathrm{mmol}$ to $0.3 \mathrm{mmol}$ ), the amount of $2 \mathrm{a}$ remains unchanged $(0.1 \mathrm{mmol})$; when changing the amount of $\mathbf{2 a}$ (from $0.1 \mathrm{mmol}$ to $0.3 \mathrm{mmol}$ ), the amount of 1a remains unchanged $(0.1 \mathrm{mmol})$. Reaction conditions 1 : 1a $(0.1-0.3 \mathrm{mmol}), 2 \mathrm{a}(0.1 \mathrm{mmol})$, $\mathrm{Ni}(d p p e) \mathrm{Cl}_{2}$ (0.005 mmol), BINAP (0.005 mmol), Zn (0.2 mmol), LiI (0.2 mmol), THF (1.0 mL), at 100 ${ }^{\circ} \mathrm{C}$ for $20 \mathrm{~min}$. Reaction conditions 2: 2a $(0.1-0.3 \mathrm{mmol}), \mathbf{1 a}(0.1 \mathrm{mmol}), \mathrm{Ni}(\mathrm{dppe}) \mathrm{Cl}_{2}(0.005 \mathrm{mmol})$, BINAP (0.005 mmol), Zn (0.2 mmol), LiI $(0.2 \mathrm{mmol})$, THF $(1.0 \mathrm{~mL})$, at $100^{\circ} \mathrm{C}$ for $20 \mathrm{~min} .{ }^{a}$ Yields were determined by ${ }^{1} \mathrm{H}$ NMR analysis using mesitylene as internal standard. 

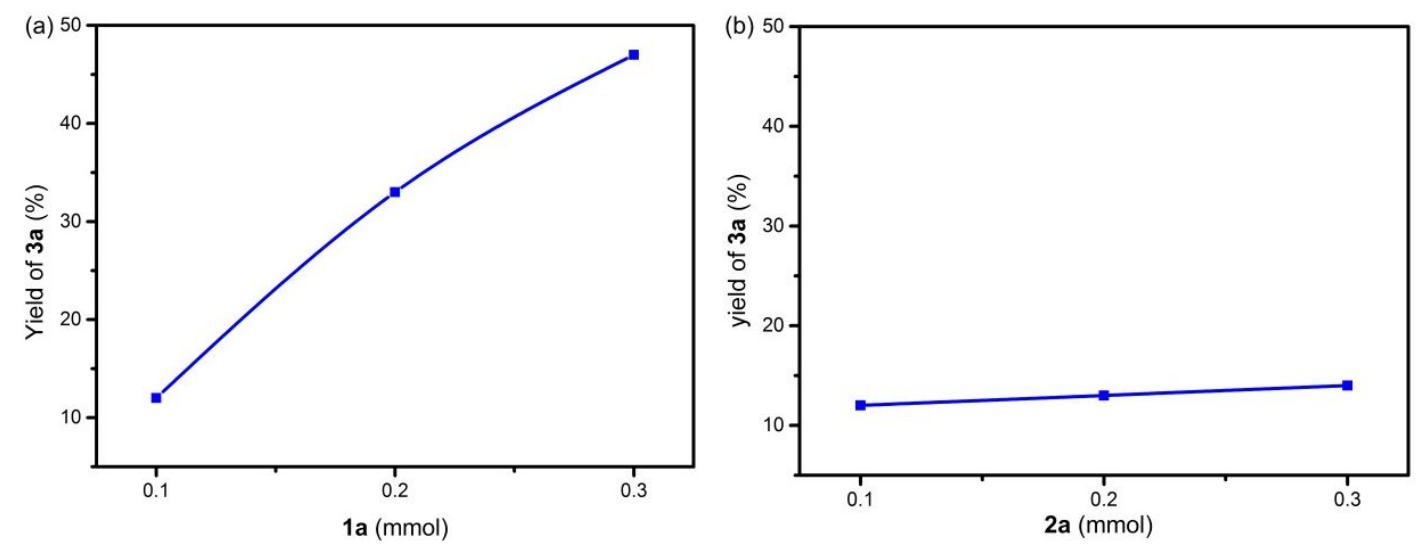

Figure S5. (a) The effect of concentration of 1a on the reductive cross-coupling of chlorostibine 1a with 1-iodo-4-methylbenzene 2a. Reaction conditions: $\mathbf{1 a}(0.1-0.3 \mathrm{mmol}), \mathbf{2 a}(0.1 \mathrm{mmol})$ remains unchanged, $\mathrm{Ni}(\mathrm{dppe}) \mathrm{Cl}_{2}(0.005 \mathrm{mmol}), \mathrm{BINAP}$ (0.005 mmol), Zn (0.2 mmol), LiI (0.2 mmol), THF (1.0 mL), at 100 ${ }^{\circ} \mathrm{C}$ for $20 \mathrm{~min}$. All yields were determined by ${ }^{1} \mathrm{H}$ NMR analysis using mesitylene as internal standard. (b) The effect of concentration of 1-iodo-4-methylbenzene $\mathbf{2 a}$ on the reductive cross-coupling of chlorostibine 1a with 1-iodo-4-methylbenzene 2a. Reaction conditions: 2a (0.1 - 0.3 mmol), 1a (0.1 mmol) remains unchanged, $\mathrm{Ni}(\mathrm{dppe}) \mathrm{Cl}_{2}(0.005 \mathrm{mmol}), \mathrm{BINAP}(0.005 \mathrm{mmol}), \mathrm{Zn}(0.2 \mathrm{mmol})$, LiI (0.2 $\mathrm{mmol})$, THF $(1.0 \mathrm{~mL})$, run at $100{ }^{\circ} \mathrm{C}$ for $20 \mathrm{~min}$. All yields were determined by ${ }^{1} \mathrm{H} \mathrm{NMR}$ analysis using mesitylene as internal standard.

Procedure (a): Under a nitrogen atmosphere, a $10 \mathrm{~mL}$ Schlenk tube equipped with a magnetic stir bar was charged successively with chlorostibine 1a $(42.7$ - $128.1 \mathrm{mg}, 0.1-0.3 \mathrm{mmol})$, 1-iodo-4methylbenzene $2 \mathbf{a}(21.8 \mathrm{mg}, 0.1 \mathrm{mmol}), \mathrm{Ni}(\mathrm{dppe}) \mathrm{Cl}_{2}(2.9 \mathrm{mg}, 0.005 \mathrm{mmol}, 5.0 \mathrm{~mol} \%)$, BINAP (3.1 mg, $0.005 \mathrm{mmol}, 5.0 \mathrm{~mol} \%$ ), LiI (26.8 mg, $0.2 \mathrm{mmol}, 2.0$ equiv), zinc powder (13 mg, $0.2 \mathrm{mmol}, 2.0$ equiv) and $1.0 \mathrm{~mL}$ of THF. The reaction mixture was stirred at $100{ }^{\circ} \mathrm{C}$ for $20 \mathrm{~min}$. The resulting mixture was diluted with $2.0 \mathrm{~mL}$ of $\mathrm{CH}_{2} \mathrm{Cl}_{2}$, filtrated, washed with $1 \mathrm{~N} \mathrm{HCl}$ solution $(3.0 \mathrm{~mL} \times 3)$, and then washed with $3.0 \mathrm{~mL}$ of deionized water. The organic layer was dried over anhydrous $\mathrm{Na}_{2} \mathrm{SO}_{4}$, concentrated under reduced pressure, and then $0.6 \mathrm{~mL}$ of $\mathrm{CDCl}_{3}$ and $12.0 \mathrm{mg}$ of mesitylene were added. The yields of products were determined by ${ }^{1} \mathrm{H}$ NMR analysis using mesitylene as an internal standard.

Procedure (b): Under a nitrogen atmosphere, a $10 \mathrm{~mL}$ Schlenk tube equipped with a magnetic stir bar was charged successively with chlorostibine $\mathbf{1 a}(42.7 \mathrm{mg}, 0.1 \mathrm{mmol}), 1$-iodo-4-methylbenzene $2 \mathbf{a}(21.8$ - $65.4 \mathrm{mg}, 0.1$ - $0.3 \mathrm{mmol}$ ), Ni(dppe)Cl 2 (2.9 mg, $0.005 \mathrm{mmol}, 5.0 \mathrm{~mol} \%$ ), BINAP (3.1 mg, $0.005 \mathrm{mmol}$, $5.0 \mathrm{~mol} \%$ ), LiI (26.8 mg, $0.2 \mathrm{mmol}, 2.0$ equiv), zinc powder (13.0 mg, $0.2 \mathrm{mmol}, 2.0$ equiv) and $1.0 \mathrm{~mL}$ of THF. The reaction mixture was stirred at $100{ }^{\circ} \mathrm{C}$ for $20 \mathrm{~min}$. The resulting mixture was diluted with $2.0 \mathrm{~mL}$ of $\mathrm{CH}_{2} \mathrm{Cl}_{2}$, filtrated, washed with $1 \mathrm{~N} \mathrm{HCl}$ solution $(3.0 \mathrm{~mL} \times 3)$, and then washed with $3.0 \mathrm{~mL}$ of deionized water. The organic layer was dried over anhydrous $\mathrm{Na}_{2} \mathrm{SO}_{4}$, concentrated under reduced pressure, and then $0.6 \mathrm{~mL}$ of $\mathrm{CDCl}_{3}$ and $12.0 \mathrm{mg}$ of mesitylene were added. The yields of products were determined by ${ }^{1} \mathrm{H}$ NMR analysis using mesitylene as an internal standard. 


\section{S4.1.3 Control Experiments}

Scheme S4. Control Experiments for the Nickel-Catalyzed Reductive Coupling of Chlorostibines with Organic Halides

(a) Competitive reaction of aryl iodides

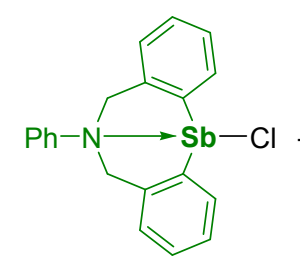

1a, $0.10 \mathrm{mmol}$
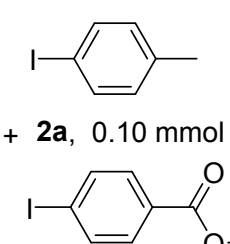

$\mathrm{Ni}(\mathrm{dppe}) \mathrm{Cl}_{2}(5.0 \mathrm{~mol} \%)$ BINAP $(5.0 \mathrm{~mol} \%)$ Zn (2.0 equiv)

Lil (2.0 equiv) THF, $100^{\circ} \mathrm{C}, 12 \mathrm{~h}$ by ${ }^{1} \mathrm{H}$ NMR analysis
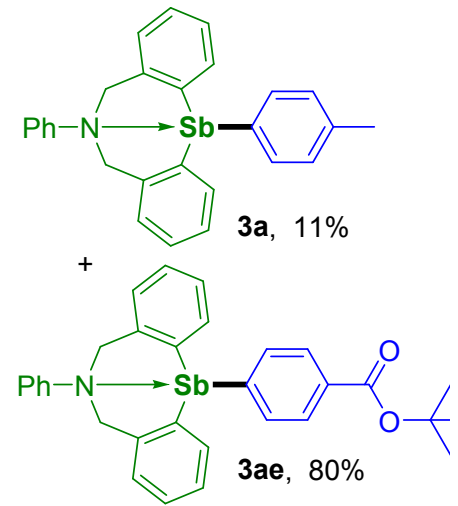

(b) Competitive reaction of stibines

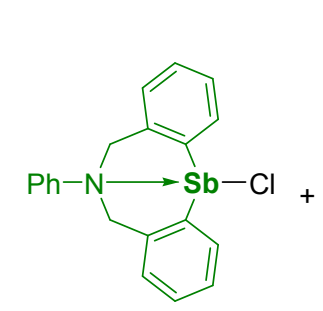

1a, $0.10 \mathrm{mmol}$

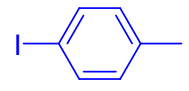

2a, $0.10 \mathrm{mmol}$

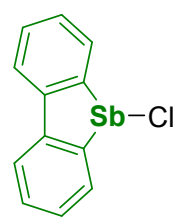

1b, $0.10 \mathrm{mmol}$

$\mathbf{3 a}: \mathbf{3 a e}=11: 80$

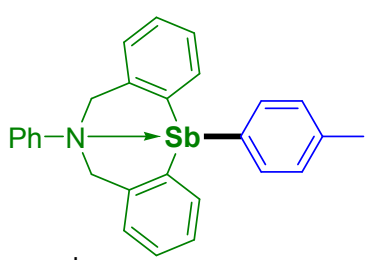

BINAP $(5.0 \mathrm{~mol} \%)$

$\mathrm{Zn}$ (2.0 equiv)

Lil ( 2.0 equiv) THF, $100{ }^{\circ} \mathrm{C}, 12 \mathrm{~h}$ by ${ }^{1} \mathrm{H}$ NMR analysis

3a $: \mathbf{3 b d}=12 \%: 86 \%=6: 43$

1a, $0.10 \mathrm{mmol}$

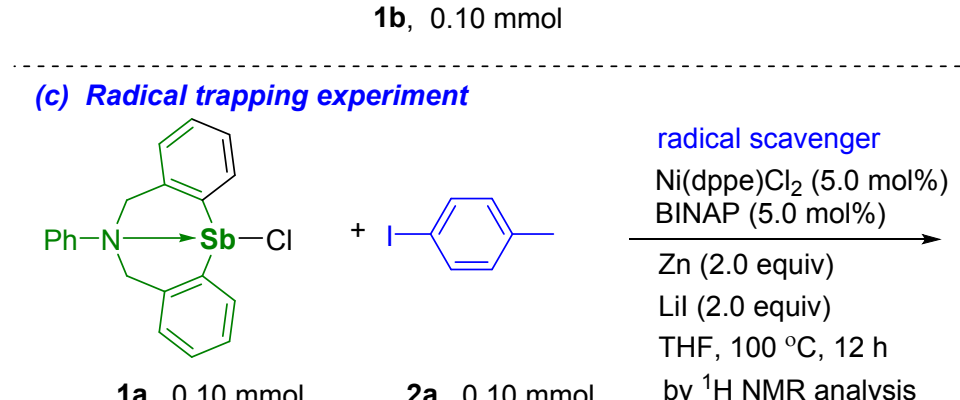

2a, $0.10 \mathrm{mmo}$

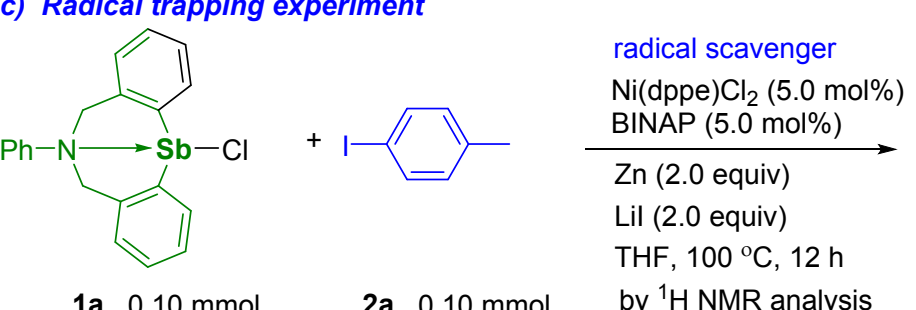

by ${ }^{1} \mathrm{H}$ NMR analysis

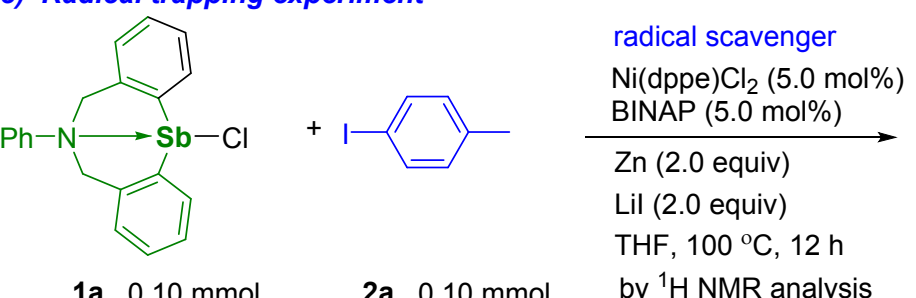

3a, $12 \%$

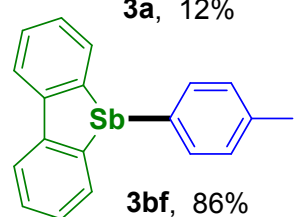

(c) Radical trapping experiment

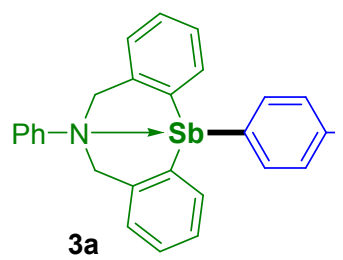

standard conditions: $90 \%$ TEMPO (2.0 equiv): $65 \%$

Procedure for Scheme S4a: Under a nitrogen atmosphere, a $10 \mathrm{~mL}$ Schlenk tube equipped with a magnetic stir bar was charged successively with chlorostibine 1a (42.7 mg, $0.1 \mathrm{mmol})$, 1-iodo-4methylbenzene 2a (21.8 mg, $0.1 \mathrm{mmol})$, tert-butyl 4-iodobenzoate 2au (30.4 mg, $0.1 \mathrm{mmol}), \mathrm{Ni}(\mathrm{dppe}) \mathrm{Cl}_{2}$ (2.9 mg, $0.005 \mathrm{mmol}, 5.0 \mathrm{~mol} \%$ ), BINAP (3.1 mg, $0.005 \mathrm{mmol}, 5.0 \mathrm{~mol} \%$ ), LiI (26.8 mg, $0.2 \mathrm{mmol}, 2.0$ equiv), zinc powder (13.0 mg, $0.2 \mathrm{mmol}, 2.0$ equiv) and $1.0 \mathrm{~mL}$ of THF. The reaction mixture was stirred at $100{ }^{\circ} \mathrm{C}$ for $12 \mathrm{~h}$. The resulting mixture was diluted with $2.0 \mathrm{~mL}$ of $\mathrm{CH}_{2} \mathrm{Cl}_{2}$, filtrated, washed with $1 \mathrm{~N}$ $\mathrm{HCl}$ solution $(3.0 \mathrm{~mL} \times 3)$, and then washed with $3.0 \mathrm{~mL}$ of deionized water. The organic layer was dried over anhydrous $\mathrm{Na}_{2} \mathrm{SO}_{4}$, concentrated under reduced pressure, and then $0.6 \mathrm{~mL}$ of $\mathrm{CDCl}_{3}$ and $7.0 \mu \mathrm{L}$ of $\mathrm{CH}_{2} \mathrm{Br}_{2}$ were added. The ratio of 3a:3ae was determined by ${ }^{1} \mathrm{H}$ NMR analysis. Ratio of 3a:3ae is 11:80. Procedure for Scheme S4b: Under a nitrogen atmosphere, a $10 \mathrm{~mL}$ Schlenk tube equipped with a magnetic stir bar was charged successively with chlorostibine 1a (42.7 mg, $0.1 \mathrm{mmol})$, chlorostibine 1b 
(30.7 mg, $0.1 \mathrm{mmol})$, 1-iodo-4-methylbenzene $2 \mathrm{a}(21.8 \mathrm{mg}, 0.1 \mathrm{mmol}), \mathrm{Ni}(\mathrm{dppe}) \mathrm{Cl}_{2}(2.9 \mathrm{mg}, 0.005$ mmol, $5.0 \mathrm{~mol} \%$ ), BINAP ( $3.1 \mathrm{mg}, 0.005 \mathrm{mmol}, 5 \mathrm{~mol} \%)$, Lil (26.8 mg, $0.2 \mathrm{mmol}, 2.0$ equiv), zinc powder $\left(13.0 \mathrm{mg}, 0.2 \mathrm{mmol}, 2.0\right.$ equiv) and $1.0 \mathrm{~mL}$ of THF. The reaction mixture was stirred at $100{ }^{\circ} \mathrm{C}$ for $12 \mathrm{~h}$. The resulting mixture was diluted with $2.0 \mathrm{~mL}$ of $\mathrm{CH}_{2} \mathrm{Cl}_{2}$, filtrated, washed with $1 \mathrm{~N} \mathrm{HCl}$ solution $(3.0 \mathrm{~mL} \times 3)$, and then washed with $3.0 \mathrm{~mL}$ of deionized water. The organic layer was dried over anhydrous $\mathrm{Na}_{2} \mathrm{SO}_{4}$, concentrated under reduced pressure, and then $0.6 \mathrm{~mL}$ of $\mathrm{CDCl}_{3}$ and $7.0 \mu \mathrm{L}$ of $\mathrm{CH}_{2} \mathrm{Br}_{2}$ were added. The ratio of 3a:3bf was determined by ${ }^{1} \mathrm{H}$ NMR analysis. Ratio of $\mathbf{3 a} \mathbf{a} \mathbf{3 b f}$ is $6: 43$.

Procedure for Scheme S4c: Under a nitrogen atmosphere, a $10 \mathrm{~mL}$ Schlenk tube equipped with a magnetic stir bar was charged successively with chlorostibine $1 \mathbf{a}(42.7 \mathrm{mg}, 0.1 \mathrm{mmol}, 1.0$ equiv), 2a (21.8 mg, $0.1 \mathrm{mmol}, 1.0$ equiv), Ni(dppe)Cl 2 (2.9 mg, $0.005 \mathrm{mmol}, 5.0 \mathrm{~mol} \%$ ), BINAP (3.1 mg, 0.005 mmol, $5.0 \mathrm{~mol} \%$ ), LiI (26.8 mg, $0.2 \mathrm{mmol}, 2.0$ equiv), zinc powder (13.0 mg, $0.2 \mathrm{mmol}, 2.0$ equiv), 2,2,6,6-tetramethylpiperidinooxy (TEMPO) $(31.3 \mathrm{mg}, 0.2 \mathrm{mmol}, 2.0$ equiv) and $1.0 \mathrm{~mL}$ of THF. The reaction mixture was stirred at $100^{\circ} \mathrm{C}$ for $12 \mathrm{~h}$. The resulting mixture was diluted with $2.0 \mathrm{~mL}$ of $\mathrm{CH}_{2} \mathrm{Cl}_{2}$, filtrated, washed with $1 \mathrm{~N} \mathrm{HCl}$ solution $(3.0 \mathrm{~mL} \times 3)$, and then washed with $3.0 \mathrm{~mL}$ of deionized water. The organic layer was dried over anhydrous $\mathrm{Na}_{2} \mathrm{SO}_{4}$, concentrated under reduced pressure, and then 0.6 $\mathrm{mL}$ of $\mathrm{CDCl}_{3}$ and $12.0 \mathrm{mg}$ of mesitylene were added. The yield of the product $3 \mathbf{a}$ was determined by ${ }^{1} \mathrm{H}$ NMR analysis. (Addition of TEMPO: 65\% yield; under standard conditions: 90\% yield).

\section{Scheme S5. Reactions of Sb-Sb Stibines with Organic Halides}


(a) Isolation, structure determination and reactions of intermediate $1 \mathrm{e}$

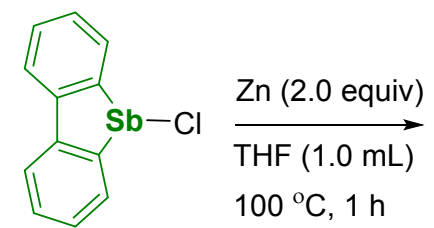

1b, $0.1 \mathrm{mmol}$

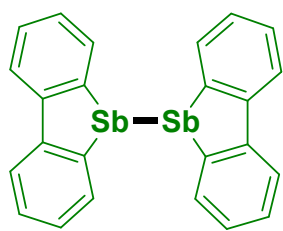

1 e, $80 \%$

HRMS $[\mathrm{M}+\mathrm{H}]^{+}$: calcd 546.9401 , found 546.9403

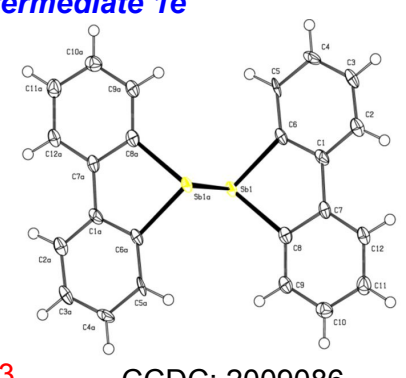

CCDC: 2009086<smiles>c1ccc2c(c1)[Se][Se](=[Se]1c3ccccc3-c3ccccc31)c1ccccc1-2</smiles>

1e, $0.05 \mathrm{mmol}$

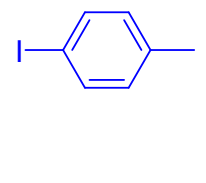

2a, $0.1 \mathrm{mmol}$
$\mathrm{Ni}(\mathrm{dppe}) \mathrm{Cl}_{2}(5.0 \mathrm{~mol} \%)$ BINAP $(5.0 \mathrm{~mol} \%)$

$\mathrm{Zn}$ (2.0 equiv)

Lil (2.0 equiv), $12 \mathrm{~h}$ THF $(1.0 \mathrm{~mL}), 100^{\circ} \mathrm{C}, \mathrm{N}_{2}$ by $\mathrm{GC}$ analysis

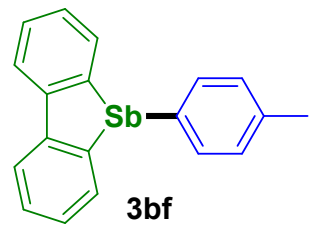

standard condition, $99 \%$

(b) Isolation and reactions of intermediate $1 f$<smiles></smiles>

1a, $0.1 \mathrm{mmol}$<smiles>c1ccc(N2Cc3ccccc3CN(Cc3ccccc3O[Sb](c3ccccc3)c3ccccc3)C2)cc1</smiles>

1f, possible structure<smiles>Cc1cccc(I)c1</smiles>
$2 \mathrm{e}, 0.1 \mathrm{mmol}$ $\underset{\mathrm{Ni}(\mathrm{dppe}) \mathrm{Cl}_{2}(5.0 \mathrm{~mol} \%)}{\mathrm{BINAP}(5.0 \mathrm{~mol} \%)} \quad 3 \mathrm{c}$

Lil (2.0 equiv) $100{ }^{\circ} \mathrm{C}, 12 \mathrm{~h}$ by ${ }^{1} \mathrm{H}$ NMR analysis

HRMS $[\mathrm{M}+\mathrm{H}]^{+}$: calcd 785.0871 , found 785.0876<smiles>Cc1cc(C)cc(N2Cc3ccccc3[Sn]3(Cl)c4ccccc4C23CSc2ccccc2)c1</smiles>

$0.05 \mathrm{mmol}$ 1c, $0.1 \mathrm{mmol}$

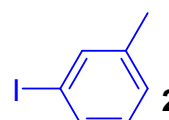

$2 \mathrm{e}, 0.1 \mathrm{mmol}$ $\mathrm{Ni}$ (dppe) $\mathrm{Cl}_{2}$ (5.0 mol\%) BINAP (5.0 mol\%)

Zn (2.0 equiv)

Lil (2.0 equiv) THF, $100{ }^{\circ} \mathrm{C}, 12 \mathrm{~h}$ by ${ }^{1} \mathrm{H}$ NMR analysis $\quad 4 \%$

Procedure for Scheme S5a (1): Under a nitrogen atmosphere, a $10 \mathrm{~mL}$ Schlenk tube equipped with a magnetic stir bar was charged successively with chlorostibine $1 \mathbf{b}$ (30.7 mg, $0.1 \mathrm{mmol})$, zinc powder (13.0 $\mathrm{mg}, 0.2 \mathrm{mmol}, 2.0$ equiv) and $1.0 \mathrm{~mL}$ of THF. The reaction mixture was stirred at $100{ }^{\circ} \mathrm{C}$ for $1 \mathrm{~h}$. The resulting mixture was left at room temperature for $2 \mathrm{~h}$. The yellow solid was largely precipitated, and then filtered to obtain distibine 1e in $80 \%$ yield. M.P.: $263.2-264.7{ }^{\circ} \mathrm{C} .{ }^{1} \mathrm{H}$ NMR $\left(400 \mathrm{MHz}, \mathrm{C}_{6} \mathrm{D}_{6}\right) \delta$ 7.52 (d, $J=7.4 \mathrm{~Hz}, 3 \mathrm{H}), 7.44$ (d, $J=7.6 \mathrm{~Hz}, 2 \mathrm{H}), 7.38-7.24(\mathrm{~m}, 4 \mathrm{H}), 7.08$ (d, $J=7.2 \mathrm{~Hz}, 4 \mathrm{H}), 7.01-$ $6.85(\mathrm{~m}, 3 \mathrm{H}) .{ }^{13} \mathrm{C}$ NMR $\left(101 \mathrm{MHz}, \mathrm{C}_{6} \mathrm{D}_{6}\right) \delta 153.0,151.1,135.3,130.1,127.9,123.2$. HRMS-ESI $(\mathrm{m} / \mathrm{z})$ $[\mathrm{M}+\mathrm{H}]^{+}$Calcd for $\mathrm{C}_{24} \mathrm{H}_{17} \mathrm{Sb}_{2}{ }^{+}, 546.9401$, Found, 546.9406. 


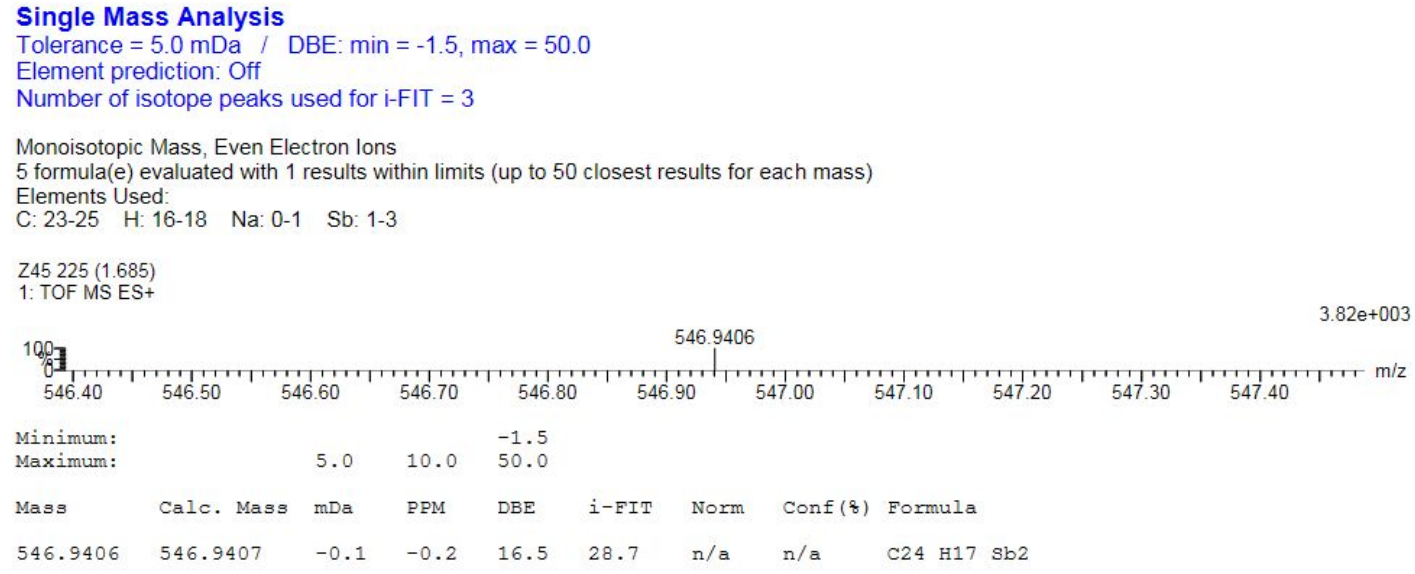

Figure S6. HRMS data of distibine 1e

Procedure for Scheme S5a (2): Under a nitrogen atmosphere, a $10 \mathrm{~mL}$ Schlenk tube equipped with a magnetic stir bar was charged successively with distibine 1e (27.3 mg, 0.05 mmol), 1-iodo-4methylbenzene $2 \mathrm{a}(21.8 \mathrm{mg}, 0.1 \mathrm{mmol}), \mathrm{Ni}(\mathrm{dppe}) \mathrm{Cl}_{2}(2.6 \mathrm{mg}, 0.005 \mathrm{mmol}, 5.0 \mathrm{~mol} \%)$, BINAP (3.1 mg, $0.005 \mathrm{mmol}, 5 \mathrm{~mol} \%$ ), LiI (26.8 mg, $0.2 \mathrm{mmol}, 2.0$ equiv), zinc powder (13.0 mg, $0.2 \mathrm{mmol}, 2.0$ equiv) and $1.0 \mathrm{~mL}$ of THF. The reaction mixture was stirred at $100{ }^{\circ} \mathrm{C}$ for $12 \mathrm{~h}$. The resulting mixture was diluted with $2.0 \mathrm{~mL}$ of $\mathrm{CH}_{2} \mathrm{Cl}_{2}$, filtrated, washed with $1 \mathrm{~N} \mathrm{HCl}$ solution $(3.0 \mathrm{~mL} \times 3)$, and then washed with $3.0 \mathrm{~mL}$ of deionized water. The organic layer was dried over anhydrous $\mathrm{Na}_{2} \mathrm{SO}_{4}$, and then $30 \mu \mathrm{L}$ of $n$-dodecane was added. Cross-coupling product $\mathbf{3 b f}$ was obtained in $99 \%$ yield.

Procedure for Scheme S5b (1): Under a nitrogen atmosphere, a $10 \mathrm{~mL}$ Schlenk tube equipped with a magnetic stir bar was charged successively with chlorostibine $1 \mathrm{a}(42.7 \mathrm{mg}, 0.1 \mathrm{mmol})$, zinc powder (13.0 $\mathrm{mg}, 0.2 \mathrm{mmol}, 2.0$ equiv) and $1.0 \mathrm{~mL}$ of THF. The reaction mixture was stirred at $100{ }^{\circ} \mathrm{C}$ for $1 \mathrm{~h}$. 1 -iodo3-methylbenzene 2e (21.8 mg, $0.1 \mathrm{mmol})$, Ni(dppe)Cl 2 (2.9 mg, $0.005 \mathrm{mmol}, 5.0 \mathrm{~mol} \%$ ), BINAP (3.1 $\mathrm{mg}, 0.005 \mathrm{mmol}, 5.0 \mathrm{~mol} \%$ ) and LiI (26.8 $\mathrm{mg}, 0.2 \mathrm{mmol}, 2.0$ equiv) were added into the tube. The reaction mixture continued to be stirred at $100{ }^{\circ} \mathrm{C}$ for $12 \mathrm{~h}$. The resulting mixture was diluted with 2.0 $\mathrm{mL}$ of $\mathrm{CH}_{2} \mathrm{Cl}_{2}$, filtrated, washed with $1 \mathrm{~N} \mathrm{HCl}$ solution $(3.0 \mathrm{~mL} \times 3)$, and then washed with $3.0 \mathrm{~mL}$ of deionized water. The organic layer was dried over anhydrous $\mathrm{Na}_{2} \mathrm{SO}_{4}$, concentrated under reduced pressure, and then $0.6 \mathrm{~mL}$ of $\mathrm{CDCl}_{3}$ and $12.0 \mathrm{mg}$ of mesitylene were added. The yield of 3c was determined by ${ }^{1} \mathrm{H}$ NMR analysis. The yield of $\mathbf{3 c}$ was $23 \%$. Distibine $\mathbf{1 f}$ was analyzed by HRMS analysis. HRMS-ESI (m/z) [M+Na $]^{+}$Calcd for $\mathrm{C}_{40} \mathrm{H}_{35} \mathrm{~N}_{2} \mathrm{Sb}_{2}{ }^{+}$, 785.0871; Found, 785.0876.

Note: (1) Compound 1 f was not dissolved in DMF- $d_{7}$, DMSO- $d_{6}$, THF- $d_{8}, \mathrm{CD}_{3} \mathrm{COD}, \mathrm{C}_{6} \mathrm{D}_{6}$, Toluene- $d_{8}$, $\mathrm{CD}_{3} \mathrm{CN}, \mathrm{CDCl}_{3}$; (2) Distibine 1f was sensitive to oxygen or moisture or air. Hence, NMR spectrum of distibine 1f could not obtain, and only HRMS data could provide. 


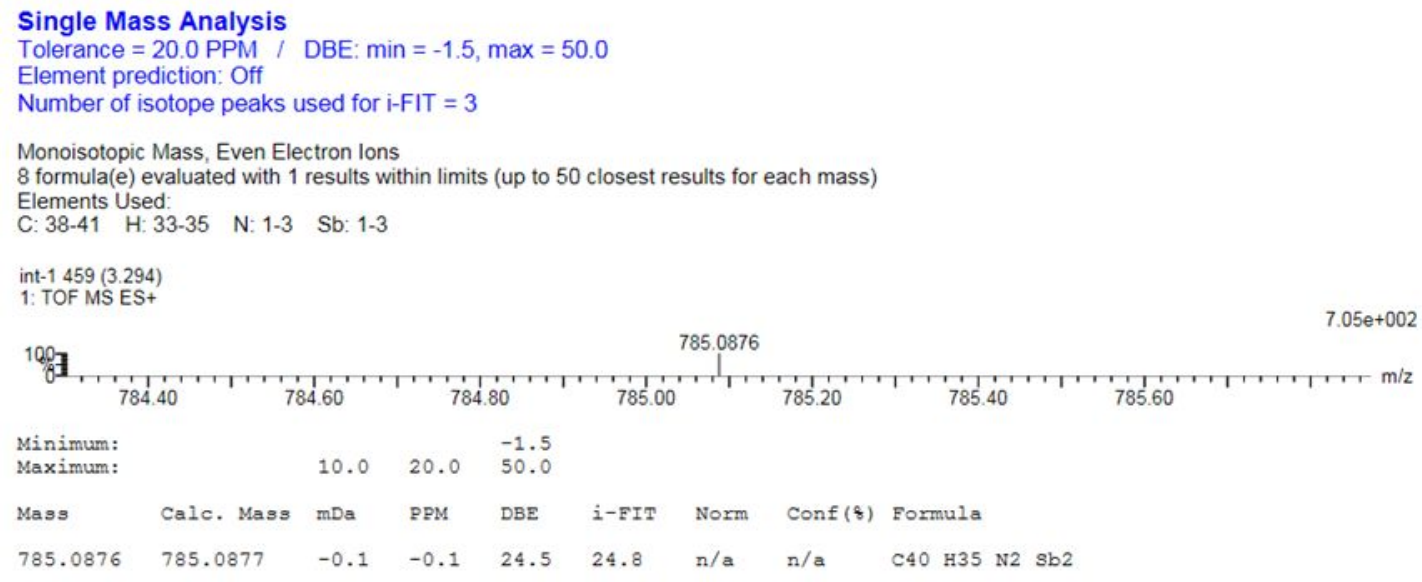

Figure S7. HRMS data of distibine 1f

Procedure for Scheme S5b (2): Under a nitrogen atmosphere, a $10 \mathrm{~mL}$ Schlenk tube equipped with a magnetic stir bar was charged successively with distibine $\mathbf{1 f}$ (39.2 $\mathrm{mg}, 0.05 \mathrm{mmol})$, chlorostibine $\mathbf{1 c}$ (45.5 mg, $0.1 \mathrm{mmol})$, 1-iodo-3-methylbenzene $2 \mathrm{e}$ (21.8 mg, $0.1 \mathrm{mmol}), \mathrm{Ni}(\mathrm{dppe}) \mathrm{Cl}_{2}$ (2.9 mg, $0.005 \mathrm{mmol}, 5.0$ mol\%), BINAP (3.1 mg, $0.005 \mathrm{mmol}, 5.0 \mathrm{~mol} \%$ ), LiI (26.8 mg, $0.2 \mathrm{mmol}, 2.0$ equiv), zinc powder (13.0 $\mathrm{mg}, 0.2 \mathrm{mmol}, 2.0$ equiv) and $1.0 \mathrm{~mL}$ of THF. The reaction mixture was stirred at $100{ }^{\circ} \mathrm{C}$ for $12 \mathrm{~h}$. The resulting mixture was diluted with $2.0 \mathrm{~mL}$ of $\mathrm{CH}_{2} \mathrm{Cl}_{2}$, filtrated, washed with $1 \mathrm{~N} \mathrm{HCl}$ solution $(3.0 \mathrm{~mL} \mathrm{x}$ 3), and then washed with $3.0 \mathrm{~mL}$ of deionized water. The organic layer was dried over anhydrous $\mathrm{Na}_{2} \mathrm{SO}_{4}$, concentrated under reduced pressure, and then $0.6 \mathrm{~mL}$ of $\mathrm{CDCl}_{3}$ and $17.4 \mathrm{mg}$ of $\mathrm{CH}_{2} \mathrm{Br}_{2}$ were added. The ratio of $\mathbf{3 c}: \mathbf{3 b a}$ were determined by ${ }^{1} \mathrm{H}$ NMR analysis $(\mathbf{3} \mathbf{c}: \mathbf{3 b a}=4 \%: 51 \%=4: 51)$.

\section{Scheme S6. Investigation on the Role of Zinc Powder}
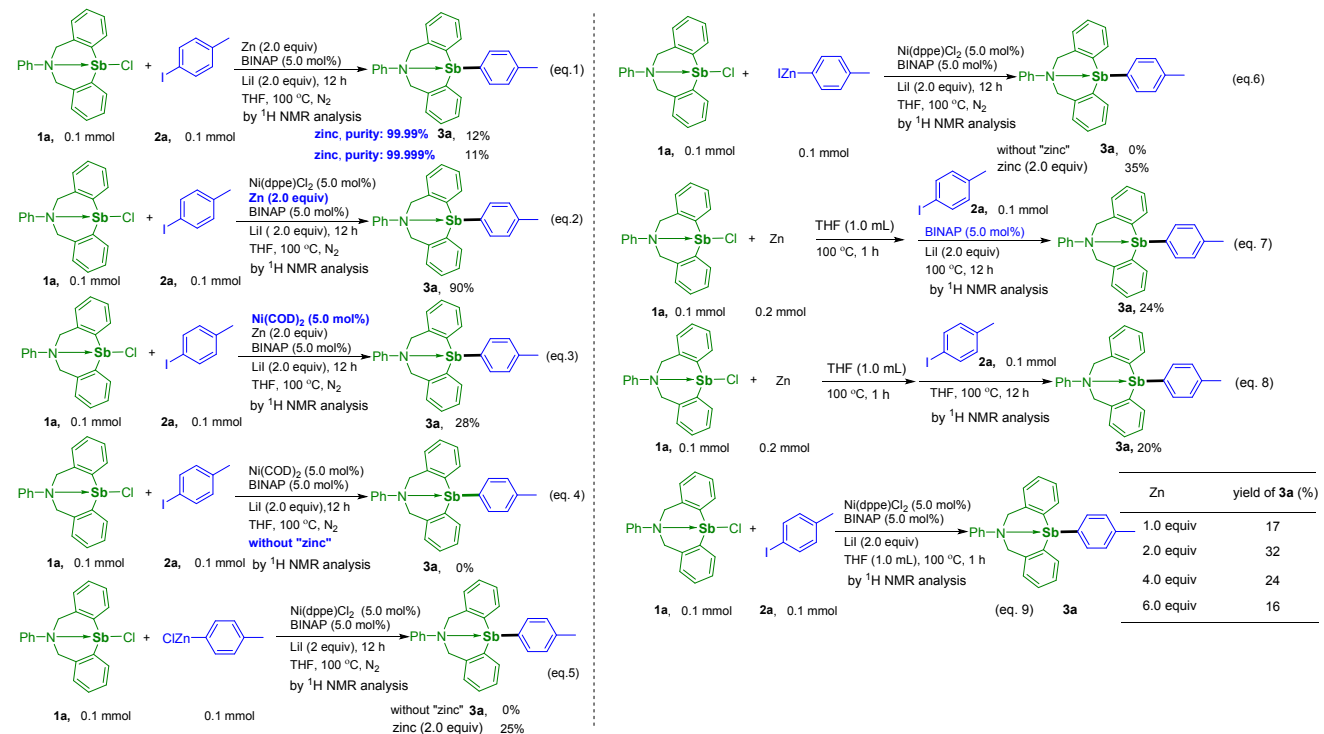

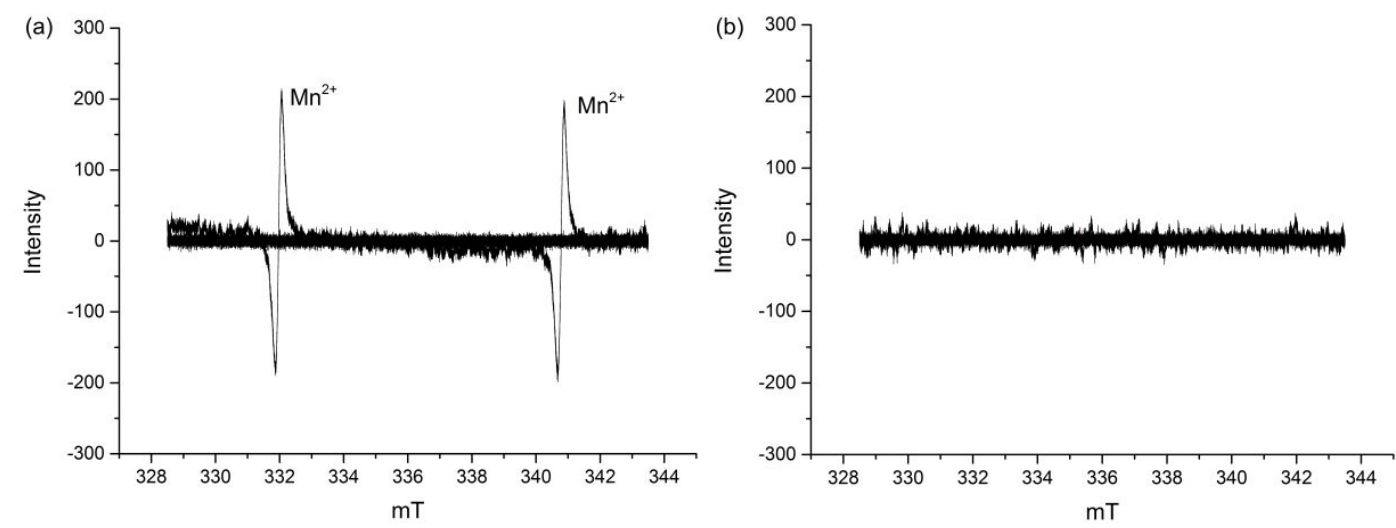

Figure S8. EPR test results at (a) $-20{ }^{\circ} \mathrm{C}, \mathrm{Mn}^{2+}$ as internal standard, and (B)-50 ${ }^{\circ} \mathrm{C}$

In order to verify the existence of $\mathrm{Ni}(\mathrm{I})$ intermediate in the system, the stoichiometric reaction between chlorostibine 1a and 1-iodo-4-methylbenzene was investigated by EPR spectroscopy. As shown in Figure $\mathrm{S} 8$, no signal peak of $\mathrm{Ni}(\mathrm{I})$ was detected at $-20{ }^{\circ} \mathrm{C}$ and $-50^{\circ} \mathrm{C}$. Ni(I) species could not be excluded $\mathrm{t}$ in this coupling.

Procedure for Figure S8: Under a nitrogen atmosphere, a $10 \mathrm{~mL}$ Schlenk tube equipped with a magnetic stir bar was charged successively with chlorostibine $1 \mathrm{a}(42.7 \mathrm{mg}, 0.1 \mathrm{mmol}), 1$-iodo-4-methylbenzene $2 \mathbf{a}(21.8 \mathrm{mg}, 0.1 \mathrm{mmol}), \mathrm{Ni}(\mathrm{dppe}) \mathrm{Cl}_{2}(52.8 \mathrm{mg}, 0.1 \mathrm{mmol}), \mathrm{LiI}(26.8 \mathrm{mg}, 0.2 \mathrm{mmol}), \mathrm{BINAP}(62.2 \mathrm{mg}$, $0.1 \mathrm{mmol})$, zinc powder $(13.0 \mathrm{mg}, 0.2 \mathrm{mmol})$, and $1.0 \mathrm{~mL}$ of THF. The reaction mixture was stirred at $100{ }^{\circ} \mathrm{C}$ for $6 \mathrm{~h}$. After $6 \mathrm{~h}$, the reaction mixture was concentrated under reduced pressure, and transferred into EPR tube. The sample was analyzed at $-20^{\circ} \mathrm{C}$ and $-50^{\circ} \mathrm{C}$ by EPR spectroscopy, respectively. 
(a)
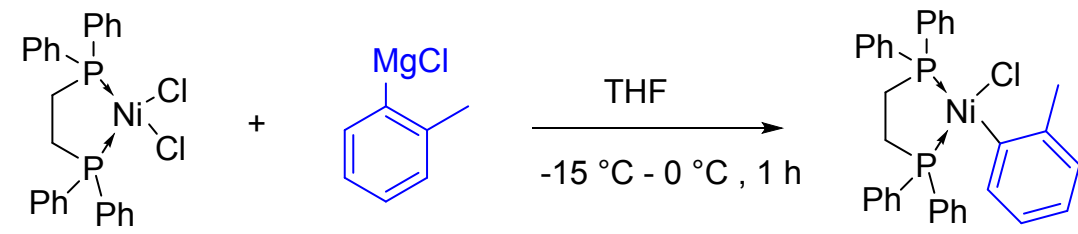

Ni(II)-1

(b)<smiles>Cc1ccccc1N1CP(c2ccccc2)(c2ccccc2)(c2ccccc2)CC1(Cl)P(c1ccccc1)c1ccccc1</smiles>

$\mathrm{Ni}(\mathrm{II})-1,0.1 \mathrm{mmol}$

1b , $0.1 \mathrm{mmol}$

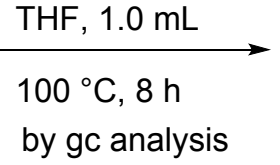

by gc analysis

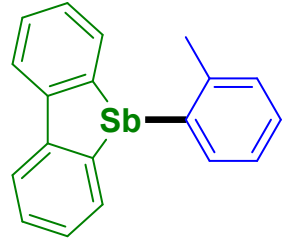

3bi, $98 \%$ yield

(c)<smiles>Cc1ccccc1N1[PH](c2ccccc2)(c2ccccc2)CC[PH]1(c1ccccc1)c1ccccc1</smiles>

$\mathrm{Ni}(\mathrm{II})-1,0.1 \mathrm{mmol}$<smiles>c1ccc2c(c1)-c1ccccc1[Sb]2[Sb]1c2ccccc2-c2ccccc21</smiles>

1e , $0.05 \mathrm{mmol}$

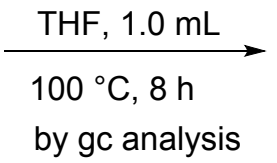

by gc analysis

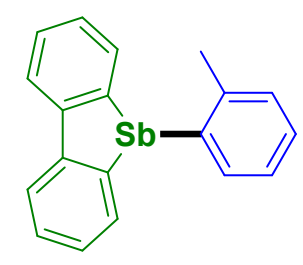

3bi, $76 \%$ yield with 2.0 equiv, $96 \%$ yield

(d)<smiles>Cc1ccccc1N1[P+](c2ccccc2)(P(Cl)c2ccccc2)CC[PH]1(Cl)c1ccccc1</smiles><smiles>Cl[Sb]1(Cl)c2ccccc2CN(c2ccccc2)Cc2ccccc21</smiles>

$\mathrm{Ni}(\mathrm{II})-1,0.1 \mathrm{mmol}$

1a, $0.1 \mathrm{mmol}$<smiles>[C+]1CCC1</smiles>
$100{ }^{\circ} \mathrm{C}, 8 \mathrm{~h}$ by gc analysis<smiles>Cc1ccccc1[Sb]1(c2ccccc2)CN2Cc3ccccc3[Sn]1(c1ccccc1)C2</smiles>

3d, $0 \%$ yield

(e)<smiles>Cc1ccccc1N1CCP(c2ccccc2)[N+]1(Cl)c1ccccc1</smiles>

$\mathrm{Ni}(\mathrm{II})-1,0.1 \mathrm{mmol}$<smiles>Cl[Sb]12Sc3ccccc3CN(Cc3ccccc31)c1ccccc12</smiles>

1a, $0.1 \mathrm{mmol}$
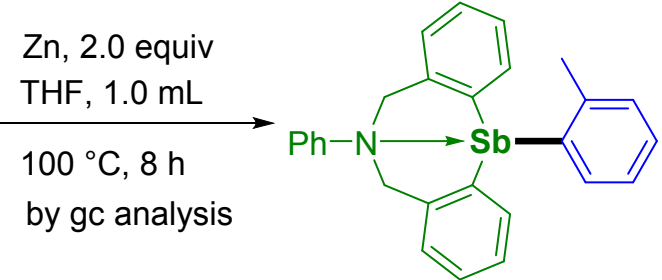

3d, $46 \%$ yield

Next, rigorous experiments were performed. The bivalent nickel compound Ni(II)-1 was synthesized according to the method reported in the literature. ${ }^{\text {S14 }}$

Procedure for Scheme S7a: Under a nitrogen atmosphere, $1.06 \mathrm{~g}$ of (dppe $)_{2} \mathrm{NiCl}_{2}$ and $100 \mathrm{~mL}$ of THF were added into $200 \mathrm{~mL}$ Schlenk tube with agitator, and the Schlenk tube was placed in a cooling bath of $-15{ }^{\circ} \mathrm{C}$ for 10 minutes. Then, $2 \mathrm{~mL}$ of 2 -methylmagnesium chloride $(1.0 \mathrm{~mol} / \mathrm{L})$ was slowly added to the reaction mixture by syringe. It was observed that the color of reaction solution changed from red to yellow, and reaction solution gradually changed into homogeneous solution. After completing the addition of the Grignard reagent, the solution was stirred for $15 \mathrm{~min}$ at $0{ }^{\circ} \mathrm{C}$. After which the stir bar was removed and the solution was concentrated under reduced pressure. $\mathrm{MeOH}(20 \mathrm{~mL})$ was added, and the 
mixture was sonicated until a uniform suspension was obtained. The suspension was cooled to $0{ }^{\circ} \mathrm{C}$ for 30 minutes, and much yellow precipitate appeared. The yellow solid was collected by vacuum filtration, washed with two portions of cold $\mathrm{MeOH}(5 \mathrm{~mL})$, and dried under high vacuum to yield Ni(II)-1 (582.1 $\mathrm{mg}$ ) as a yellow solid. ${ }^{1} \mathrm{H}$ NMR $(400 \mathrm{MHz}$, Chloroform- $d) \delta 8.21-8.13(\mathrm{~m}, 4 \mathrm{H}), 7.80-7.69(\mathrm{~m}, 2 \mathrm{H})$, $7.55-7.40(\mathrm{~m}, 9 \mathrm{H}), 7.33-7.28(\mathrm{~m}, 1 \mathrm{H}), 7.25-7.19(\mathrm{~m}, 1 \mathrm{H}), 7.06(\mathrm{t}, J=7.6 \mathrm{~Hz}, 2 \mathrm{H}), 6.70(\mathrm{t}, J=9.3$ $\mathrm{Hz}, 2 \mathrm{H}), 6.66-6.52(\mathrm{~m}, 2 \mathrm{H}), 6.40(\mathrm{~d}, J=7.3 \mathrm{~Hz}, 2 \mathrm{H}), 2.45-2.33(\mathrm{~m}, 2 \mathrm{H}), 2.29-2.18(\mathrm{~m}, 4 \mathrm{H}), 1.35-$ $1.15(\mathrm{~m}, 1 \mathrm{H})$.

As shown in Scheme S7b, chlorostibine 1b could react with Ni(II)-1 to produce $\mathbf{3 b i}$ in $98 \%$ GC yield. Similarly, transmetalation between distibine 1e and nickel compound Ni(II)-1 also occurred, and the product 3 bi was obtained in $76 \%$ yield in Scheme S7c. These two experimental results show that the reaction involves two possible transmetalation processes. As shown in Scheme S7d, Reaction between chlorostibine 1a and Ni(II)-1 did not take place in the absence of zinc. In contrast, using zinc as an additive, reaction between chlorostibine 1a and Ni(II)-1 smoothly occurred to give the desired product 3d in $46 \%$ yiled in Scheme S7e, which implies that zinc is crucial in this coupling.

Table S16. The Effect of $\mathrm{Zn}$ or $\mathrm{ZnCl}_{2}$ on This Coupling

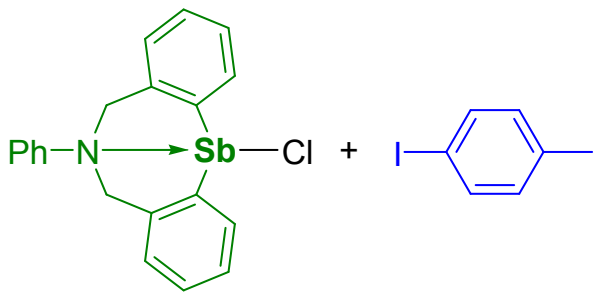

1a, $0.1 \mathrm{mmol}$

2a, $0.1 \mathrm{mmol}$

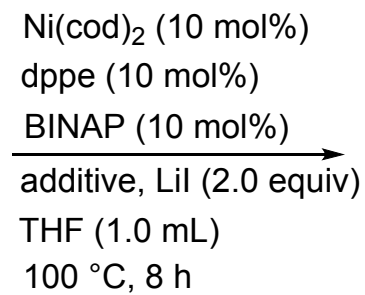

$100{ }^{\circ} \mathrm{C}, 8 \mathrm{~h}$

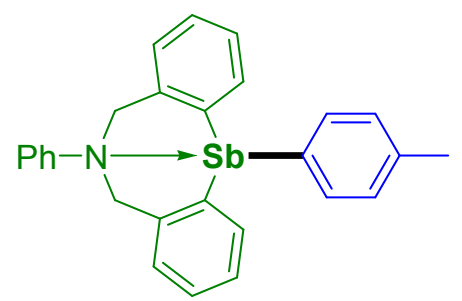

$3 \mathbf{a}$

\begin{tabular}{lll}
\hline Entry & Additive & Yield of 3a $(\%)^{a}$ \\
\hline 1 & Without additive & 21 \\
2 & $\mathrm{Zn}$ (1.0 equiv) & 49 \\
3 & $\mathrm{Zn}$ (2.0 equiv) & 80 \\
4 & $\mathrm{ZnCl}_{2}$ (0.1 equiv) & 20 \\
5 & $\mathrm{ZnCl}_{2}$ (0.5 equiv) & 16 \\
6 & $\mathrm{ZnCl}_{2}$ (1.0 equiv) & 11 \\
7 & $\mathrm{ZnCl}_{2}$ (2.0 equiv) & 3
\end{tabular}

Reaction conditions: 1a $(0.1 \mathrm{mmol}), \mathbf{2 a}(0.1 \mathrm{mmol}), \mathrm{Ni}(\mathrm{cod})_{2}(0.01 \mathrm{mmol}, 10 \mathrm{~mol} \%)$, dppe $(0.01 \mathrm{mmol}$, $10 \mathrm{~mol} \%)$, BINAP (0.01 mmol, $10 \mathrm{~mol} \%)$, without or with additive, LiI (0.2 mmol, 2.0 equiv), THF (1.0 $\mathrm{mL}$ ), at $100{ }^{\circ} \mathrm{C}$ for $8 \mathrm{~h} .{ }^{a}$ Yields were determined by ${ }^{1} \mathrm{H}$ NMR analysis using mesitylene as an internal standard.

Table S17. The Effect of $\mathrm{Zn}$ or $\mathrm{ZnCl}_{2}$ on This Coupling 


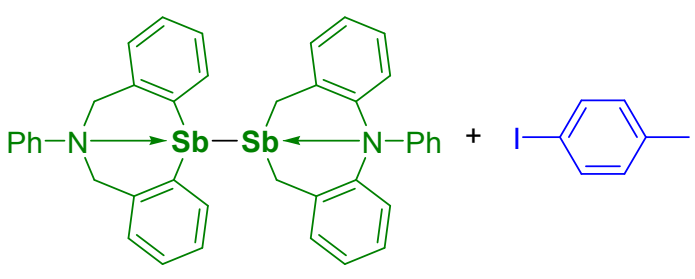

1f, $0.05 \mathrm{mmol}$

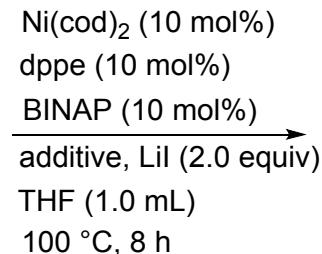

$100{ }^{\circ} \mathrm{C}, 8 \mathrm{~h}$

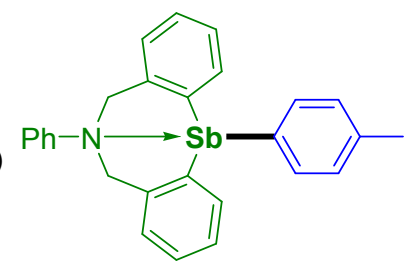

$3 a$

\begin{tabular}{lll}
\hline Entry & Additive & ${\text { Yield of 3a }(\%)^{a}}^{a}$ \\
\hline 1 & Without additive & 31 \\
2 & $\mathrm{Zn} \mathrm{(1.0} \mathrm{equiv)}$ & 36 \\
3 & $\mathrm{Zn} \mathrm{(2.0} \mathrm{equiv)}$ & 47 \\
4 & $\mathrm{ZnCl}_{2}$ (0.1 equiv) & 31 \\
5 & $\mathrm{ZnCl}_{2}$ (0.5 equiv) & 27 \\
6 & $\mathrm{ZnCl}_{2}$ (1.0 equiv) & 19 \\
7 & $\mathrm{ZnCl}_{2}$ (2.0 equiv) & 7
\end{tabular}

Reaction conditions: 1f $(0.05 \mathrm{mmol}), \mathbf{2 a}(0.1 \mathrm{mmol}), \mathrm{Ni}(\operatorname{cod})_{2}(0.01 \mathrm{mmol}, 10 \mathrm{~mol} \%)$, dppe $(0.01 \mathrm{mmol}$, $10 \mathrm{~mol} \%)$, BINAP ( $0.01 \mathrm{mmol}, 10 \mathrm{~mol} \%)$, without or with an additive, LiI ( $0.2 \mathrm{mmol}, 2.0$ equiv), THF $(1.0 \mathrm{~mL})$, at $100{ }^{\circ} \mathrm{C}$ for $8 \mathrm{~h} .{ }^{a}$ Yields were determined by ${ }^{1} \mathrm{H}$ NMR analysis using mesitylene as an internal standard.

To test whether $\mathrm{Zn}$ or $\mathrm{ZnCl}_{2}$ is crucial in the reaction, we performed several control experiments, and the results are summarized in Tables S16 and S17. As depicted in Table S16, using chlorostibine 1a as the substrate, the yield increased significantly from $21 \%$ to $80 \%$ with the addition of 2.0 equivalents of $\mathrm{Zn}$. In contrast, the yield of 3a basically remained unchanged when 0.1 equivalent of $\mathrm{ZnCl}_{2}$ was added and as a matter of fact decreased with the increased presence of $\mathrm{ZnCl}_{2}$ (Table S16, entries 4-7). It is noted that the yield of $\mathbf{3 a}$ was $3 \%$ in the presence of 2.0 equivalents of $\mathrm{ZnCl}_{2}$ (Table S16, entry 7). In another example of using distibine $\mathbf{1 f}$ as the substrate in the presence of Zn (Table S17, entry 3), the yield of 3a was increased from $31 \%$ to $47 \%$. In the case of using 0.1 equivalent of $\mathrm{ZnCl}_{2}$, the yield of 3a remained basically not changed, and decreased with the increase of $\mathrm{ZnCl}_{2}$ (Table S17, entry 5-7). The results of Tables $\mathrm{S} 16$ and $\mathrm{S} 17$ demonstrate that $\mathrm{Zn}$ is indispensable for reaction promotion, whereas $\mathrm{ZnCl}_{2}$ does not have such positive effect on this cross-coupling reaction. 


\section{S4.1.4 Proposed Mechanism}

Scheme S8. Proposed Mechanism for the Nickel-Catalyzed Reductive Coupling of Chlorostibines with Organic Halides

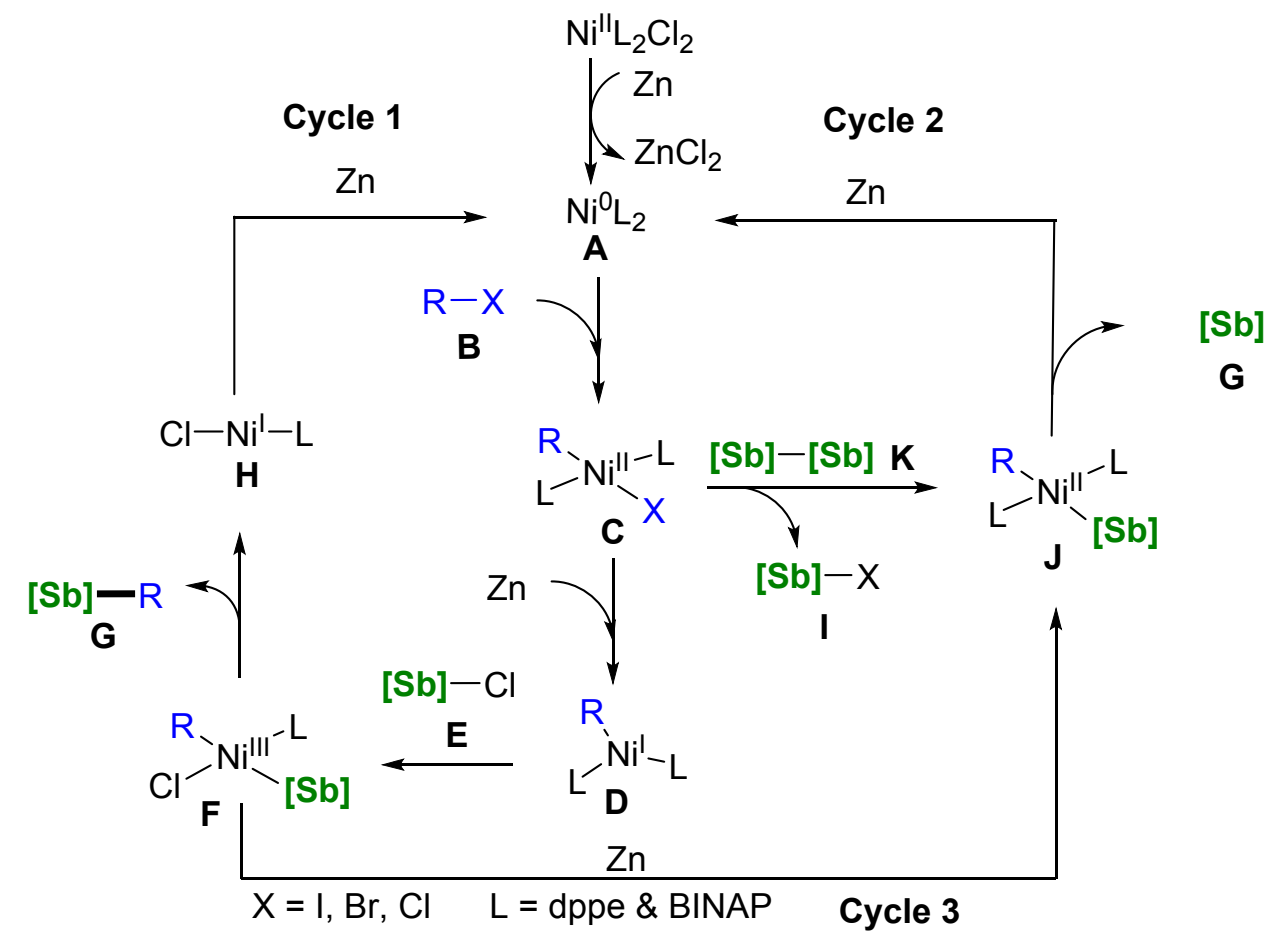

The reaction begins with the oxidative addition of $\mathrm{Ni}(0) \mathrm{L}_{2}(\mathbf{A})$ that is in situ generated during zinc reduction of aryl halides. In cycle 1, the reduction of intermediate $\mathrm{C}$ by zinc provides intermediate $\mathrm{RNi}(\mathrm{I}) \mathrm{L}_{2}(\mathbf{D})$, which undergoes oxidative addition with chlorostibines $\mathbf{E}$ to generate RNi(III)[Sb]ClL (F). Subsequently, intermediate $\mathbf{F}$ undergoes reductive elimination to form organostibines $[\mathrm{Sb}]-\mathrm{R}(\mathbf{G})$, as well as intermediate $\mathrm{Ni}(\mathrm{I}) \mathrm{ClL}(\mathbf{H})$. Finally, intermediate $\mathbf{H}$ is reduced by zinc to $\mathbf{A}$. In cycle 2, transmetalation of intermediate $\mathbf{C}$ with distibine $\mathbf{K}$ provides $\mathrm{RNi}(\mathrm{II})[\mathrm{Sb}] \mathrm{L}_{2}(\mathbf{J})$, which undergoes reductive elimination to afford the desired product $\mathbf{G}$, together with the regeneration of A. In cycle 3, the reduction of intermediate $\mathbf{F}$ by $\mathrm{Zn}$ gives intermediate J. The latter provides the desired product $\mathbf{G}$ through same route for cycle 2 .

S4.2 Mechanism Study on Palladium-Catalyzed Cross-Coupling of Sb-aryl Stibines with Aryl Halides

S4.2.1 Kinetic Research on Palladium-Catalyzed Cross-Coupling of Sb-aryl Stibines with Aryl Halides

Table S18. Time-Course Monitoring Results 


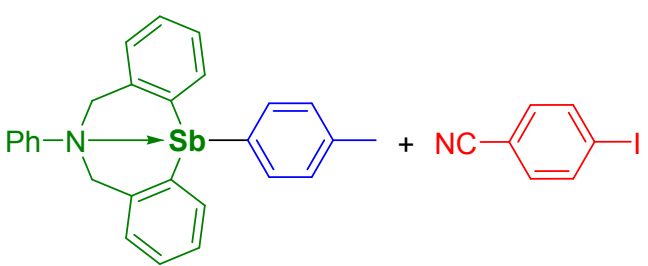

3a, $\quad 0.11 \mathrm{mmol}$

2b, $0.1 \mathrm{mmol}$
$\mathrm{Pd}(\mathrm{OAc})_{2}(5.0 \mathrm{~mol} \%)$ $n-\mathrm{Bu}_{4} \mathrm{PBr}(20 \mathrm{~mol} \%)$

$\mathrm{Cs}_{2} \mathrm{CO}_{3}$ (2.0 equiv)

THF: $\mathrm{H}_{2} \mathrm{O}=50: 1(\mathrm{v}: \mathrm{v})$

$110^{\circ} \mathrm{C}, \mathrm{xx}$ min

by $\mathrm{GC}$ analysis

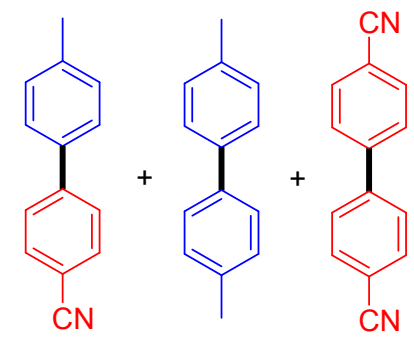

$5 a \quad 4 a \quad 4 b$

\begin{tabular}{lllllllllll}
\hline Time (min) & 0 & 5 & 10 & 20 & 40 & 60 & 120 & 180 & 240 & 360 \\
\hline Yield of 5a (\%) $)^{a}$ & 0 & 5 & 13 & 27 & 48 & 63 & 81 & 90 & 92 & 94 \\
Yield of 4a (\%) $)^{a}$ & 0 & 0 & 0 & 0 & 0 & 0 & 0 & 1 & 2 & 3 \\
Yield of 4b (\%) $^{a}$ & 0 & 1 & 1 & 2 & 2 & 3 & 3 & 4 & 5 & 5 \\
Surplus of 2b (\%) $^{a}$ & 100 & 95 & 87 & 73 & 52 & 37 & 19 & 8 & 4 & 0
\end{tabular}

Reaction conditions: 3a $(0.11 \mathrm{mmol}), \mathbf{2 b}(0.10 \mathrm{mmol}), \mathrm{Pd}(\mathrm{OAc})_{2}(0.005 \mathrm{mmol}), n-\mathrm{Bu}_{4} \mathrm{PBr}(0.02 \mathrm{mmol})$, $\mathrm{Cs}_{2} \mathrm{CO}_{3}(0.20 \mathrm{mmol})$, THF $(1.0 \mathrm{~mL}), \mathrm{H}_{2} \mathrm{O}(20 \mu \mathrm{L}), 110{ }^{\circ} \mathrm{C}, 0-360 \min (6 \mathrm{~h}) .{ }^{a}$ Yields were determined by $\mathrm{GC}$ analysis using $n$-dodecane as an internal standard.

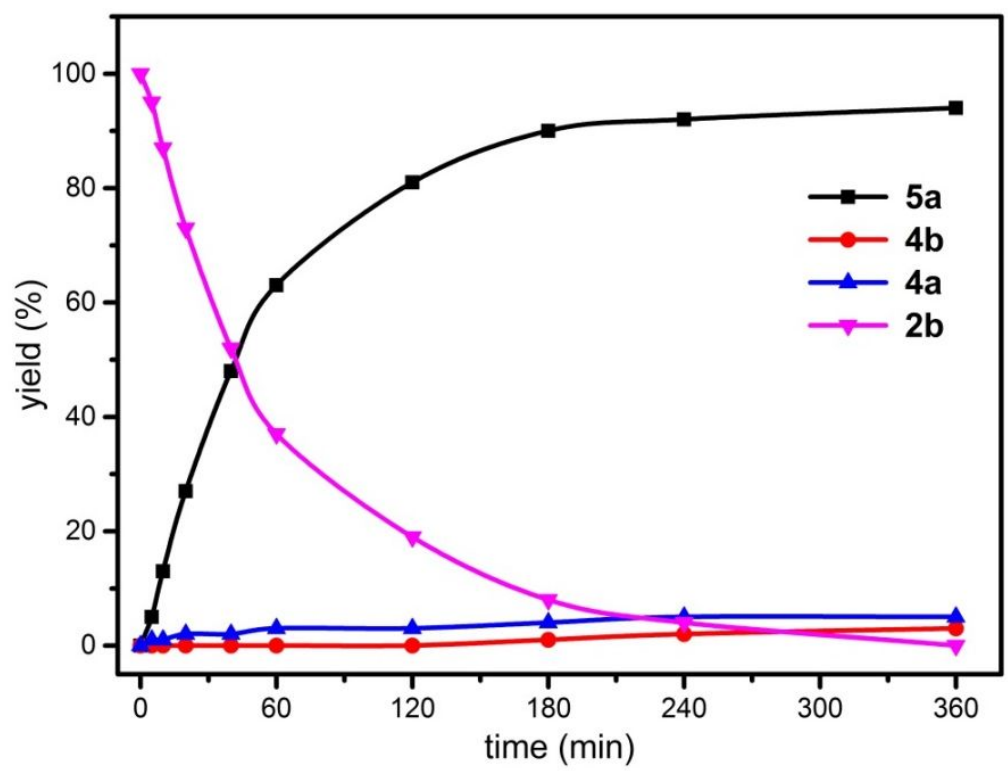

Figure S9. Time-course plots of cross-coupling of $\mathbf{3 a}$ with $\mathbf{2 b}$. Reaction conditions: $\mathbf{3 a}(0.11 \mathrm{mmol}), \mathbf{2 b}$ (0.10 mmol), $\mathrm{Pd}(\mathrm{OAc})_{2}(0.005 \mathrm{mmol}), n-\mathrm{Bu}_{4} \mathrm{PBr}(0.02 \mathrm{mmol}), \mathrm{Cs}_{2} \mathrm{CO}_{3}(0.20 \mathrm{mmol}), \mathrm{THF}(1.0 \mathrm{~mL}), \mathrm{H}_{2} \mathrm{O}$ $(20 \mu \mathrm{L}), 110^{\circ} \mathrm{C}, 0-360 \mathrm{~min}(6 \mathrm{~h})$.

Procedure: Under a nitrogen atmosphere, a $10 \mathrm{~mL}$ Schlenk tube equipped with a magnetic stir bar was charged successively with stibine $\mathbf{3 a}(53.1 \mathrm{mg}, 0.11 \mathrm{mmol})$, 4-iodobenzonitrile $2 \mathbf{b}$ (22.9 mg, $0.1 \mathrm{mmol})$, $\mathrm{Pd}(\mathrm{OAc})_{2}(1.1 \mathrm{mg}, 0.005 \mathrm{mmol}, 5.0 \mathrm{~mol} \%), n-\mathrm{Bu}_{4} \mathrm{PBr}$ (6.8 mg, $\left.0.02 \mathrm{mmol}, 20 \mathrm{~mol} \%\right), \mathrm{Cs}_{2} \mathrm{CO}_{3}(65.0 \mathrm{mg}$, $0.2 \mathrm{mmol}, 2.0$ equiv), $1.0 \mathrm{~mL}$ of THF, $20 \mu \mathrm{L}$ of $\mathrm{H}_{2} \mathrm{O}$ and $30 \mu \mathrm{L}$ of $n$-dodecane. The reaction mixture was stirred at $110{ }^{\circ} \mathrm{C}$ for $0-360 \mathrm{~min}$. The resulting mixture was monitored, and the reaction progress was traced by GC analysis. 
S4.2.2 The Effect of Concentration of Substrates on Palladium-Catalyzed Cross-Coupling of SbAryl Stibines with Aryl Halides

Table S19. Results of the Effect of Concentration of Substrates (10 min)
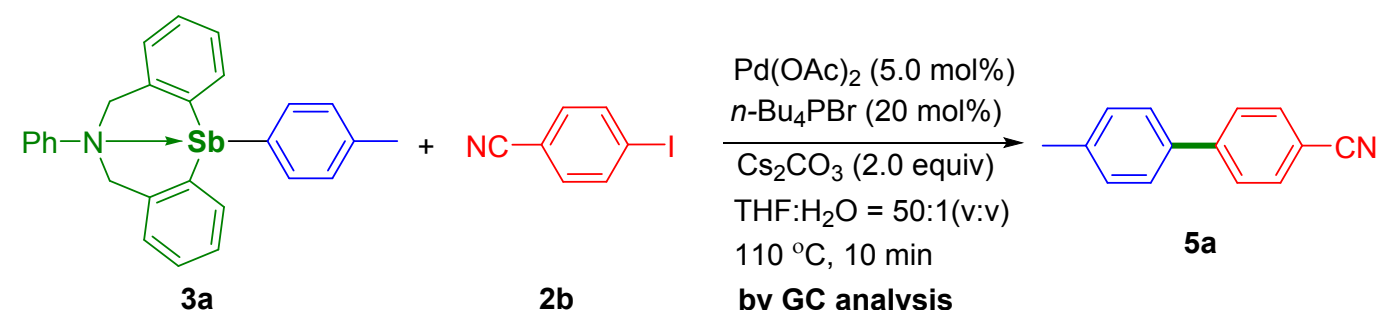

\begin{tabular}{llll}
\hline Entry & $\mathbf{3 a}(\mathrm{mmol})$ & $\mathbf{2 b}(\mathrm{mmol})$ & ${\text { Yield of } \mathbf{5 a}(\%)^{a}}^{a}$ \\
\hline 1 & 0.1 & 0.1 & 18 \\
2 & 0.2 & 0.1 & 19 \\
3 & 0.3 & 0.1 & 20 \\
4 & 0.4 & 0.1 & 22 \\
5 & 0.1 & 0.2 & 20 \\
6 & 0.1 & 0.3 & 21 \\
7 & 0.1 & 0.4 & 21 \\
\hline
\end{tabular}

Note: When changing the amount of $\mathbf{3 a}$ (from $0.1 \mathrm{mmol}$ to $0.4 \mathrm{mmol})$, the amount of $\mathbf{2 b}(0.1 \mathrm{mmol})$ remains unchanged; when changing the amount of $\mathbf{2 b}$ (from $0.1 \mathrm{mmol}$ to $0.4 \mathrm{mmol}$ ), the amount of $\mathbf{3 a}$ (0.1 mmol) remains unchanged. Reaction conditions $1: \mathbf{3 a}(0.10-0.40 \mathrm{mmol}), 2 \mathbf{b}(0.10 \mathrm{mmol}), \mathrm{Pd}(\mathrm{OAc})_{2}$ (0.005 mmol), $n-\mathrm{Bu}_{4} \mathrm{PBr}(0.02 \mathrm{mmol}), \mathrm{Cs}_{2} \mathrm{CO}_{3}(0.20 \mathrm{mmol}), \mathrm{THF}(1.0 \mathrm{~mL}), \mathrm{H}_{2} \mathrm{O}(20 \mu \mathrm{L}), 110{ }^{\circ} \mathrm{C}, 10$ min. Reaction conditions 2: $2 \mathbf{b}(0.10-0.40 \mathrm{mmol}), \mathbf{3 a}(0.10 \mathrm{mmol}), \mathrm{Pd}(\mathrm{OAc})_{2}(0.005 \mathrm{mmol}), n-\mathrm{Bu}_{4} \mathrm{PBr}$ $(0.02 \mathrm{mmol}), \mathrm{Cs}_{2} \mathrm{CO}_{3}(0.20 \mathrm{mmol}), \mathrm{THF}(1.0 \mathrm{~mL}), \mathrm{H}_{2} \mathrm{O}(20 \mu \mathrm{L}), 110^{\circ} \mathrm{C}, 10 \mathrm{~min} .{ }^{a}$ Yields were determined by GC analysis using $n$-dodecane as an internal standard. 
Table S20. Results of the Effect of Concentration of Substrates (20 min)

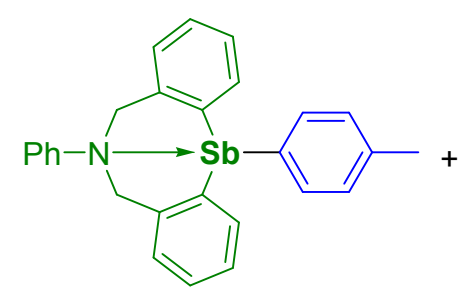

3a

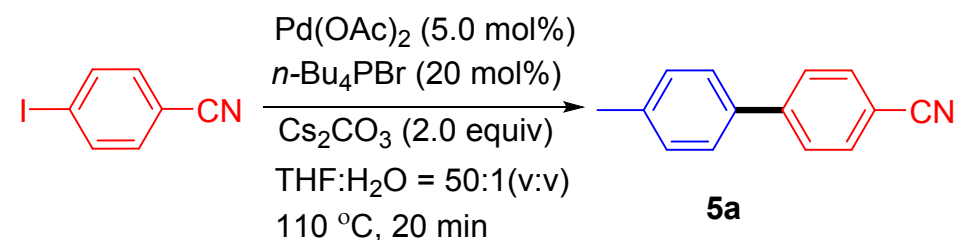

2b

\begin{tabular}{llll}
\hline Entry & $\mathbf{3 a}(\mathrm{mmol})$ & $\mathbf{2 b}(\mathrm{mmol})$ & ${\text { Yield of } \mathbf{5 a}(\%)^{a}}^{a}$ \\
\hline 1 & 0.1 & 0.1 & 35 \\
2 & 0.2 & 0.1 & 36 \\
3 & 0.3 & 0.1 & 37 \\
4 & 0.4 & 0.1 & 37 \\
5 & 0.1 & 0.2 & 35 \\
6 & 0.1 & 0.3 & 36 \\
7 & 0.1 & 0.4 & 36 \\
\hline
\end{tabular}

Note: When changing the amount of $\mathbf{3 a}$ (from $0.1 \mathrm{mmol}$ to $0.4 \mathrm{mmol})$, the amount of $\mathbf{2 b}(0.1 \mathrm{mmol})$ remains unchanged; when changing the amount of $\mathbf{2} \mathbf{b}$ (from $0.1 \mathrm{mmol}$ to $0.4 \mathrm{mmol}), \mathbf{3 a}(0.1 \mathrm{mmol})$ remains unchanged. Reaction conditions 1: 3a $(0.10-0.40 \mathrm{mmol}), \mathbf{2 b}(0.10 \mathrm{mmol}), \mathrm{Pd}(\mathrm{OAc})_{2}(0.005$ mmol), $n$ - $\mathrm{Bu}_{4} \mathrm{PBr}(0.02 \mathrm{mmol}), \mathrm{Cs}_{2} \mathrm{CO}_{3}(0.20 \mathrm{mmol})$, THF $(1.0 \mathrm{~mL}), \mathrm{H}_{2} \mathrm{O}(20 \mu \mathrm{L}), 110{ }^{\circ} \mathrm{C}, 20 \mathrm{~min}$. Reaction conditions $2: \mathbf{2 b}(0.10-0.40 \mathrm{mmol}), \mathbf{3 a}(0.10 \mathrm{mmol}), \mathrm{Pd}(\mathrm{OAc})_{2}(0.005 \mathrm{mmol}), n-\mathrm{Bu} 4 \mathrm{PBr}(0.02$ $\mathrm{mmol}), \mathrm{Cs}_{2} \mathrm{CO}_{3}(0.20 \mathrm{mmol})$, THF $(1.0 \mathrm{~mL}), \mathrm{H}_{2} \mathrm{O}(20 \mu \mathrm{L}), 110^{\circ} \mathrm{C}, 20 \mathrm{~min} .{ }^{a}$ Yields were determined by $\mathrm{GC}$ analysis using $n$-dodecane as an internal standard.
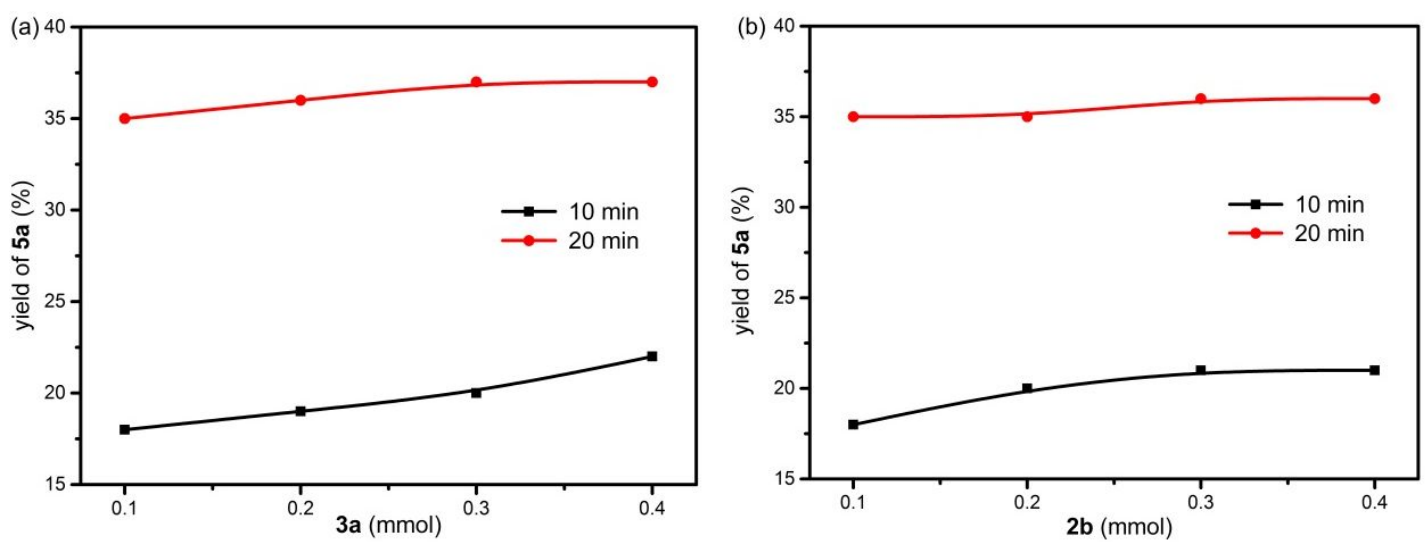

Figure S10 (a) The effect of concentration of $\mathbf{3 a}$ on the cross-coupling reaction of stibines 3a with 4iodobenzonitrile $\mathbf{2 b}$. Reaction conditions: 3a $(0.1-0.4 \mathrm{mmol})$, $2 \mathbf{b}(0.1 \mathrm{mmol})$ remains unchanged, $\mathrm{Pd}(\mathrm{OAc})_{2}(0.005 \mathrm{mmol}), n$ - $\mathrm{Bu}_{4} \mathrm{PBr}(0.02 \mathrm{mmol}), \mathrm{Cs}_{2} \mathrm{CO}_{3}(0.2 \mathrm{mmol}), \mathrm{THF}(1.0 \mathrm{~mL}), \mathrm{H}_{2} \mathrm{O}(20 \mu \mathrm{L})$, monitoring at $100{ }^{\circ} \mathrm{C}$ for $10 \mathrm{~min}$ and $20 \mathrm{~min}$, respectively. All yields were determined by GC analysis using $n$-dodecane as internal standard. (b) The effect of concentration of $\mathbf{2} \mathbf{b}$ on the cross-coupling 
reaction of stibine 3a with 4 -iodobenzonitrile $\mathbf{2 b}$. Reaction conditions: 2 b $(0.1-0.4 \mathrm{mmol}), \mathbf{3 a}(0.1$ mmol) remains unchanged, $\mathrm{Pd}(\mathrm{OAc})_{2}(0.005 \mathrm{mmol}), n-\mathrm{Bu}_{4} \mathrm{PBr}(0.02 \mathrm{mmol}), \mathrm{Cs}_{2} \mathrm{CO}_{3}(0.2 \mathrm{mmol})$, THF $(1.0 \mathrm{~mL}), \mathrm{H}_{2} \mathrm{O}(20 \mu \mathrm{L})$, monitoring at $100{ }^{\circ} \mathrm{C}$ for $10 \mathrm{~min}$ and $20 \mathrm{~min}$, respectively. All yields were determined by GC analysis using $n$-dodecane as internal standard.

Procedure (a): Under a nitrogen atmosphere, a $10 \mathrm{~mL}$ Schlenk tube equipped with a magnetic stir bar was charged successively with stibine 3a (48.3 - $193.2 \mathrm{mg}, 0.10-0.40 \mathrm{mmol})$, 4-iodobenzonitrile 2b (22.9 mg, $0.10 \mathrm{mmol}, 1.0$ equiv), $\mathrm{Pd}(\mathrm{OAc})_{2}$ (1.1 mg, $\left.0.005 \mathrm{mmol}, 5 \mathrm{~mol} \%\right), n-\mathrm{Bu}_{4} \mathrm{PBr}(6.8 \mathrm{mg}, 0.02$ mmol, $20 \mathrm{~mol} \%$ ), $\mathrm{Cs}_{2} \mathrm{CO}_{3}$ (65 mg, $0.2 \mathrm{mmol}, 2.0$ equiv), $1.0 \mathrm{~mL}$ of THF, $20 \mu \mathrm{L}$ of $\mathrm{H}_{2} \mathrm{O}$ and $30 \mu \mathrm{L}$ of $n$ dodecane. The reaction mixture was stirred at $110^{\circ} \mathrm{C}$ for $10 \mathrm{~min}$ or $20 \mathrm{~min}$. The resulting mixture was analyzed by GC analysis.

Procedure (b): Under a nitrogen atmosphere, a $10 \mathrm{~mL}$ Schlenk tube equipped with a magnetic stir bar was charged successively with stibine $\mathbf{3 a}$ (48.3 $\mathrm{mg}, 0.10 \mathrm{mmol}, 0.10$ equiv), 4-iodobenzonitrile $\mathbf{2 b}$ (22.9 - $91.6 \mathrm{mg}, 0.10-0.40 \mathrm{mmol}), \mathrm{Pd}(\mathrm{OAc})_{2}$ (1.1 mg, $\left.0.005 \mathrm{mmol}, 5 \mathrm{~mol} \%\right), n-\mathrm{Bu}_{4} \mathrm{PBr}(6.8 \mathrm{mg}, 0.02 \mathrm{mmol}$, $20 \mathrm{~mol} \%$ ), $\mathrm{Cs}_{2} \mathrm{CO}_{3}\left(65 \mathrm{mg}, 0.20 \mathrm{mmol}, 2.0\right.$ equiv), $1.0 \mathrm{~mL}$ of THF, $20 \mu \mathrm{L}$ of $\mathrm{H}_{2} \mathrm{O}$ and $30 \mu \mathrm{L}$ of $n$ dodecane. The reaction mixture was stirred at $110{ }^{\circ} \mathrm{C}$ for $10 \mathrm{~min}$ or $20 \mathrm{~min}$. The resulting mixture was analyzed by GC analysis.

\section{S4.2.3 Control Experiments}

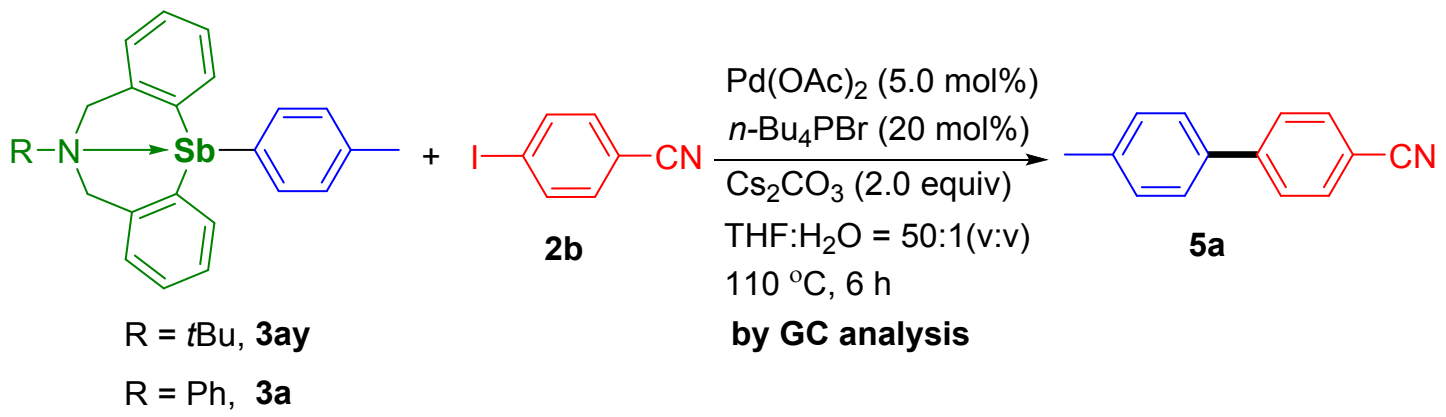

Table S21. Time-Course Data Using 3ay as a Partner

\begin{tabular}{|l|l|l|l|l|l|l|l|l|l|}
\hline Time (min) & 5 & 10 & 20 & 40 & 60 & 120 & 180 & 240 & 360 \\
\hline Yield of 5a (\%) & 15 & 25 & 54 & 70 & 80 & 92 & 94 & 94 & 94 \\
\hline
\end{tabular}

Reaction conditions: 3ay (0.1 mmol), $2 \mathbf{b}(0.1 \mathrm{mmol}), \mathrm{Pd}(\mathrm{OAc})_{2}(0.005 \mathrm{mmol}), n-\mathrm{Bu}_{4} \mathrm{PBr}(0.02 \mathrm{mmol})$, $\mathrm{Cs}_{2} \mathrm{CO}_{3}(0.2 \mathrm{mmol})$, THF $(1.0 \mathrm{~mL}), \mathrm{H}_{2} \mathrm{O}(20 \mu \mathrm{L})$, monitoring at $100{ }^{\circ} \mathrm{C}$ for $5-360 \mathrm{~min} .{ }^{a}$ Yields were determined by GC analysis using $n$-dodecane as an internal standard.

Table S22. Time-Course Data Using 3a as a Partner

\begin{tabular}{|l|l|l|l|l|l|l|l|l|l|}
\hline Time (min) & 5 & 10 & 20 & 40 & 60 & 120 & 180 & 240 & 360 \\
\hline Yield of 5a (\%) & 5 & 13 & 27 & 48 & 63 & 81 & 90 & 92 & 94 \\
\hline
\end{tabular}


Reaction conditions: 3a $(0.1 \mathrm{mmol}), 2 \mathbf{b}(0.1 \mathrm{mmol}), \mathrm{Pd}(\mathrm{OAc})_{2}(0.005 \mathrm{mmol}), n-\mathrm{Bu}_{4} \mathrm{PBr}(0.02 \mathrm{mmol})$, $\mathrm{Cs}_{2} \mathrm{CO}_{3}(0.2 \mathrm{mmol})$, THF $(1.0 \mathrm{~mL}), \mathrm{H}_{2} \mathrm{O}(20 \mu \mathrm{L})$, monitoring at $100{ }^{\circ} \mathrm{C}$ for $5-360 \mathrm{~min}$. $a$ Yields were determined by GC analysis using $n$-dodecane as an internal standard.

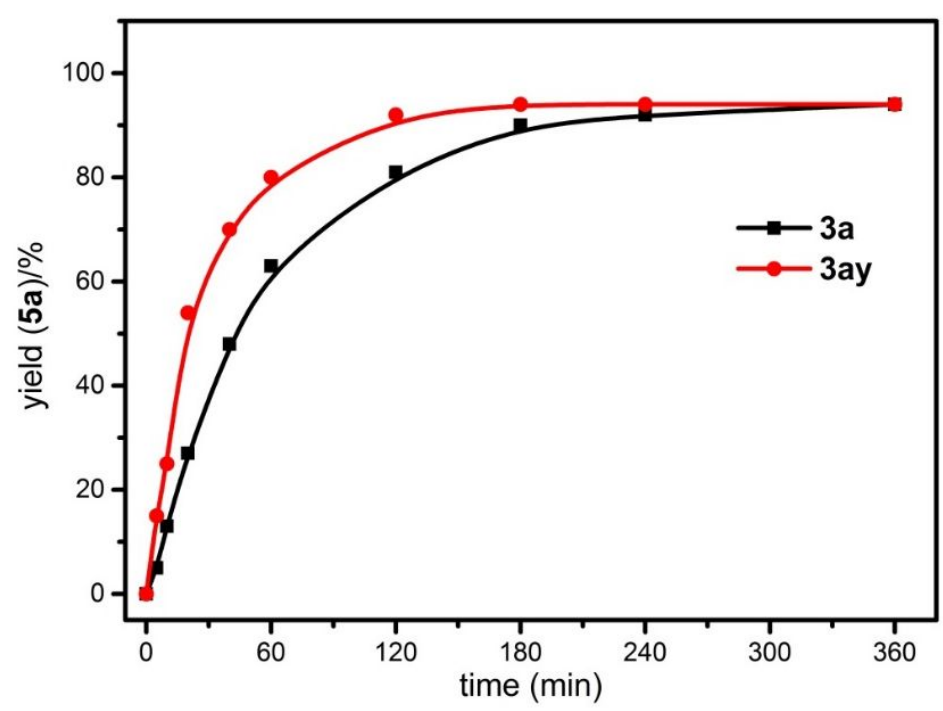

Figure S11. Time course plots of the reaction of $\mathbf{3 a}$ with $\mathbf{2 b}$ and reaction of $\mathbf{3 a y}$ and $\mathbf{2 b}$. All yields were determined by GC analysis using $n$-dodecane as an internal standard. Reaction condition 1: 3ay (0.10 mmol), $2 \mathbf{b}$ (0.1 mmol), $\mathrm{Pd}(\mathrm{OAc})_{2}(0.005 \mathrm{mmol}), n-\mathrm{Bu}_{4} \mathrm{PBr}(0.02 \mathrm{mmol}), \mathrm{Cs}_{2} \mathrm{CO}_{3}(0.2 \mathrm{mmol}), \mathrm{THF}$ (1.0 $\mathrm{mL}), \mathrm{H}_{2} \mathrm{O}(20 \mu \mathrm{L})$, monitoring $6 \mathrm{~h}$ (360 min). Reaction condition 2: 3a $(0.11 \mathrm{mmol}), 2 \mathbf{b}(0.1 \mathrm{mmol})$, $\mathrm{Pd}(\mathrm{OAc})_{2}(0.005 \mathrm{mmol}), n-\mathrm{Bu}_{4} \mathrm{PBr}(0.02 \mathrm{mmol}), \mathrm{Cs}_{2} \mathrm{CO}_{3}(0.2 \mathrm{mmol}), \mathrm{THF}(1.0 \mathrm{~mL}), \mathrm{H}_{2} \mathrm{O}(20 \mu \mathrm{L})$, at $110^{\circ} \mathrm{C}$ for $5-360 \mathrm{~min}$.

Procedure (1): Under a nitrogen atmosphere, a $10 \mathrm{~mL}$ Schlenk tube equipped with a magnetic stir bar was charged successively with stibine 3 ay ( $46.3 \mathrm{mg}, 0.10 \mathrm{mmol}, 1.0$ equiv), 4-iodobenzonitrile $2 \mathbf{b}$ (22.9 $\mathrm{mg}, 0.10 \mathrm{mmol}, 1.0$ equiv), $\mathrm{Pd}(\mathrm{OAc})_{2}(1.1 \mathrm{mg}, 0.005 \mathrm{mmol}, 5 \mathrm{~mol} \%), n-\mathrm{Bu}_{4} \mathrm{PBr}(6.8 \mathrm{mg}, 0.02 \mathrm{mmol}, 20$ mol\%), $\mathrm{Cs}_{2} \mathrm{CO}_{3}$ (0.20 mmol, 2.0 equiv), $1.0 \mathrm{~mL}$ of THF, $20 \mu \mathrm{L}$ of $\mathrm{H}_{2} \mathrm{O}$ and $30 \mu \mathrm{L}$ of $n$-dodecane. The reaction mixture was stirred at $110^{\circ} \mathrm{C}$ for 5-360 $\mathrm{min}$. The resulting mixture was analyzed by GC analysis. Procedure (2): Under a nitrogen atmosphere, a $10 \mathrm{~mL}$ Schlenk tube equipped with a magnetic stir bar was charged successively with stibine $\mathbf{3 a}$ (48.3 $\mathrm{mg}, 0.10 \mathrm{mmol}, 1.0$ equiv), 4-iodobenzonitrile $\mathbf{2 b}$ (22.9 $\mathrm{mg}, 0.10 \mathrm{mmol}, 1.0$ equiv), $\mathrm{Pd}(\mathrm{OAc})_{2}(1.1 \mathrm{mg}, 0.005 \mathrm{mmol}, 5.0 \mathrm{~mol} \%), n-\mathrm{Bu}_{4} \mathrm{PBr}(0.02 \mathrm{mmol}, 20 \mathrm{~mol} \%)$, $\mathrm{Cs}_{2} \mathrm{CO}_{3}$ (65 mg, $0.20 \mathrm{mmol}, 2.0$ equiv), $1.0 \mathrm{~mL}$ of THF, $20 \mu \mathrm{L}$ of $\mathrm{H}_{2} \mathrm{O}$ and $30 \mu \mathrm{L}$ of $n$-dodecane. The reaction mixture was stirred at $110^{\circ} \mathrm{C}$ for $5-360 \mathrm{~min}$. The resulting mixture was analyzed by GC analysis.

\section{Scheme S9. Control Experiments of the Palladium-Catalyzed Cross-Coupling of Sb-Aryl Stibines with Aryl Iodides}


(a) Competitive reaction of aryl iodides

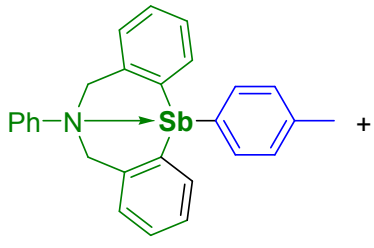

3a, $0.1 \mathrm{mmol}$

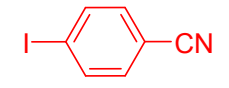

2b, $0.1 \mathrm{mmol}$

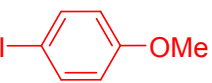

2h, $0.1 \mathrm{mmo}$
$\mathrm{Pd}(\mathrm{OAc})_{2}(5.0 \mathrm{~mol} \%)$ $n-\mathrm{Bu}_{4} \mathrm{PBr}(20 \mathrm{~mol} \%)$ $\mathrm{Cs}_{2} \mathrm{CO}_{3}$ (2.0 equiv.) THF: $\mathrm{H}_{2} \mathrm{O}=50: 1$ (v:v) $110{ }^{\circ} \mathrm{C}, 8 \mathrm{~h}$ by $\mathrm{GC}$ analysis $5 \mathbf{a}: \mathbf{5 c}=72 \%: 18 \%=4: 1$

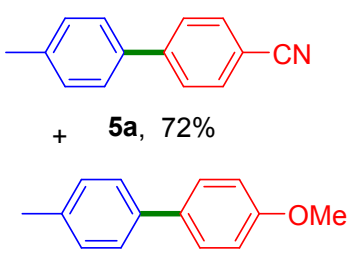

5c, $18 \%$

(b) Competitive reaction of organostibines<smiles>Cc1ccc(S(=O)(=O)c2ccccc2CN(Cc2ccccc2)C(C)(C)C)cc1</smiles>

3ay, $0.1 \mathrm{mmol}$<smiles>CCc1ccc(S(=O)(=O)c2ccccc2CN2Cc3ccccc3C2)cc1</smiles>
2b, $0.1 \mathrm{mmol} 110^{\circ} \mathrm{C}, 8 \mathrm{~h}$

$\mathrm{Pd}(\mathrm{OAc})_{2}(5.0 \mathrm{~mol} \%)$ $n-\mathrm{Bu}_{4} \mathrm{PBr}(20 \mathrm{~mol} \%)$ $\mathrm{Cs}_{2} \mathrm{CO}_{3}$ (2.0 equiv.) THF: $\mathrm{H}_{2} \mathrm{O}=50: 1(\mathrm{v}: \mathrm{v})$ by $\mathrm{GC}$ analysis

5a $: \mathbf{5 i}=65 \%: 26 \%=5: 2$<smiles>Cc1ccc(-c2ccc(C#N)cc2)cc1</smiles>

5a, $65 \%$<smiles>CCc1ccc(-c2ccc(C#N)cc2)cc1</smiles>

5i, $26 \%$

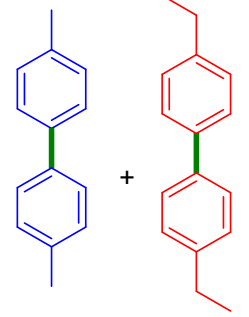

$\mathbf{4 a}, 3.2 \% \mathbf{4 f}, 5.0 \%$

3t, $0.1 \mathrm{mmol}$

(c) Radical trapping experiments

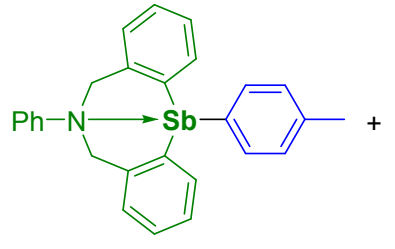

3a, $0.1 \mathrm{mmol}$

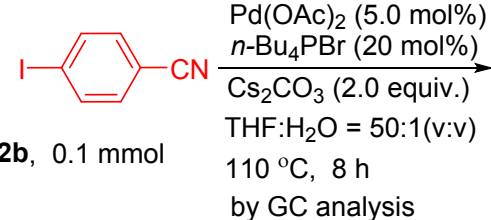

by $\mathrm{GC}$ analysis

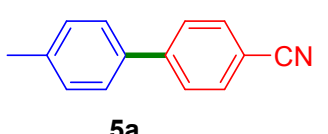

standard conditions : $90 \%$ TEMPO (2.0 equiv) : $69 \%$ BHT (2.0 equiv) : $57 \%$

(d) Stoichiometric reaction of organostibines with $\mathrm{Pd}(\mathrm{OAc})_{2}$

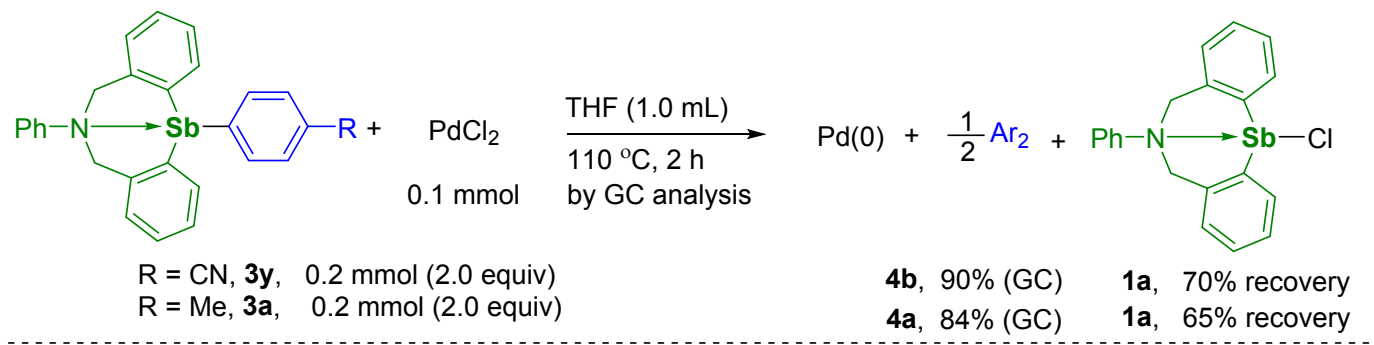

(e) Investigation on catalytic activity of the palladium species in situ generated

$\mathrm{PdCl}_{2}$

$0.005 \mathrm{mmol}$

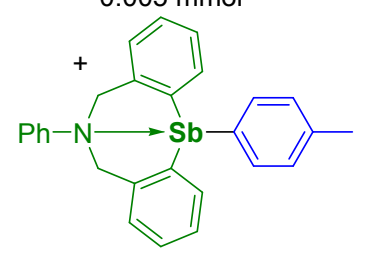

3a, $0.01 \mathrm{mmol}$

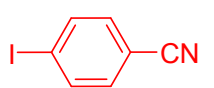

2b, $0.1 \mathrm{mmol}$

3a, $0.1 \mathrm{mmol}$ $n-\mathrm{Bu}_{4} \mathrm{PBr}(20 \mathrm{~mol} \%)$

$\mathrm{Cs}_{2} \mathrm{CO}_{3}$ (2.0 equiv)

THF: $\mathrm{H}_{2} \mathrm{O}=50: 1$ (v:v)

$110^{\circ} \mathrm{C}, 8 \mathrm{~h}$

by $\mathrm{GC}$ analysis

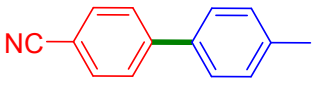

5a, $88 \%$ (GC)

Procedure for Scheme S9a: Under nitrogen, a $10 \mathrm{~mL}$ Schlenk tube of equipped with a magnetic stir bar was charged successively with stibine $\mathbf{3 a}(48.3 \mathrm{mg}, 0.1 \mathrm{mmol})$, 4-iodobenzonitrile $\mathbf{2 b}(22.9 \mathrm{mg}, 0.10$ 
mmol), 1-iodo-4-methoxybenzene $2 \mathbf{h}\left(23.4 \mathrm{mg}, 0.10 \mathrm{mmol}, 1.0\right.$ equiv), $\mathrm{Pd}(\mathrm{OAc})_{2}(1.1 \mathrm{mg}, 0.005 \mathrm{mmol}$, $5.0 \mathrm{~mol} \%$ ), $n-\mathrm{Bu}_{4} \mathrm{PBr}$ (6.8 mg, $\left.0.02 \mathrm{mmol}, 20 \mathrm{~mol} \%\right), \mathrm{Cs}_{2} \mathrm{CO}_{3}$ ( $65 \mathrm{mg}, 0.2 \mathrm{mmol}, 2.0$ equiv), $1.0 \mathrm{~mL}$ of THF, $20 \mu \mathrm{L}$ of $\mathrm{H}_{2} \mathrm{O}$ and $30 \mu \mathrm{L}$ of $n$-dodecane. The reaction mixture was stirred at $110{ }^{\circ} \mathrm{C}$ for $8 \mathrm{~h}$. The resulting mixture was analyzed by GC analysis. Ratio of $\mathbf{5 a}: \mathbf{5 c}$ is $4: 1$.

Procedure for Scheme S9b: Under a nitrogen atmosphere, a $10 \mathrm{~mL}$ Schlenk tube equipped with a magnetic stir bar was charged successively with stibine 3 ay ( $46.3 \mathrm{mg}, 0.1 \mathrm{mmol}, 1.0$ equiv), stibine $3 \mathrm{t}$ (49.7 mg, $0.1 \mathrm{mmol}, 1.0$ equiv), 4-iodobenzonitrile $2 \mathbf{b}$ ( $22.9 \mathrm{mg}, 0.1 \mathrm{mmol}, 1.0$ equiv), $\mathrm{Pd}(\mathrm{OAc})_{2}(1.1$ $\mathrm{mg}, 0.005 \mathrm{mmol}, 5.0 \mathrm{~mol} \%$ ), $n$ - $\mathrm{Bu} 4 \mathrm{PBr}\left(6.8 \mathrm{mg}, 0.02 \mathrm{mmol}, 20 \mathrm{~mol} \%\right.$ ), $\mathrm{Cs}_{2} \mathrm{CO}_{3}(65 \mathrm{mg}, 0.2 \mathrm{mmol}, 2.0$ equiv), $1.0 \mathrm{~mL}$ of THF, $20 \mu \mathrm{L}$ of $\mathrm{H}_{2} \mathrm{O}$ and $30 \mu \mathrm{L}$ of $n$-dodecane. The reaction mixture was stirred at $110{ }^{\circ} \mathrm{C}$ for $8 \mathrm{~h}$. The resulting mixture was analyzed by GC analysis. Ratio of $\mathbf{5 a}: 5 \mathbf{i}$ is 5:2.

Procedure for Scheme S9c (1): Under a nitrogen atmosphere, a $10 \mathrm{~mL}$ Schlenk tube equipped with a magnetic stir bar was charged successively with stibine $\mathbf{3 a}$ ( $48.3 \mathrm{mg}, 0.10 \mathrm{mmol})$, 4-iodobenzonitrile $\mathbf{2 b}$ (22.9 mg, $0.1 \mathrm{mmol}), \mathrm{Pd}(\mathrm{OAc})_{2}(1.1 \mathrm{mg}, 0.005 \mathrm{mmol}, 5.0 \mathrm{~mol} \%), n-\mathrm{Bu}_{4} \mathrm{PBr}(6.8 \mathrm{mg}, 0.02 \mathrm{mmol}, 20$ mol\%), $\mathrm{Cs}_{2} \mathrm{CO}_{3}$ (65 mg, $0.2 \mathrm{mmol}, 2.0$ equiv), TEMPO (31.3 mg, $0.2 \mathrm{mmol}, 2.0$ equiv), $1.0 \mathrm{~mL}$ of THF, $20 \mu \mathrm{L}$ of $\mathrm{H}_{2} \mathrm{O}$ and $30 \mu \mathrm{L}$ of $n$-dodecane. The reaction mixture was stirred at $110^{\circ} \mathrm{C}$ for $8 \mathrm{~h}$. The resulting mixture was analyzed by GC analysis. The yield of $\mathbf{5 a}$ was $69 \%$.

Procedure for Scheme S9c (2): Under a nitrogen atmosphere, a $10 \mathrm{~mL}$ Schlenk tube equipped with a magnetic stir bar was charged successively with stibine $\mathbf{3 a}$ ( $48.3 \mathrm{mg}, 0.11 \mathrm{mmol})$, 4-iodobenzonitrile $\mathbf{2 b}$ (22.9 mg, $0.1 \mathrm{mmol}$ ), $\mathrm{Pd}(\mathrm{OAc})_{2}$ (1.1 mg, $0.005 \mathrm{mmol}, 5.0 \mathrm{~mol} \%$ ), $n$ - $\mathrm{Bu}_{4} \mathrm{PBr}(6.8 \mathrm{mg}, 0.02 \mathrm{mmol}, 20$ mol\%), $\mathrm{Cs}_{2} \mathrm{CO}_{3}$ (65 mg, $0.2 \mathrm{mmol}, 2.0$ equiv), BHT (44.0 mg, $0.2 \mathrm{mmol}, 2.0$ equiv), $1.0 \mathrm{~mL}$ of THF, 20 $\mu \mathrm{L}$ of $\mathrm{H}_{2} \mathrm{O}$ and $30 \mu \mathrm{L}$ of $n$-dodecane. The reaction mixture was stirred at $110^{\circ} \mathrm{C}$ for $8 \mathrm{~h}$. The resulting mixture was analyzed by GC analysis. The yield of $\mathbf{5 a}$ was $57 \%$.

Procedure for Scheme S9d (1): Under nitrogen, a $10 \mathrm{~mL}$ Schlenk tube equipped with a magnetic stir bar was charged successively with stibine $3 \mathbf{y}$ ( $98.8 \mathrm{mg}, 0.2 \mathrm{mmol}, 2.0$ equiv), $\mathrm{PdCl}_{2}(17.7 \mathrm{mg}, 0.1 \mathrm{mmol}$, 1.0 equiv), $1.0 \mathrm{~mL}$ of THF and $30 \mathrm{~mL}$ of $n$-dodecane. The reaction mixture was stirred at $110{ }^{\circ} \mathrm{C}$ for 2 $\mathrm{h}$. The yield of $\mathbf{4 b}$ was determined by GC analysis, and chlorostibine 1a was isolated by flash chromatography on silica gel using a mixture solvent of $\mathrm{CH}_{2} \mathrm{Cl}_{2}: \mathrm{MeOH}(5: 1(\mathrm{v}: \mathrm{v}))$. Product $\mathbf{4 b}$ was detected in $90 \%$ yield and chlorostibine 1a was recycled in $70 \%$ yield.

Procedure for Scheme S9d (2): Under a nitrogen atmosphere, a $10 \mathrm{~mL}$ Schlenk tube equipped with a magnetic stir bar was charged successively with stibine $3 \mathbf{a}$ ( $96.6 \mathrm{mg}, 0.2 \mathrm{mmol}, 2.0$ equiv), $\mathrm{PdCl}_{2}$ (17.7 $\mathrm{mg}, 0.1 \mathrm{mmol}, 1.0$ equiv), $1.0 \mathrm{~mL}$ of THF and $30 \mathrm{~mL}$ of $n$-dodecane. The reaction mixture was stirred at $110^{\circ} \mathrm{C}$ for $2 \mathrm{~h}$. The yield of $\mathbf{4 a}$ was determined by GC analysis, and chlorostibine $1 \mathbf{a}$ was isolated by flash chromatography on silica gel using the mixed solvent of $\mathrm{CH}_{2} \mathrm{Cl}_{2}: \mathrm{MeOH}(5: 1(\mathrm{v}: \mathrm{v}))$. Product $4 \mathrm{a}$ was detected in $84 \%$ yield and chlorostibine 1a was recycled in $65 \%$ yield.

Procedure for Scheme S9e: Under a nitrogen atmosphere, a $10 \mathrm{~mL}$ Schlenk tube equipped with a magnetic stir bar was charged successively with stibine $3 \mathbf{a}$ ( $4.8 \mathrm{mg}, 0.01 \mathrm{mmol}), \mathrm{PdCl}_{2}(0.9 \mathrm{mg}, 0.005$ mmol), $1.0 \mathrm{~mL}$ of THF and $30 \mu \mathrm{L}$ of $n$-dodecane. The reaction mixture was stirred at $110{ }^{\circ} \mathrm{C}$ for $2 \mathrm{~h}$. After $2 \mathrm{~h}$, stibine 3a (48.3 mg, $0.1 \mathrm{mmol}), n-\mathrm{Bu}_{4} \mathrm{PBr}(6.8 \mathrm{mg}, 0.02 \mathrm{mmol}, 20 \mathrm{~mol} \%), \mathrm{Cs}_{2} \mathrm{CO}_{3}(65 \mathrm{mg}$, $0.2 \mathrm{mmol}, 2.0$ equiv), $1.0 \mathrm{~mL}$ of THF and $20 \mu \mathrm{L}$ of $\mathrm{H}_{2} \mathrm{O}$ were successively added into the Schlenk tube. 
The reaction mixture was stirred at $110^{\circ} \mathrm{C}$ for $8 \mathrm{~h}$. The resulting mixture was analyzed by GC analysis. Cross-coupling product 5a was detected in $88 \%$ GC yield.

Scheme S10. Transmetalation between Stibine 3a and a Pd(II) Species

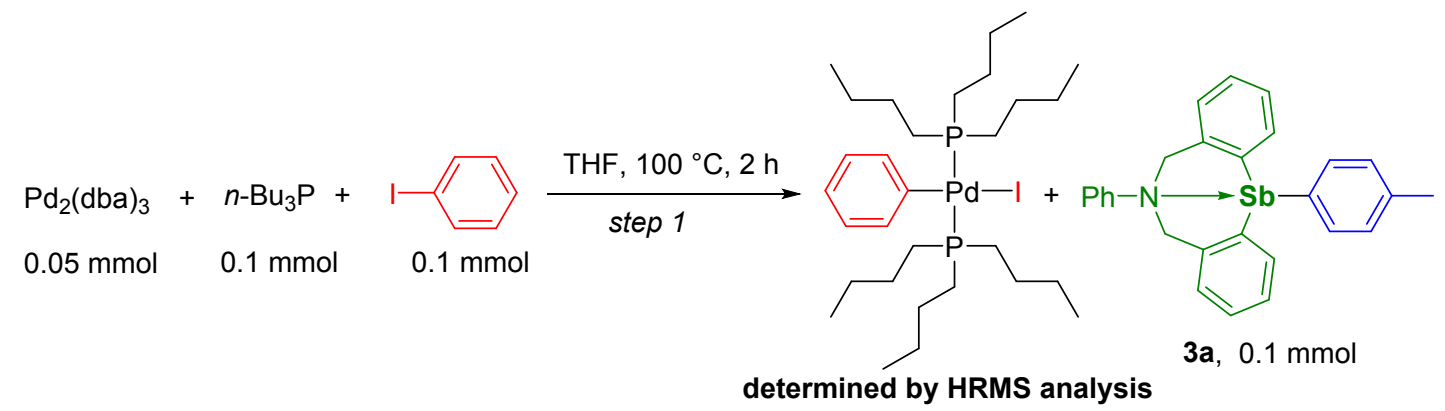

[M] Chemical Formula: $\mathrm{C}_{30} \mathrm{H}_{59} \mathrm{IP}{ }_{2} \mathrm{Pd}, 714.2172$, Found, 714.2166
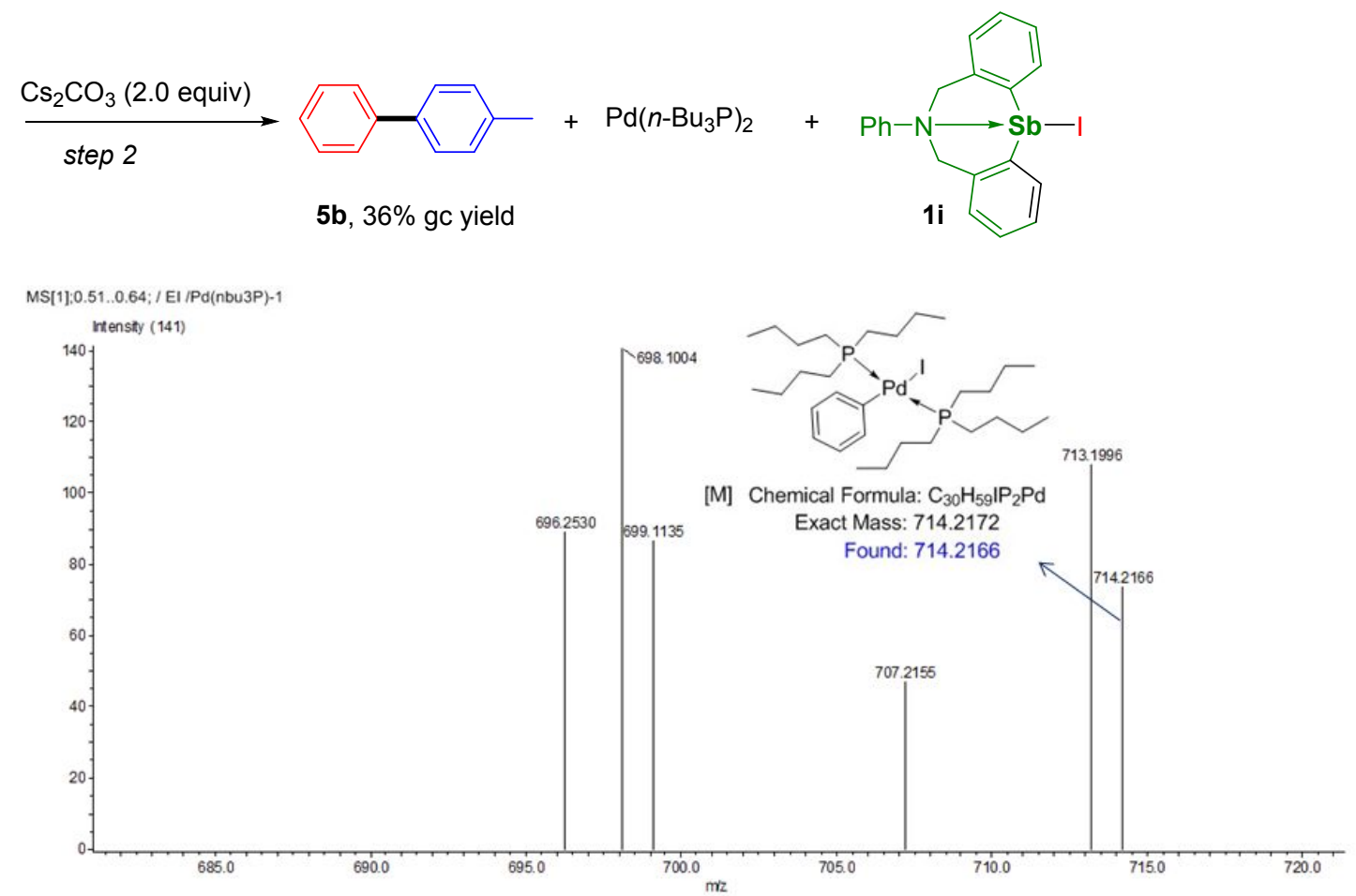

Figure S12. HRMS (EI) data of $\mathrm{Pd}\left(n-\mathrm{Bu}_{3} \mathrm{P}\right)_{2}-\mathrm{PhI}$ 


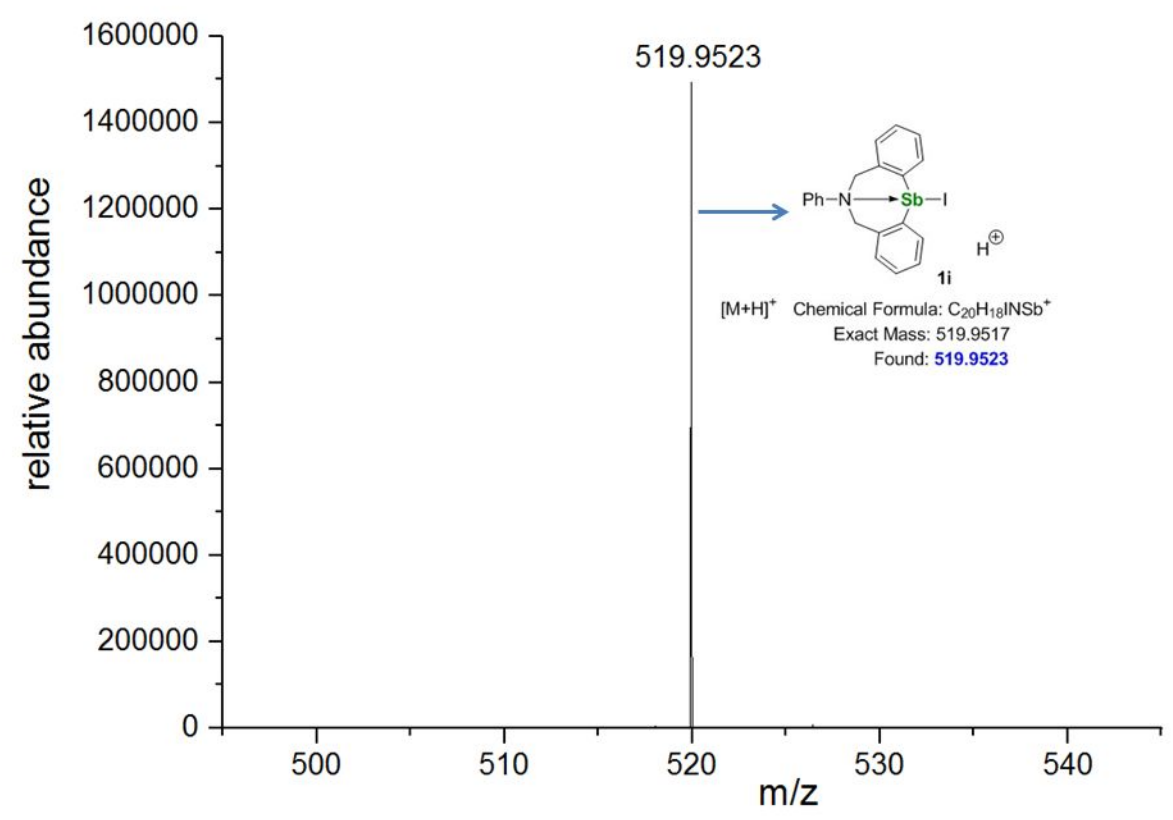

Figure S13. HRMS (ESI) data of Sb-I

Oxidative addition between iodobenzene and $\mathrm{Pd}_{2}(\mathrm{dba})_{3}, n-\mathrm{Bu}_{3} \mathrm{P}$ occurred to give possible intermediate $\mathrm{Pd}\left(n-\mathrm{Bu}_{3} \mathrm{P}\right)_{2}-\mathrm{PhI}$ in the step 1 , which was determined by HRMS analysis (HRMS-EI (m/z) [M] Calcd for $\mathrm{C}_{30} \mathrm{H}_{59} \mathrm{IP}_{2} \mathrm{Pd}, 714.2172$; Found, 714.2166). Biaryl $\mathbf{5 b}$ was formed in $36 \%$ yield (by gc analysis), and iodostibine was detected by HRMS analysis (HRMS-ESI $(\mathrm{m} / \mathrm{z})[\mathrm{M}+\mathrm{H}]^{+} \mathrm{Calcd}$ for $\mathrm{C}_{20} \mathrm{H}_{18} \mathrm{INSb}^{+}$, 519.9517; Found, 519.9648). These experimental results indicate that transmetalation between a $\mathrm{Pd}(\mathrm{II})$ imternediate and an stibine successfully proceeded. 


\section{S4.2.4 Proposed Mechanism}

Scheme S11. Proposed Mechanism for the Palladium-Catalyzed Cross-Coupling of Sb-Aryl Stibines with Aryl Iodides

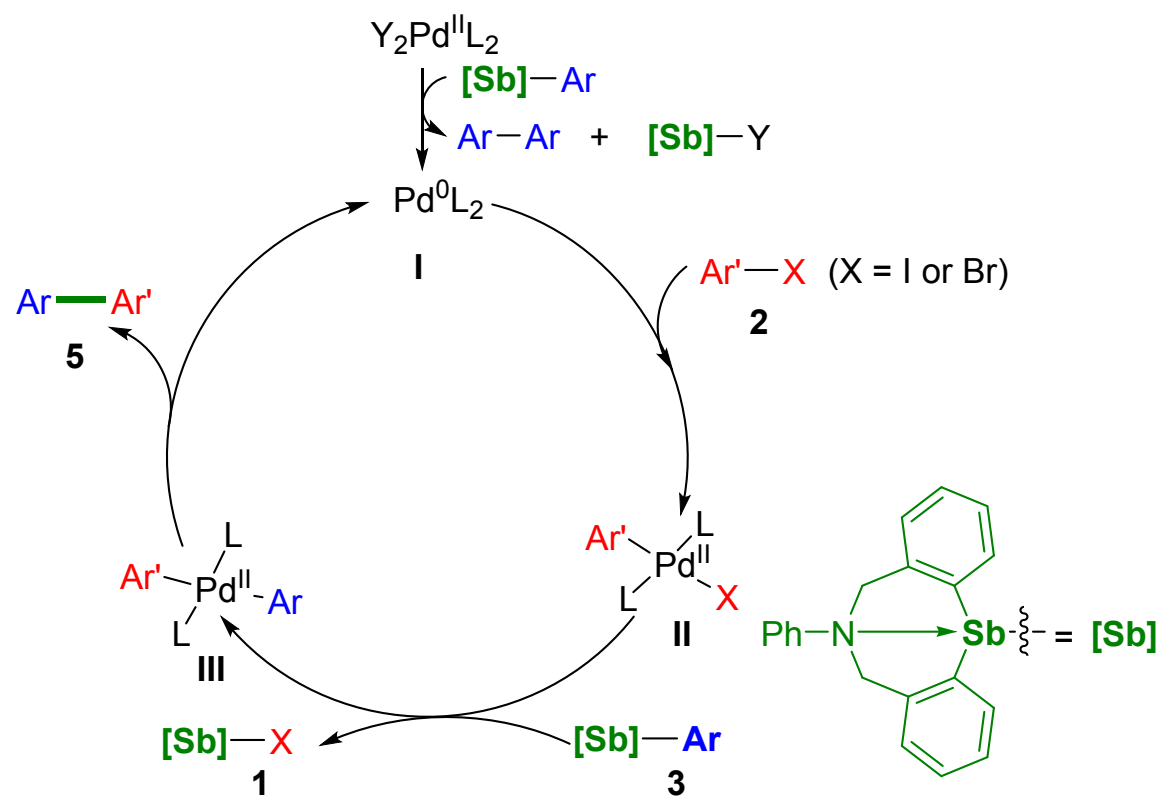

\section{S5. X-ray Crystallographic Data}

X-ray crystallographic data for compounds 1e was recorded at $296 \mathrm{~K}$ on a Rigaku Oxford Diffraction Supernova Dual Source, $\mathrm{Cu}$ at Zero equipped with an AtlasS2 CCD using $\mathrm{Cu}$ Ka radiation. Data reduction was carried out with the diffractometer software. ${ }^{\mathrm{S} 15}$ The struceture was solved by direct methods using Olex2 software ${ }^{\mathrm{S} 16}$ and the non-hydrogen atoms were located from the trial structure and then refined anisotropically with SHELXL-2014 ${ }^{\mathrm{S} 17}$ using a full-matrix least squares procedure based on $F_{2}$. The weighted $R$ factor, $w R$ and goodness-of-fit $S$ values were obtained based on $F_{2}$. The hydrogen atom positions were fixed geometrically at the calculated distances and allowed to ride on their parent atoms. Crystallographic data for the structure reported in this paper has been deposited at the Cambridge Crystallographic Data Center and allocated with the deposition numbers: 2009086. 


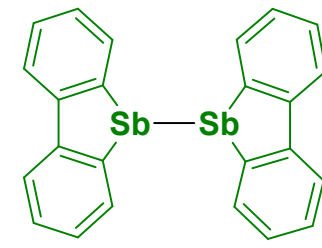

$1 e$

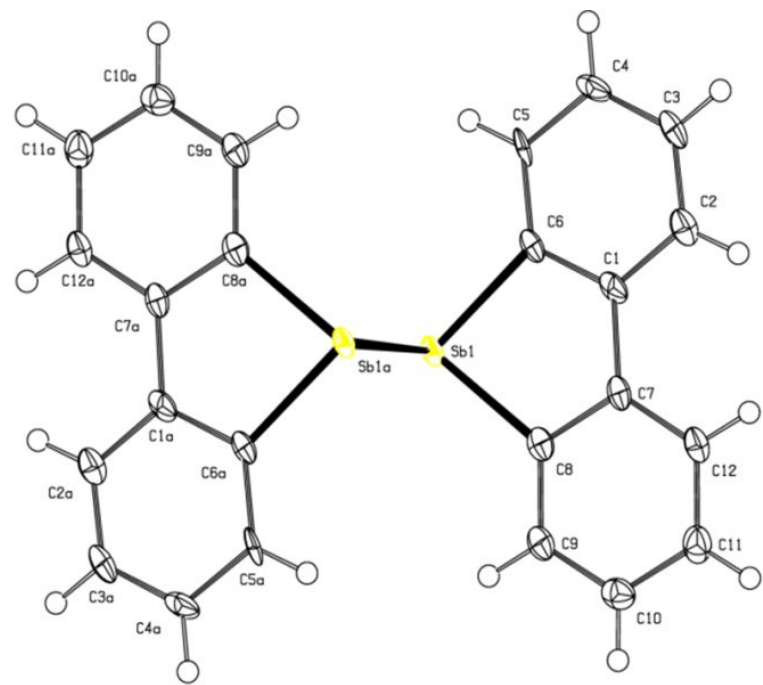

Figure S14. ORTEP drawing of 1e

Table S23. Crystal Data and Structure Refinement for 1e

Identification code

Empirical formula

Formula weight

Temperature/K

Crystal system

Space group

$\mathrm{a} / \AA$

$\mathrm{b} / \AA$

$\mathrm{c} / \AA$

$\alpha{ }^{\circ}$

$\beta /{ }^{\circ}$

$\gamma /{ }^{\circ}$

Volume/Å3

Z

pcalcg/cm 3

$\mu / \mathrm{mm}-1$

$\mathrm{F}(000)$

Crystal size/mm3

Radiation
$1 \mathrm{e}$

C24H16Sb2

547.87

100.01(10)

monoclinic

$\mathrm{P} 21 / \mathrm{n}$

11.9115(7)

5.2479(3)

14.9482(8)

90

103.680(5)

90

907.91(9)

2

2.004

23.608

524.0

$0.11 \times 0.1 \times 0.09$

$\operatorname{CuK} \alpha(\lambda=1.54184)$

$2 \Theta$ range for data collection ${ }^{\circ} 8.57$ to 147.576

Index ranges

$-14 \leq \mathrm{h} \leq 13,-4 \leq \mathrm{k} \leq 6,-18 \leq 1 \leq 18$ 
Reflections collected

Independent reflections

Data/restraints/parameters

Goodness-of-fit on F2

Final $\mathrm{R}$ indexes $[\mathrm{I}>=2 \sigma(\mathrm{I})]$

Final $\mathrm{R}$ indexes [all data]

Largest diff. peak/hole / e Å-3 2.46/-1.26
$1816[$ Rint $=0.0593$, Rsigma $=0.0445]$

$1816 / 0 / 118$

1.058

$\mathrm{R} 1=0.0466, \mathrm{wR} 2=0.1199$

$\mathrm{R} 1=0.0485, \mathrm{wR} 2=0.1234$

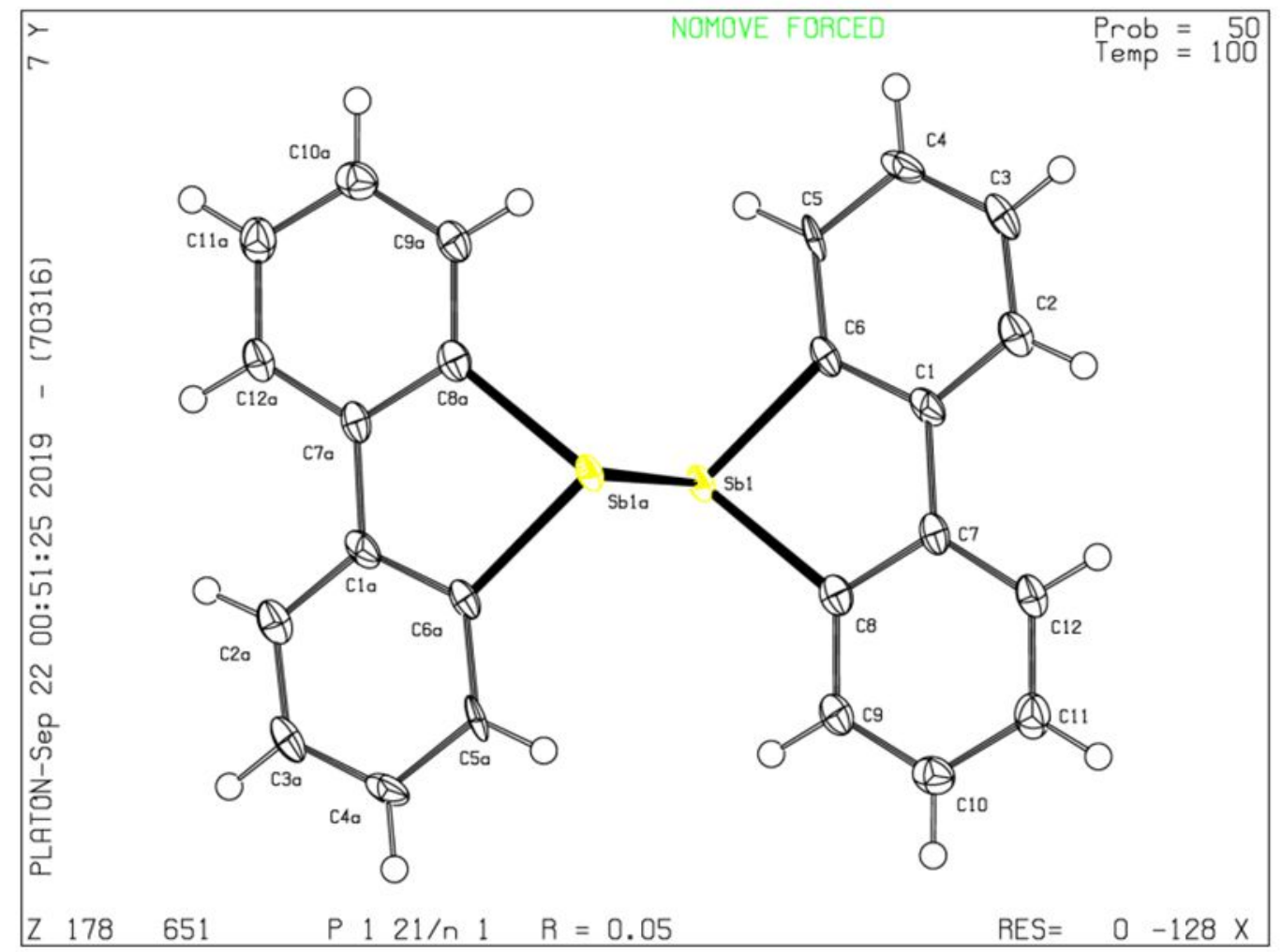

The ellipsoid contour \% probability level in the caption for the image of the structure is $50 \%$.

\section{General procedure for preparation of single crystal}

1e $\mathbf{( 5 0} \mathrm{mg}$ ) was dissolved in $4.0 \mathrm{~mL}$ of tetrahydrofurane (THF) in $25 \mathrm{~mL}$ of round bottom flask, and then $0.5 \mathrm{~mL}$ of $n$-hexane was added into the flask. Subsequently, the round bottom flask was sealed with sealing film and placed in a $-5^{\circ} \mathrm{C}$ refrigerator to give 1e as single crystal in 1 day. 


\section{S6. Reference}

(S1) Zhang, D.; Le, L.; Qiu, R.; Wong, W.-Y.; Kambe, N., Nickel- and Palladium-Catalyzed CrossCoupling Reactions of Stibines with Organoboronic Acids. Angew. Chem. Int. Ed. 2021, 60, 3104-3114.

(S2) Kakusawa, N.; Kurita, J. Hypervalent Organoantimony Compound 12Aryltetrahydrodibenz $[c, f][1,5]$ azastibocine: New Transmetalating Agent for Palladium-Catalyzed Arylation of Organic Halides. Heterocycles 2006, 68, 1335-1348.

(S3) Ackerman, L. K. G.; Anka-Lufford, L. L.; Naodovic, M.; Weix, D. J., Cobalt co-catalysis for crosselectrophile coupling: diarylmethanes from benzyl mesylates and aryl halides. Chem. Sci. 2015, 6, 1115-1119.

(S4) Prehn Jr, F. C.; Etz, B. D.; Price, D.; Trainor, A.; Reese, C. J.; Vyas, S.; Boyes, S. G., Chain-growth polycondensation via the substituent effect: Investigation in to the role of initiator and base on the synthesis of poly(N-octyl benzamide). J. Polym Sci. 2020, 58, 2407-2422.

(S5) Fyfe, J. W. B.; Fazakerley, N. J.; Watson, A. J. B., Chemoselective Suzuki-Miyaura Cross-Coupling via Kinetic Transmetallation. Angew. Chem. Int. Ed. 2017, 56, 1249-1253.

(S6) Liu, N.; Wang, L.; Wang, Z.-X., Room-temperature nickel-catalysed cross-couplings of aryl chlorides with arylzincs. Chem. Commun. 2011, 47, 1598-1600.

(S7) Delaney, C. P.; Kassel, V. M.; Denmark, S. E., Potassium Trimethylsilanolate Enables Rapid, Homogeneous Suzuki-Miyaura Cross-Coupling of Boronic Esters. ACS Catal. 2020, 10, 73-80.

(S8) Liu, C.; Liu, C.; Li, X.-M.; Gao, Z.-M.; Jin, Z.-L., Oxygen-promoted Pd/C-catalyzed SuzukiMiyaura reaction of potassium aryltrifluoroborates. Chin. Chem. Lett. 2016, 27, 631-634.

(S9) Kirsch, P.; Bremer, M. Nematic Liquid Crystals for Active Matrix Displays: Molecular Design and Synthesis. Angew. Chem., Int. Ed. 2000, 39, 4216-4235.

(S10) Li, X.; Liu, C.; Wang, L.; Ye, Q.; Jin, X.; Jin, Z., Temperature-controlled sequential SuzukiMiyaura reactions for preparing unsymmetrical terphenyls. Org. Biomol. Chem. 2018, 16, 8719-8723.

(S11) Nanayakkara, P.; Alper, H., Synthesis of 3-Substituted Furans by Hydroformylation. Adv. Synth. Catal. 2006, 348, 545-550.

(S12) Chen, W.-B.; Xing, C.-H.; Dong, J.; Hu, Q.-S. Electron-Poor, Fluoro-Containing Arylboronic Acids as Efficient Coupling Partners for Bis(1,5-cyclooctadiene)nickel(0)/TricyclohexylphosphineCatalyzed Cross-Coupling Reactions of Aryl Arenesulfonates. Adv. Syn. Catal. 2016, 358, 2072-2076.

(S13) Zheng, K.; Xiao, G.; Guo, T.; Ding, Y.; Wang, C.; Loh, T.-P.; Wu, X. Intermolecular Reductive Heck Reaction of Unactivated Aliphatic Alkenes with Organohalides. Org. Lett. 2020, 22, 694-699.

(S14) Standley, E. A.; Smith, S. J.; Müller, P.; Jamison, T. F. A Broadly Applicable Strategy for Entryinto Homogeneous Nickel(0) Catalysts from Air-Stable Nickel(II) Complexes. Organometallics 2014, 33, 2012-2018.

(S15) Agilent Technologies, CrysAlisPRO, Version 1.171.36.28, 2013.

(S16) Dolomanov, O. V.; Bourhis, L. J.; Gildea, R. J. OLEX2: a complete structure solution, refinement and analysis program. J. Appl. Cryst. 2009, 42, 339-341.

(S17) Kratzert, D.; Holstein, J. J.; Krossing, I. DSR: enhanced modelling and refinement of disordered structures with SHELXL. J. Appl. Cryst. 2015, 48, 933-938. 
S7. Copies of NMR Spectrum of All Compounds

${ }^{1} \mathrm{H}$ NMR $\left(\mathrm{C}_{6} \mathrm{D}_{6}\right)$ spectrum of compound $\mathbf{1 e}$ 


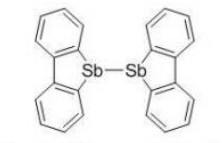

Chemical Formula: $\mathrm{C}_{24} \mathrm{H}_{16} \mathrm{Sb}_{2}$ Exact Mass: 545.9328

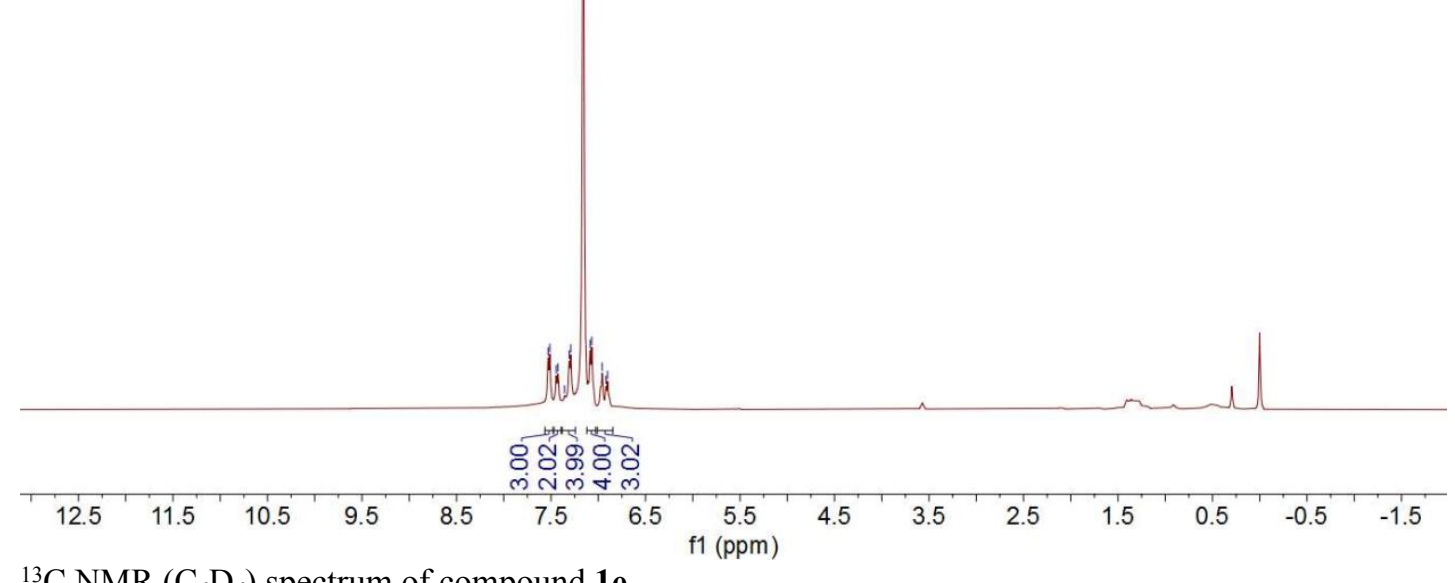

${ }^{13} \mathrm{C}$ NMR $\left(\mathrm{C}_{6} \mathrm{D}_{6}\right)$ spectrum of compound $\mathbf{1 e}$

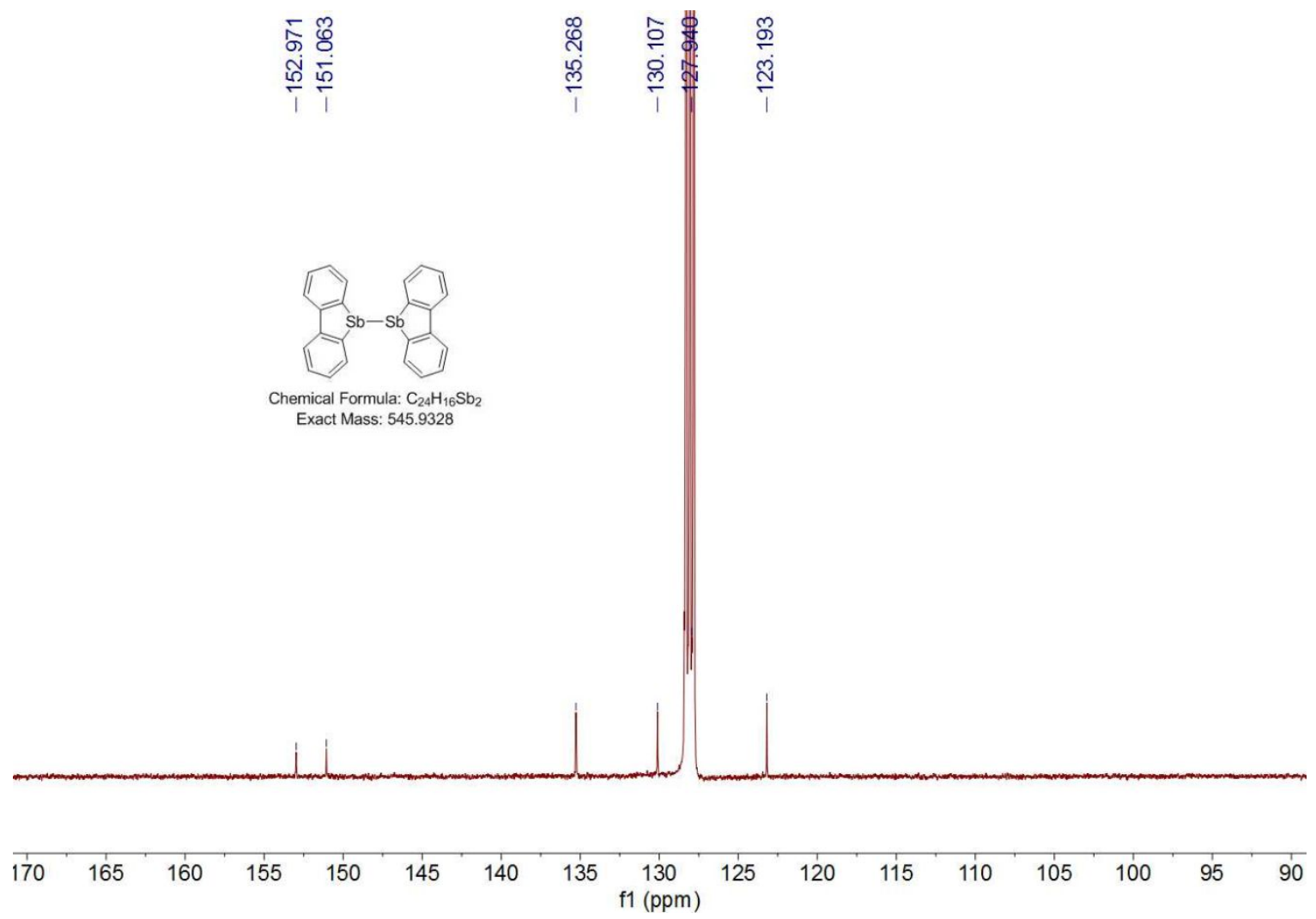

${ }^{1} \mathrm{H} \mathrm{NMR}\left(\mathrm{CDCl}_{3}\right)$ spectrum of compound $\mathbf{1 g}$ 

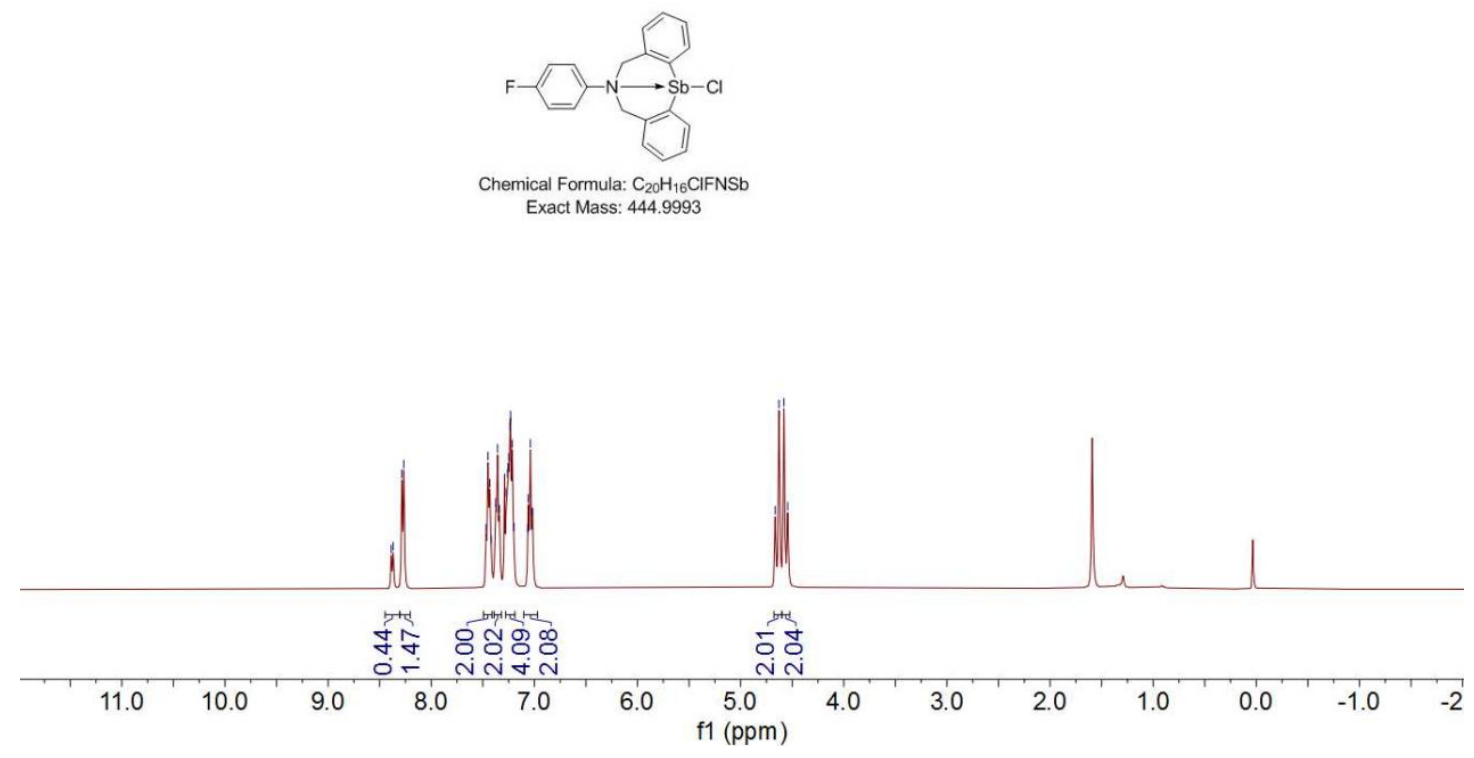

${ }^{13} \mathrm{C} \mathrm{NMR}\left(\mathrm{CDCl}_{3}\right)$ spectrum of compound $\mathbf{1 g}$
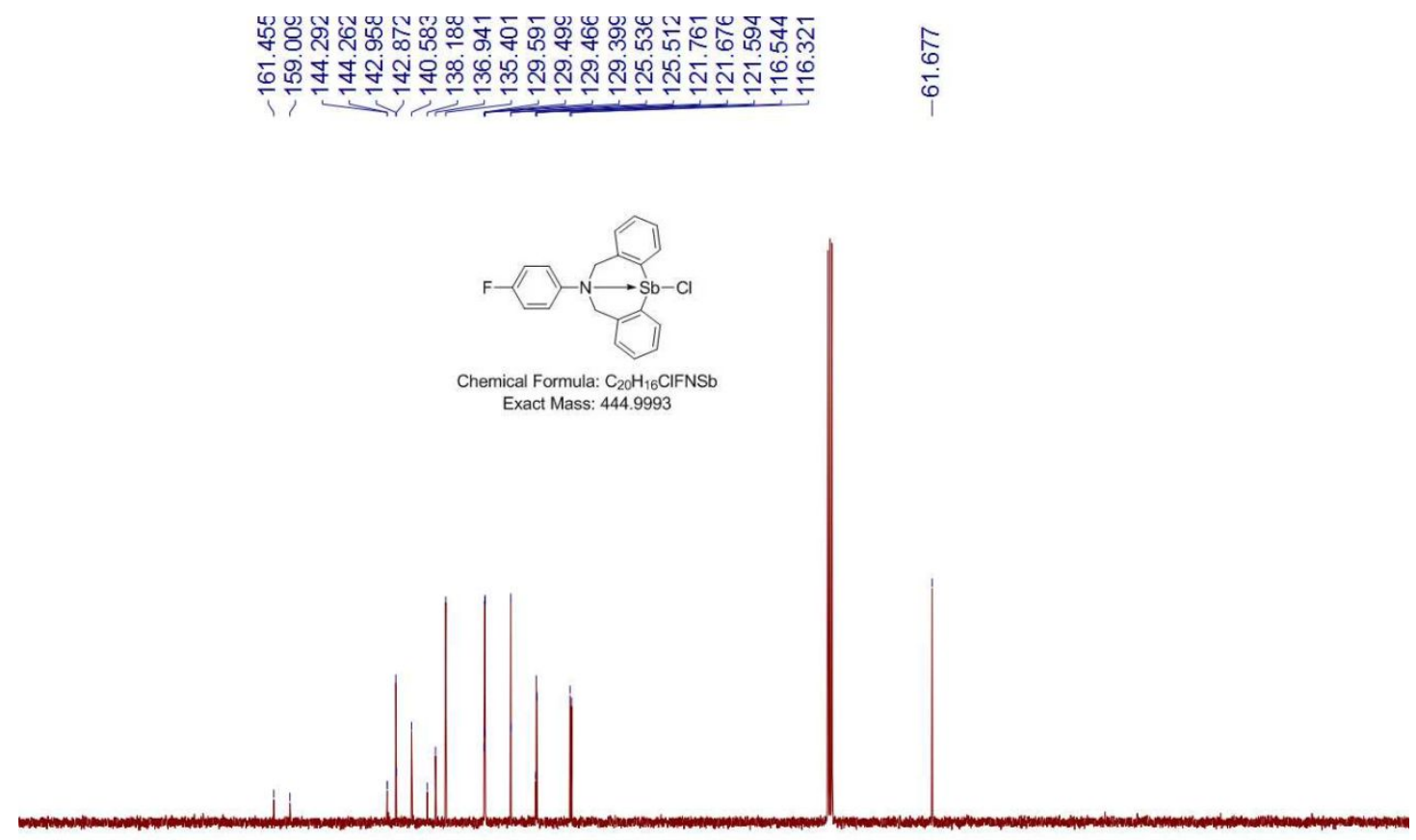

\begin{tabular}{rllllll|l|l|l|llllllllllllll}
\hline 0 & 190 & 180 & 170 & 160 & 150 & 140 & 130 & 120 & 110 & $\begin{array}{r}100 \\
\mathrm{f} 1(\mathrm{ppm})\end{array}$ & 90 & 80 & 70 & 60 & 50 & 40 & 30 & 20 & 10 & 0 & -1
\end{tabular}

${ }^{19} \mathrm{~F}$ NMR $\left(\mathrm{CDCl}_{3}\right)$ spectrum of compound $\mathbf{1 g}$ 


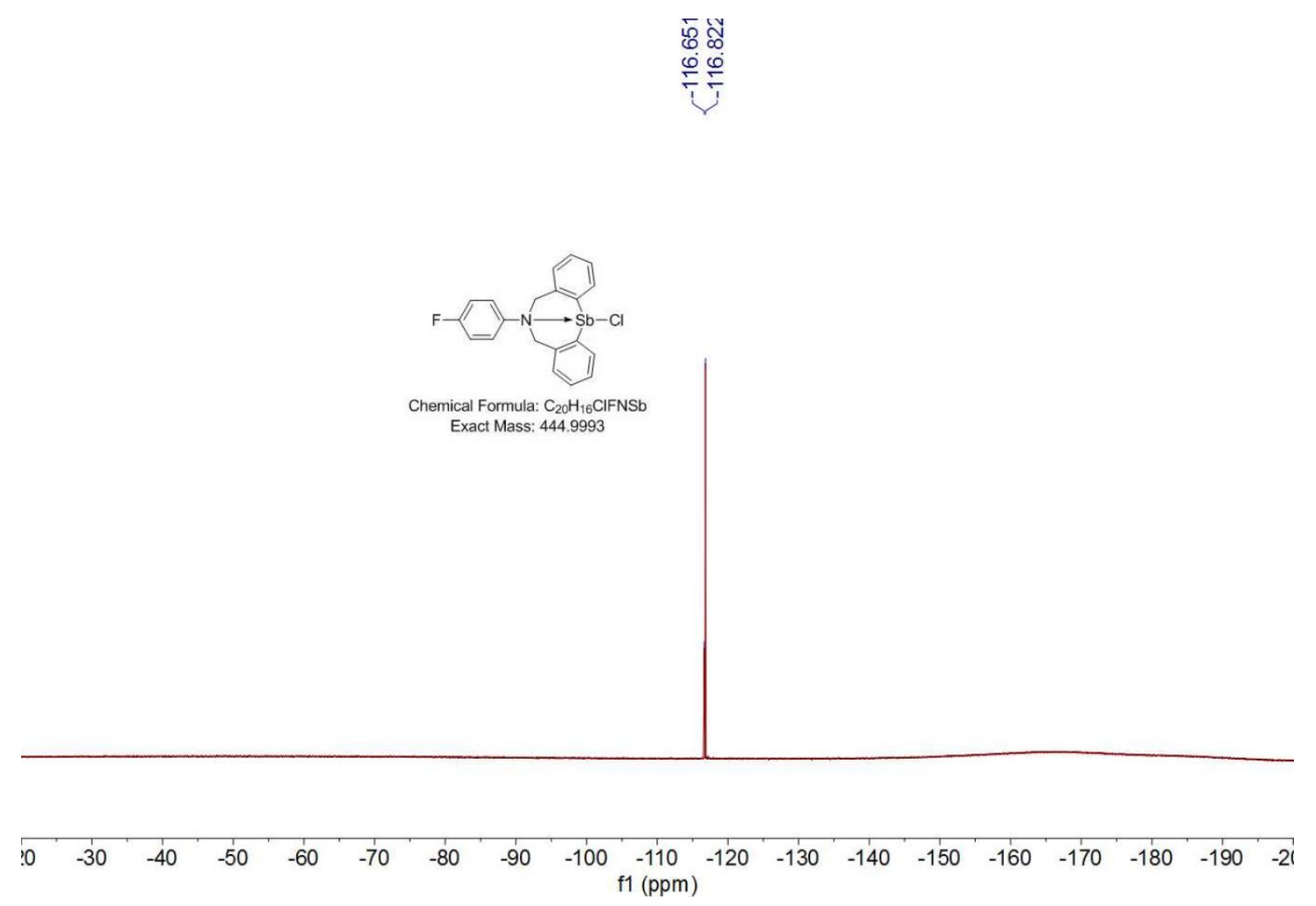

${ }^{1} \mathrm{H}$ NMR $\left(\mathrm{CDCl}_{3}\right)$ spectrum of compound Ni(II)-1

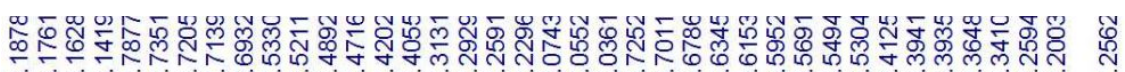

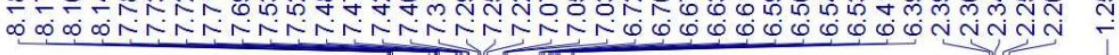

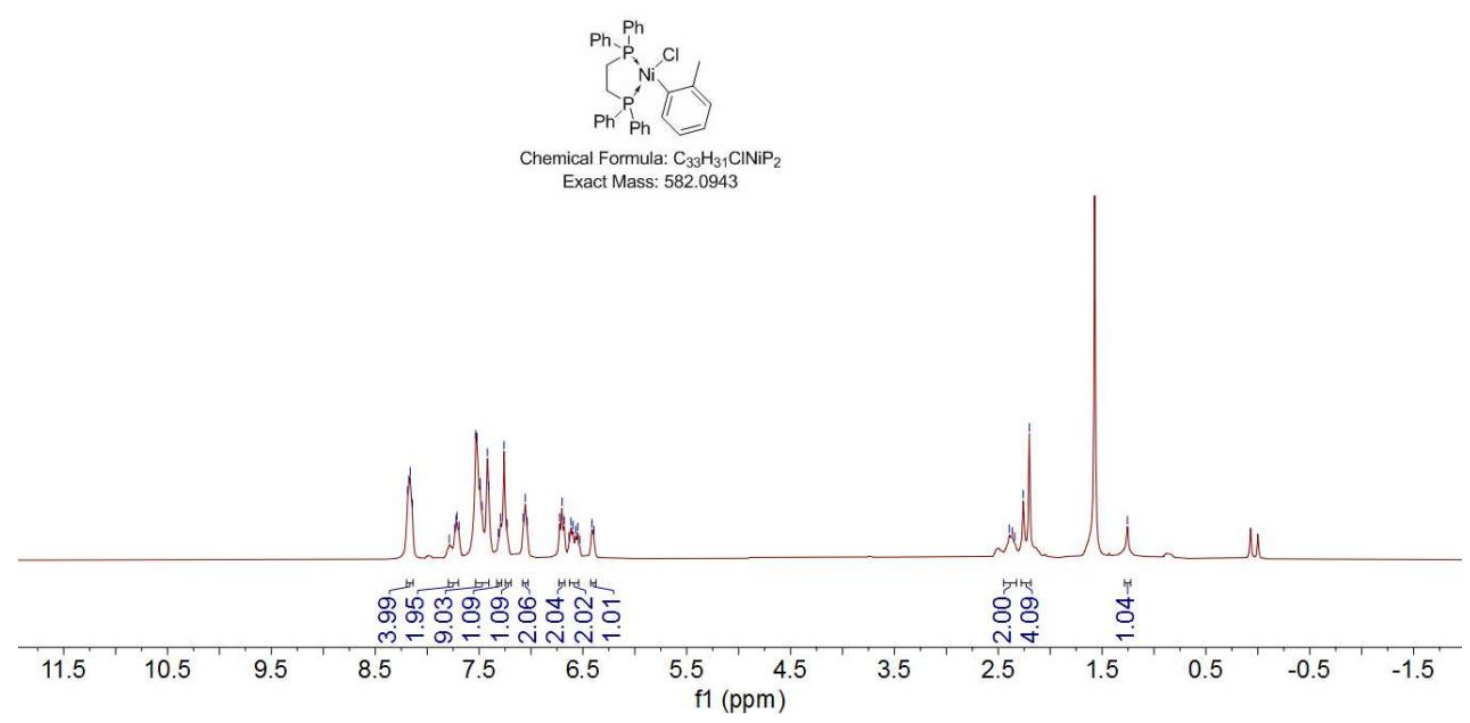

${ }^{1} \mathrm{H}$ NMR $\left(\mathrm{CDCl}_{3}\right)$ spectrum of compound $\mathbf{2 b a}$ 


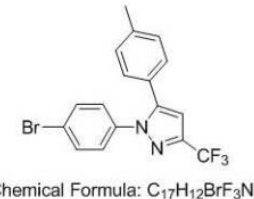

Exact Mass: 380.0136

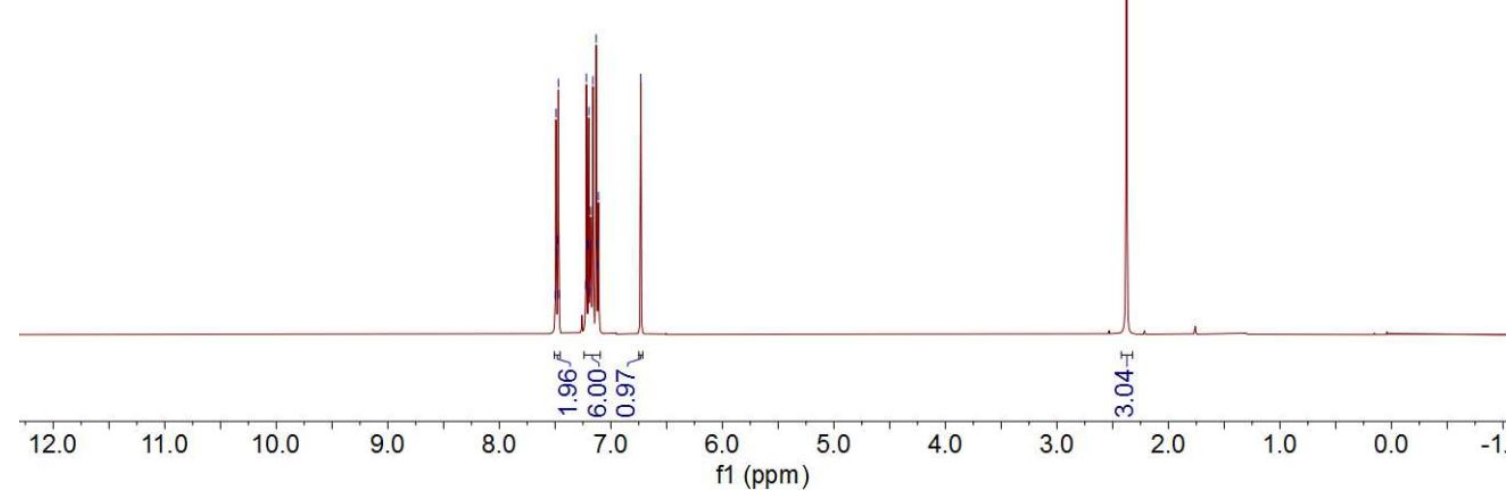

${ }^{13} \mathrm{C} \mathrm{NMR}\left(\mathrm{CDCl}_{3}\right)$ spectrum of compound $\mathbf{2 b a}$

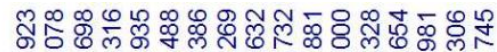

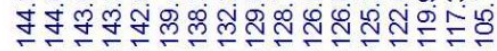

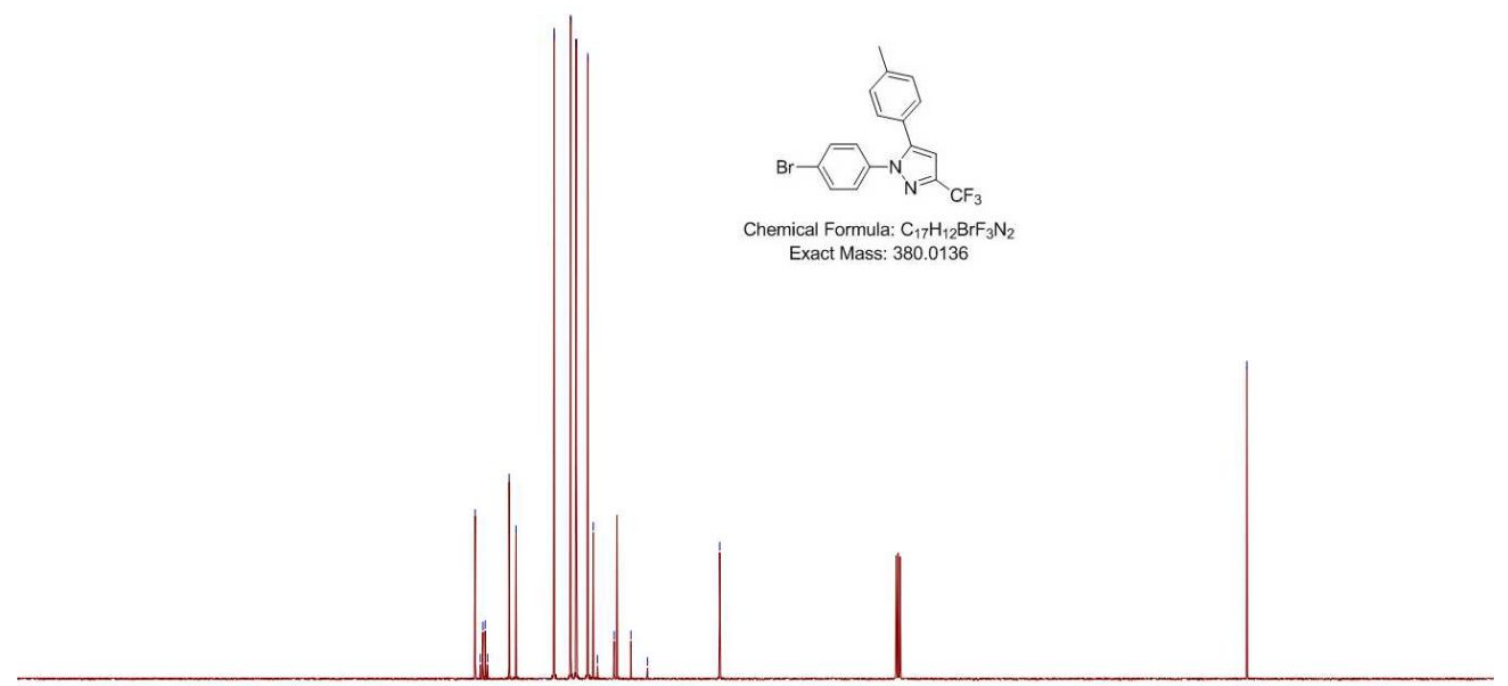

$\begin{array}{lllllllllllllllllllllllllll}210 & 200 & 190 & 180 & 170 & 160 & 150 & 140 & 130 & 120 & 110 & 100 & 90 & 80 & 70 & 60 & 50 & 40 & 30 & 20 & 10 & 0 & -10\end{array}$

${ }^{19} \mathrm{~F} \mathrm{NMR}\left(\mathrm{CDCl}_{3}\right)$ spectrum of compound $\mathbf{2 b a}$ 


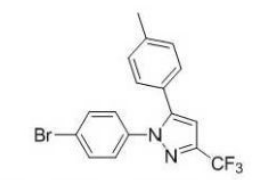

Chemical Formula: $\mathrm{C}_{17} \mathrm{H}_{12} \mathrm{BrF}_{3} \mathrm{~N}_{2}$ Exact Mass: 380.0136
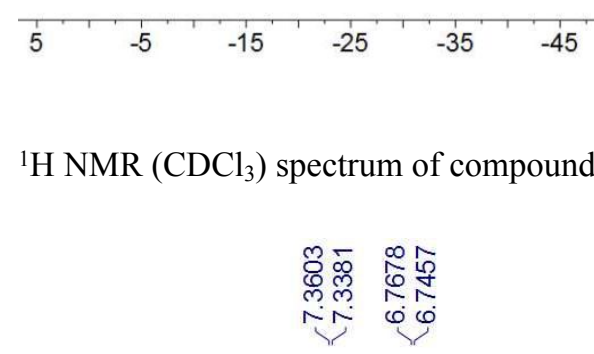

$\begin{array}{cc}-55 & -65 \\ & f 1(\mathrm{ppm})\end{array}$

$\begin{array}{lllllll}-75 & -85 & -95 & -105 & -115 & -125 & -13\end{array}$



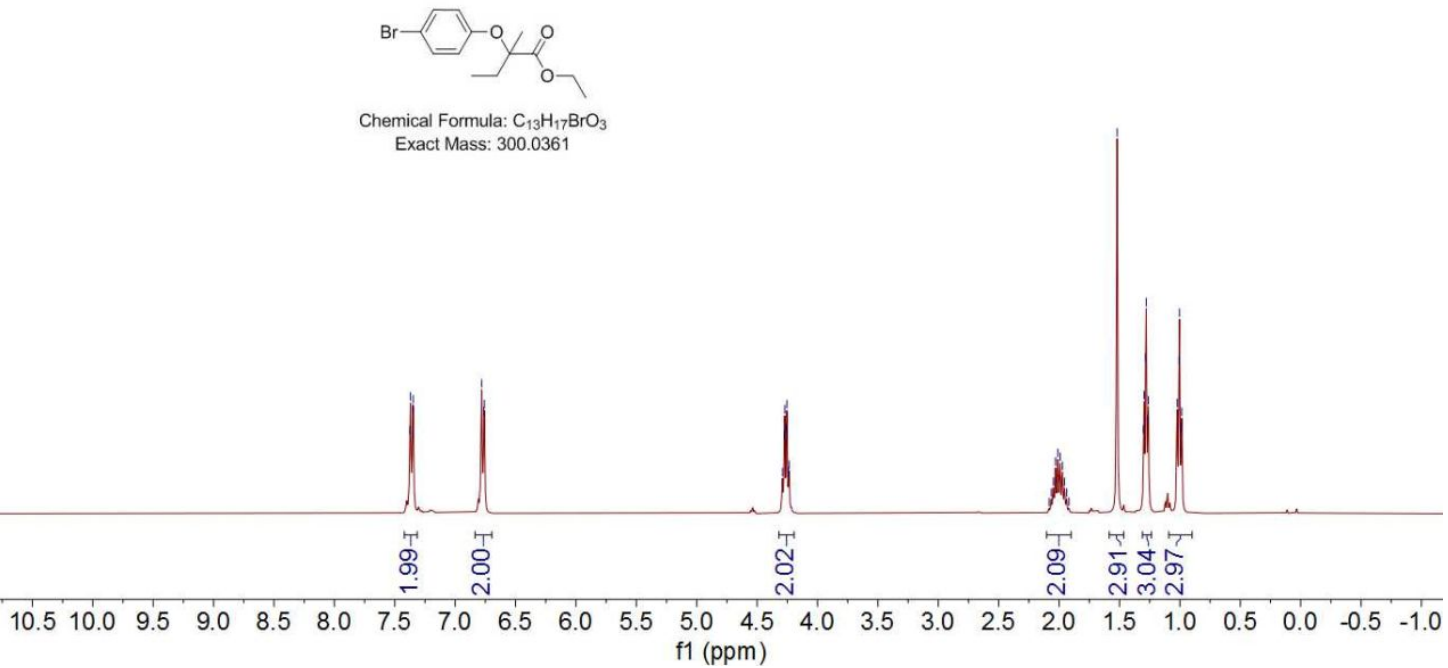

${ }^{1} \mathrm{H}$ NMR $\left(\mathrm{CDCl}_{3}\right)$ spectrum of compound $\mathbf{2 b d}$

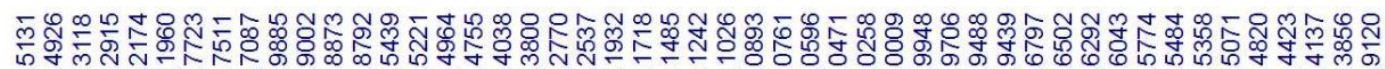

i m

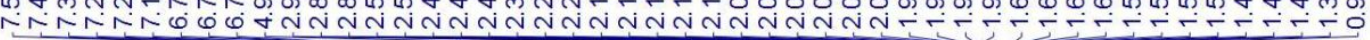
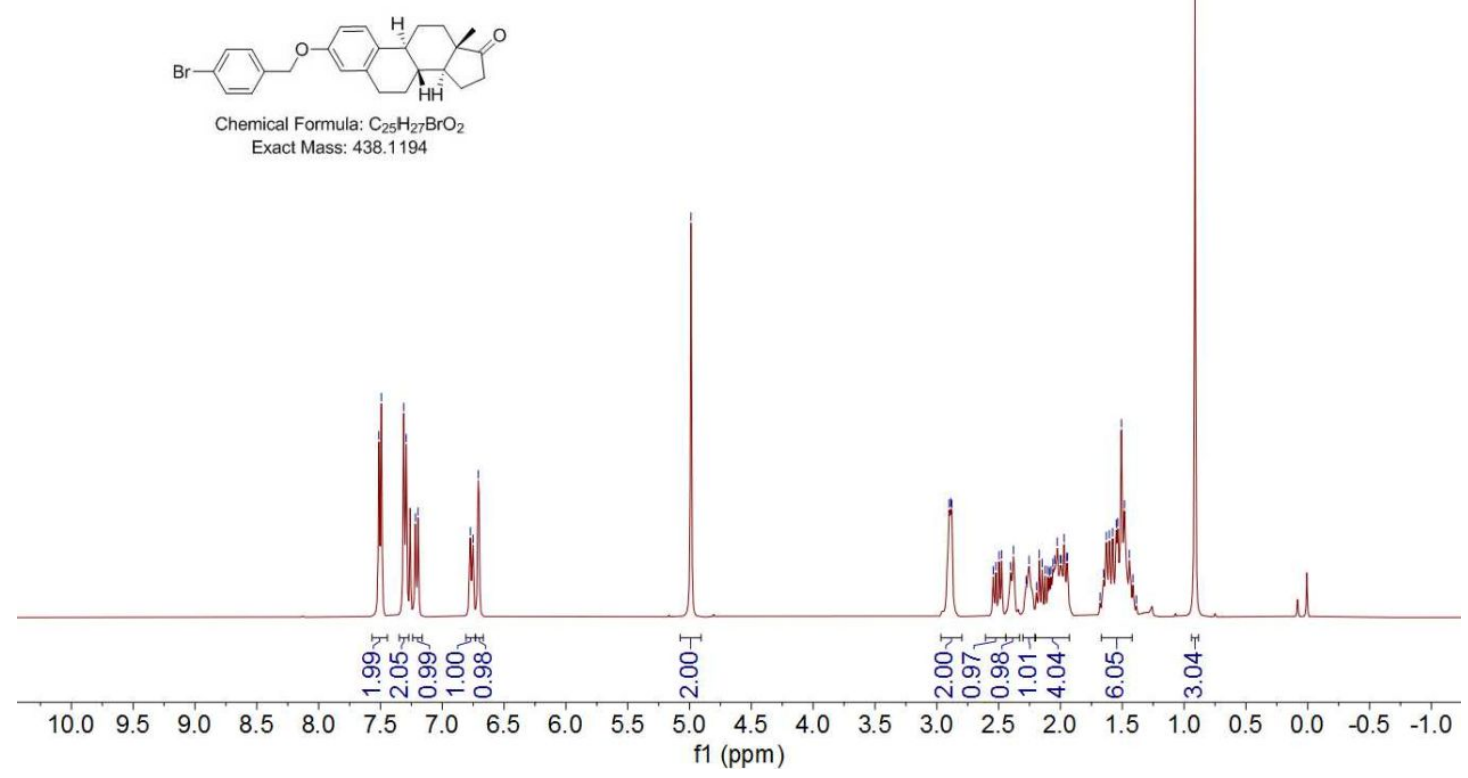

${ }^{13} \mathrm{C} \mathrm{NMR}\left(\mathrm{CDCl}_{3}\right)$ spectrum of compound $\mathbf{2} \mathbf{b d}$ 


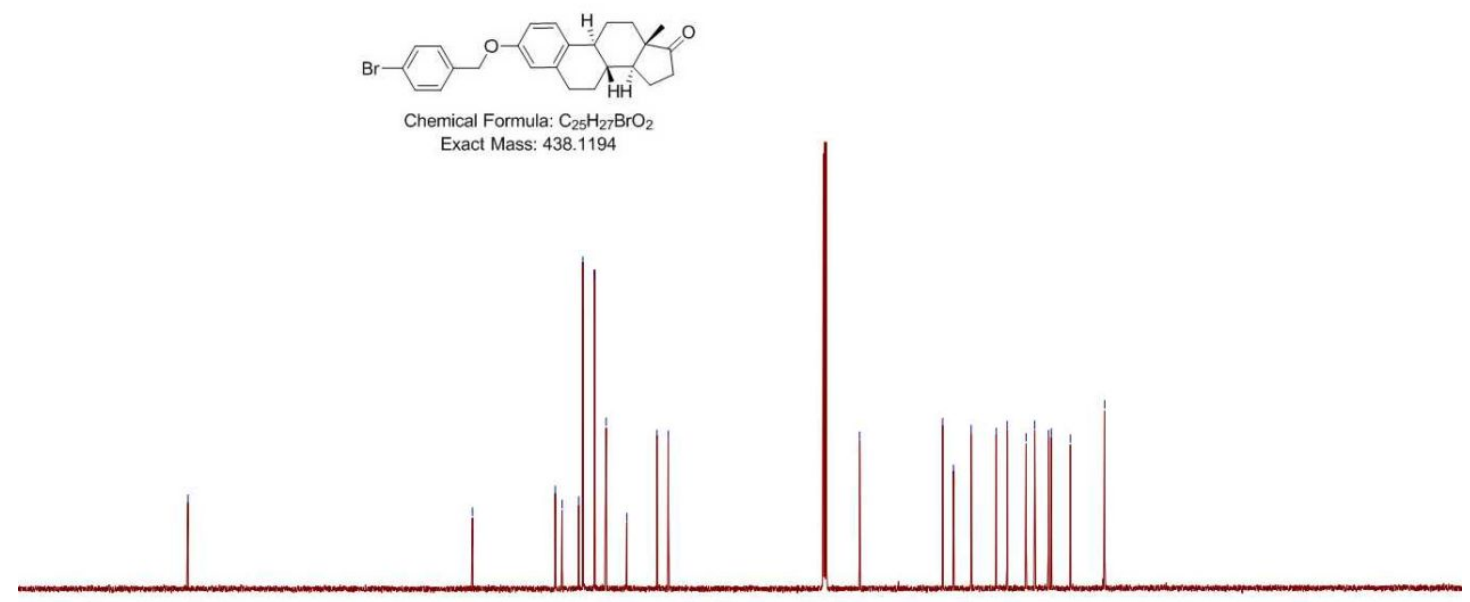

\begin{tabular}{llllllllllllllllllll}
\hline 240 & 220 & 200 & 180 & 160 & 140 & 120 & $\begin{array}{l}100 \\
\mathrm{f} 1(\mathrm{ppm})\end{array}$ & 80 & 60 & 40 & 20 & 0 & -20 & -40 & -60
\end{tabular}

${ }^{1} \mathrm{H}$ NMR $\left(\mathrm{CDCl}_{3}\right)$ spectrum of compound $2 \mathrm{cc}$

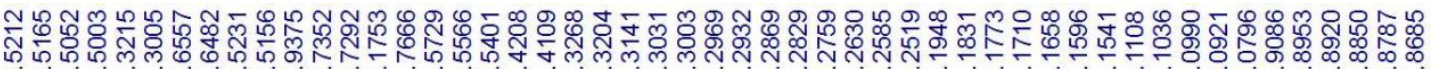

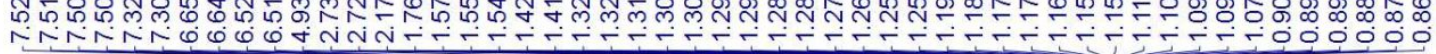
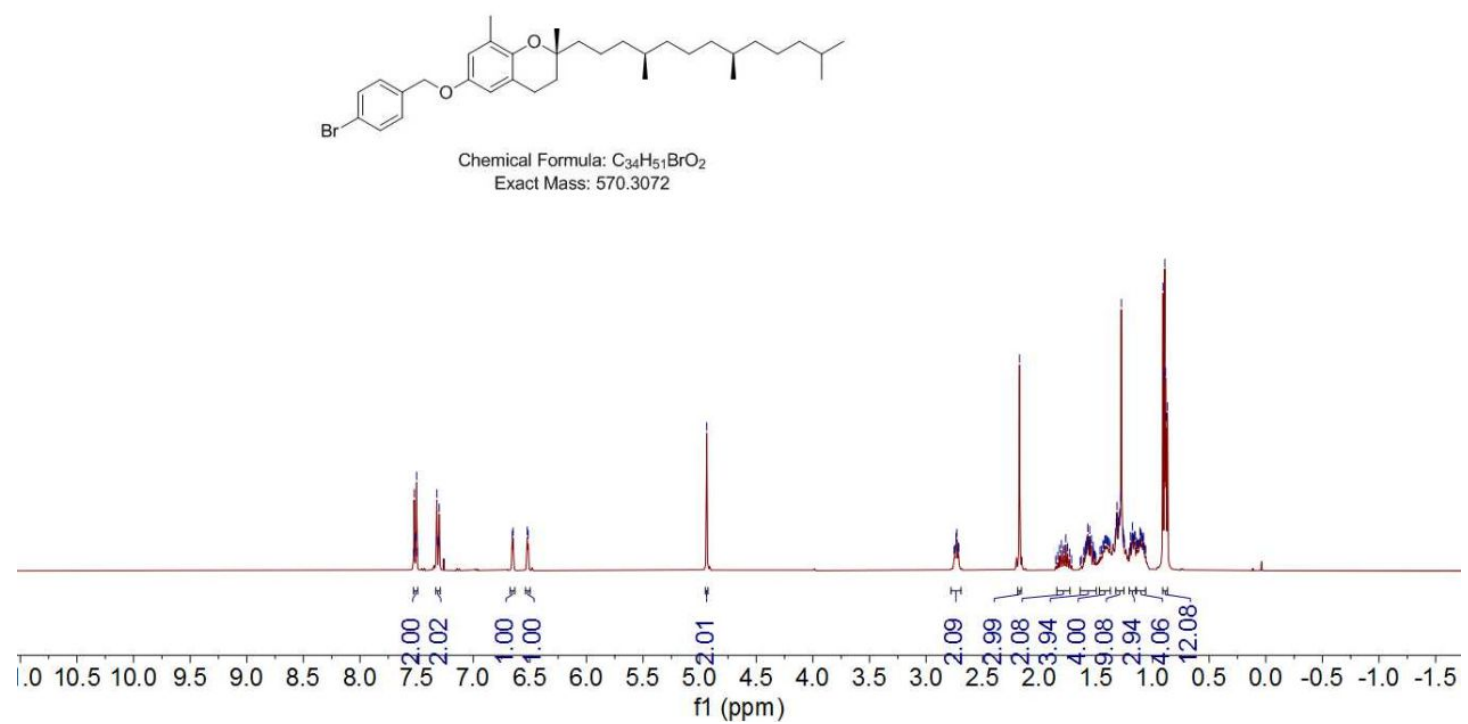

${ }^{13} \mathrm{C} \mathrm{NMR}\left(\mathrm{CDCl}_{3}\right)$ spectrum of compound $2 \mathrm{cc}$ 


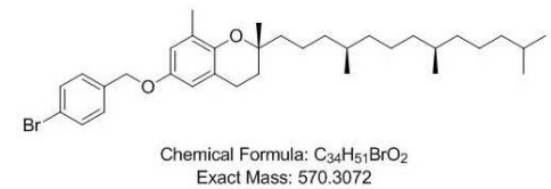
Exact Mass: 570.3072

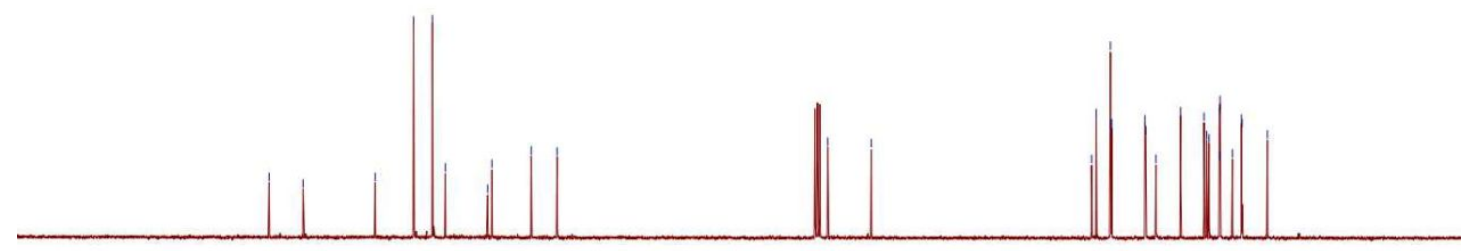

$\begin{array}{lllllllllllllllllll}180 & 170 & 160 & 150 & 140 & 130 & 120 & 110 & 100 & \begin{array}{c}90 \\ \mathrm{f} 1(\mathrm{ppm})\end{array} & 80 & 70 & 60 & 50 & 40 & 30 & 20 & 10 & 0\end{array}$

${ }^{1} \mathrm{H}$ NMR $\left(\mathrm{CDCl}_{3}\right)$ spectrum of compound $\mathbf{2 d a}$
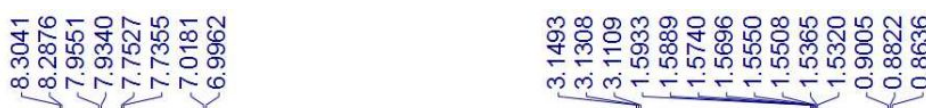

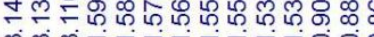

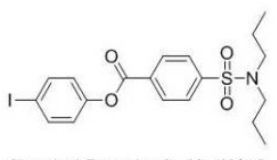

Chemical Formula: $\mathrm{C}_{19} \mathrm{H}_{22} 2 \mathrm{NO}_{4} \mathrm{~S}$ Exact Mass: 487.0314

${ }^{13} \mathrm{C}$ NMR $\left(\mathrm{CDCl}_{3}\right)$ spectrum of compound $2 \mathrm{da}$ 


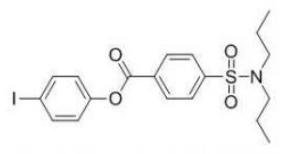

Chemical Formula: $\mathrm{C}_{19} \mathrm{H}_{22} \mathrm{INO}_{4} \mathrm{~S}$

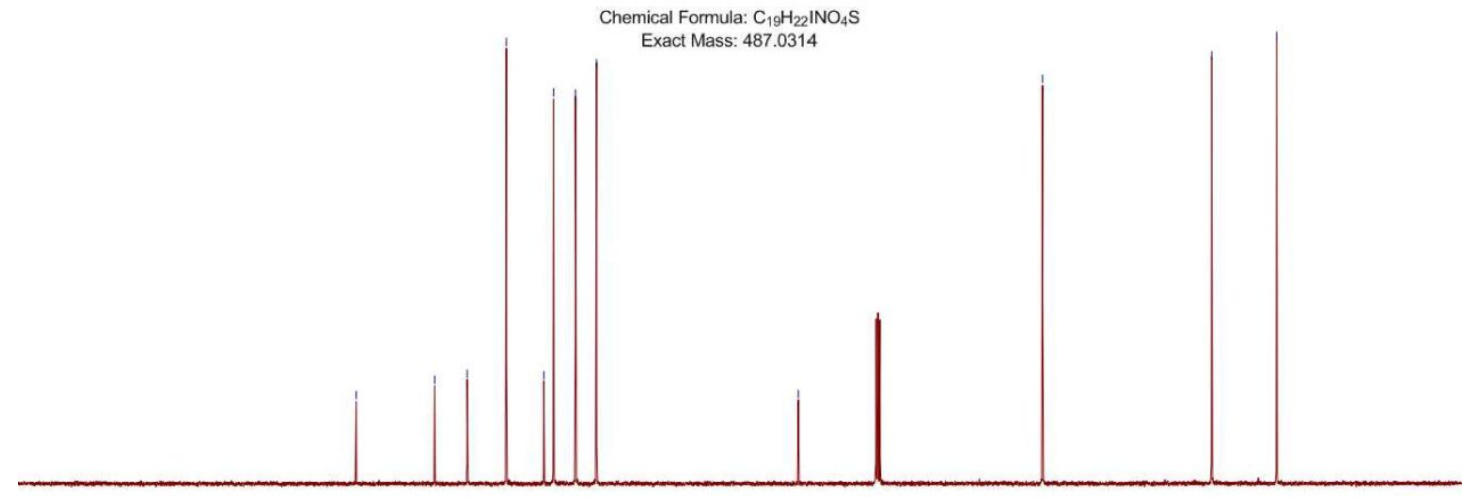

$\begin{array}{llllllllllllllllllllllllllll}210 & 200 & 190 & 180 & 170 & 160 & 150 & 140 & 130 & 120 & 110 & 100 & 90 & 80 & 70 & 60 & 50 & 40 & 30 & 20 & 10 & 0 & -10\end{array}$ f1 (ppm)

${ }^{1} \mathrm{H}$ NMR $\left(\mathrm{CDCl}_{3}\right)$ spectrum of compound $\mathbf{2 d b}$
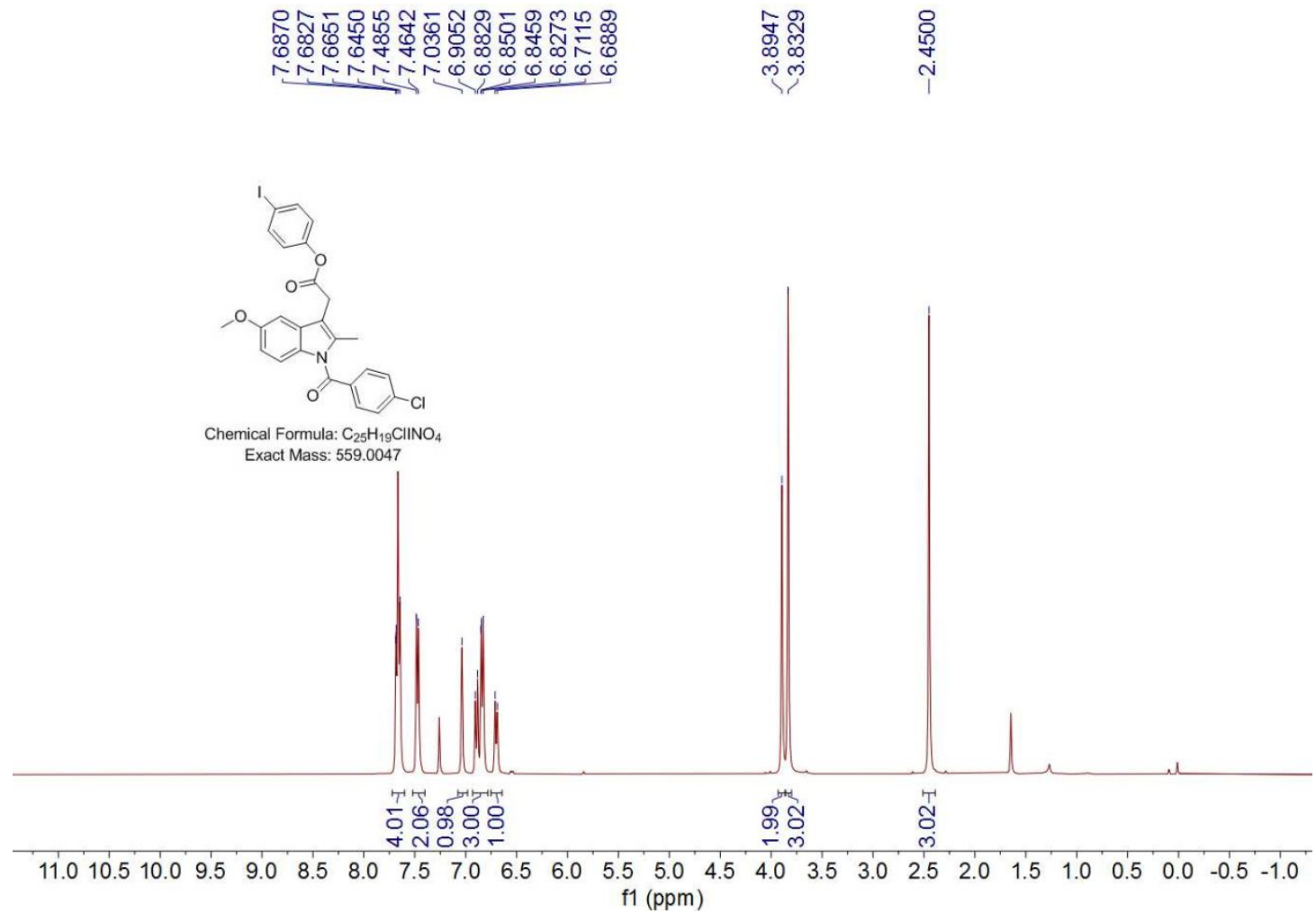

${ }^{13} \mathrm{C} \mathrm{NMR}\left(\mathrm{CDCl}_{3}\right)$ spectrum of compound $\mathbf{2 d \mathbf { b }}$ 


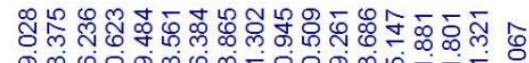

ஜ은

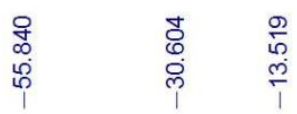

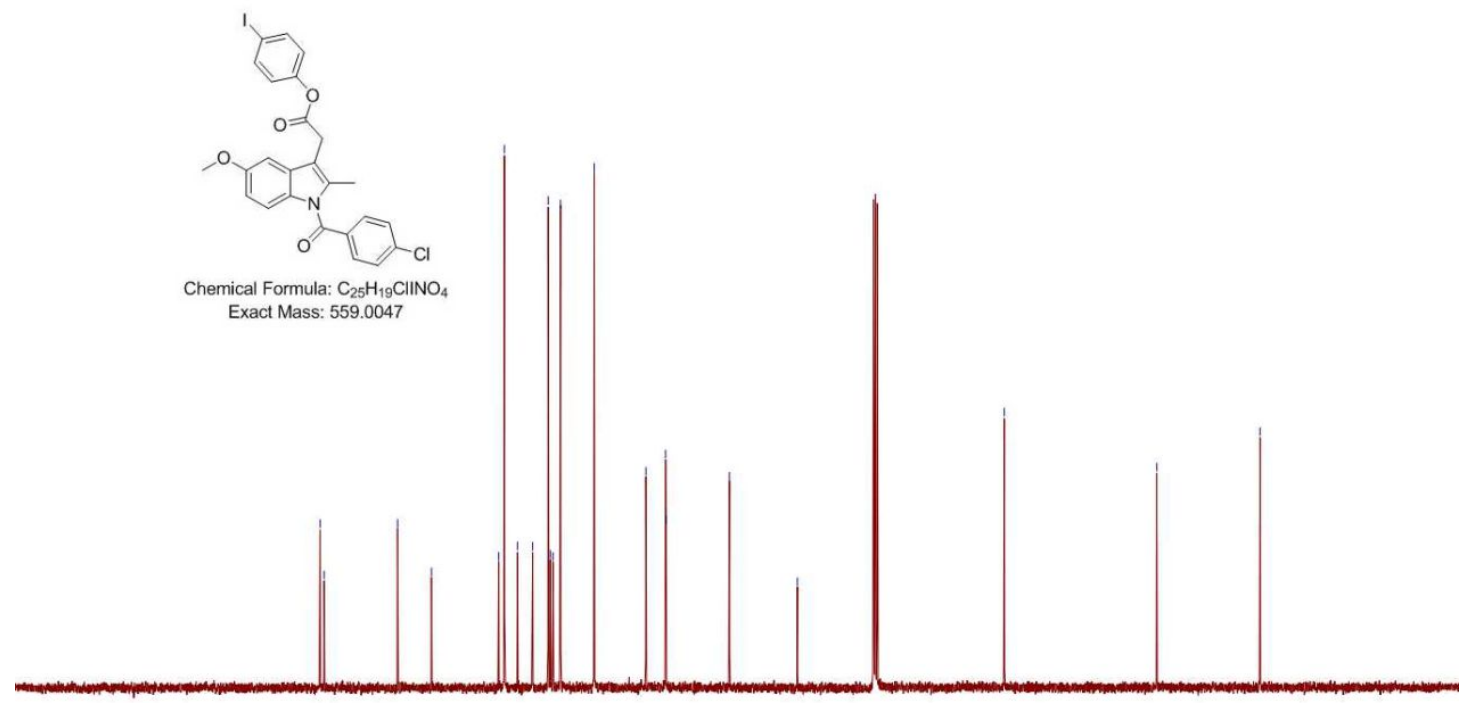

$\begin{array}{llllllllllllllllllllllllll}210 & 200 & 190 & 180 & 170 & 160 & 150 & 140 & 130 & 120 & 110 & 100 & 90 & 80 & 70 & 60 & 50 & 40 & 30 & 20 & 10 & 0 & -10\end{array}$ f1 (ppm)

${ }^{1} \mathrm{H}$ NMR $\left(\mathrm{CDCl}_{3}\right)$ spectrum of compound $\mathbf{2 d c}$
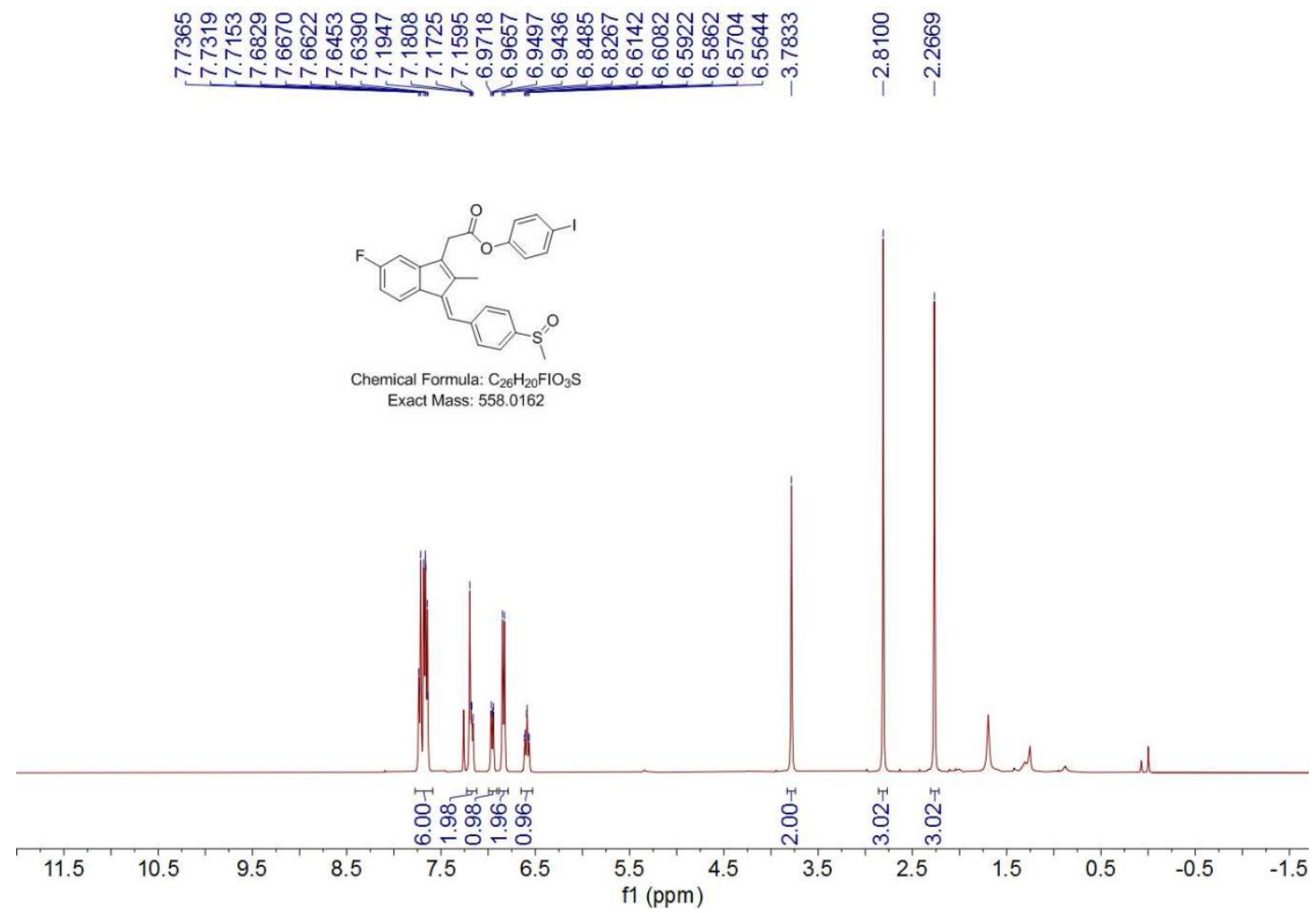

${ }^{13} \mathrm{C}$ NMR $\left(\mathrm{CDCl}_{3}\right)$ spectrum of compound $\mathbf{2 d c}$ 


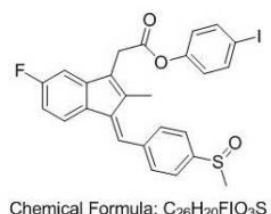

Exact Mass: 558.0162

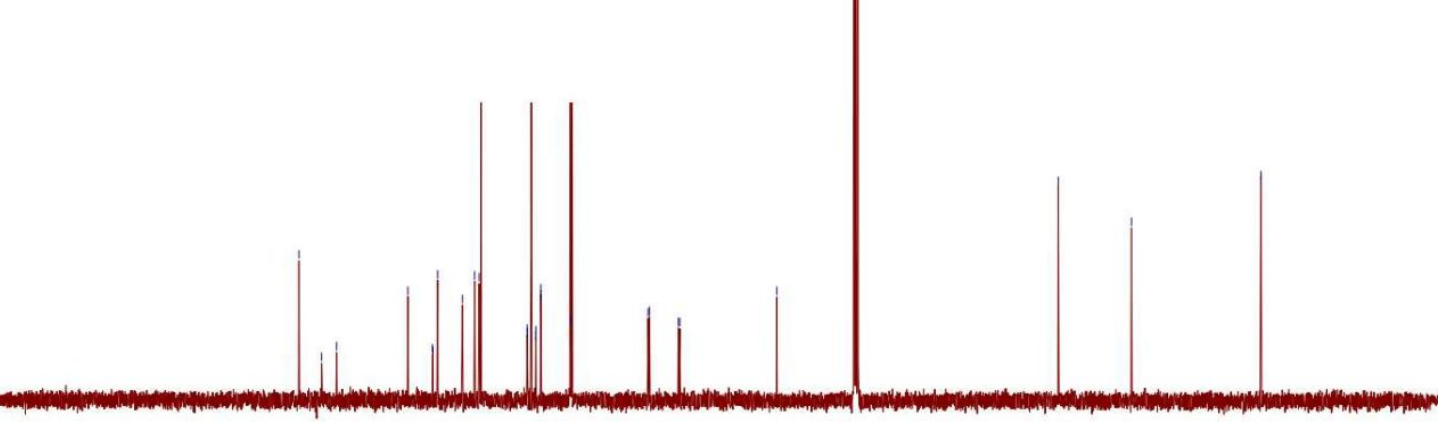

$\begin{array}{lllllllllllllllllllllllllllll}210 & 200 & 190 & 180 & 170 & 160 & 150 & 140 & 130 & 120 & 110 & 100 & 90 & 80 & 70 & 60 & 50 & 40 & 30 & 20 & 10 & 0 & -10\end{array}$ f1 (ppm)

${ }^{19} \mathrm{~F}$ NMR $\left(\mathrm{CDCl}_{3}\right)$ spectrum of compound $\mathbf{2 d c}$

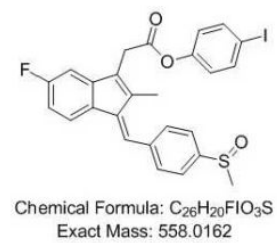

$\begin{array}{llllllllllllllllllllllllllllllll}10 & 0 & -10 & -20 & -30 & -40 & -50 & -60 & -70 & -80 & -90 & -100 & -110 & -120 & -130 & -140 & -150 & -160 & -170 & -180 & -190 & -200 & -210\end{array}$

${ }^{1} \mathrm{H}$ NMR $\left(\mathrm{CDCl}_{3}\right)$ spectrum of compound $\mathbf{2 d d}$ 


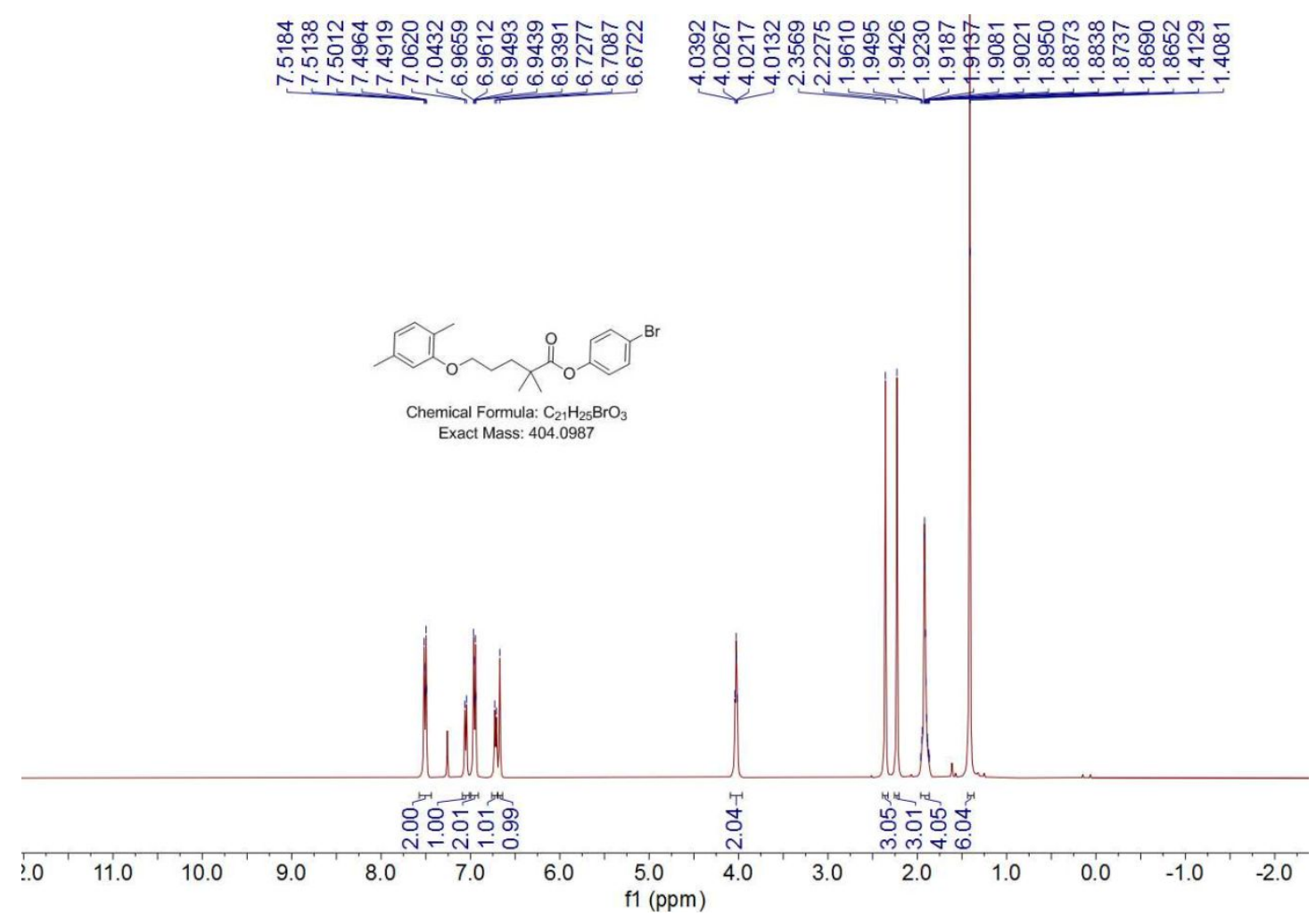

${ }^{13} \mathrm{C}$ NMR $\left(\mathrm{CDCl}_{3}\right)$ spectrum of compound $\mathbf{2 d d}$

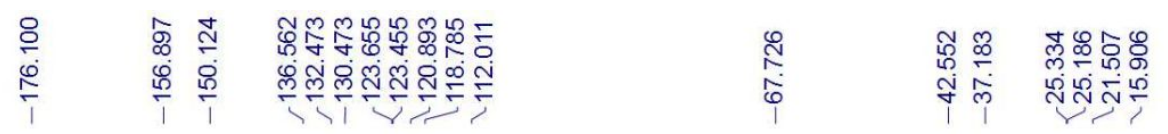

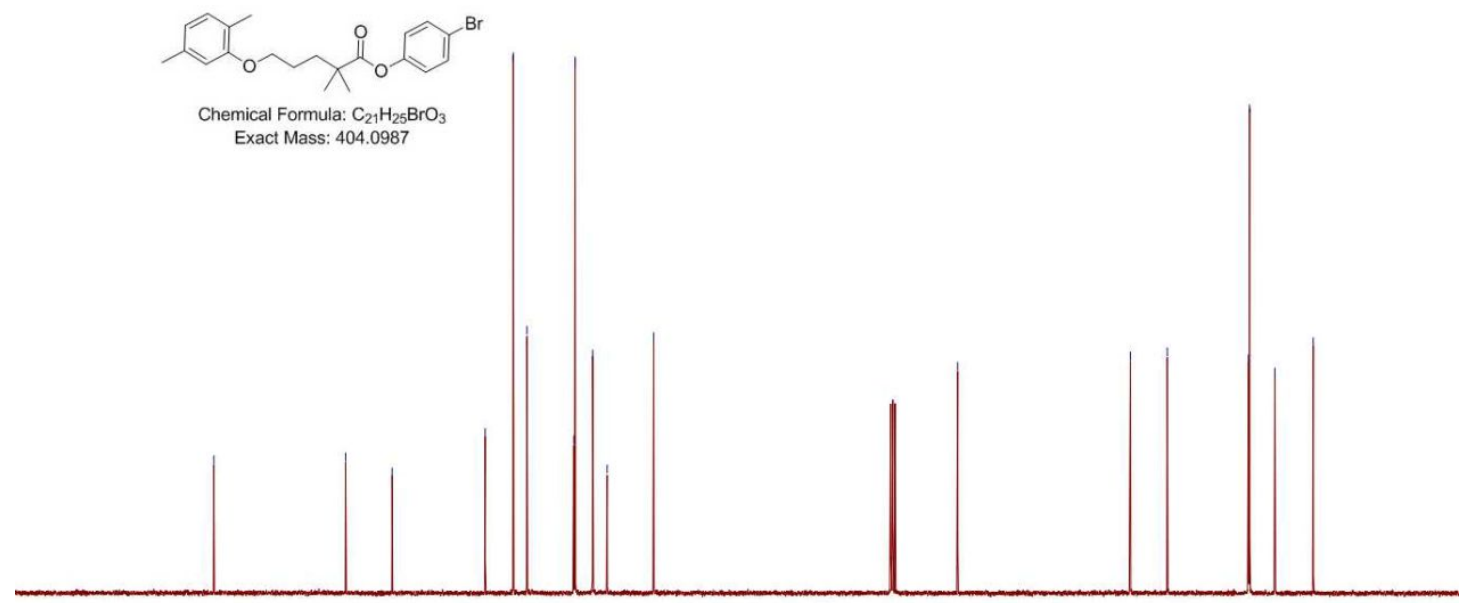

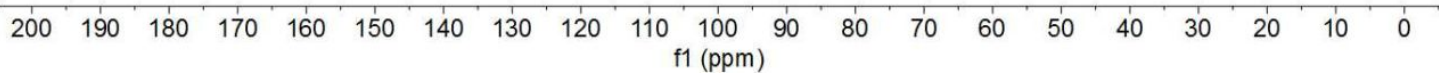

${ }^{1} \mathrm{H}$ NMR $\left(\mathrm{CDCl}_{3}\right)$ spectrum of compound 2 de 


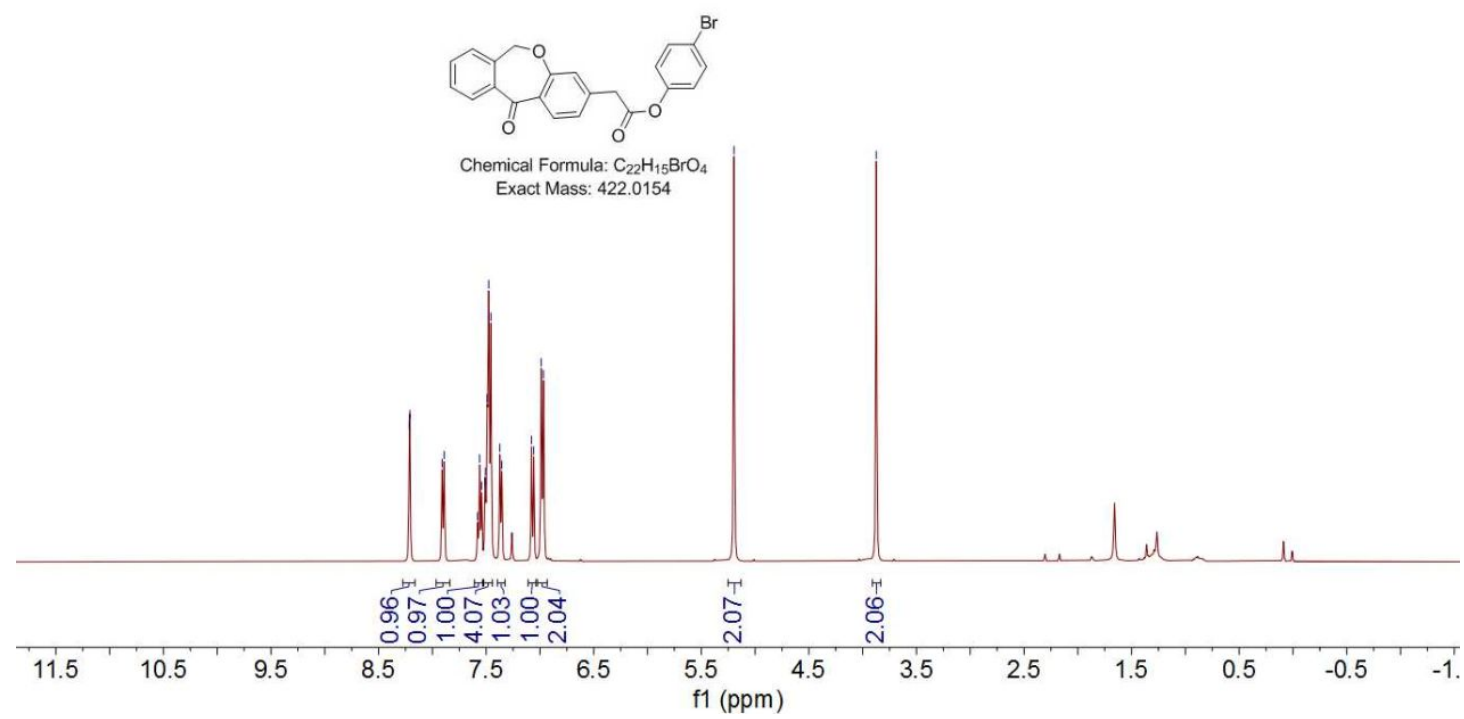

${ }^{13} \mathrm{C} \mathrm{NMR}\left(\mathrm{CDCl}_{3}\right)$ spectrum of compound $2 \mathrm{de}$
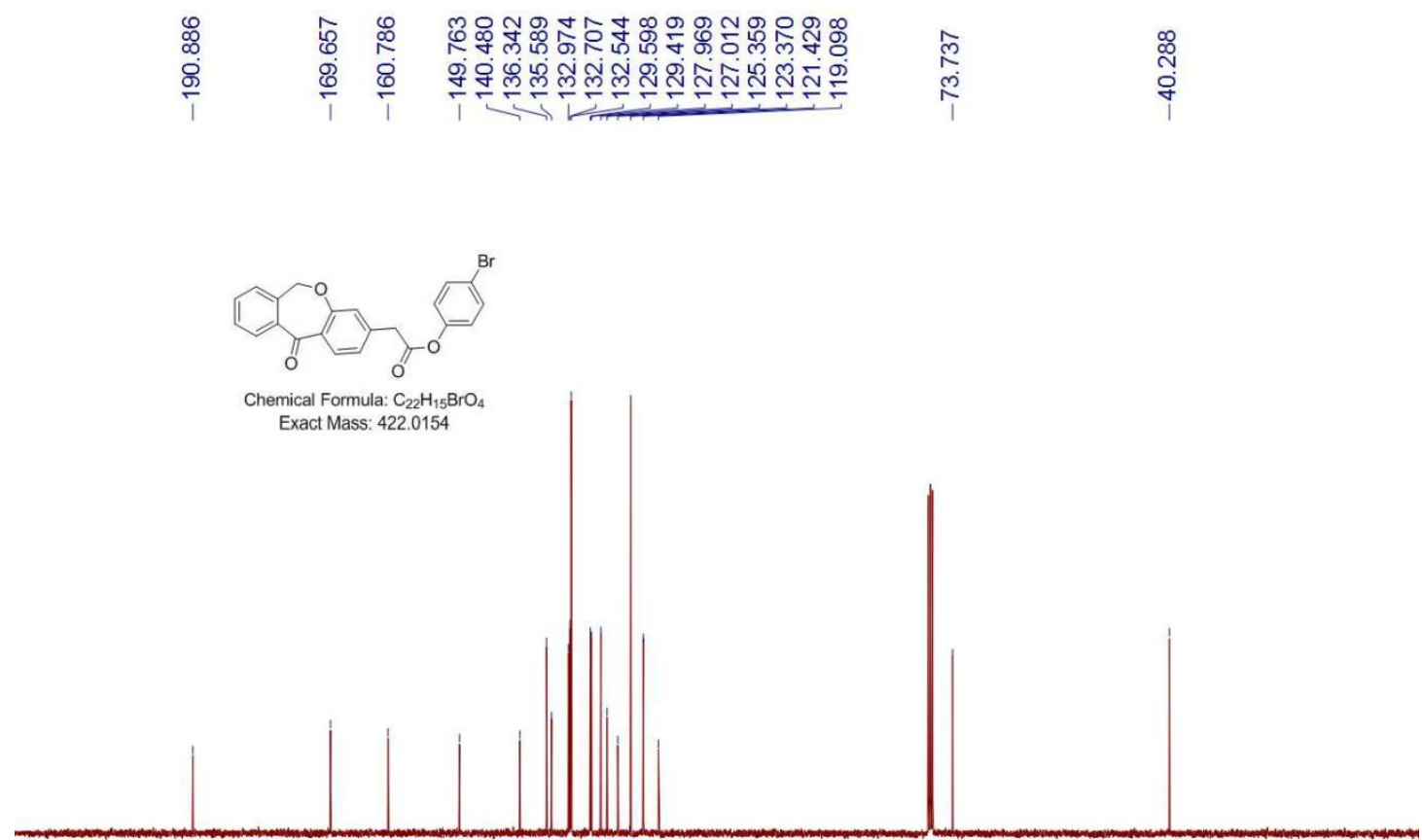

$\begin{array}{lllllllllllllllllllll}210 & 200 & 190 & 180 & 170 & 160 & 150 & 140 & 130 & 120 & \begin{array}{c}110 \\ \mathrm{f} 1(\mathrm{ppm})\end{array} & 100 & 90 & 80 & 70 & 60 & 50 & 40 & 30 & 20 & 10\end{array}$

${ }^{1} \mathrm{H}$ NMR $\left(\mathrm{CDCl}_{3}\right)$ spectrum of compound $\mathbf{2 d f}$ 


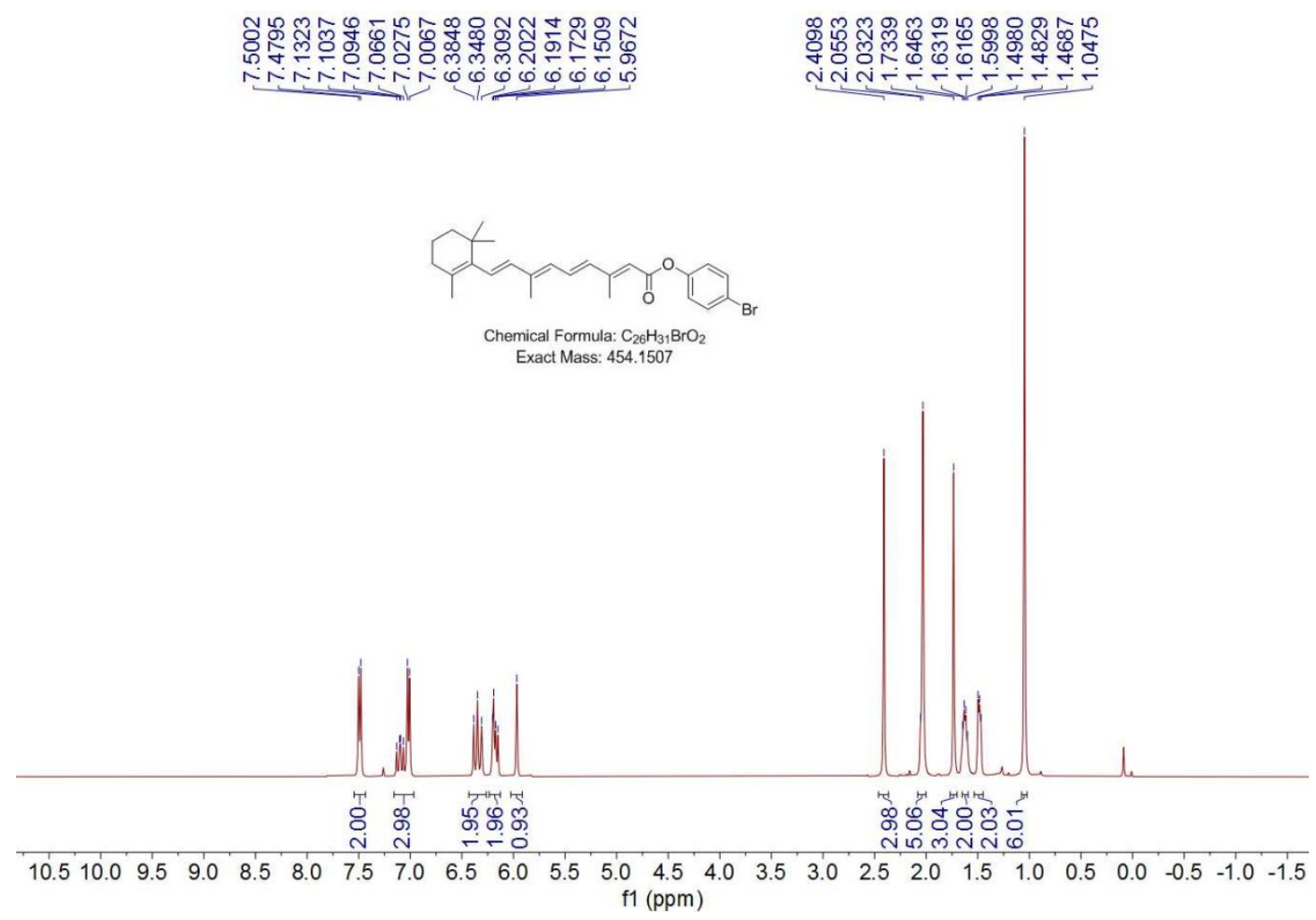

${ }^{13} \mathrm{C}$ NMR $\left(\mathrm{CDCl}_{3}\right)$ spectrum of compound $\mathbf{2 d f}$

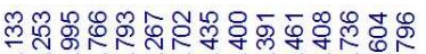

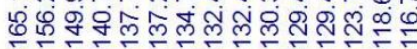

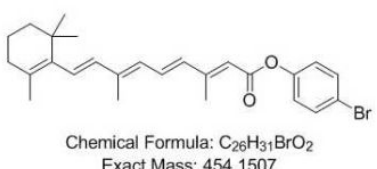

Exact Mass: 454.1507

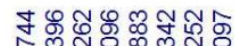

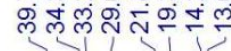

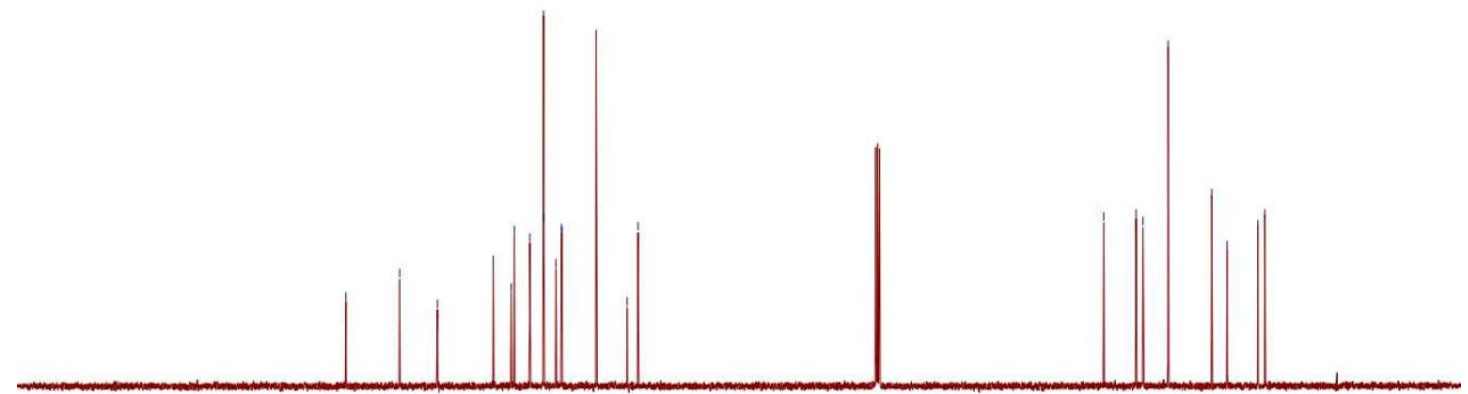

$\begin{array}{llllllllllllllllllllllll}210 & 200 & 190 & 180 & 170 & 160 & 150 & 140 & 130 & 120 & 110 & 100 & 90 & 80 & 70 & 60 & 50 & 40 & 30 & 20 & 10 & 0 & -10\end{array}$ $\mathrm{f} 1(\mathrm{ppm})$

${ }^{1} \mathrm{H}$ NMR $\left(\mathrm{CDCl}_{3}\right)$ spectrum of compound 3a 


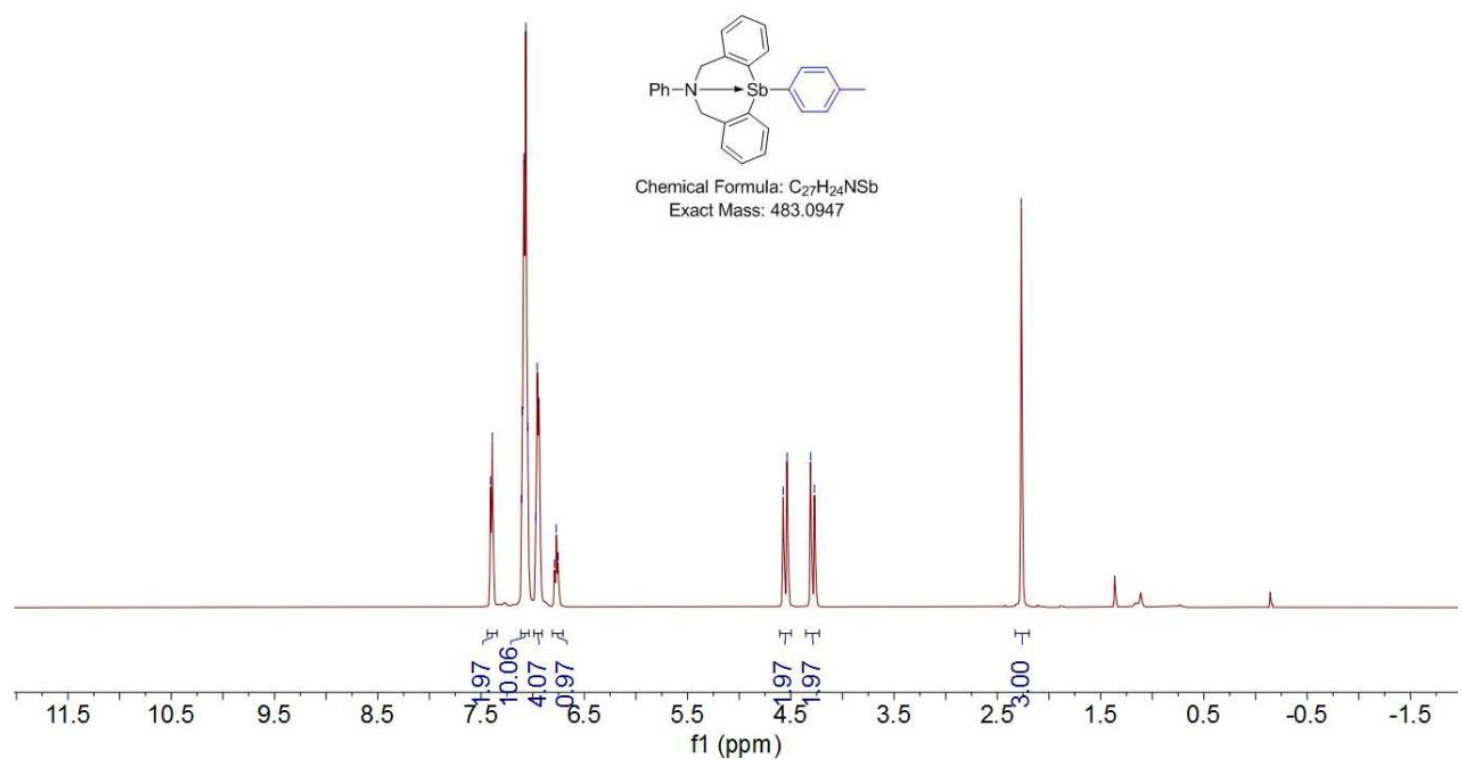

${ }^{13} \mathrm{C}$ NMR $\left(\mathrm{CDCl}_{3}\right)$ spectrum of compound $\mathbf{3 a}$

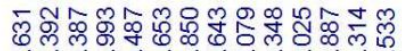

过过宓品
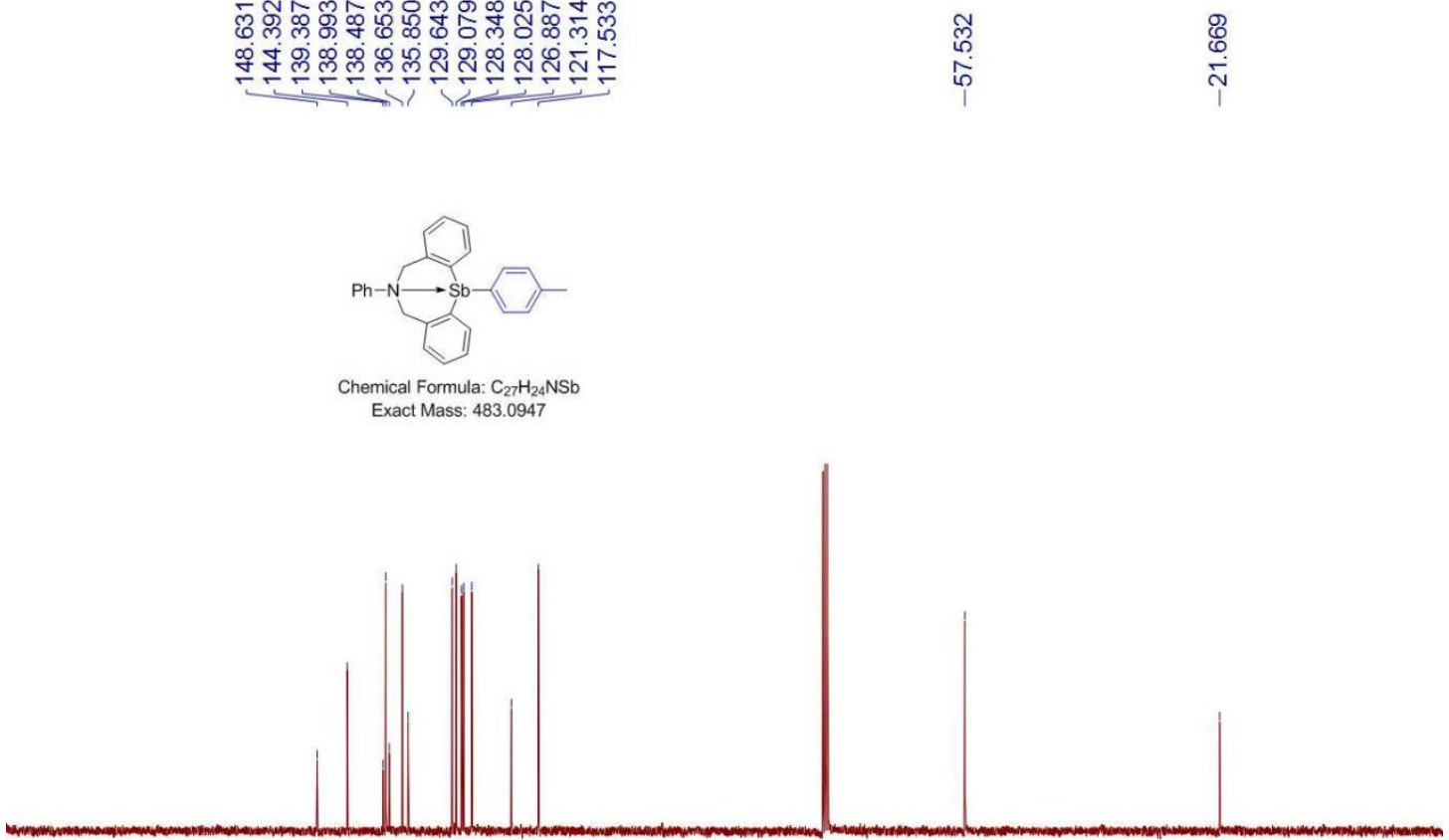

$\begin{array}{llllllllllllllllllll}190 & 180 & 170 & 160 & 150 & 140 & 130 & 120 & 110 & 100 & 90 & 80 & 70 & 60 & 50 & 40 & 30 & 20 & 10 & 0\end{array}$

${ }^{1} \mathrm{H}$ NMR $\left(\mathrm{CDCl}_{3}\right)$ spectrum of compound $\mathbf{3 b}$ 


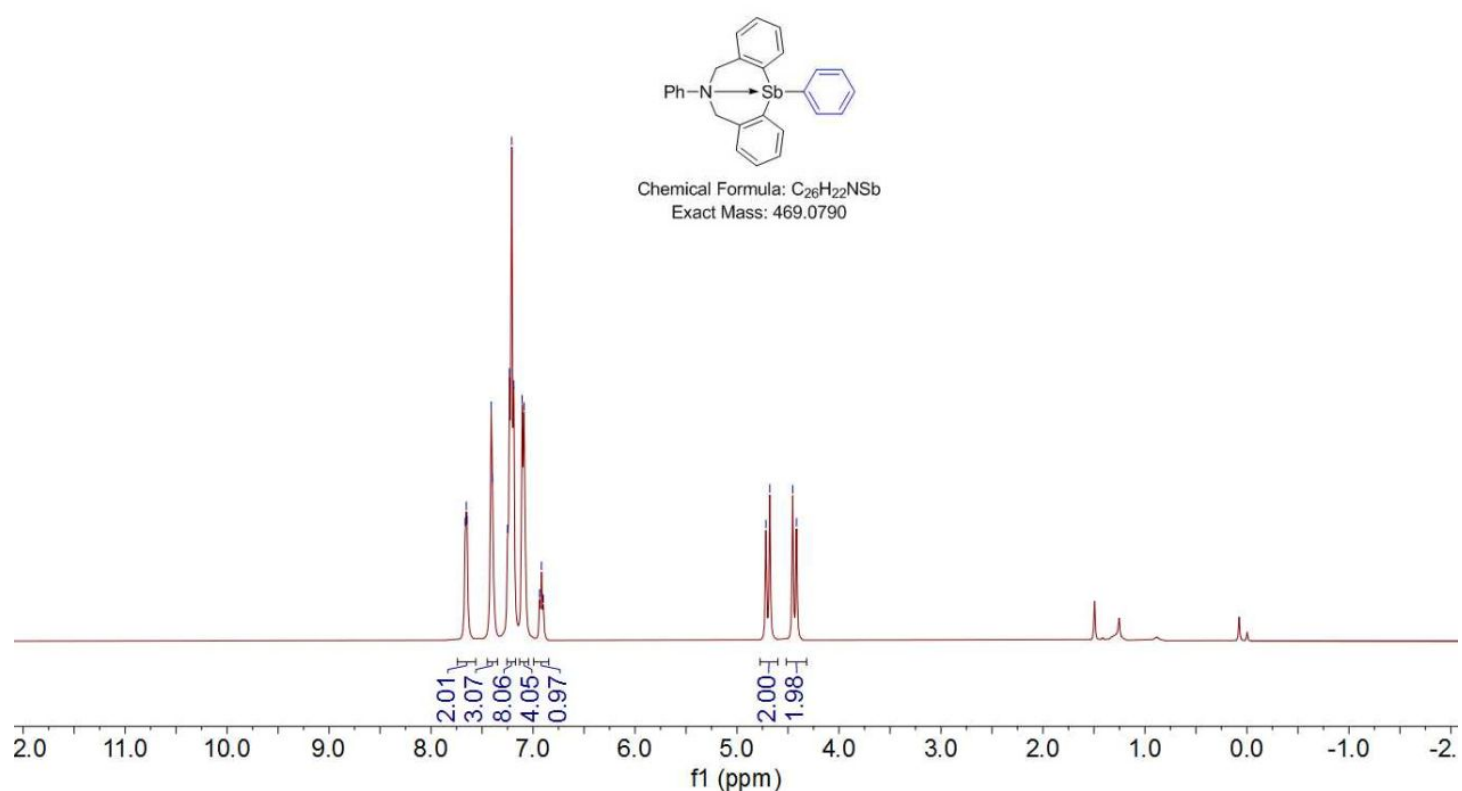

${ }^{13} \mathrm{C}$ NMR $\left(\mathrm{CDCl}_{3}\right)$ spectrum of compound $\mathbf{3 b}$

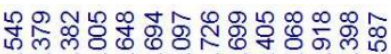

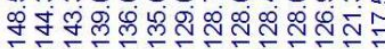

?ำ

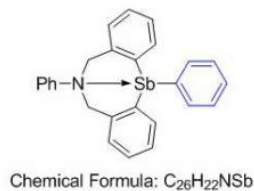

Exact Mass: 469.0790

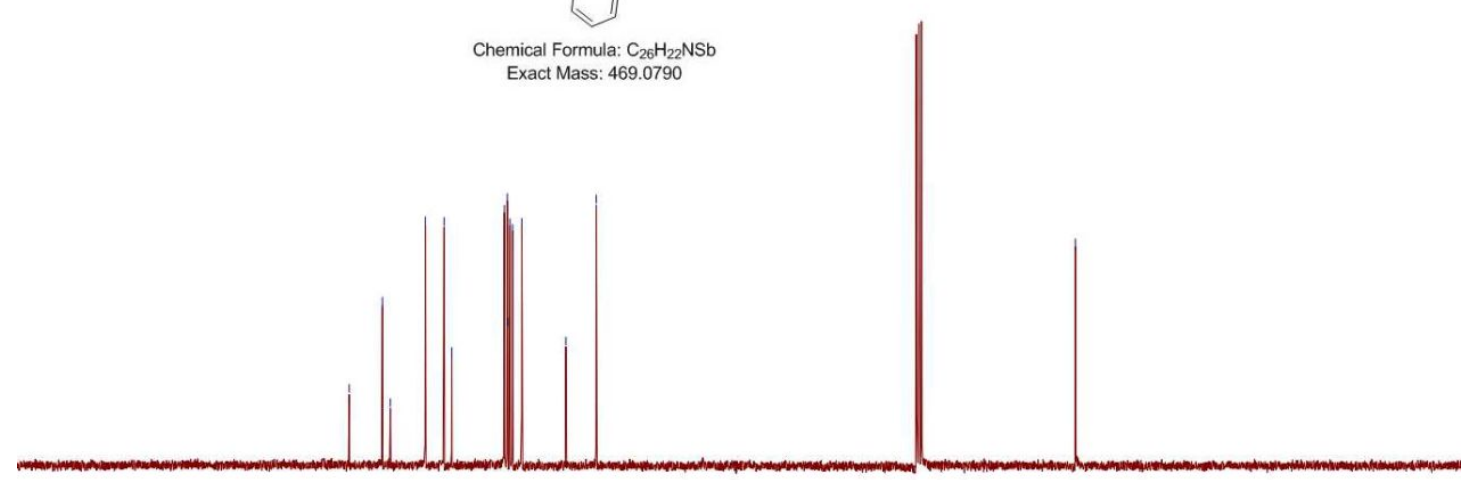

\begin{tabular}{lllllllllllllllllll}
\hline 30 & 180 & 170 & 160 & 150 & 140 & 130 & 120 & 110 & $\begin{array}{c}100 \\
\mathrm{f} 1(\mathrm{ppm})\end{array}$ & 90 & 80 & 70 & 60 & 50 & 40 & 30 & 20 & 1(
\end{tabular}

${ }^{1} \mathrm{H}$ NMR $\left(\mathrm{CDCl}_{3}\right)$ spectrum of compound $\mathbf{3 c}$ 


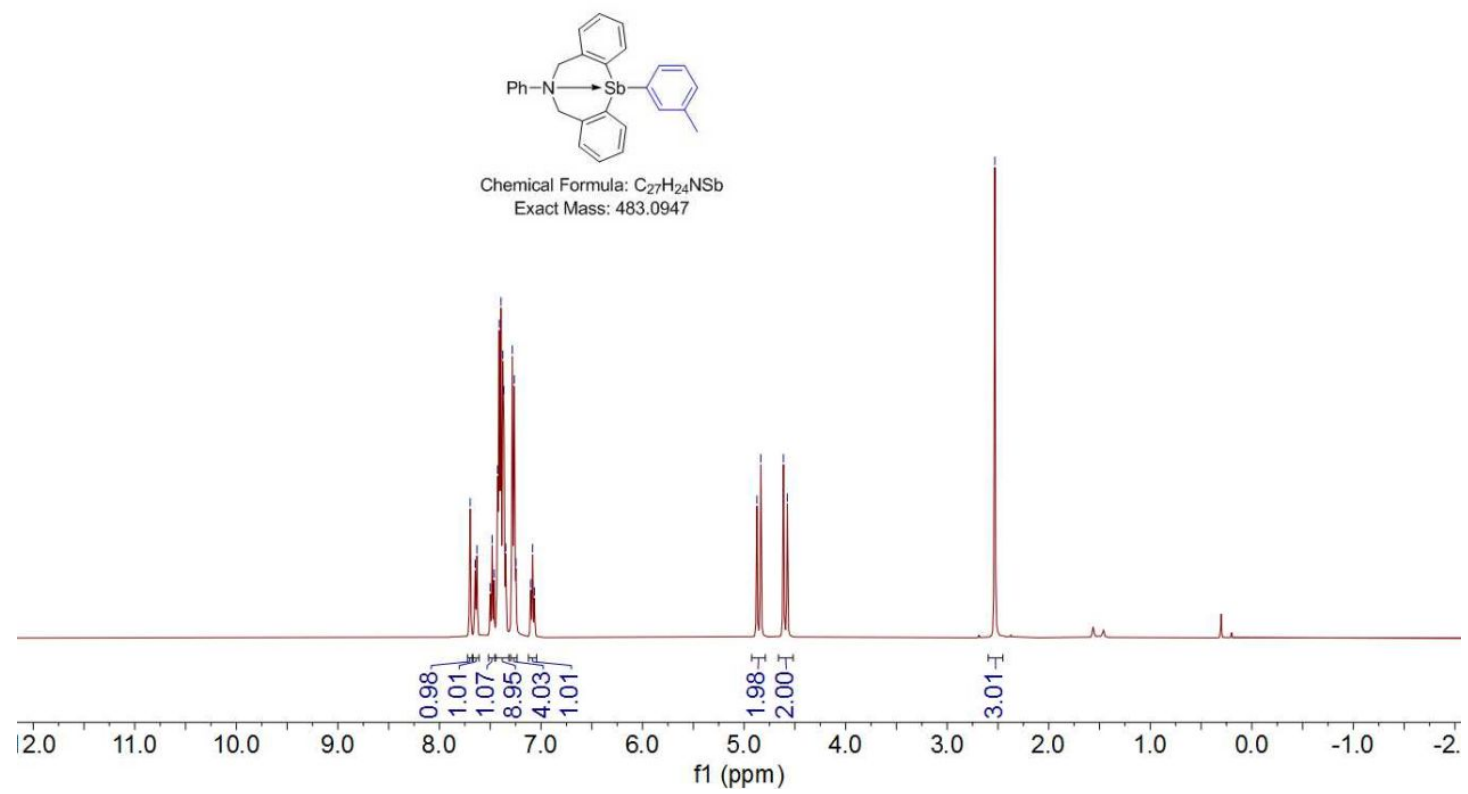

${ }^{13} \mathrm{C} \mathrm{NMR}\left(\mathrm{CDCl}_{3}\right)$ spectrum of compound $3 \mathbf{c}$

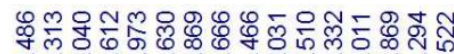

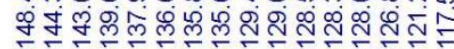

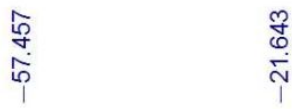
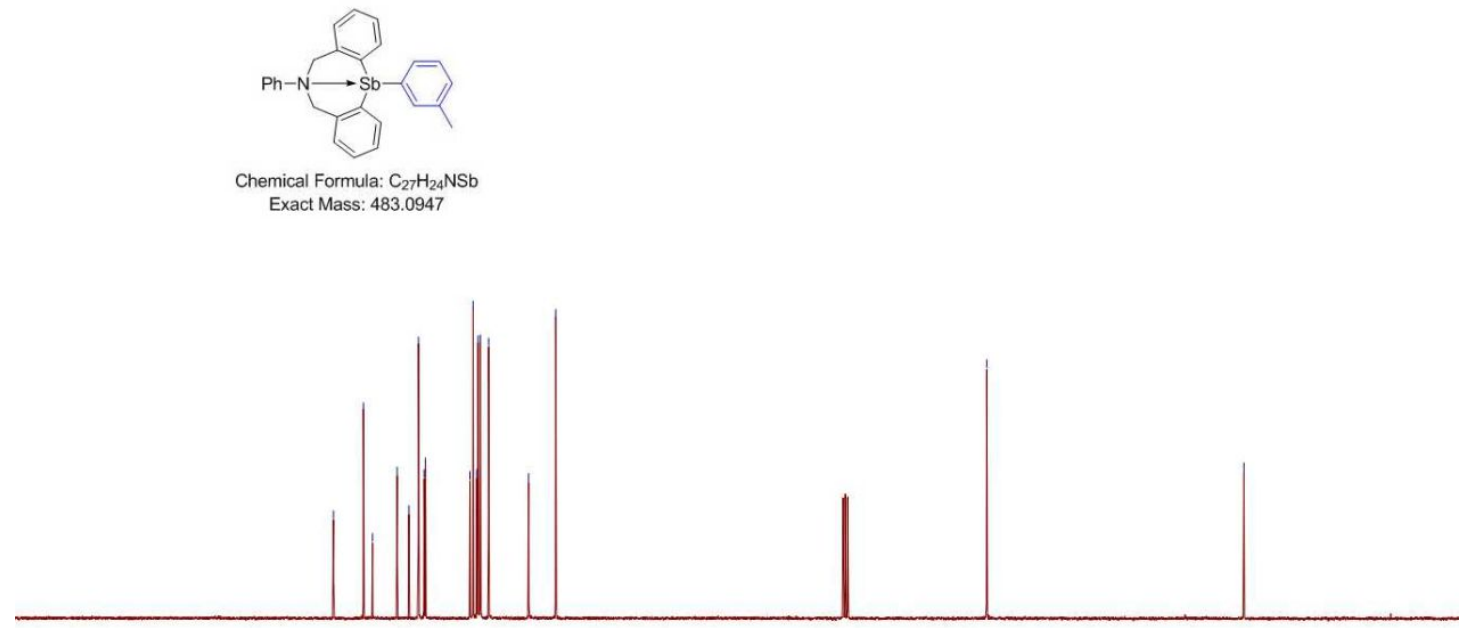

$\begin{array}{lllllllllllllllllllll}190 & 180 & 170 & 160 & 150 & 140 & 130 & 120 & 110 & \begin{array}{c}100 \\ \mathrm{f} 1(\mathrm{ppm})\end{array} & 80 & 70 & 60 & 50 & 40 & 30 & 20 & 10 & 0\end{array}$

${ }^{1} \mathrm{H}$ NMR $\left(\mathrm{CDCl}_{3}\right)$ spectrum of compound 3d 


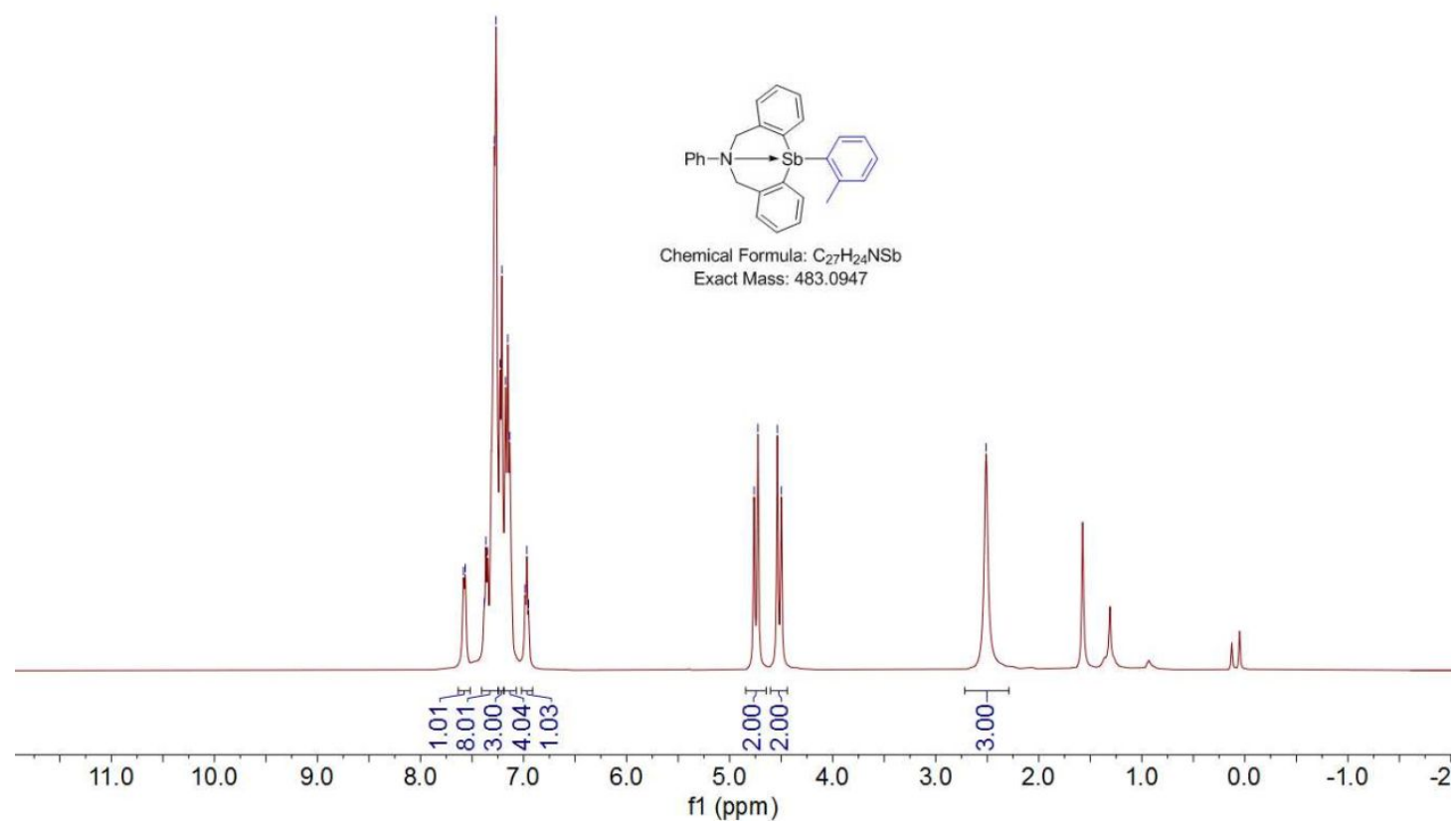

${ }^{13} \mathrm{C}$ NMR $\left(\mathrm{CDCl}_{3}\right)$ spectrum of compound $\mathbf{3 d}$

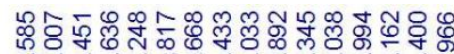

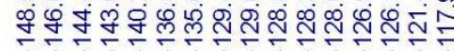

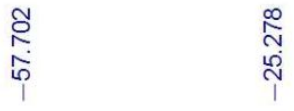

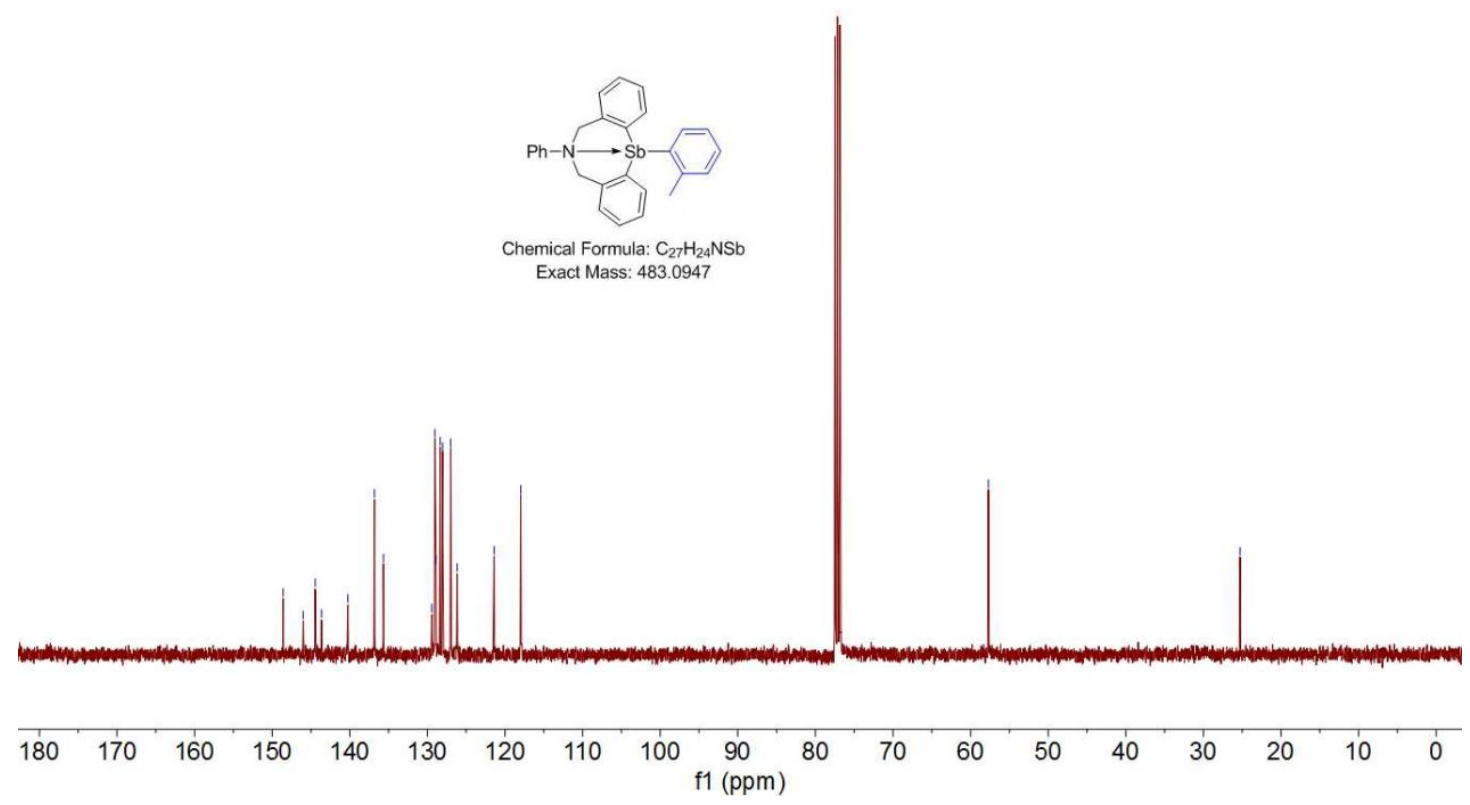

${ }^{1} \mathrm{H}$ NMR $\left(\mathrm{CDCl}_{3}\right)$ spectrum of compound $\mathbf{3 e}$ 

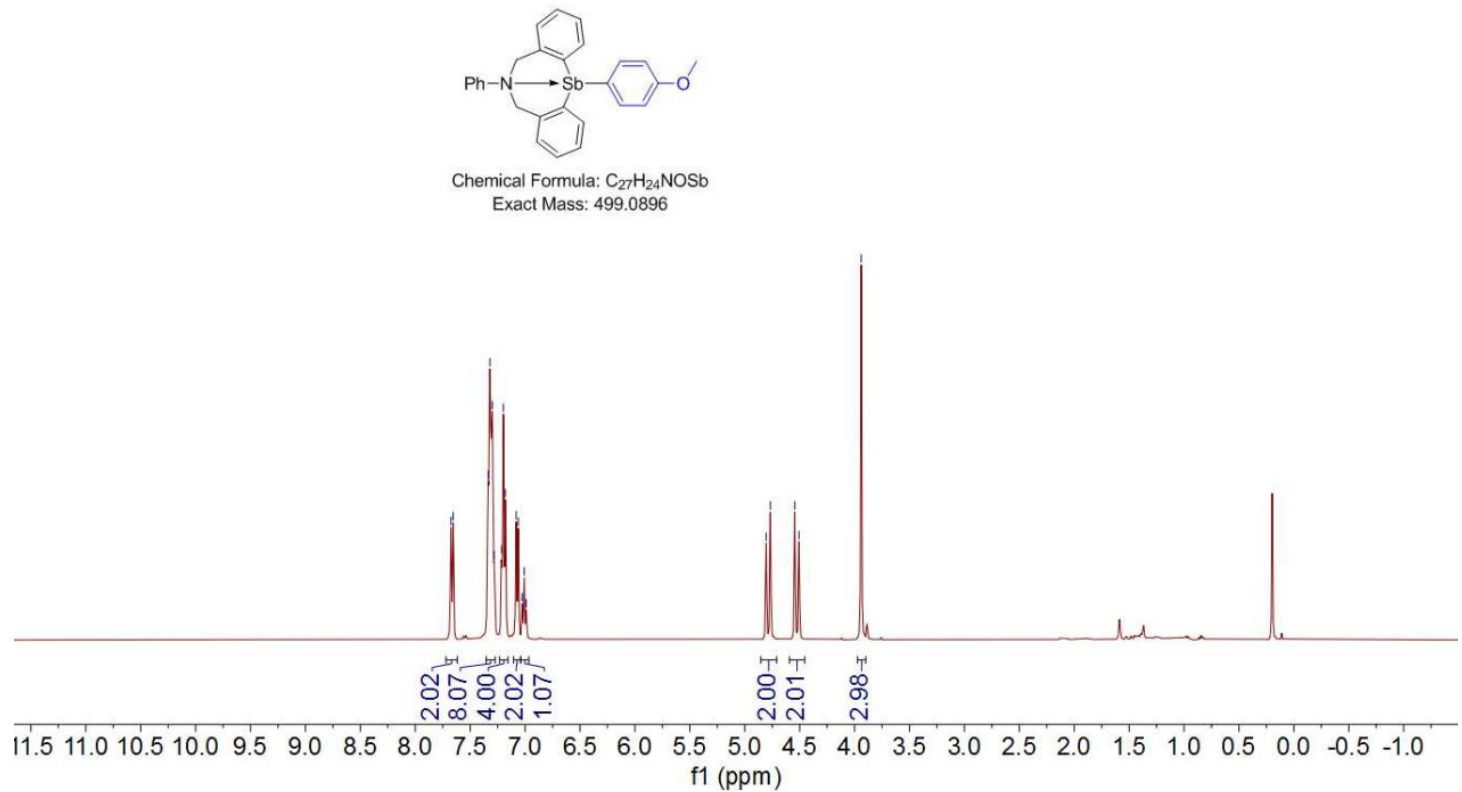

${ }^{13} \mathrm{C}$ NMR $\left(\mathrm{CDCl}_{3}\right)$ spectrum of compound $\mathbf{3 e}$

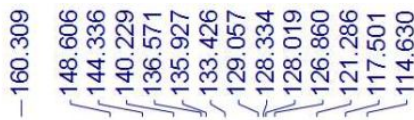

思总

ถึ่

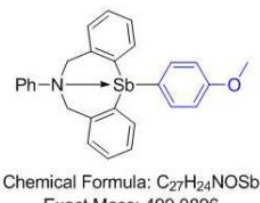

Exact Mass: 499.0896

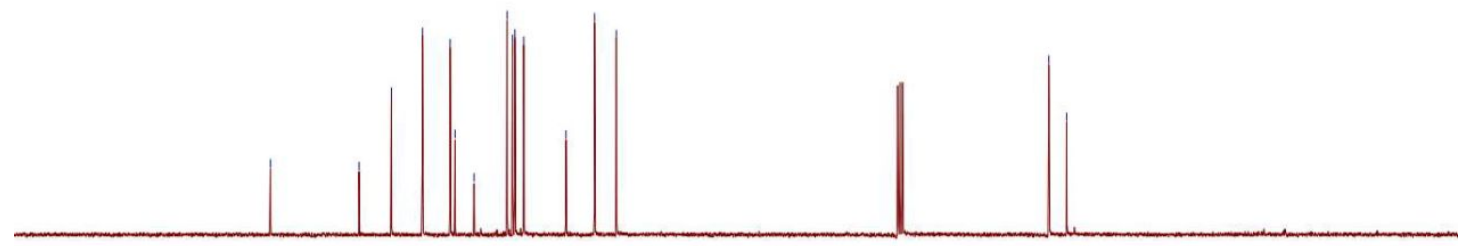

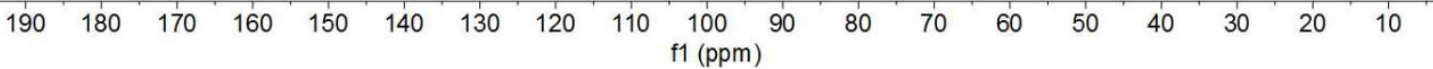

${ }^{1} \mathrm{H}$ NMR $\left(\mathrm{CDCl}_{3}\right)$ spectrum of compound $\mathbf{3 f}$ 


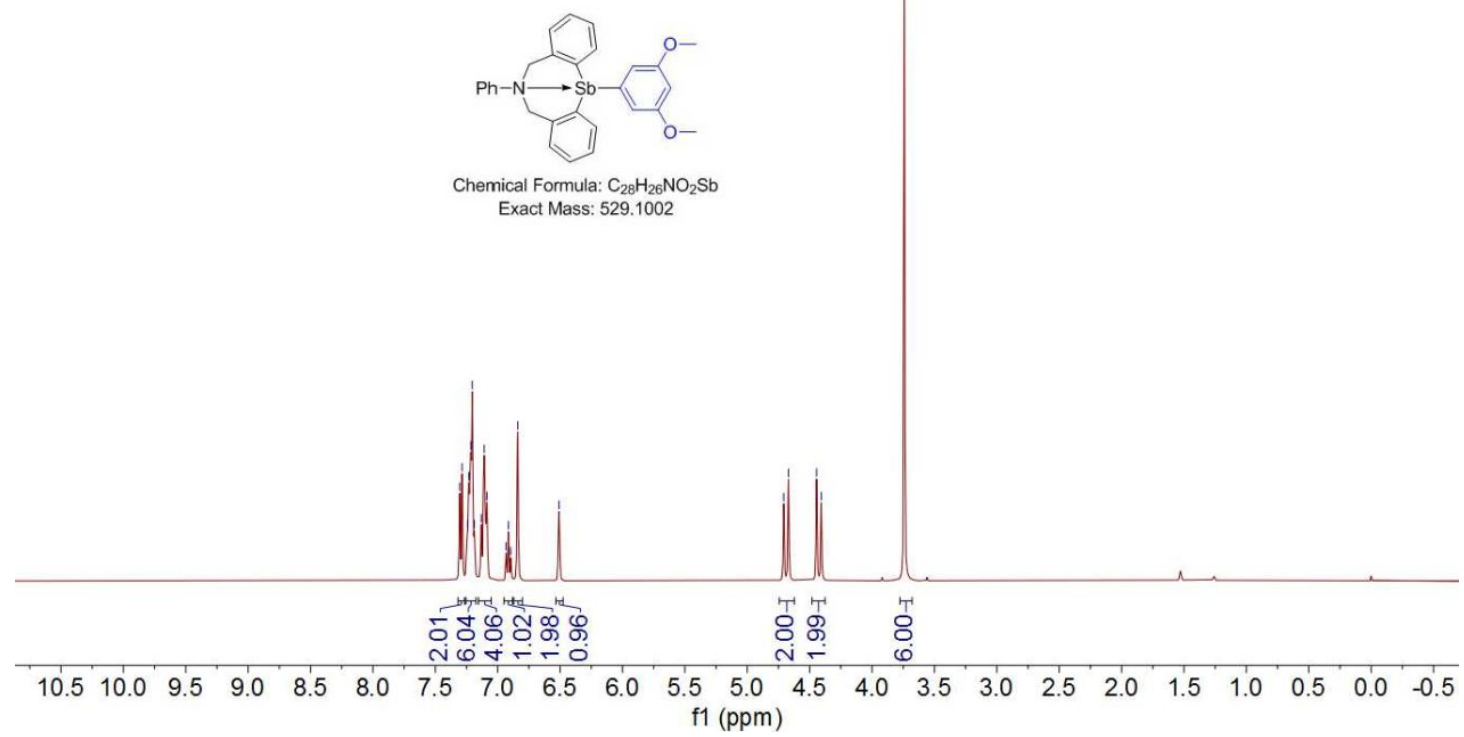

${ }^{13} \mathrm{C}$ NMR $\left(\mathrm{CDCl}_{3}\right)$ spectrum of compound $\mathbf{3 f}$

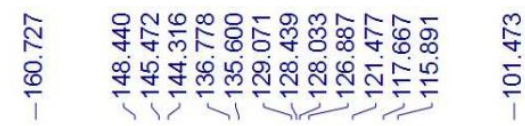

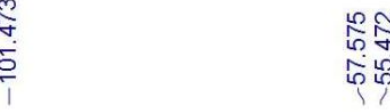

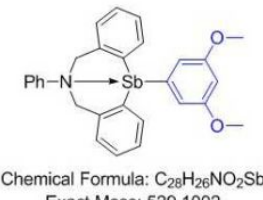

Exact Mass: 529.1002

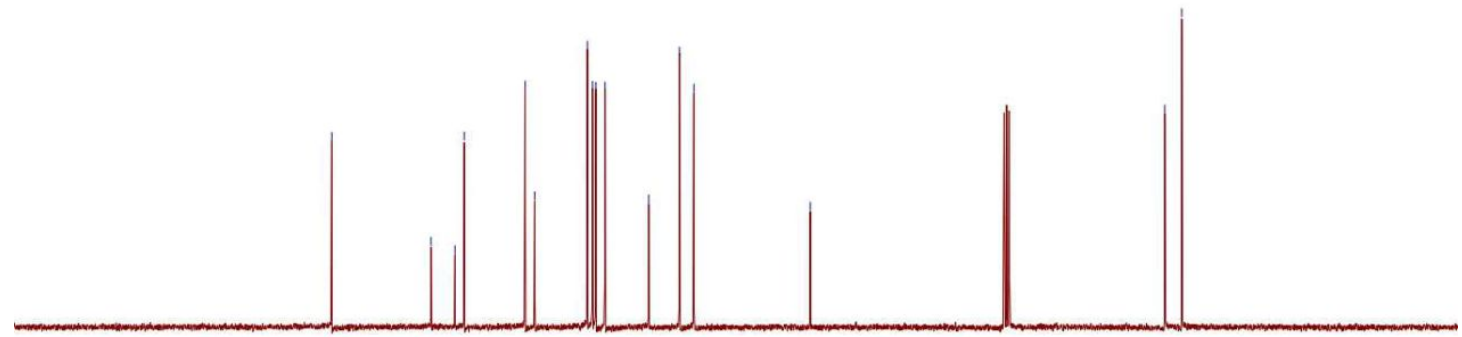

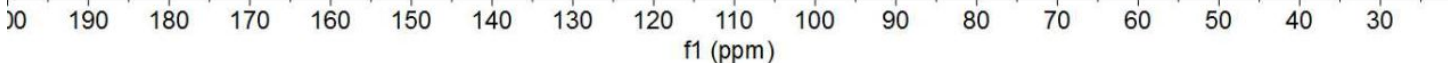

${ }^{1} \mathrm{H}$ NMR $\left(\mathrm{CDCl}_{3}\right)$ spectrum of compound $\mathbf{3 g}$ 


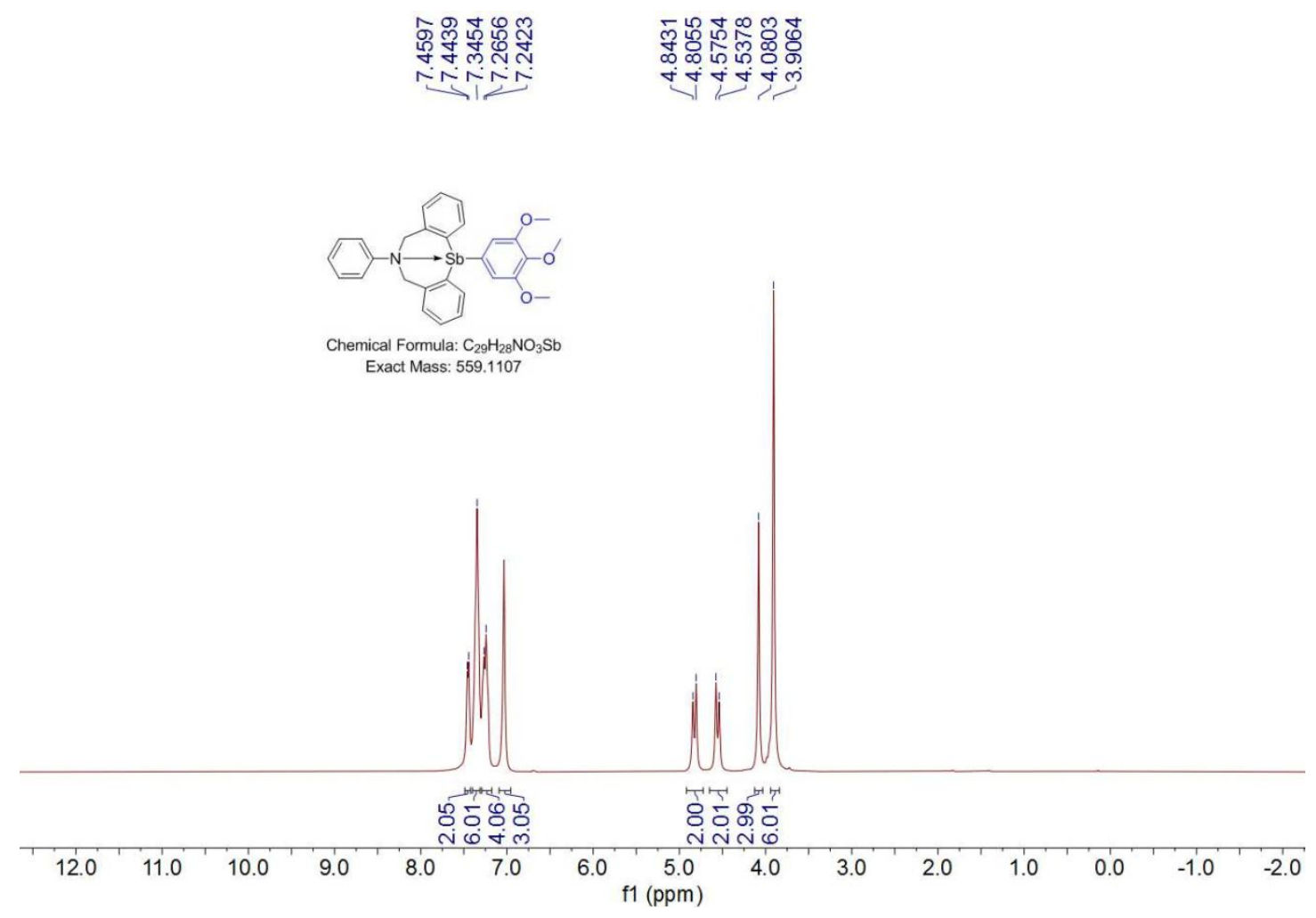

${ }^{13} \mathrm{C} \mathrm{NMR}\left(\mathrm{CDCl}_{3}\right)$ spectrum of compound $\mathbf{3 g}$

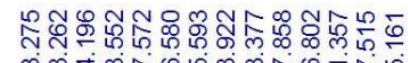

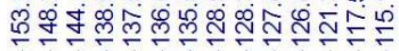

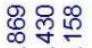

छํํㅇ
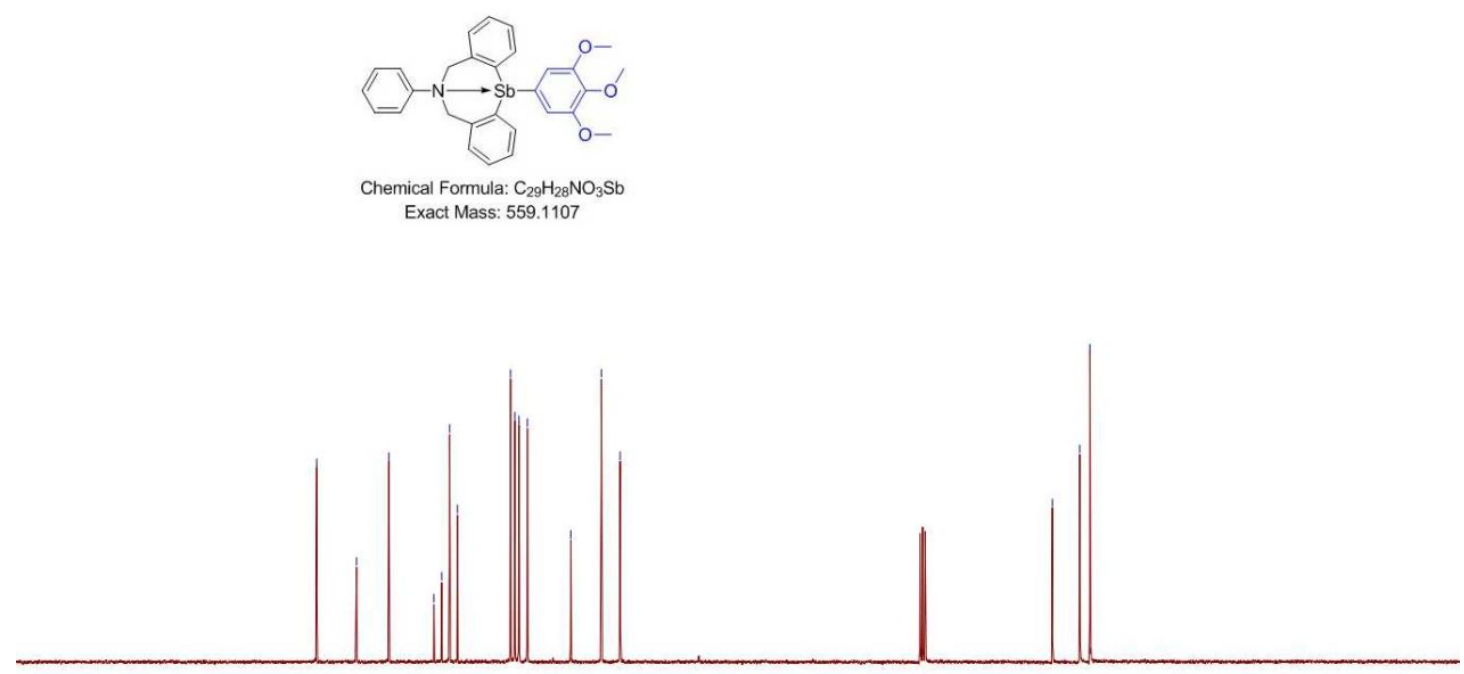

$\begin{array}{lllllllllllllllllll}90 & 180 & 170 & 160 & 150 & 140 & 130 & 120 & 110 & \begin{array}{c}100 \\ \mathrm{f} 1(\mathrm{ppm})\end{array} & 90 & 80 & 70 & 60 & 50 & 40 & 30 & 20 & 11\end{array}$

${ }^{1} \mathrm{H} \mathrm{NMR}\left(\mathrm{CDCl}_{3}\right)$ spectrum of compound $\mathbf{3 h}$ 

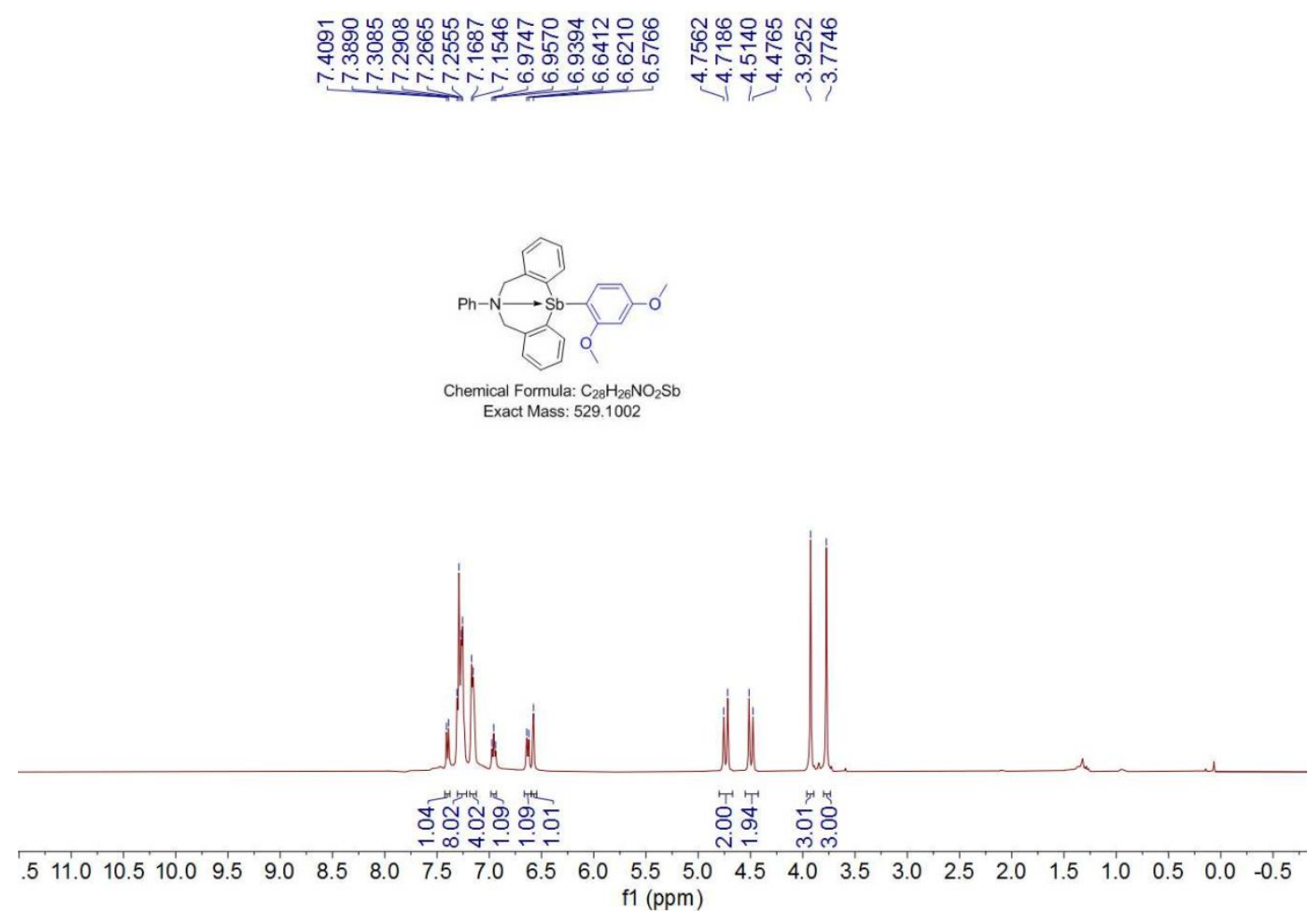

${ }^{13} \mathrm{C} \mathrm{NMR}\left(\mathrm{CDCl}_{3}\right)$ spectrum of compound $\mathbf{3 h}$
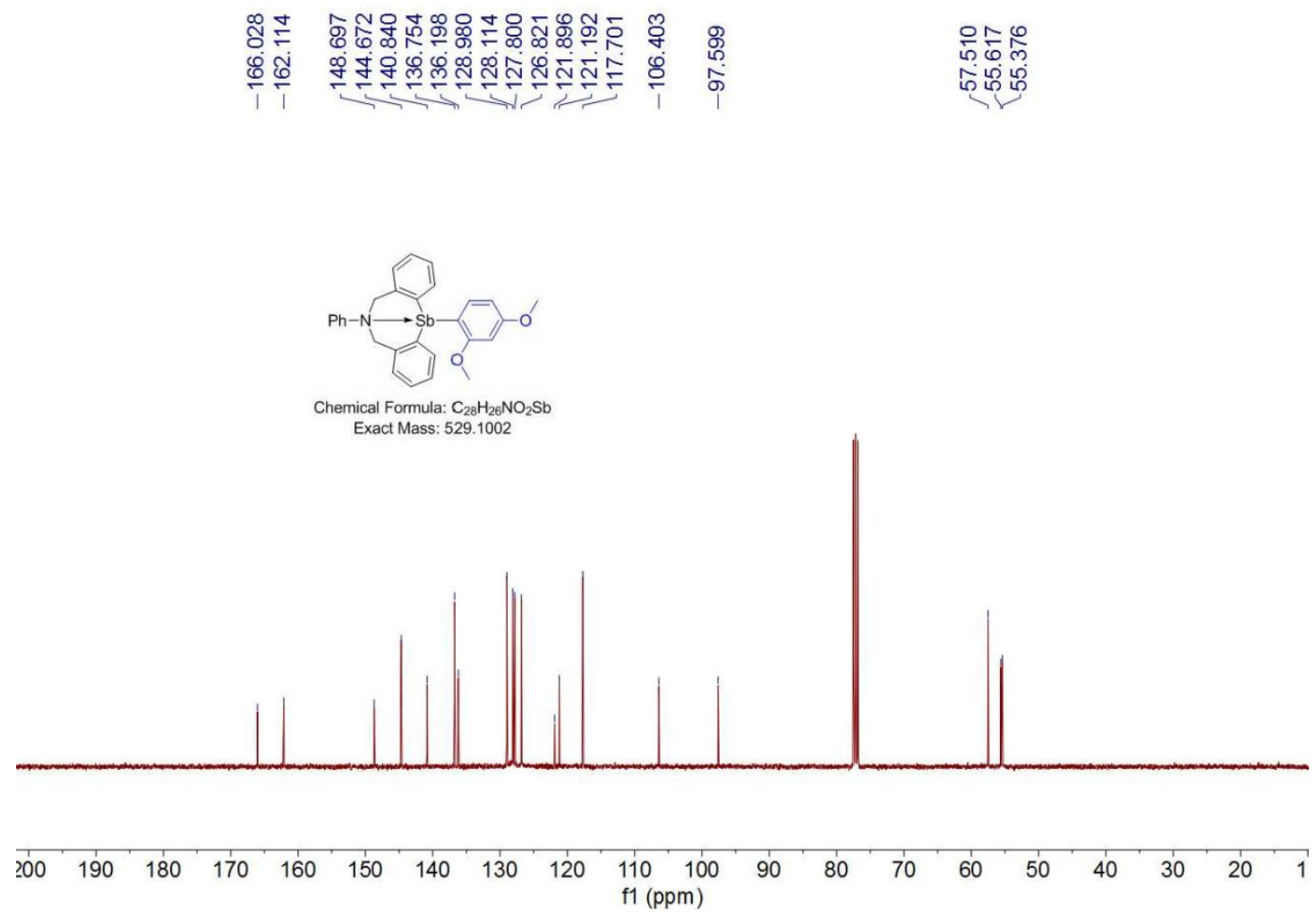

${ }^{1} \mathrm{H}$ NMR $\left(\mathrm{CDCl}_{3}\right)$ spectrum of compound $\mathbf{3 i}$ 


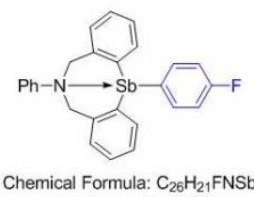

Exact Mass: 487.0696

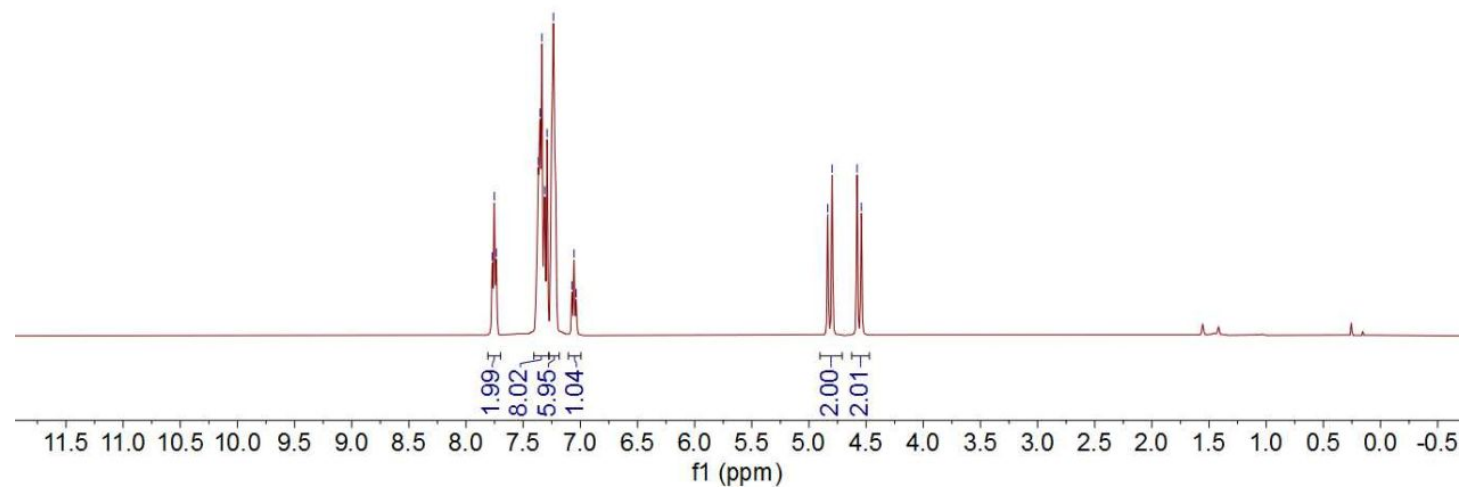

${ }^{13} \mathrm{C}$ NMR $\left(\mathrm{CDCl}_{3}\right)$ spectrum of compound $\mathbf{3 i}$

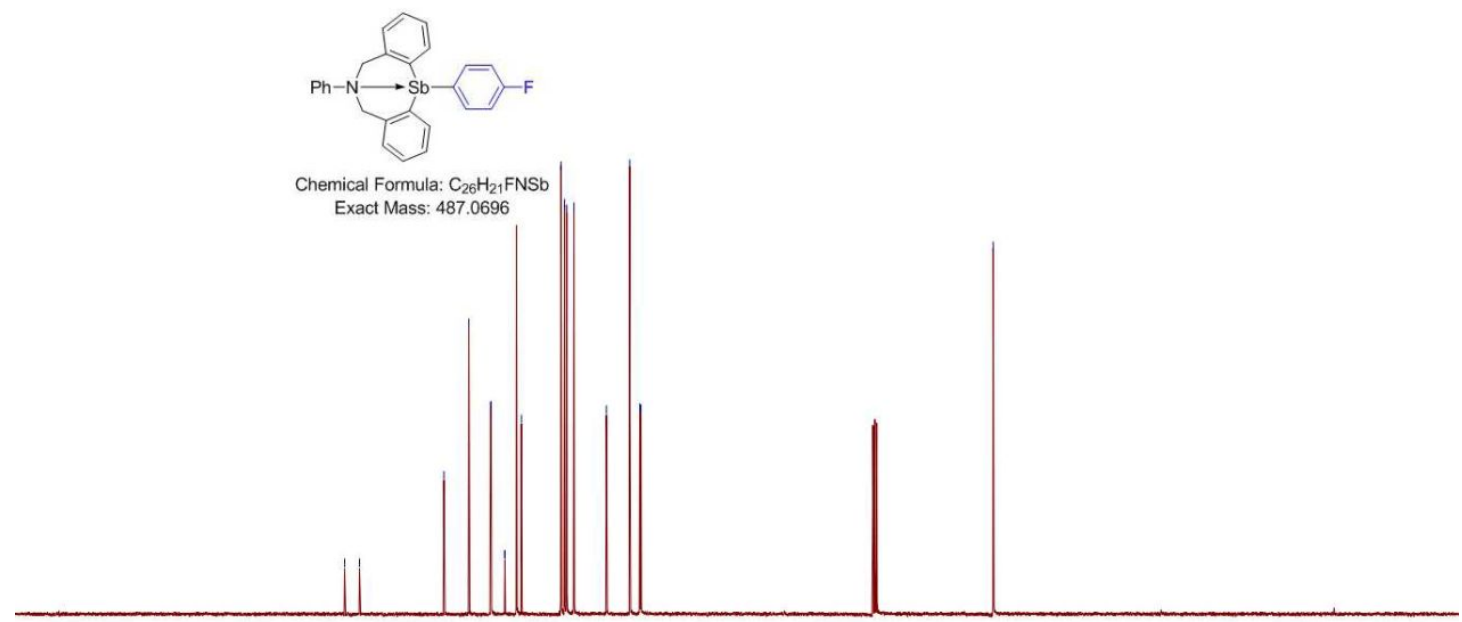

$\begin{array}{lllllllllllllllllllllllll}210 & 200 & 190 & 180 & 170 & 160 & 150 & 140 & 130 & 120 & 110 & 100 & 90 & 80 & 70 & 60 & 50 & 40 & 30 & 20 & 10 & 0 & -10\end{array}$ f1 (ppm)

${ }^{19} \mathrm{~F}$ NMR $\left(\mathrm{CDCl}_{3}\right)$ spectrum of compound $\mathbf{3 i}$ 

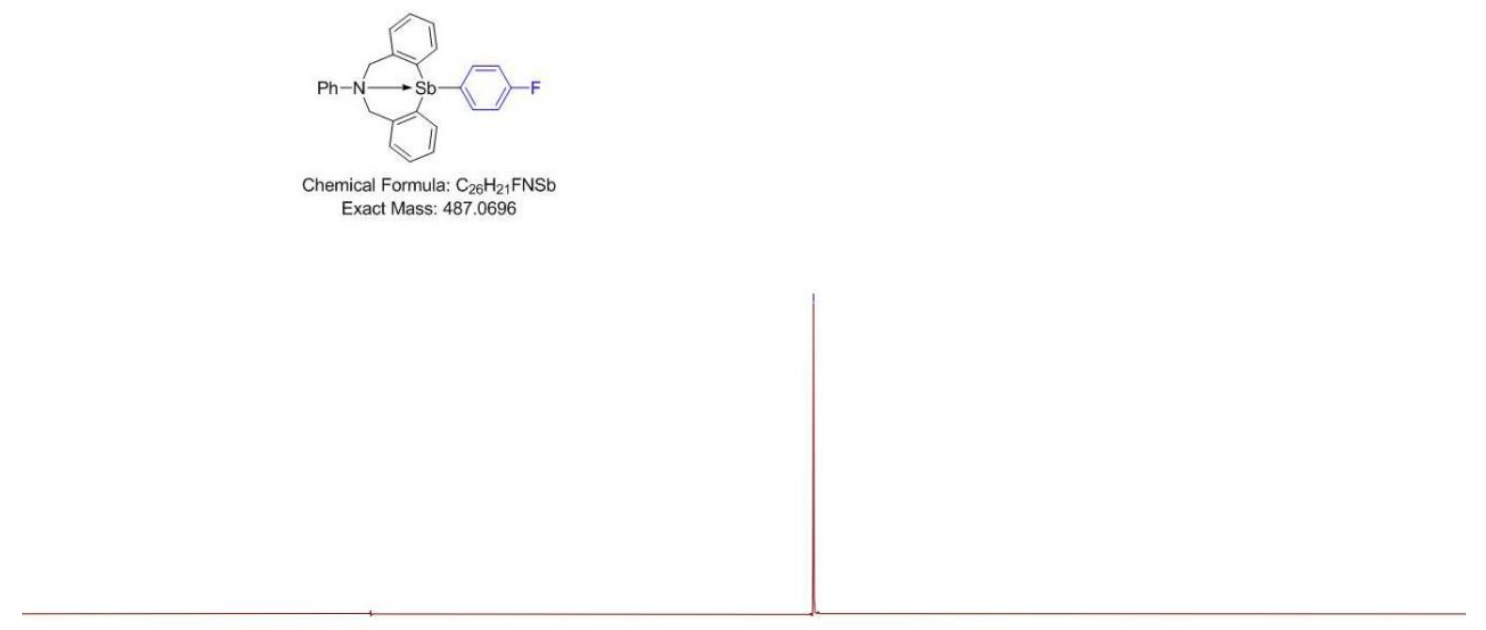

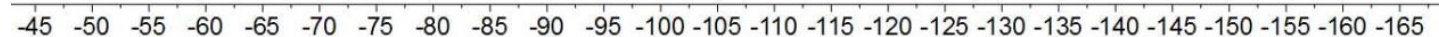
$\mathrm{f} 1(\mathrm{ppm})$

${ }^{1} \mathrm{H}$ NMR $\left(\mathrm{CDCl}_{3}\right)$ spectrum of compound $\mathbf{3 j}$
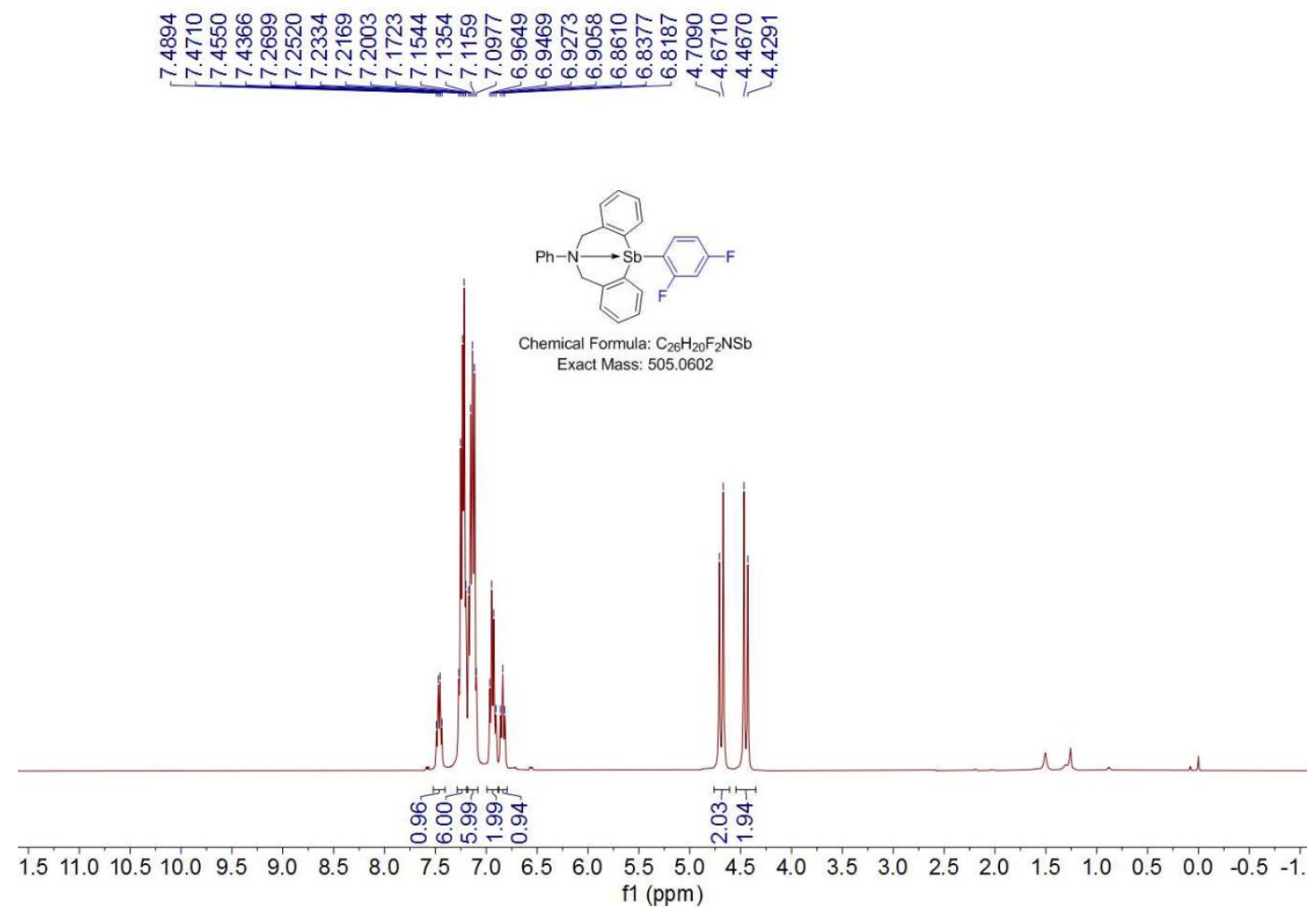

${ }^{13} \mathrm{C}$ NMR $\left(\mathrm{CDCl}_{3}\right)$ spectrum of compound $\mathbf{3 j}$ 


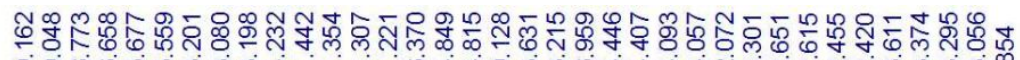

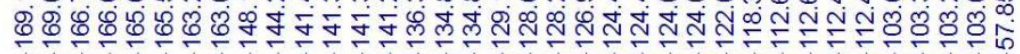

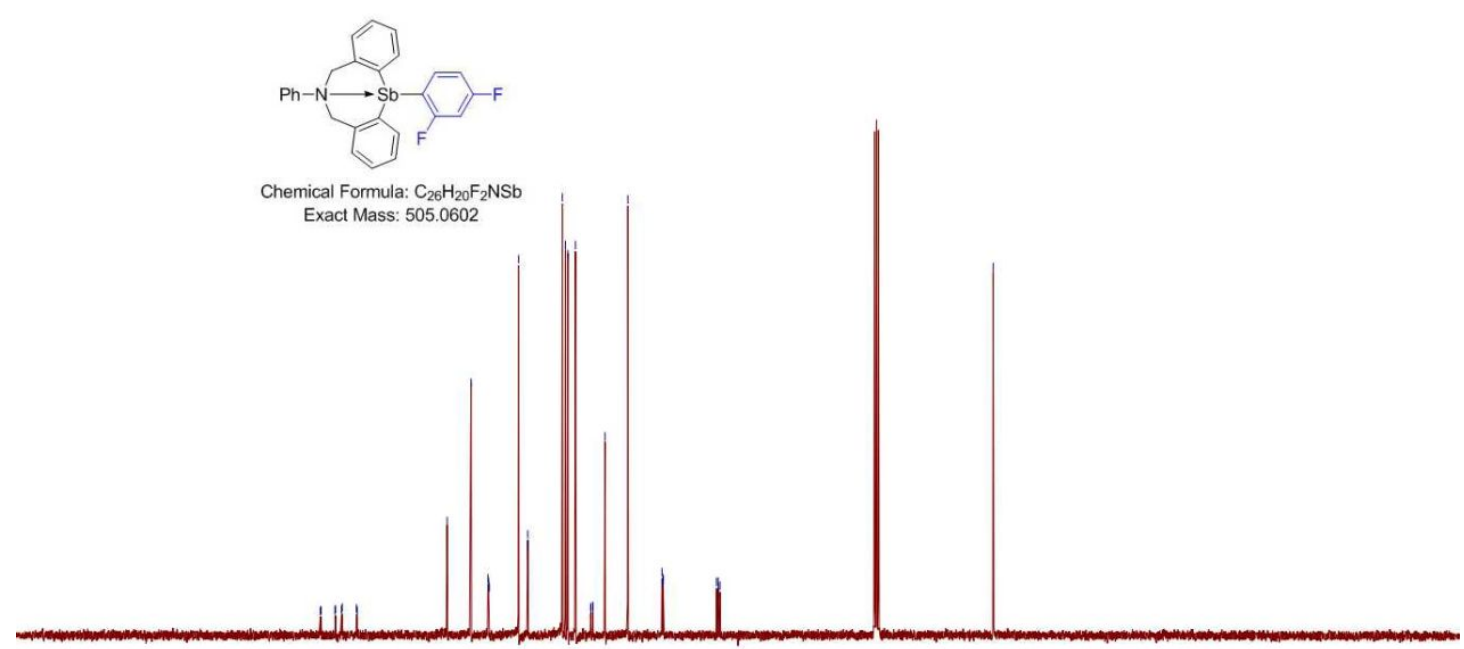

$\begin{array}{lllllllllllllllllllllllll}1 & 110 & 200 & 190 & 180 & 170 & 160 & 150 & 140 & 130 & 120 & 110 & 100 & 90 & 80 & 70 & 60 & 50 & 40 & 30 & 20 & 10 & 0 & -10\end{array}$ $\mathrm{f1}(\mathrm{ppm})$

${ }^{19} \mathrm{~F} \mathrm{NMR}\left(\mathrm{CDCl}_{3}\right)$ spectrum of compound $\mathbf{3 j}$

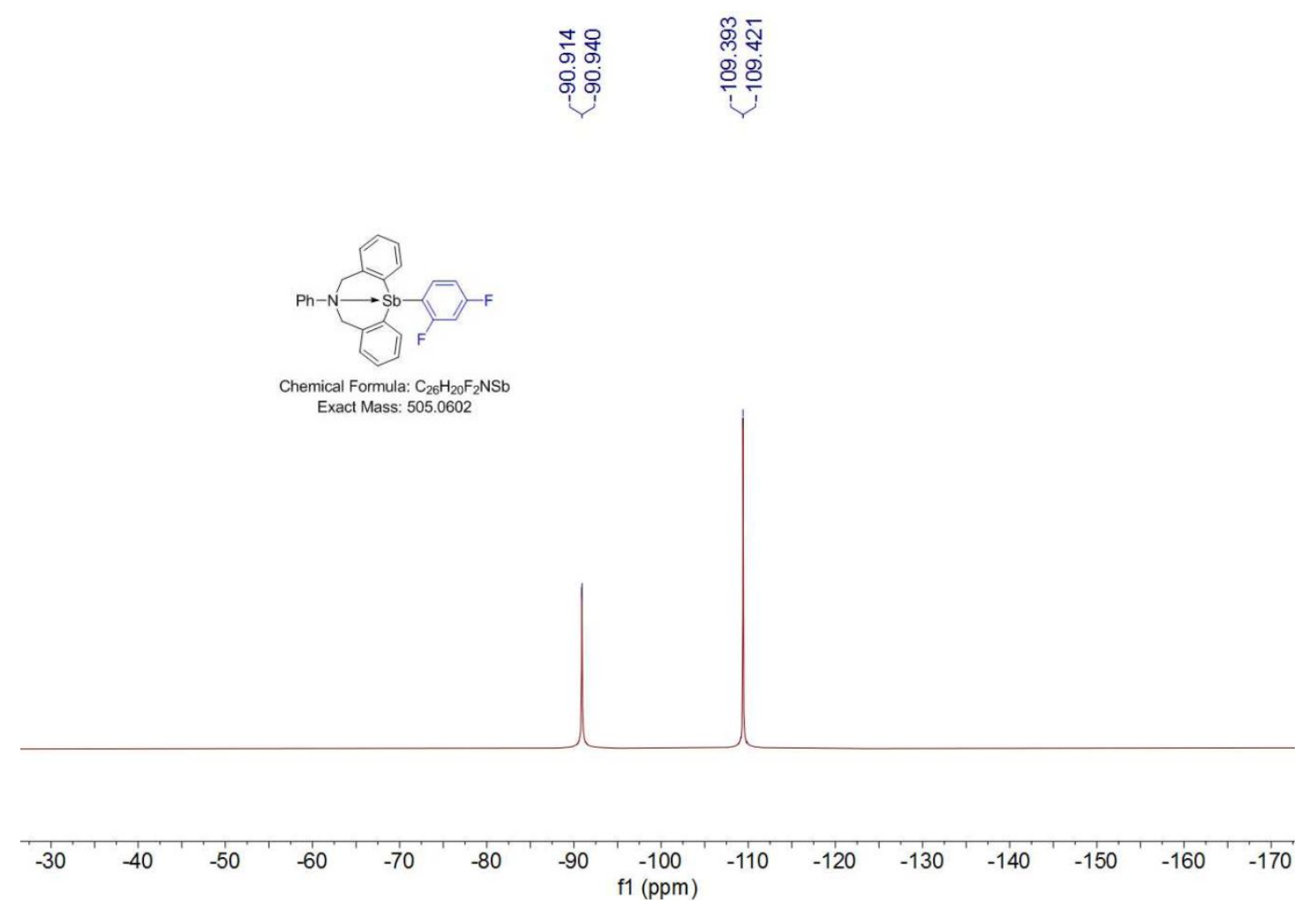

${ }^{1} \mathrm{H} \mathrm{NMR}\left(\mathrm{CDCl}_{3}\right)$ spectrum of compound $\mathbf{3 k}$ 

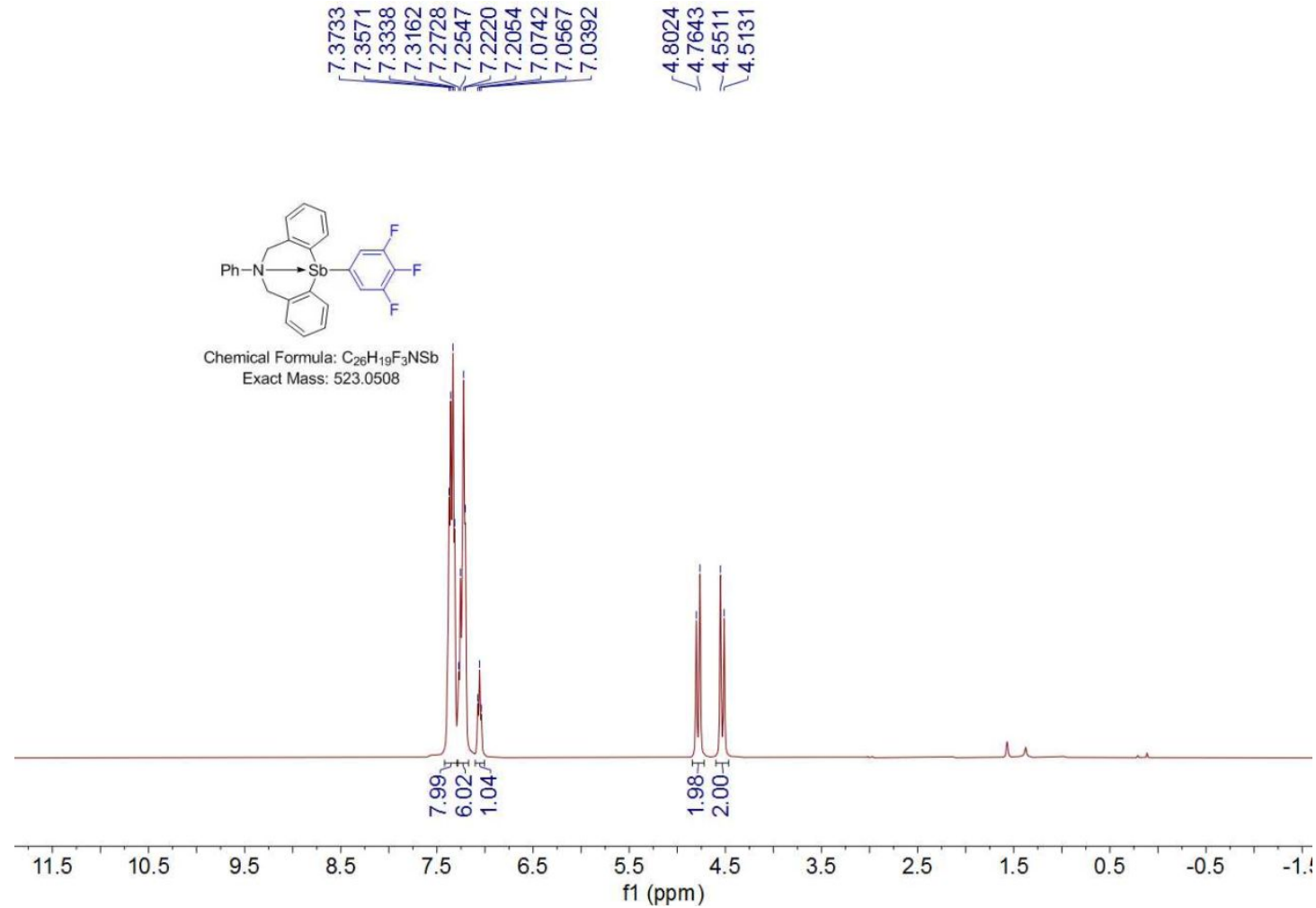

${ }^{13} \mathrm{C} \mathrm{NMR}\left(\mathrm{CDCl}_{3}\right)$ spectrum of compound $\mathbf{3 k}$

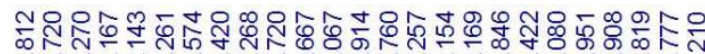

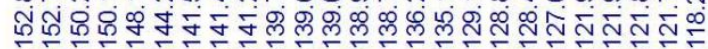

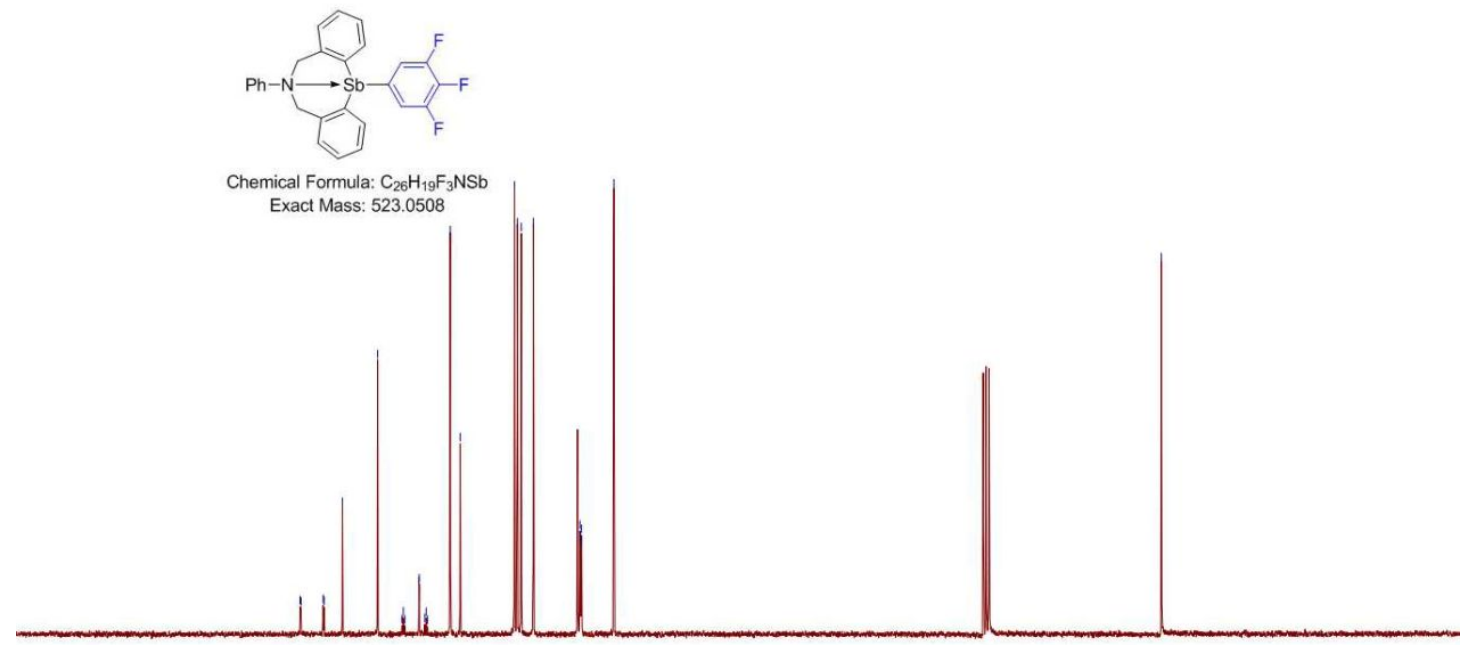

180

$\begin{array}{lllll}170 & 160 & 150 & 140 & 130\end{array}$

$120 \quad 110$

f1 (ppm)

${ }^{19} \mathrm{~F}$ NMR $\left(\mathrm{CDCl}_{3}\right)$ spectrum of compound $\mathbf{3 k}$ 


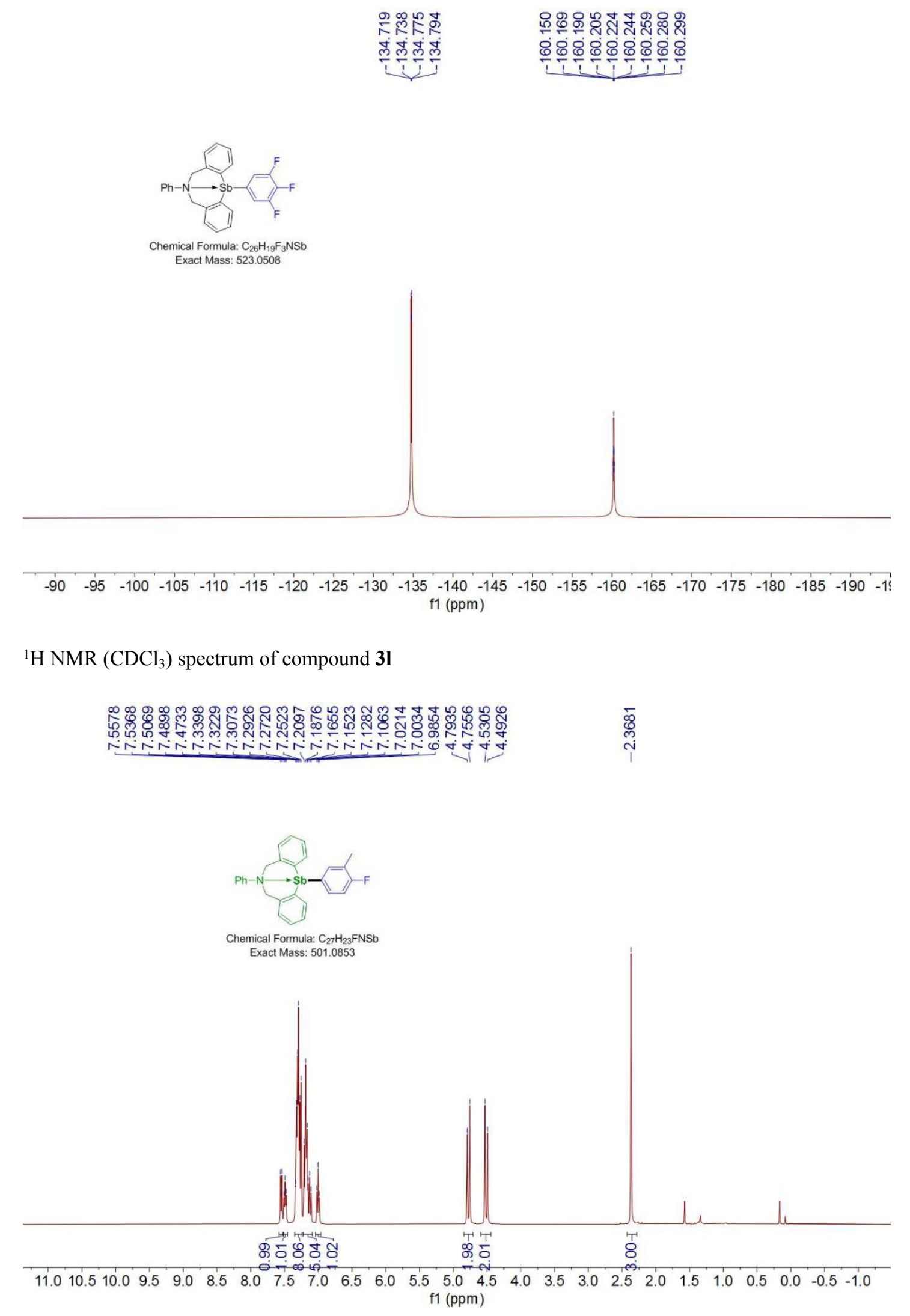

${ }^{13} \mathrm{C}$ NMR $\left(\mathrm{CDCl}_{3}\right)$ spectrum of compound $3 \mathbf{I}$ 


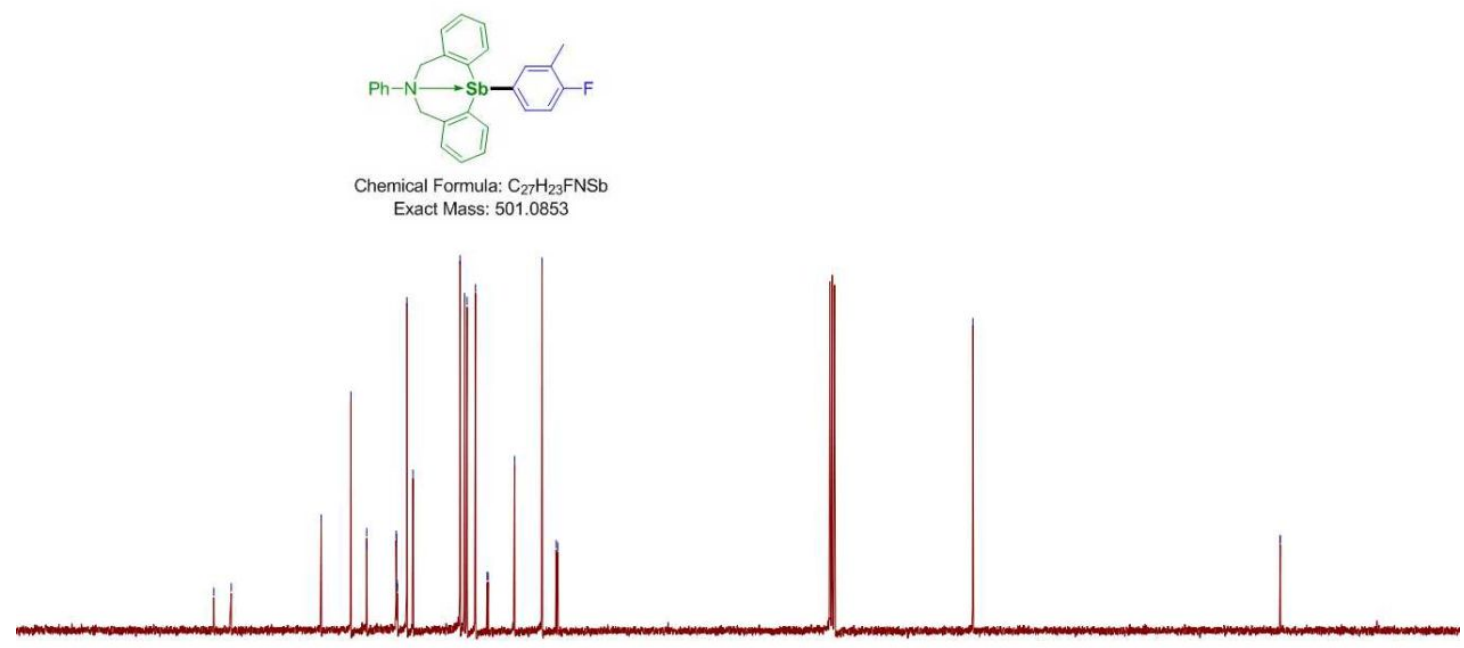

\begin{tabular}{lllllllllllllllllllllll}
\hline 90 & 180 & 170 & 160 & 150 & 140 & 130 & 120 & 110 & 100 & 90 & 80 & 70 & 60 & 50 & 40 & 30 & 20 & 10 & 0 & -1
\end{tabular}

${ }^{19} \mathrm{~F}$ NMR $\left(\mathrm{CDCl}_{3}\right)$ spectrum of compound $3 \mathbf{I}$

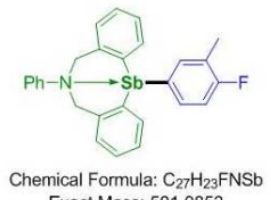

Exact Mass: 501.0853

\begin{tabular}{lllllllllllllllllllll}
\hline 20 & -30 & -40 & -50 & -60 & -70 & -80 & -90 & -100 & -110 & -120 & -130 & -140 & -150 & -160 & -170 & -180 & -190 & -21 \\
& & & & & &
\end{tabular}

${ }^{1} \mathrm{H}$ NMR $\left(\mathrm{CDCl}_{3}\right)$ spectrum of compound $\mathbf{3 m}$ 

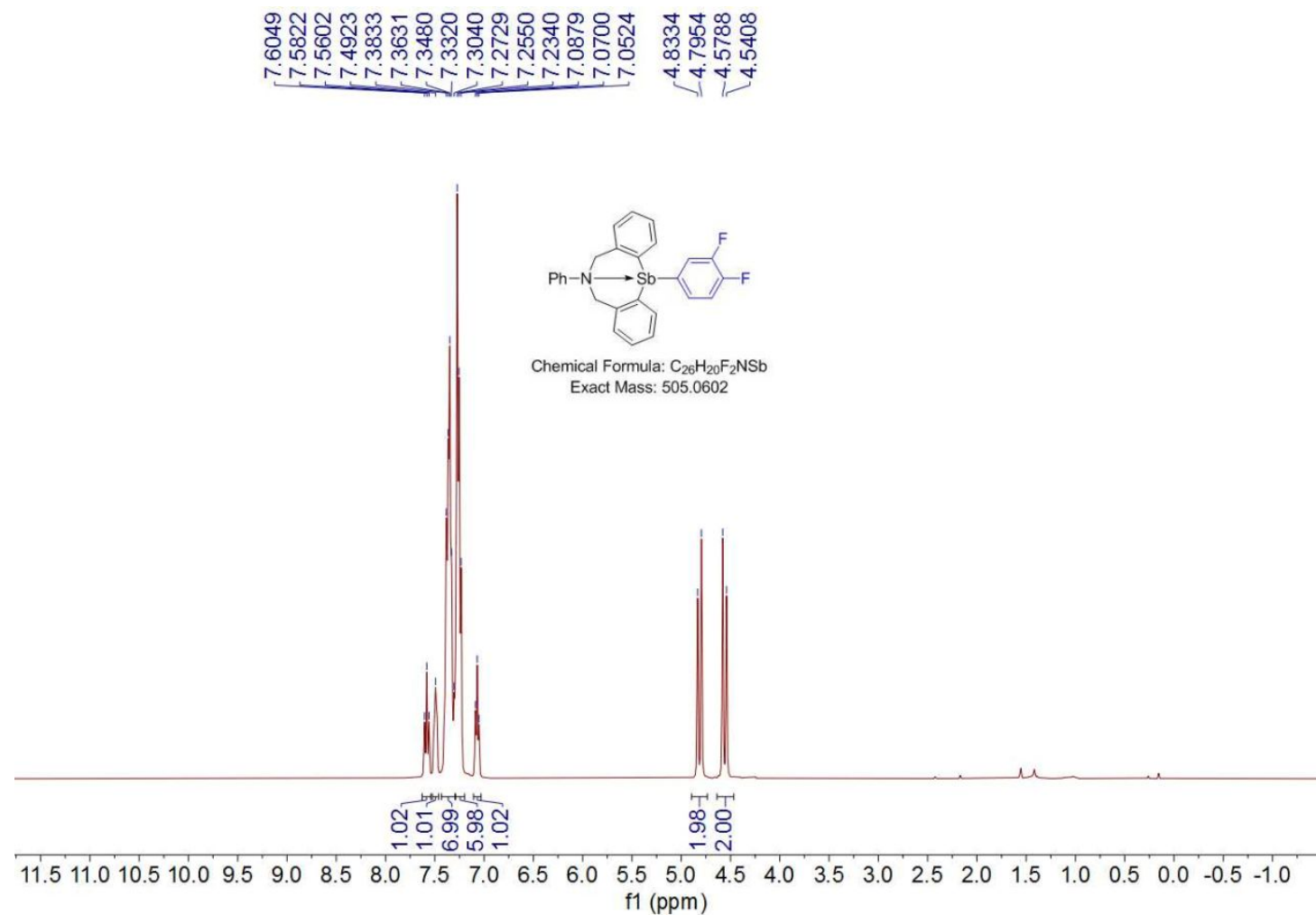

${ }^{13} \mathrm{C}$ NMR $\left(\mathrm{CDCl}_{3}\right)$ spectrum of compound $\mathbf{3 m}$

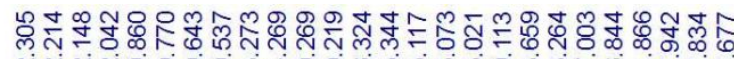

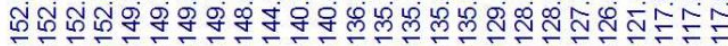

$\stackrel{\infty}{\stackrel{\infty}{6}}$

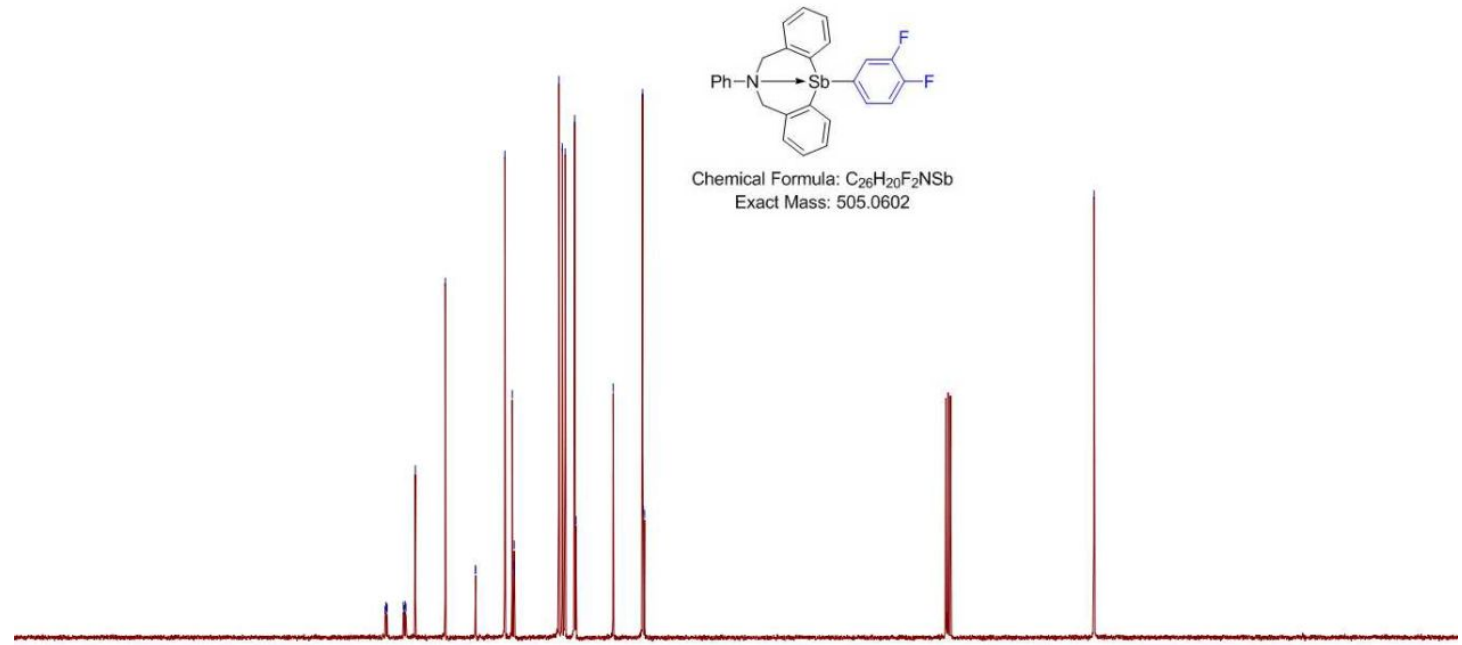

$\begin{array}{lllllllllllllllllllll}200 & 190 & 180 & 170 & 160 & 150 & 140 & 130 & 120 & 110 & 100 & 90 & 80 & 70 & 60 & 50 & 40 & 30 & 20 & 16\end{array}$ $\mathrm{f1}(\mathrm{ppm})$

${ }^{19} \mathrm{~F}$ NMR $\left(\mathrm{CDCl}_{3}\right)$ spectrum of compound $\mathbf{3 m}$ 


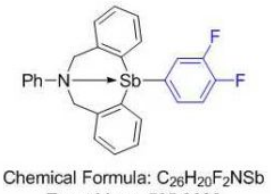

Exact Mass: 505.0602

\begin{tabular}{llllllllllllllllllllllllll}
\hline 0 & -85 & -90 & -95 & -100 & -105 & -110 & -115 & -120 & -125 & -130 & -135 & -140 & -145 & -150 & -155 & -160 & -165 & -170 & -175 & -180
\end{tabular} $\mathrm{f1}(\mathrm{ppm})$

${ }^{1} \mathrm{H}$ NMR $\left(\mathrm{CDCl}_{3}\right)$ spectrum of compound $\mathbf{3 n}$
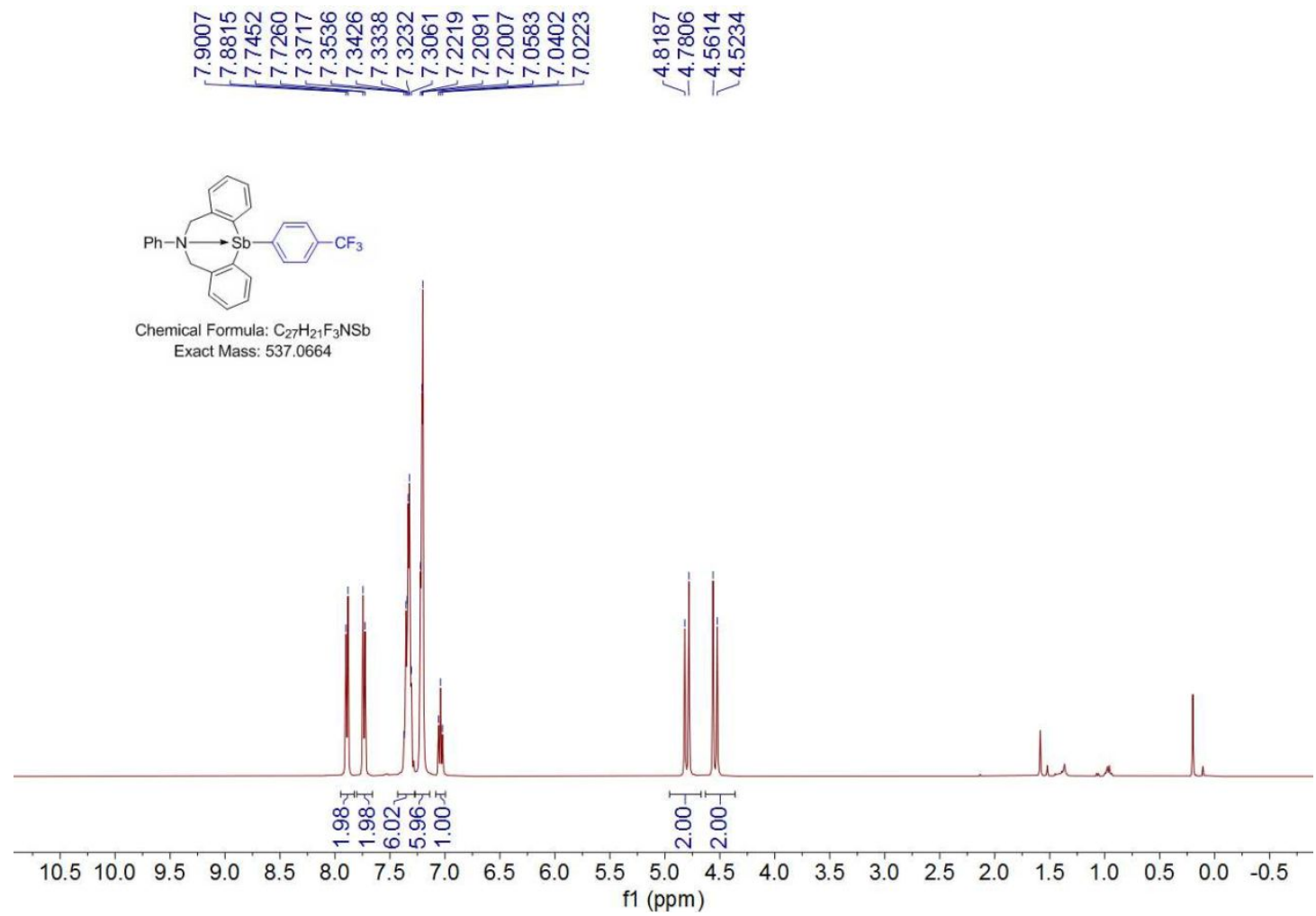

${ }^{13} \mathrm{C} \mathrm{NMR}\left(\mathrm{CDCl}_{3}\right)$ spectrum of compound $\mathbf{3 n}$ 


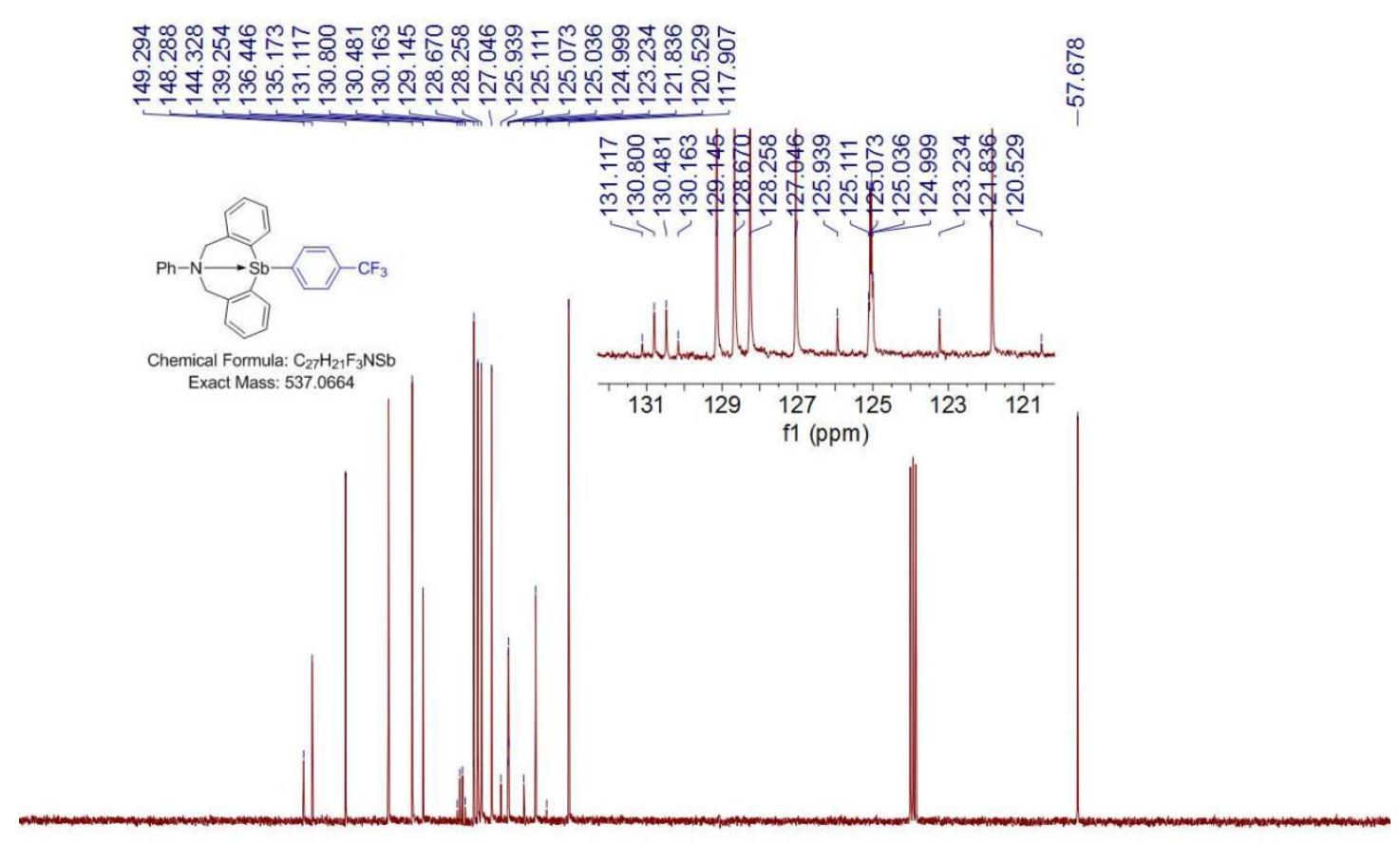

\begin{tabular}{llllllllllllllll}
\hline 180 & 170 & 160 & 150 & 140 & 130 & 120 & 110 & $\begin{array}{c}100 \\
\mathrm{f} 1(\mathrm{ppm})\end{array}$ & 90 & 80 & 70 & 60 & 50 & 40 & 30
\end{tabular}

${ }^{19} \mathrm{~F}$ NMR $\left(\mathrm{CDCl}_{3}\right)$ spectrum of compound $\mathbf{3 n}$

ֻัฐ

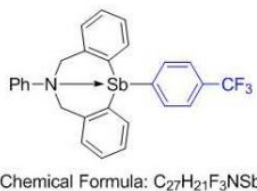

Chemical Formula: $\mathrm{C}_{27} \mathrm{H}_{21} \mathrm{~F}_{3} \mathrm{NSb}$

Exact Mass: 537.0664

$\begin{array}{lllllllllllllllllllllllllllllllll}-45 & -46 & -47 & -48 & -49 & -50 & -51 & -52 & -53 & -54 & -55 & -56 & -57 & -58 & -59 & -60 & -61 & -62 & -63 & -64 & -65 & -66 & -67 & -68 & -69 & -70 & -71 & -72 & -73 & -74 & -75 & -76\end{array}$ $\mathrm{f1}(\mathrm{ppm})$

${ }^{1} \mathrm{H}$ NMR $\left(\mathrm{CDCl}_{3}\right)$ spectrum of compound $\mathbf{3 o}$ 


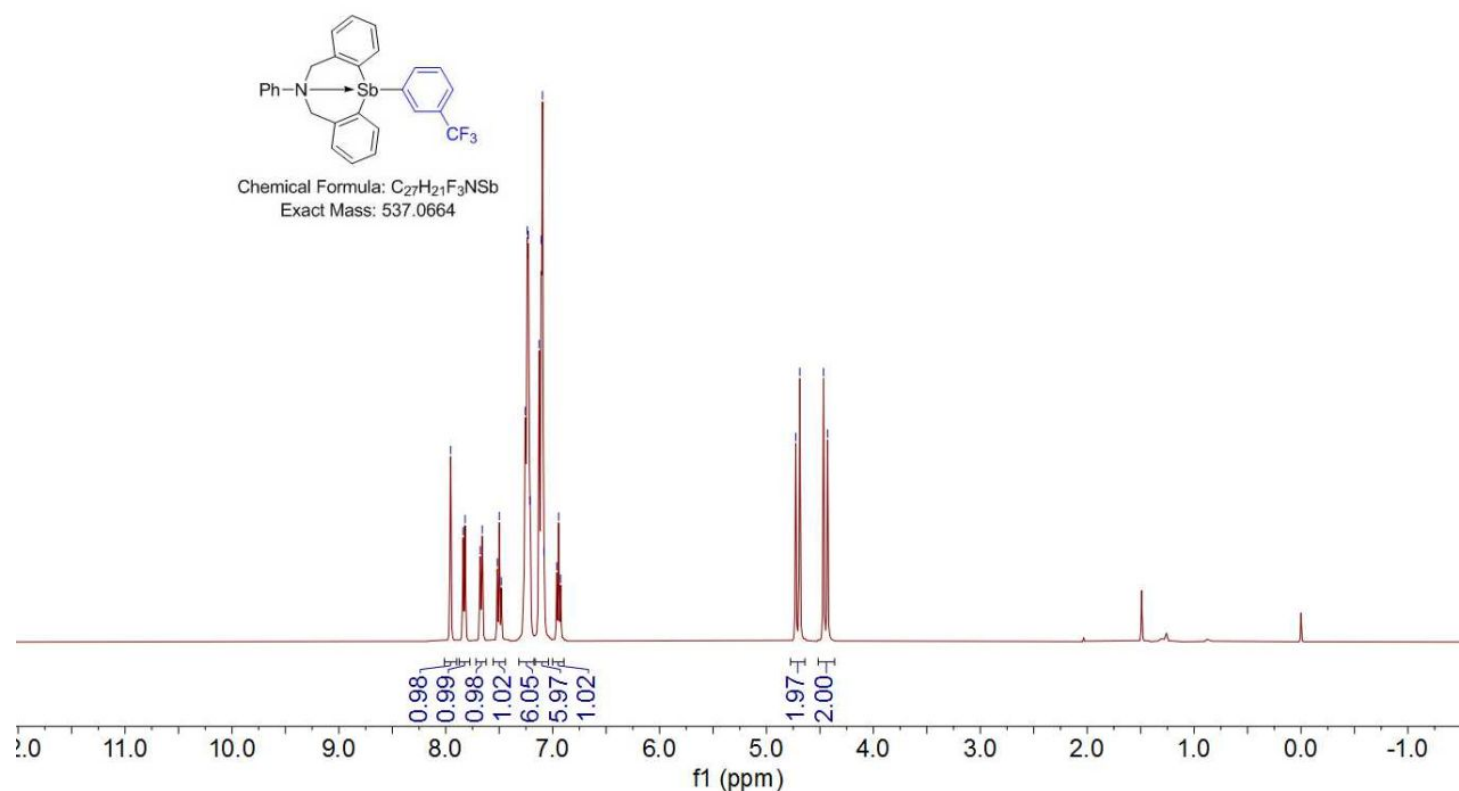

${ }^{13} \mathrm{C}$ NMR $\left(\mathrm{CDCl}_{3}\right)$ spectrum of compound 3o

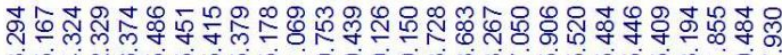

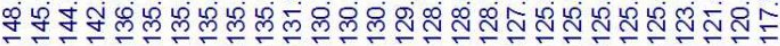

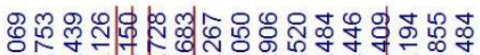

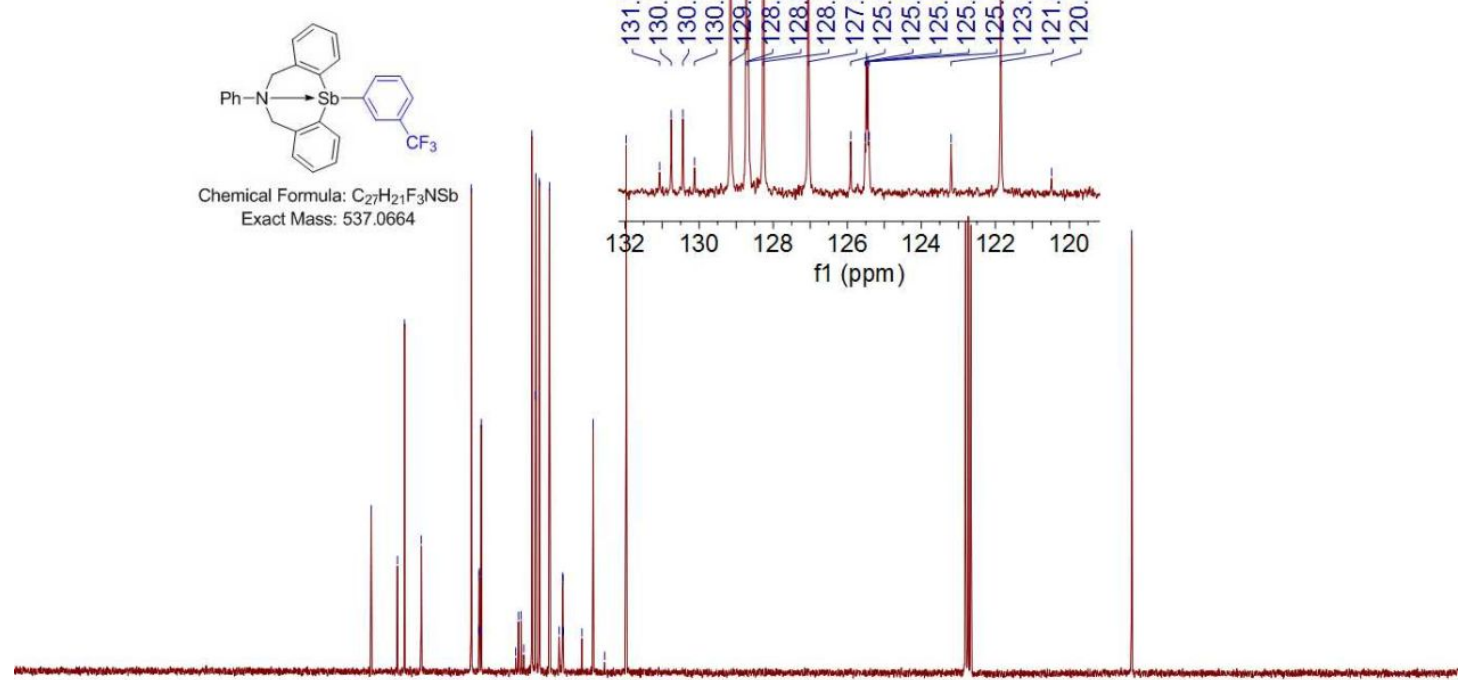

Chemical Formula: $\mathrm{C}_{27} \mathrm{H}_{21} \mathrm{~F}_{3} \mathrm{NSb}$ Exact Mass: 537.0664

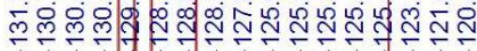

$\begin{array}{llllllllllllllllll}90 & 180 & 170 & 160 & 150 & 140 & 130 & 120 & 110 & 100 & 90 & 80 & 70 & 60 & 50 & 40 & 30 & 20\end{array}$

${ }^{19} \mathrm{~F}$ NMR $\left(\mathrm{CDCl}_{3}\right)$ spectrum of compound $\mathbf{3 o}$ 


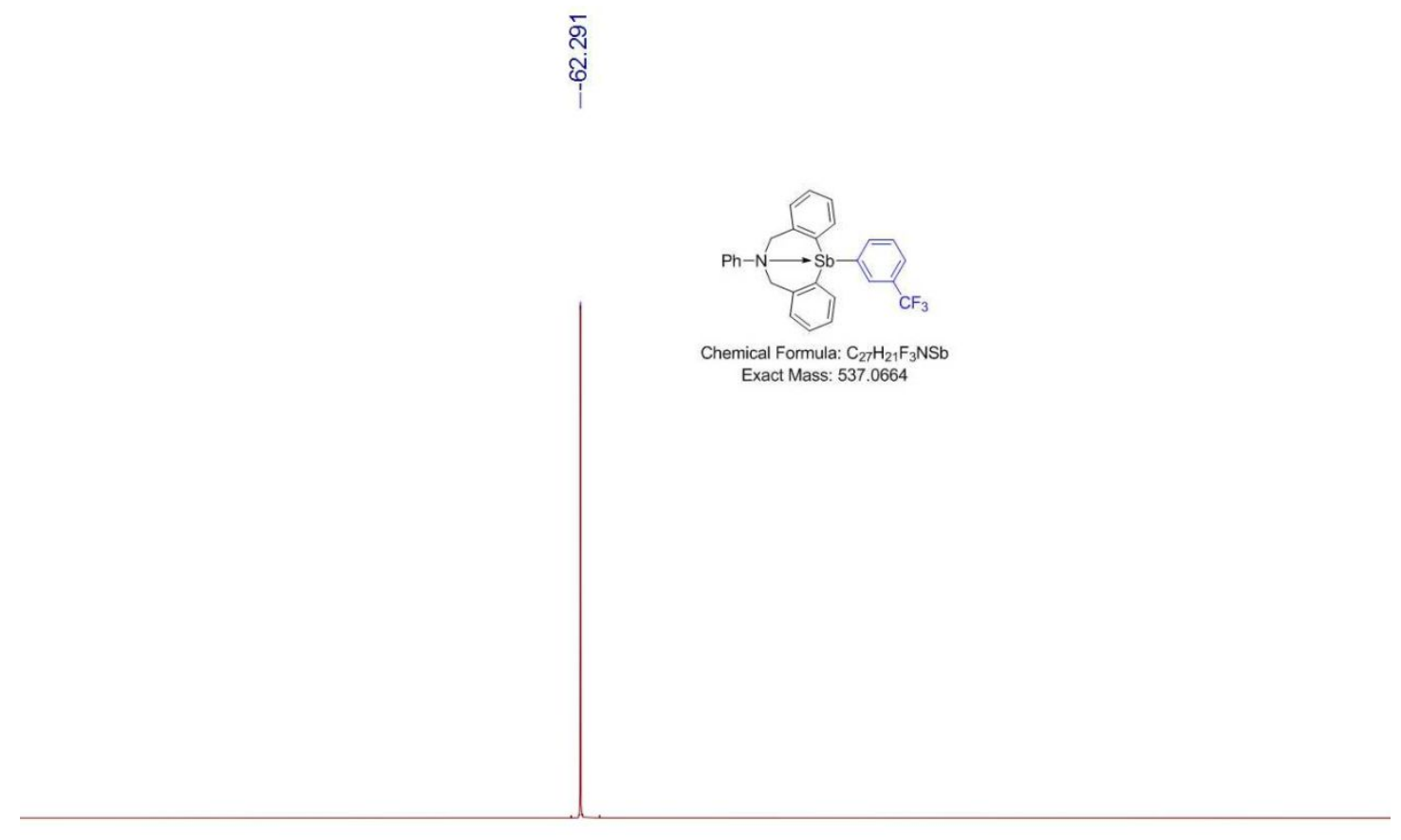

$-49-50-51-52-53-54-55-56-57-58-59-60-61-62-63-64-65-66-67-68-69-70-71-72-73-74-75-76-77-78-79-80-81-82$ $\mathrm{f1}(\mathrm{ppm})$

${ }^{1} \mathrm{H}$ NMR $\left(\mathrm{CDCl}_{3}\right)$ spectrum of compound 3p

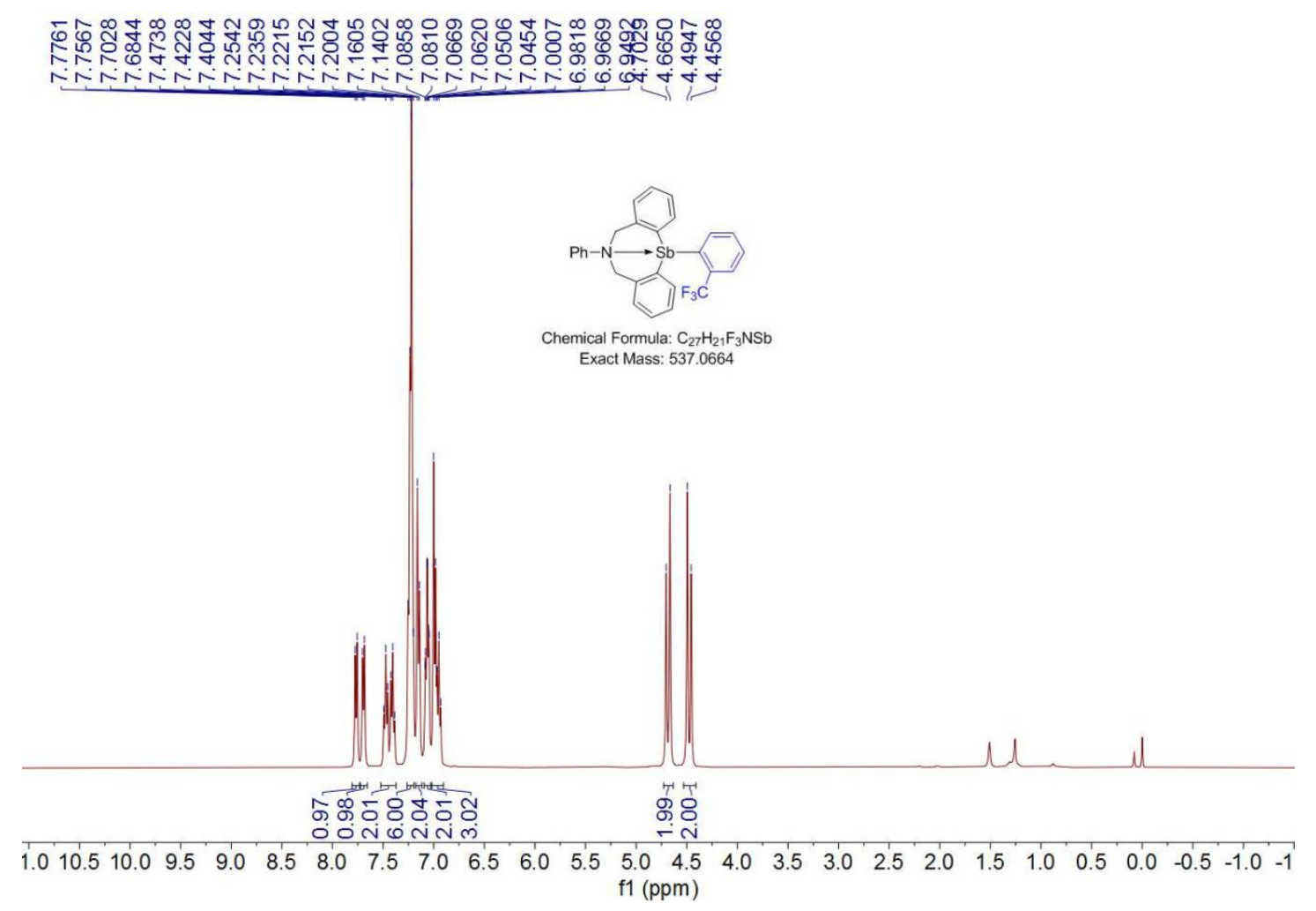

${ }^{13} \mathrm{C}$ NMR $\left(\mathrm{CDCl}_{3}\right)$ spectrum of compound $\mathbf{3 p}$ 


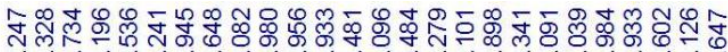

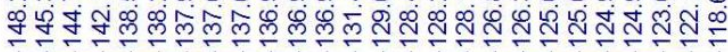

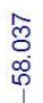

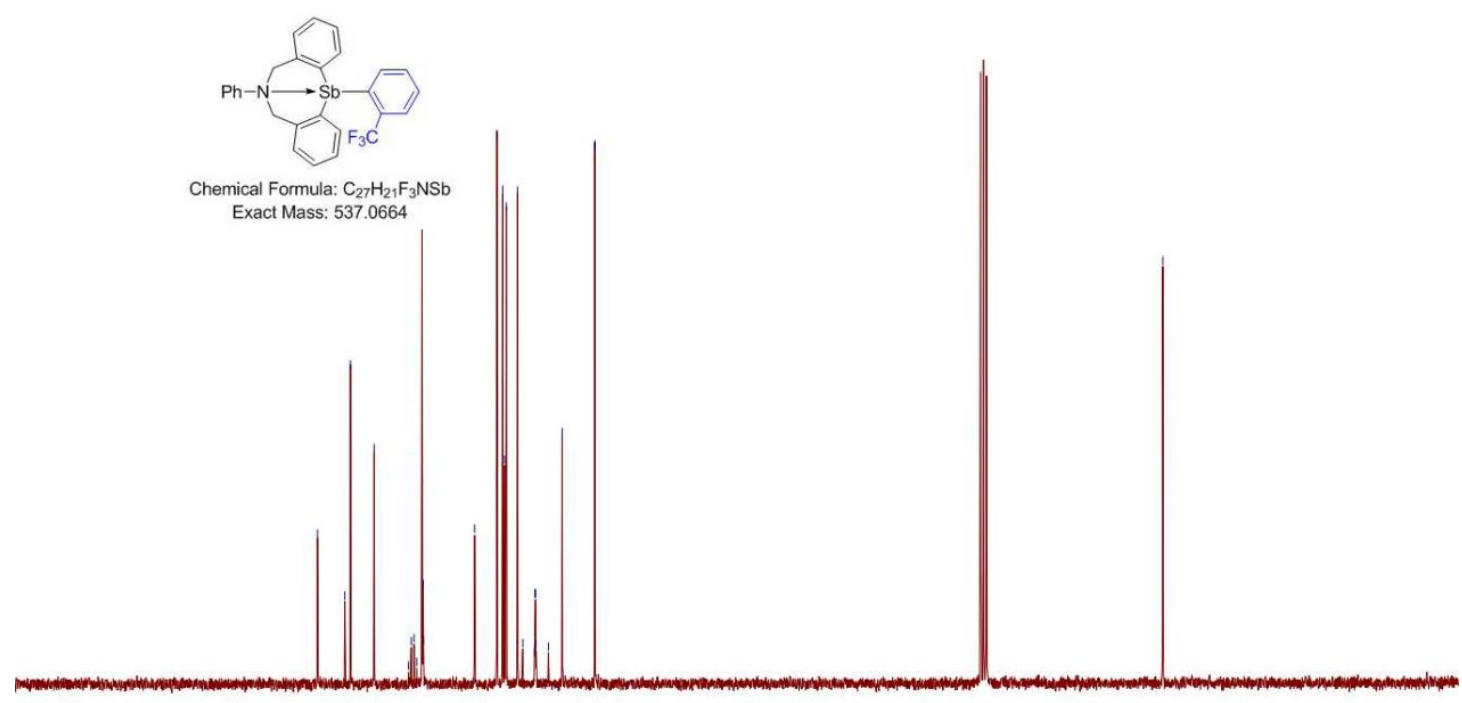

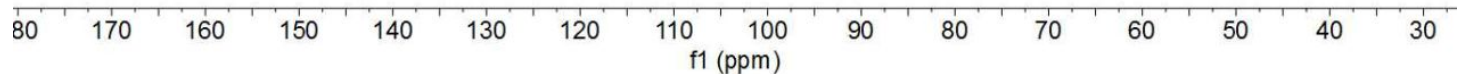

${ }^{19} \mathrm{~F}$ NMR $\left(\mathrm{CDCl}_{3}\right)$ spectrum of compound $\mathbf{3 p}$

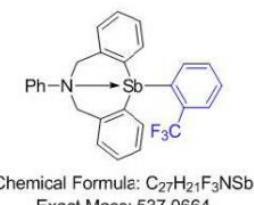

Exact Mass: 537.0664

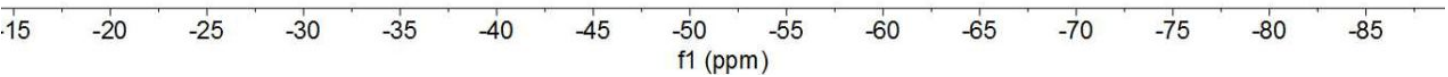

${ }^{1} \mathrm{H} \mathrm{NMR}\left(\mathrm{CDCl}_{3}\right)$ spectrum of compound $\mathbf{3 q}$ 


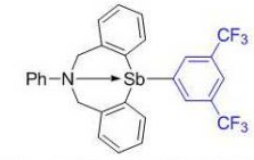

Chemical Formula: $\mathrm{C}_{28} \mathrm{H}_{20} \mathrm{~F}_{6} \mathrm{NS}$ Exact Mass: 605.0538

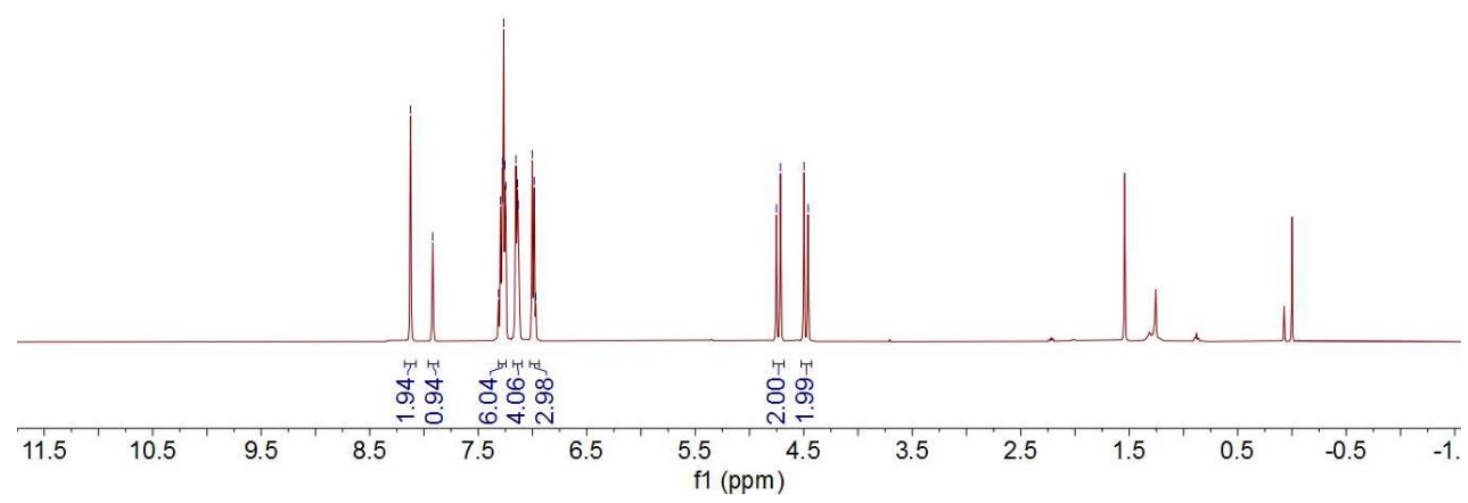

${ }^{13} \mathrm{C} \mathrm{NMR}\left(\mathrm{CDCl}_{3}\right)$ spectrum of compound $\mathbf{3 q}$

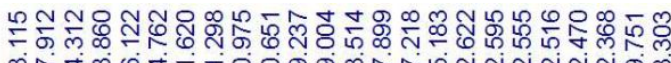

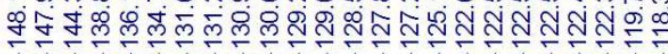

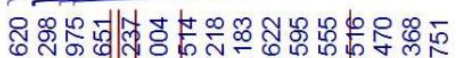

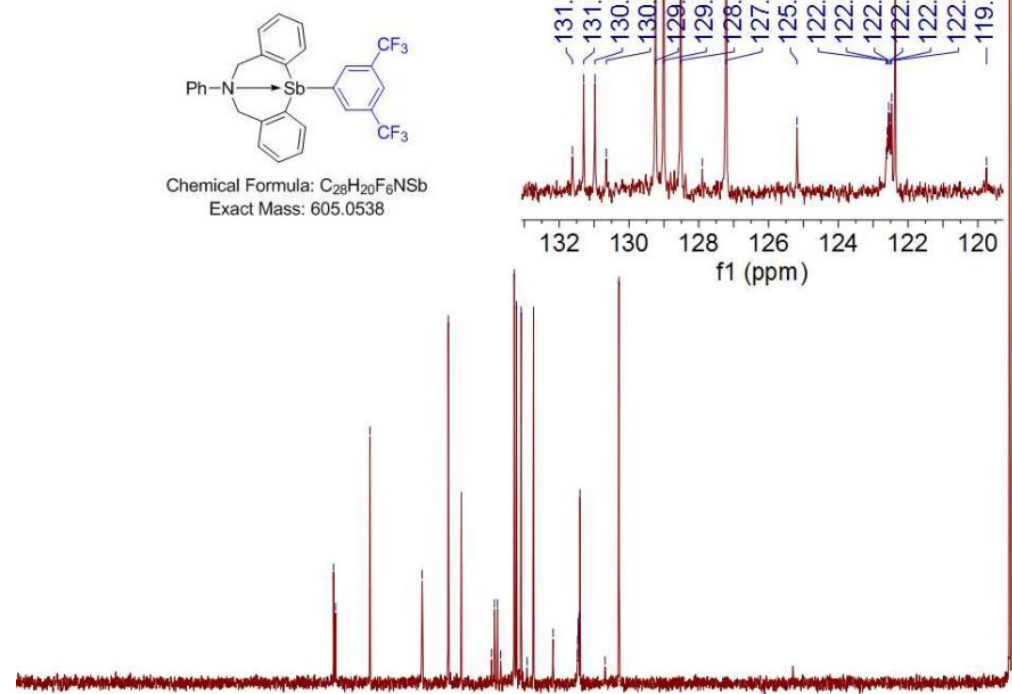

Chemical Formula: $\mathrm{C}_{28} \mathrm{H}_{20} \mathrm{~F}_{6} \mathrm{NSb}$ Exact Mass: 605.0538

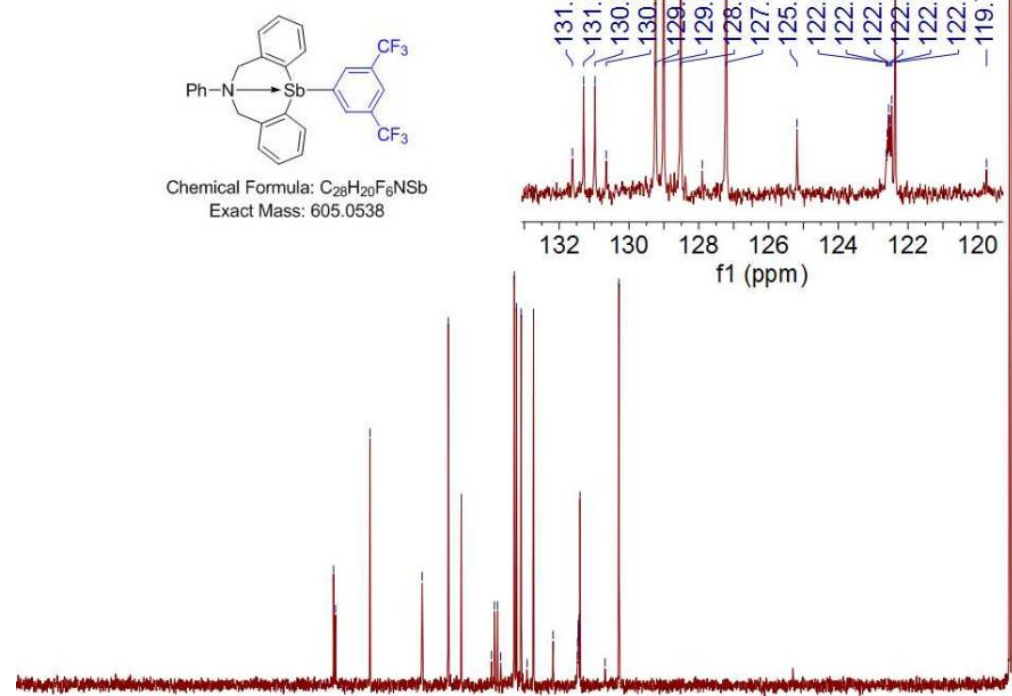

${ }^{19} \mathrm{~F}$ NMR $\left(\mathrm{CDCl}_{3}\right)$ spectrum of compound $\mathbf{3 q}$ 


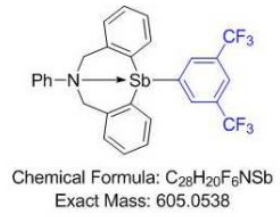

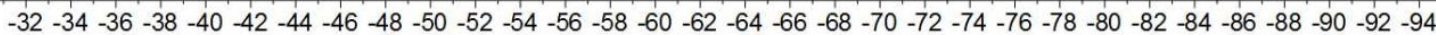
$\mathrm{f1}(\mathrm{ppm})$

${ }^{1} \mathrm{H}$ NMR $\left(\mathrm{CDCl}_{3}\right)$ spectrum of compound $\mathbf{3 r}$

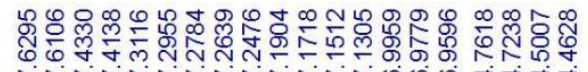

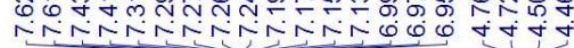
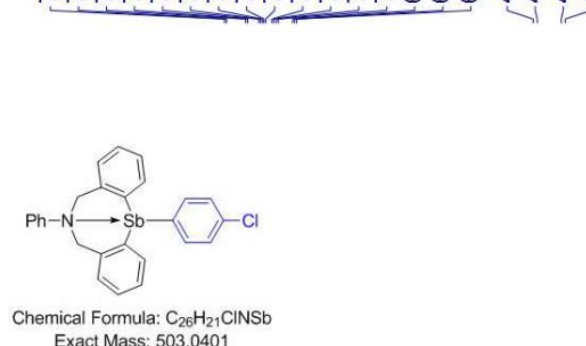

Exact Mass: 503.0401

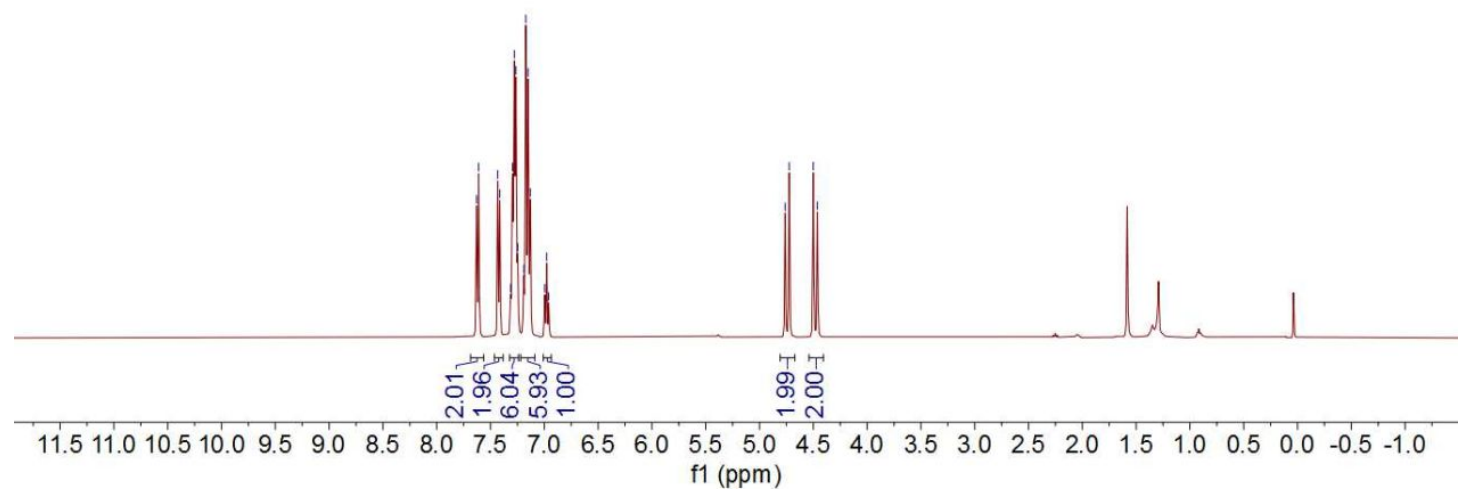

${ }^{13} \mathrm{C} \mathrm{NMR}\left(\mathrm{CDCl}_{3}\right)$ spectrum of compound $\mathbf{3 r}$ 

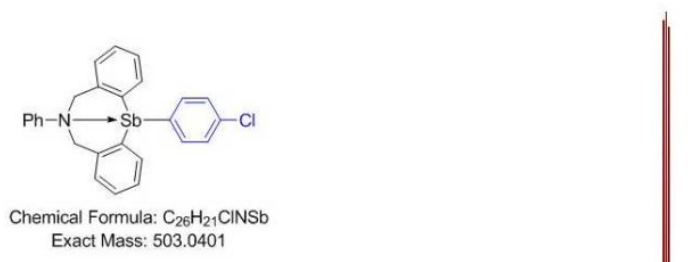
Exact Mass: 503.040

\begin{tabular}{lllllllllllllllllll}
\hline 90 & 180 & 170 & 160 & 150 & 140 & 130 & 120 & 110 & $\begin{array}{r}100 \\
\mathrm{f} 1(\mathrm{ppm})\end{array}$ & 90 & 80 & 70 & 60 & 50 & 40 & 30 & 20
\end{tabular}

${ }^{1} \mathrm{H}$ NMR $\left(\mathrm{CDCl}_{3}\right)$ spectrum of compound 3s
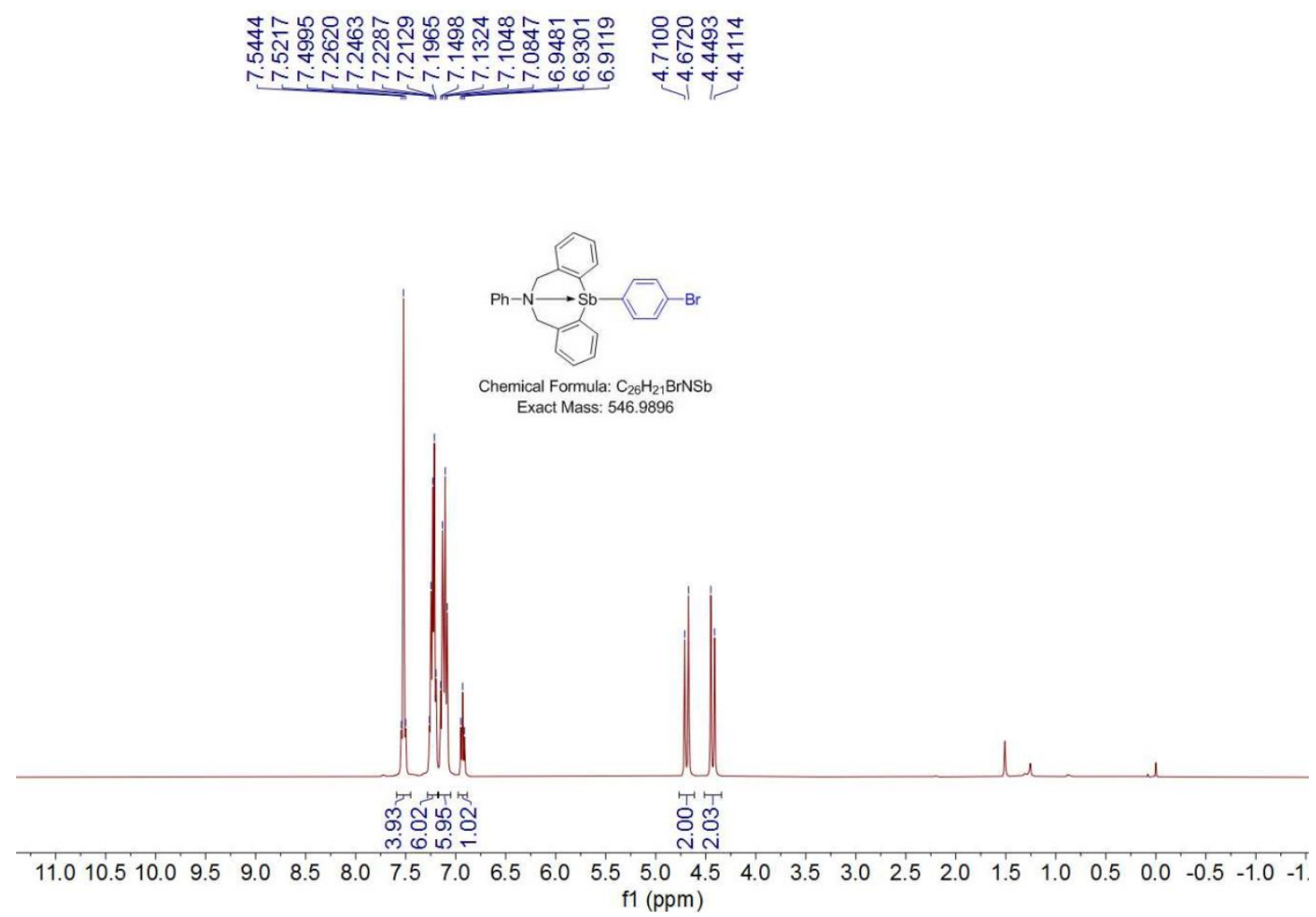

${ }^{13} \mathrm{C} \mathrm{NMR}\left(\mathrm{CDCl}_{3}\right)$ spectrum of compound $\mathbf{3 s}$ 


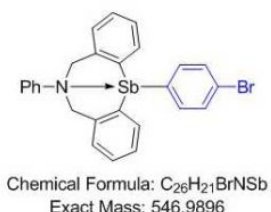

Exact Mass: 546.9896

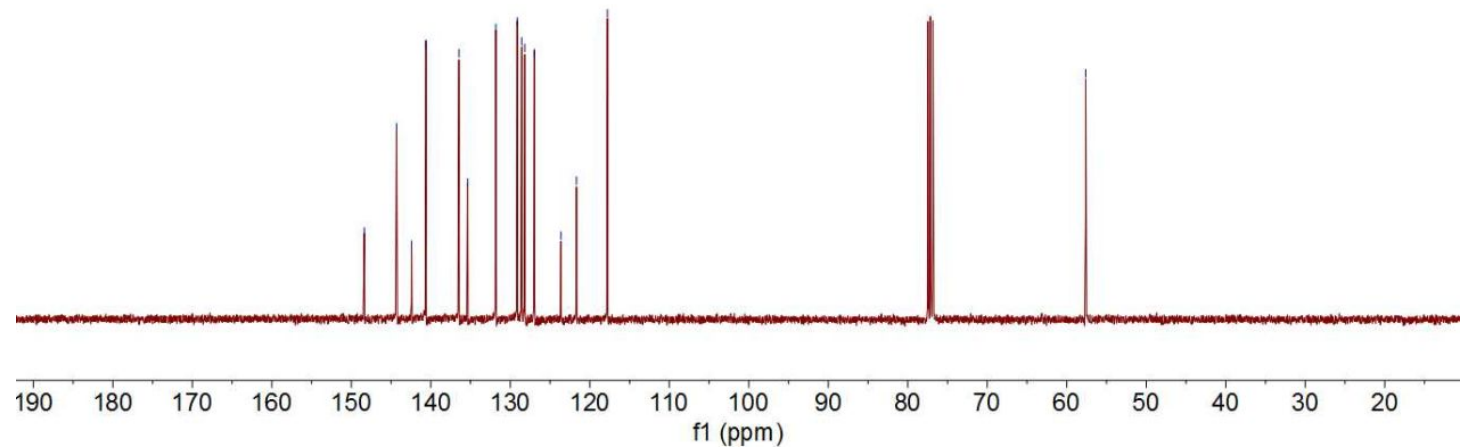

${ }^{1} \mathrm{H}$ NMR $\left(\mathrm{CDCl}_{3}\right)$ spectrum of compound $\mathbf{3 t}$
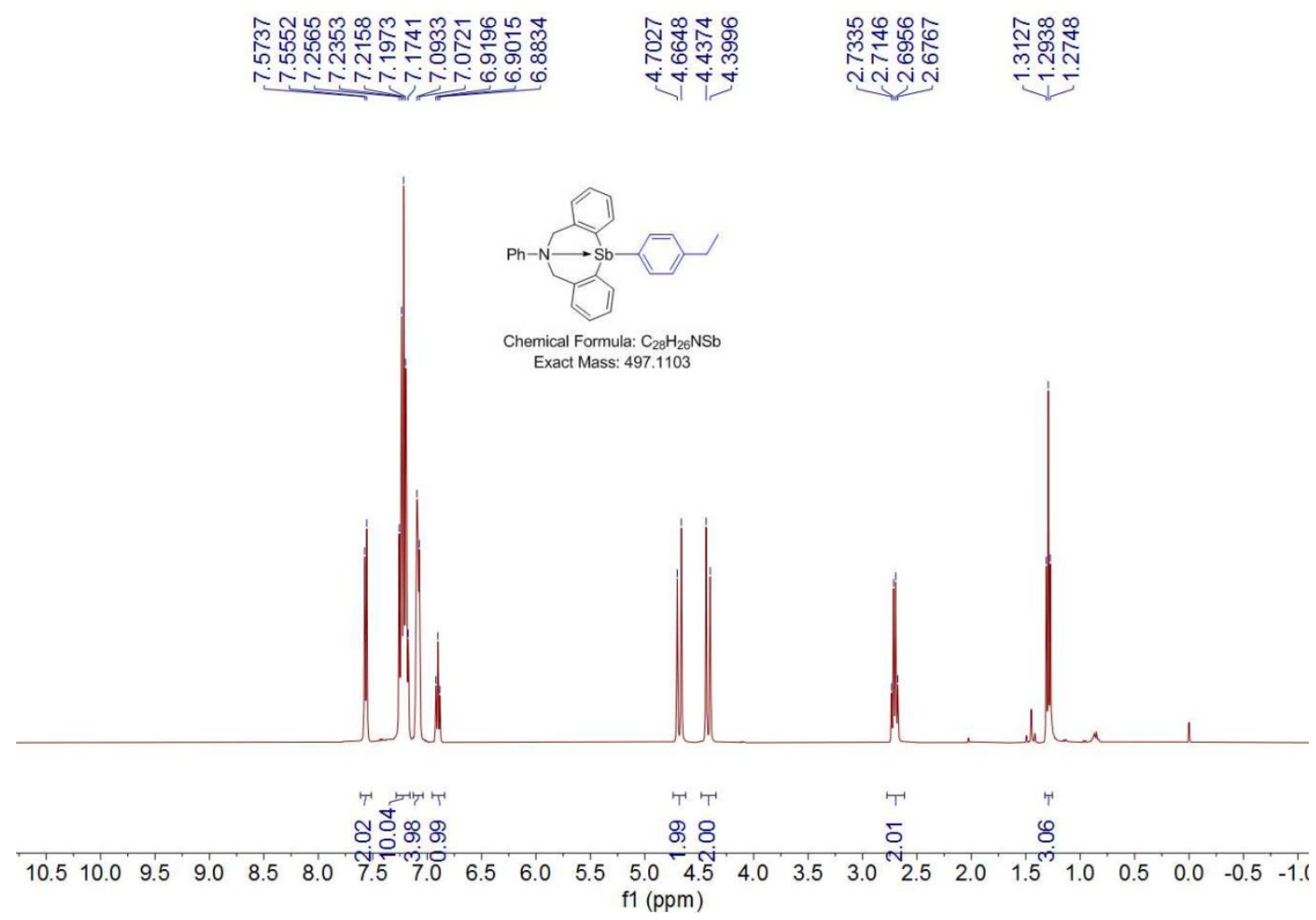

${ }^{13} \mathrm{C} \mathrm{NMR}\left(\mathrm{CDCl}_{3}\right)$ spectrum of compound $\mathbf{3 t}$ 


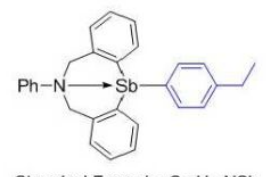

Chemical Formula: $\mathrm{C}_{28} \mathrm{H}_{26} \mathrm{NSb}$ Exact Mass: 497.1103

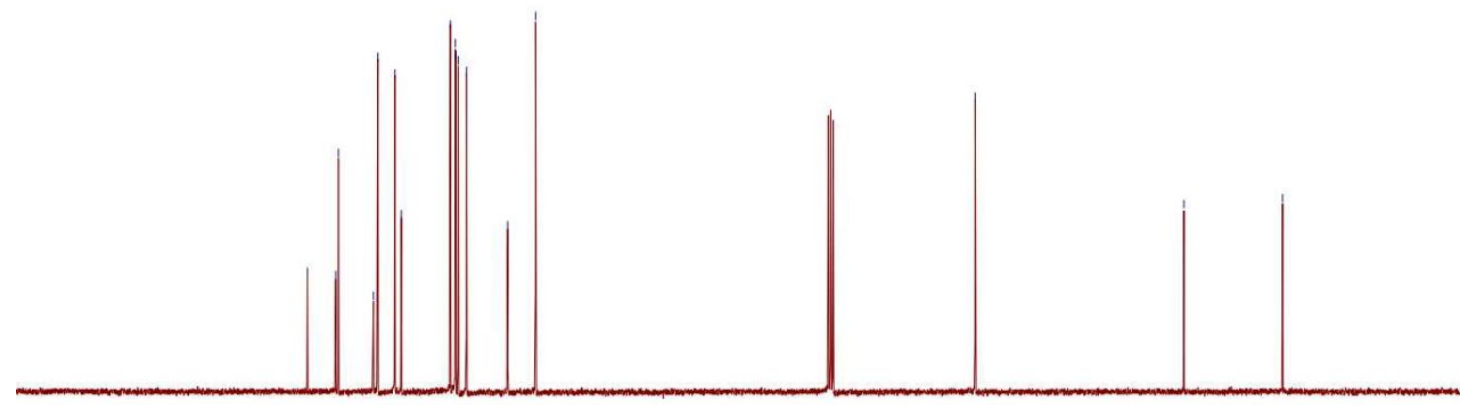

$\begin{array}{llllllllllllllllllll}180 & 170 & 160 & 150 & 140 & 130 & 120 & 110 & 100 & \begin{array}{c}90 \\ \mathrm{f} 1(\mathrm{ppm})\end{array} & 80 & 70 & 60 & 50 & 40 & 30 & 20 & 10 & 0\end{array}$

${ }^{1} \mathrm{H} \mathrm{NMR}\left(\mathrm{CDCl}_{3}\right)$ spectrum of compound $\mathbf{3 u}$
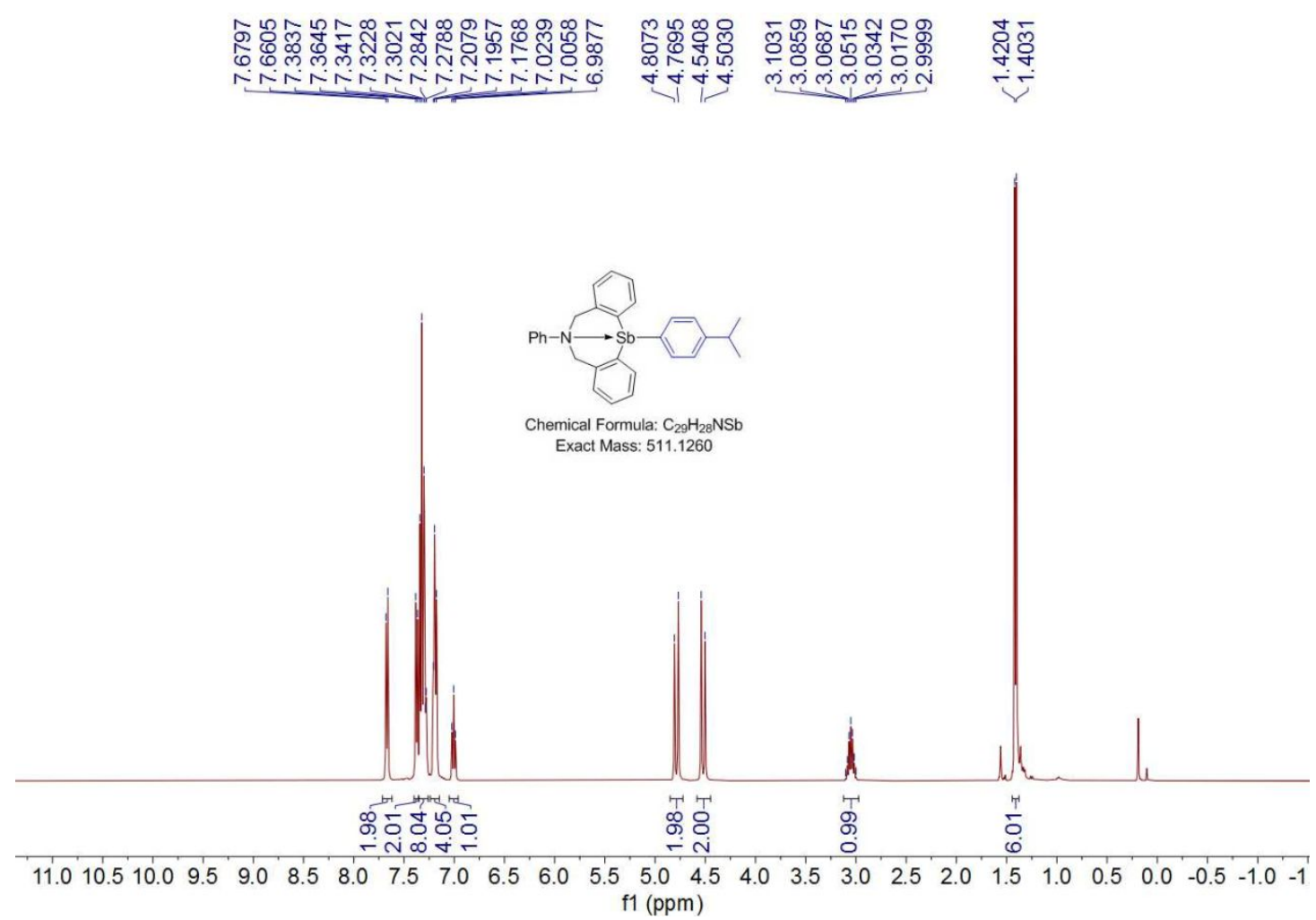

${ }^{13} \mathrm{C} \mathrm{NMR}\left(\mathrm{CDCl}_{3}\right)$ spectrum of compound $\mathbf{3 u}$ 


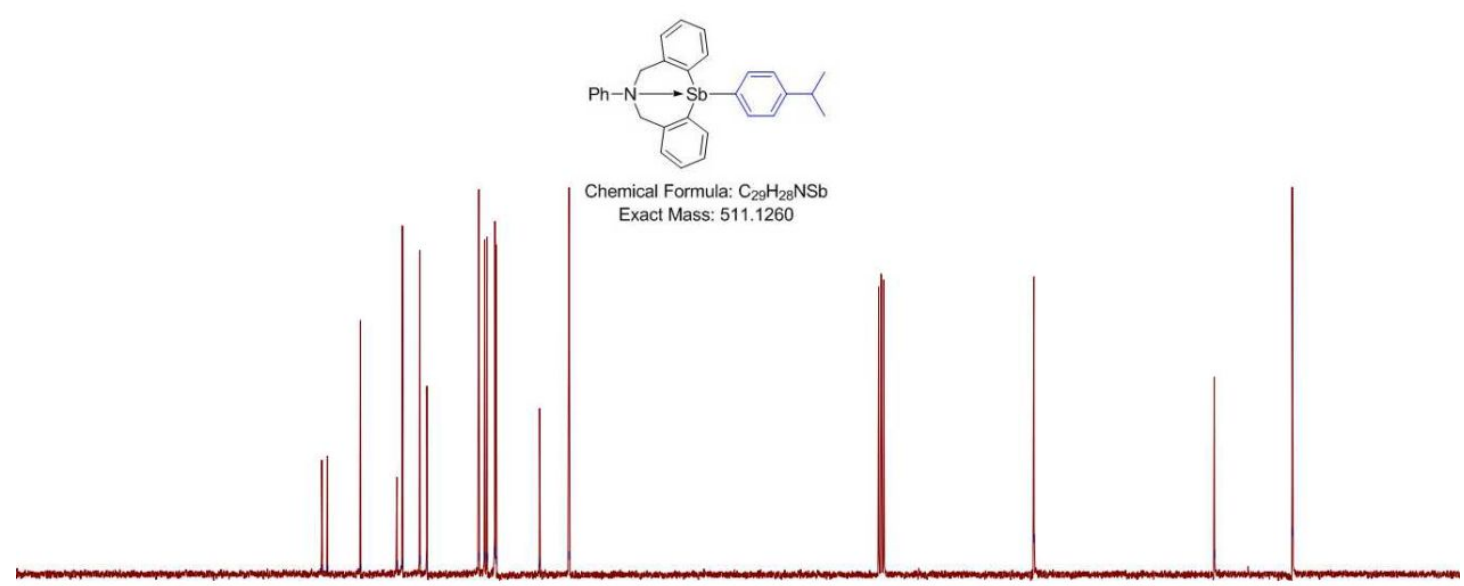

$\begin{array}{llllllllllllllllll}180 & 170 & 160 & 150 & 140 & 130 & 120 & 110 & \begin{array}{l}100 \\ \mathrm{f} 1(\mathrm{ppm})\end{array} & 80 & 70 & 60 & 50 & 40 & 30 & 20 & 10\end{array}$

${ }^{1} \mathrm{H}$ NMR $\left(\mathrm{CDCl}_{3}\right)$ spectrum of compound $\mathbf{3 v}$
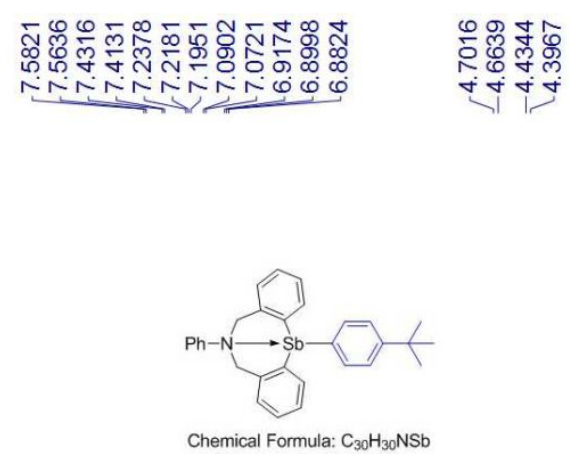

Exact Mass: 525.1416

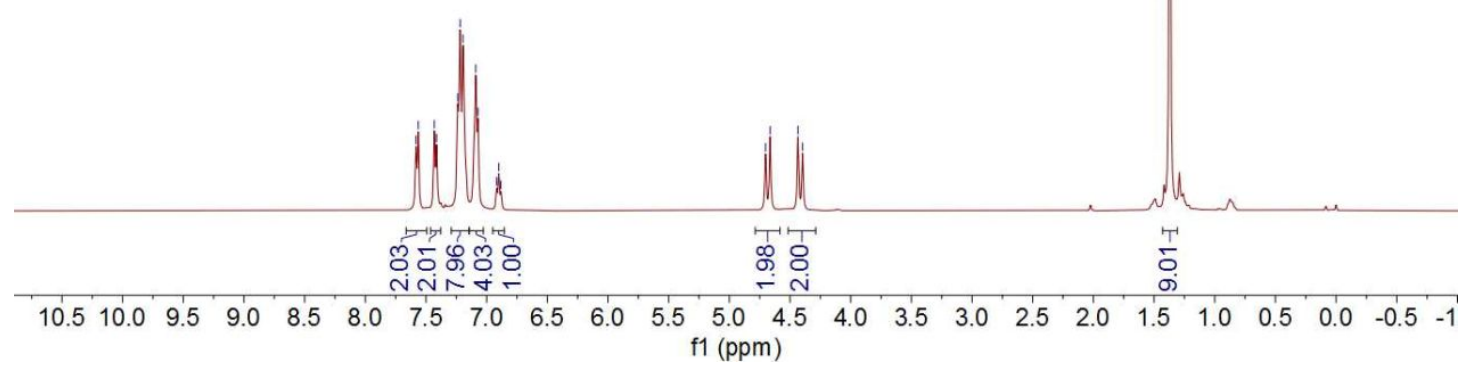

${ }^{13} \mathrm{C} \mathrm{NMR}\left(\mathrm{CDCl}_{3}\right)$ spectrum of compound $\mathbf{3 v}$ 

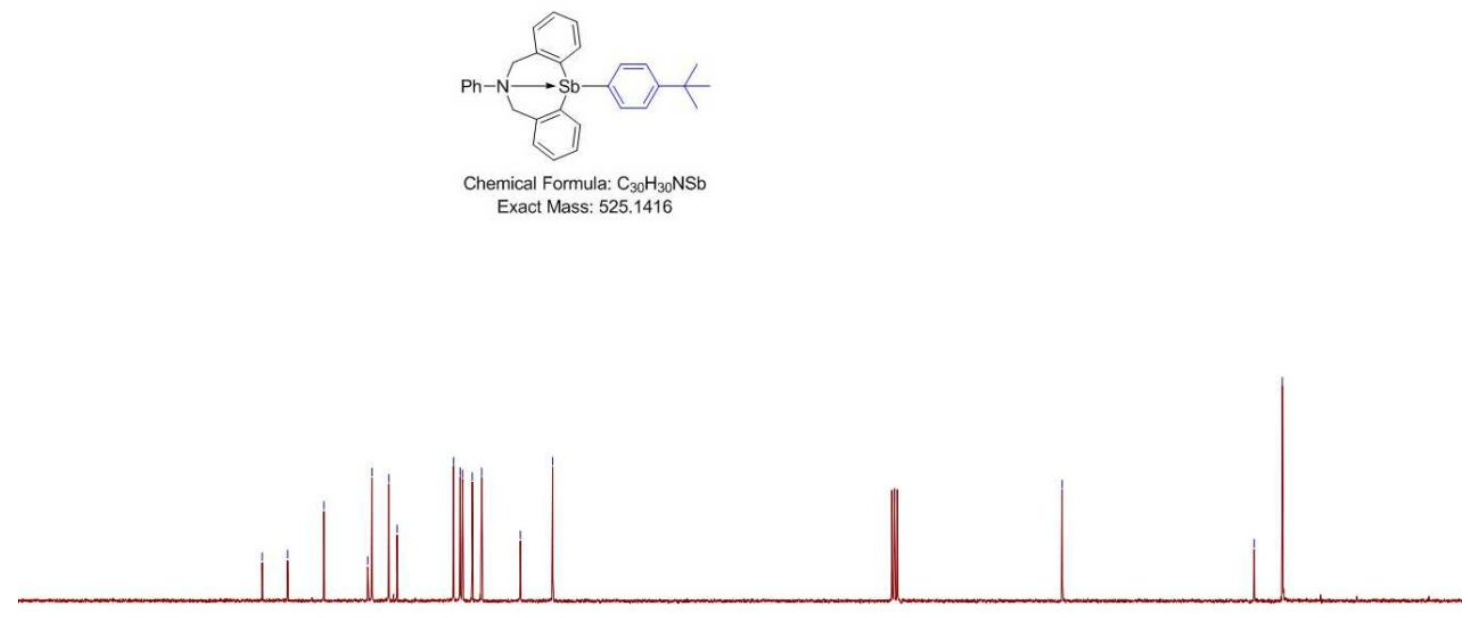

\begin{tabular}{lllllllllllllllll}
\hline 30 & 170 & 160 & 150 & 140 & 130 & 120 & 110 & $\begin{array}{c}100 \\
\mathrm{f} 1(\mathrm{ppm})\end{array}$ & 80 & 70 & 60 & 50 & 40 & 30 & 20
\end{tabular}

${ }^{1} \mathrm{H}$ NMR $\left(\mathrm{CDCl}_{3}\right)$ spectrum of compound $\mathbf{3 w}$

\begin{tabular}{|c|c|c|}
\hline 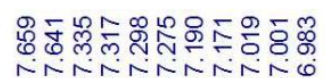 & 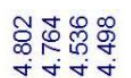 & 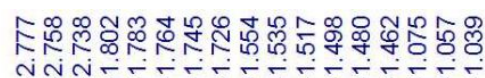 \\
\hline & & \\
\hline
\end{tabular}

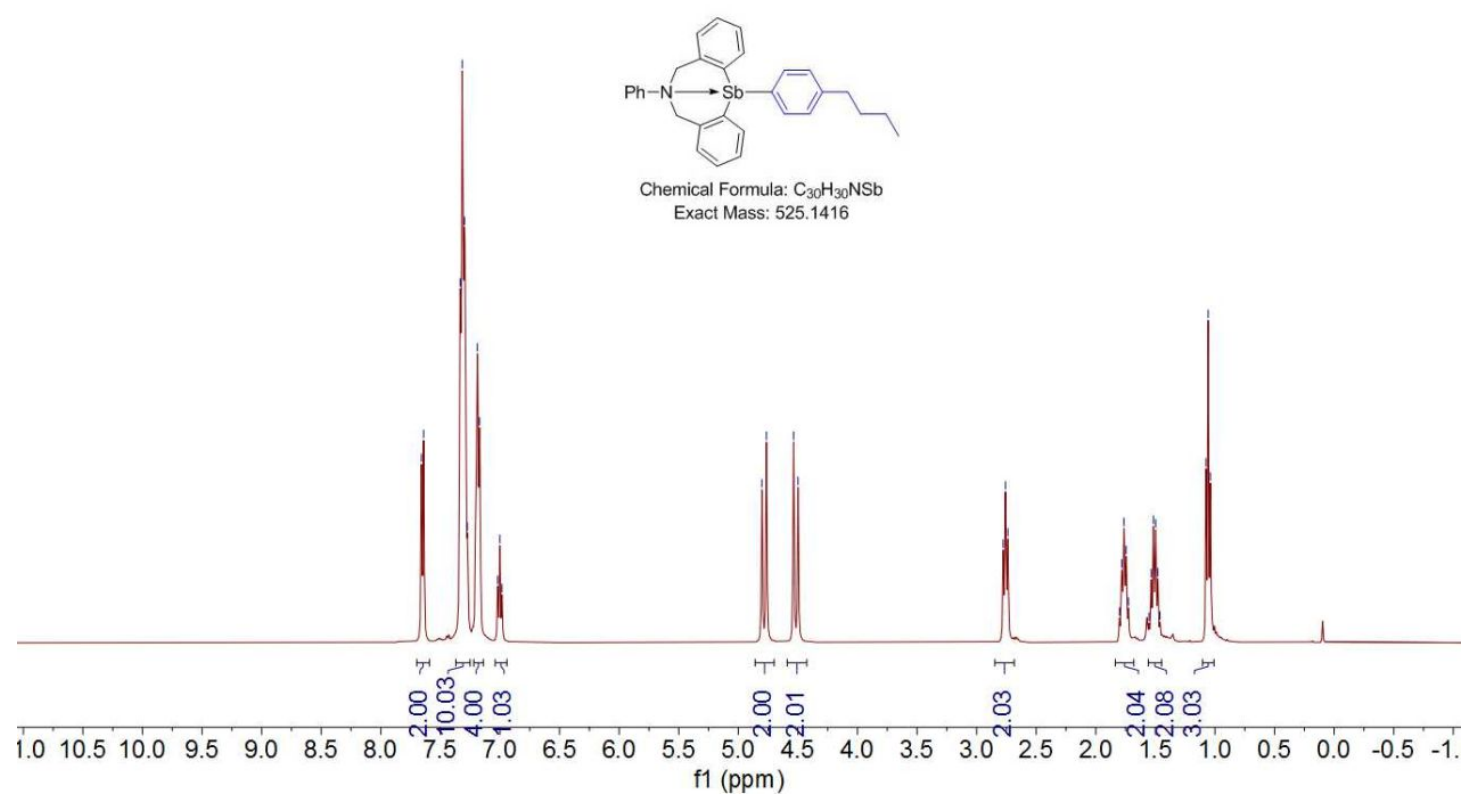

${ }^{13} \mathrm{C} \mathrm{NMR}\left(\mathrm{CDCl}_{3}\right)$ spectrum of compound $\mathbf{3 w}$ 

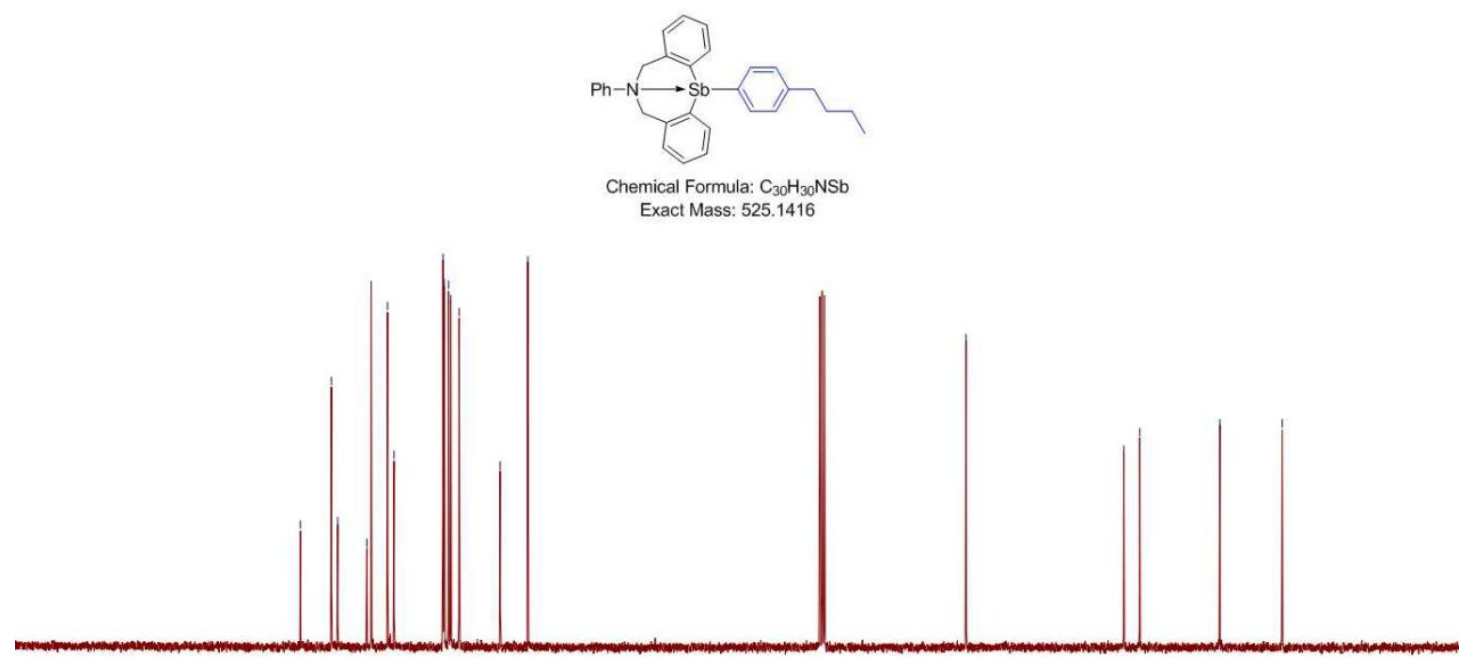

$\begin{array}{lllllllllllllllllllllllll}180 & 170 & 160 & 150 & 140 & 130 & 120 & 110 & 100 & 90 & 80 & 70 & 60 & 50 & 40 & 30 & 20 & 10 & 0\end{array}$

${ }^{1} \mathrm{H}$ NMR $\left(\mathrm{CDCl}_{3}\right)$ spectrum of compound $\mathbf{3 x}$

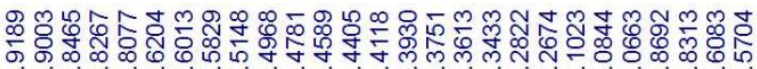

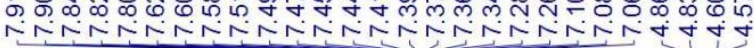

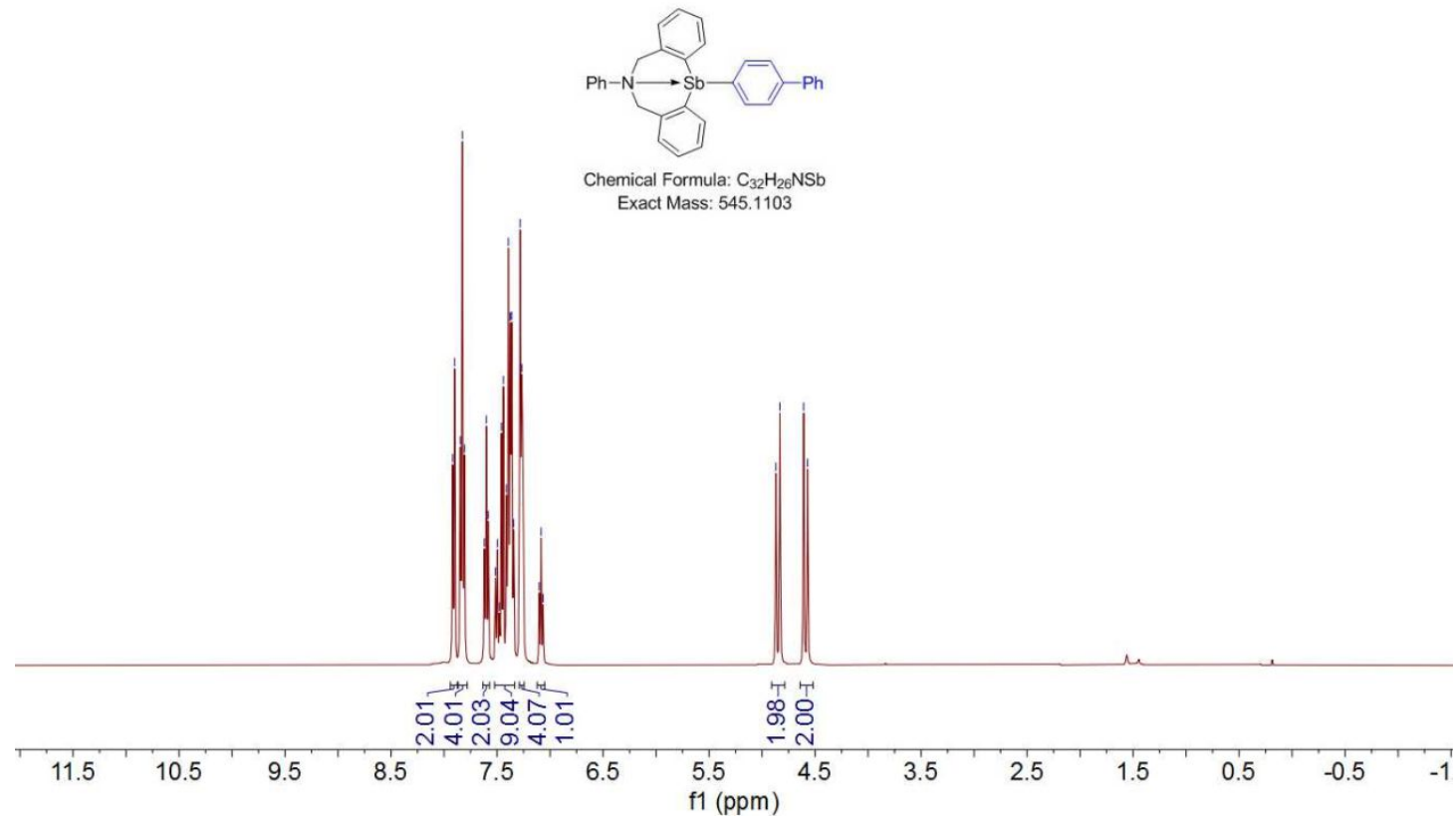

${ }^{13} \mathrm{C} \mathrm{NMR}\left(\mathrm{CDCl}_{3}\right)$ spectrum of compound $\mathbf{3 x}$ 


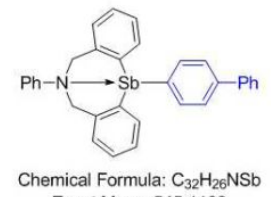
Exact Mass: 545.1103

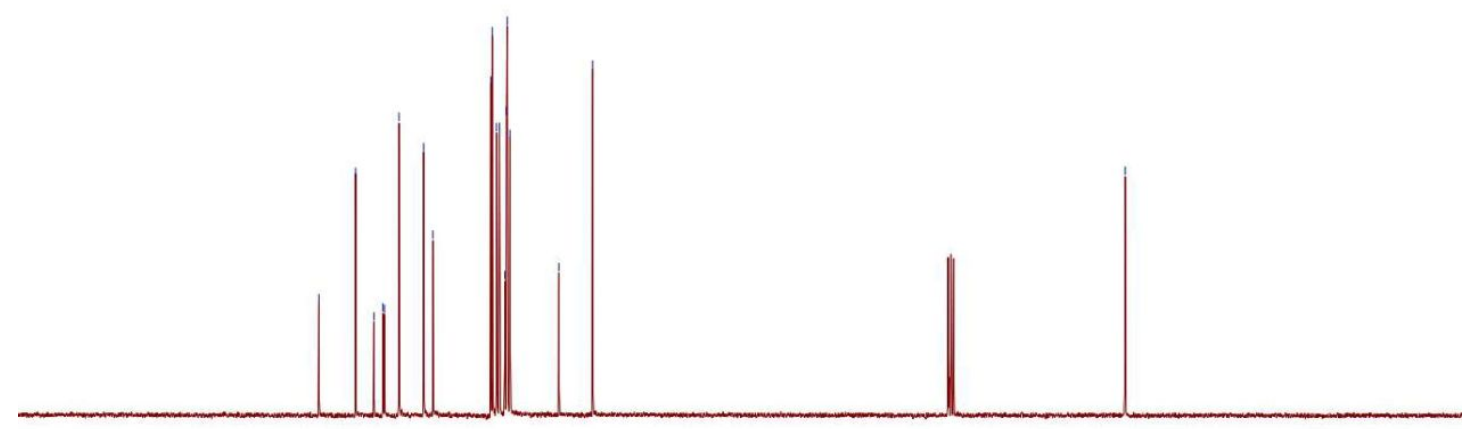

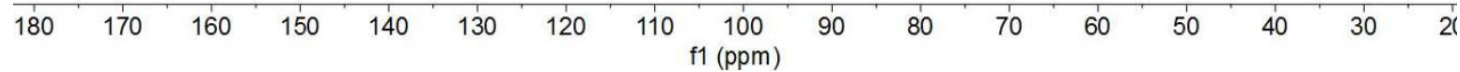

${ }^{1} \mathrm{H}$ NMR $\left(\mathrm{CDCl}_{3}\right)$ spectrum of compound $\mathbf{3 y}$
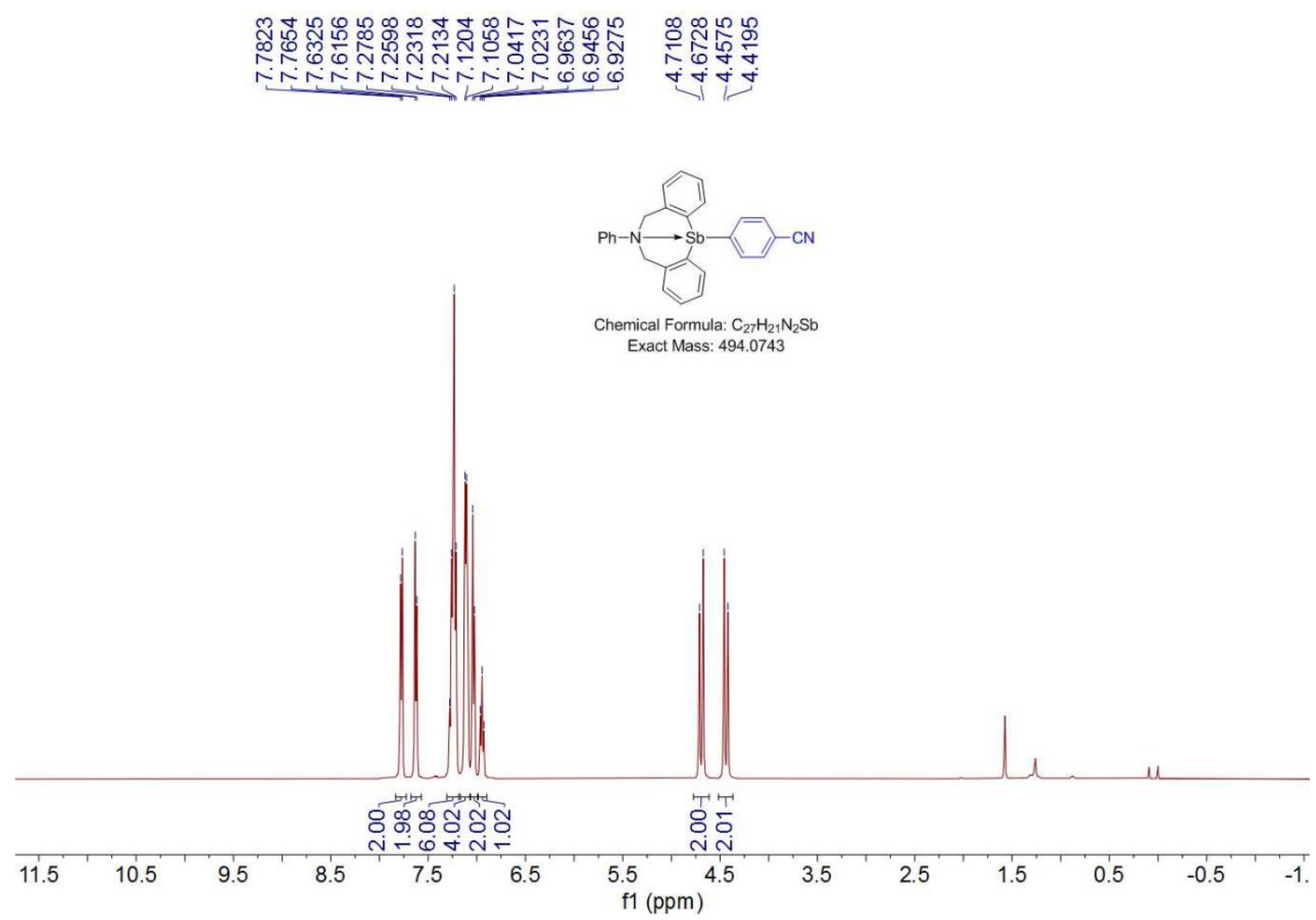

${ }^{13} \mathrm{C} \mathrm{NMR}\left(\mathrm{CDCl}_{3}\right)$ spectrum of compound $\mathbf{3 y}$ 


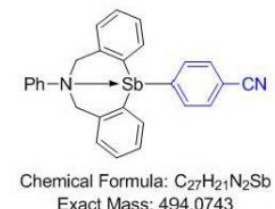

Exact Mass: 494.0743

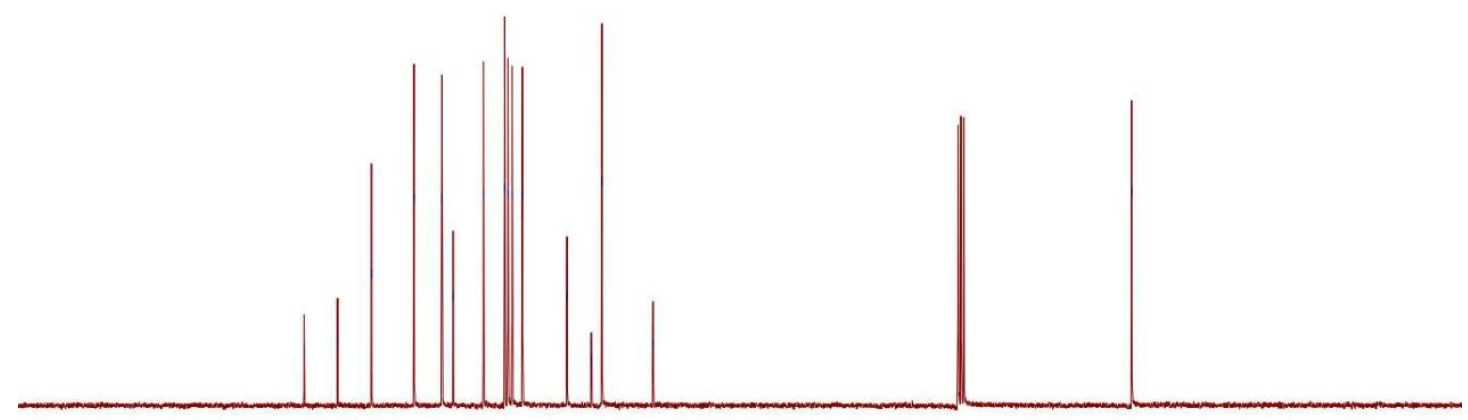

\begin{tabular}{llllllllllllllll}
\hline 180 & 170 & 160 & 150 & 140 & 130 & 120 & $110 \begin{array}{r}100 \\
\mathrm{f} 1(\mathrm{ppm})\end{array}$ & 90 & 80 & 70 & 60 & 50 & 40 & 30
\end{tabular}

${ }^{1} \mathrm{H}$ NMR $\left(\mathrm{CDCl}_{3}\right)$ spectrum of compound $\mathbf{3 z}$

\section{\&}

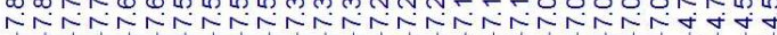

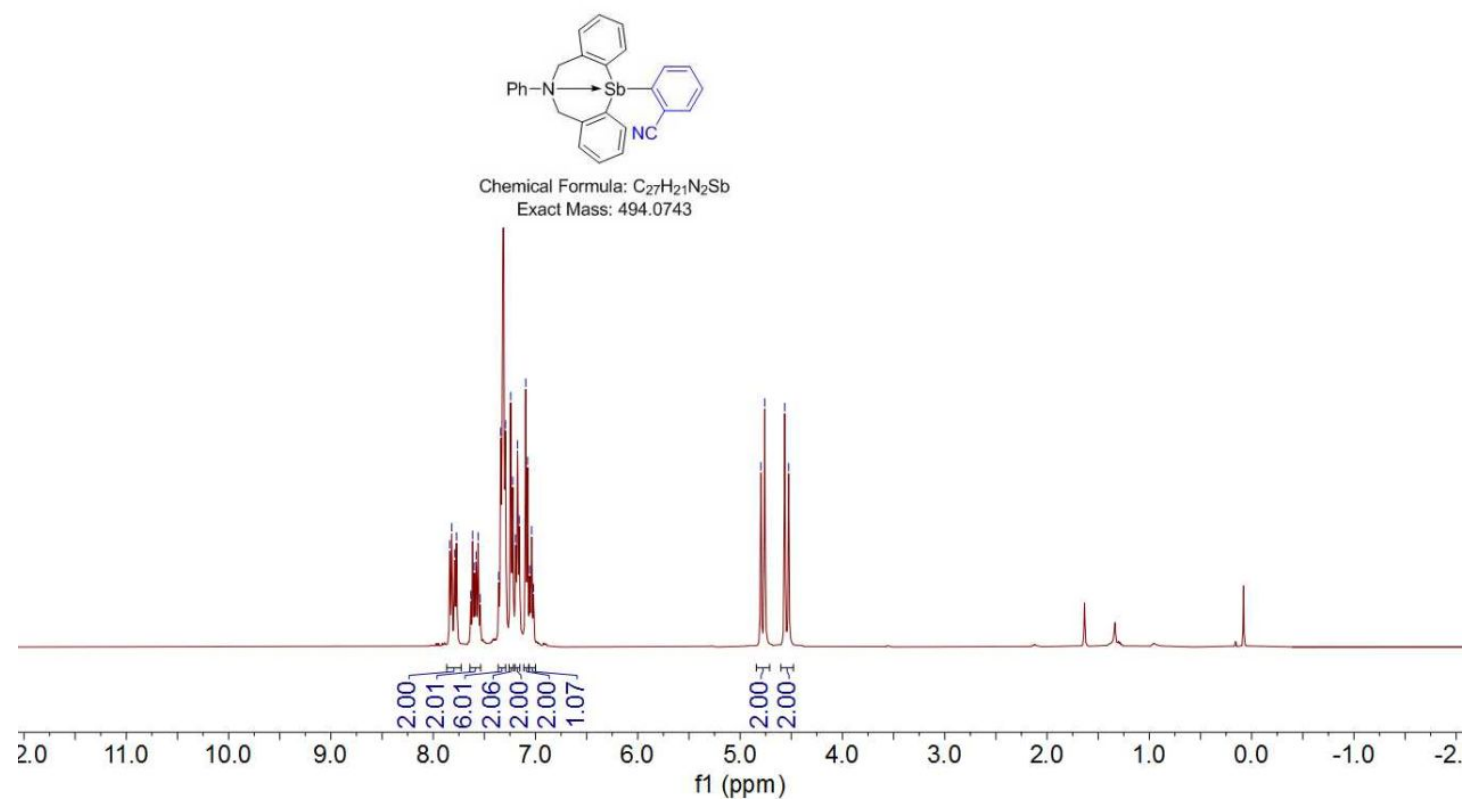

${ }^{13} \mathrm{C} \mathrm{NMR}\left(\mathrm{CDCl}_{3}\right)$ spectrum of compound $\mathbf{3 z}$ 


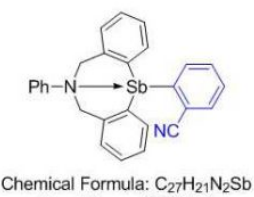

Exact Mass: 494.0743

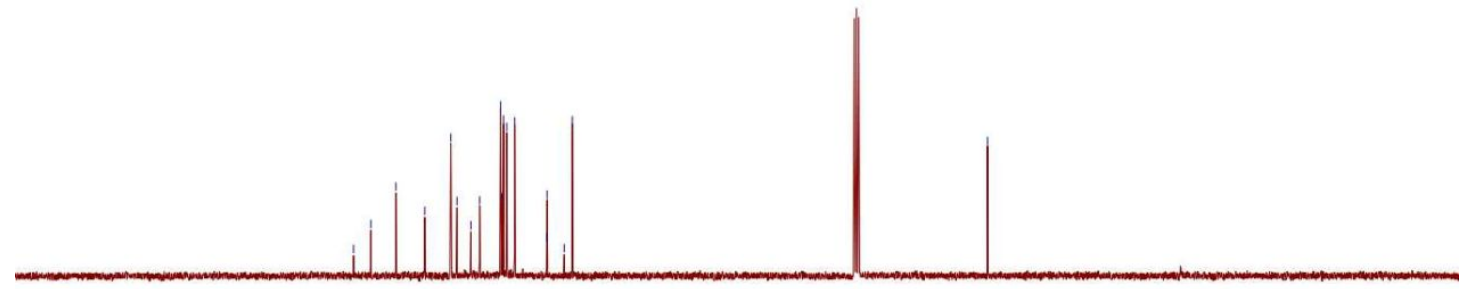

\begin{tabular}{llllllllllllllllllllllllll}
\hline 0 & 190 & 180 & 170 & 160 & 150 & 140 & 130 & 120 & 110 & $\begin{array}{r}100 \\
\mathrm{f} 1(\mathrm{ppm})\end{array}$ & 90 & 80 & 70 & 60 & 50 & 40 & 30 & 20 & 10 & 0 & -1
\end{tabular}

${ }^{1} \mathrm{H}$ NMR $\left(\mathrm{CDCl}_{3}\right)$ spectrum of compound 3aa

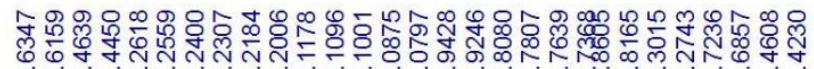

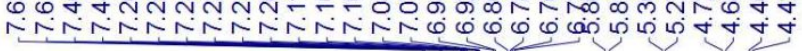

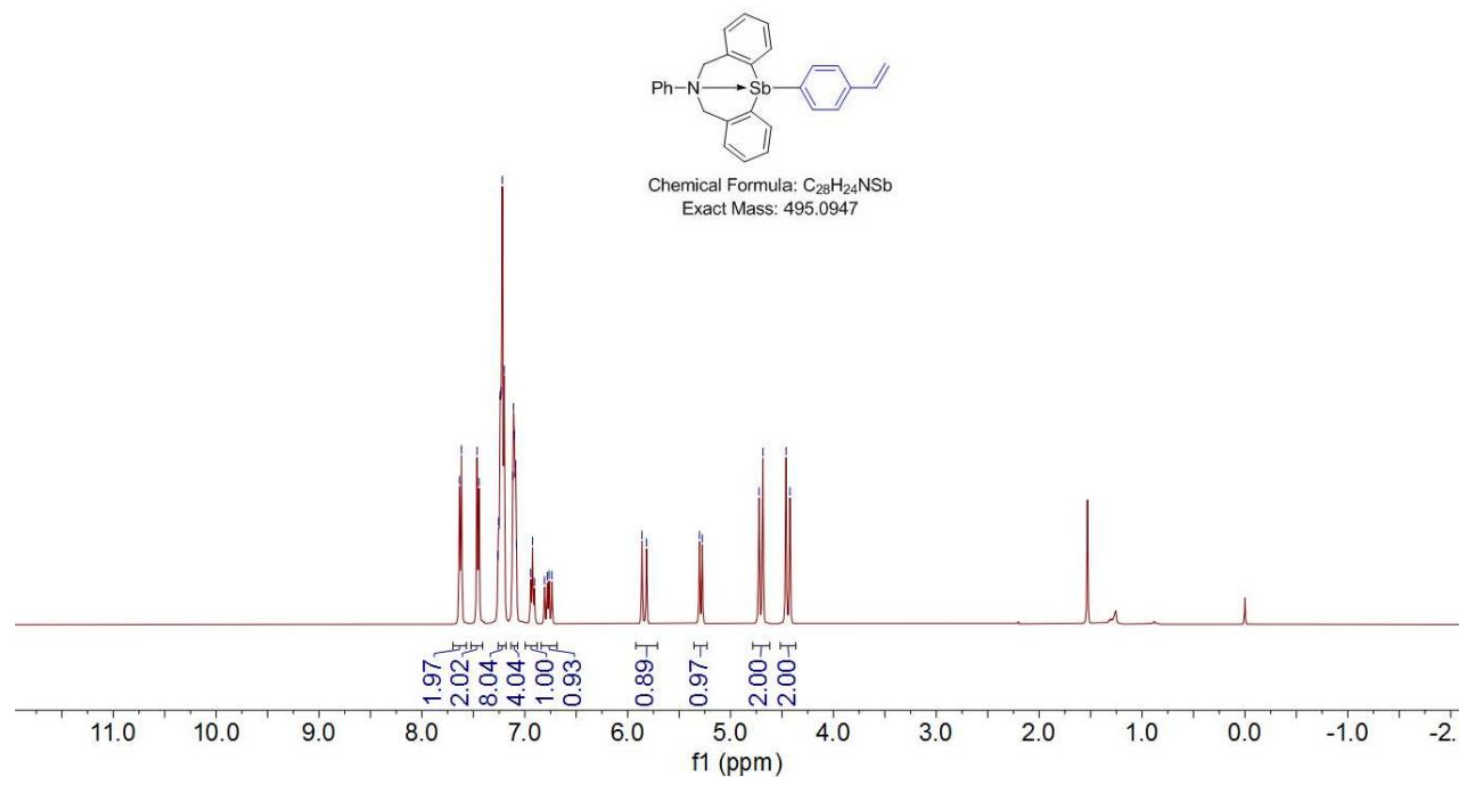

${ }^{13} \mathrm{C} \mathrm{NMR}\left(\mathrm{CDCl}_{3}\right)$ spectrum of compound 3aa 


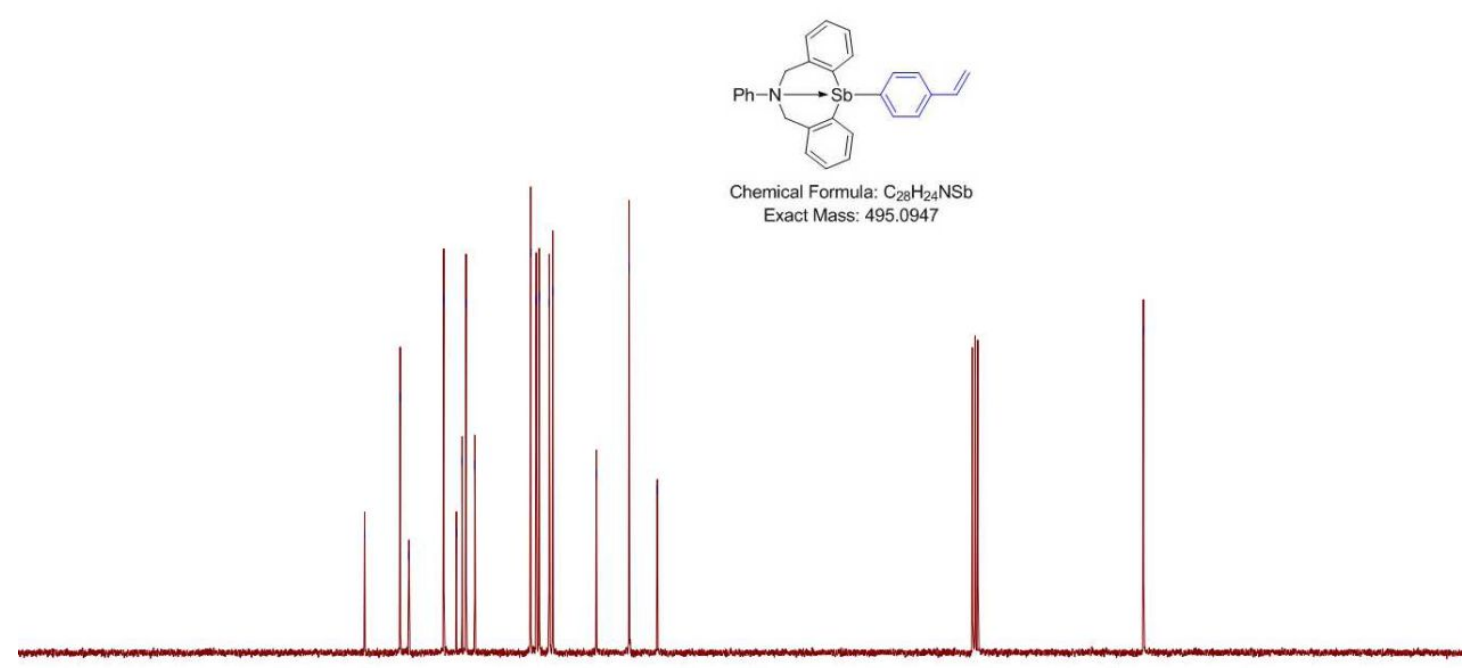

$\begin{array}{llllllllllllllll}180 & 170 & 160 & 150 & 140 & 130 & 120 & \begin{array}{c}110 \\ \mathrm{f} 1(\mathrm{ppm})\end{array} & 90 & 80 & 70 & 60 & 50 & 40 & 30\end{array}$

${ }^{1} \mathrm{H}$ NMR $\left(\mathrm{CDCl}_{3}\right)$ spectrum of compound 3ab

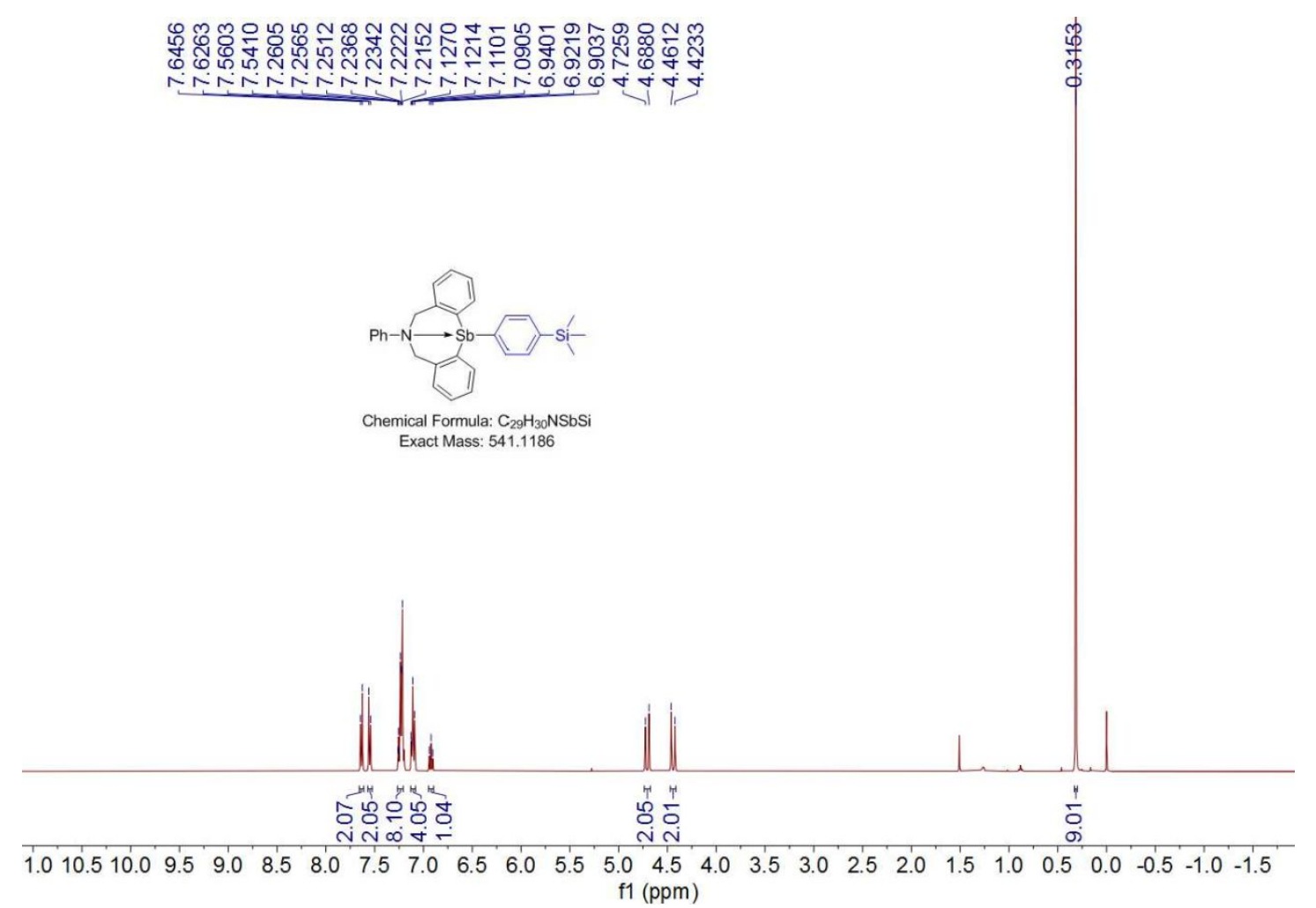


${ }^{13} \mathrm{C} \mathrm{NMR}\left(\mathrm{CDCl}_{3}\right)$ spectrum of compound 3ab

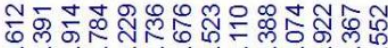

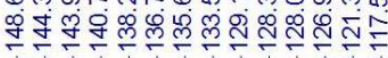

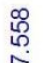

ֻ

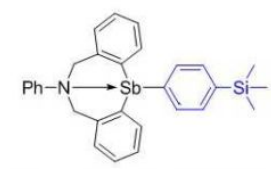

Chemical Formula: $\mathrm{C}_{29} \mathrm{H}_{30} \mathrm{NSbS}$ Exact Mass: 541.1186

$\begin{array}{llllllllllllllllllllll}180 & 170 & 160 & 150 & 140 & 130 & 120 & 110 & 100 & \begin{array}{c}90 \\ \mathrm{f} 1(\mathrm{ppm})\end{array} & \begin{array}{c}1 \\ 1\end{array} & 70 & 60 & 50 & 40 & 30 & 20 & 10 & 0 & -10\end{array}$

${ }^{1} \mathrm{H}$ NMR $\left(\mathrm{CDCl}_{3}\right)$ spectrum of compound 3ac
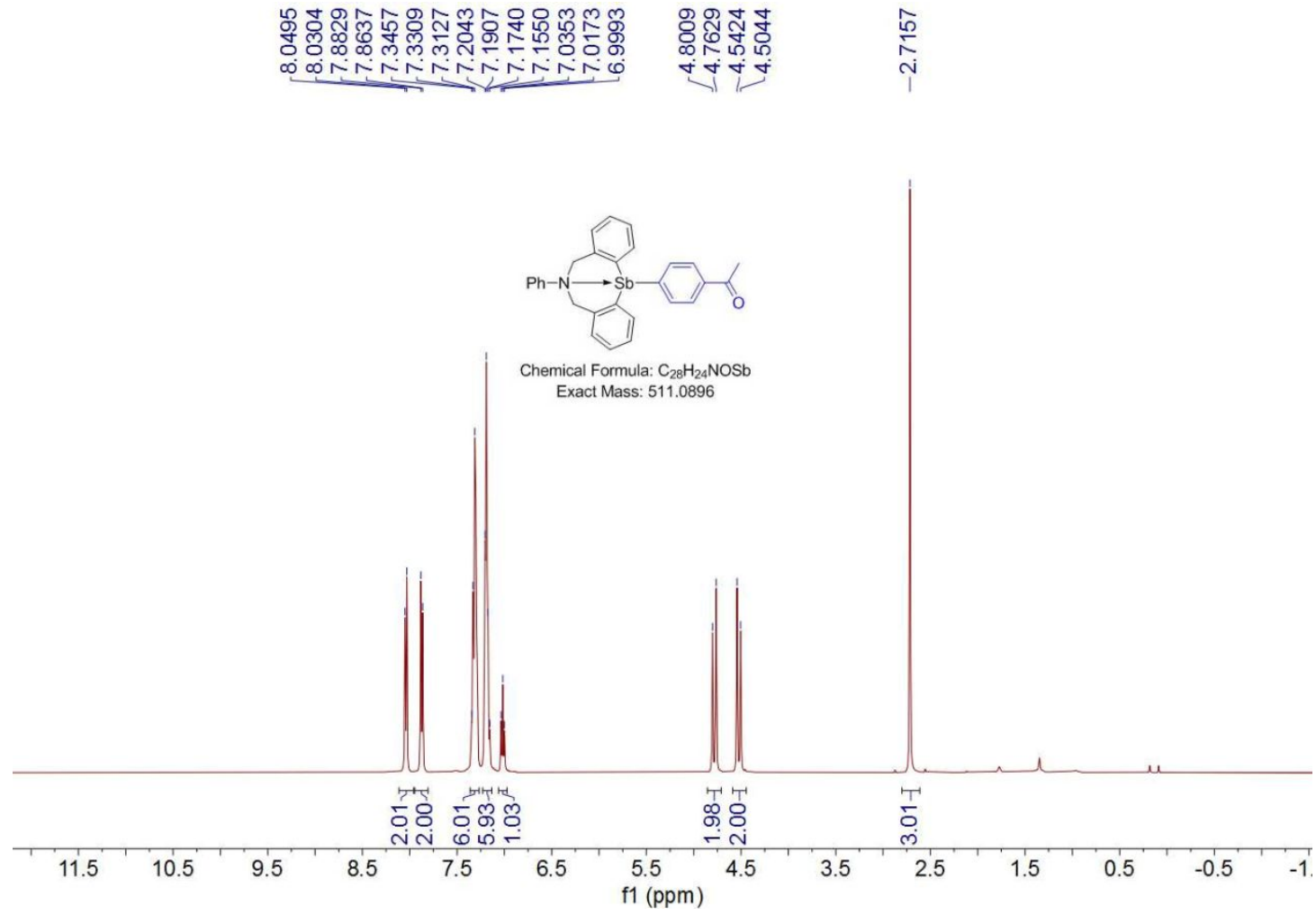

${ }^{13} \mathrm{C} \mathrm{NMR}\left(\mathrm{CDCl}_{3}\right)$ spectrum of compound $\mathbf{3 a c}$ 


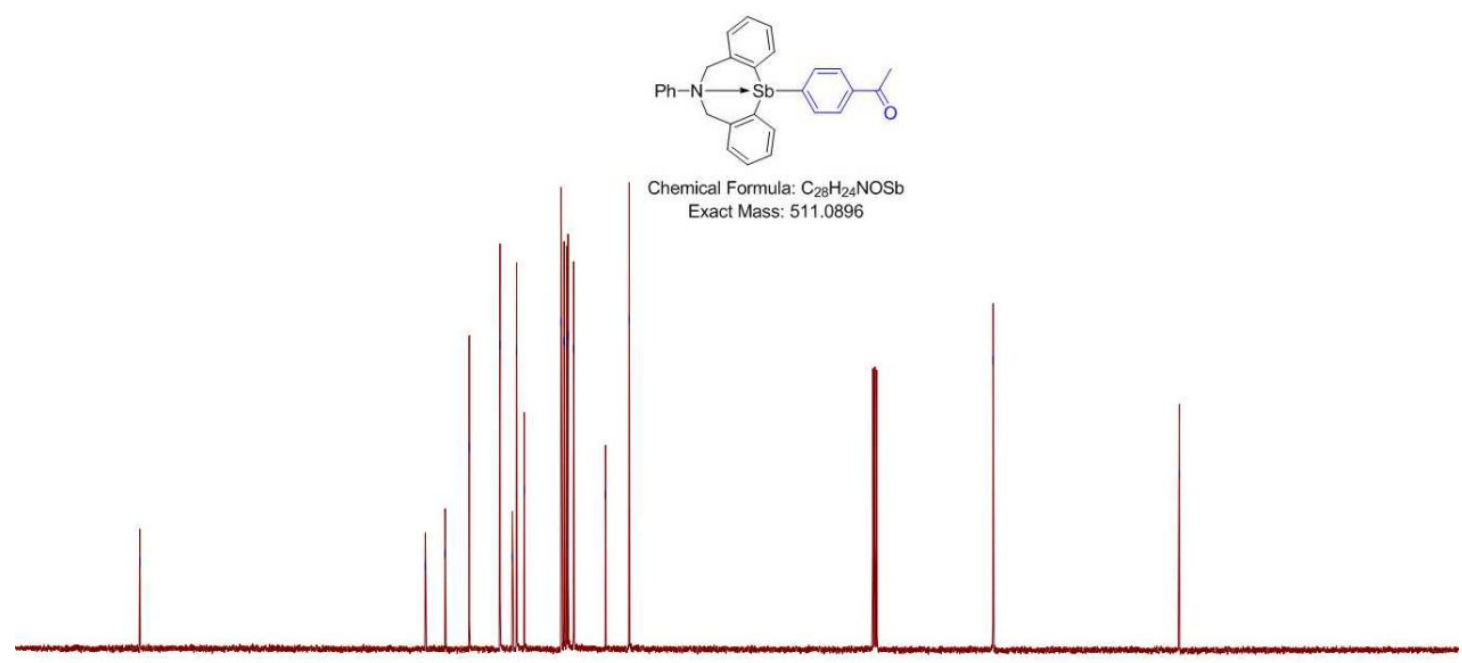

$\begin{array}{lllllllllllllllllllllllllll}210 & 200 & 190 & 180 & 170 & 160 & 150 & 140 & 130 & 120 & 110 & 100 & 90 & 80 & 70 & 60 & 50 & 40 & 30 & 20 & 10 & 0 & -10\end{array}$ f1 (ppm)

${ }^{1} \mathrm{H}$ NMR $\left(\mathrm{CDCl}_{3}\right)$ spectrum of compound 3ad

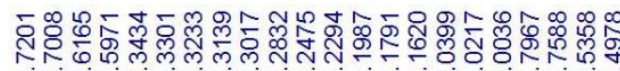

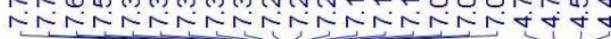

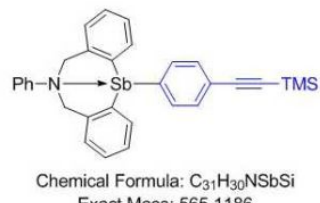

Exact Mass: 565.1186

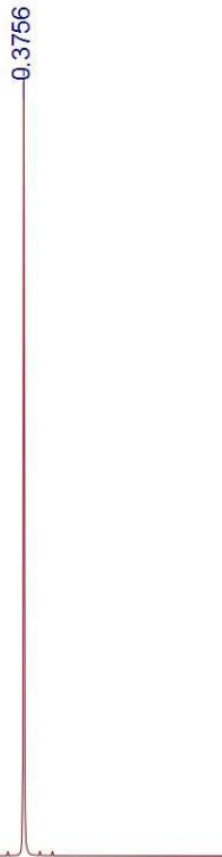

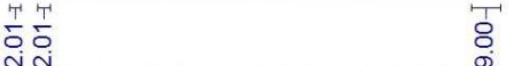

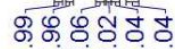

$\begin{array}{lllllllllllllllllllllllllllll}11.5 & 11.0 & 10.5 & 10.0 & 9.5 & 9.0 & 8.5 & 8.0 & 7.5 & 7.0 & 6.5 & 6.0 & 5.5 & 5.0 & 4.5 & 4.0 & 3.5 & 3.0 & 2.5 & 2.0 & 1.5 & 1.0 & 0.5 & 0.0 & -0.5 & -1.0\end{array}$ $\mathrm{f1}(\mathrm{ppm})$ 
${ }^{13} \mathrm{C}$ NMR $\left(\mathrm{CDCl}_{3}\right)$ spectrum of compound 3ad

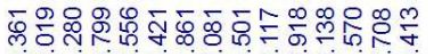

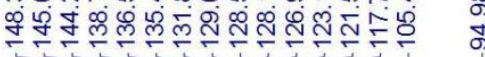

总

$\frac{\bar{\sigma}}{i}$

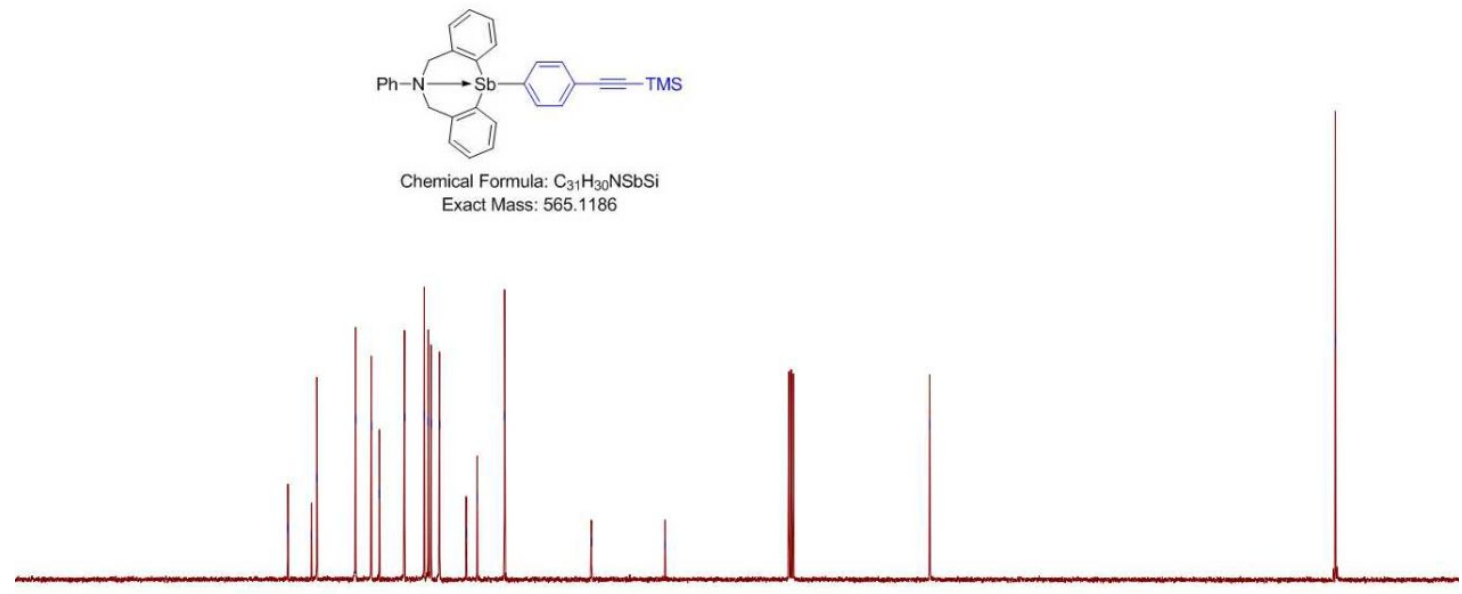

焉

$\begin{array}{lllllllllllllllllllll}180 & 170 & 160 & 150 & 140 & 130 & 120 & 110 & 100 & \begin{array}{c}90 \\ \mathrm{f} 1(\mathrm{ppm})\end{array} & 70 & 60 & 50 & 40 & 30 & 20 & 10 & 0 & -10\end{array}$

${ }^{1} \mathrm{H}$ NMR $\left(\mathrm{CDCl}_{3}\right)$ spectrum of compound 3ae
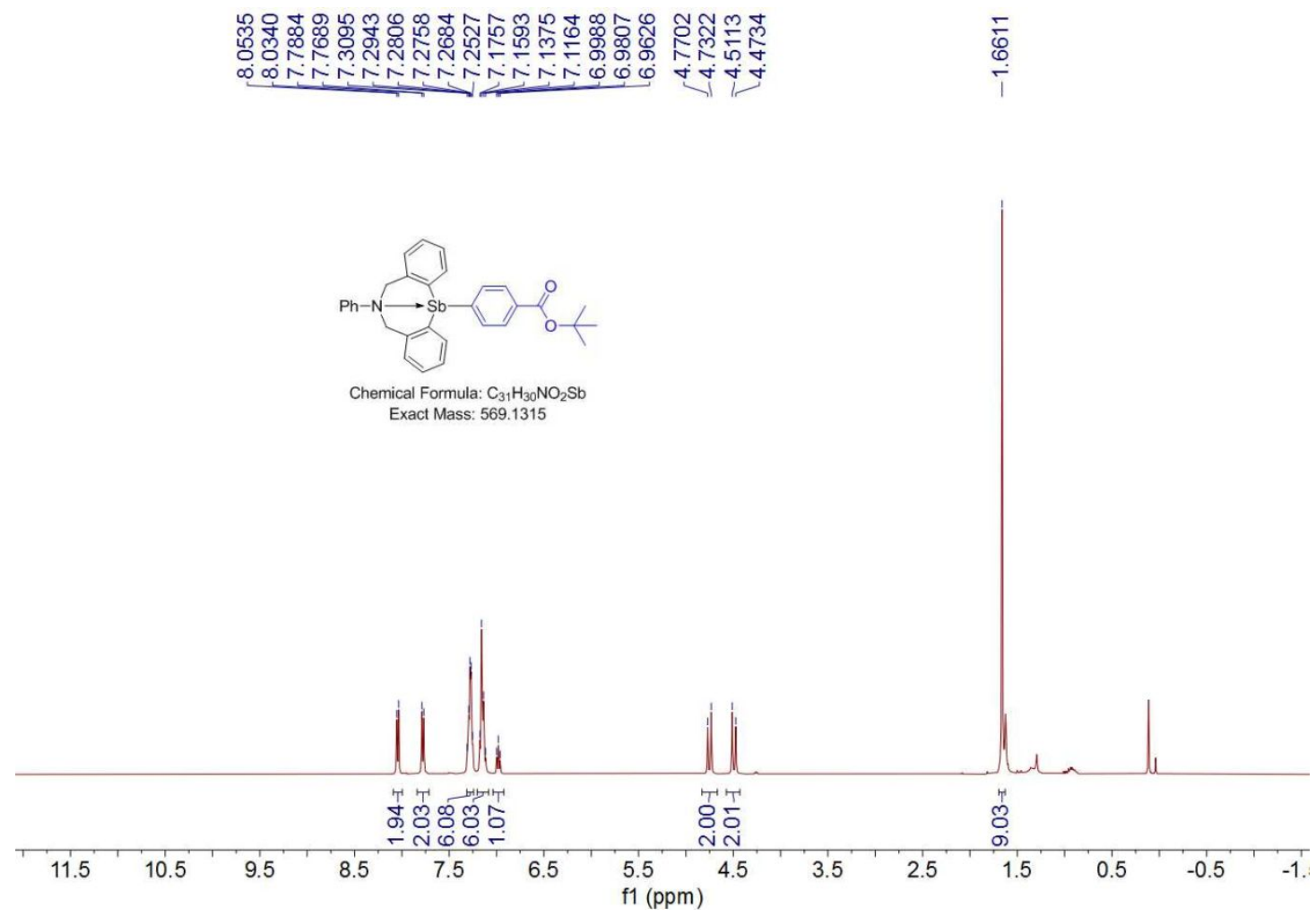
${ }^{13} \mathrm{C}$ NMR $\left(\mathrm{CDCl}_{3}\right)$ spectrum of compound 3ae

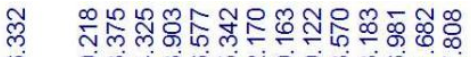

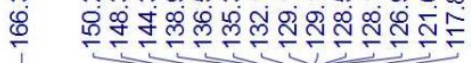

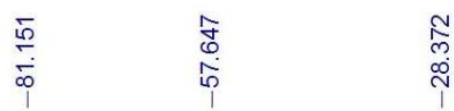

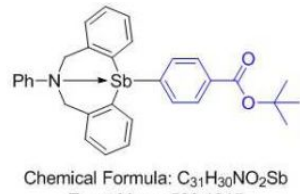
Exact Mass: 569.1315

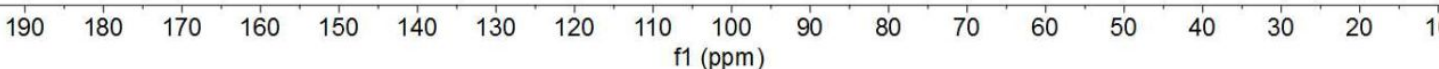

${ }^{1} \mathrm{H}$ NMR $\left(\mathrm{CDCl}_{3}\right)$ spectrum of compound 3af

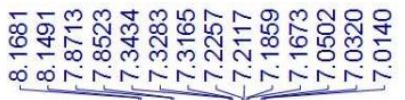

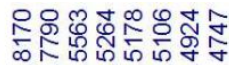

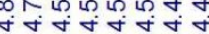

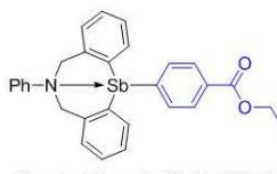

Chemical Formula: $\mathrm{C}_{29} \mathrm{H}_{26} \mathrm{NO}_{2} \mathrm{Sb}$ Exact Mass: 541.1002

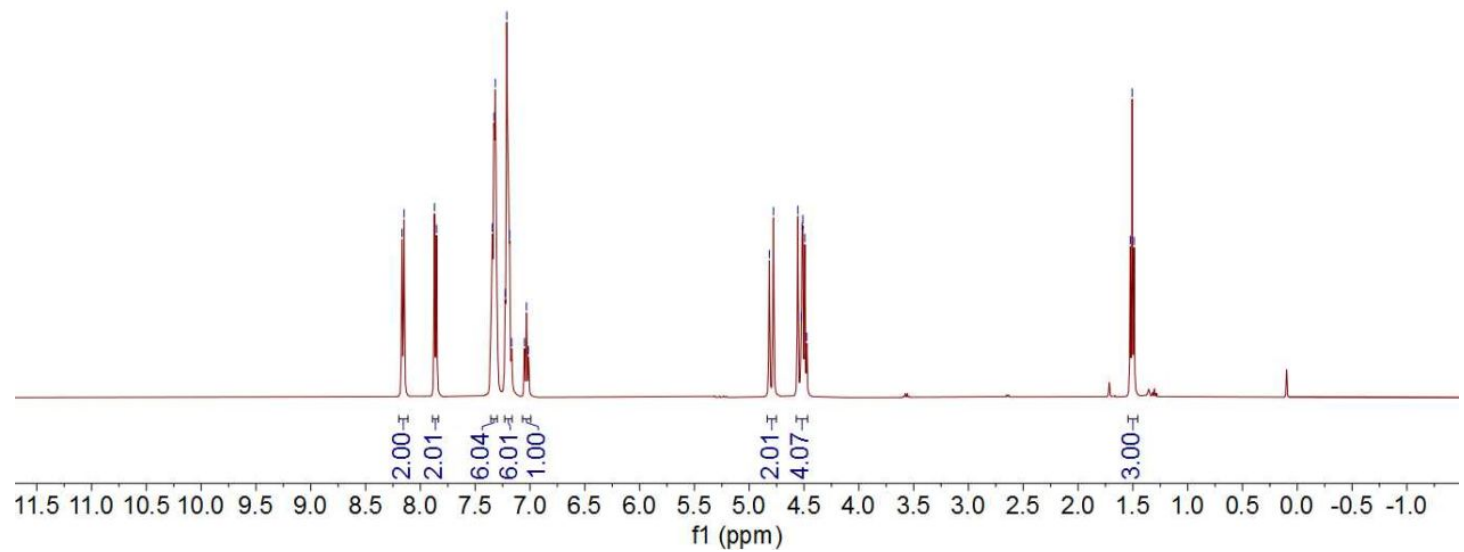


${ }^{13} \mathrm{C}$ NMR $\left(\mathrm{CDCl}_{3}\right)$ spectrum of compound 3af

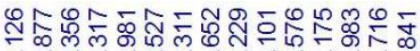

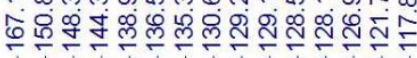

ஜ유유.

iิ

$\underset{\substack{\infty \\ \stackrel{\infty}{i}}}{\stackrel{\infty}{+}}$

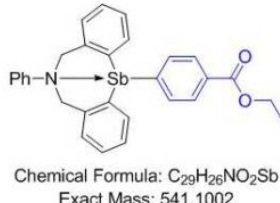

Exact Mass: 541.1002

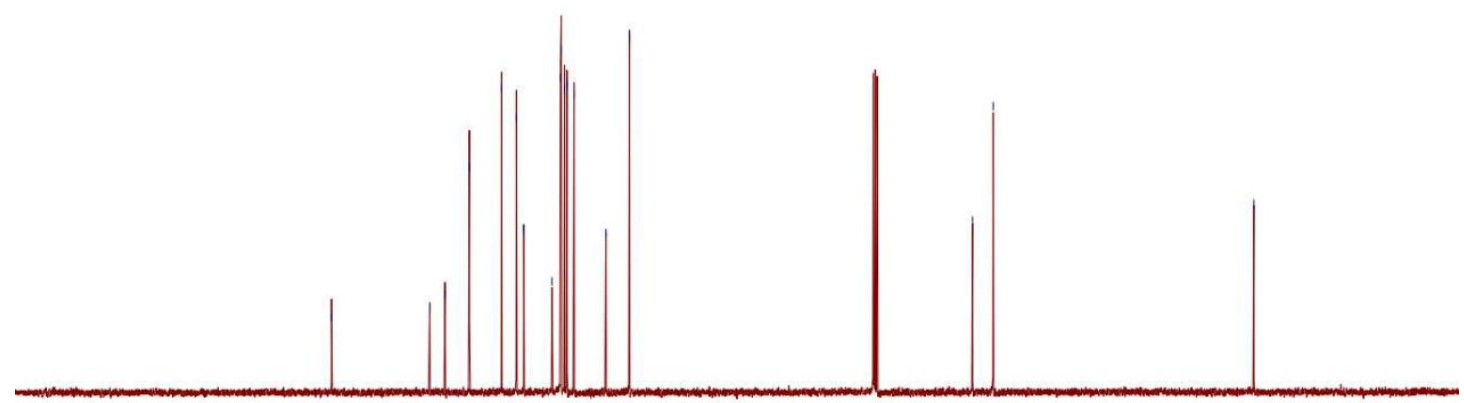

$\begin{array}{lllllllllllllllllllllllll}210 & 200 & 190 & 180 & 170 & 160 & 150 & 140 & 130 & 120 & 110 & 100 & 90 & 80 & 70 & 60 & 50 & 40 & 30 & 20 & 10 & 0 & -10\end{array}$

${ }^{1} \mathrm{H}$ NMR $\left(\mathrm{CDCl}_{3}\right)$ spectrum of compound 3ag

फू

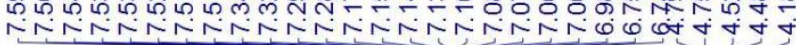

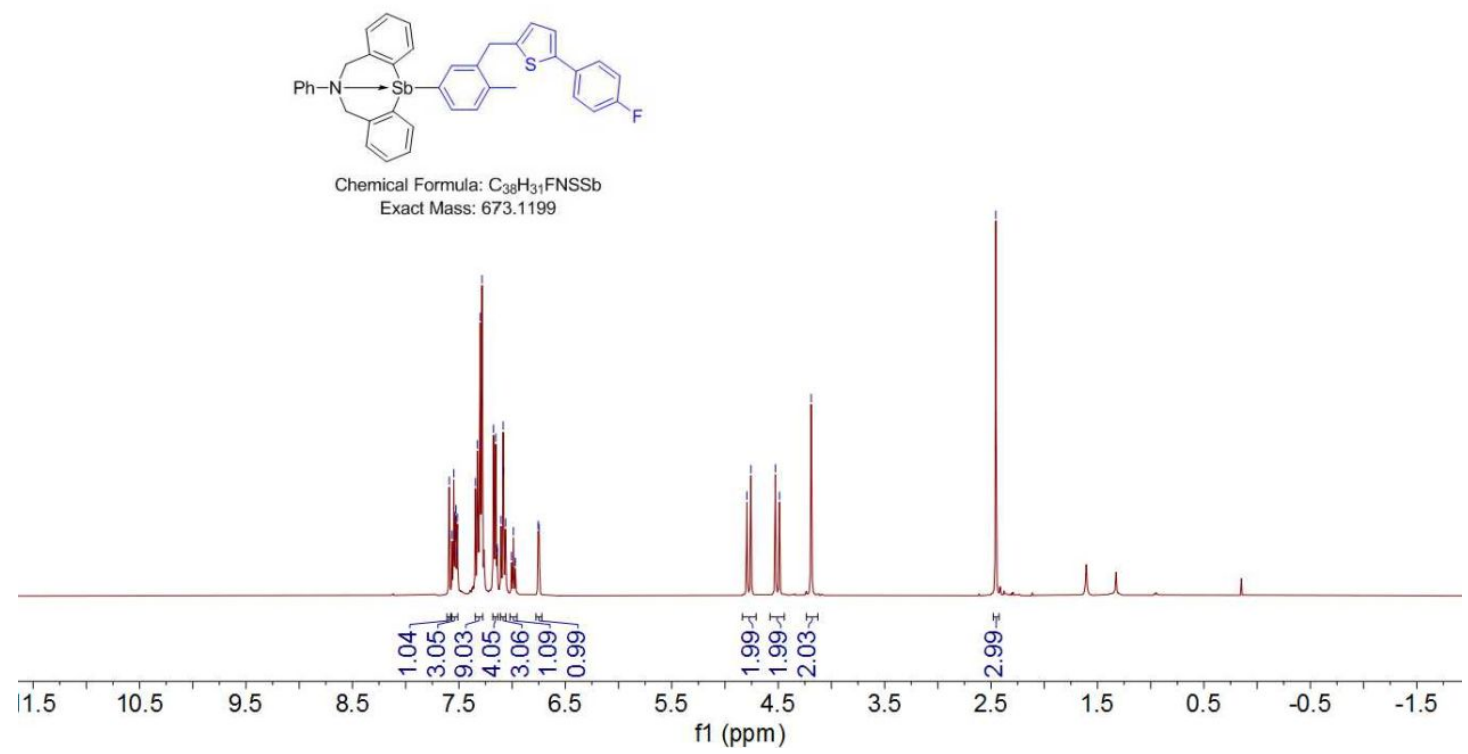


${ }^{13} \mathrm{C} \mathrm{NMR}\left(\mathrm{CDCl}_{3}\right)$ spectrum of compound $\mathbf{3 a g}$

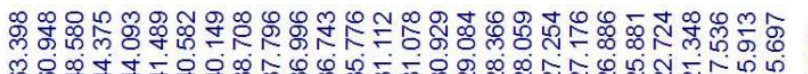

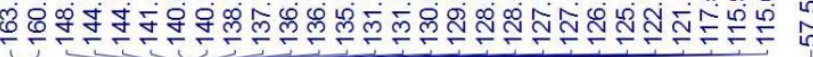

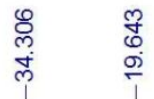
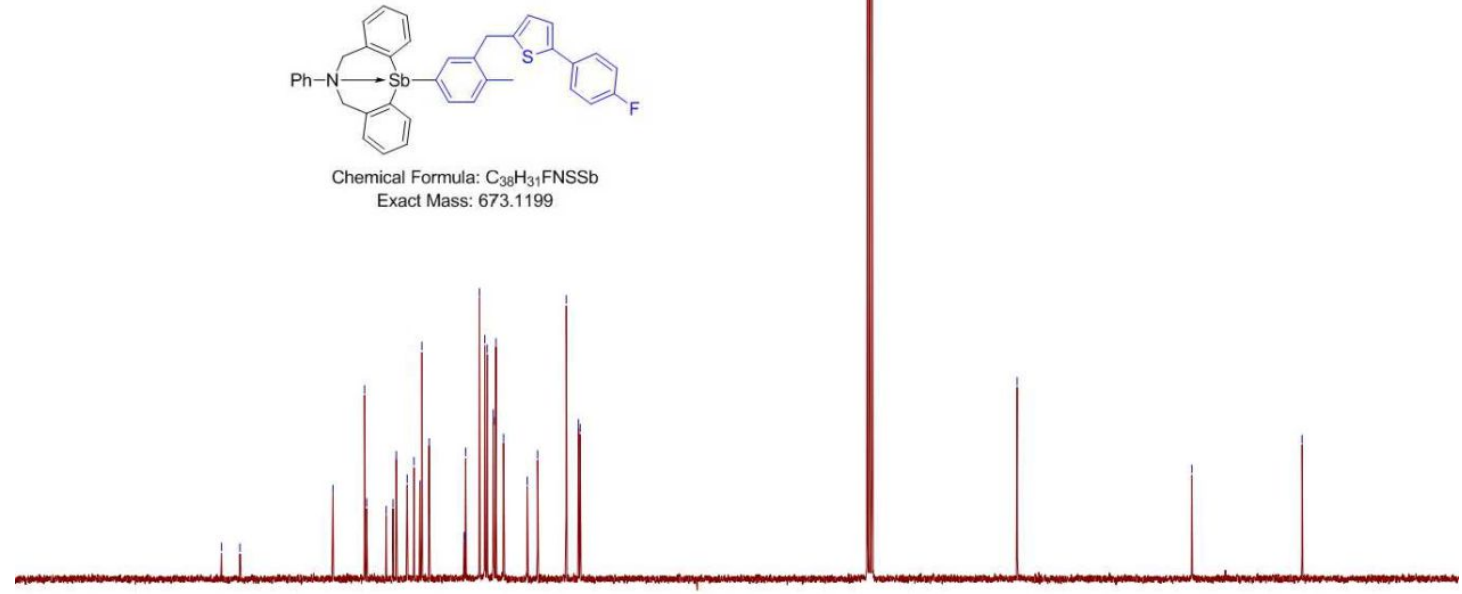

\begin{tabular}{llllllllllllllllllllll}
\hline 90 & 180 & 170 & 160 & 150 & 140 & 130 & 120 & 110 & $\begin{array}{r}100 \\
\mathrm{f} 1(\mathrm{ppm})\end{array}$ & 80 & 70 & 60 & 50 & 40 & 30 & 20 & 10 & 0
\end{tabular}

${ }^{19} \mathrm{~F}$ NMR $\left(\mathrm{CDCl}_{3}\right)$ spectrum of compound $\mathbf{3 a g}$
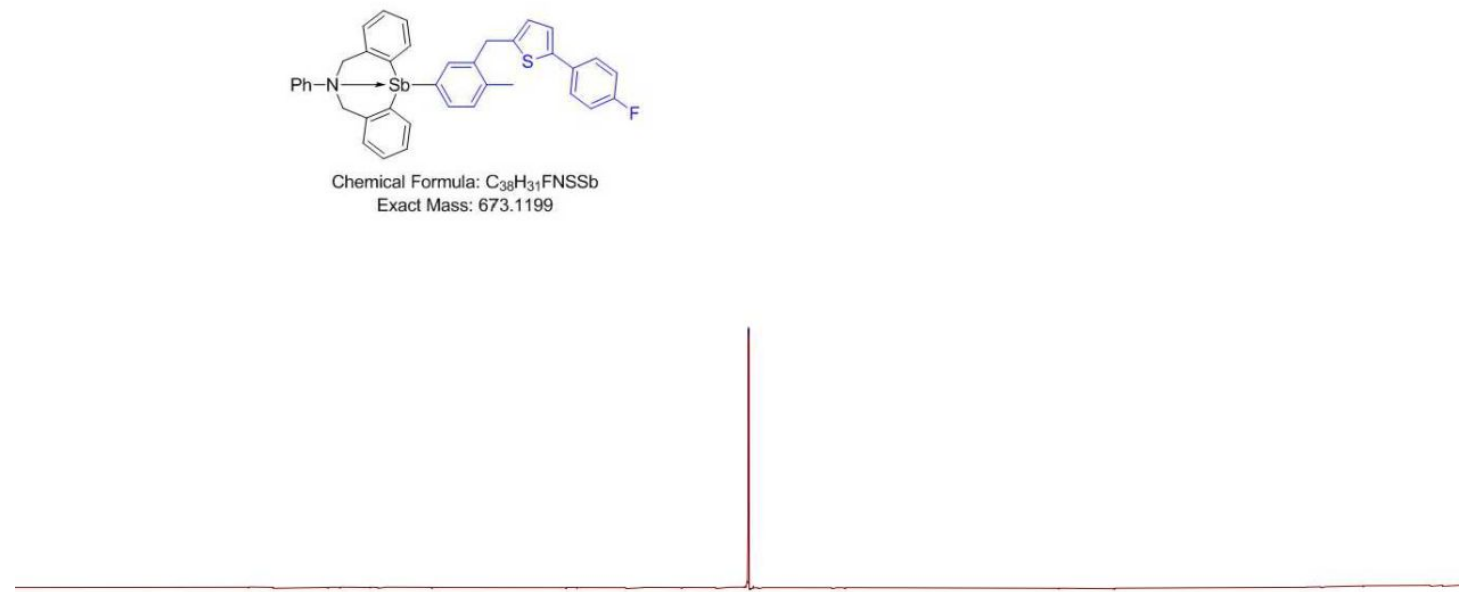

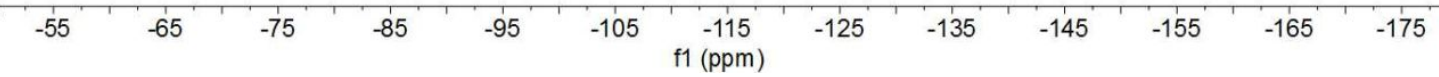


${ }^{1} \mathrm{H}$ NMR $\left(\mathrm{CDCl}_{3}\right)$ spectrum of compound 3ah

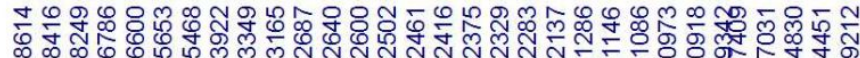

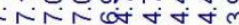

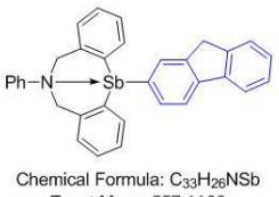
Exact Mass: 557.1103

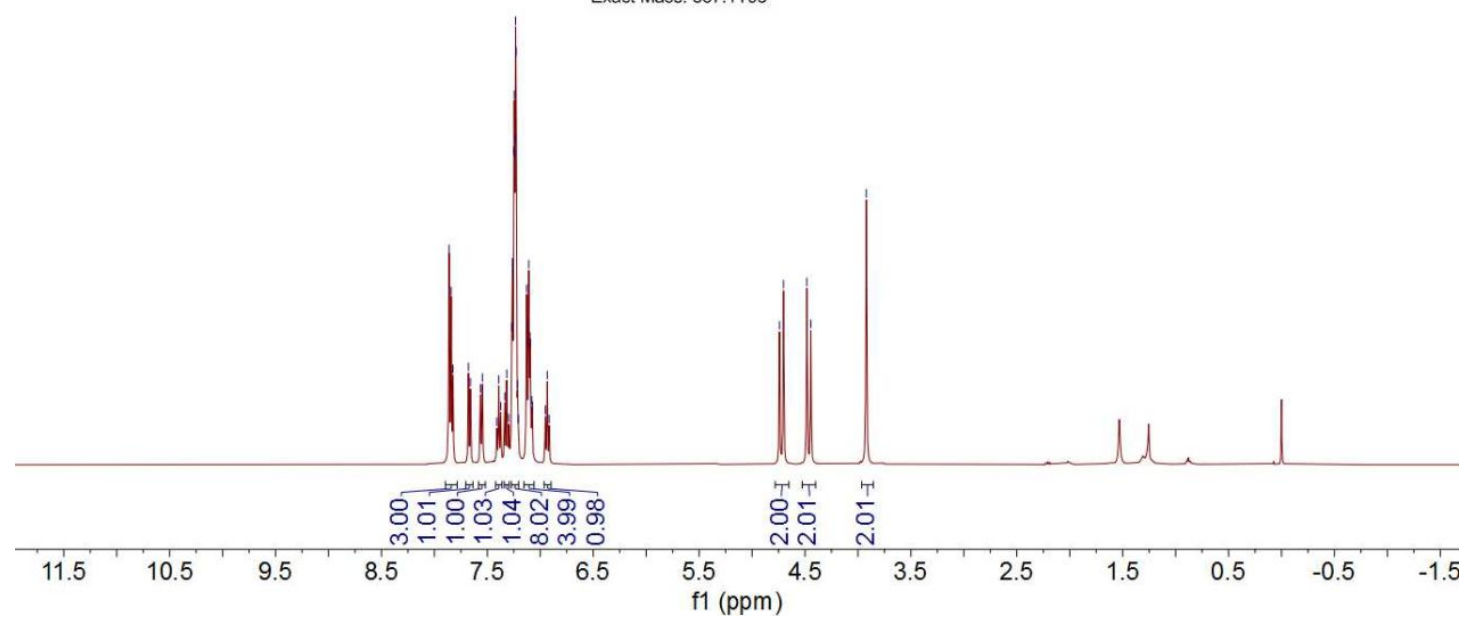

${ }^{13} \mathrm{C} \mathrm{NMR}\left(\mathrm{CDCl}_{3}\right)$ spectrum of compound 3ah

舟

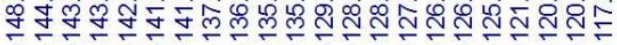

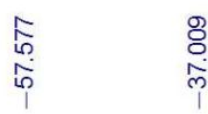

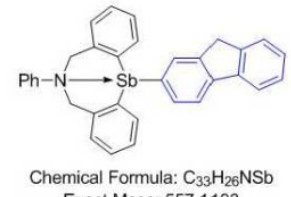
Exact Mass: 557.1103

$\begin{array}{llllllllll}80 & 170 & 160 & 150 & 140 & 130 & 120 & 110 & \begin{array}{c}100 \\ \mathrm{f} 1(\mathrm{ppm})\end{array}\end{array}$ 
${ }^{1} \mathrm{H} \mathrm{NMR}\left(\mathrm{CDCl}_{3}\right)$ spectrum of compound 3ai

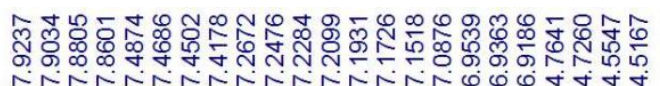

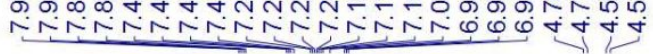

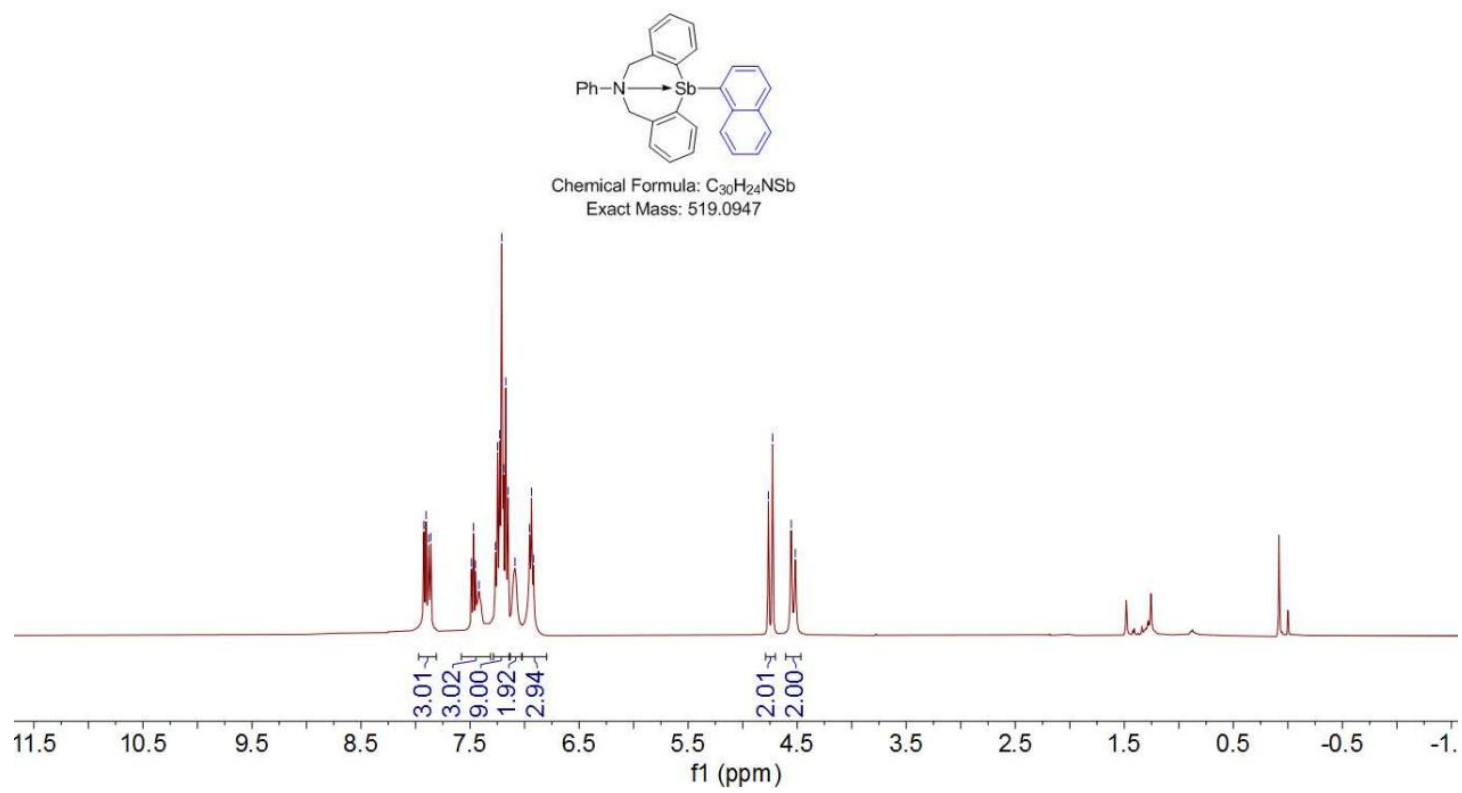

${ }^{13} \mathrm{C} \mathrm{NMR}\left(\mathrm{CDCl}_{3}\right)$ spectrum of compound 3ai

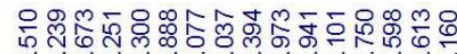

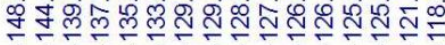

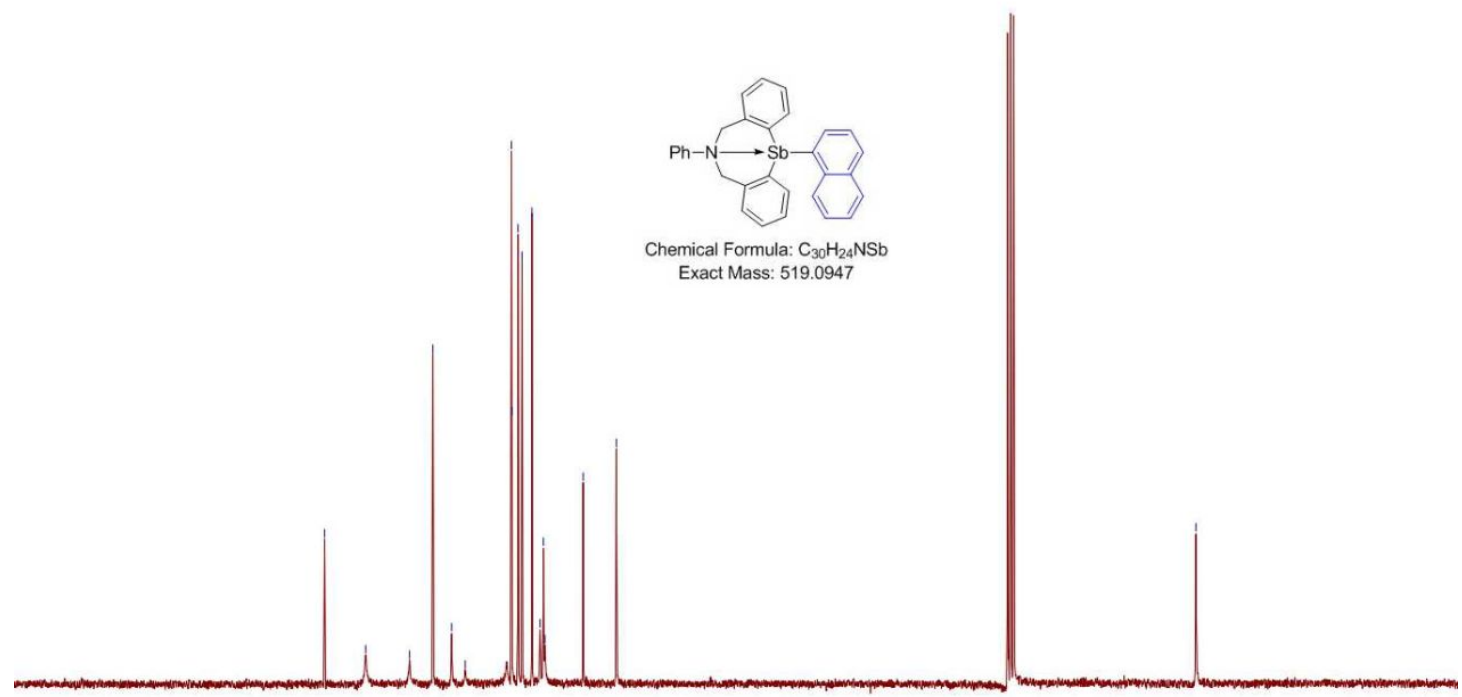

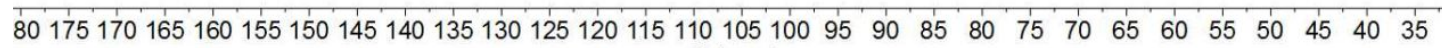
$\mathrm{f} 1(\mathrm{ppm})$ 
${ }^{1} \mathrm{H}$ NMR $\left(\mathrm{CDCl}_{3}\right)$ spectrum of compound 3aj

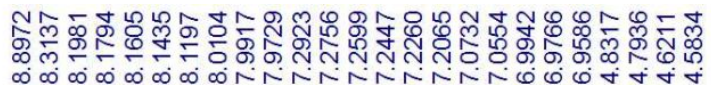

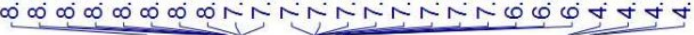

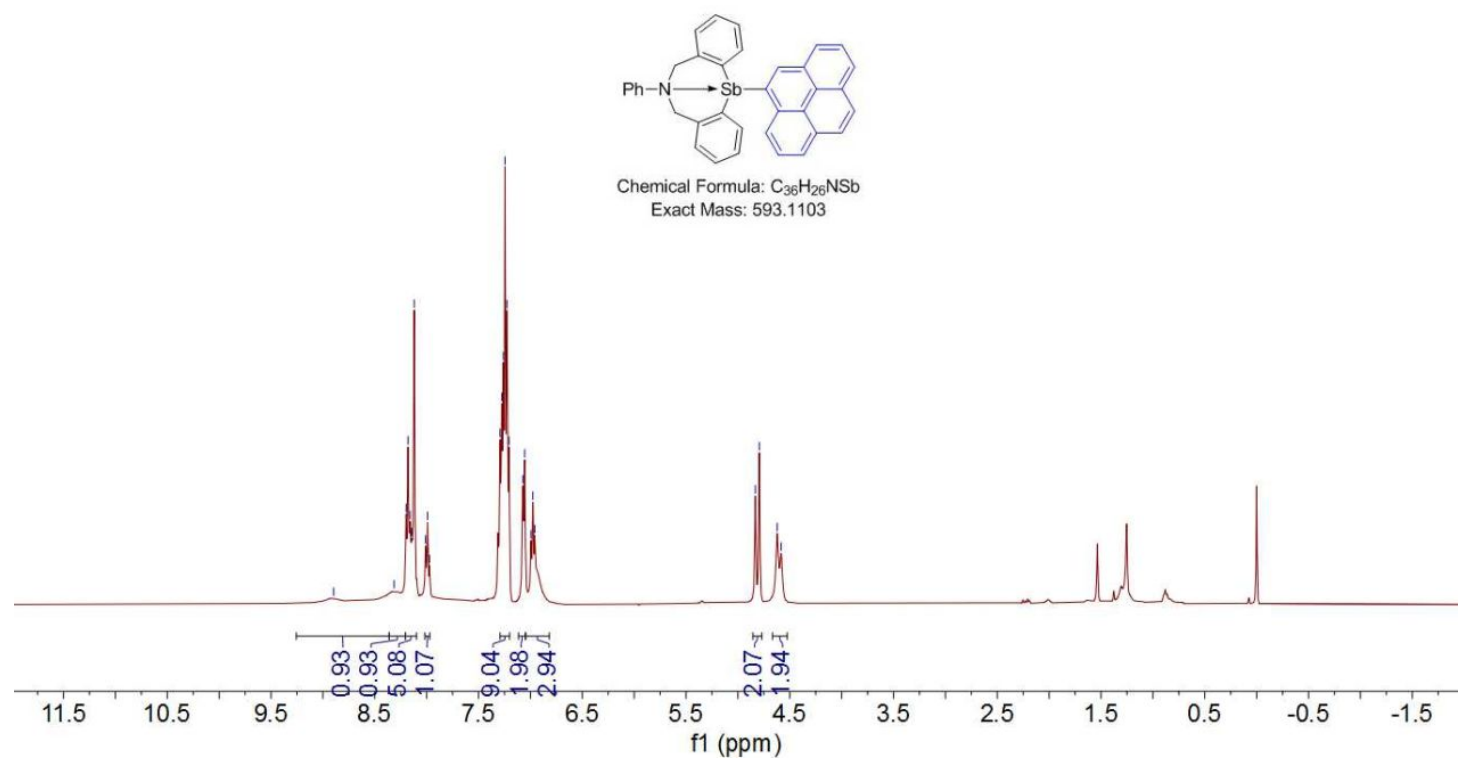

${ }^{13} \mathrm{C} \mathrm{NMR}\left(\mathrm{CDCl}_{3}\right)$ spectrum of compound $\mathbf{3 a j}$

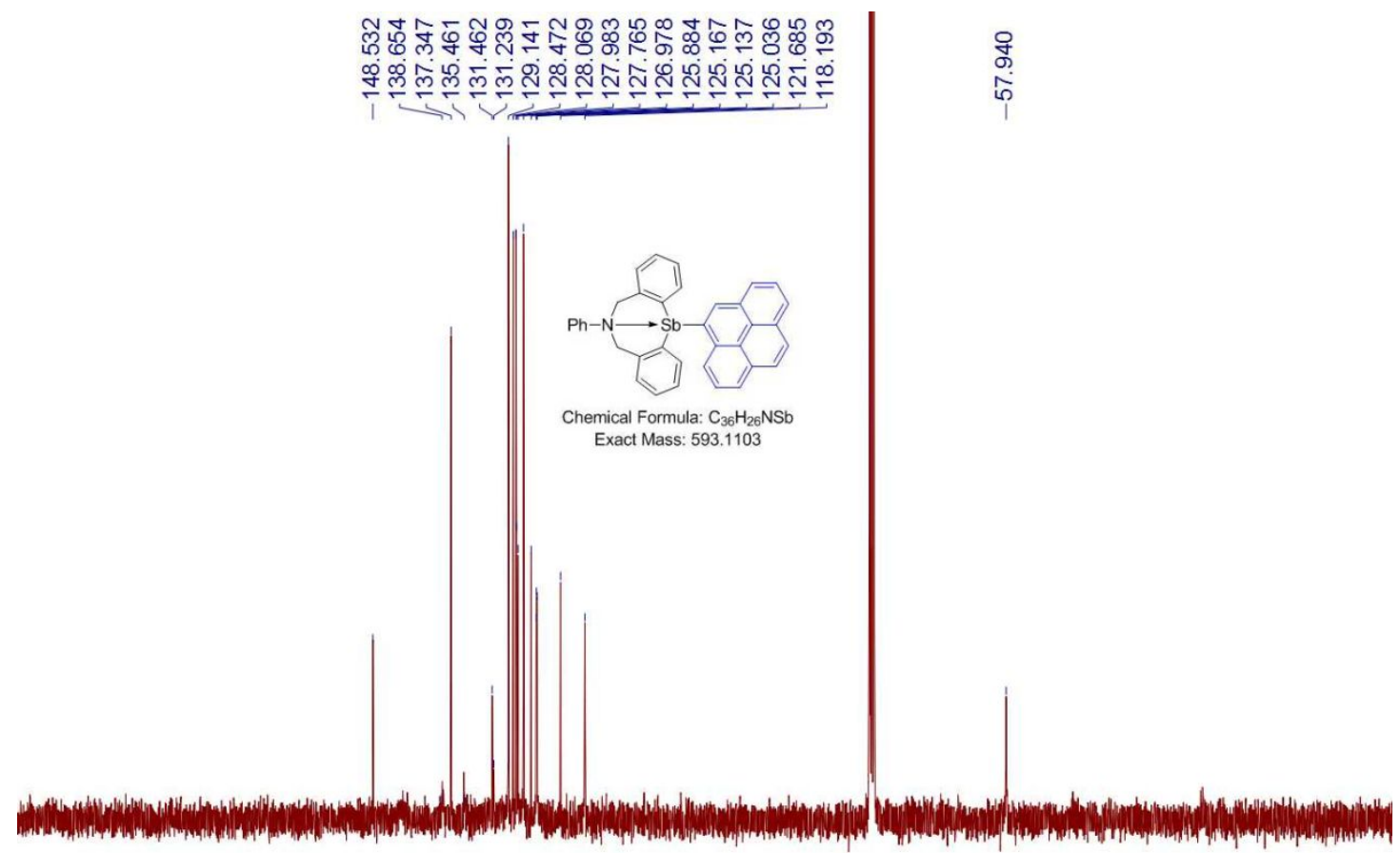

$\begin{array}{lllllllllllllllllll}190 & 180 & 170 & 160 & 150 & 140 & 130 & 120 & 110 & \begin{array}{l}100 \\ \mathrm{f} 1(\mathrm{ppm})\end{array} & 90 & 80 & 70 & 60 & 50 & 40 & 30 & 20 & 10\end{array}$ 
${ }^{1} \mathrm{H}$ NMR $\left(\mathrm{CDCl}_{3}\right)$ spectrum of compound 3ak
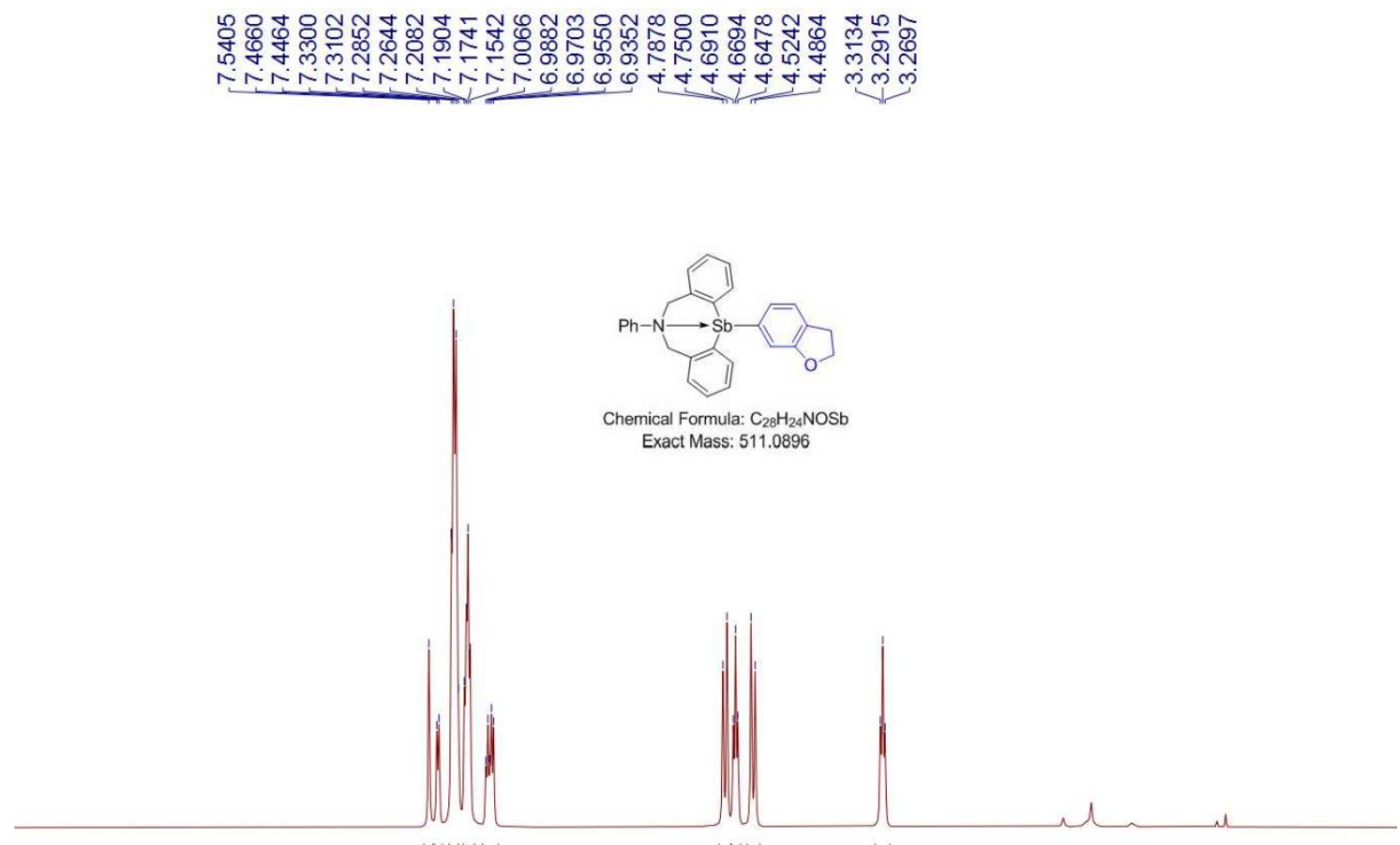
88800 808 \&

$\begin{array}{llllllllllllllllllllllllllllll}11.0 & 10.5 & 10.0 & 9.5 & 9.0 & 8.5 & 8.0 & 7.5 & 7.0 & 6.5 & 6.0 & 5.5 & 5.0 & 4.5 & 4.0 & 3.5 & 3.0 & 2.5 & 2.0 & 1.5 & 1.0 & 0.5 & 0.0 & -0.5 & -1.0 & -1\end{array}$ f1 $(\mathrm{ppm})$

${ }^{13} \mathrm{C} \mathrm{NMR}\left(\mathrm{CDCl}_{3}\right)$ spectrum of compound 3ak

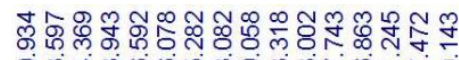

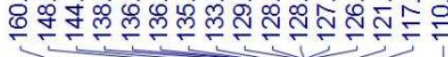

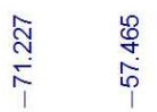

लָ

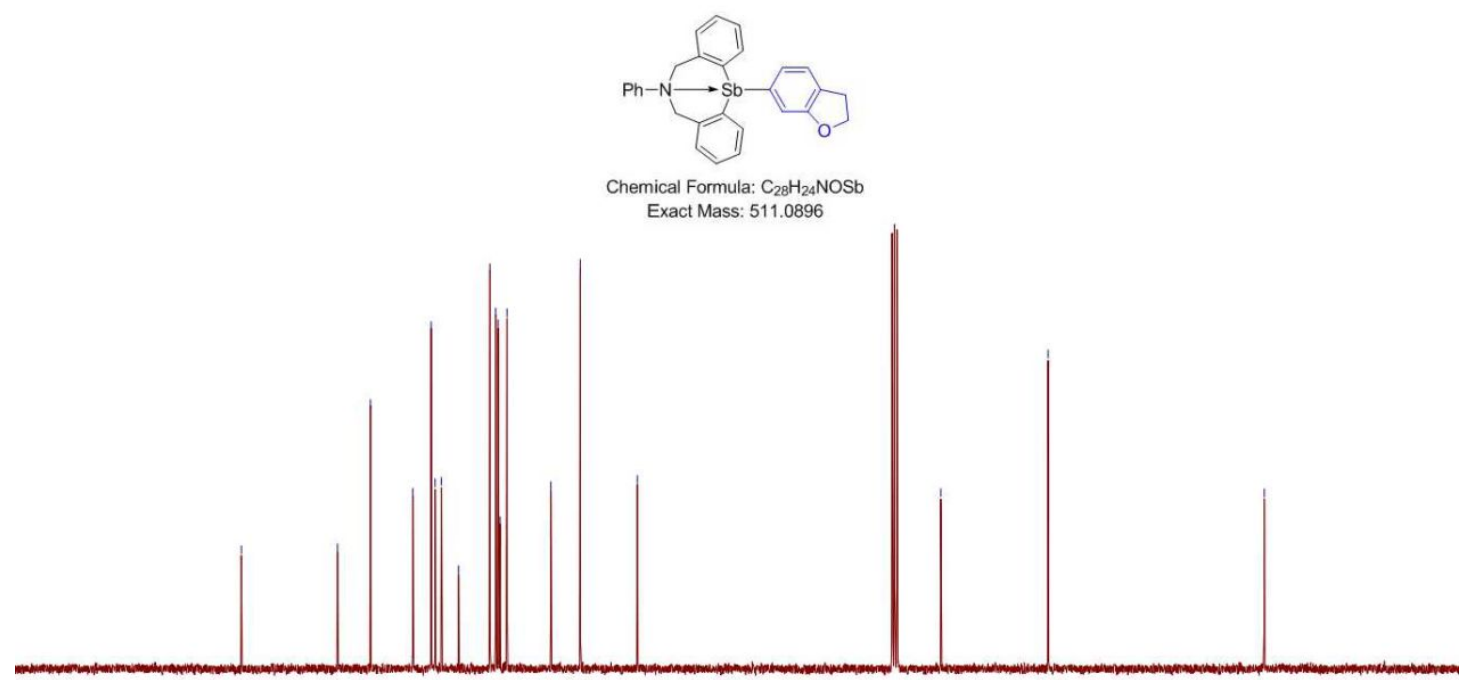

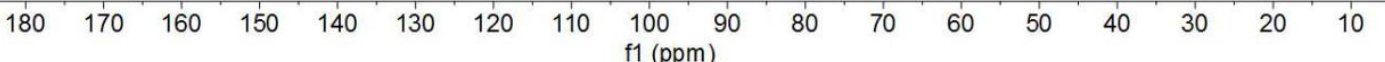


${ }^{1} \mathrm{H}$ NMR $\left(\mathrm{CDCl}_{3}\right)$ spectrum of compound 3al
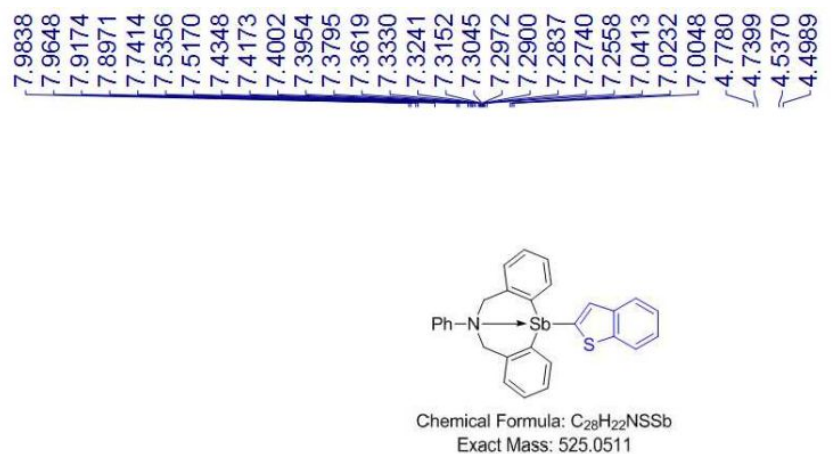

Exact Mass: 525.0511

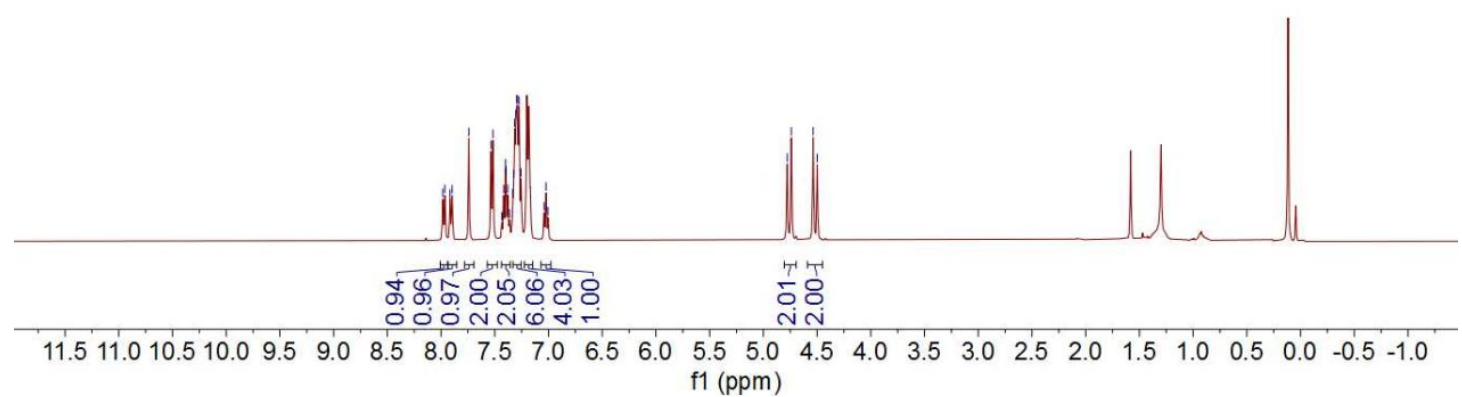

${ }^{13} \mathrm{C}$ NMR $\left(\mathrm{CDCl}_{3}\right)$ spectrum of compound 3al

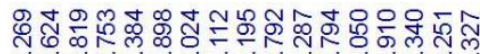

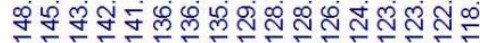

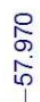

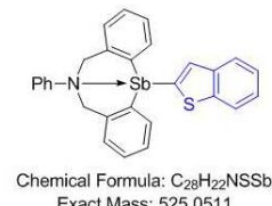

Exact Mass: 525.0511

$\begin{array}{lllllllll}80 & 170 & 160 & 150 & 140 & 130 & 120 & 110 \begin{array}{r}100 \\ \mathrm{f} 1(\mathrm{ppm})\end{array}\end{array}$

$\begin{array}{lllllll}90 & 80 & 70 & 60 & 50 & 40 & 30\end{array}$ 
${ }^{1} \mathrm{H}$ NMR $\left(\mathrm{CDCl}_{3}\right)$ spectrum of compound 3am
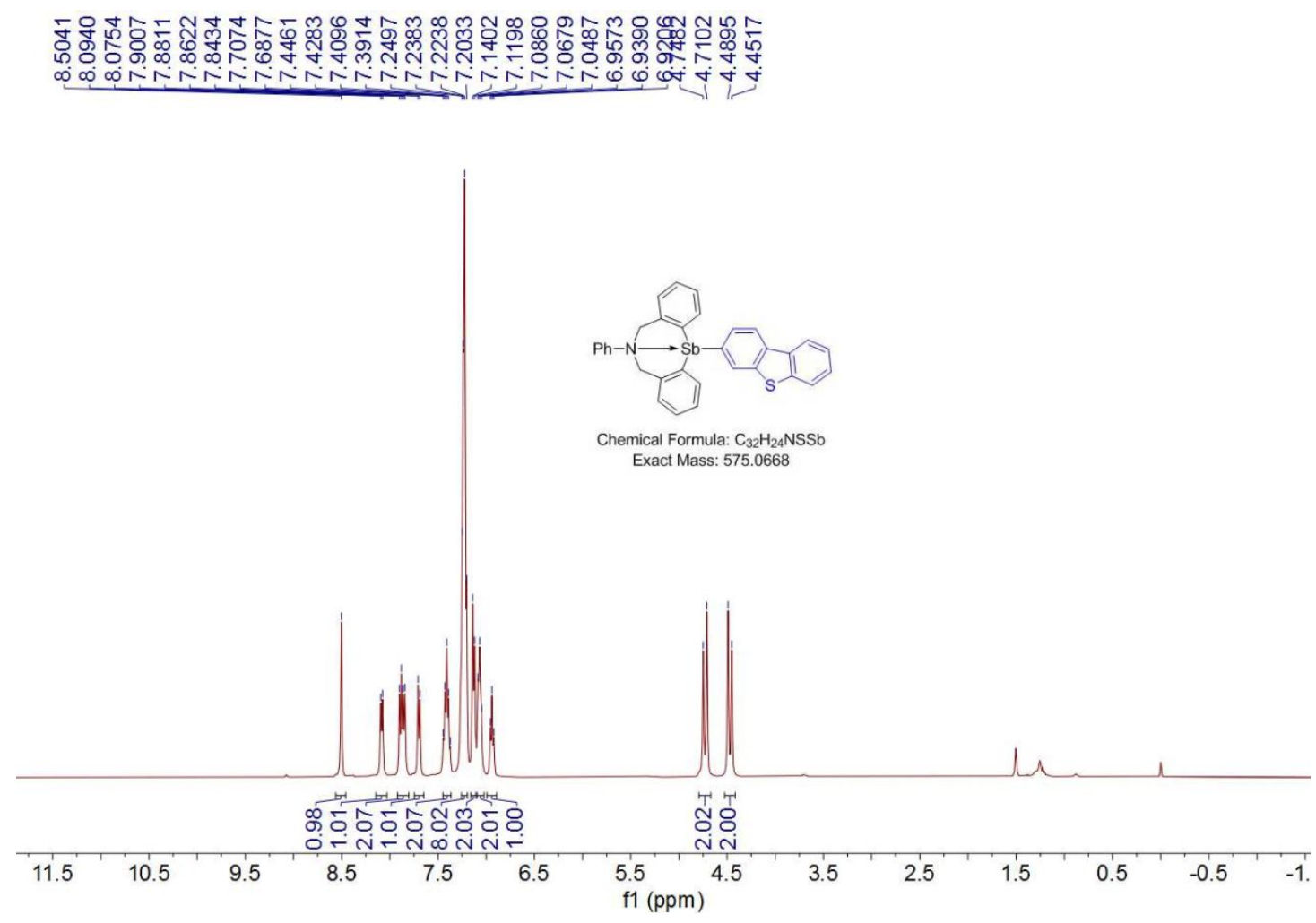

${ }^{13} \mathrm{C}$ NMR $\left(\mathrm{CDCl}_{3}\right)$ spectrum of compound 3am

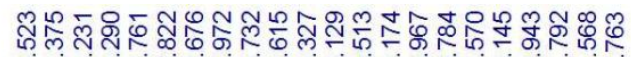

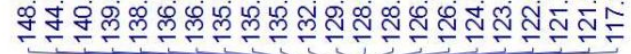

$\underset{\substack{i \\ i}}{i}$

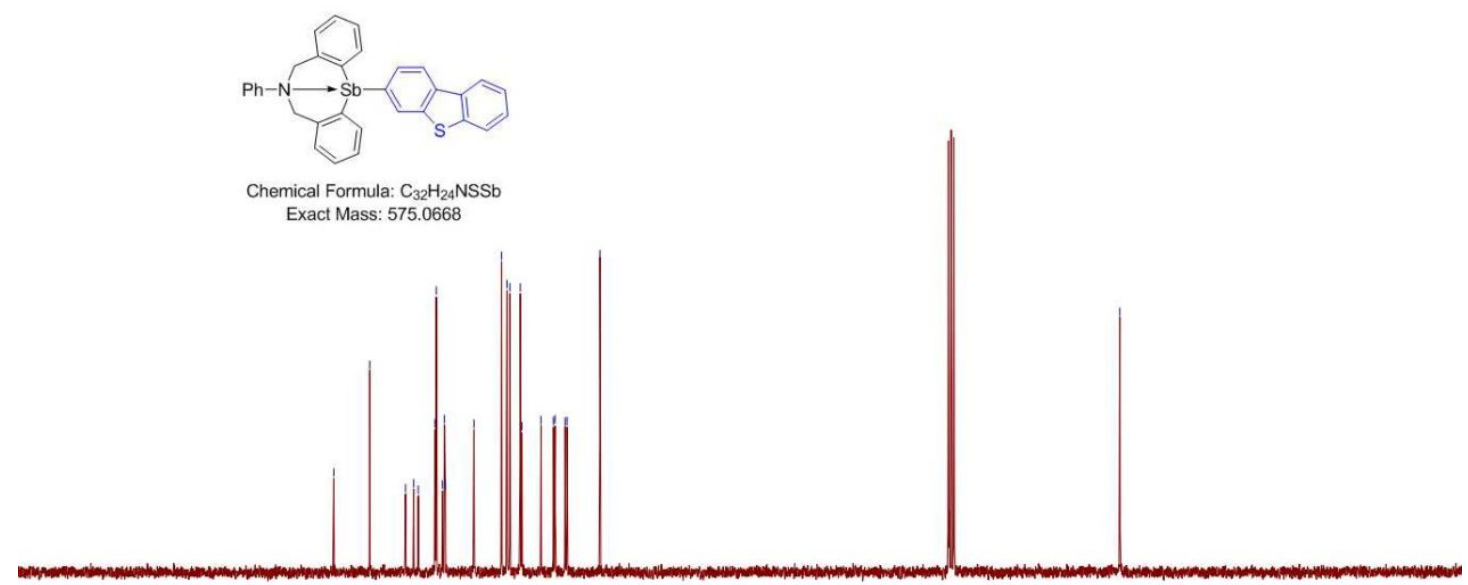

$\begin{array}{lllllllllllllllll}180 & 170 & 160 & 150 & 140 & 130 & 120 & 110 \begin{array}{c}100 \\ \mathrm{f} 1(\mathrm{ppm})\end{array} & 90 & 80 & 70 & 60 & 50 & 40 & 30 & 20\end{array}$ 
${ }^{1} \mathrm{H}$ NMR $\left(\mathrm{CDCl}_{3}\right)$ spectrum of compound 3an
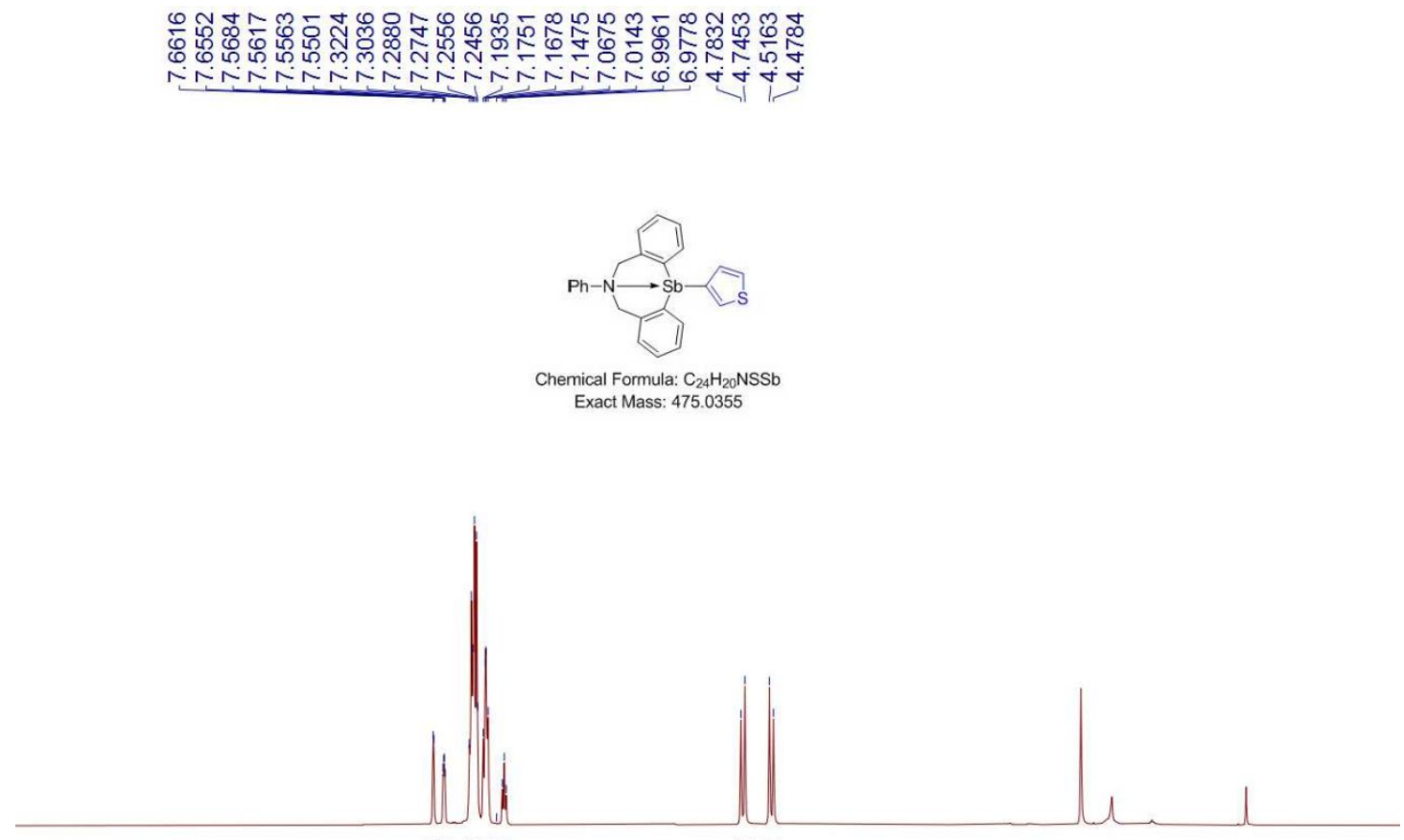

काष्ठक्ष

事苾

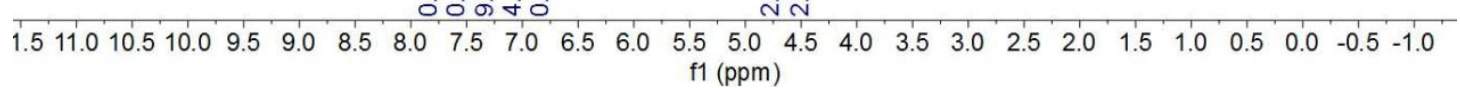

${ }^{13} \mathrm{C}$ NMR $\left(\mathrm{CDCl}_{3}\right)$ spectrum of compound 3an

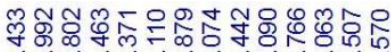

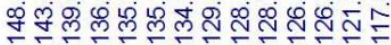

$\stackrel{\sim ్}{\sim}$

i̊

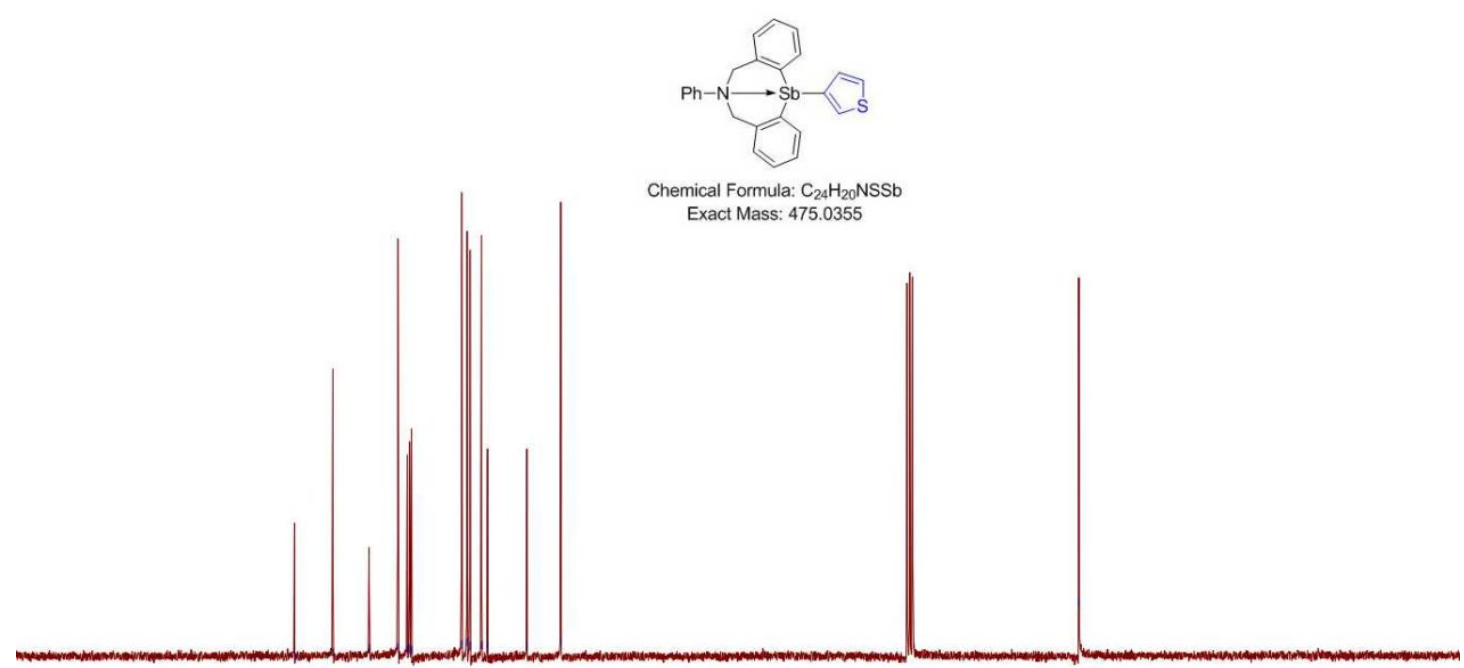

80

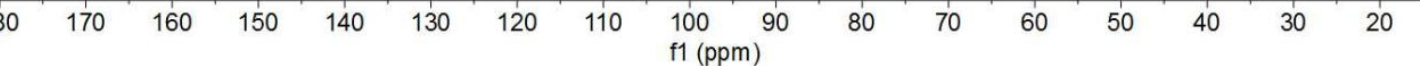


${ }^{1} \mathrm{H}$ NMR $\left(\mathrm{CDCl}_{3}\right)$ spectrum of compound 3ao

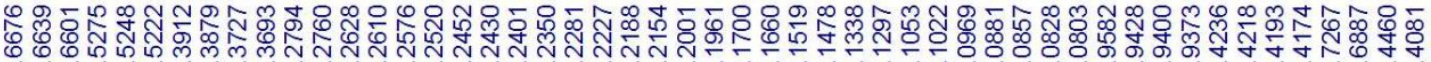

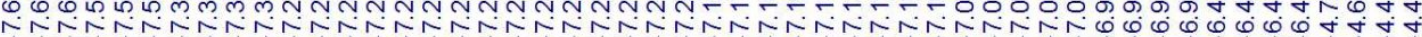

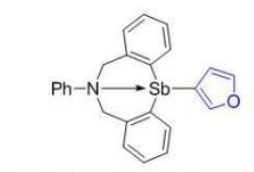

Chemical Formula: $\mathrm{C}_{24} \mathrm{H}_{20} \mathrm{NOS}$

Exact Mass: 459.0583

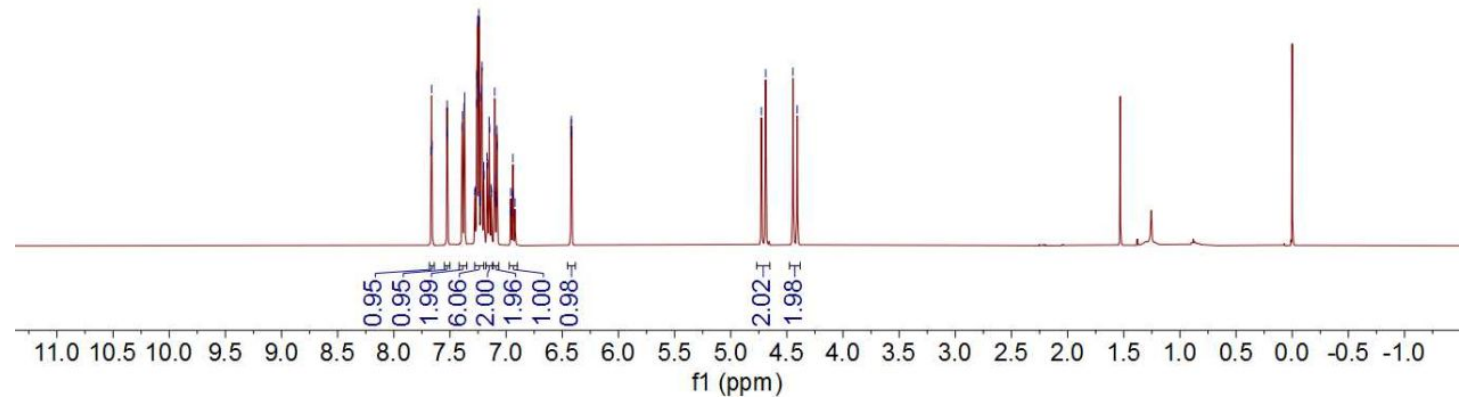

${ }^{13} \mathrm{C} \mathrm{NMR}\left(\mathrm{CDCl}_{3}\right)$ spectrum of compound $\mathbf{3 a o}$

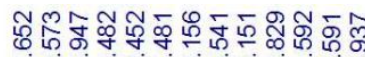

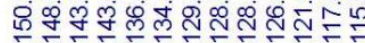

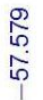

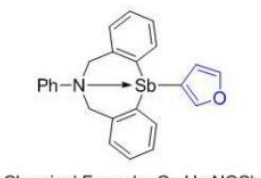

Chemical Formula: $\mathrm{C}_{24} \mathrm{H}_{20} \mathrm{NOS}$ Exact Mass: 459.0583

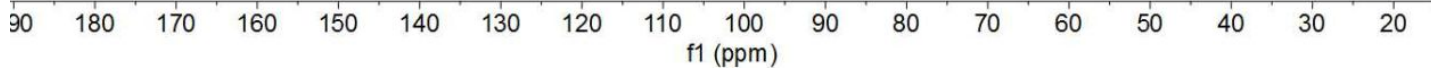


${ }^{1} \mathrm{H}$ NMR $\left(\mathrm{CDCl}_{3}\right)$ spectrum of compound 3ap
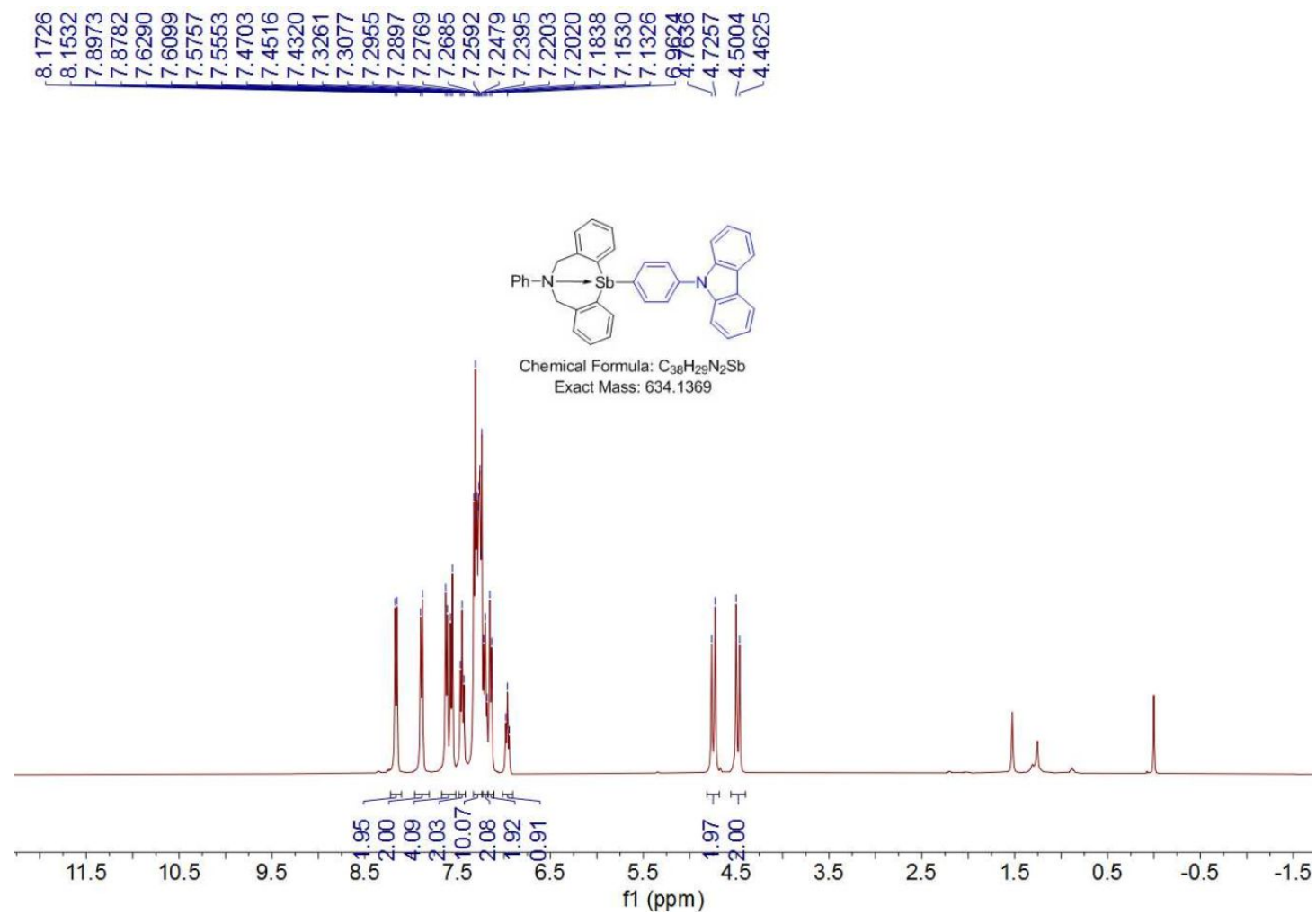

${ }^{13} \mathrm{C} \mathrm{NMR}\left(\mathrm{CDCl}_{3}\right)$ spectrum of compound 3ap

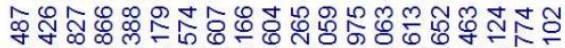

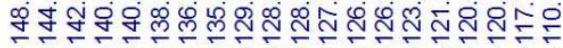

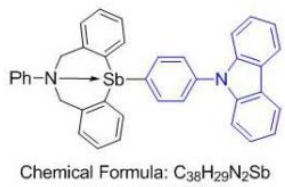

Exact Mass: 634.1369

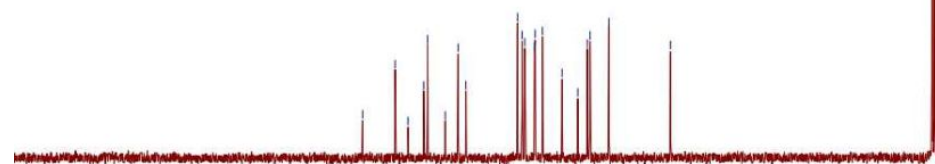

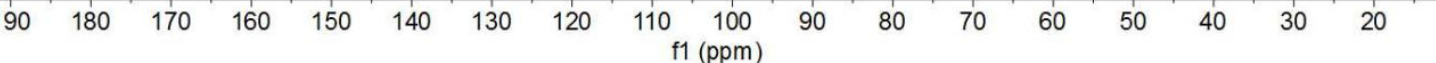


${ }^{1} \mathrm{H}$ NMR $\left(\mathrm{CDCl}_{3}\right)$ spectrum of compound 3aq

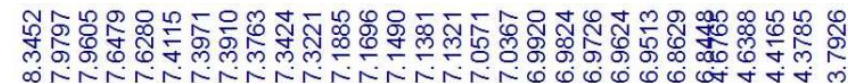

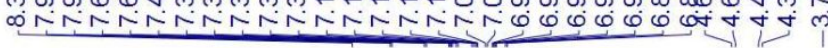

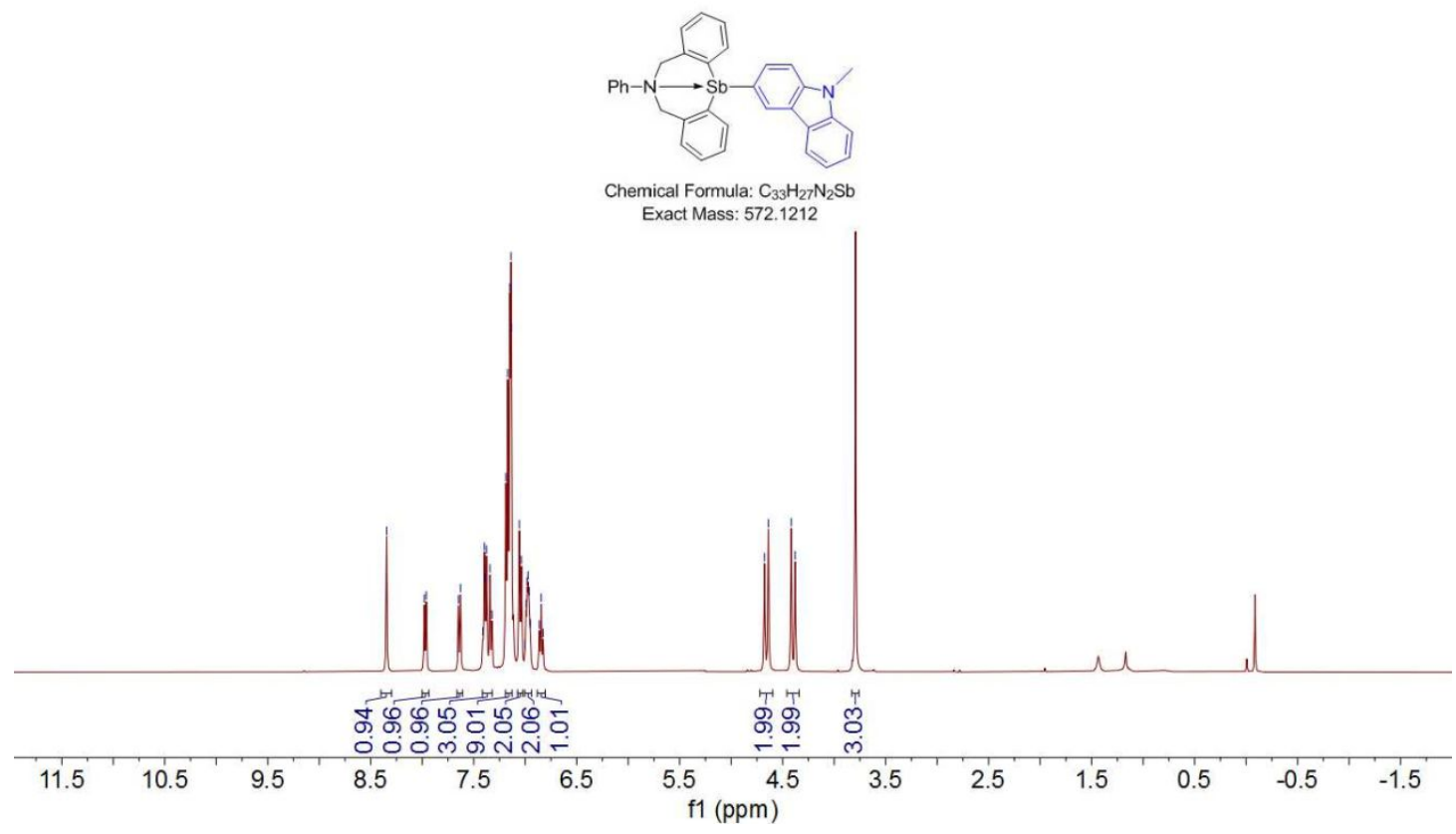

${ }^{13} \mathrm{C}$ NMR $\left(\mathrm{CDCl}_{3}\right)$ spectrum of compound 3aq

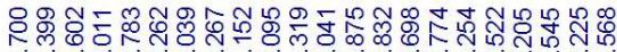

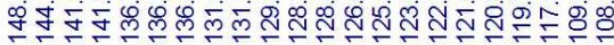

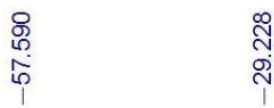
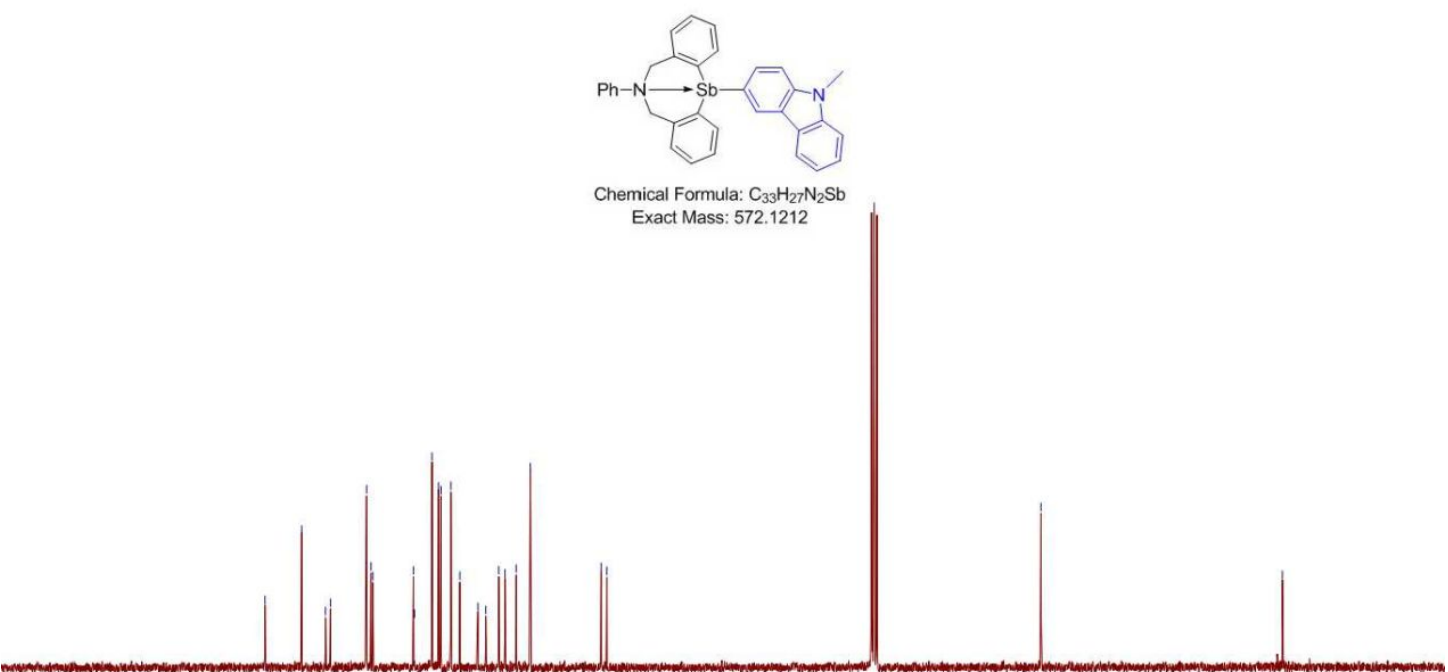

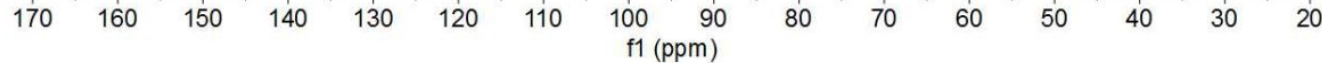


${ }^{1} \mathrm{H}$ NMR $\left(\mathrm{CDCl}_{3}\right)$ spectrum of compound 3ar
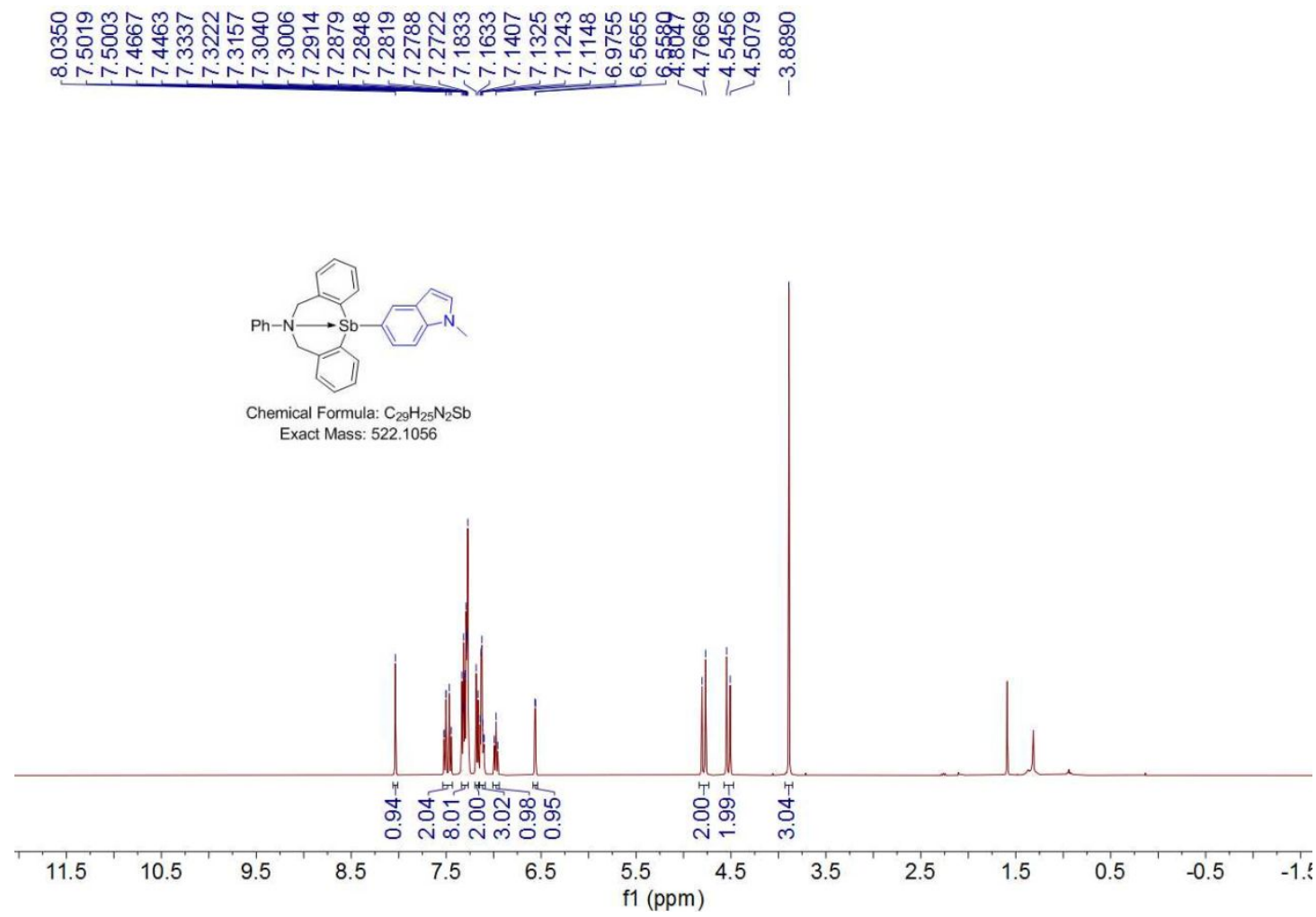

${ }^{13} \mathrm{C}$ NMR $\left(\mathrm{CDCl}_{3}\right)$ spectrum of compound $3 \mathrm{ar}$

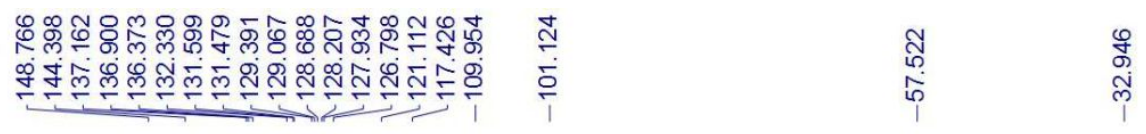

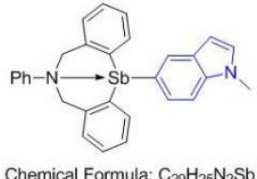

Exact Mass: 522.1056

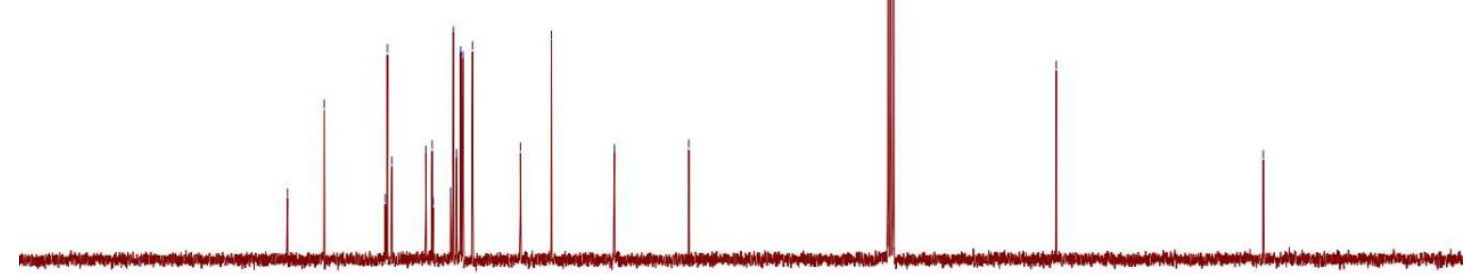

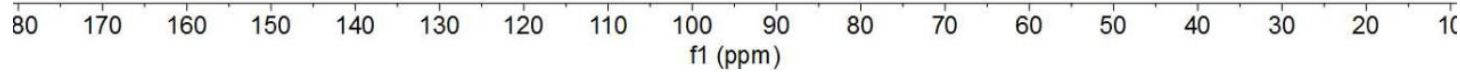


${ }^{1} \mathrm{H}$ NMR $\left(\mathrm{CDCl}_{3}\right)$ spectrum of compound 3as

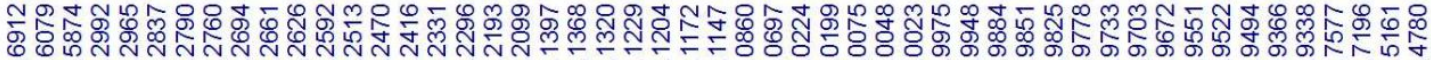

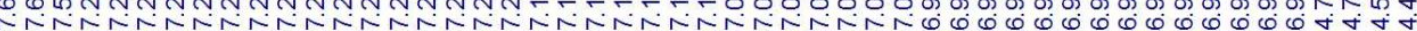

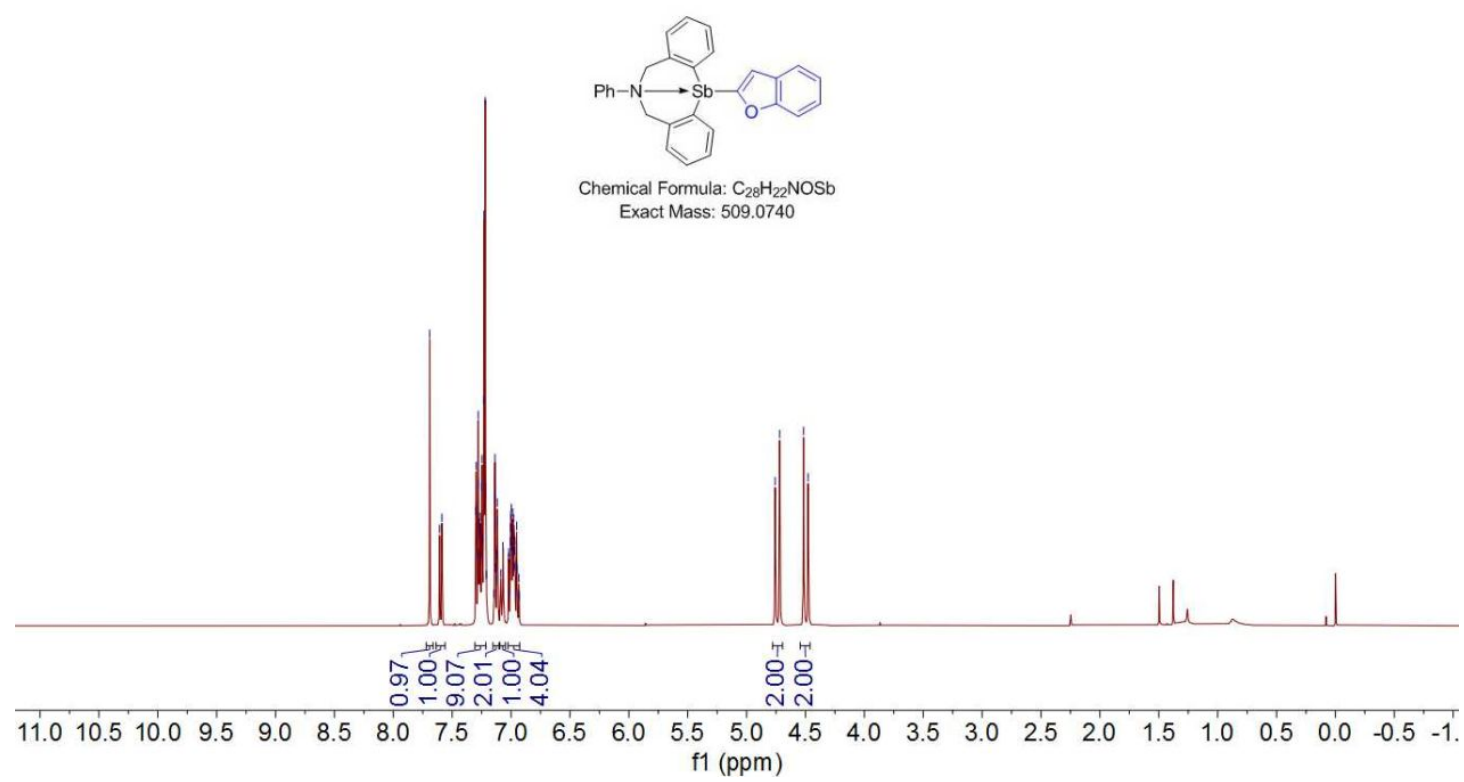

${ }^{13} \mathrm{C} \mathrm{NMR}\left(\mathrm{CDCl}_{3}\right)$ spectrum of compound 3as

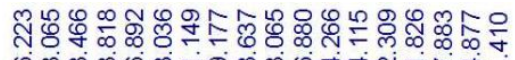

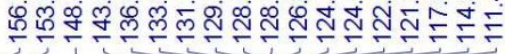

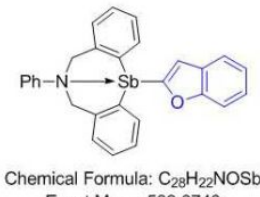

Exact Mass: 509.0740

$18017517016516015515014514013513012512011511010510095 \quad 90 \quad 85 \quad 80 \quad 75 \quad 70 \quad 65 \quad 60 \begin{array}{lllllll}5 & 50 & 45 & 40 & 35\end{array}$ f1 (ppm) 
${ }^{1} \mathrm{H} \mathrm{NMR}\left(\mathrm{CDCl}_{3}\right)$ spectrum of compound 3au
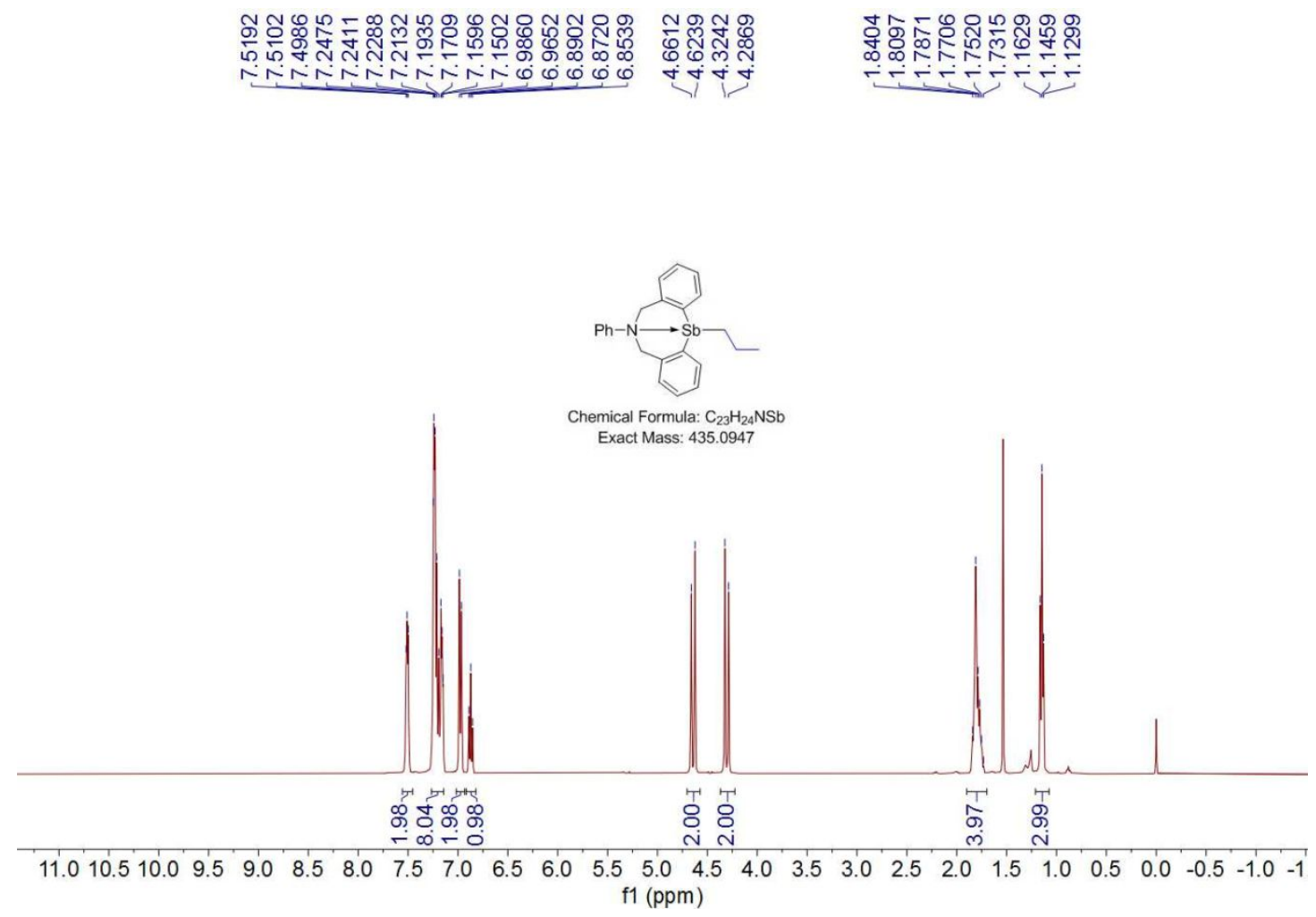

${ }^{13} \mathrm{C} \mathrm{NMR}\left(\mathrm{CDCl}_{3}\right)$ spectrum of compound 3au
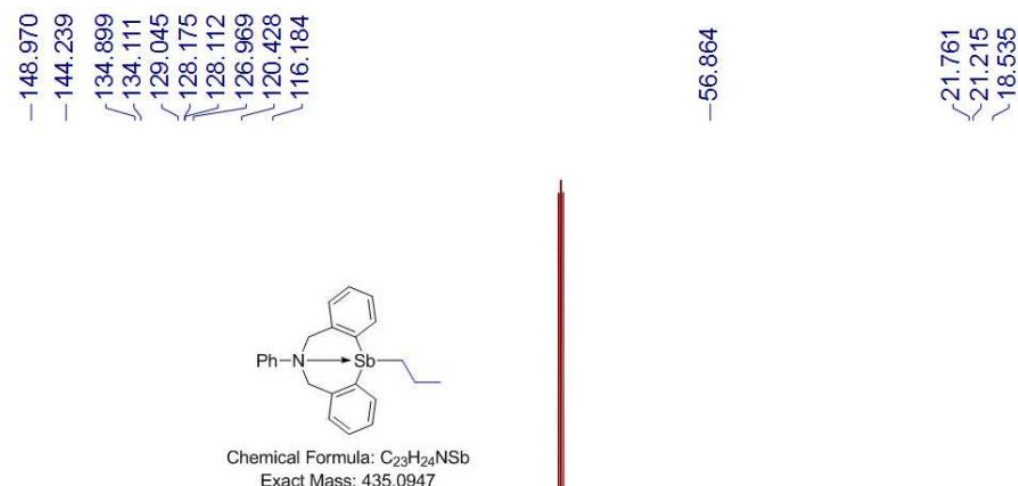

$\begin{array}{llllllllll}180 & 170 & 160 & 150 & 140 & 130 & 120 & 110 & 100 & 90 \\ \mathrm{f} 1(\mathrm{ppm})\end{array}$

${ }^{1} \mathrm{H}$ NMR $\left(\mathrm{CDCl}_{3}\right)$ spectrum of compound 3av 

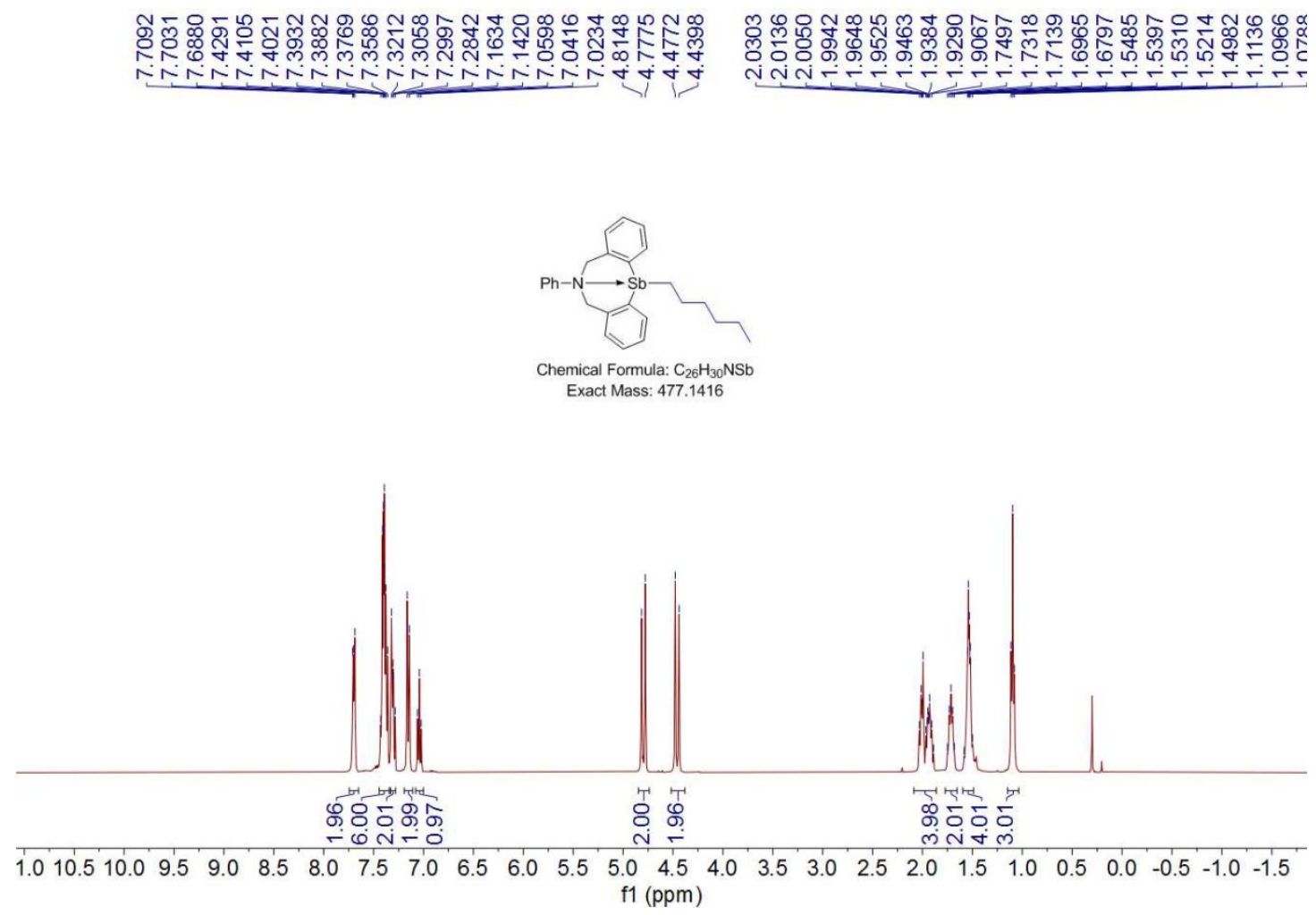

${ }^{13} \mathrm{C} \mathrm{NMR}\left(\mathrm{CDCl}_{3}\right)$ spectrum of compound $\mathbf{3 a v}$

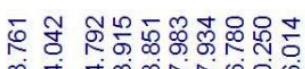

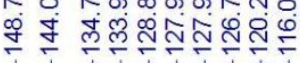

:

फ़

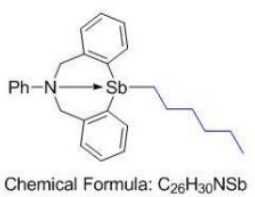

Exact Mass: 477.1416

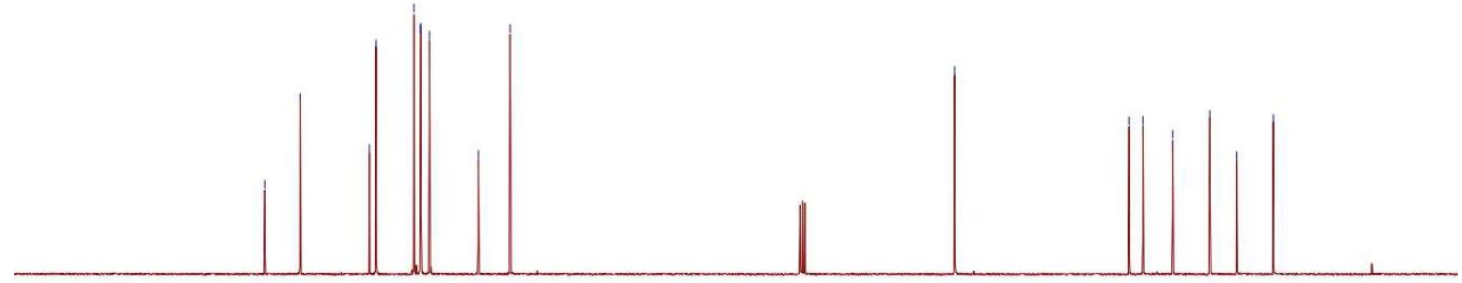

\begin{tabular}{llllllllllllllllllll}
\hline 180 & 170 & 160 & 150 & 140 & 130 & 120 & 110 & 100 & $\begin{array}{c}90 \\
\mathrm{f} 1(\mathrm{ppm})\end{array}$ & 70 & 60 & 50 & 40 & 30 & 20 & 10 & 0 & -1
\end{tabular}

${ }^{1} \mathrm{H}$ NMR $\left(\mathrm{CDCl}_{3}\right)$ spectrum of compound 3aw 

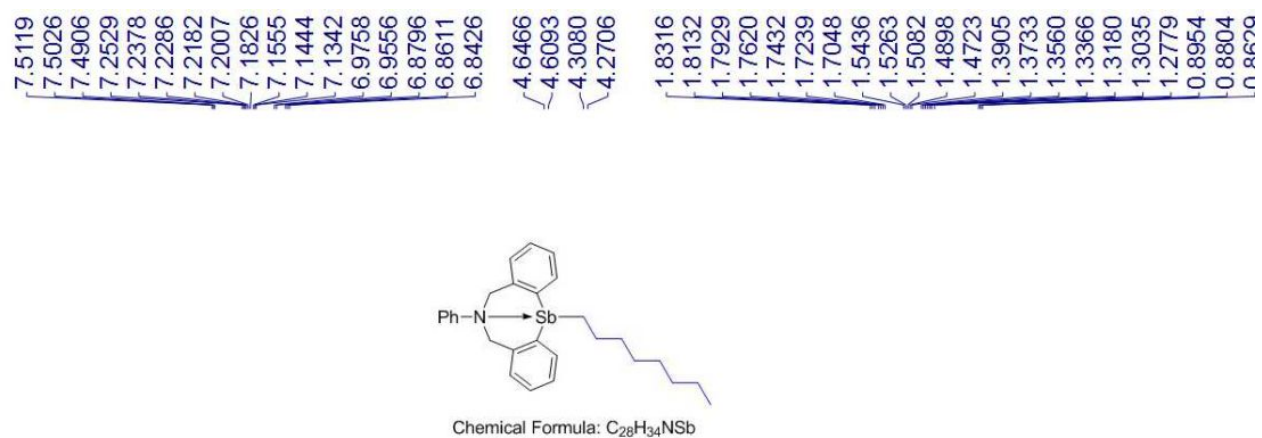

Exact Mass: 505.1729

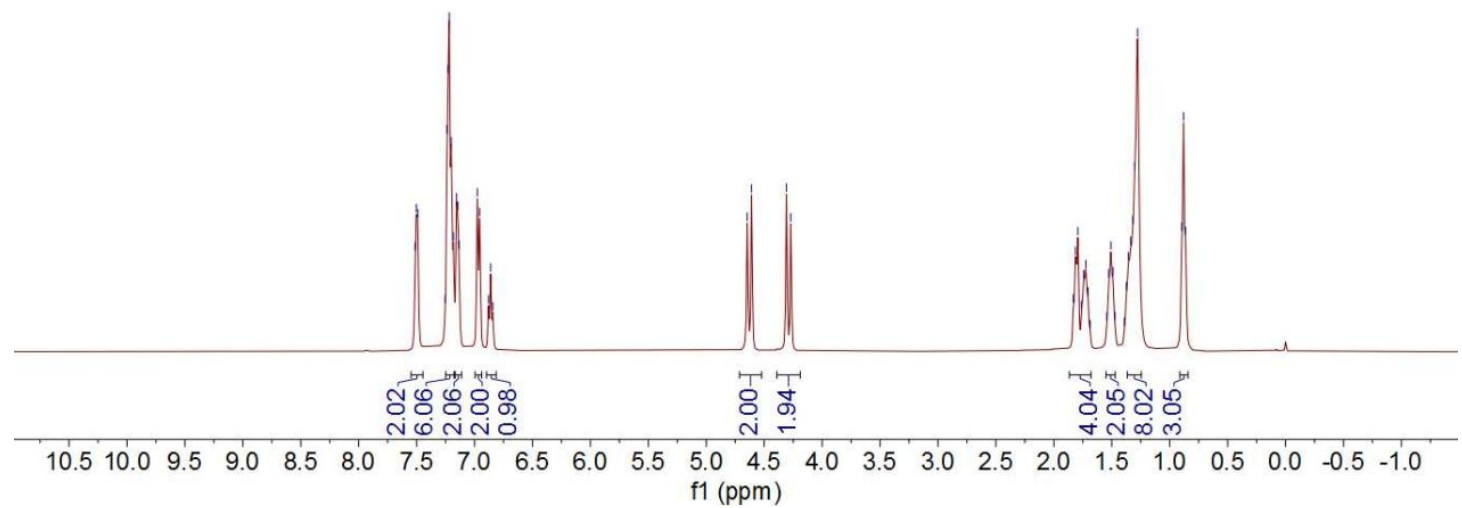

${ }^{13} \mathrm{C}$ NMR $\left(\mathrm{CDCl}_{3}\right)$ spectrum of compound 3aw

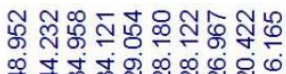

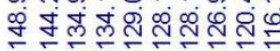

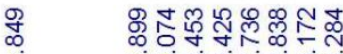

i

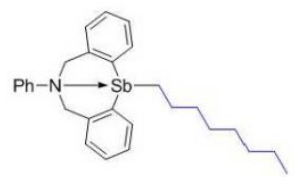

Chemical Formula: $\mathrm{C}_{28} \mathrm{H}_{34} \mathrm{NSb}$ Exact Mass: 505.1729

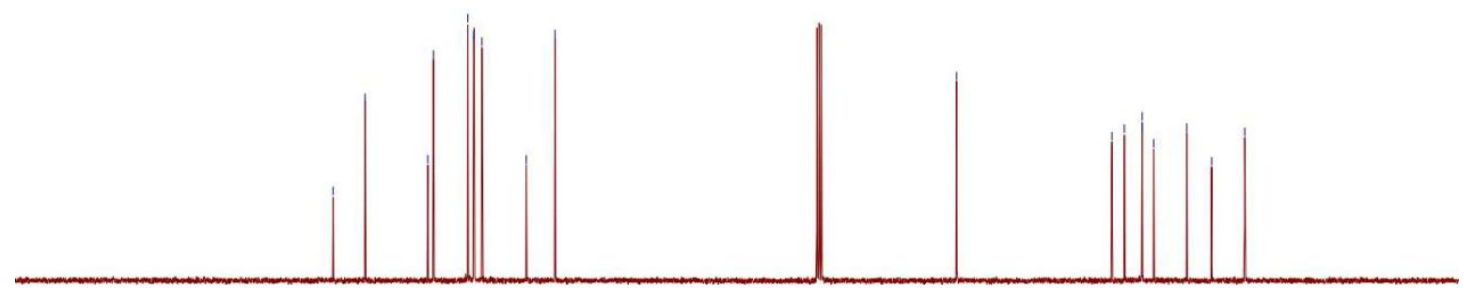

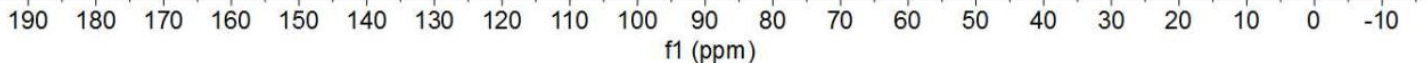

${ }^{1} \mathrm{H}$ NMR $\left(\mathrm{CDCl}_{3}\right)$ spectrum of compound 3ay 


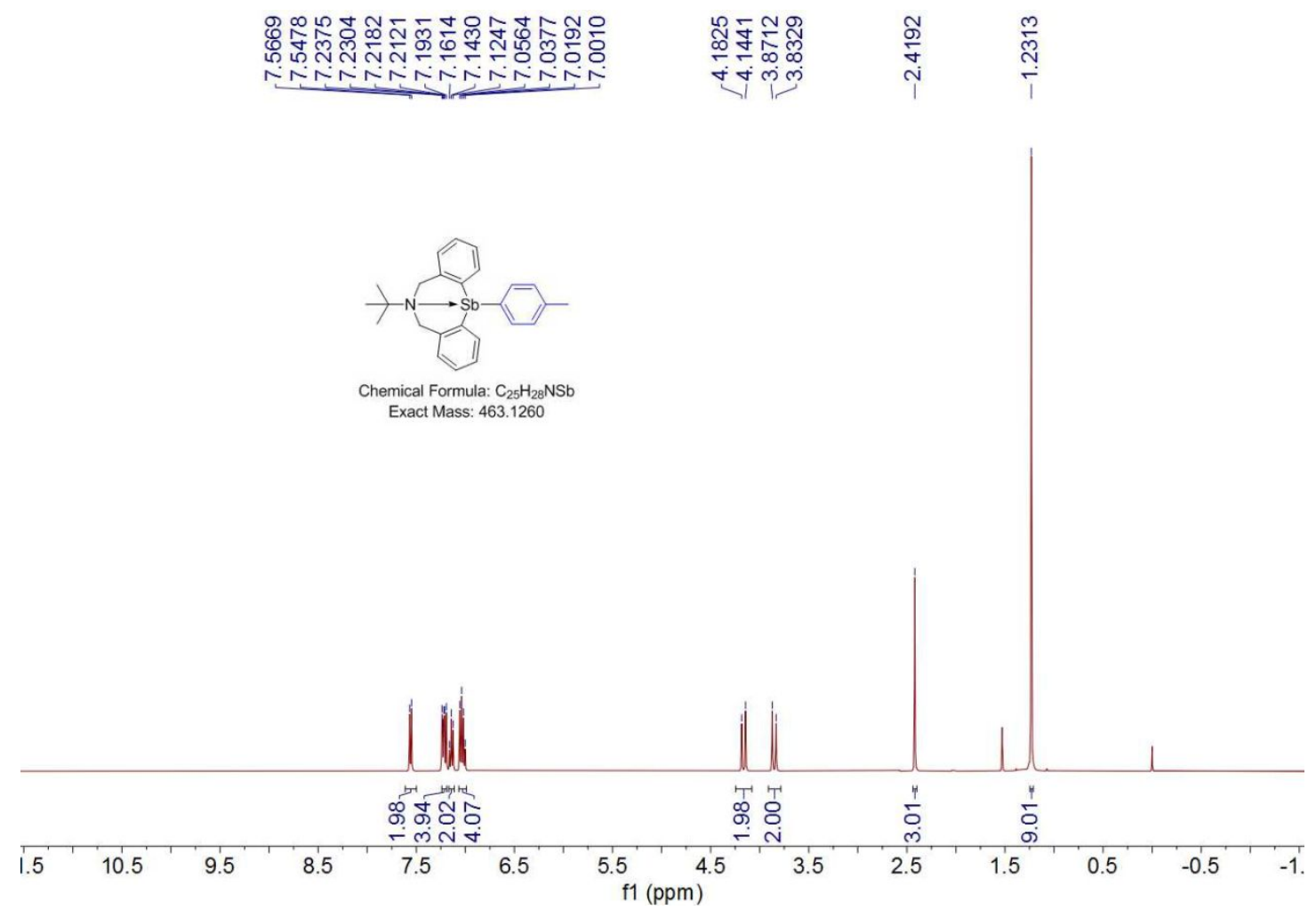

${ }^{13} \mathrm{C} \mathrm{NMR}\left(\mathrm{CDCl}_{3}\right)$ spectrum of compound 3ay

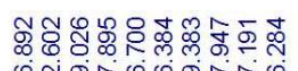

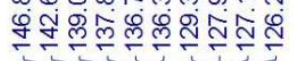

Chemical Formula: $\mathrm{C}_{25} \mathrm{H}_{28} \mathrm{NSb}$ Exact Mass: 463.1260

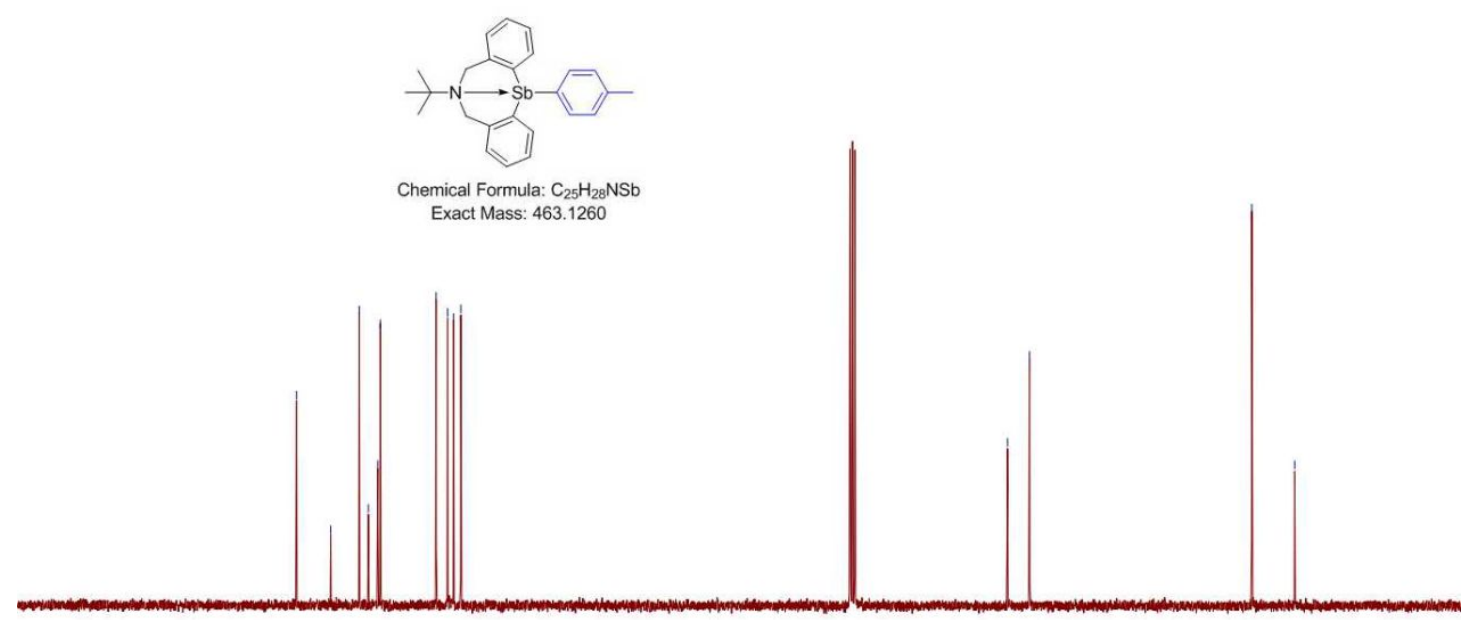

눙

กิ่

范

180

${ }^{1} \mathrm{H}$ NMR $\left(\mathrm{CDCl}_{3}\right)$ spectrum of compound $\mathbf{3 a z}$ 


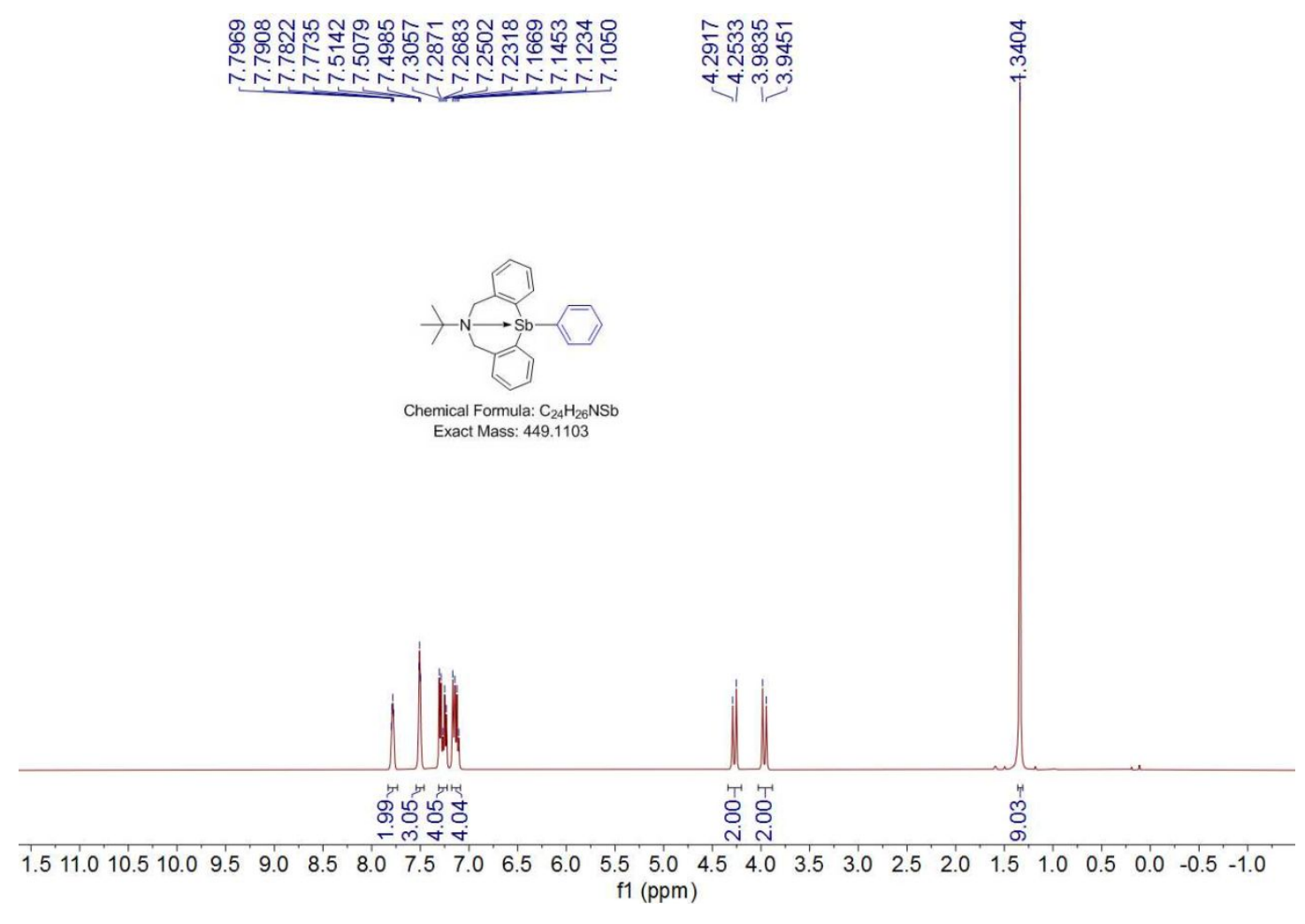

${ }^{13} \mathrm{C}$ NMR $\left(\mathrm{CDCl}_{3}\right)$ spectrum of compound $\mathbf{3 a z}$

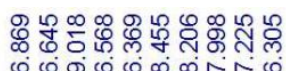

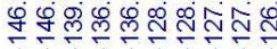

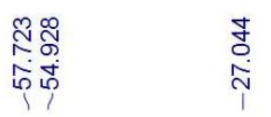
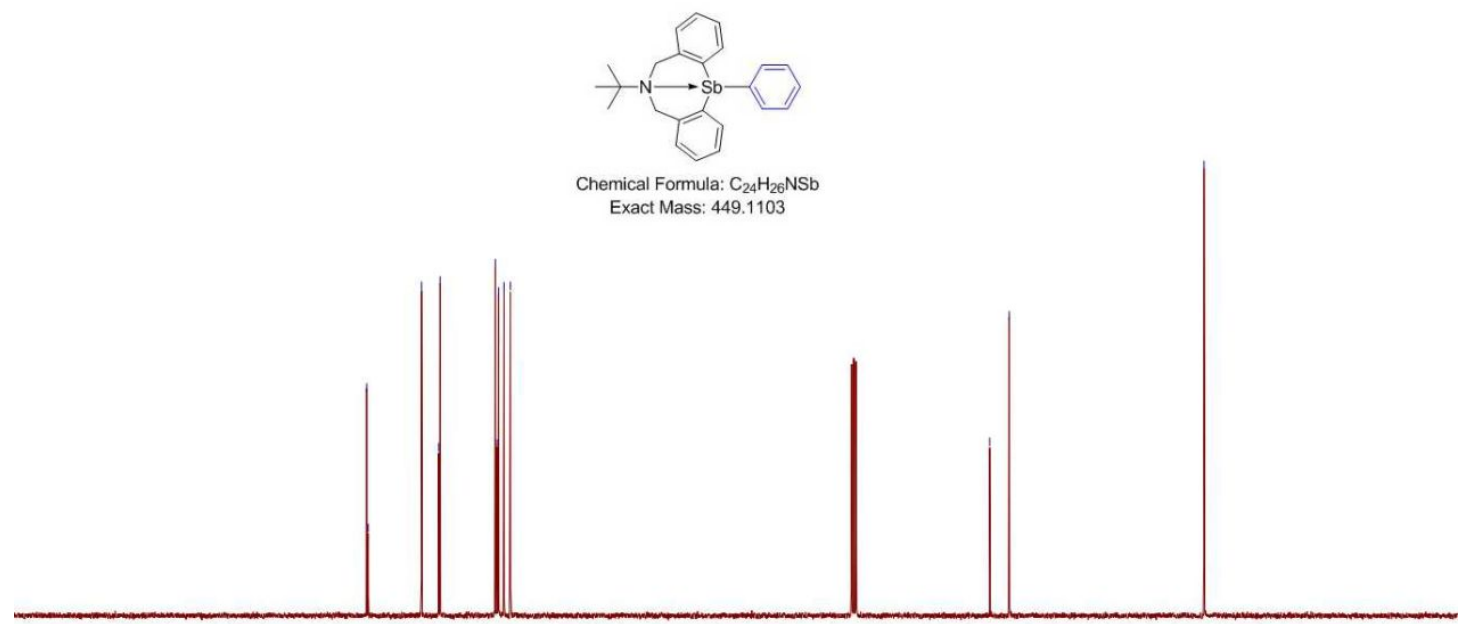

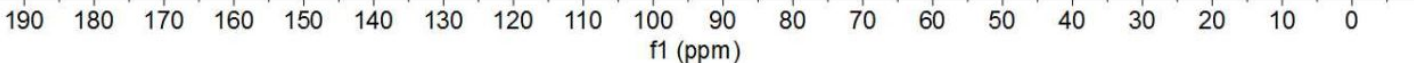

${ }^{1} \mathrm{H}$ NMR $\left(\mathrm{CDCl}_{3}\right)$ spectrum of compound $\mathbf{3 b a}$ 


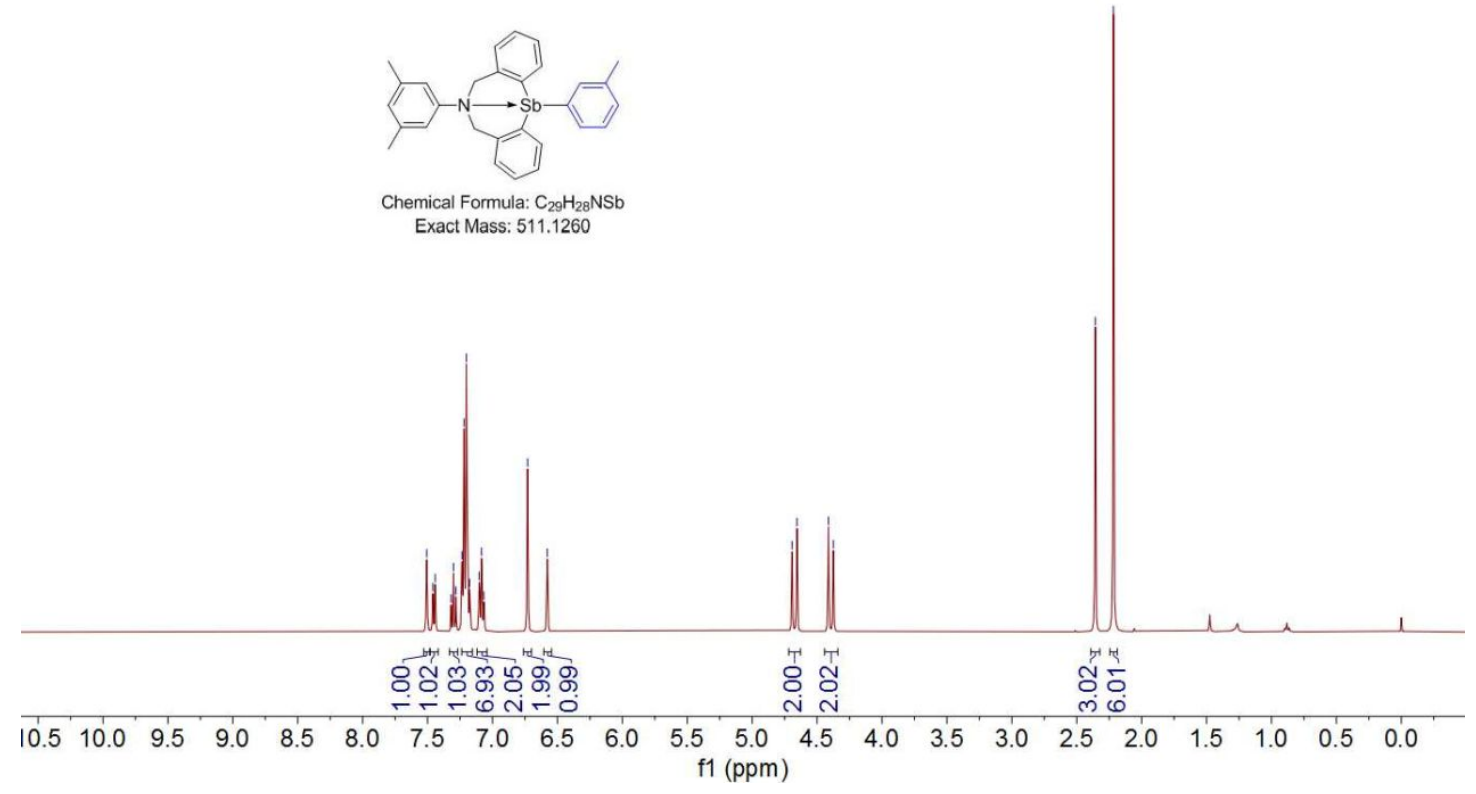

Chemical Formula: $\mathrm{C}_{29} \mathrm{H}_{28} \mathrm{NS}$ Exact Mass: 511.1260

${ }^{13} \mathrm{C}$ NMR $\left(\mathrm{CDCl}_{3}\right)$ spectrum of compound $\mathbf{3 b a}$

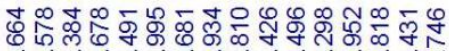

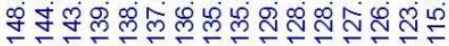
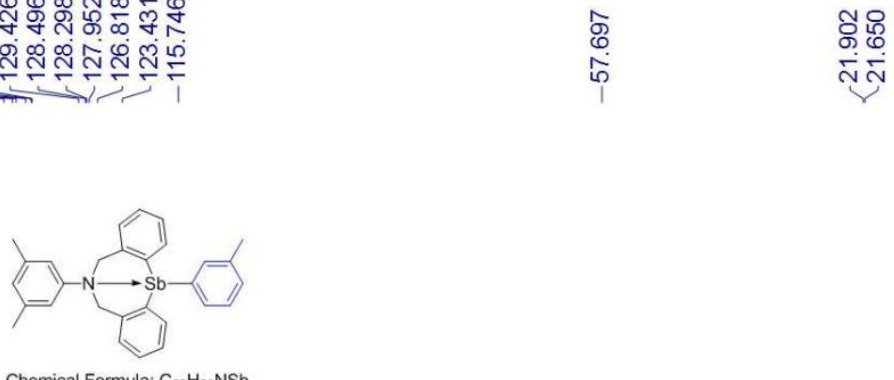

Chemical Formula: $\mathrm{C}_{29} \mathrm{H}_{28} \mathrm{NSb}$ Exact Mass: 511.1260

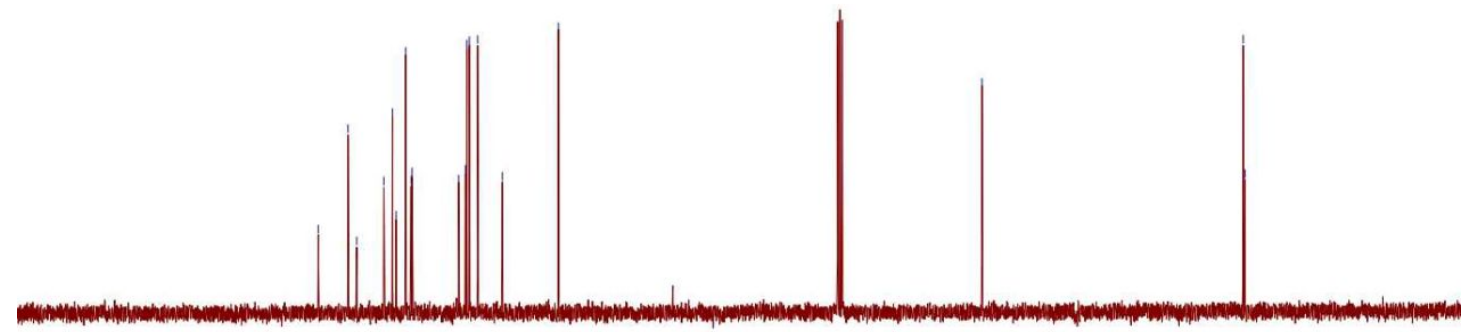

$\begin{array}{llllllllllllllllll}180 & 170 & 160 & 150 & 140 & 130 & 120 & 110 & 100 \underset{\mathrm{f} 1(\mathrm{ppm})}{90} & 80 & 70 & 60 & 50 & 40 & 30 & 20 & 10 & 0\end{array}$

${ }^{1} \mathrm{H}$ NMR $\left(\mathrm{CDCl}_{3}\right)$ spectrum of compound $\mathbf{3 b b}$ 


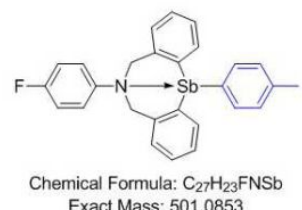

Exact Mass: 501.0853

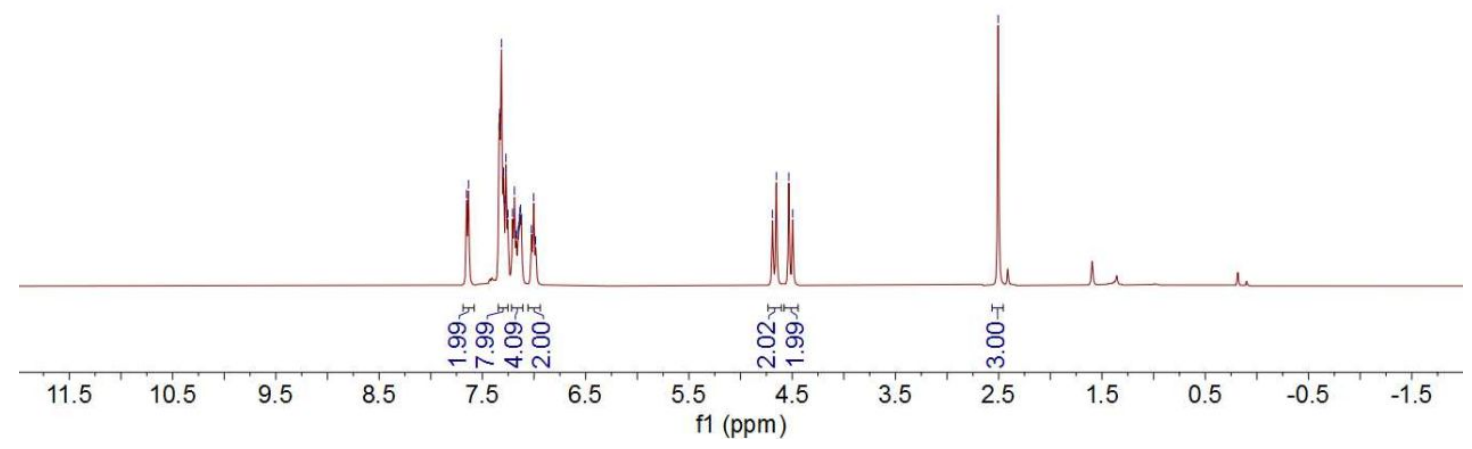

${ }^{13} \mathrm{C} \mathrm{NMR}\left(\mathrm{CDCl}_{3}\right)$ spectrum of compound $\mathbf{3 b b}$
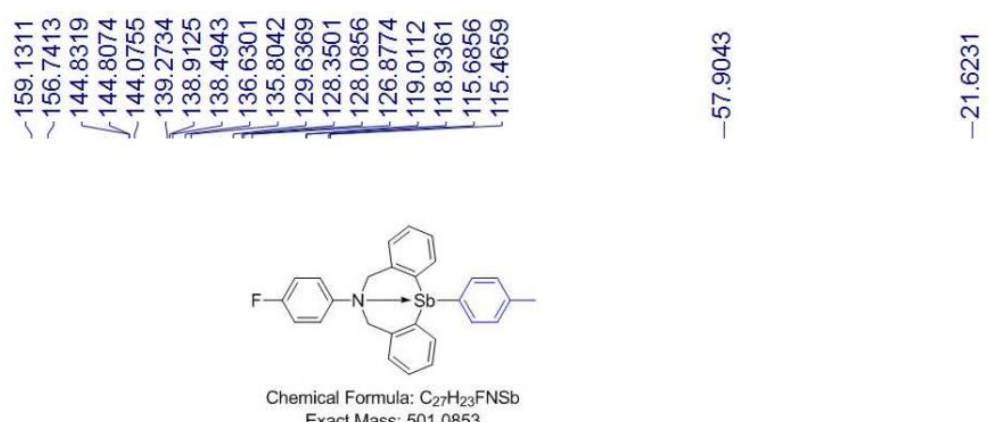

Exact Mass: 501.0853

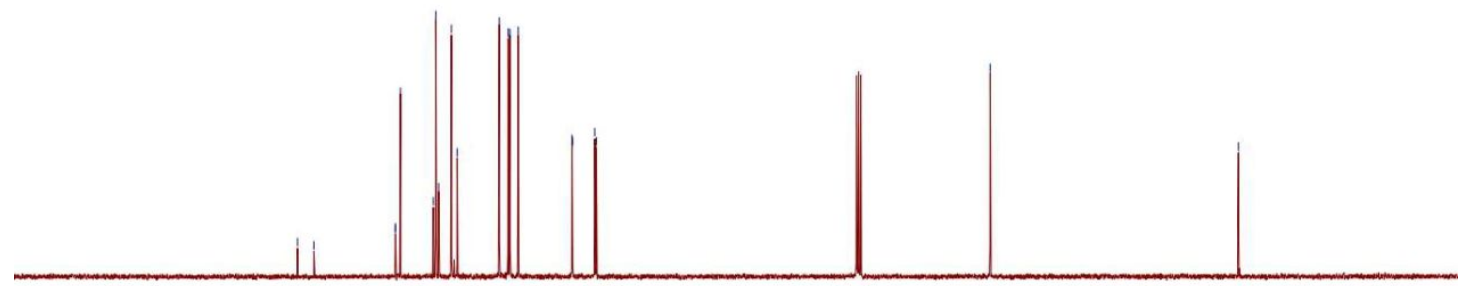

$\begin{array}{llllllllllllllllllllllll}30 & 190 & 180 & 170 & 160 & 150 & 140 & 130 & 120 & 110 & 100 & 90 & 80 & 70 & 60 & 50 & 40 & 30 & 20 & 10 & 0 & -1\end{array}$ f1 (ppm)

${ }^{19} \mathrm{~F}$ NMR $\left(\mathrm{CDCl}_{3}\right)$ spectrum of compound $\mathbf{3 b b}$ 


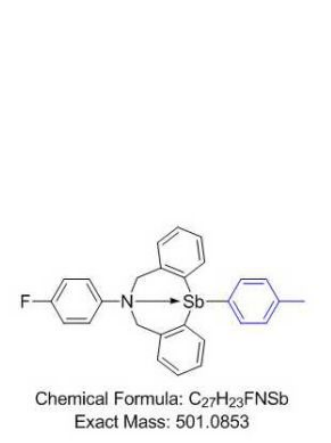

Chemical Formula: $\mathrm{C}_{27} \mathrm{H}_{23} \mathrm{FNS}$

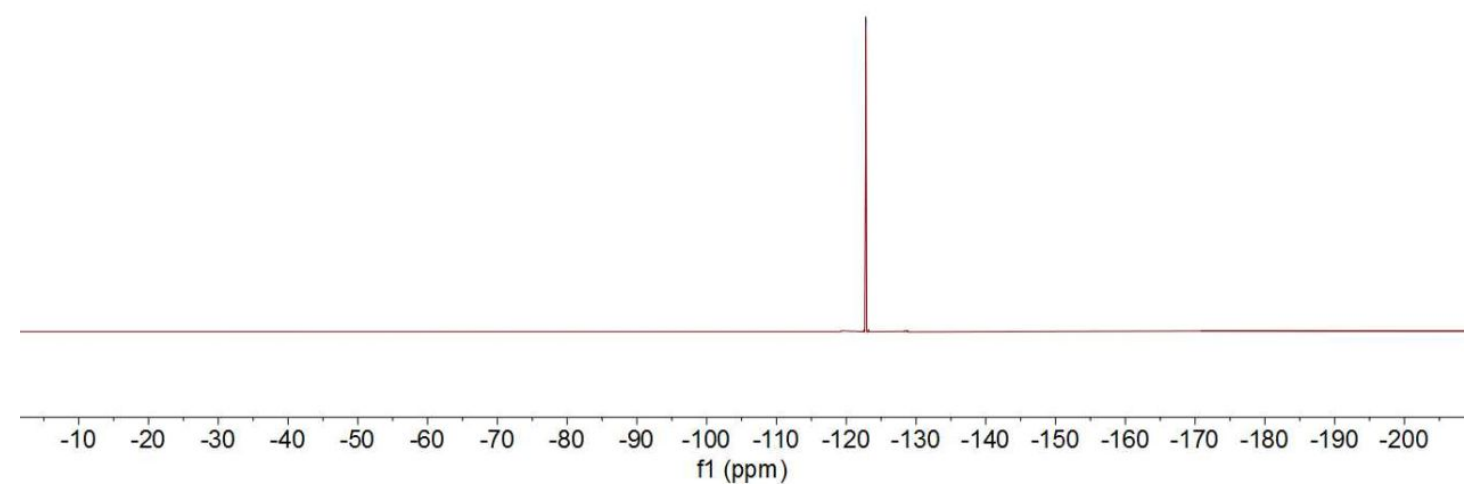

${ }^{1} \mathrm{H}$ NMR $\left(\mathrm{CDCl}_{3}\right)$ spectrum of compound 3bc
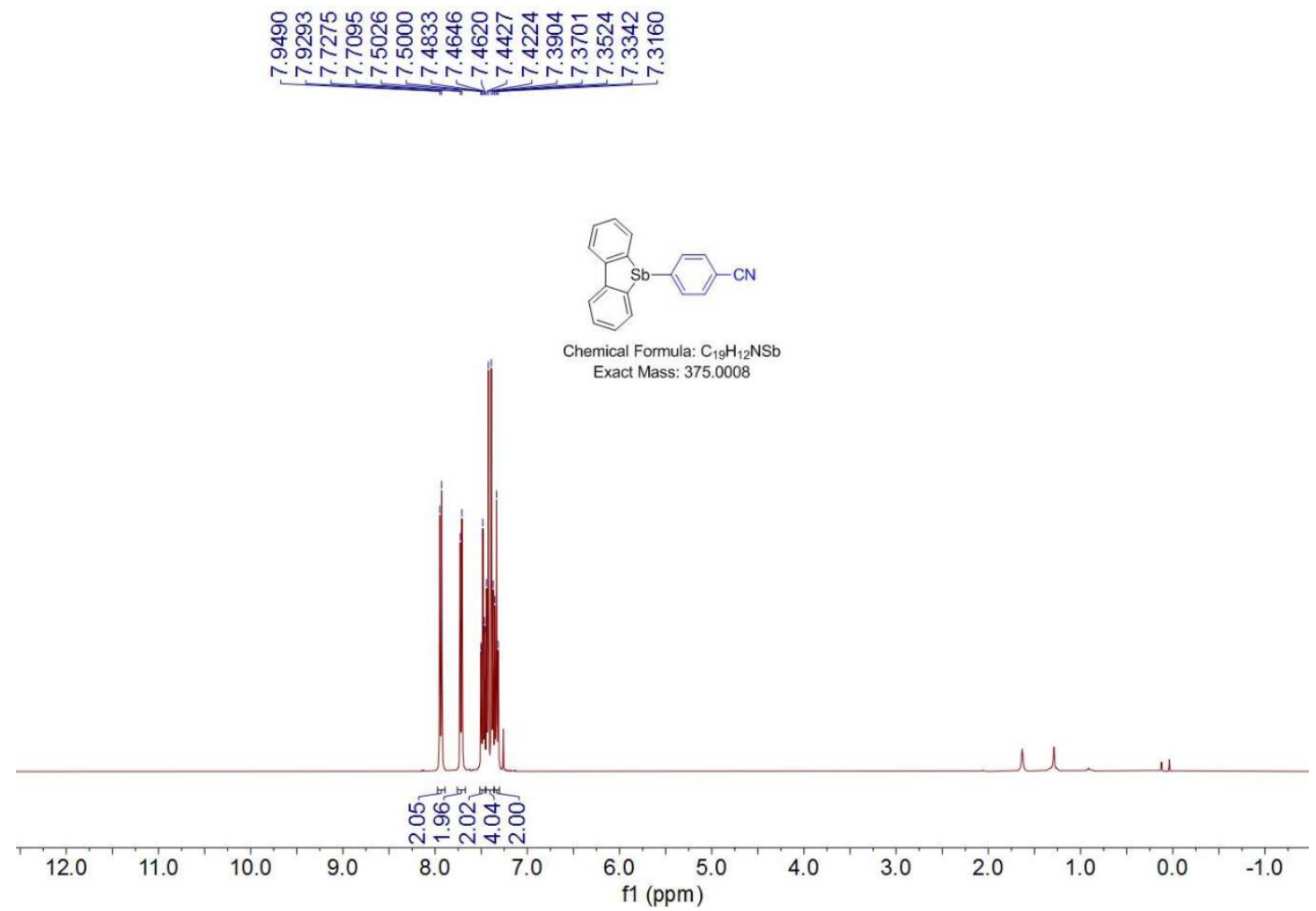

${ }^{13} \mathrm{C}$ NMR $\left(\mathrm{CDCl}_{3}\right)$ spectrum of compound $\mathbf{3 b c}$ 


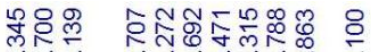

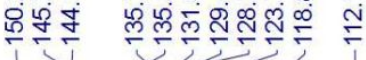

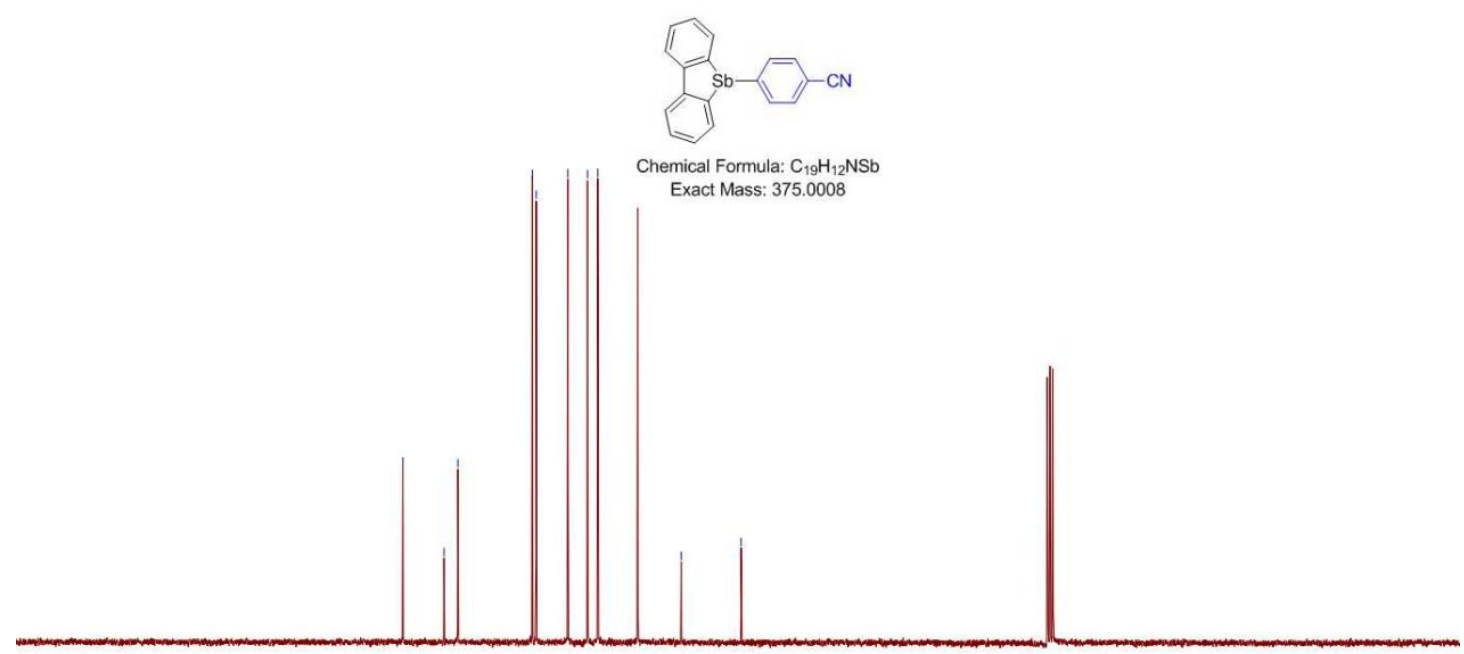

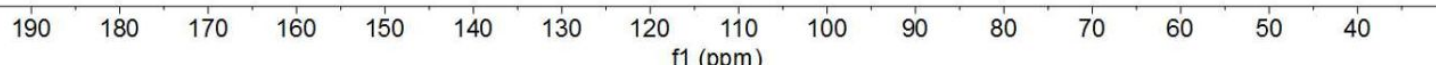

${ }^{1} \mathrm{H}$ NMR $\left(\mathrm{CDCl}_{3}\right)$ spectrum of compound $\mathbf{3 b d}$
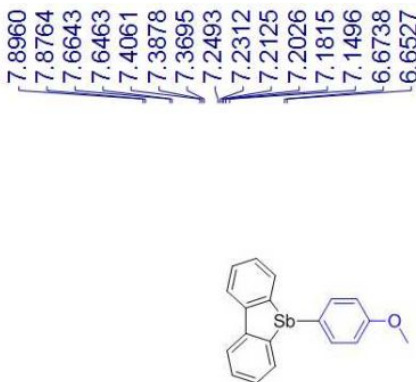

Chemical Formula: $\mathrm{C}_{19} \mathrm{H}_{15} \mathrm{OS}$ Exact Mass: 380.0161

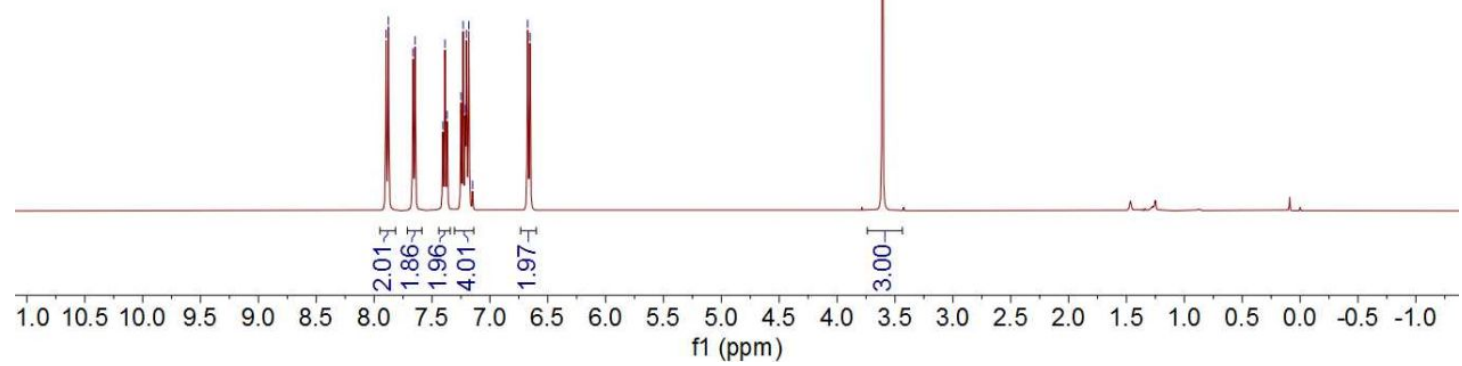

${ }^{13} \mathrm{C}$ NMR $\left(\mathrm{CDCl}_{3}\right)$ spectrum of compound $\mathbf{3 b d}$ 


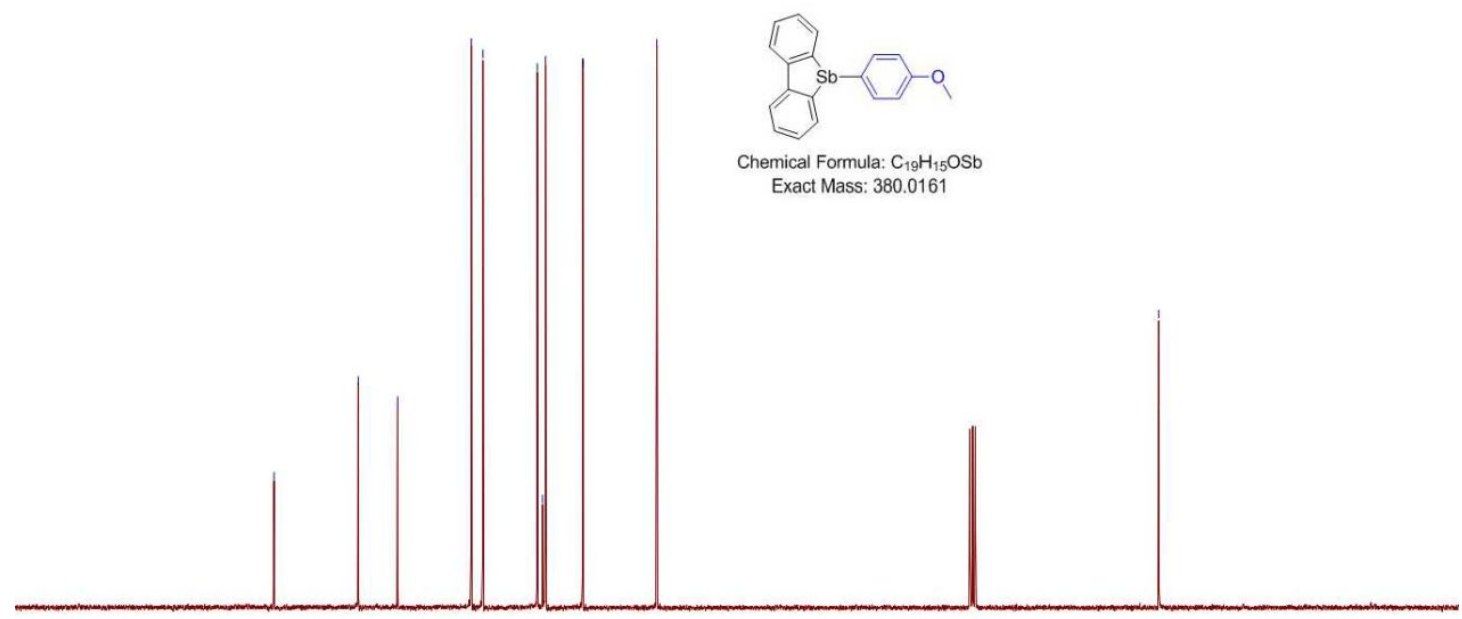

\begin{tabular}{llllllllllllllllll}
\hline 90 & 180 & 170 & 160 & 150 & 140 & 130 & 120 & $\begin{array}{c}110 \\
\mathrm{f} 1(\mathrm{ppm})\end{array}$ & 100 & 90 & 80 & 70 & 60 & 50 & 40 & 30 & 26
\end{tabular}

${ }^{1} \mathrm{H}$ NMR $\left(\mathrm{CDCl}_{3}\right)$ spectrum of compound $\mathbf{3 b e}$
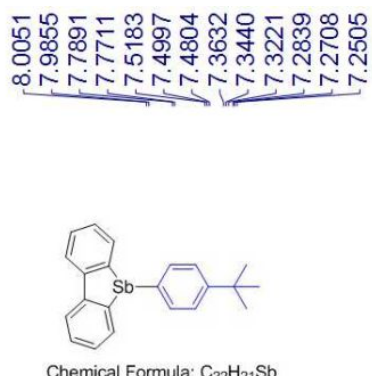

Exact Mass: 406.0681

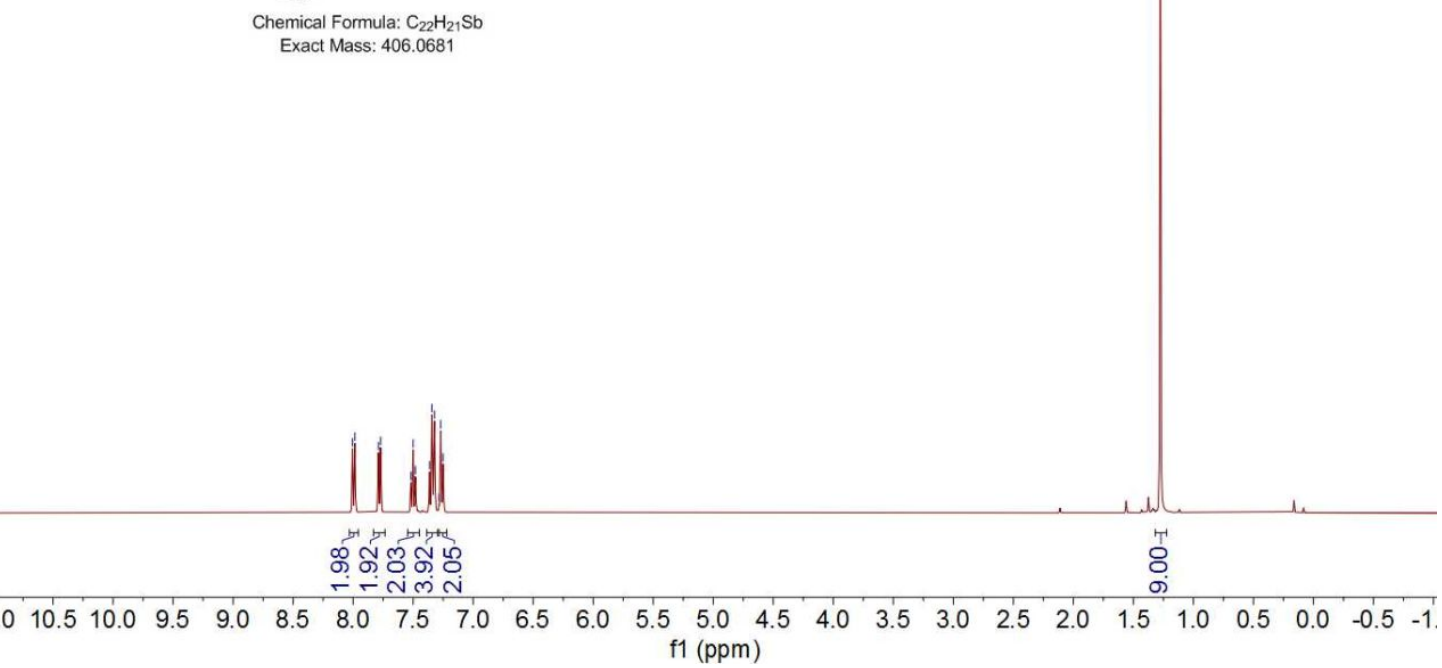

${ }^{13} \mathrm{C}$ NMR $\left(\mathrm{CDCl}_{3}\right)$ spectrum of compound 3be 


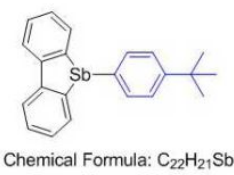
Chemical Formula: $\mathrm{C}_{22} \mathrm{H}_{21}$

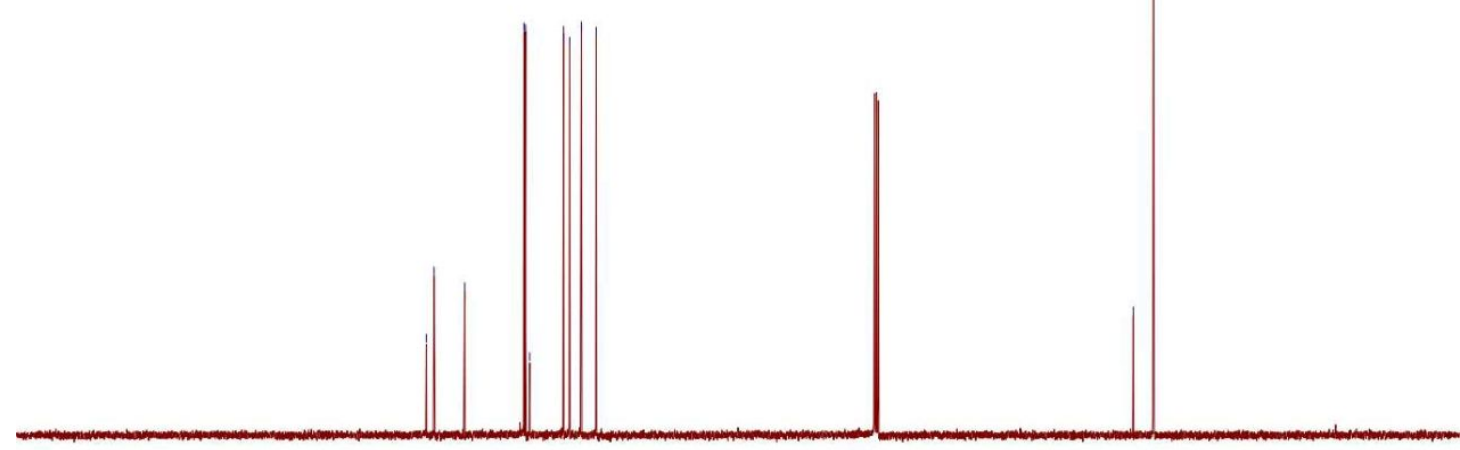

$\begin{array}{lllllllllllllllllllllllll}210 & 200 & 190 & 180 & 170 & 160 & 150 & 140 & 130 & 120 & 110 & 100 & 90 & 80 & 70 & 60 & 50 & 40 & 30 & 20 & 10 & 0 & -10\end{array}$ $\mathrm{f1}(\mathrm{ppm})$

${ }^{1} \mathrm{H}$ NMR $\left(\mathrm{CDCl}_{3}\right)$ spectrum of compound $\mathbf{3 b f}$
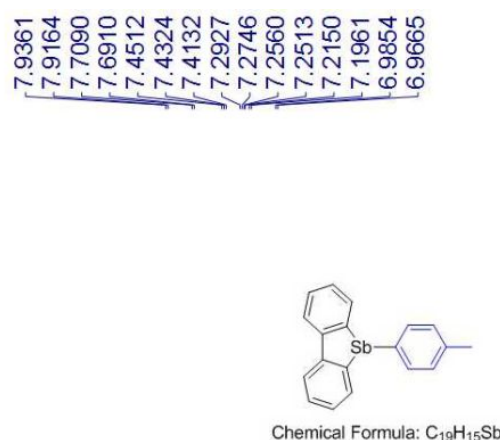

Exact Mass: 364.0212

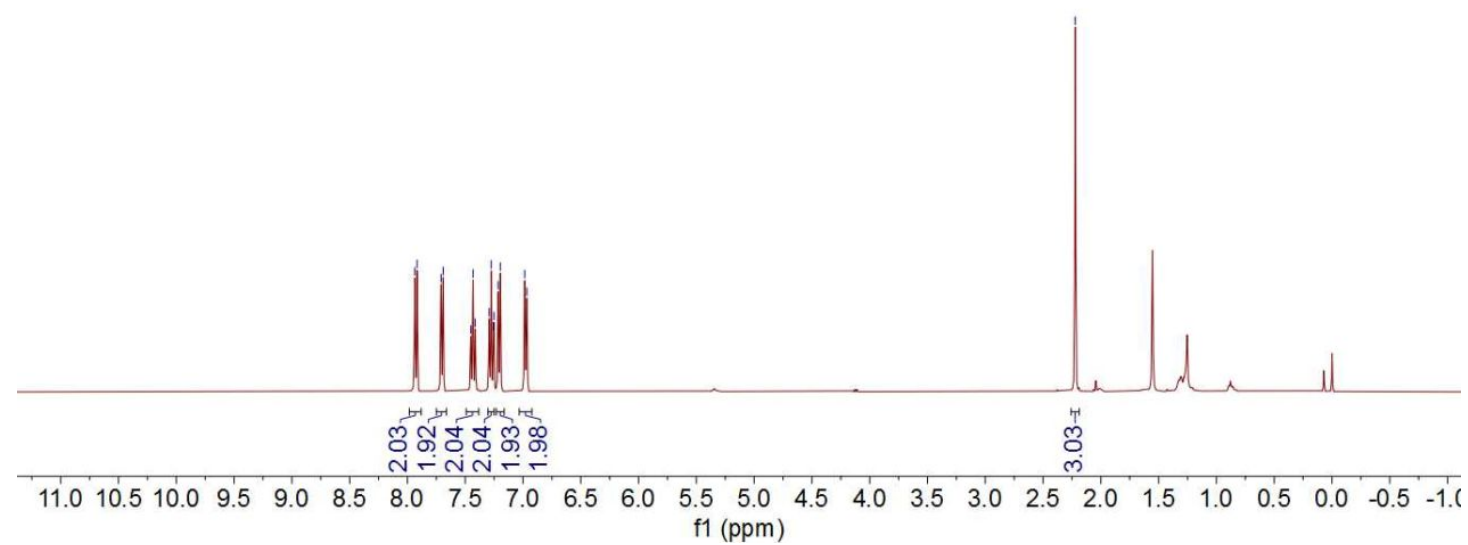

${ }^{13} \mathrm{C}$ NMR $\left(\mathrm{CDCl}_{3}\right)$ spectrum of compound $\mathbf{3 b f}$ 


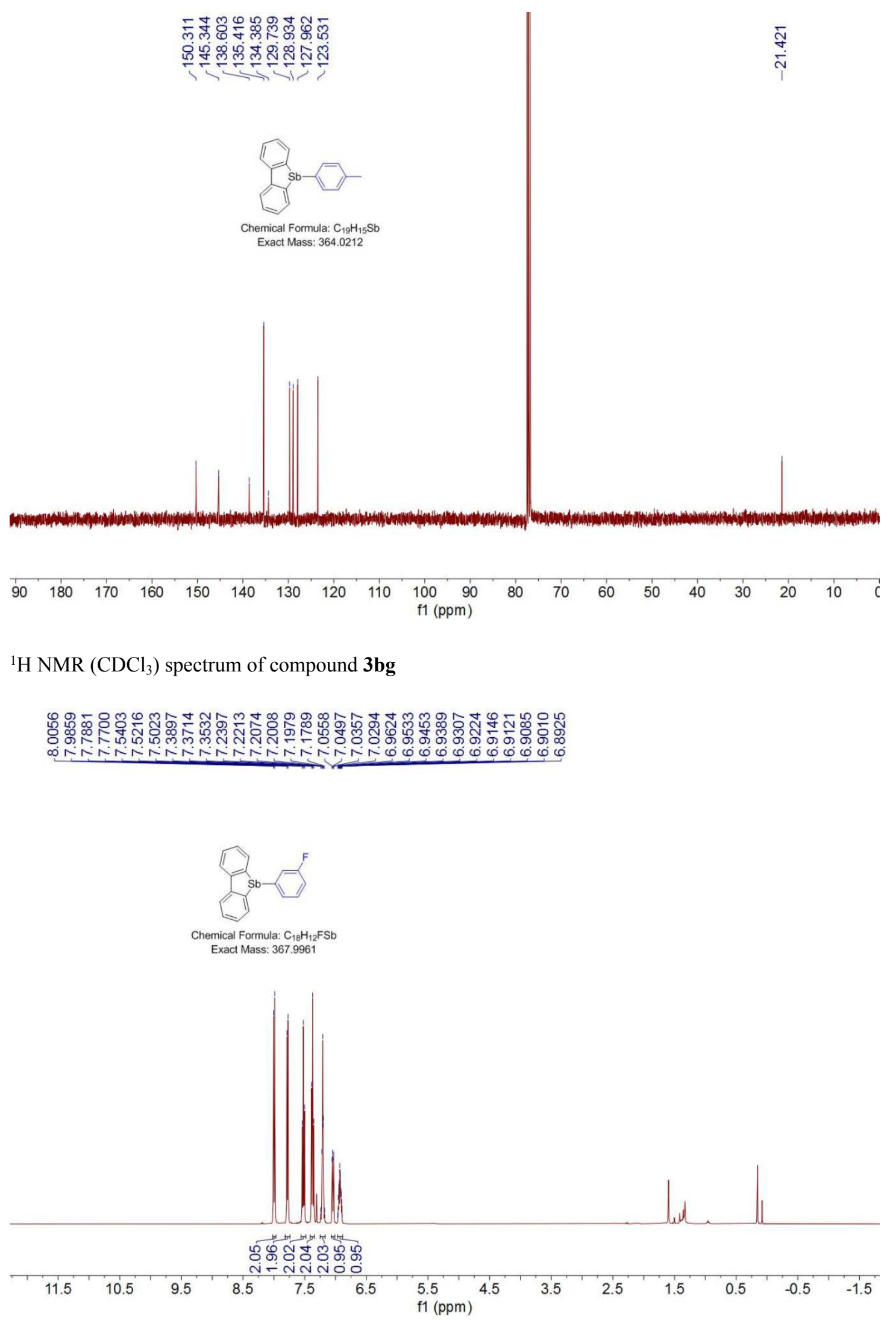

${ }^{13} \mathrm{C} \mathrm{NMR}\left(\mathrm{CDCl}_{3}\right)$ spectrum of compound $\mathbf{3 b g}$ 


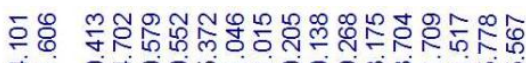

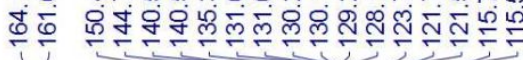

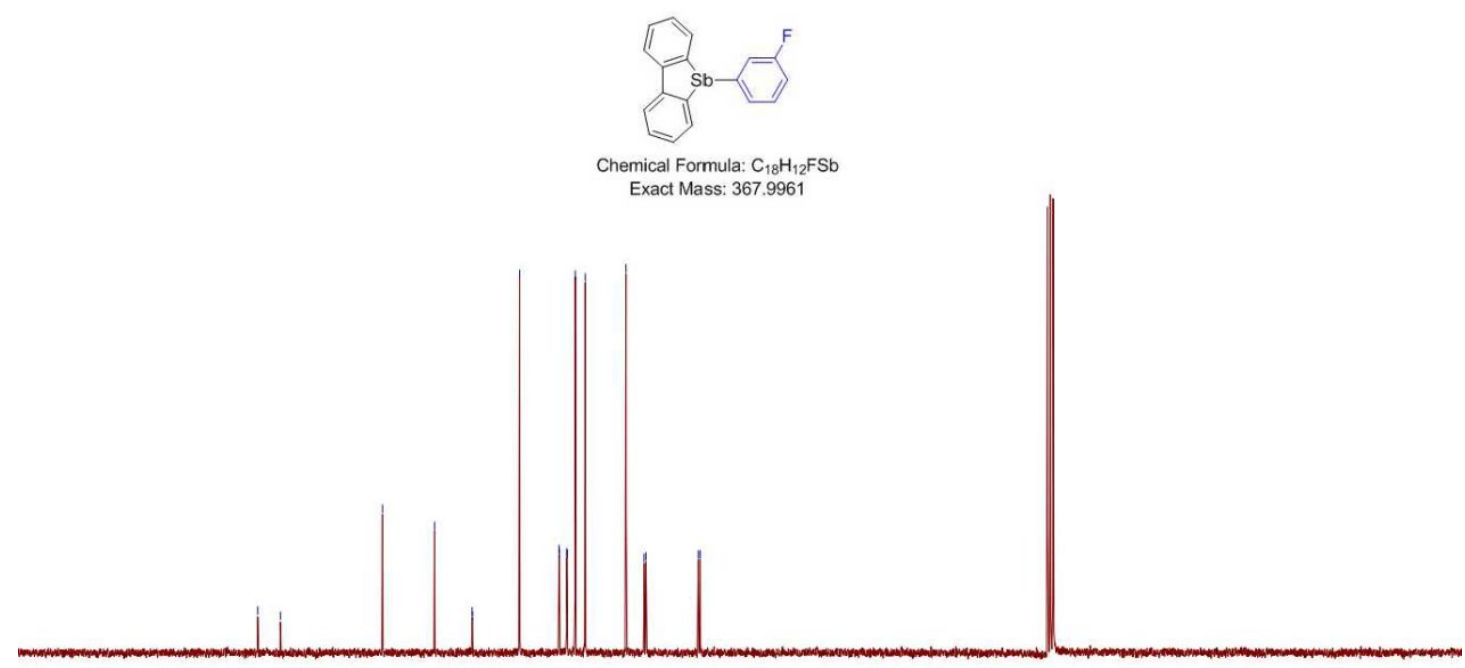

\begin{tabular}{lllllllllllllllll}
\hline 90 & 180 & 170 & 160 & 150 & 140 & 130 & 120 & $\begin{array}{c}110 \\
\mathrm{f} 1(\mathrm{ppm})\end{array}$ & 100 & 90 & 80 & 70 & 60 & 50 & 40
\end{tabular}

${ }^{19} \mathrm{~F} \mathrm{NMR}\left(\mathrm{CDCl}_{3}\right)$ spectrum of compound $\mathbf{3 b g}$

$\stackrel{\stackrel{2}{+}}{\stackrel{+}{T}}$

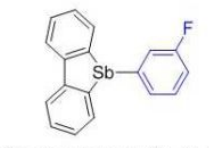

Chemical Formula: $\mathrm{C}_{18} \mathrm{H}_{12} \mathrm{FS}$

Exact Mass: 367.9961

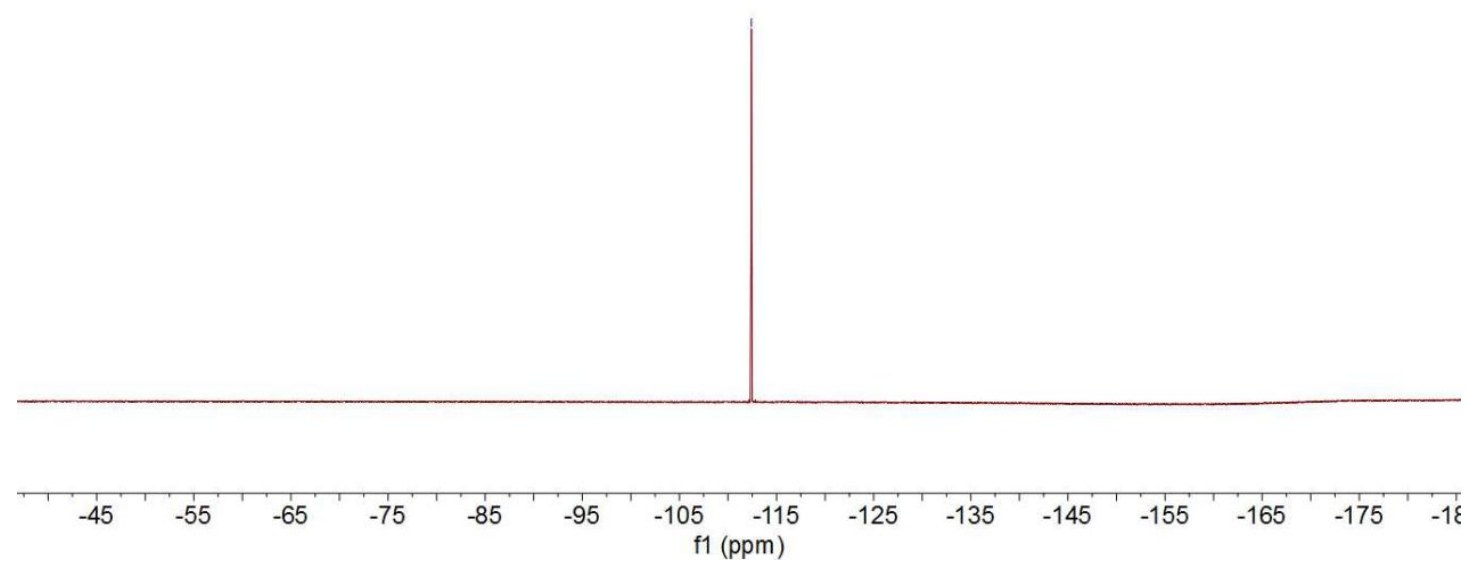


${ }^{1} \mathrm{H}$ NMR $\left(\mathrm{CDCl}_{3}\right)$ spectrum of compound $\mathbf{3 b h}$
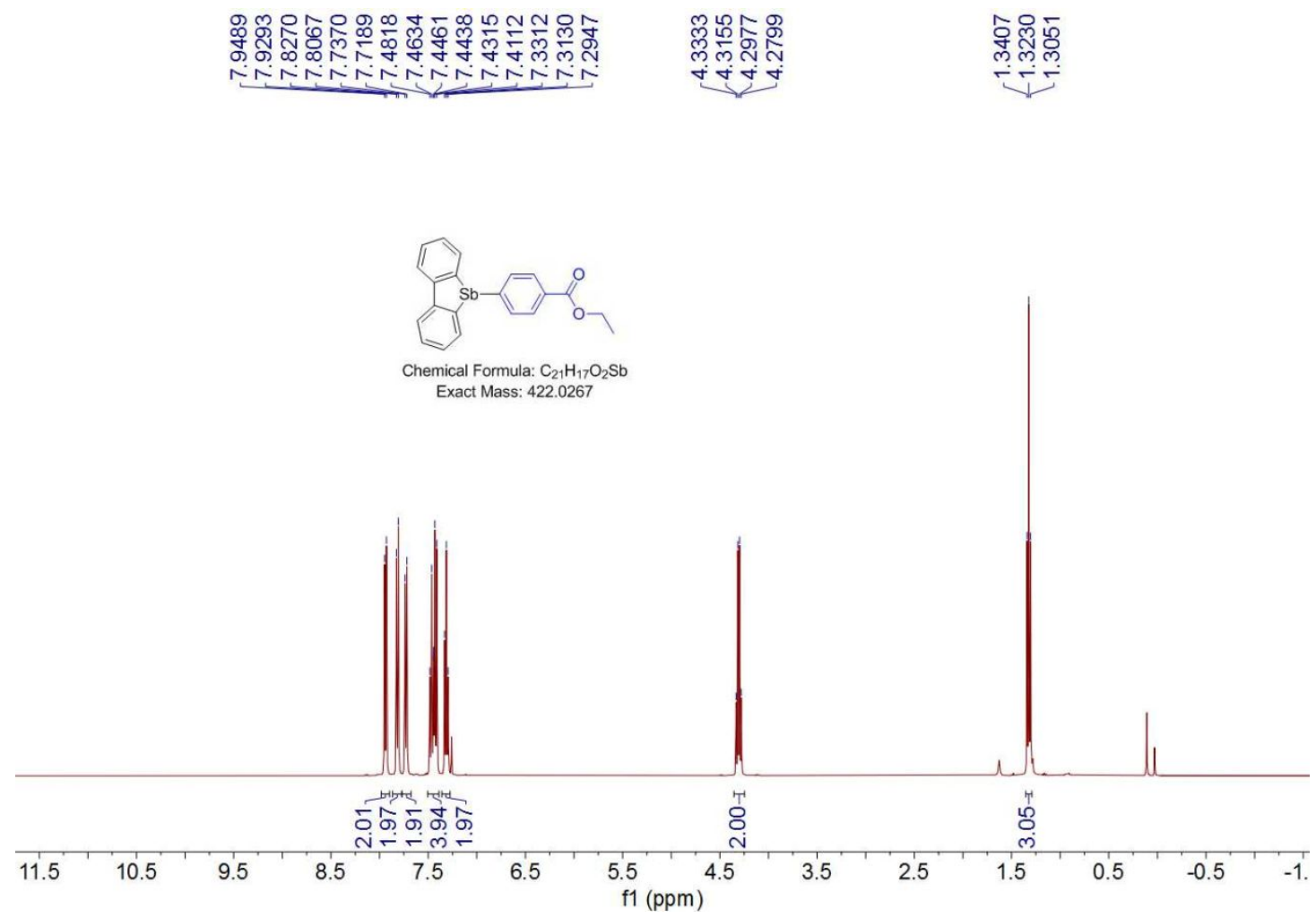

Exact Mass: 422.0267

${ }^{13} \mathrm{C} \mathrm{NMR}\left(\mathrm{CDCl}_{3}\right)$ spectrum of compound $\mathbf{3 b h}$

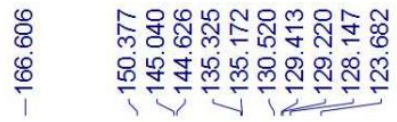

$\Sigma_{0}$
0
0
0

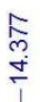
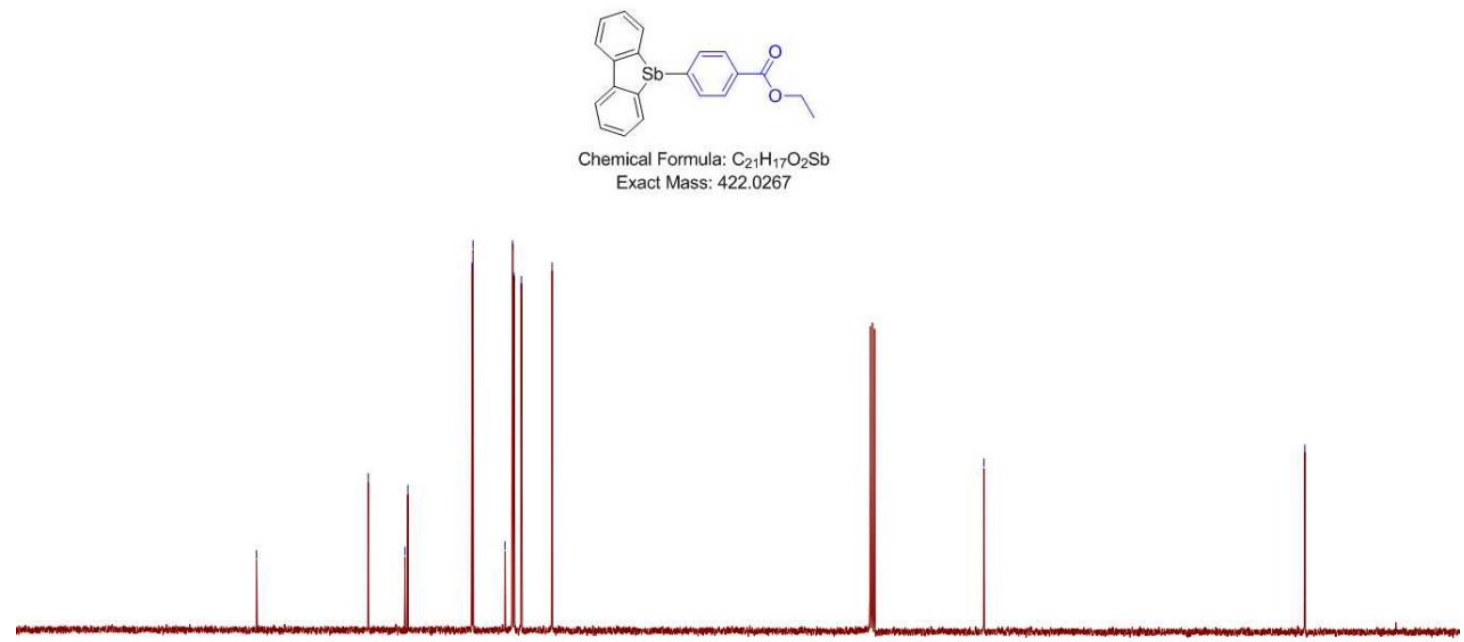

$\begin{array}{lllllllllllllllllllll}100 & 190 & 180 & 170 & 160 & 150 & 140 & 130 & 120 & 110 & \begin{array}{l}100 \\ \mathrm{f} 1(\mathrm{ppm})\end{array} & 80 & 70 & 60 & 50 & 40 & 30 & 20 & 10 & 0\end{array}$

${ }^{1} \mathrm{H}$ NMR $\left(\mathrm{CDCl}_{3}\right)$ spectrum of compound $\mathbf{3 b i}$ 


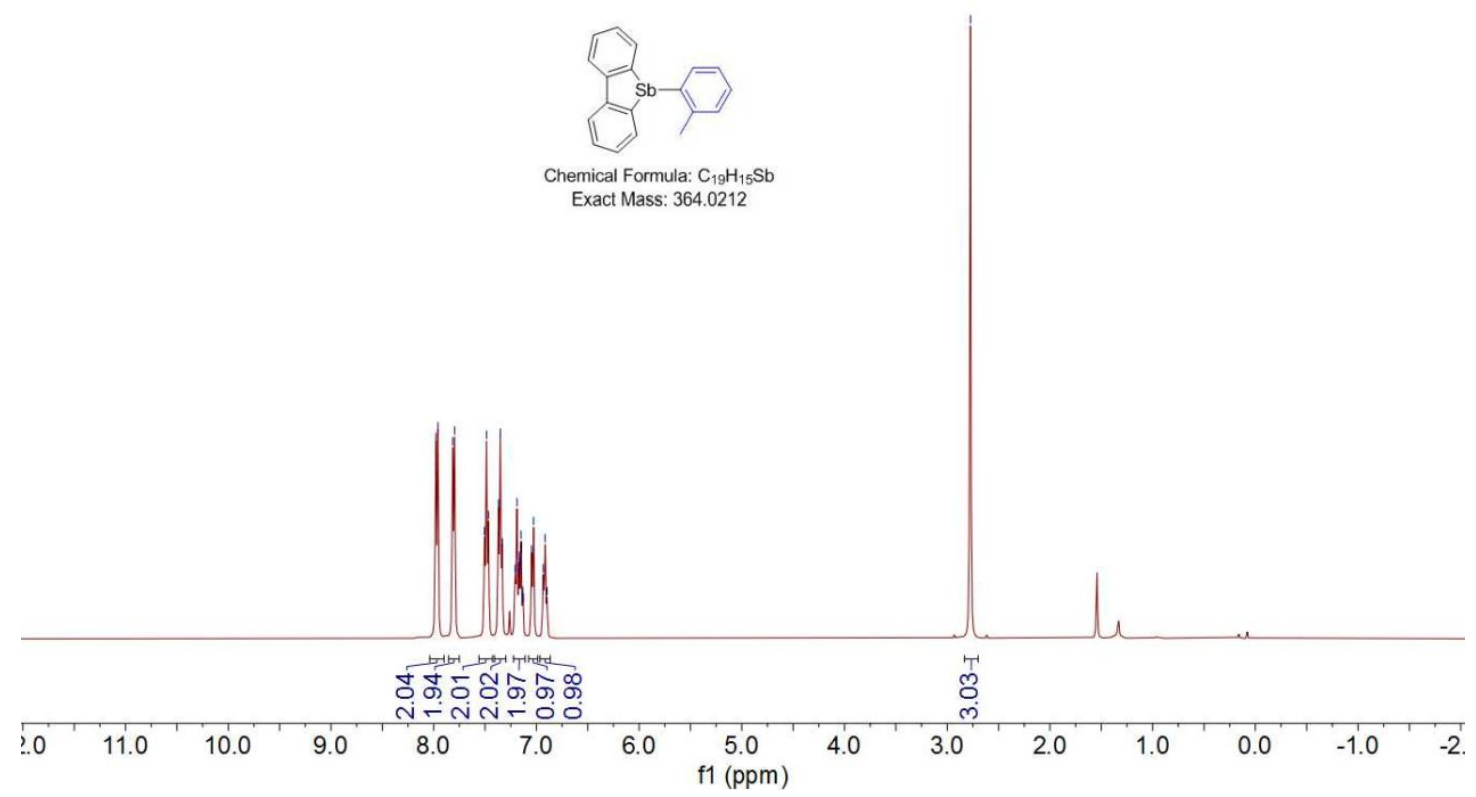

${ }^{13} \mathrm{C} \mathrm{NMR}\left(\mathrm{CDCl}_{3}\right)$ spectrum of compound $3 \mathbf{b i}$

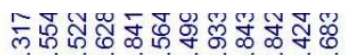

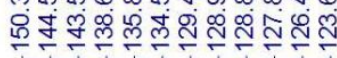
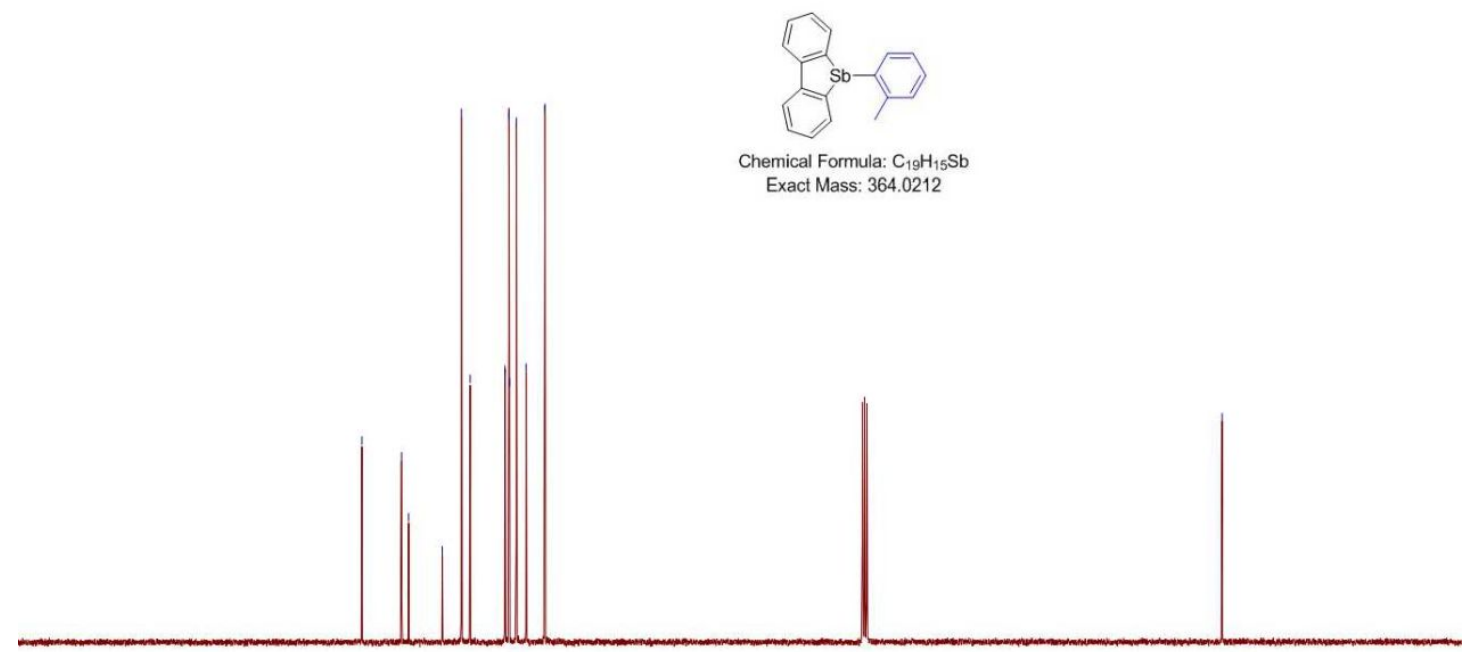

\begin{tabular}{llllllllllll}
\hline 0 & 190 & 180 & 170 & 160 & 150 & 140 & 130 & 120 & 110 & 100 & 90
\end{tabular}

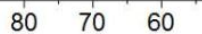

f1 (ppm)

${ }^{1} \mathrm{H} \mathrm{NMR}\left(\mathrm{CDCl}_{3}\right)$ spectrum of compound $\mathbf{3 b j}$ 


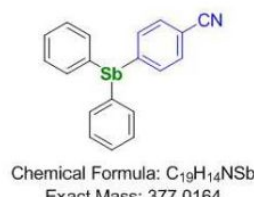

Exact Mass: 377.0164

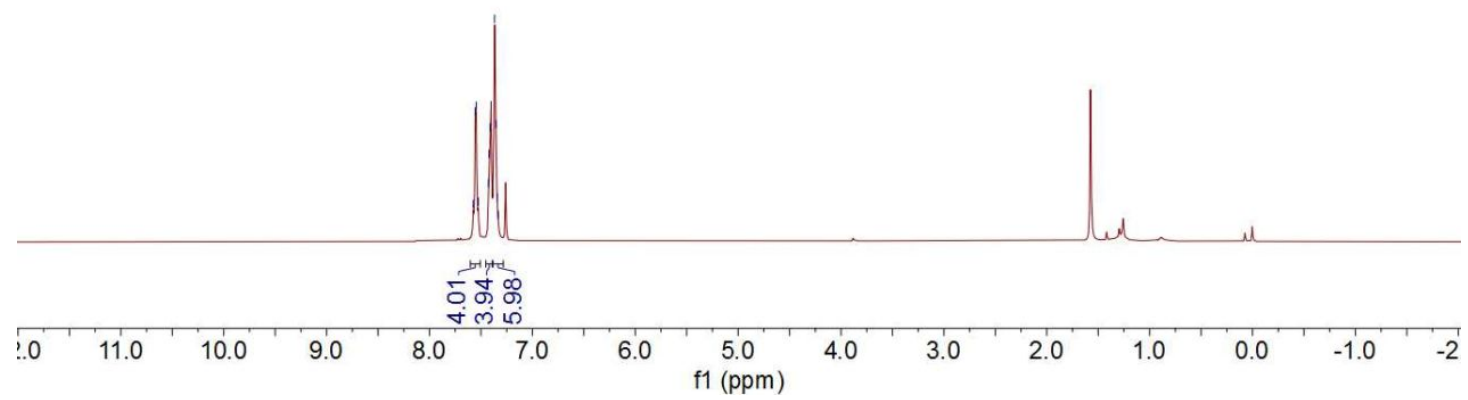

${ }^{1} \mathrm{H}$ NMR $\left(\mathrm{CDCl}_{3}\right)$ spectrum of compound $\mathbf{3 b k}$

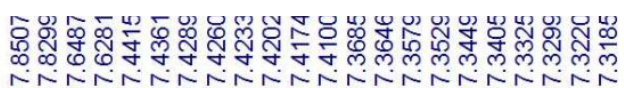

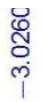

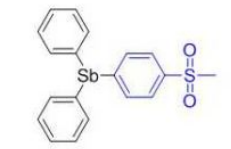

Chemical Formula: $\mathrm{C}_{19} \mathrm{H}_{17} \mathrm{O}_{2} \mathrm{SSb}$ Exact Mass: 429.9987

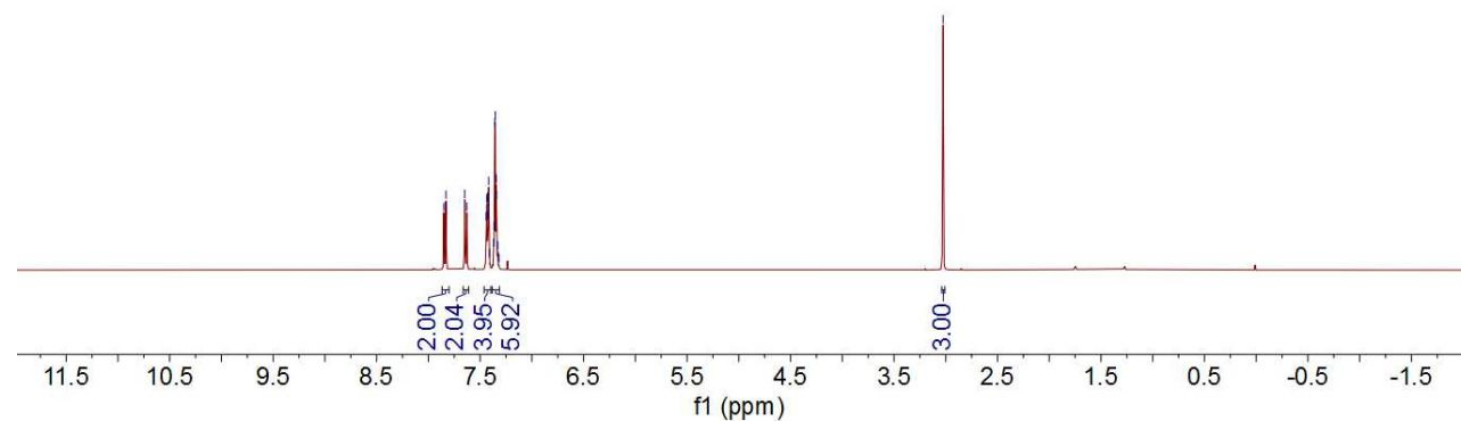

${ }^{1} \mathrm{H}$ NMR $\left(\mathrm{CDCl}_{3}\right)$ spectrum of compound $\mathbf{3 b l}$ 

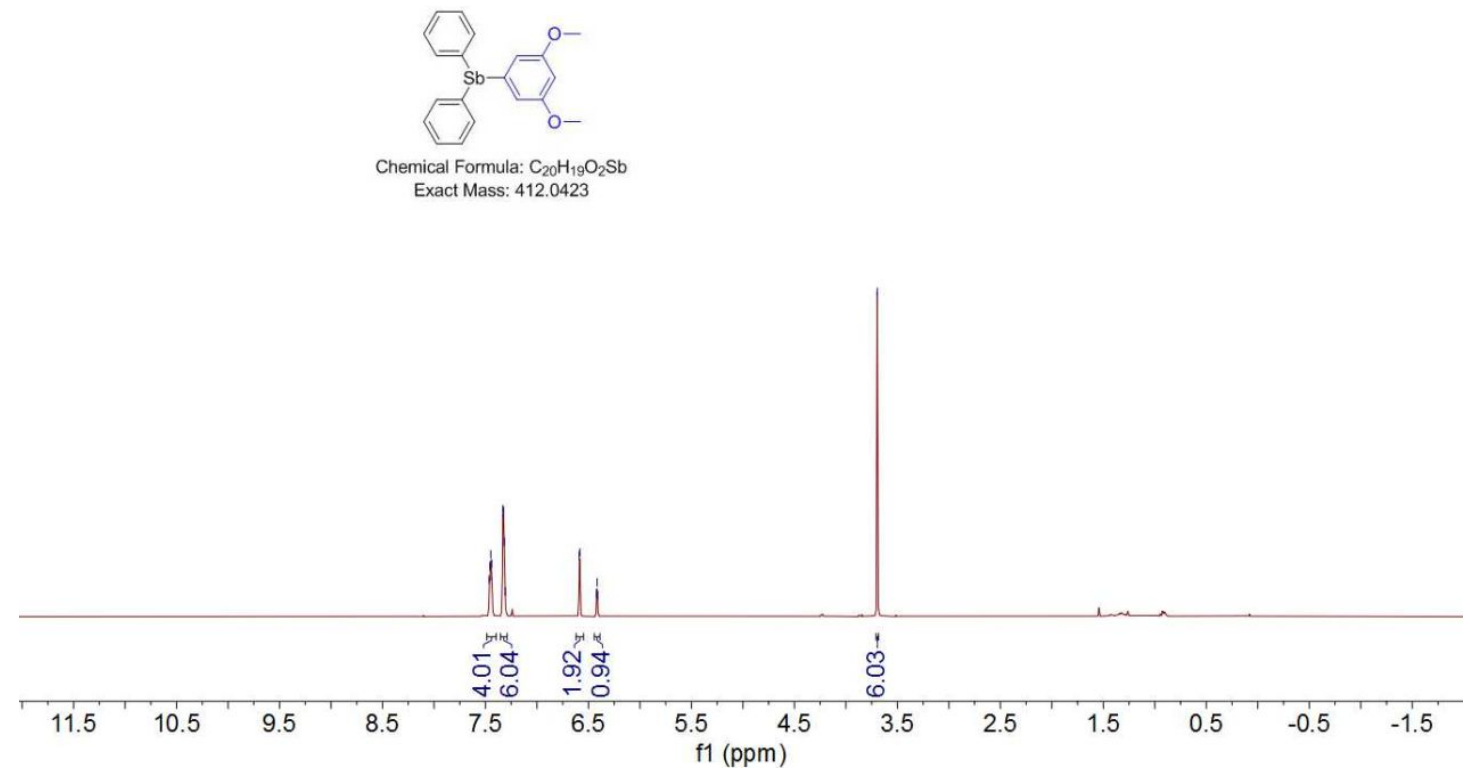

${ }^{1} \mathrm{H}$ NMR $\left(\mathrm{CDCl}_{3}\right)$ spectrum of compound $\mathbf{3 b m}$

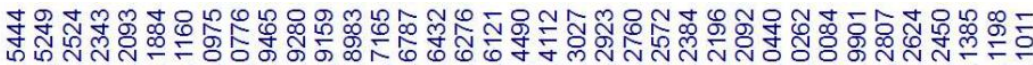

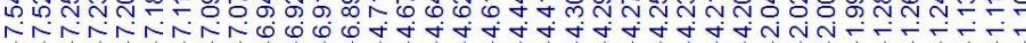

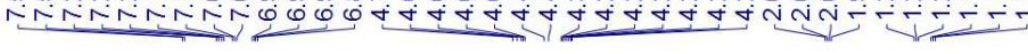

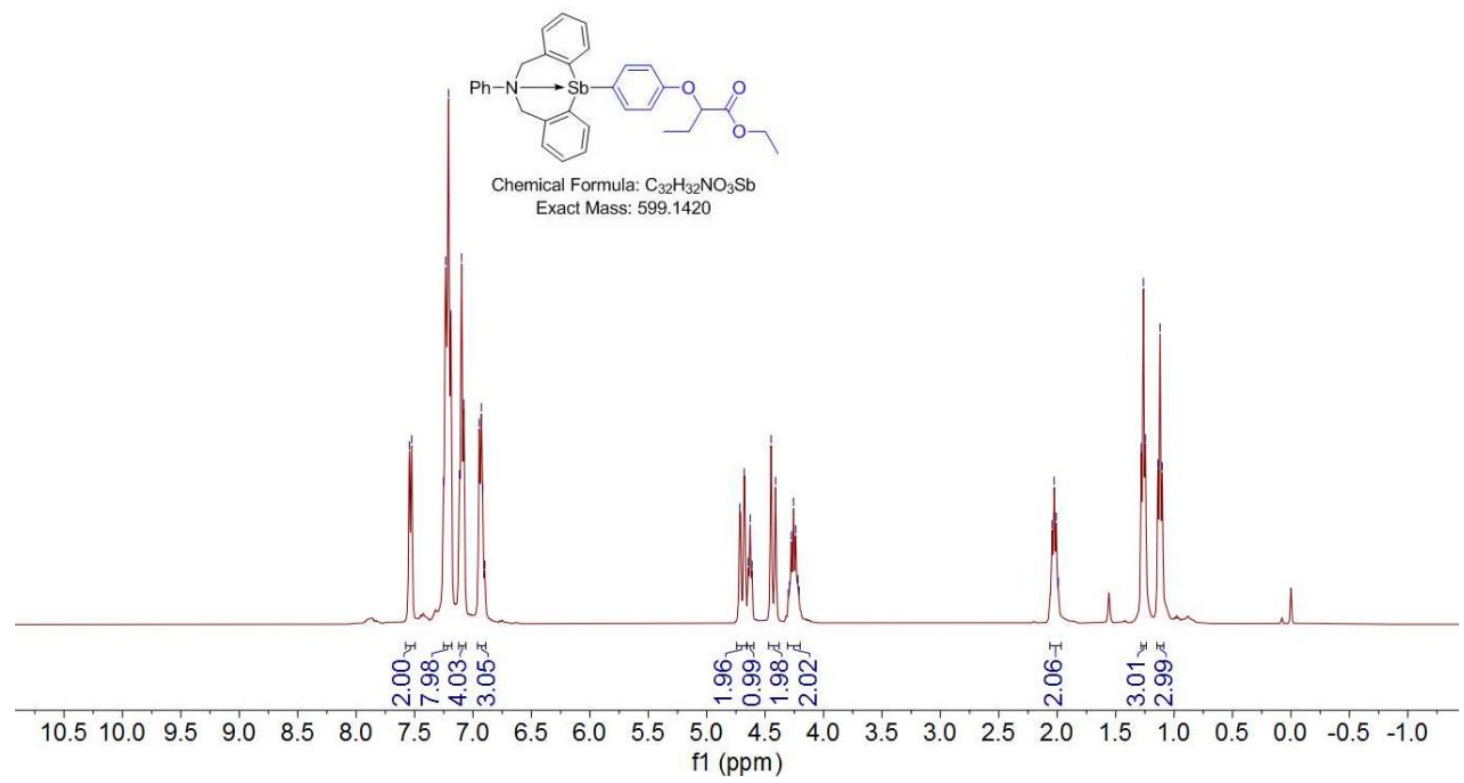


${ }^{13} \mathrm{C} \mathrm{NMR}\left(\mathrm{CDCl}_{3}\right)$ spectrum of compound $\mathbf{3 b m}$

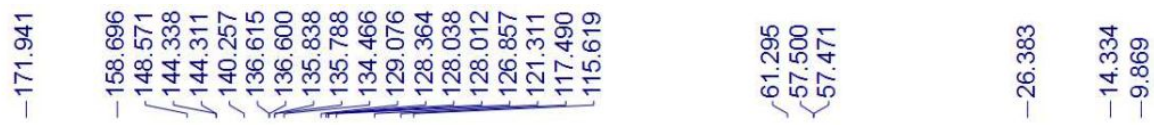

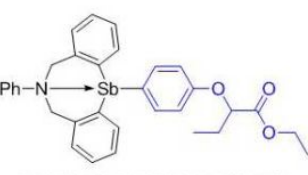

Chemical Formula: $\mathrm{C}_{32} \mathrm{H}_{32} \mathrm{NO}_{3} \mathrm{Sb}$ Exact Mass: 599.1420

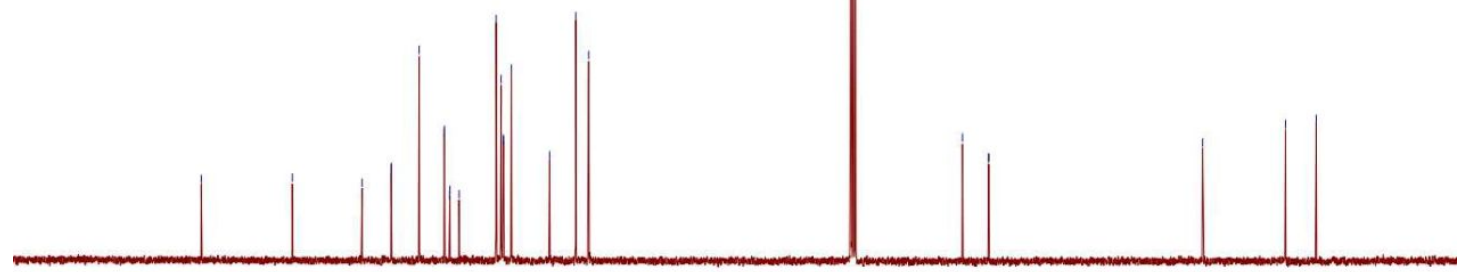

$\begin{array}{llllllllllllllllllllllllll}190 & 180 & 170 & 160 & 150 & 140 & 130 & 120 & 110 & 100 & 90 & 80 & 70 & 60 & 50 & 40 & 30 & 20 & 10 & 0 & -1\end{array}$

${ }^{1} \mathrm{H}$ NMR $\left(\mathrm{CDCl}_{3}\right)$ spectrum of compound 3bn

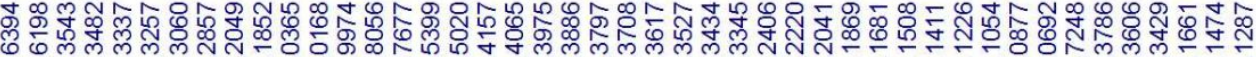

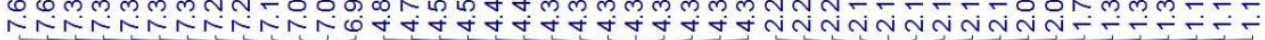

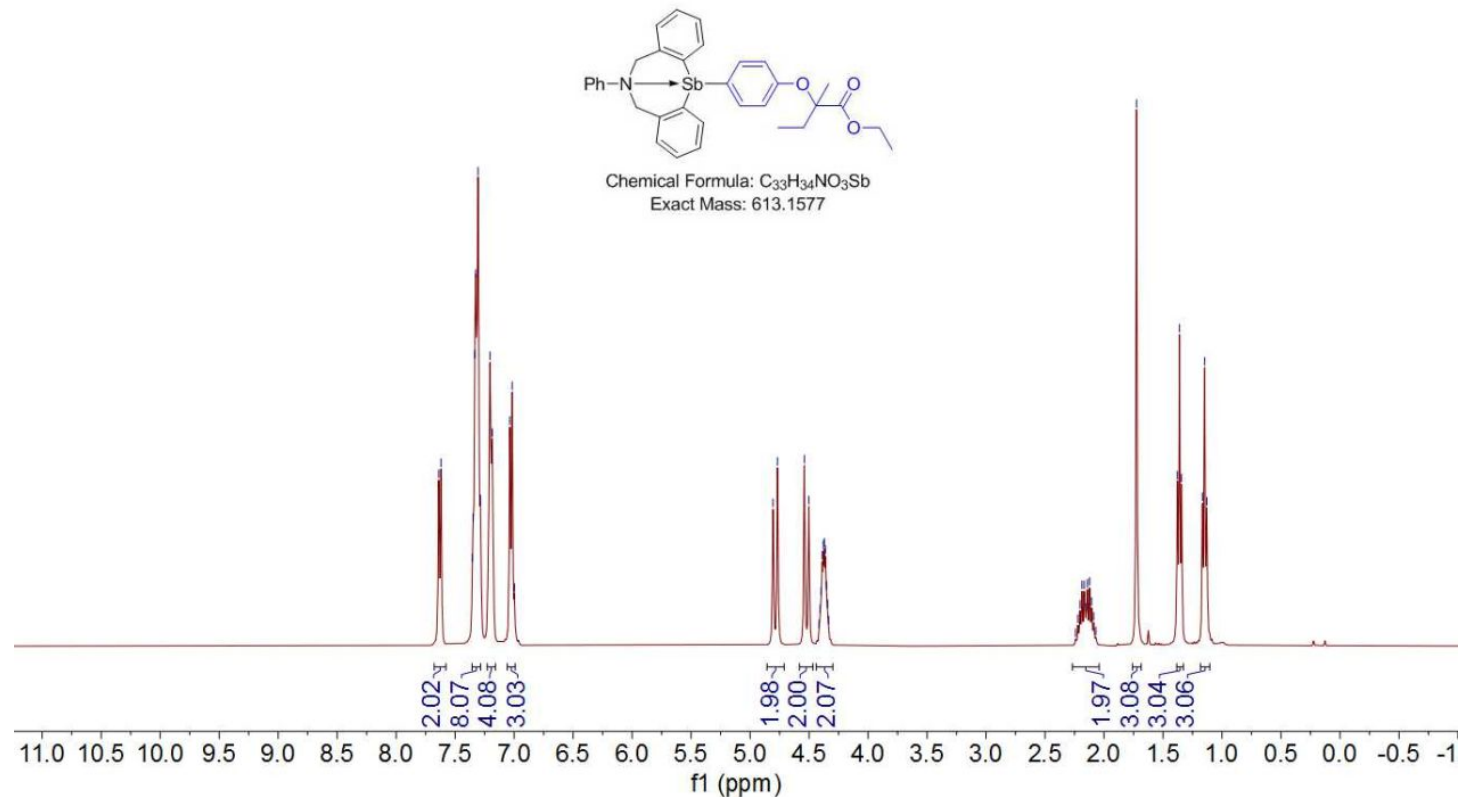


${ }^{13} \mathrm{C} \mathrm{NMR}\left(\mathrm{CDCl}_{3}\right)$ spectrum of compound $\mathbf{3 b n}$

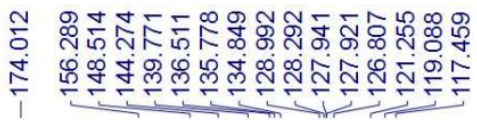

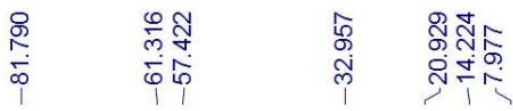

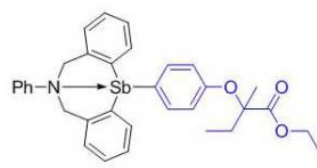

Chemical Formula: $\mathrm{C}_{33} \mathrm{H}_{34} \mathrm{NO}_{3} \mathrm{Sb}$ Exact Mass: 613.1577

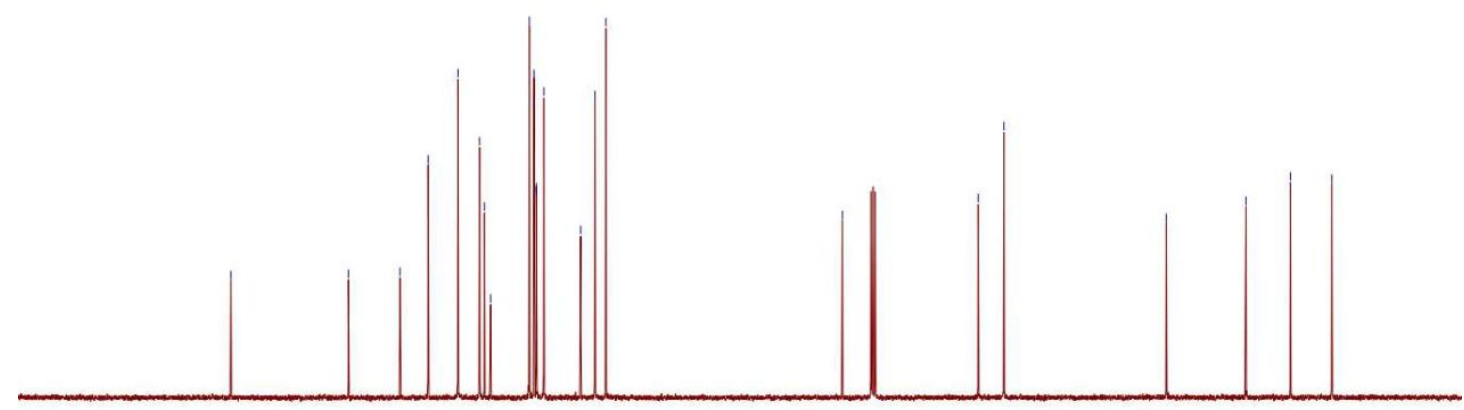

$\begin{array}{llllllllllllllllllllllllllllllll}200 & 190 & 180 & 170 & 160 & 150 & 140 & 130 & 120 & 110 & 100 & 90 & 80 & 70 & 60 & 50 & 40 & 30 & 20 & 10 & 0 & -10\end{array}$

${ }^{1} \mathrm{H}$ NMR $\left(\mathrm{CDCl}_{3}\right)$ spectrum of compound $\mathbf{3 b o}$

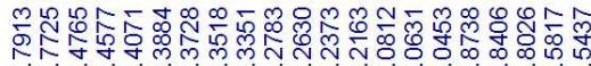

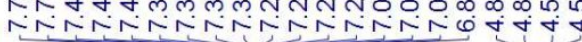

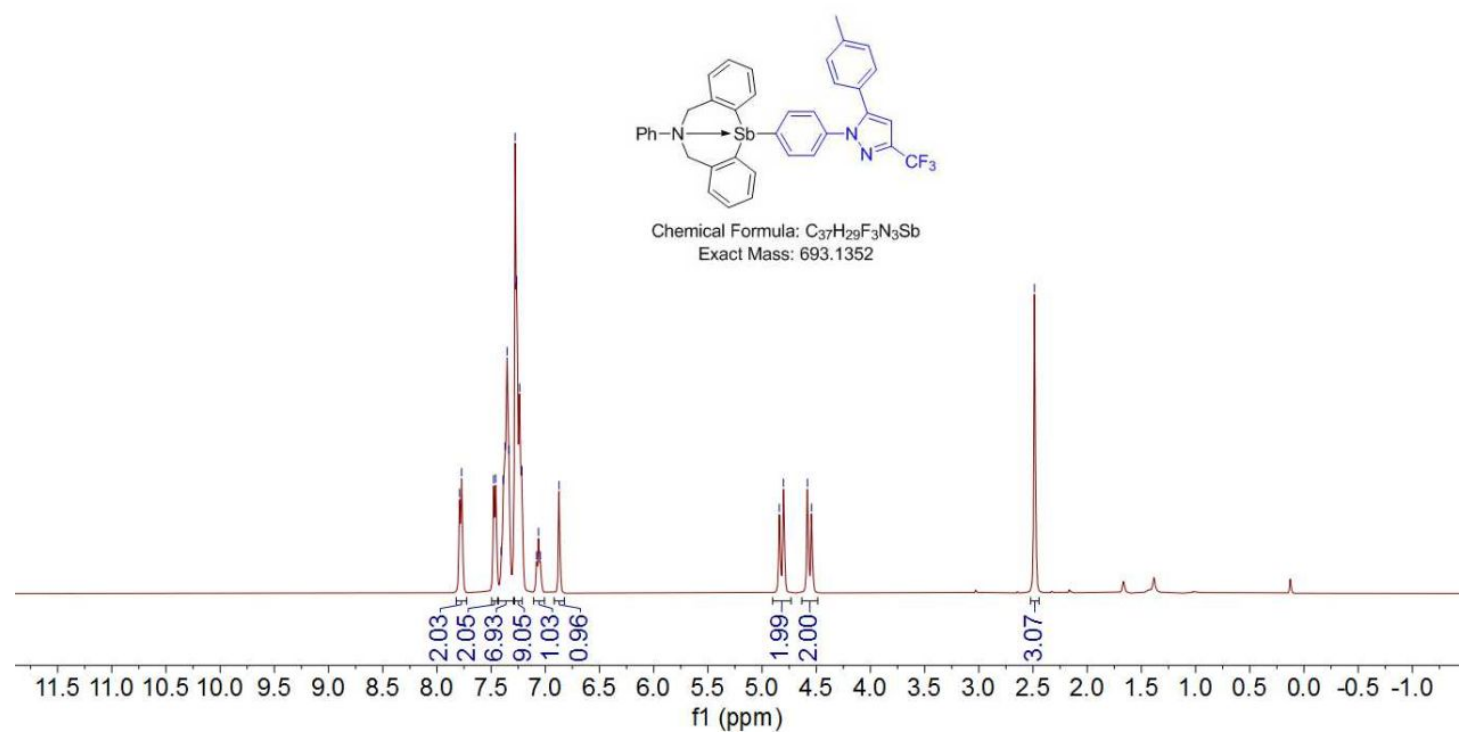


${ }^{13} \mathrm{C}$ NMR $\left(\mathrm{CDCl}_{3}\right)$ spectrum of compound 3 bo

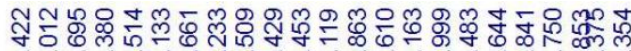

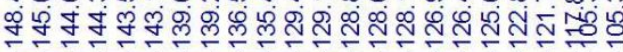

$\stackrel{8}{i}$

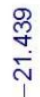

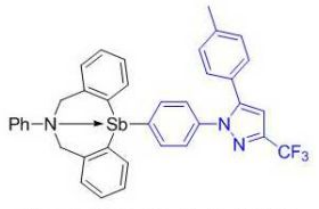

Chemical Formula: $\mathrm{C}_{37} \mathrm{H}_{29} \mathrm{~F}_{3} \mathrm{~N}_{3} \mathrm{Sb}$ Exact Mass: 693.1352

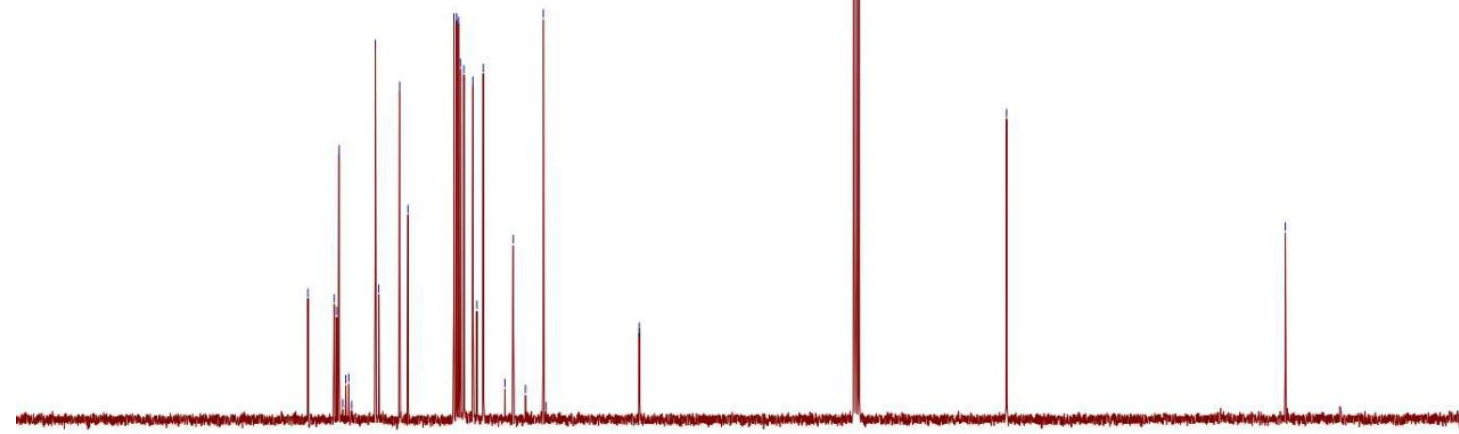

$\begin{array}{lllllllllllllllllllll}180 & 170 & 160 & 150 & 140 & 130 & 120 & 110 & 100 & 90 & 80 & 70 & 60 & 50 & 40 & 30 & 20 & 10 & 0\end{array}$

${ }^{19} \mathrm{~F}$ NMR $\left(\mathrm{CDCl}_{3}\right)$ spectrum of compound $3 \mathbf{b o}$

$\overline{8}$
ஸें

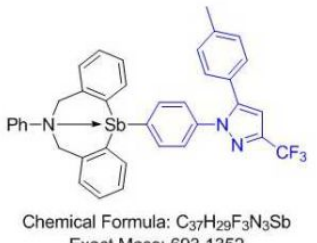

Exact Mass: 693.1352

\begin{tabular}{llllllllllllllll}
\hline 0 & -10 & -20 & -30 & -40 & -50 & -60 & -70 & -80 & -90 & -100 & -110 & -120 & -130 & -140 & -150 \\
$\mathrm{f} 1(\mathrm{ppm})$ & & & & &
\end{tabular}


${ }^{1} \mathrm{H}$ NMR $\left(\mathrm{CDCl}_{3}\right)$ spectrum of compound $\mathbf{3 b p}$
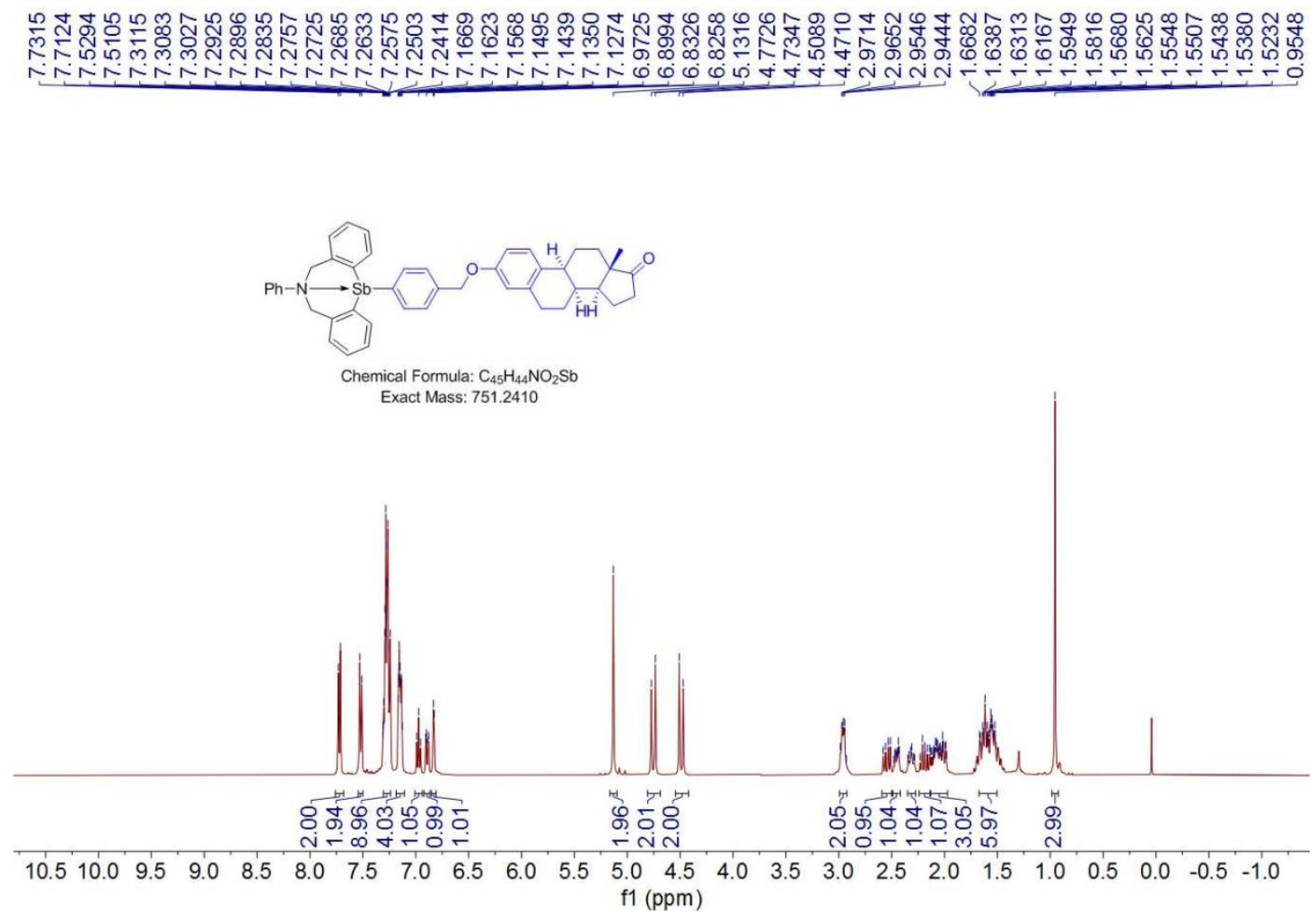

${ }^{13} \mathrm{C}$ NMR $\left(\mathrm{CDCl}_{3}\right)$ spectrum of compound $\mathbf{3 b p}$

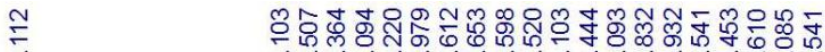

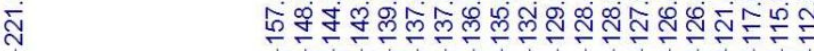

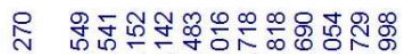

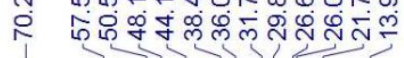
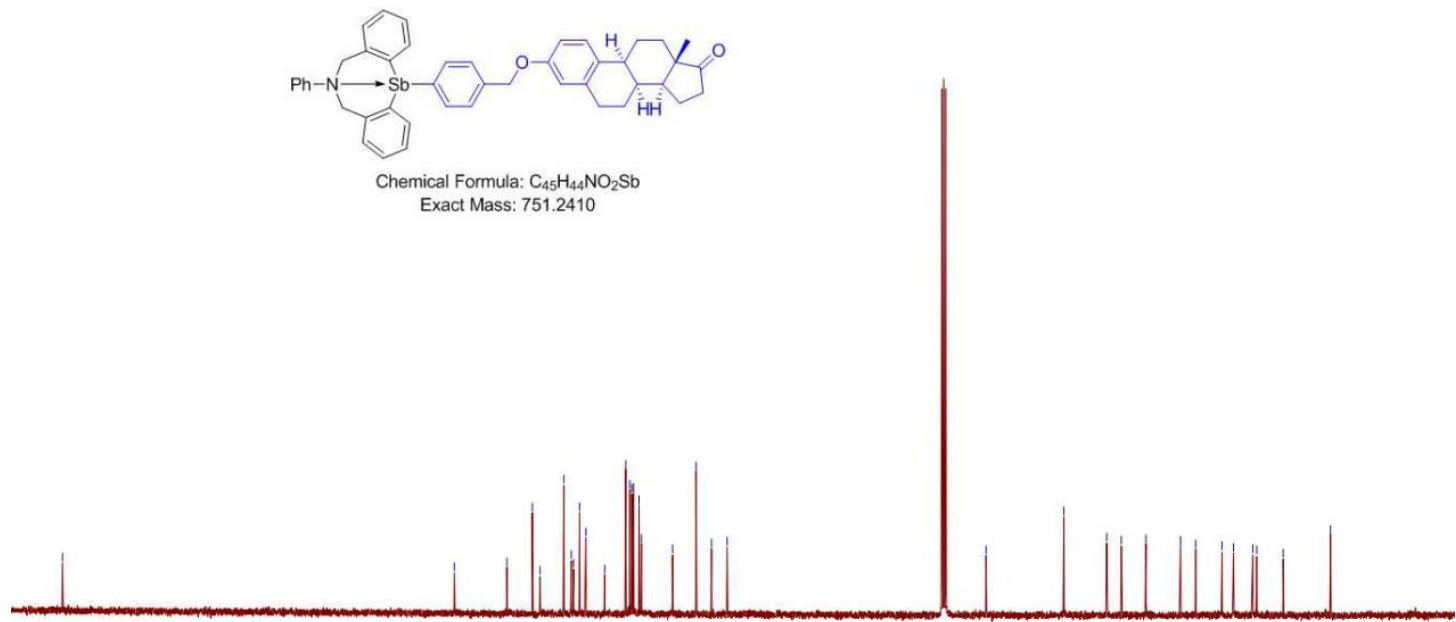

$\begin{array}{lllllllllllllllllllllllll}220 & 210 & 200 & 190 & 180 & 170 & 160 & 150 & 140 & 130 & 120 & 110 & 100 & 90 & 80 & 70 & 60 & 50 & 40 & 30 & 20 & 10 & 0\end{array}$ 
${ }^{1} \mathrm{H}$ NMR $\left(\mathrm{CDCl}_{3}\right)$ spectrum of compound $\mathbf{3 b q}$
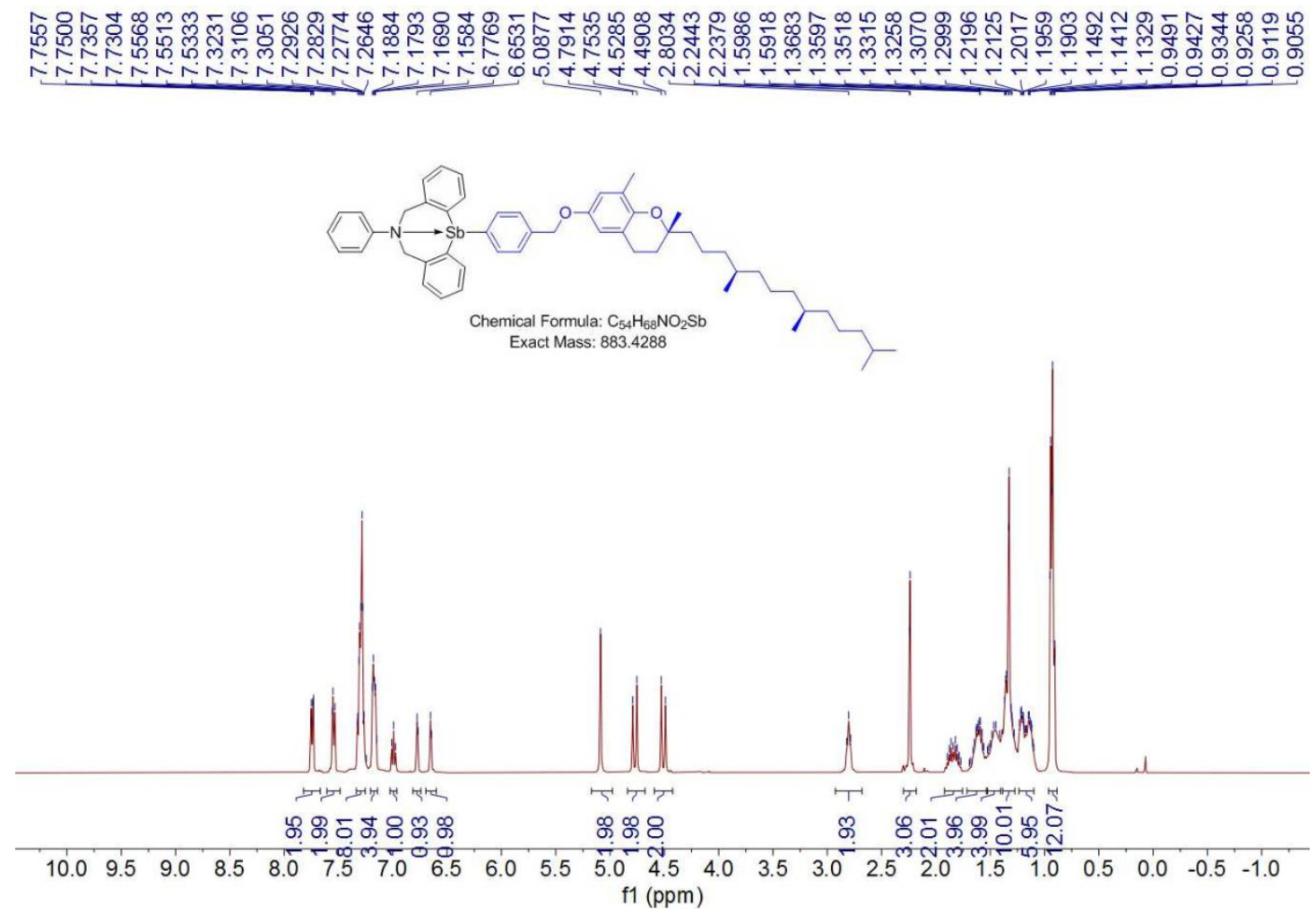

${ }^{13} \mathrm{C}$ NMR $\left(\mathrm{CDCl}_{3}\right)$ spectrum of compound $\mathbf{3 b q}$

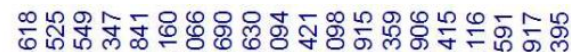

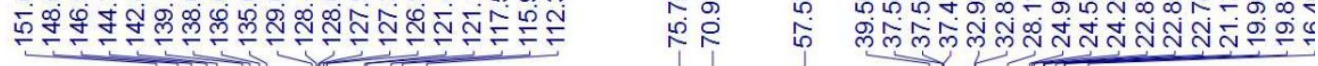

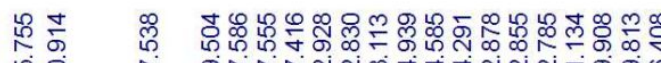

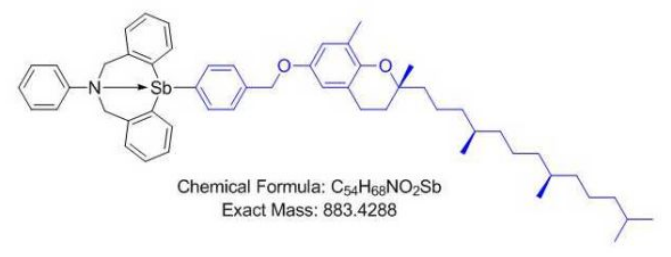

$\overline{190} 180$

$180 \quad 170 \quad 160 \quad 150$

$150 \quad 140 \quad 130 \quad 120$

$\begin{array}{lll}110 & 100\end{array}$ $\mathrm{f1}(\mathrm{ppm})$ 
${ }^{1} \mathrm{H}$ NMR $\left(\mathrm{CDCl}_{3}\right)$ spectrum of compound $\mathbf{3 b r}$
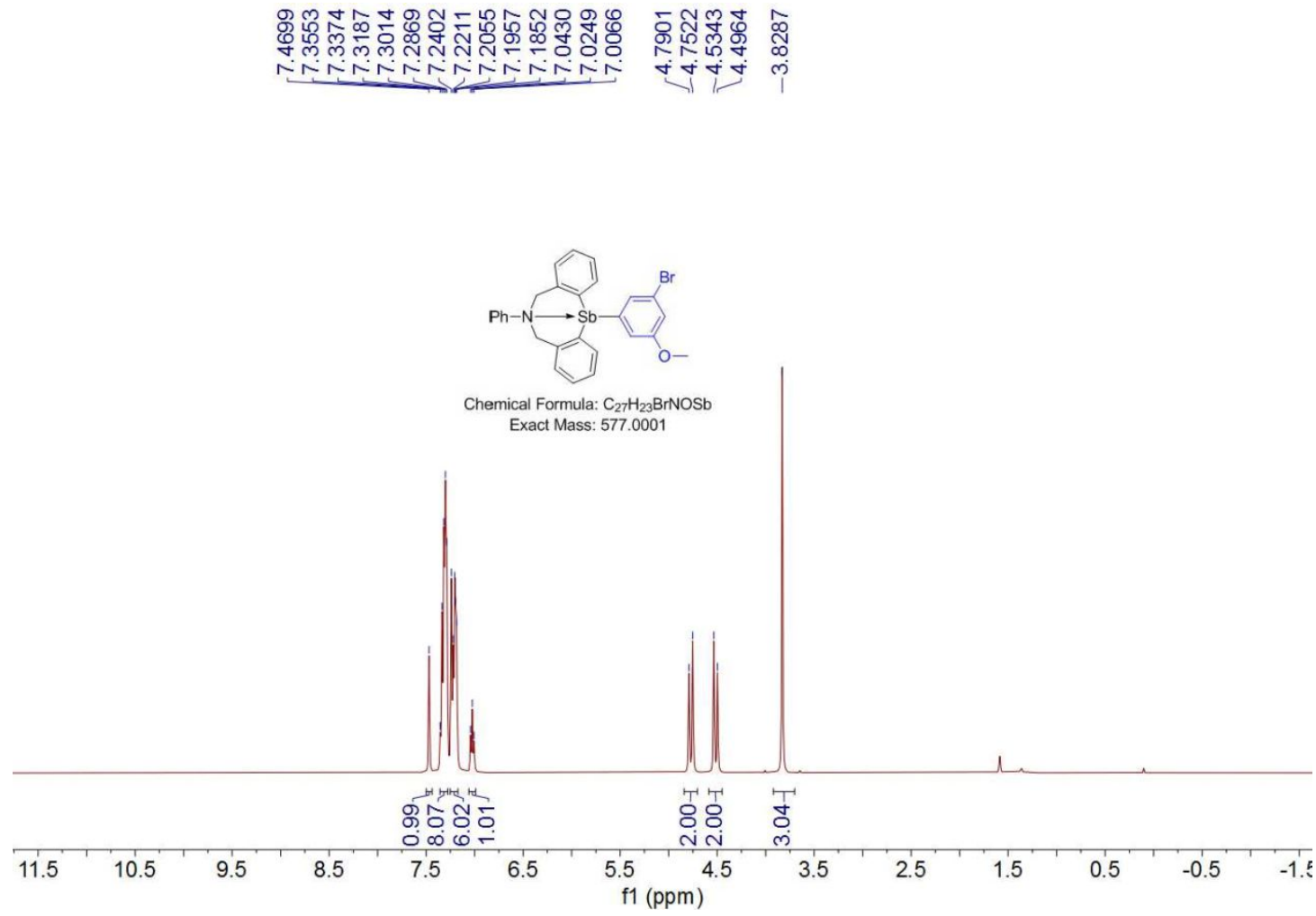

${ }^{13} \mathrm{C} \mathrm{NMR}\left(\mathrm{CDCl}_{3}\right)$ spectrum of compound $\mathbf{3 b r}$

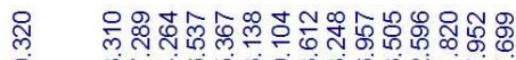

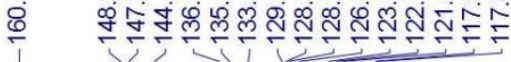

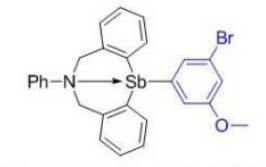

Chemical Formula: $\mathrm{C}_{27} \mathrm{H}_{23} \mathrm{BrNOSb}$ Exact Mass: 577.0001

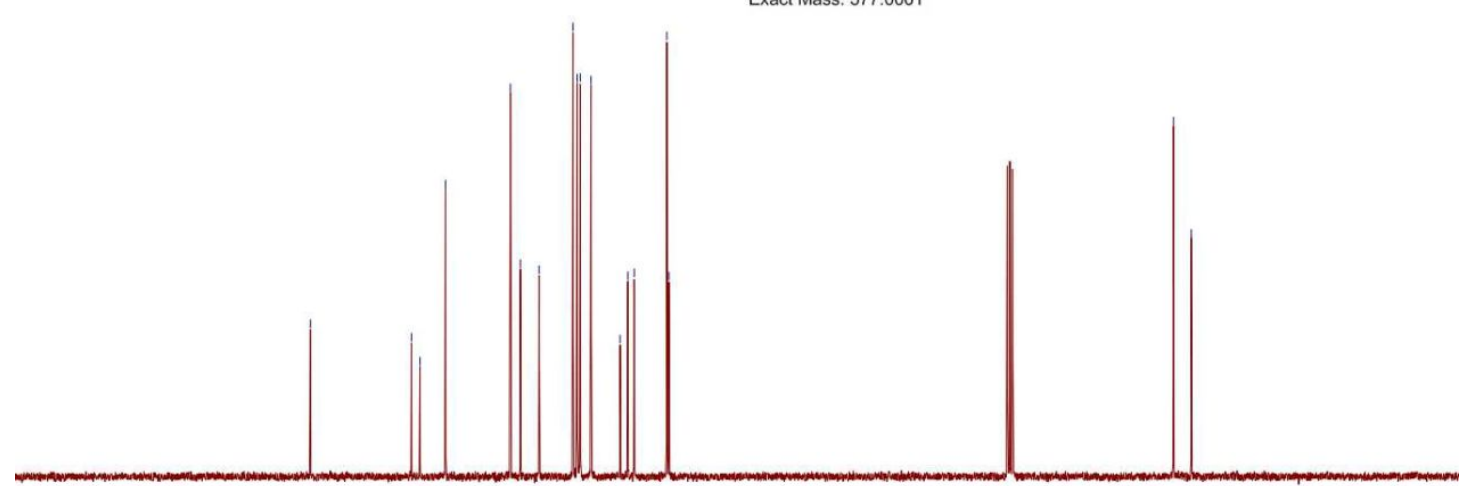

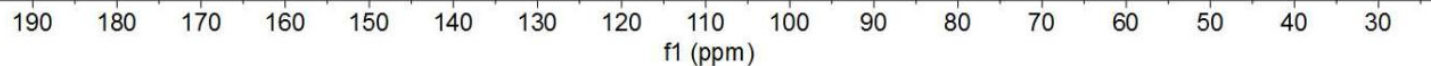


${ }^{1} \mathrm{H} \mathrm{NMR}\left(\mathrm{CDCl}_{3}\right)$ spectrum of compound $\mathbf{3 b s}$
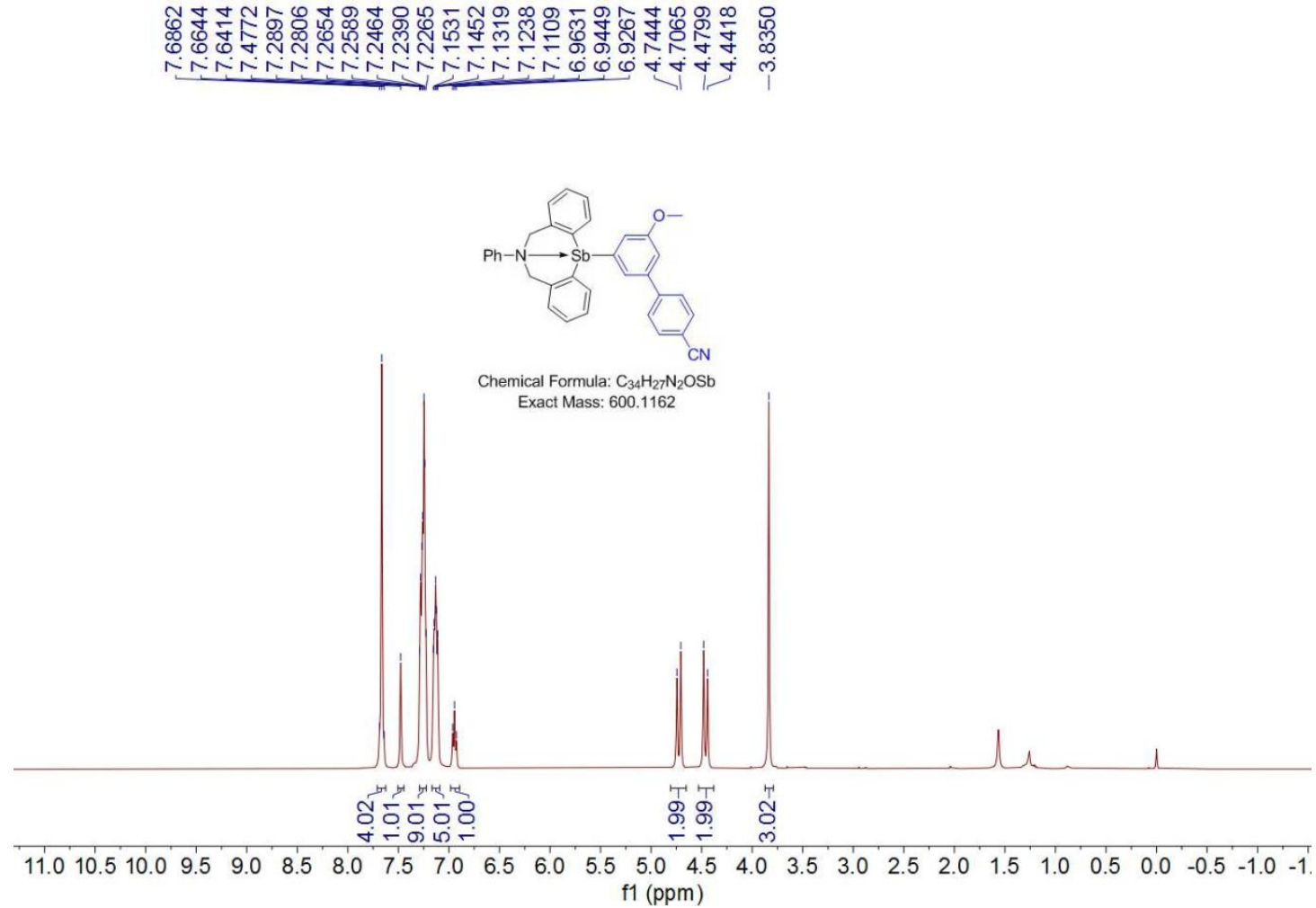

${ }^{13} \mathrm{C}$ NMR $\left(\mathrm{CDCl}_{3}\right)$ spectrum of compound $\mathbf{3 b s}$

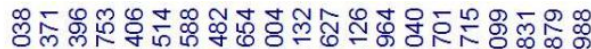

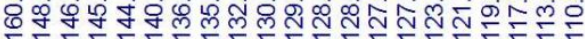

뚀용

นิ

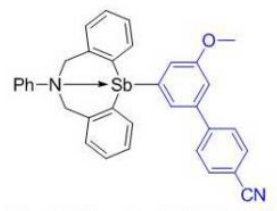

Chemical Formula: $\mathrm{C}_{34} \mathrm{H}_{27} \mathrm{~N}_{2} \mathrm{OSb}$ Exact Mass: 600.1162

\begin{tabular}{llllllllllllllllll}
\hline 90 & 180 & 170 & 160 & 150 & 140 & 130 & 120 & $\begin{array}{c}110 \\
\mathrm{f} 1(\mathrm{ppm})\end{array}$ & 90 & 80 & 70 & 60 & 50 & 40 & 30 & 2(
\end{tabular} 
${ }^{1} \mathrm{H}$ NMR $\left(\mathrm{CDCl}_{3}\right)$ spectrum of compound $\mathbf{3 b t}$
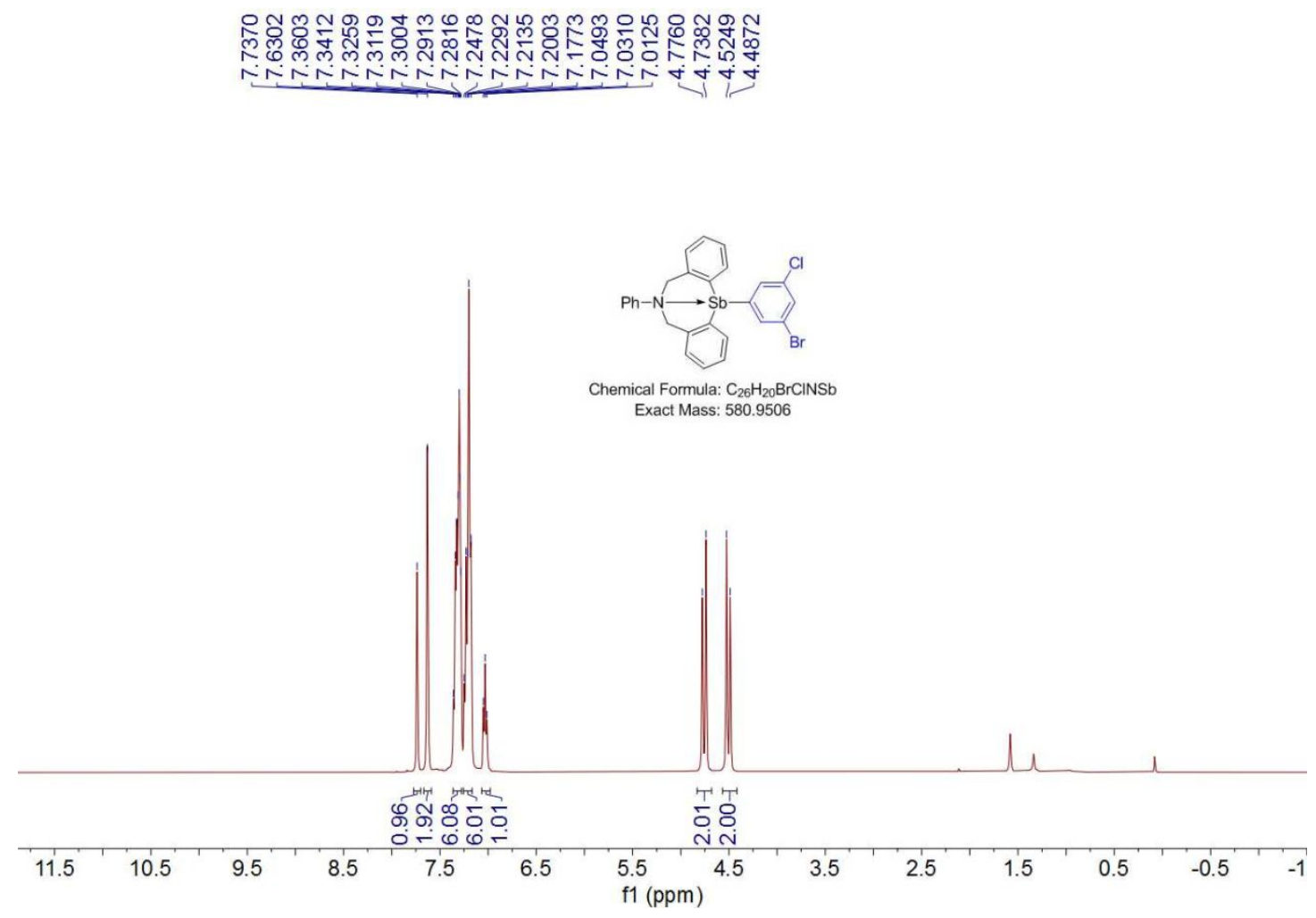

${ }^{13} \mathrm{C} \mathrm{NMR}\left(\mathrm{CDCl}_{3}\right)$ spectrum of compound $\mathbf{3 b t}$

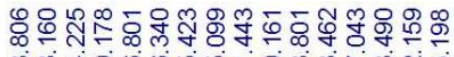

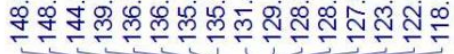

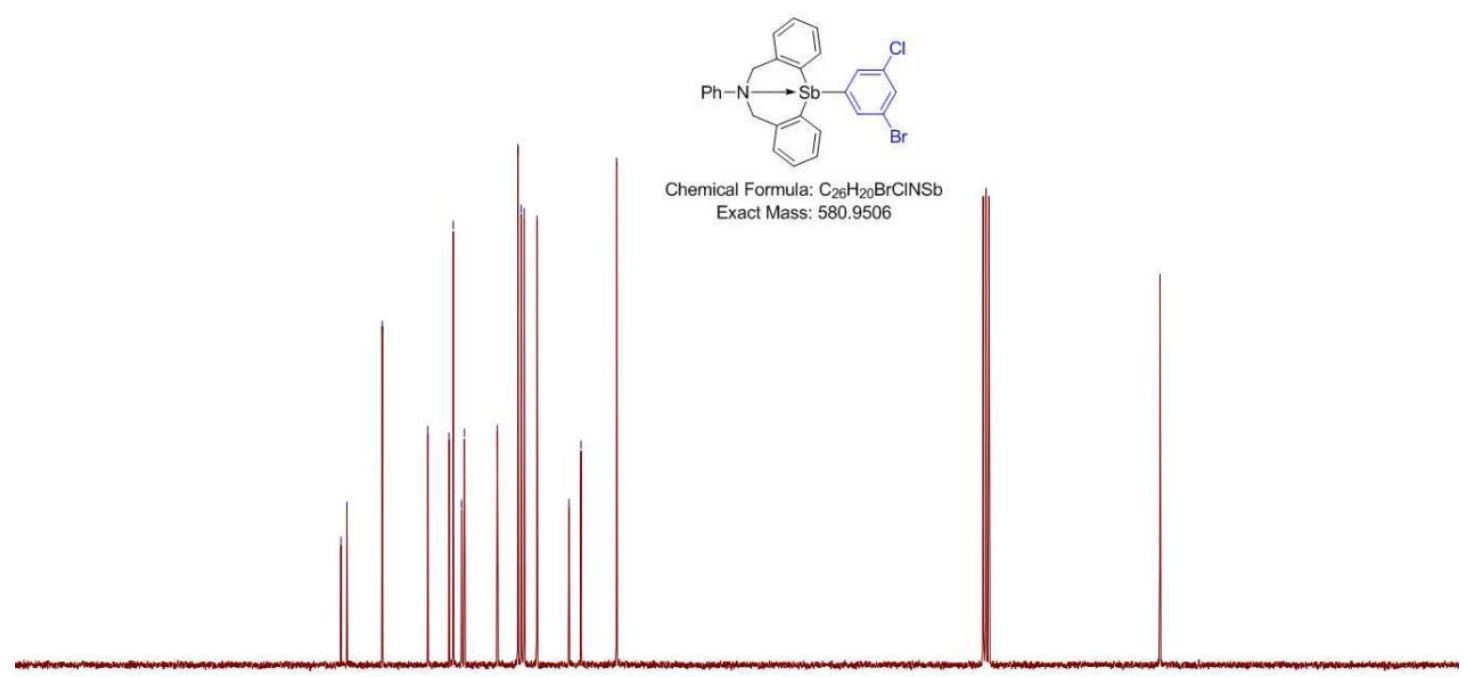

180

$\begin{array}{llllllll}170 & 160 & 150 & 140 & 130 & 120 & 110 & 100\end{array}$

f1 (ppm) 
${ }^{1} \mathrm{H}$ NMR $\left(\mathrm{CDCl}_{3}\right)$ spectrum of compound $\mathbf{3 b u}$

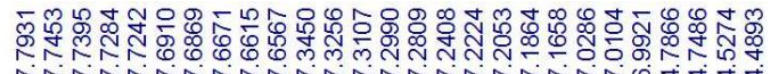

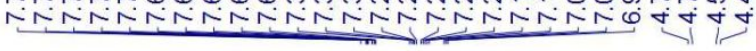

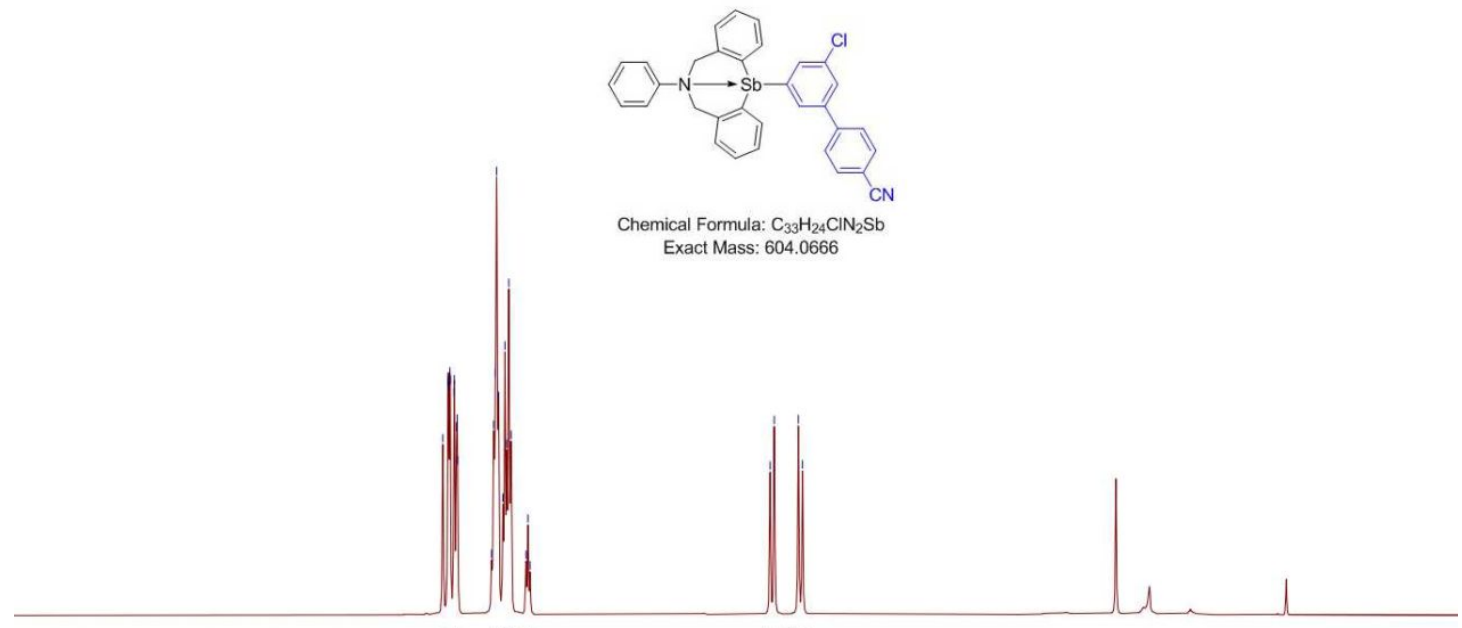

tTing

8

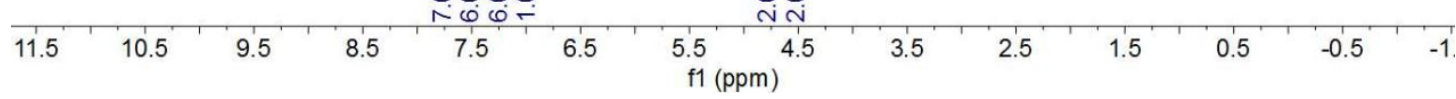

${ }^{13} \mathrm{C} \mathrm{NMR}\left(\mathrm{CDCl}_{3}\right)$ spectrum of compound $\mathbf{3 b u}$

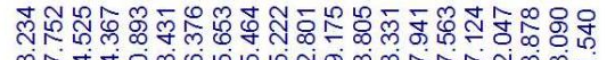

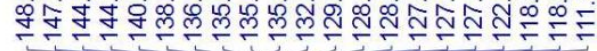

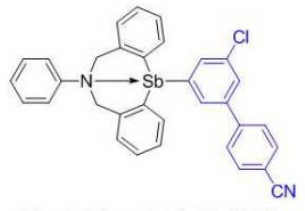

Chemical Formula: $\mathrm{C}_{33} \mathrm{H}_{24} \mathrm{CIN}_{2} \mathrm{Sb}$ Exact Mass: 604.0666

$180 \quad 17$

$140 \quad 130 \quad 120$

$110 \quad 100$ $\mathrm{f1}(\mathrm{ppm})$ 
${ }^{1} \mathrm{H}$ NMR $\left(\mathrm{CDCl}_{3}\right)$ spectrum of compound $\mathbf{3 b v}$

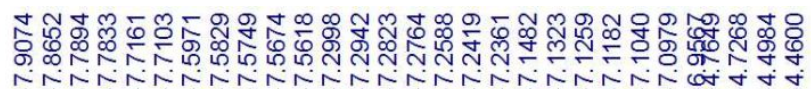

o

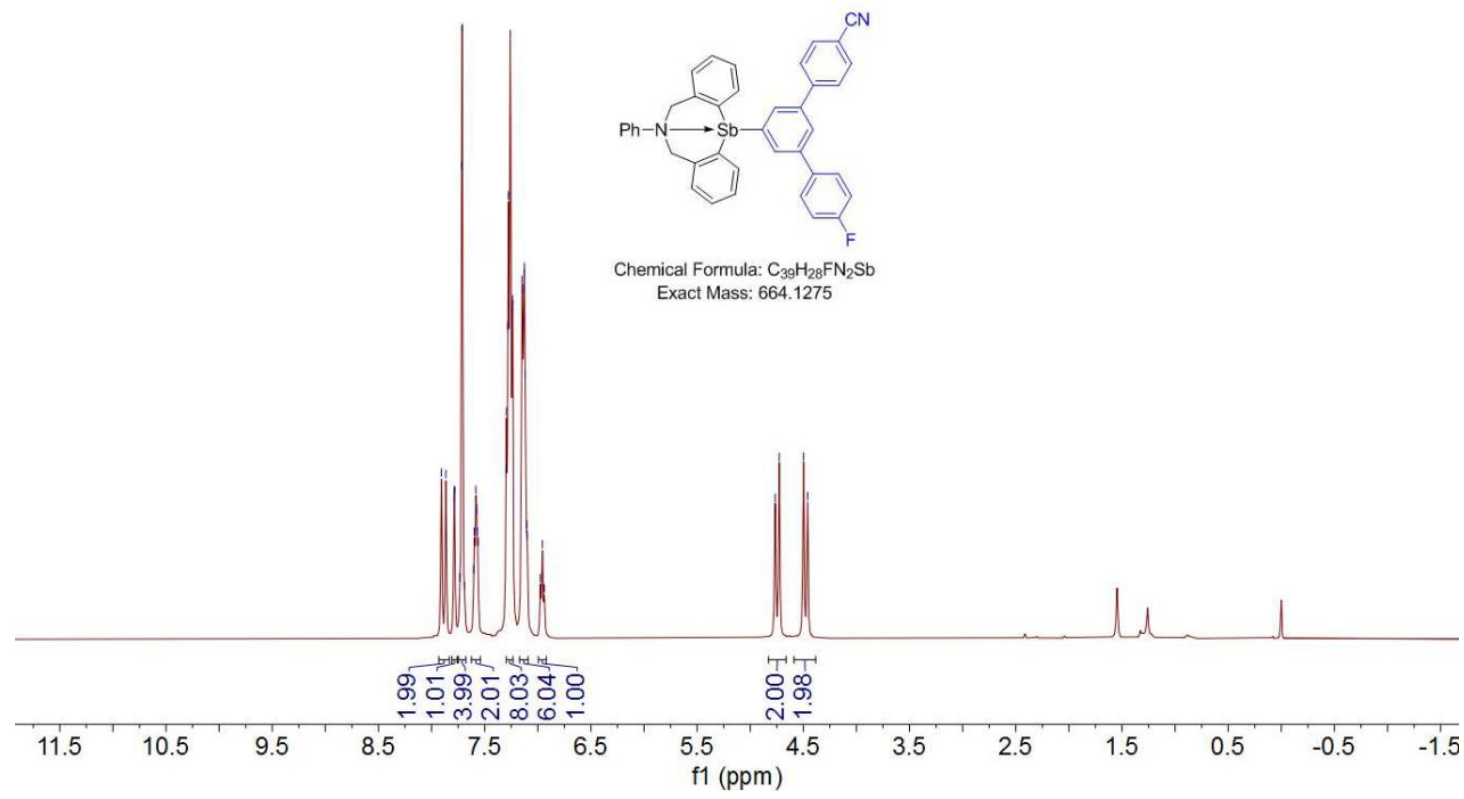

${ }^{13} \mathrm{C}$ NMR $\left(\mathrm{CDCl}_{3}\right)$ spectrum of compound $\mathbf{3 b v}$

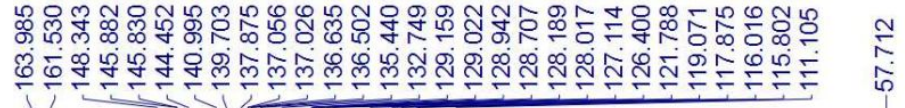

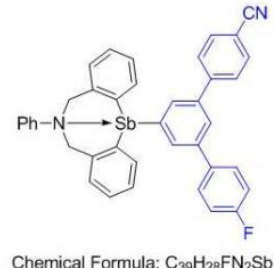

Exact Mass: 664.1275

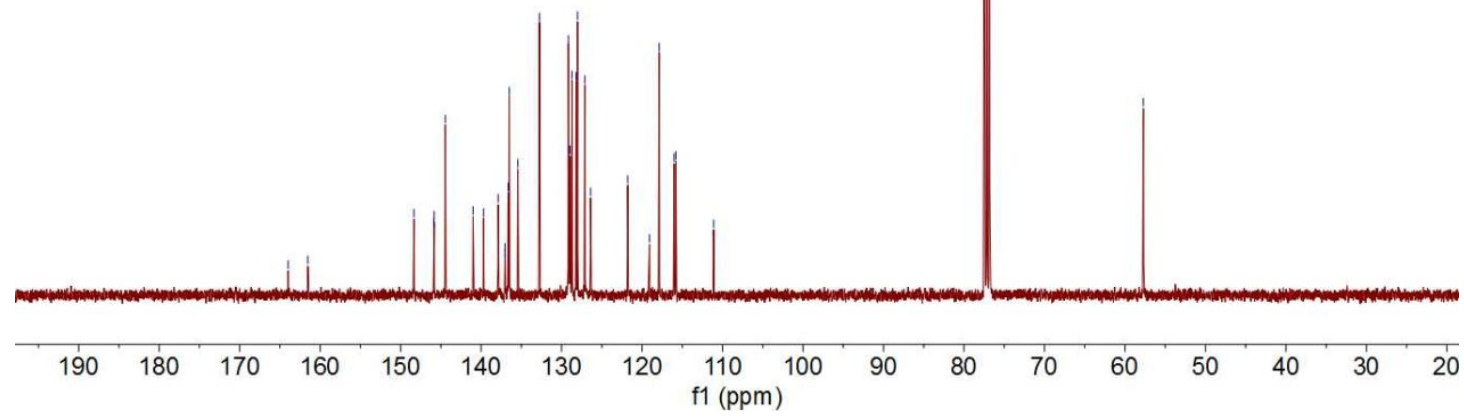


${ }^{19} \mathrm{~F}$ NMR $\left(\mathrm{CDCl}_{3}\right)$ spectrum of compound $\mathbf{3 b v}$
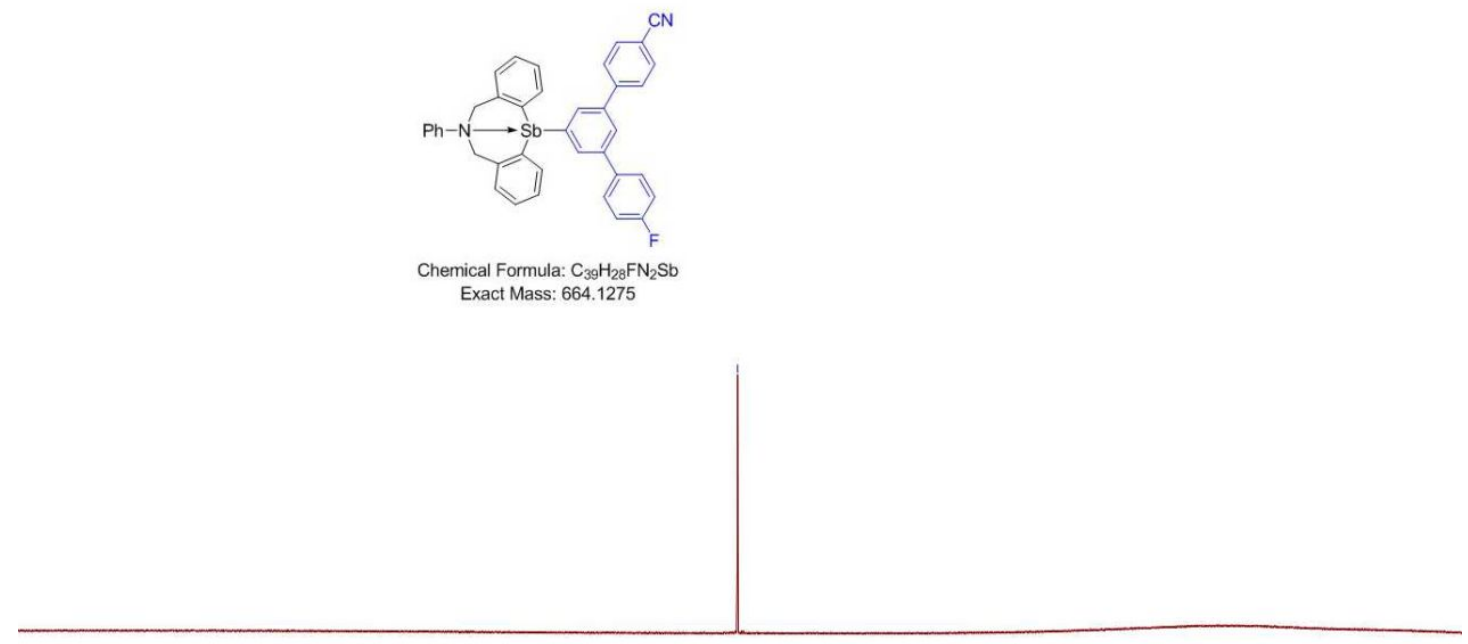

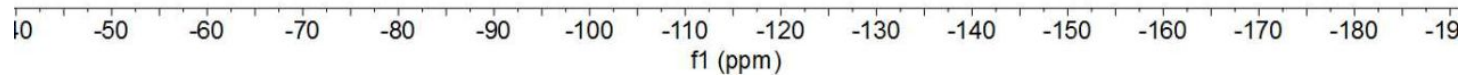

${ }^{1} \mathrm{H}$ NMR $\left(\mathrm{CDCl}_{3}\right)$ spectrum of compound $\mathbf{3 b w}$

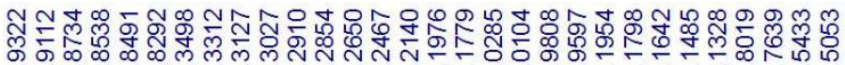

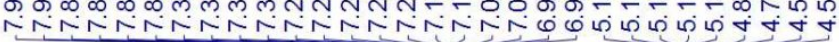

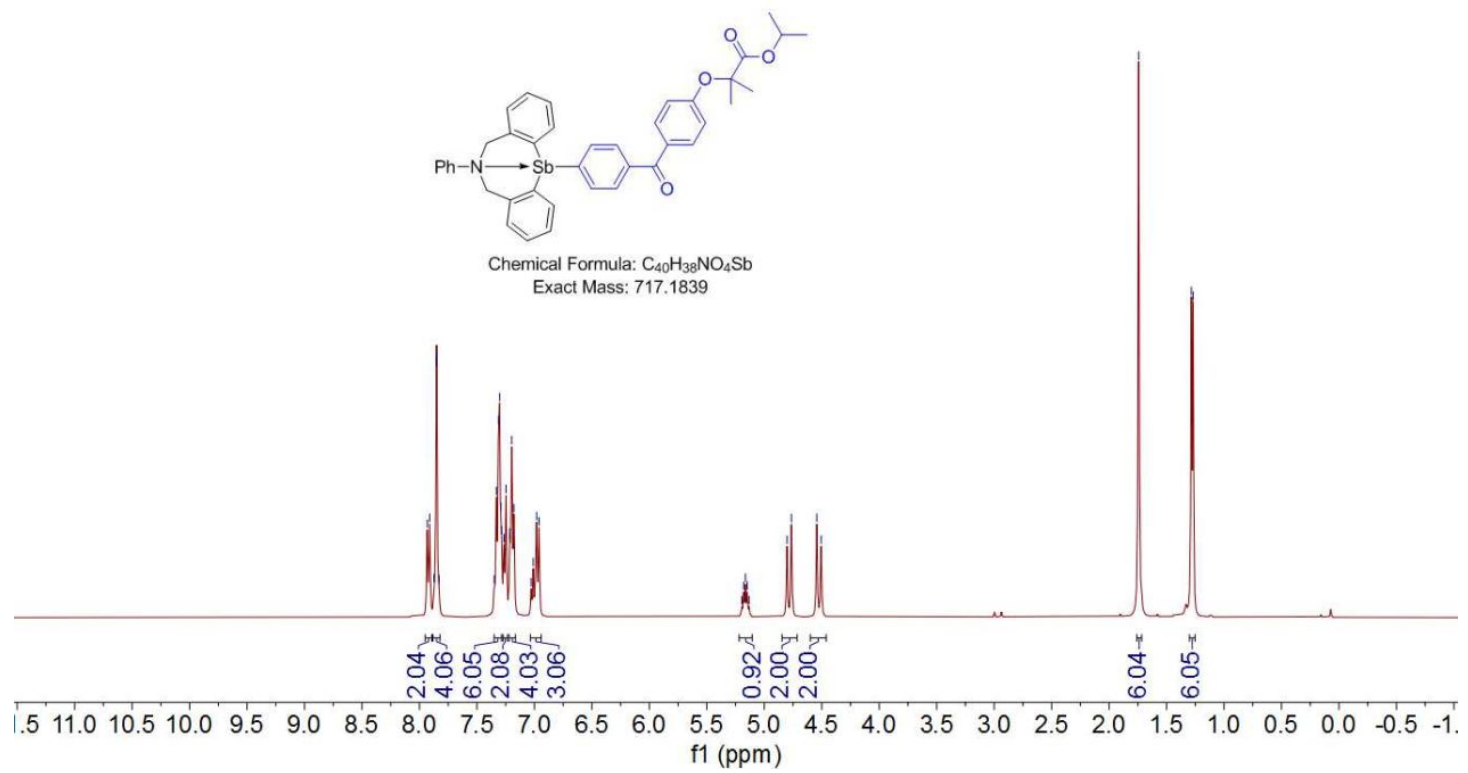


${ }^{13} \mathrm{C} \mathrm{NMR}\left(\mathrm{CDCl}_{3}\right)$ spectrum of compound $\mathbf{3 b w}$

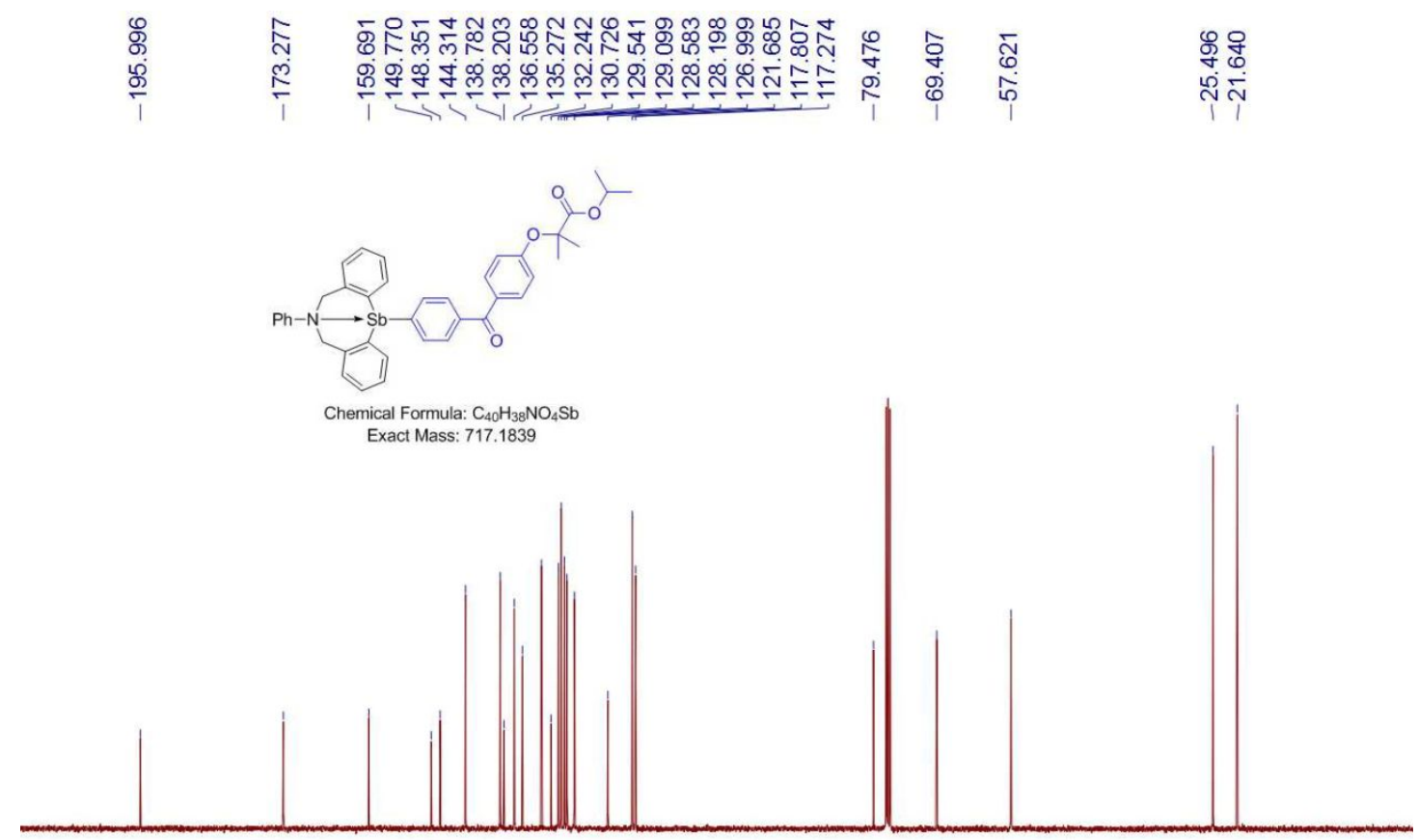

\begin{tabular}{llllllllllllllllllllllll}
\hline 210 & 200 & 190 & 180 & 170 & 160 & 150 & 140 & 130 & 120 & $\begin{array}{r}110 \\
\mathrm{f} 1(\mathrm{ppm})\end{array}$ & 90 & 80 & 70 & 60 & 50 & 40 & 30 & 20 & 10 & 0
\end{tabular}

${ }^{1} \mathrm{H}$ NMR $\left(\mathrm{CDCl}_{3}\right)$ spectrum of compound 5a

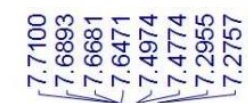

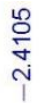

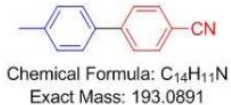

Exact Mass: 193.0891

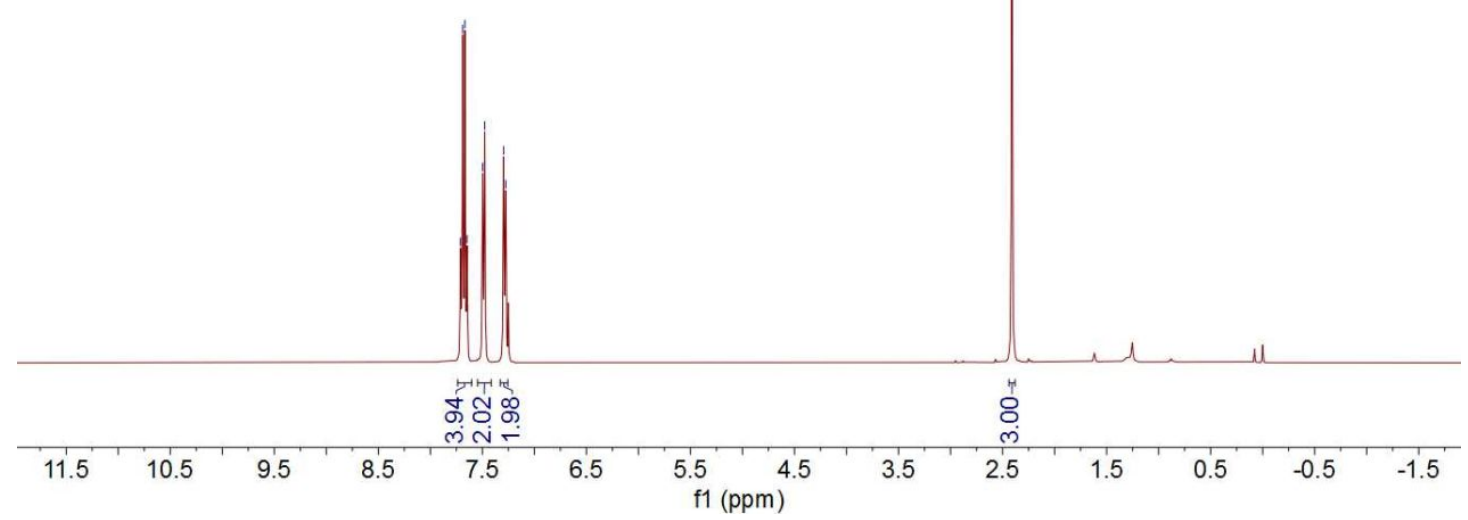


${ }^{13} \mathrm{C} \mathrm{NMR}\left(\mathrm{CDCl}_{3}\right)$ spectrum of compound $\mathbf{5 a}$

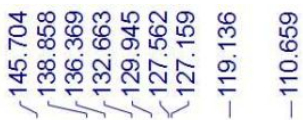

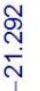

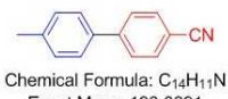

Exact Mass: 193.0891

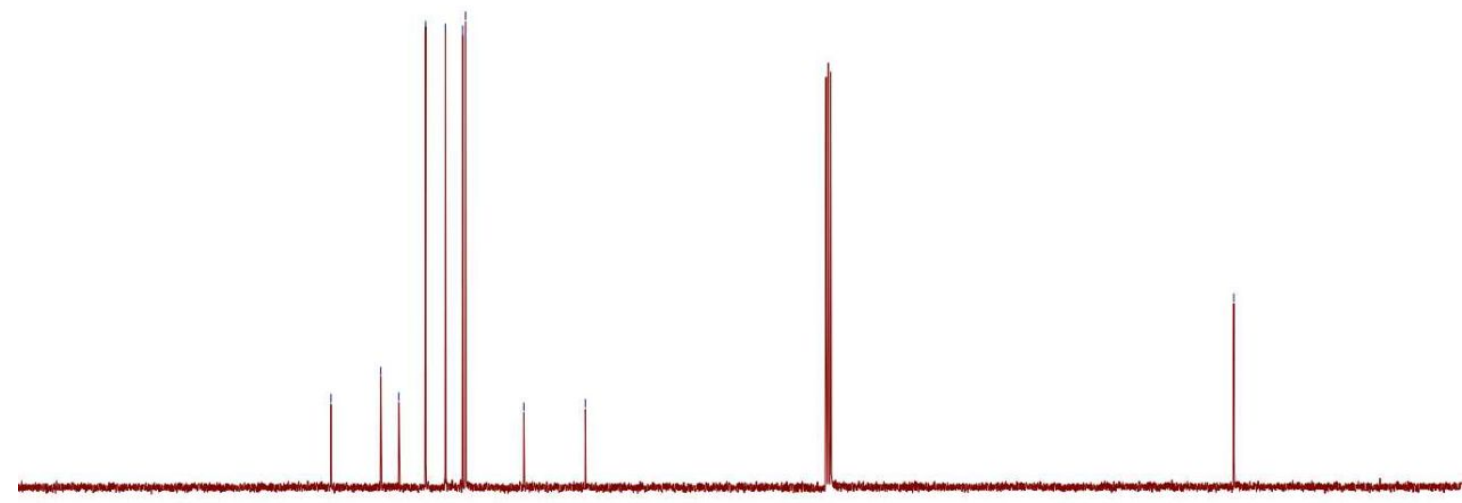

$\begin{array}{llllllllllllllllllll}180 & 170 & 160 & 150 & 140 & 130 & 120 & 110 & 100 & 90 & 80 & 70 & 60 & 50 & 40 & 30 & 20 & 10 & 0 & -1\end{array}$

${ }^{1} \mathrm{H}$ NMR $\left(\mathrm{CDCl}_{3}\right)$ spectrum of compound $\mathbf{5 b}$

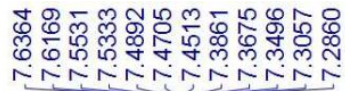

$\underset{\substack{\text { \& } \\ \text { N }}}{1}$

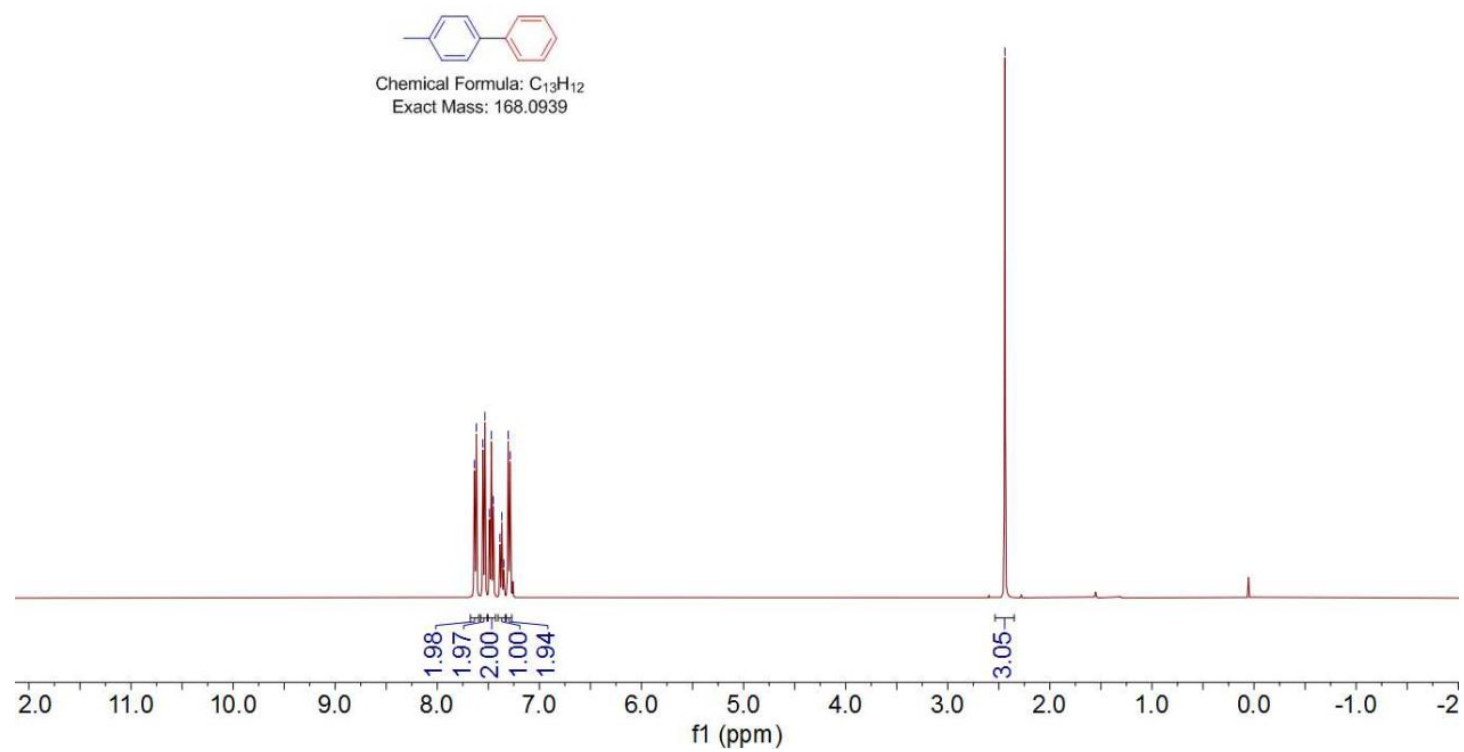

Exact Mass: $168 . \mathrm{C}_{13} \mathrm{H}$ 
${ }^{13} \mathrm{C} \mathrm{NMR}\left(\mathrm{CDCl}_{3}\right)$ spectrum of compound $\mathbf{5 b}$

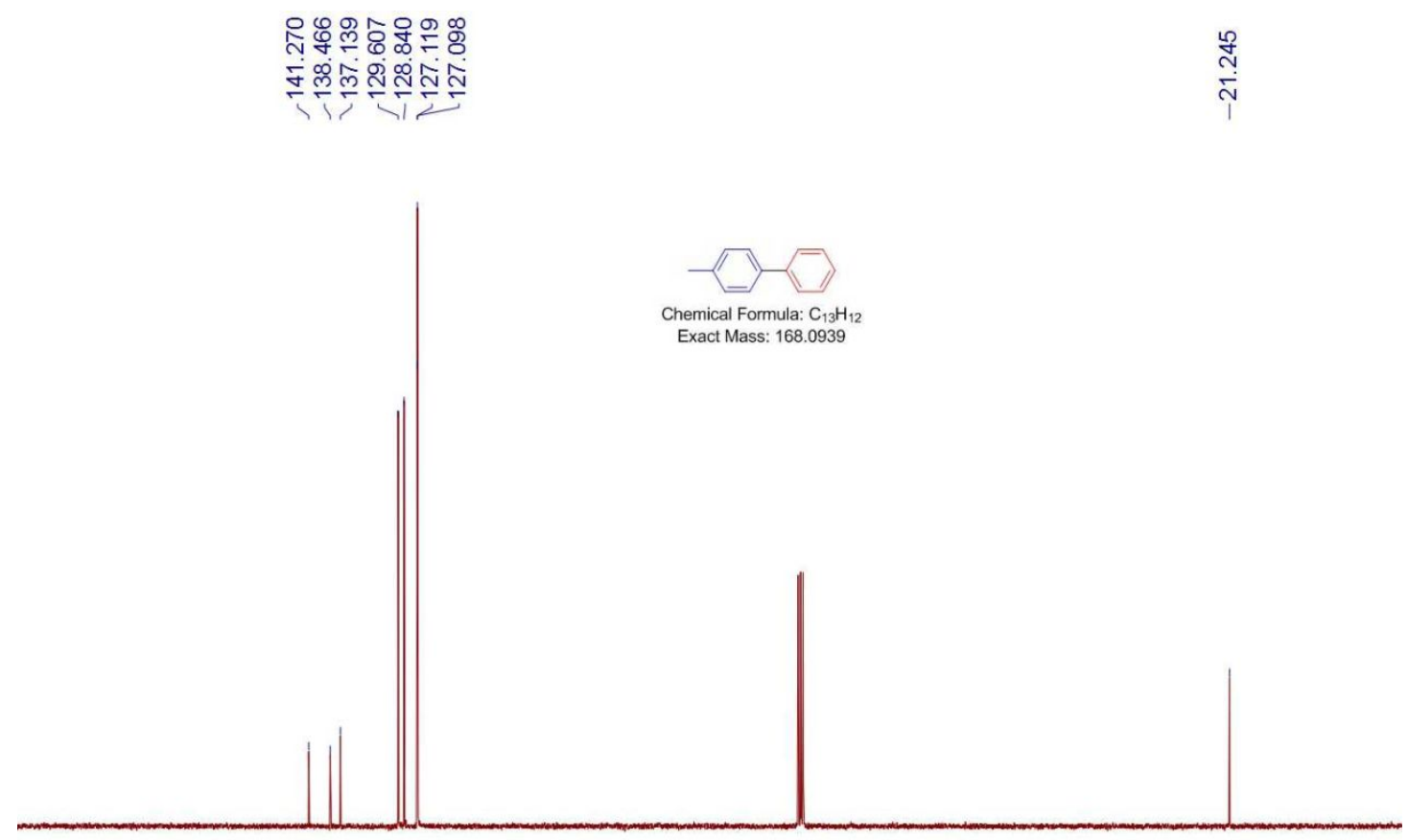

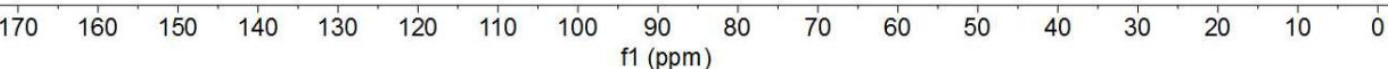

${ }^{1} \mathrm{H}$ NMR $\left(\mathrm{CDCl}_{3}\right)$ spectrum of compound $\mathbf{5 e}$
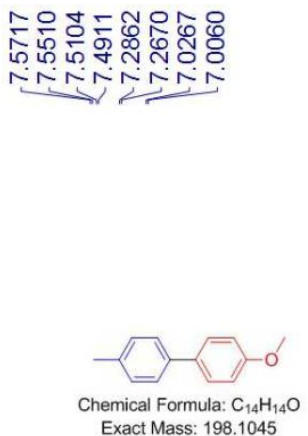

Exact Mass: 198.1045

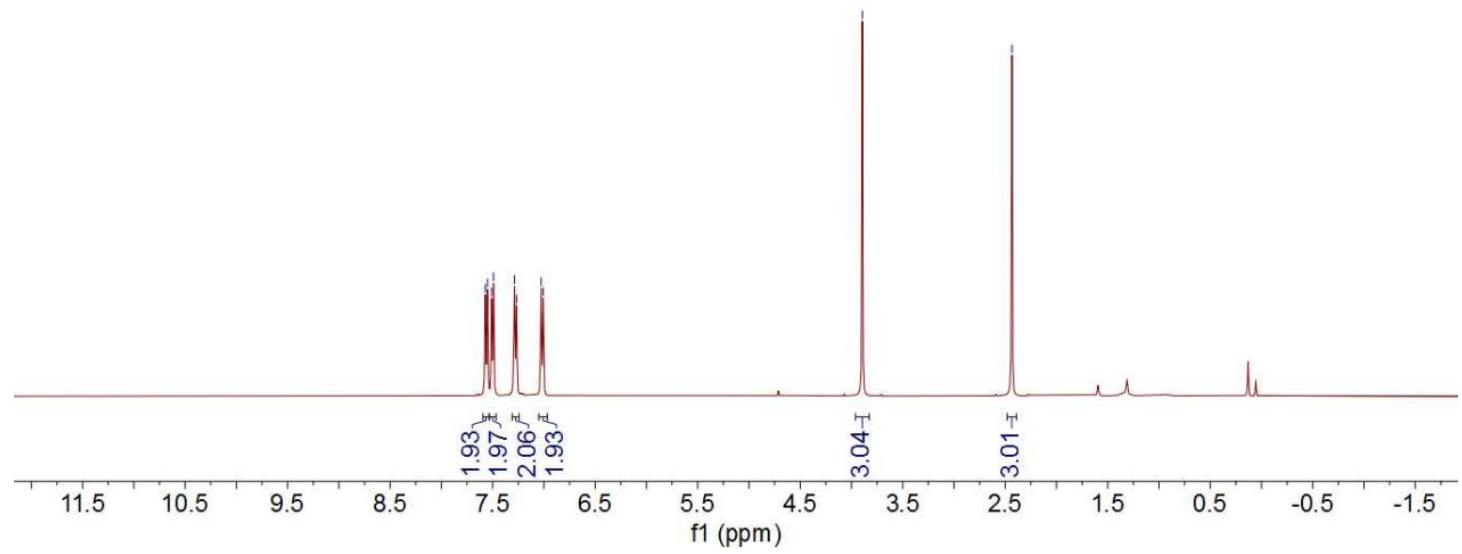


${ }^{13} \mathrm{C}$ NMR $\left(\mathrm{CDCl}_{3}\right)$ spectrum of compound $\mathbf{5 d}$

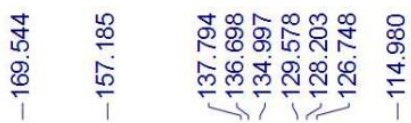

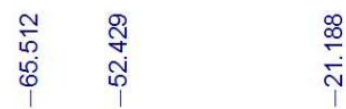

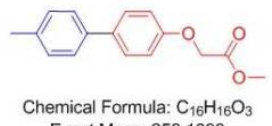

Exact Mass: 256.1099

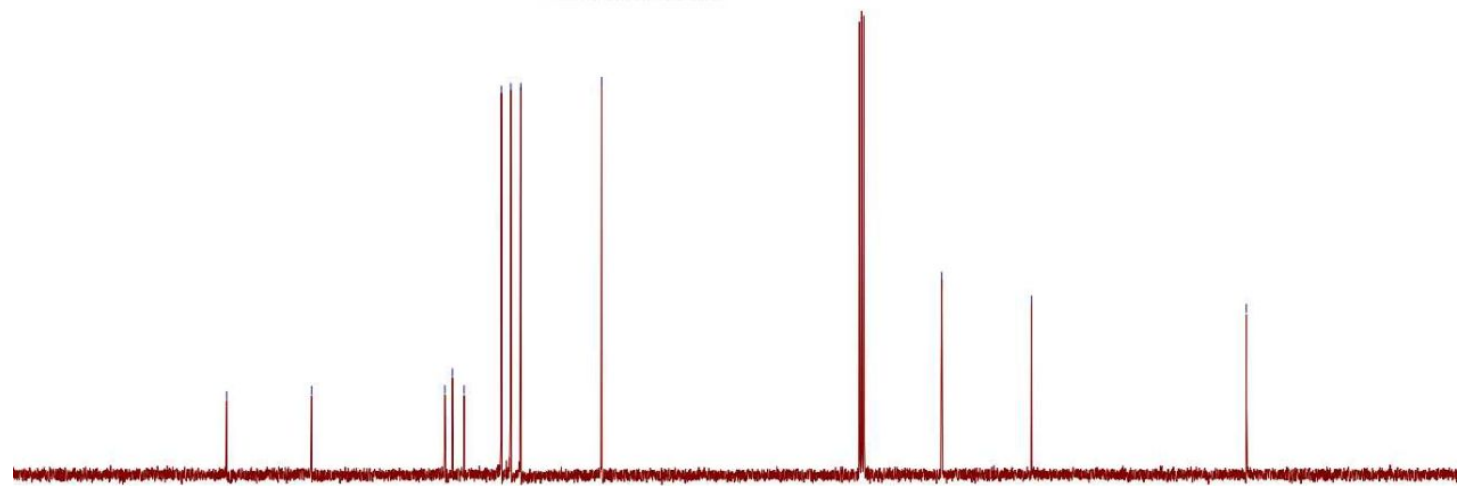

\begin{tabular}{lllllllllllllllllllll}
\hline 0 & 190 & 180 & 170 & 160 & 150 & 140 & 130 & 120 & 110 & $\begin{array}{l}100 \\
\mathrm{f} 1(\mathrm{ppm})\end{array}$ & 90 & 80 & 60 & 50 & 40 & 30 & 20 & 10 & 0 &
\end{tabular}

${ }^{1} \mathrm{H}$ NMR $\left(\mathrm{CDCl}_{3}\right)$ spectrum of compound $\mathbf{5 e}$

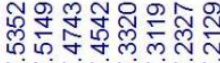

Nininging

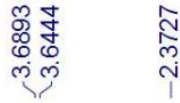

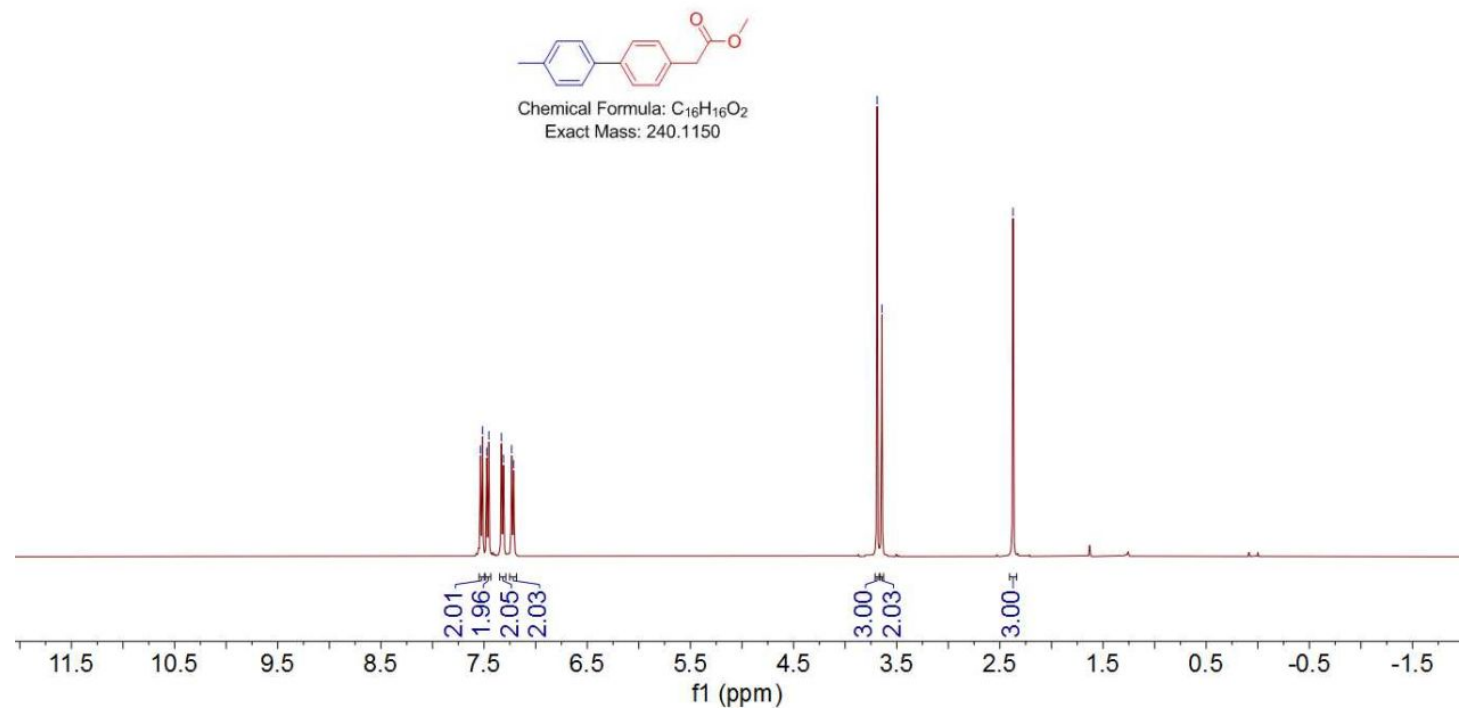


${ }^{13} \mathrm{C}$ NMR $\left(\mathrm{CDCl}_{3}\right)$ spectrum of compound $\mathbf{5 e}$

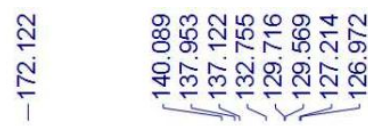

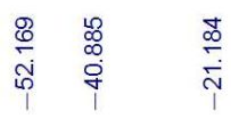
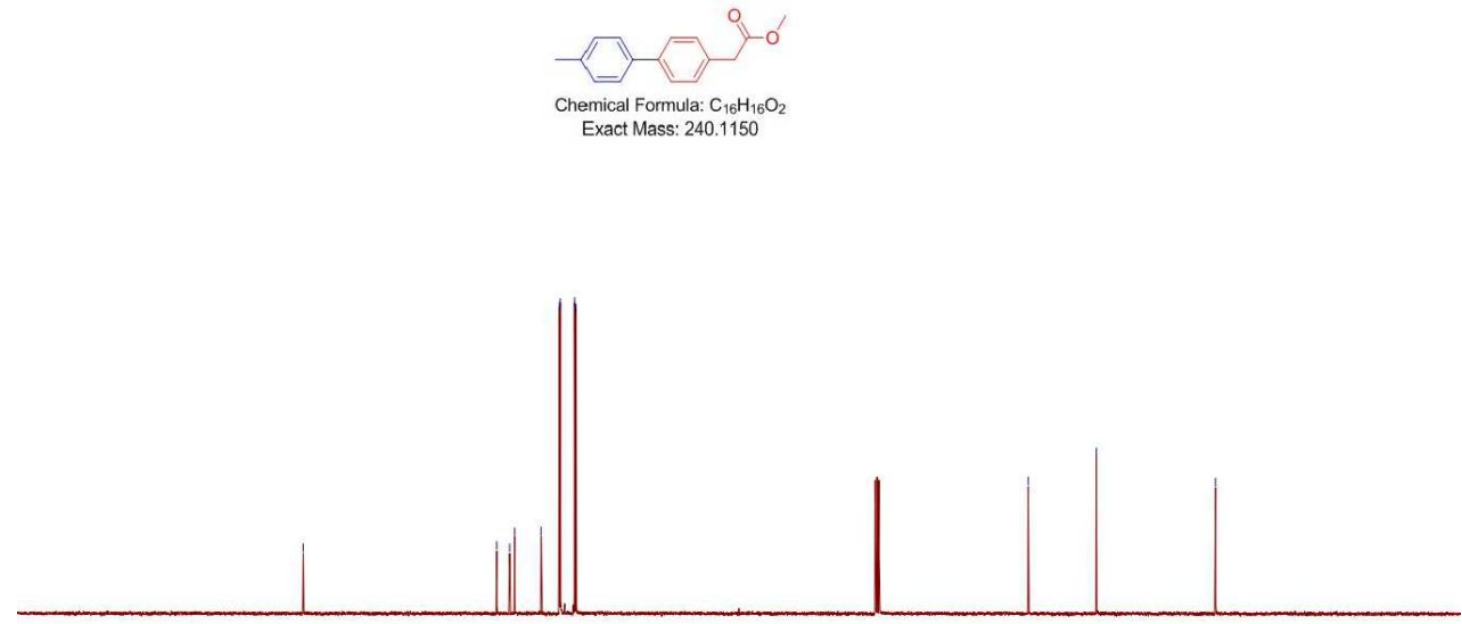

$\begin{array}{lllllllllllllllllllllllllll}210 & 200 & 190 & 180 & 170 & 160 & 150 & 140 & 130 & 120 & 110 & 100 & 90 & 80 & 70 & 60 & 50 & 40 & 30 & 20 & 10 & 0 & -10\end{array}$ f1 (ppm)

${ }^{1} \mathrm{H} \mathrm{NMR}\left(\mathrm{CDCl}_{3}\right)$ spectrum of compound $\mathbf{5 f}$
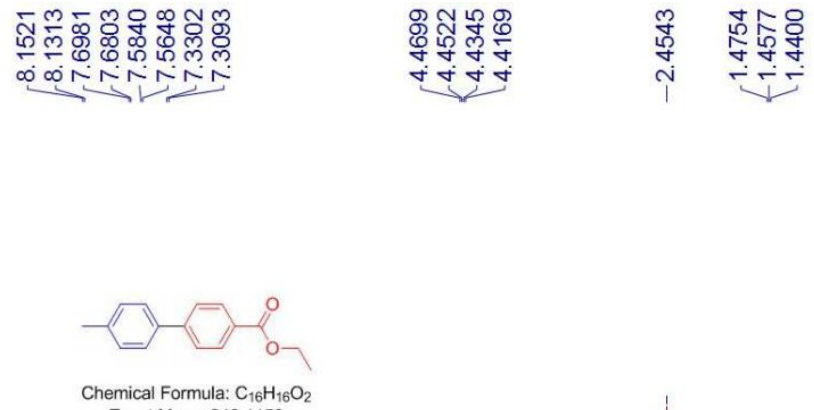
Exact Mass: 240.1150

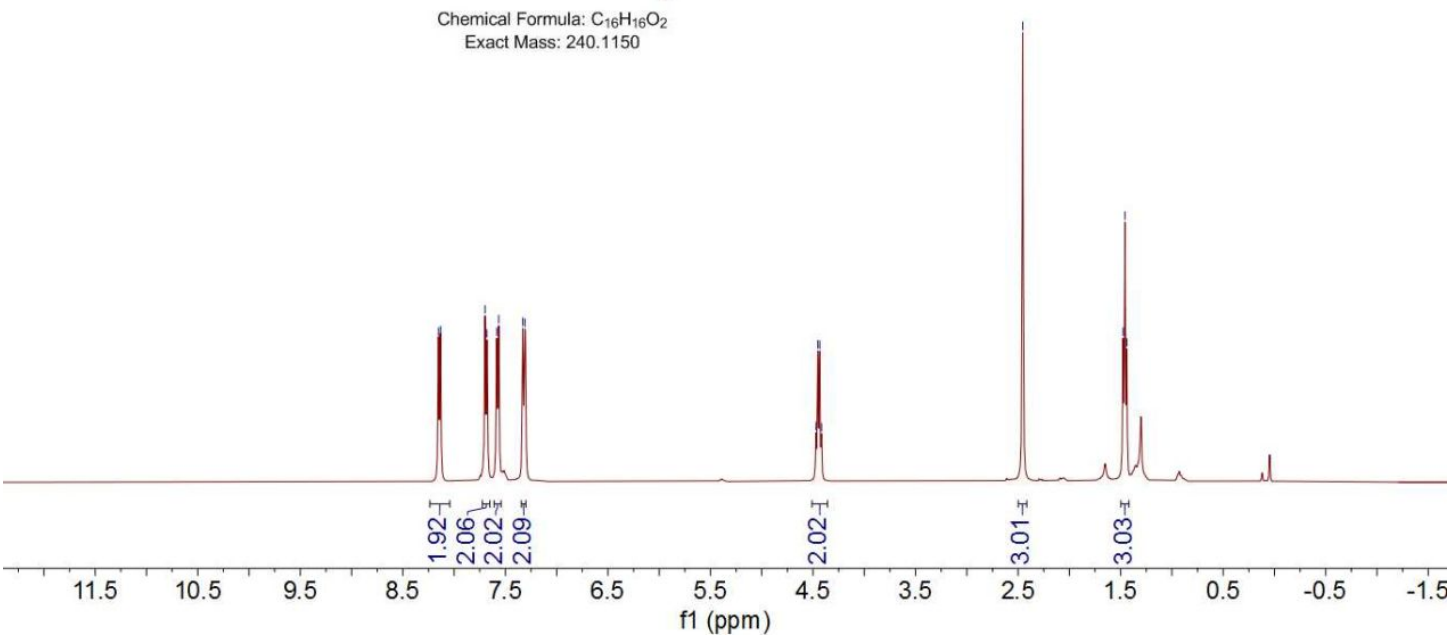


${ }^{13} \mathrm{C} \mathrm{NMR}\left(\mathrm{CDCl}_{3}\right)$ spectrum of compound $\mathbf{5 f}$
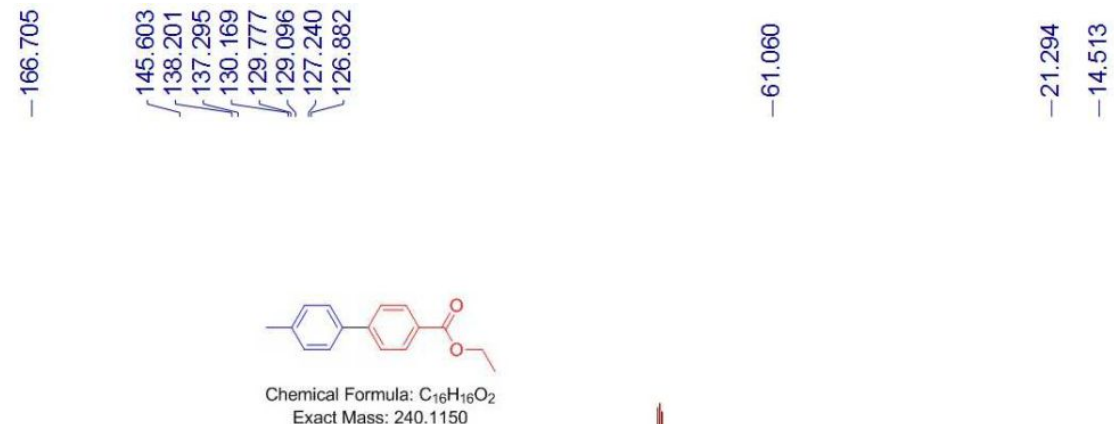
Exact Mass: 240.1150

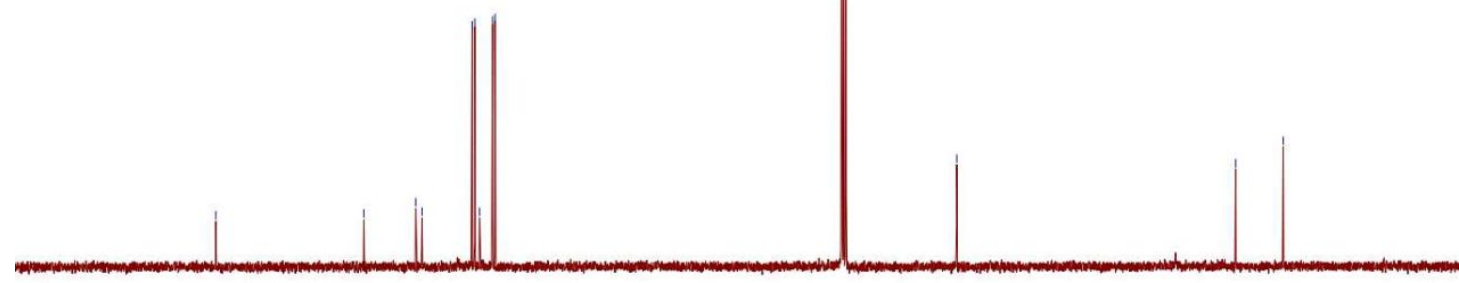

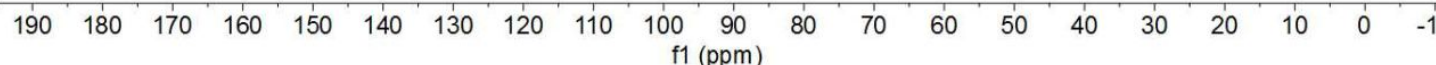

${ }^{1} \mathrm{H}$ NMR $\left(\mathrm{CDCl}_{3}\right)$ spectrum of compound $\mathbf{5 g}$
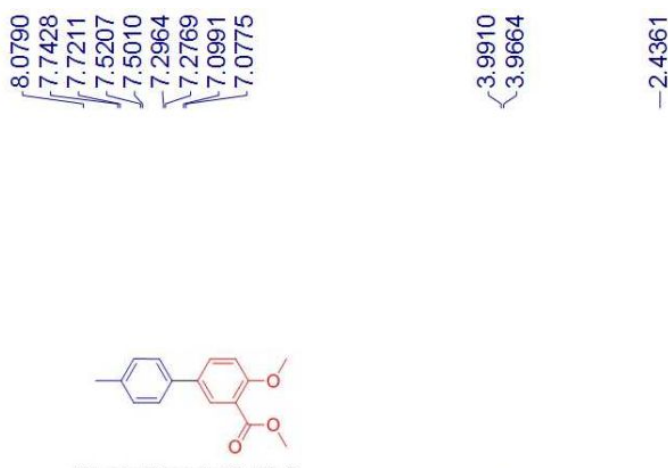

Chemical Formula: $\mathrm{C}_{16} \mathrm{H}_{16} \mathrm{O}_{3}$ Exact Mass: 256.1099

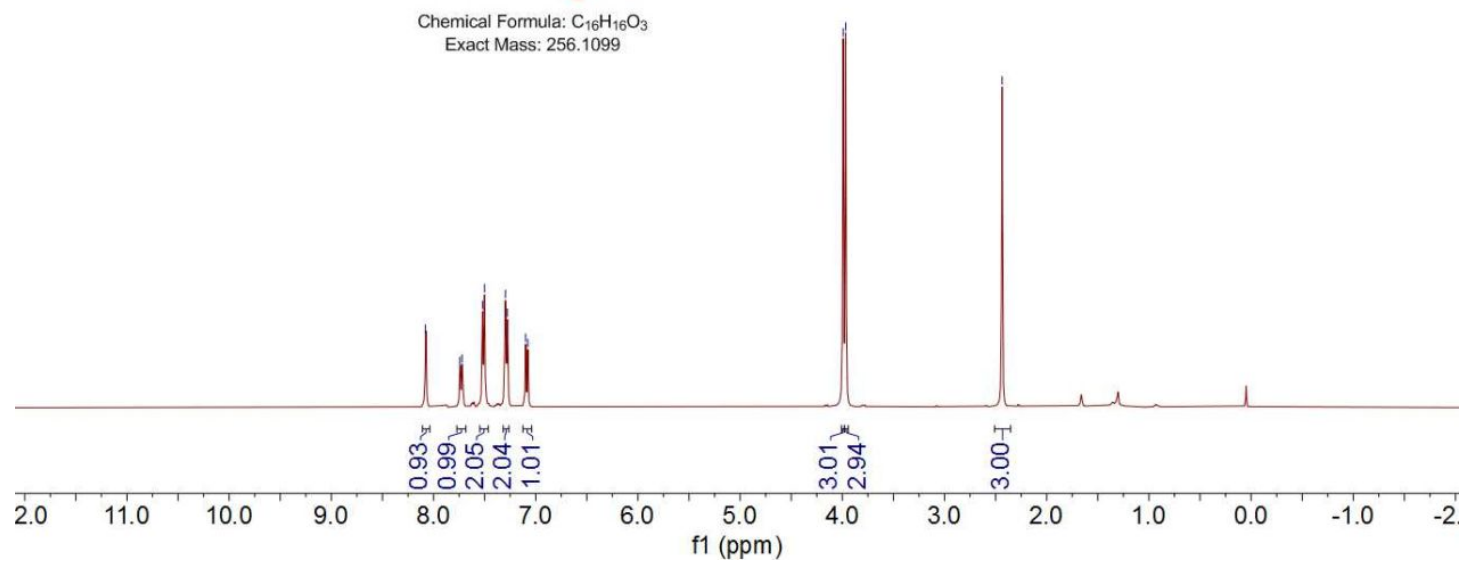


${ }^{13} \mathrm{C} \mathrm{NMR}\left(\mathrm{CDCl}_{3}\right)$ spectrum of compound $\mathbf{5 g}$

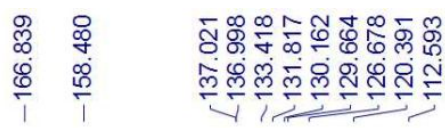

mृ $\frac{\text { m }}{\mathrm{N}}$

เึ่

$\frac{\text { g }}{\frac{1}{1}}$

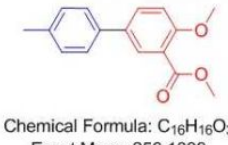

emical Formula: $\mathrm{C}_{16} \mathrm{H}_{16} \mathrm{O}_{3}$

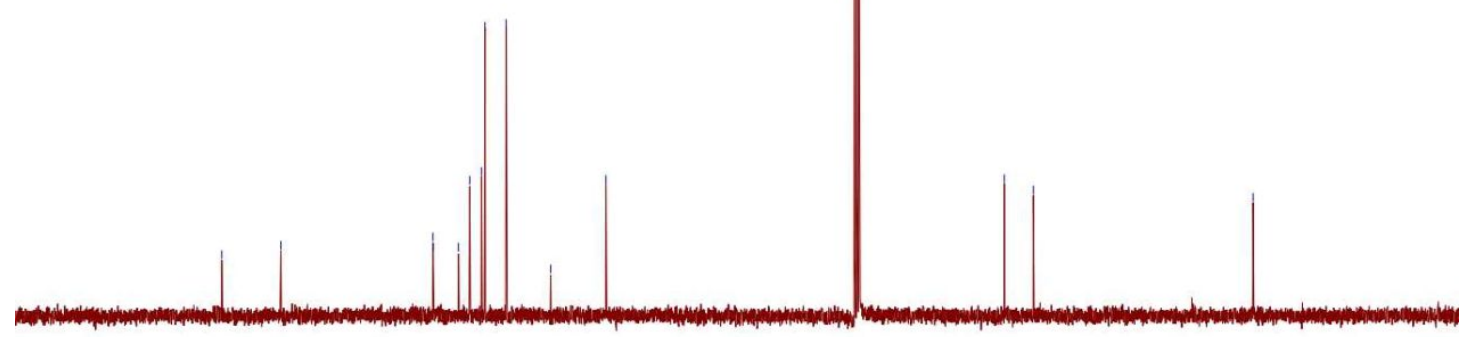

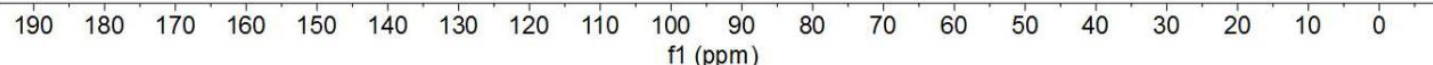

${ }^{1} \mathrm{H}$ NMR $\left(\mathrm{CDCl}_{3}\right)$ spectrum of compound $\mathbf{5 h}$

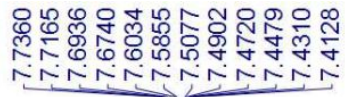

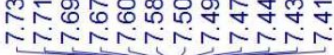

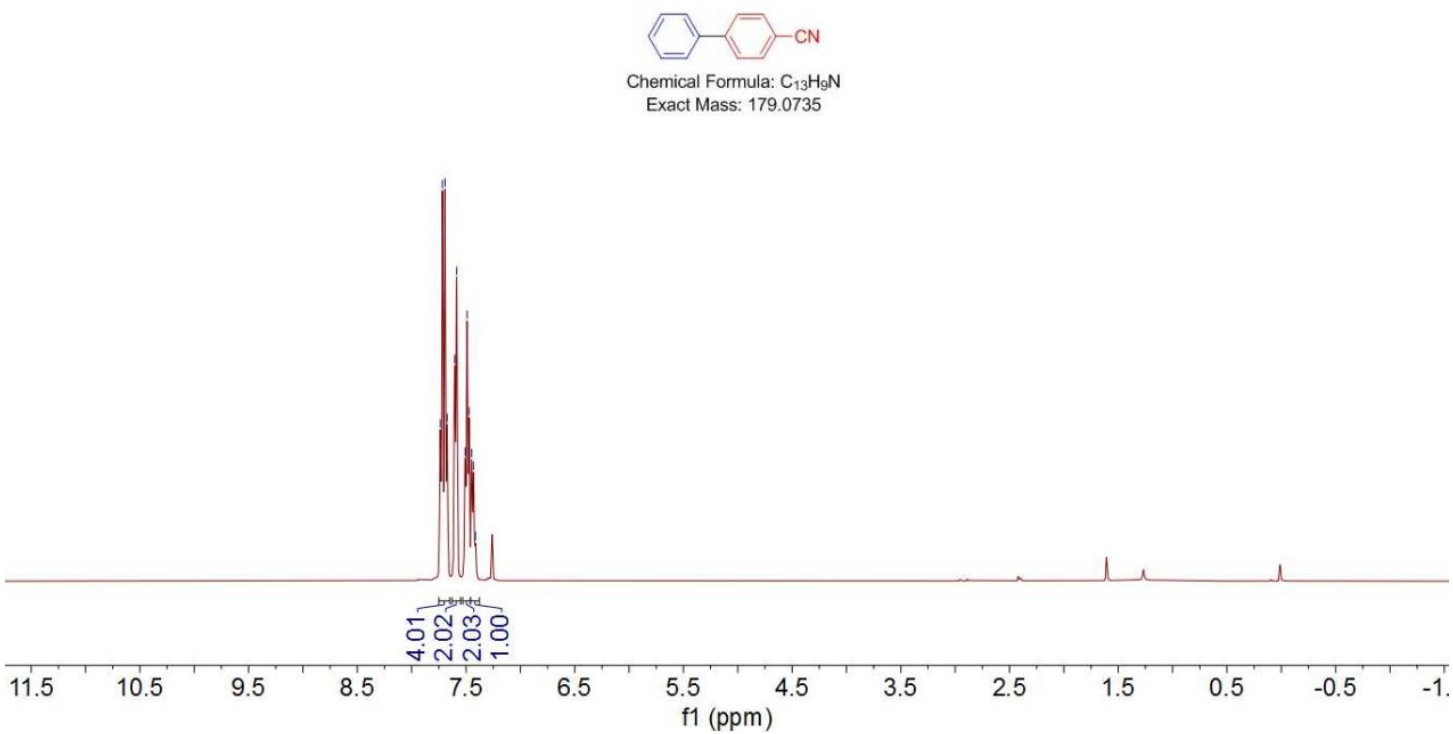


${ }^{13} \mathrm{C} \mathrm{NMR}\left(\mathrm{CDCl}_{3}\right)$ spectrum of compound $\mathbf{5 h}$

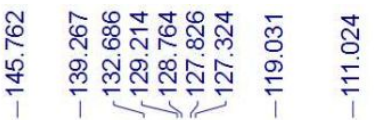

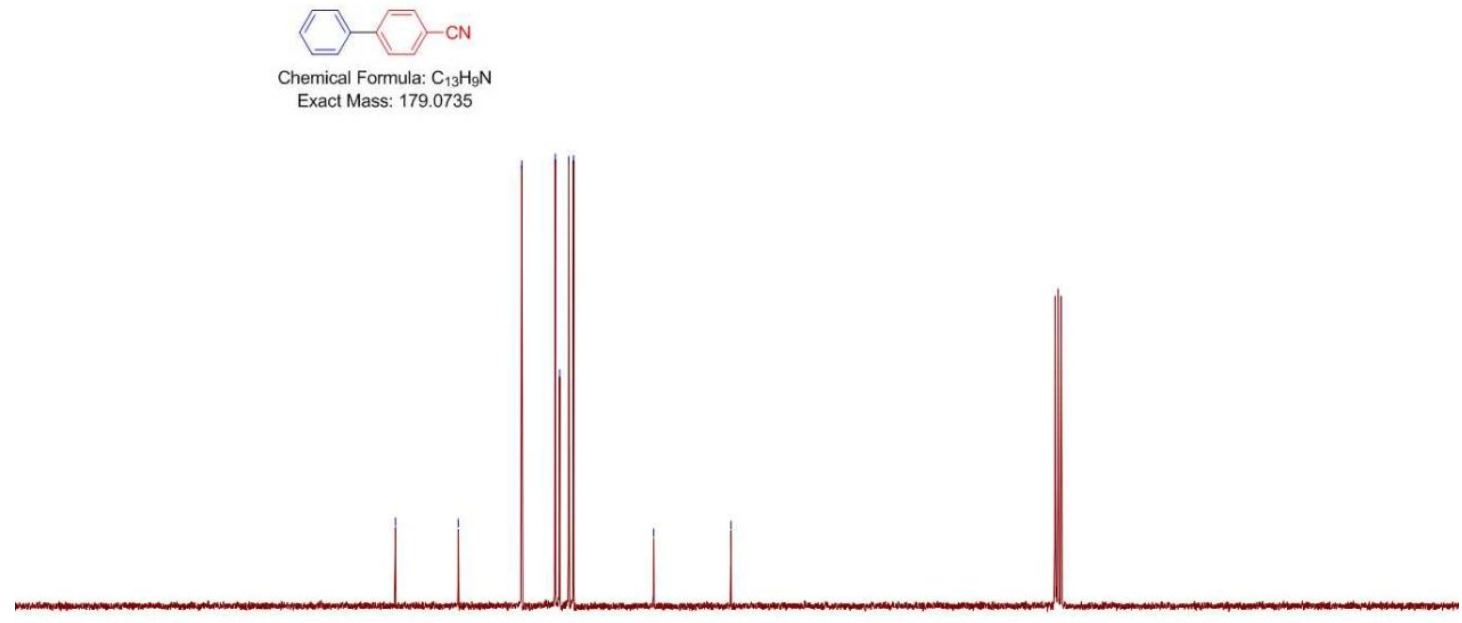

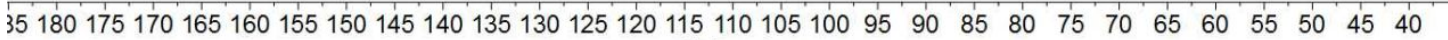
f1 (ppm)

${ }^{1} \mathrm{H}$ NMR $\left(\mathrm{CDCl}_{3}\right)$ spectrum of compound $\mathbf{5 i}$
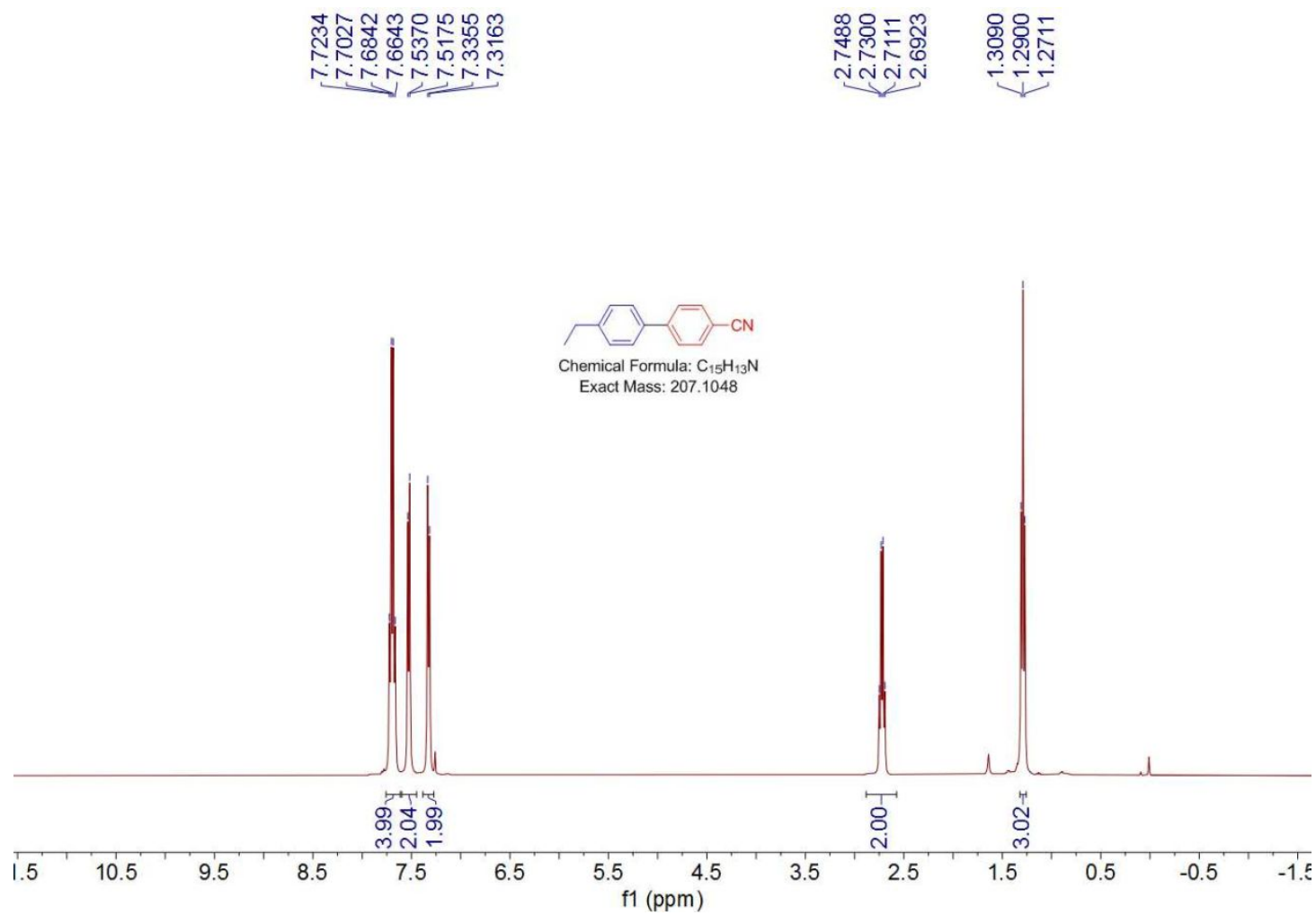
${ }^{13} \mathrm{C}$ NMR $\left(\mathrm{CDCl}_{3}\right)$ spectrum of compound $\mathbf{5 i}$

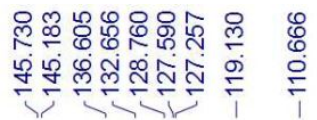

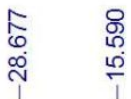
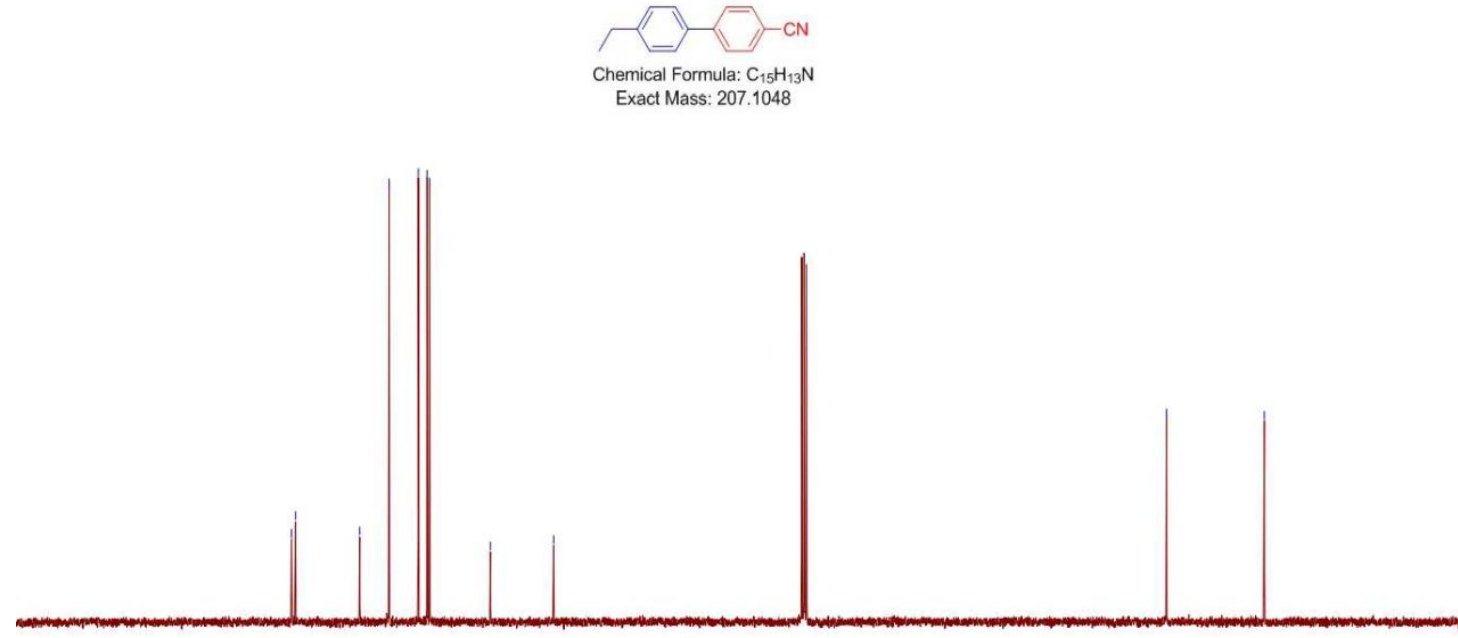

$\begin{array}{lllllllllllllllllllll}180 & 170 & 160 & 150 & 140 & 130 & 120 & 110 & 100 & \begin{array}{c}90 \\ \mathrm{f} 1(\mathrm{ppm})\end{array} & 80 & 60 & 50 & 40 & 30 & 20 & 10 & 0 & -1\end{array}$

${ }^{1} \mathrm{H}$ NMR $\left(\mathrm{CDCl}_{3}\right)$ spectrum of compound $\mathbf{5} \mathbf{j}$

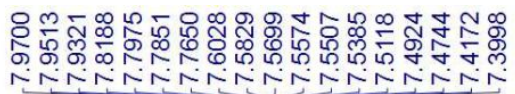

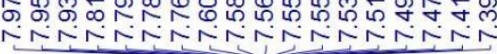

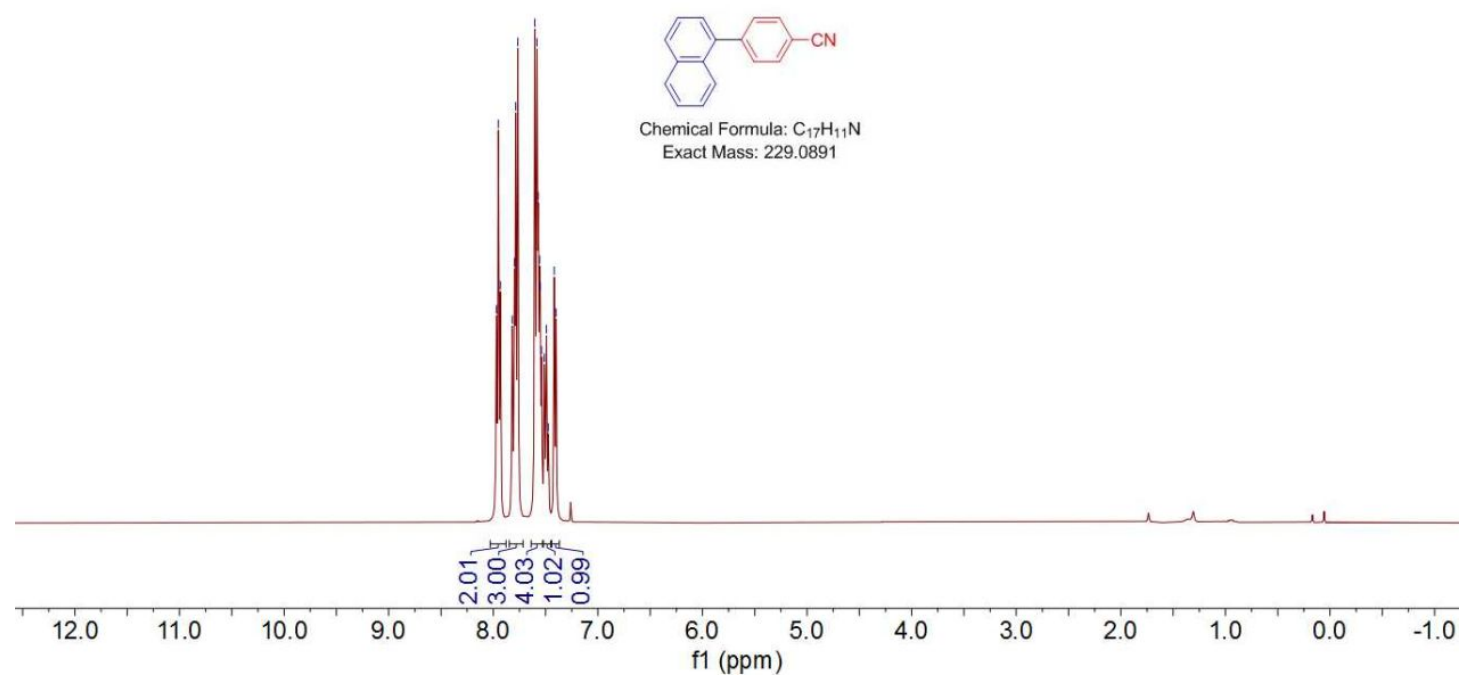


${ }^{13} \mathrm{C}$ NMR $\left(\mathrm{CDCl}_{3}\right)$ spectrum of compound $\mathbf{5 j}$
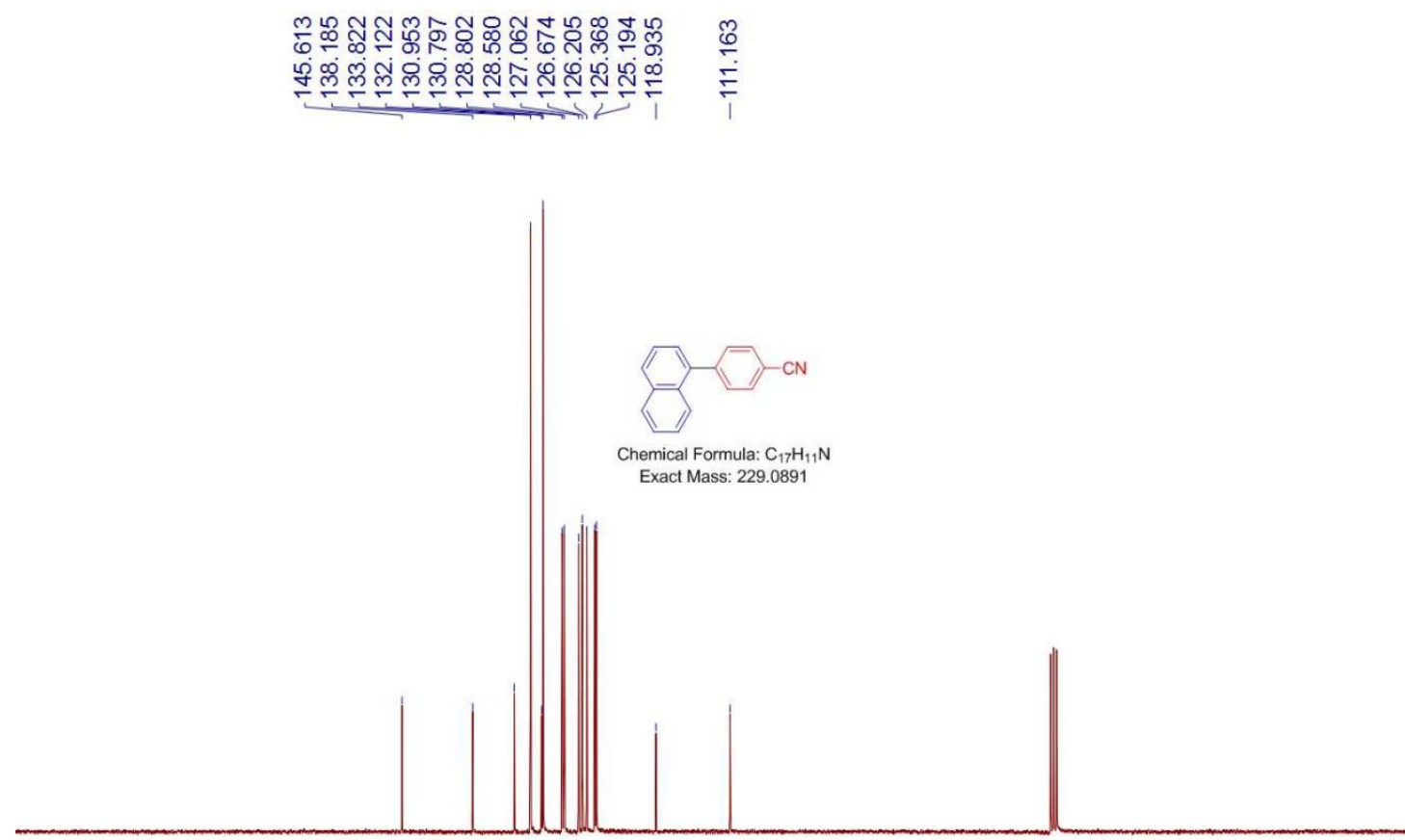

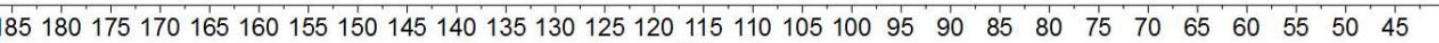
f1 (ppm)

${ }^{1} \mathrm{H}$ NMR $\left(\mathrm{CDCl}_{3}\right)$ spectrum of compound $\mathbf{5 k}$

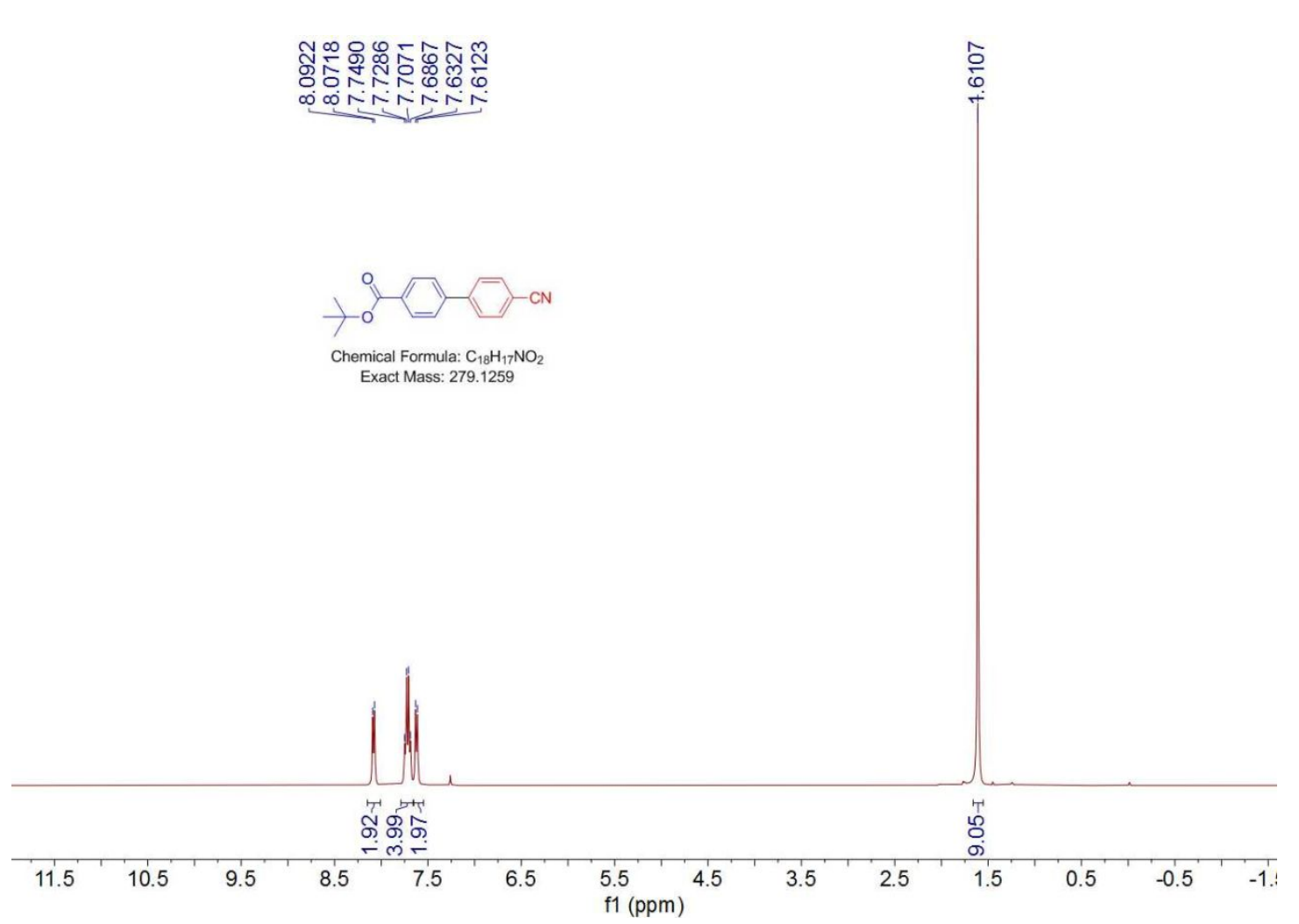

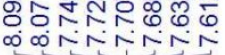

\section{$\underline{8}$} $\stackrel{F}{\digamma}$ 
${ }^{13} \mathrm{C} \mathrm{NMR}\left(\mathrm{CDCl}_{3}\right)$ spectrum of compound $\mathbf{5 k}$

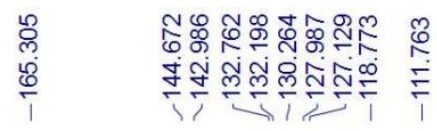

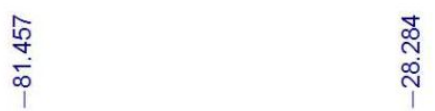

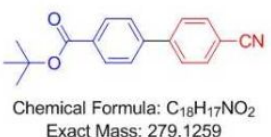
Exact Mass: 279.1259

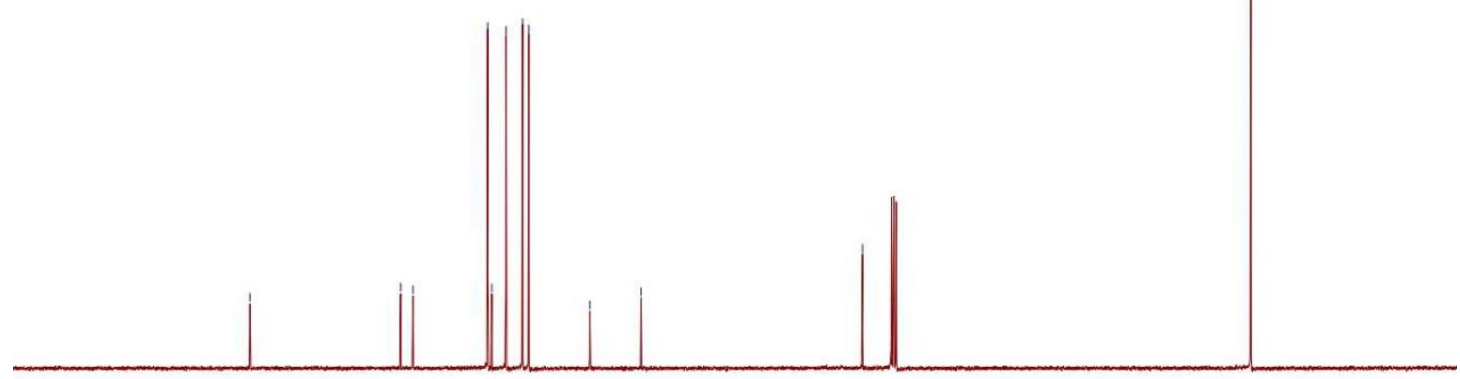

$\begin{array}{llllllllllllllllllll}190 & 180 & 170 & 160 & 150 & 140 & 130 & 120 & 110 & \begin{array}{c}100 \\ \mathrm{f} 1(\mathrm{ppm})\end{array} & 90 & 80 & 70 & 60 & 50 & 40 & 30 & 20 & 10 & 10\end{array}$

${ }^{1} \mathrm{H}$ NMR $\left(\mathrm{CDCl}_{3}\right)$ spectrum of compound $\mathbf{5 l}$

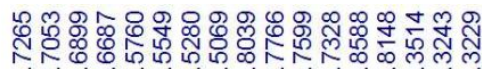

Nog L

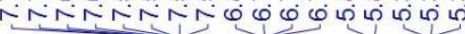

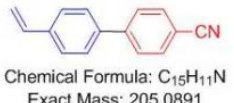

Exact Mass: 205.0891

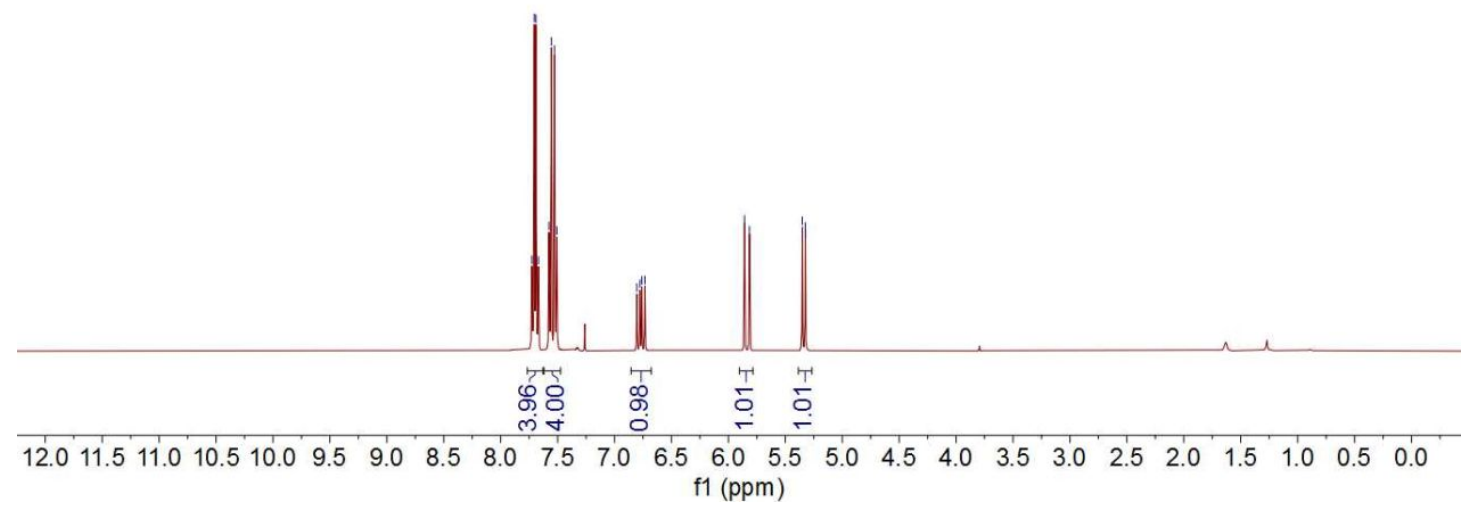


${ }^{13} \mathrm{C}$ NMR $\left(\mathrm{CDCl}_{3}\right)$ spectrum of compound $\mathbf{5 l}$

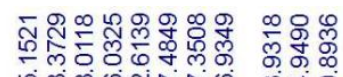

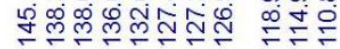

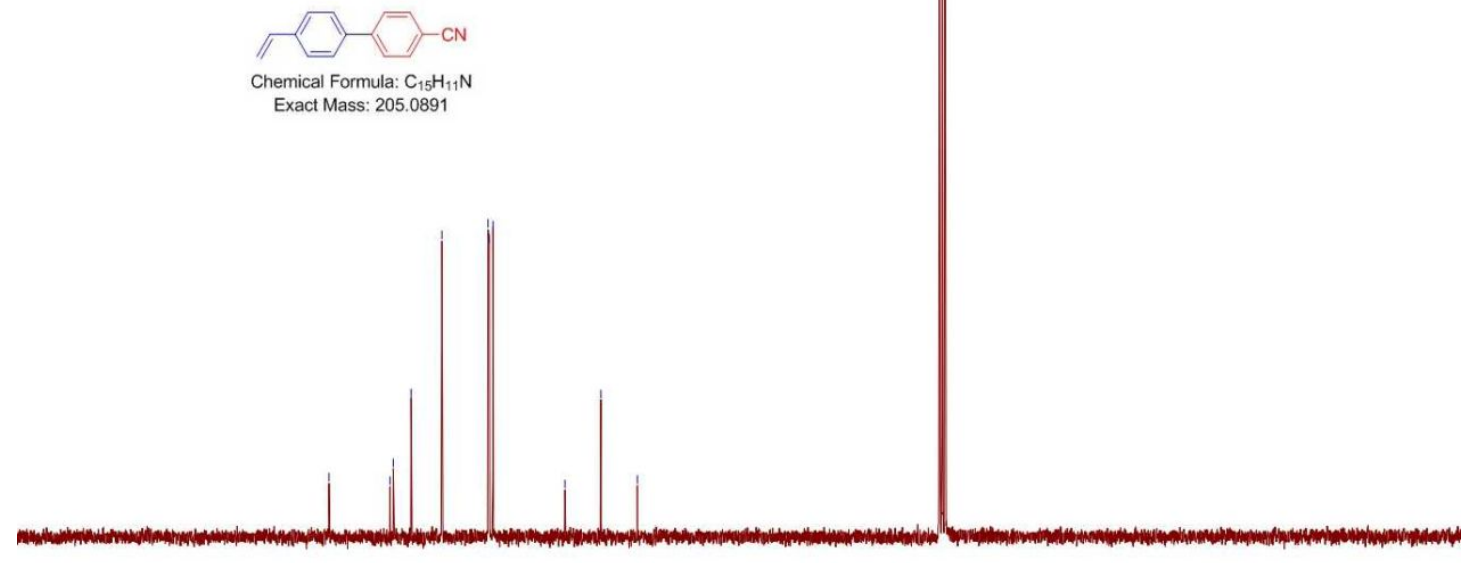

$110 \quad 100$

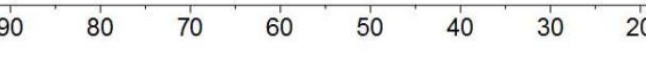

${ }^{1} \mathrm{H}$ NMR $\left(\mathrm{CDCl}_{3}\right)$ spectrum of compound $\mathbf{5 m}$

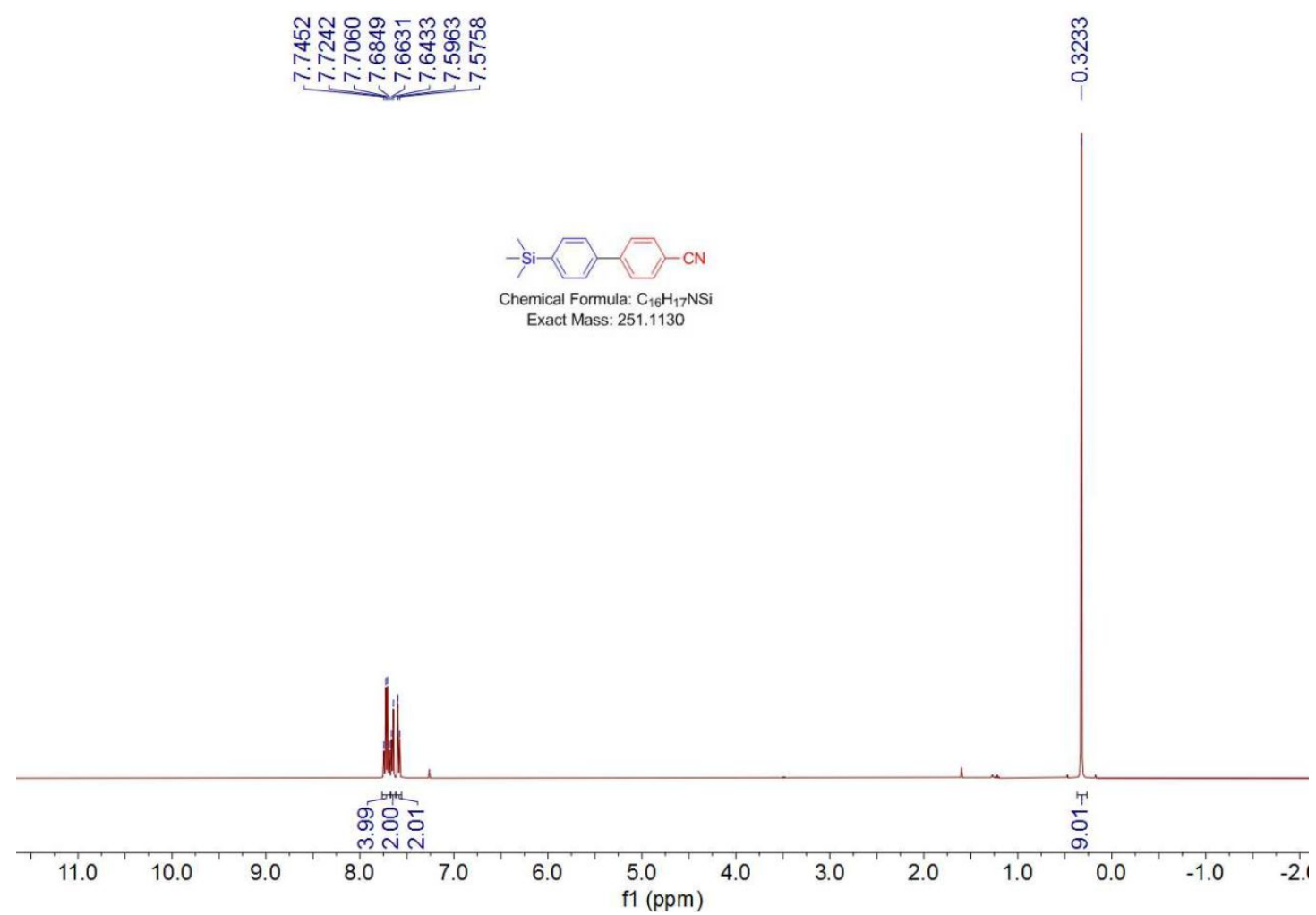


${ }^{13} \mathrm{C}$ NMR $\left(\mathrm{CDCl}_{3}\right)$ spectrum of compound $\mathbf{5 m}$
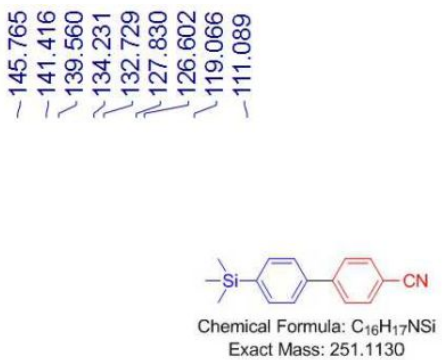

Exact Mass: 251.1130

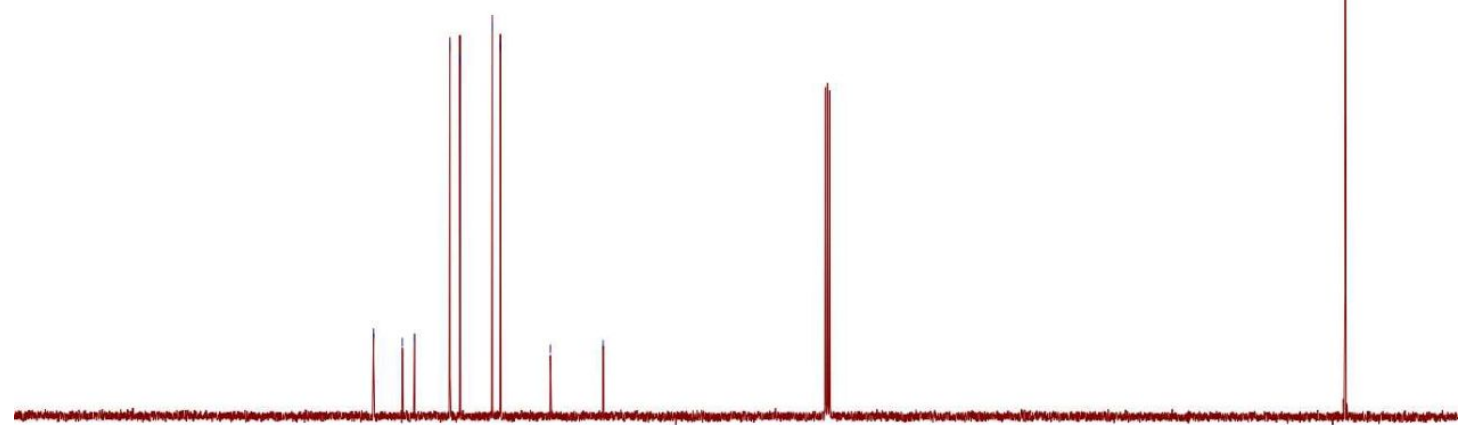

\begin{tabular}{llllllllllllllllllllllll}
\hline 0 & 190 & 180 & 170 & 160 & 150 & 140 & 130 & 120 & 110 & 100 & 90 & 80 & 70 & 60 & 50 & 40 & 30 & 20 & 10 & 0 & -10
\end{tabular}

${ }^{1} \mathrm{H}$ NMR $\left(\mathrm{CDCl}_{3}\right)$ spectrum of compound $\mathbf{5 n}$

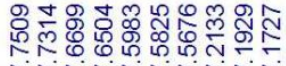

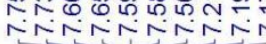

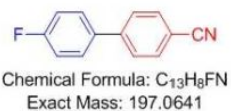

Chemical Formula: $\mathrm{C}_{13} \mathrm{H}_{8} \mathrm{FN}$

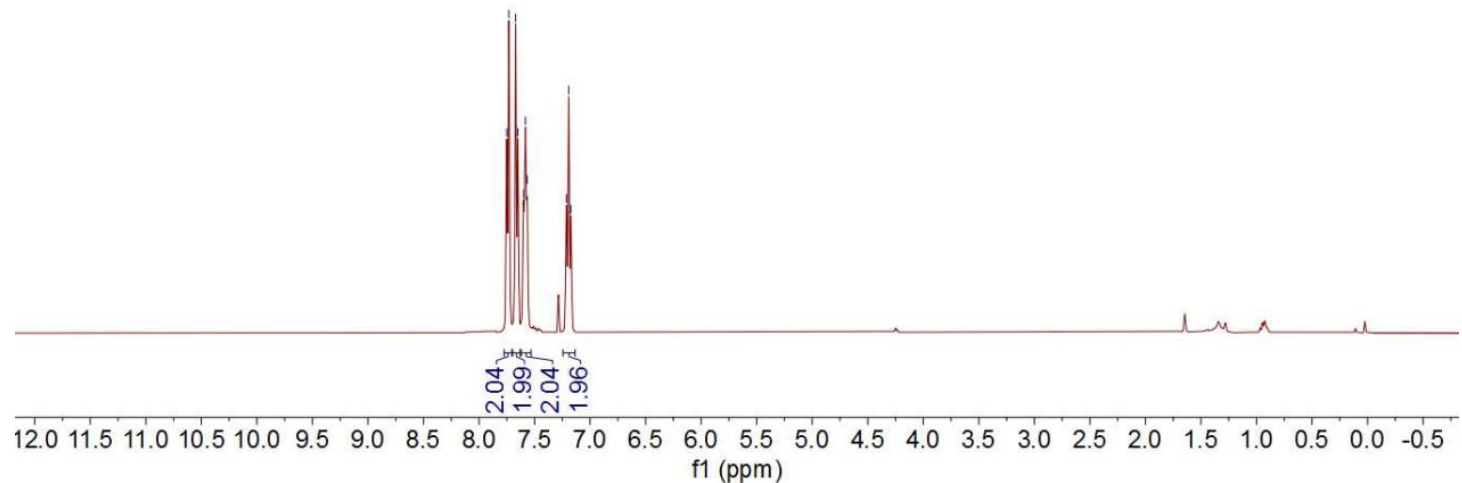


${ }^{13} \mathrm{C} \mathrm{NMR}\left(\mathrm{CDCl}_{3}\right)$ spectrum of compound $\mathbf{5 n}$
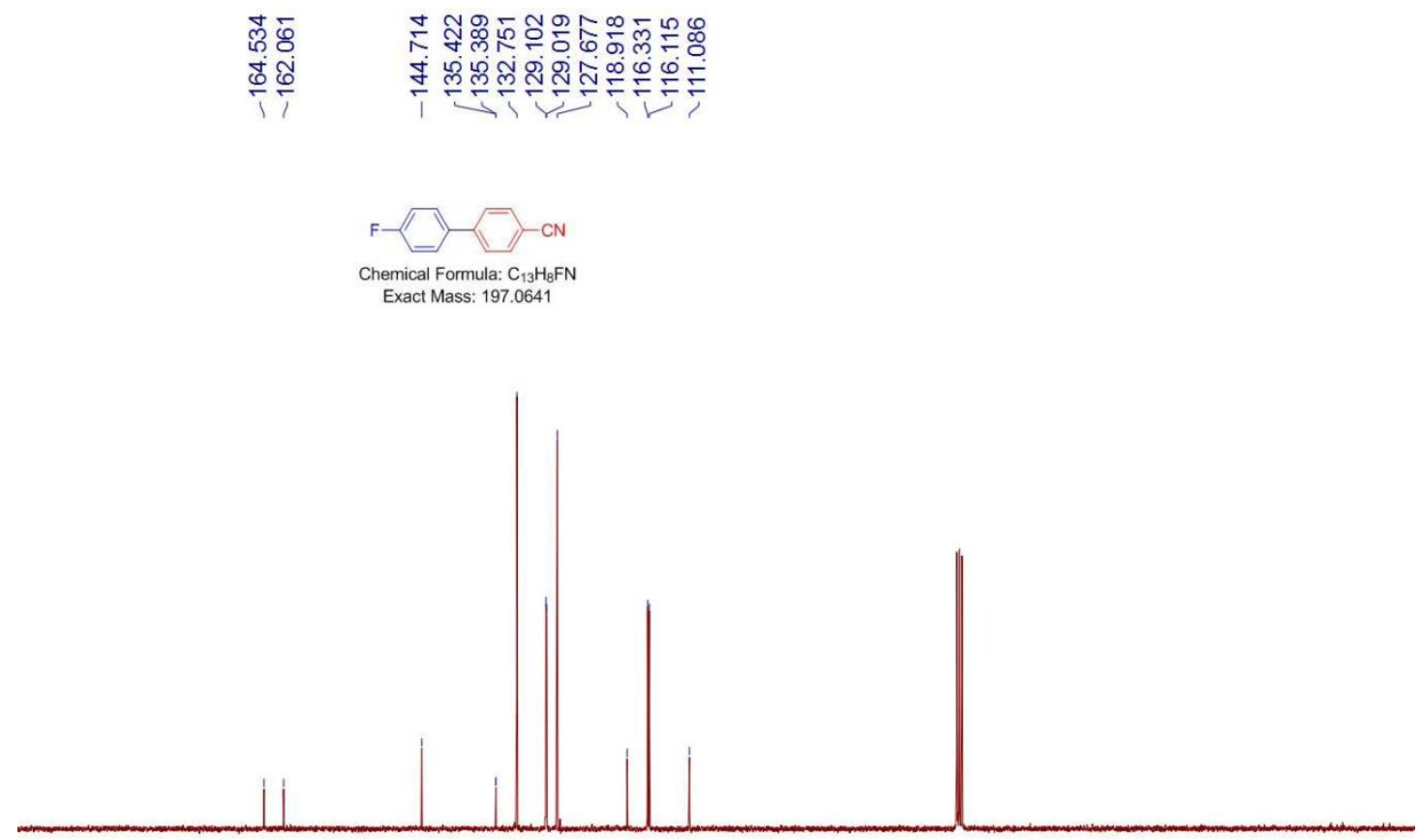

$\begin{array}{lllllllllllllllllll}190 & 180 & 170 & 160 & 150 & 140 & 130 & 120 & \begin{array}{c}110 \\ \mathrm{f} 1(\mathrm{ppm})\end{array} & 100 & 90 & 80 & 70 & 60 & 50 & 40 & 30 & 2\end{array}$

${ }^{19} \mathrm{~F}$ NMR $\left(\mathrm{CDCl}_{3}\right)$ spectrum of compound $\mathbf{5 n}$

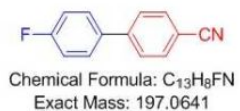

Exact Mass: 197.0641

$\begin{array}{lllllllllllllllllllllll}-55 & -60 & -65 & -70 & -75 & -80 & -85 & -90 & -95 & -100 & -105 & -110 & -115 & -120 & -125 & -130 & -135 & -140 & -145 & -150 & -155 & -16\end{array}$ 
${ }^{1} \mathrm{H}$ NMR $\left(\mathrm{CDCl}_{3}\right)$ spectrum of compound $\mathbf{5 o}$

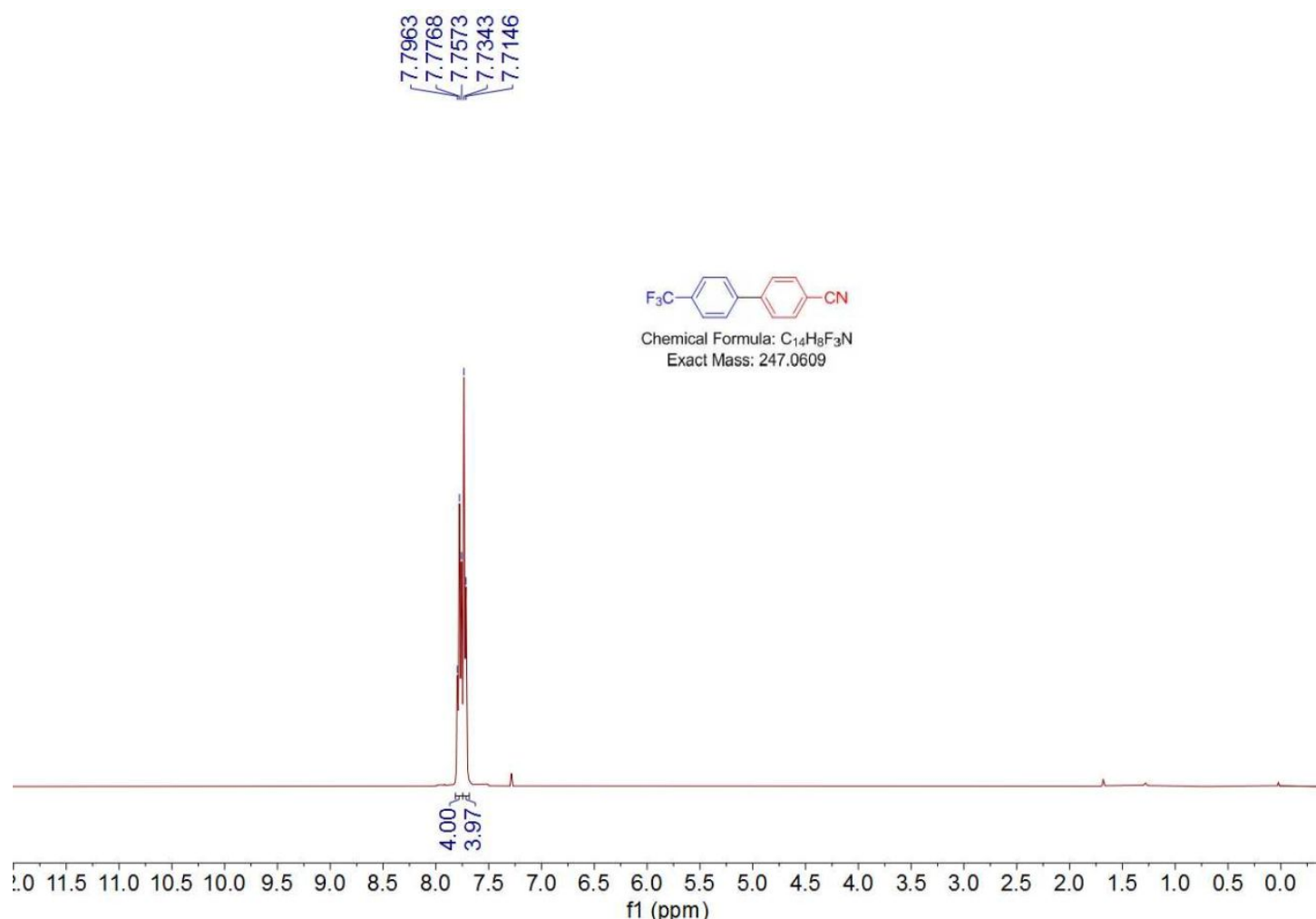

${ }^{13} \mathrm{C}$ NMR $\left(\mathrm{CDCl}_{3}\right)$ spectrum of compound $\mathbf{5 o}$

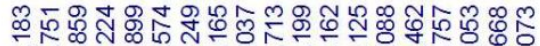

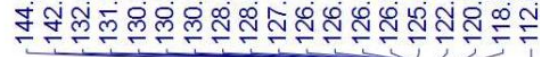

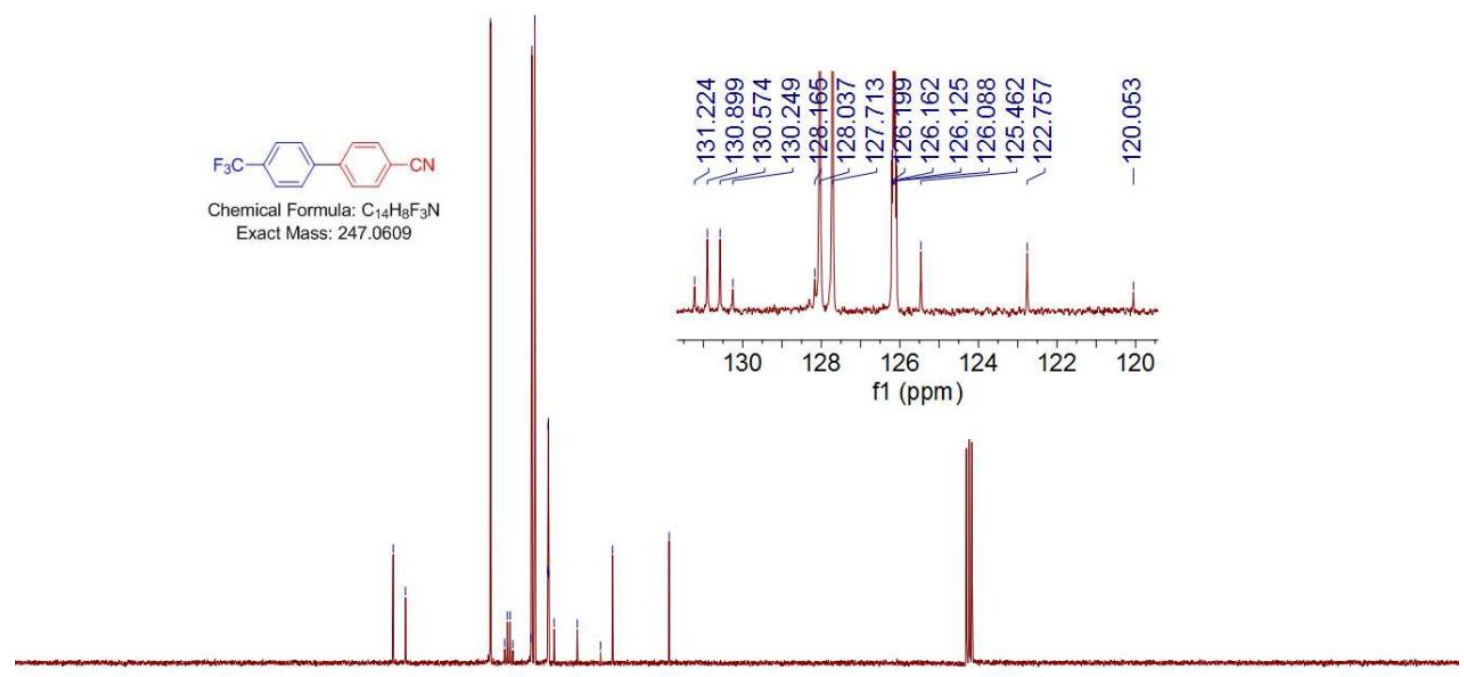

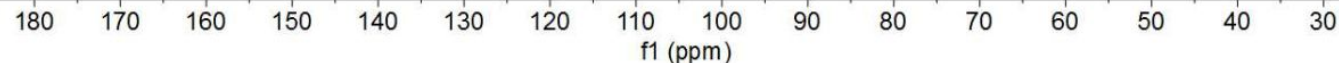


${ }^{19} \mathrm{~F}$ NMR $\left(\mathrm{CDCl}_{3}\right)$ spectrum of compound $\mathbf{5 0}$

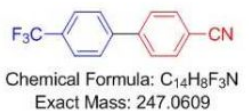

$\begin{array}{lllllllllllllllll}-58.8 & -59.2 & -59.6 & -60.0 & -60.4 & -60.8 & -61.2 & -61.6 & -62.0 & -62.4 & -62.8 & -63.2 & -63.6 & -64.0 & -64.4 & -64.8 & -65.2\end{array}$

${ }^{1} \mathrm{H}$ NMR $\left(\mathrm{CDCl}_{3}\right)$ spectrum of compound $\mathbf{5 p}$
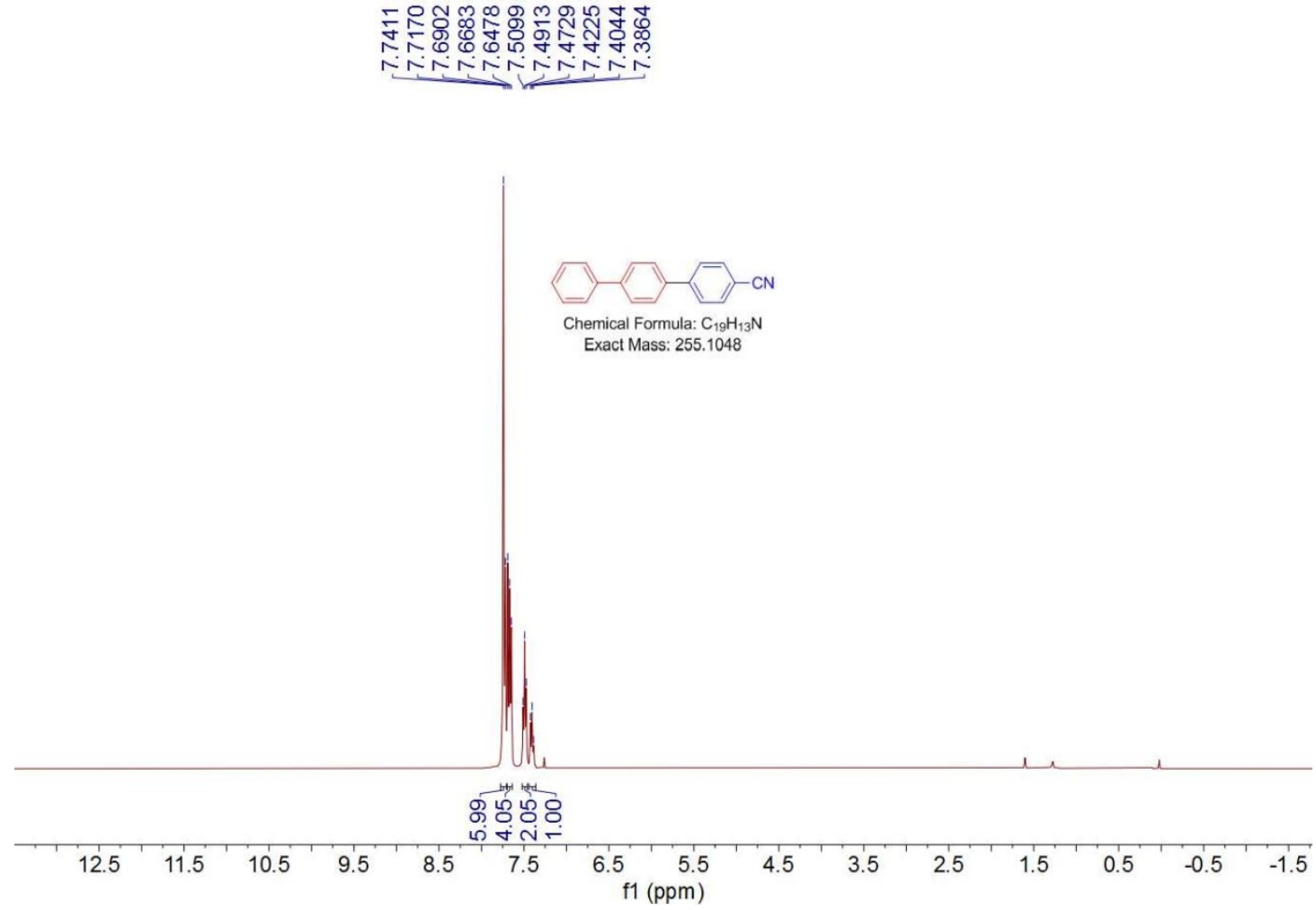
${ }^{13} \mathrm{C} \mathrm{NMR}\left(\mathrm{CDCl}_{3}\right)$ spectrum of compound $\mathbf{5 p}$

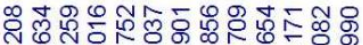

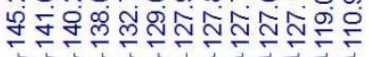
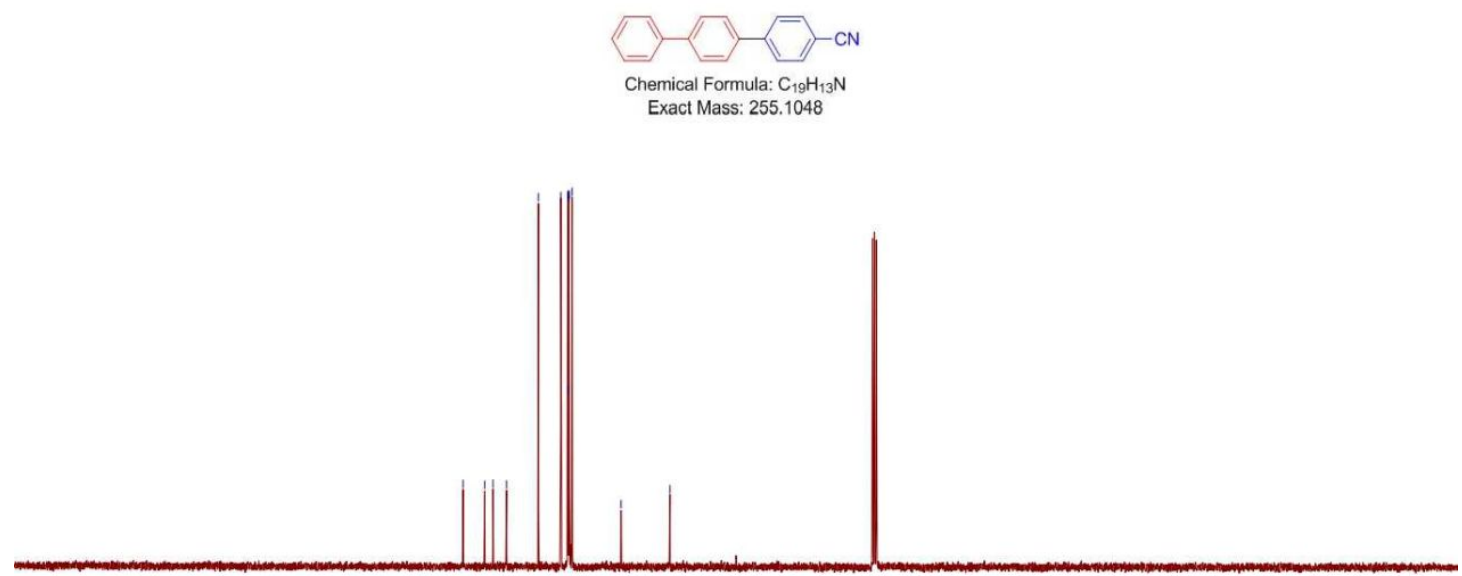

$\begin{array}{lllllllllllllllllllllll}210 & 200 & 190 & 180 & 170 & 160 & 150 & 140 & 130 & 120 & 110 & 100 & 90 & 80 & 70 & 60 & 50 & 40 & 30 & 20 & 10 & 0 & -10\end{array}$ f1 (ppm)

${ }^{1} \mathrm{H} \mathrm{NMR}\left(\mathrm{CDCl}_{3}\right)$ spectrum of compound $\mathbf{5 q}$

\section{๘

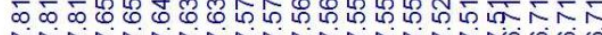

NiNiNiNiNiNiNiNo600

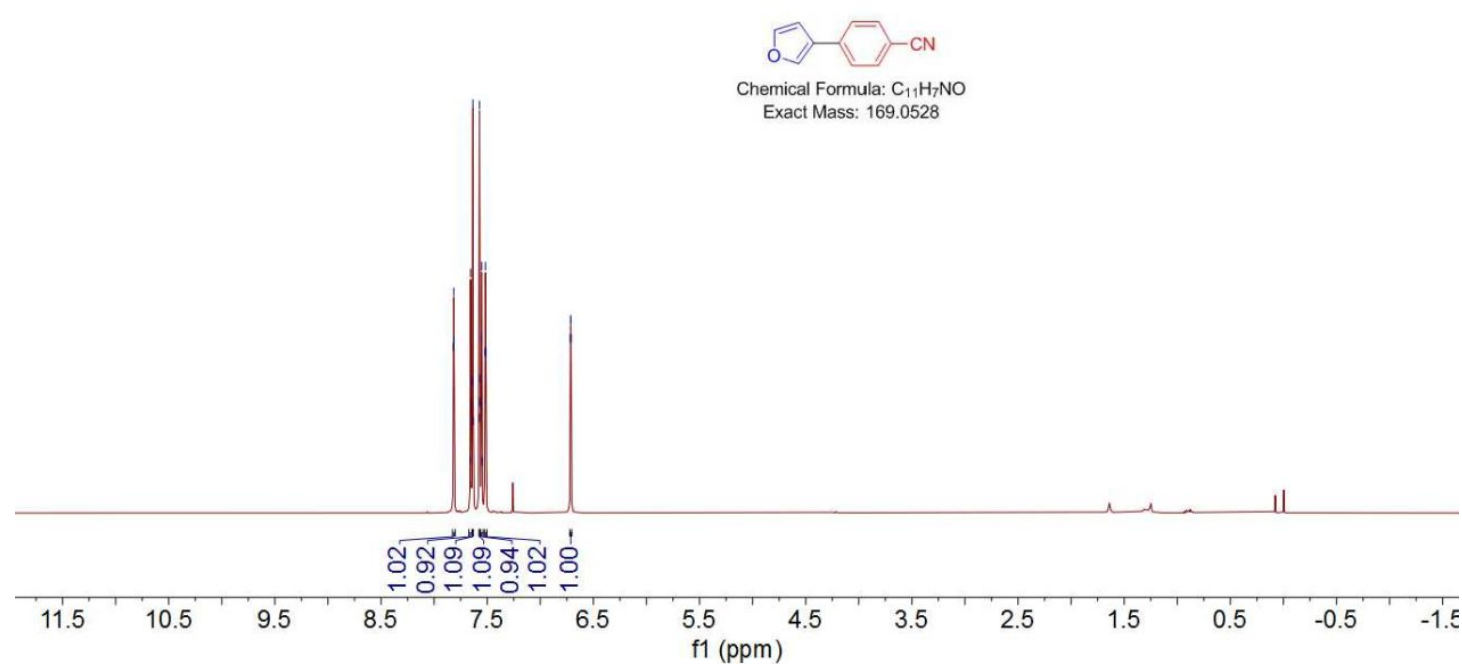


${ }^{13} \mathrm{C}$ NMR $\left(\mathrm{CDCl}_{3}\right)$ spectrum of compound $\mathbf{5 q}$

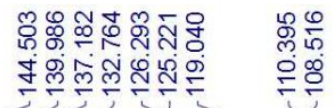

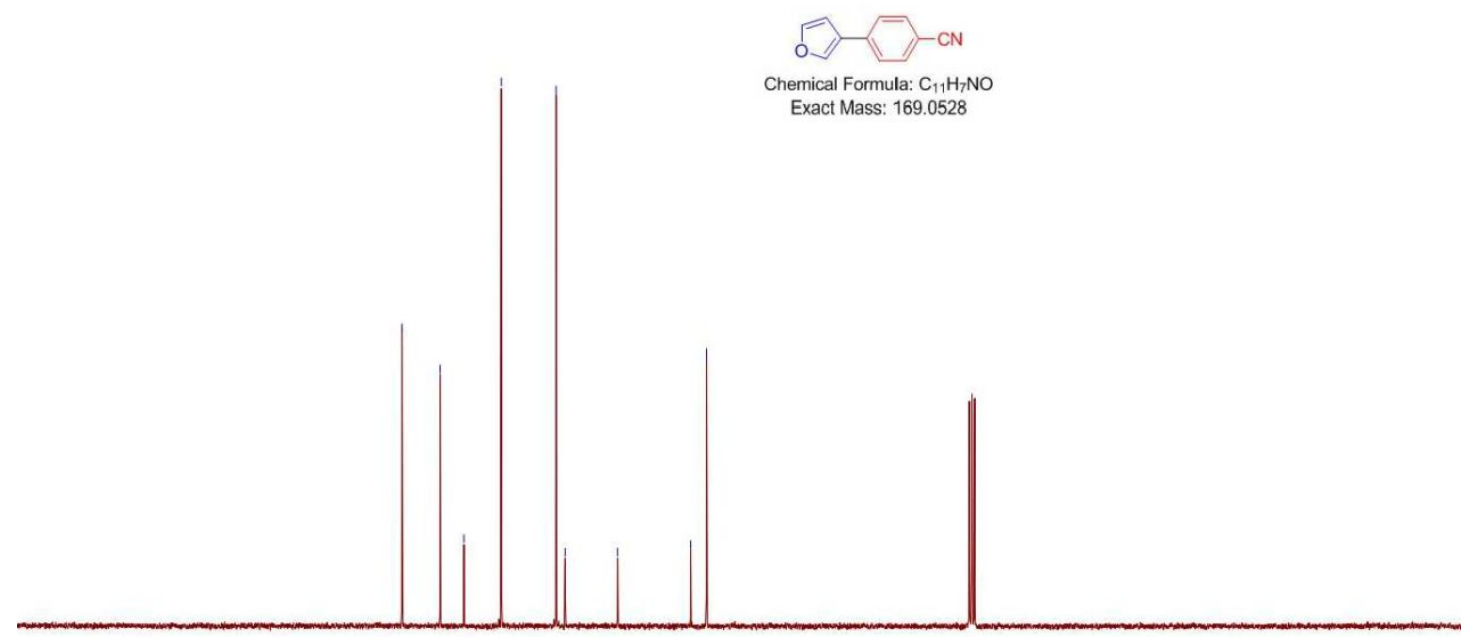

$\begin{array}{lllllllllllllllll}180 & 170 & 160 & 150 & 140 & 130 & 120 & 110 & 100 & 90 & 80 & 70 & 60 & 50 & 40 & 30 & 21\end{array}$

${ }^{1} \mathrm{H}$ NMR $\left(\mathrm{CDCl}_{3}\right)$ spectrum of compound $\mathbf{5 r}$

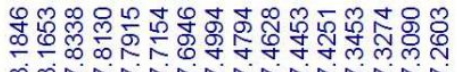

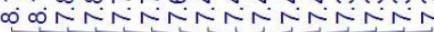

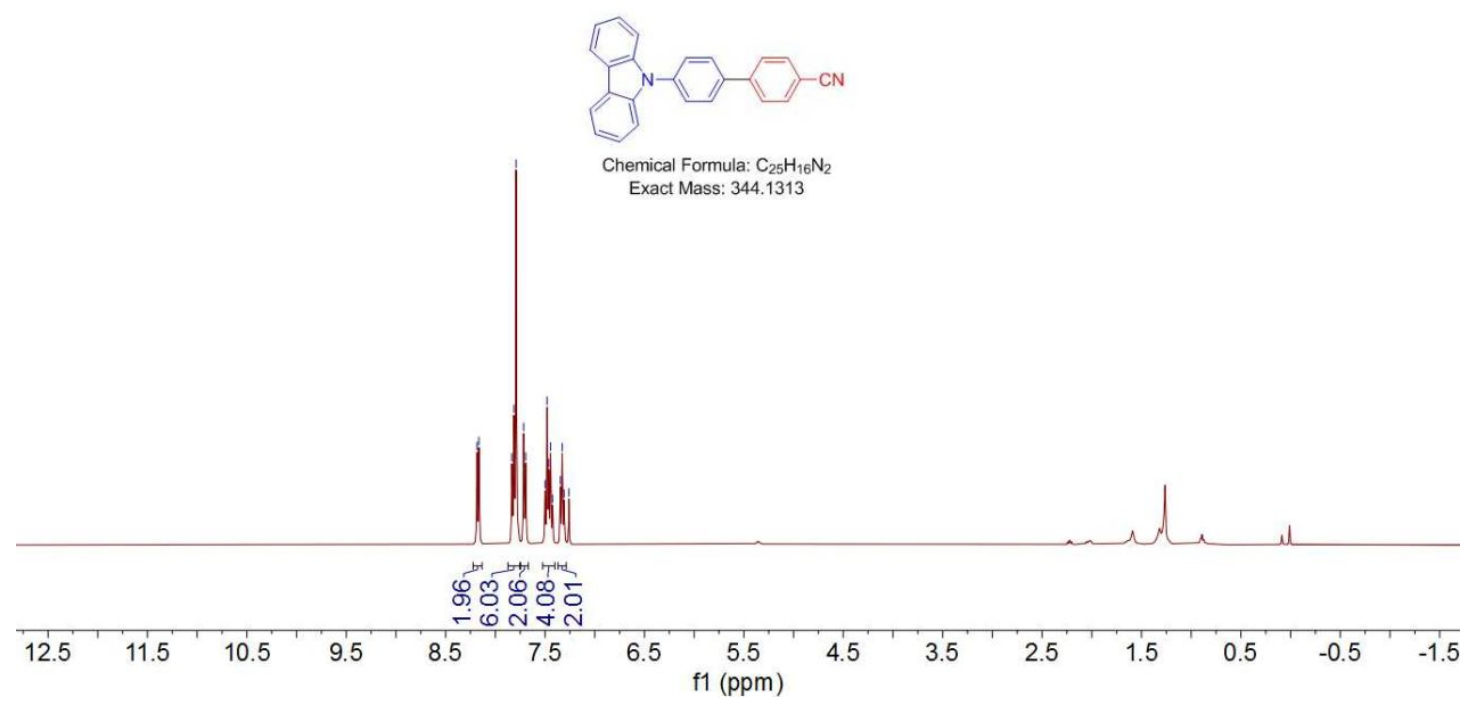


${ }^{13} \mathrm{C} \mathrm{NMR}\left(\mathrm{CDCl}_{3}\right)$ spectrum of compound $\mathbf{5 r}$

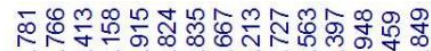

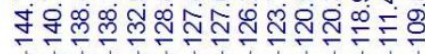

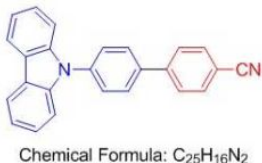

Exact Mass: 344.1313

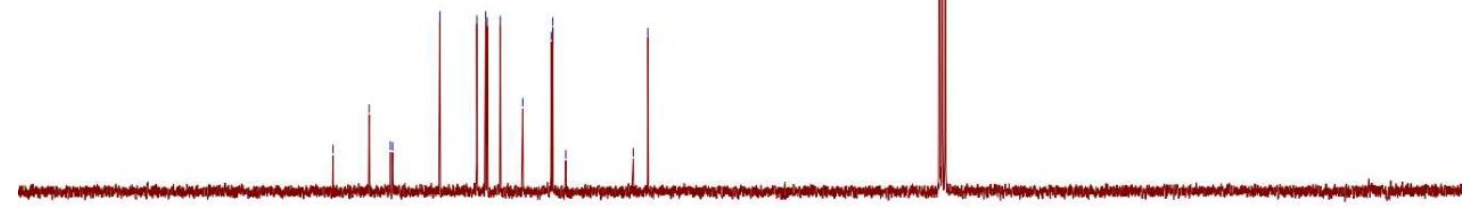

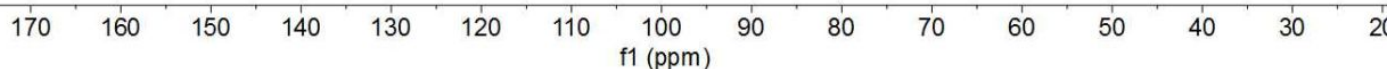

${ }^{1} \mathrm{H} \mathrm{NMR}\left(\mathrm{CDCl}_{3}\right)$ spectrum of compound $\mathbf{5 s}$

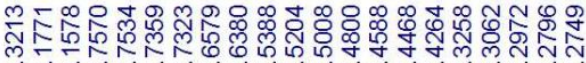

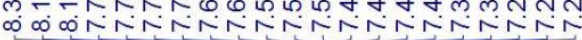

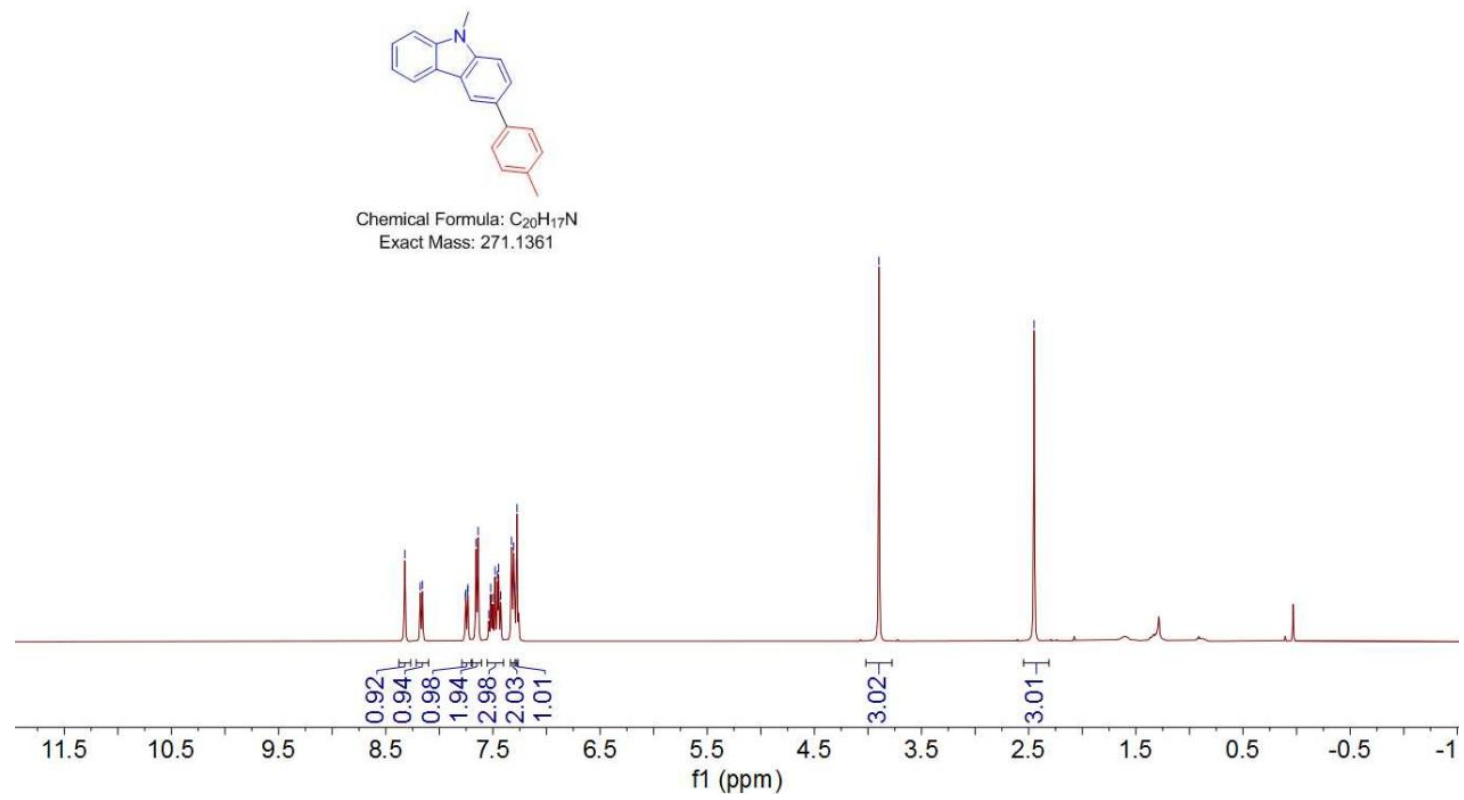


${ }^{13} \mathrm{C} \mathrm{NMR}\left(\mathrm{CDCl}_{3}\right)$ spectrum of compound $\mathbf{5 s}$

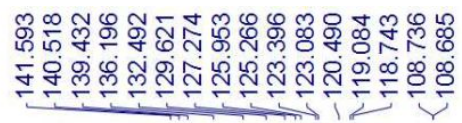

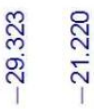

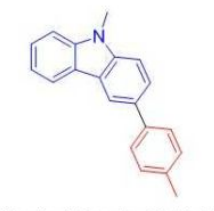

Chemical Formula: $\mathrm{C}_{20} \mathrm{H}_{17} \mathrm{~N}$ Exact Mass: 271.136

\begin{tabular}{llllllllllllllllll}
\hline 80 & 170 & 160 & 150 & 140 & 130 & 120 & 110 & 100 & 90 & 80 & 70 & 60 & 50 & 40 & 30 & 20 & 10
\end{tabular}

${ }^{1} \mathrm{H}$ NMR $\left(\mathrm{CDCl}_{3}\right)$ spectrum of compound $\mathbf{5 t}$
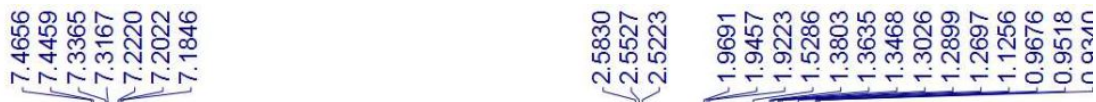

ninniñ

NiN Tr-

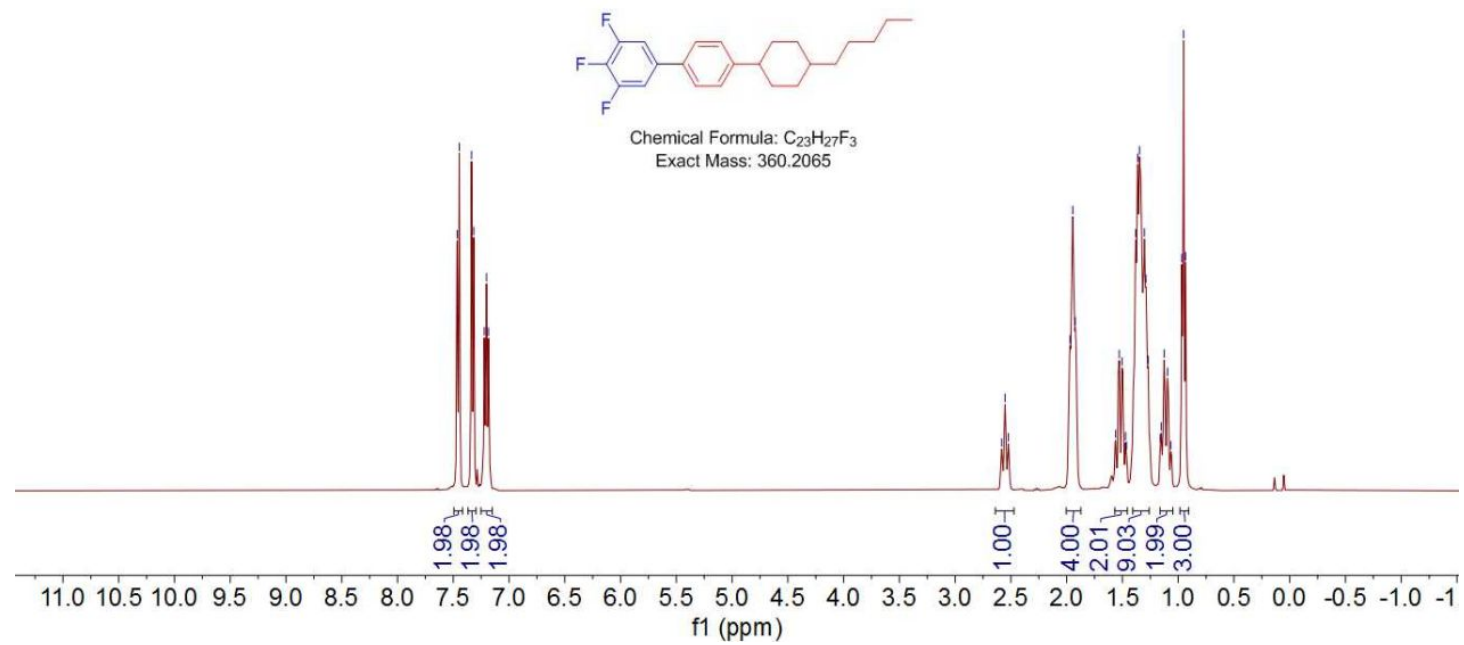


${ }^{13} \mathrm{C} \mathrm{NMR}\left(\mathrm{CDCl}_{3}\right)$ spectrum of compound $\mathbf{5 t}$

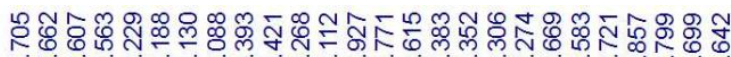

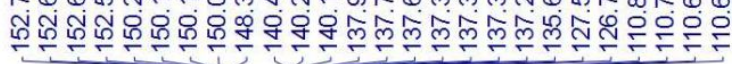

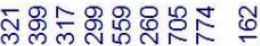

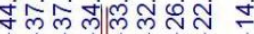

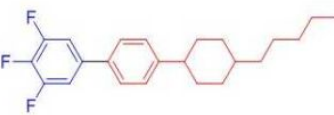

Chemical Formula: $\mathrm{C}_{23} \mathrm{H}_{27} \mathrm{~F}_{3}$ Exact Mass: 360.2065

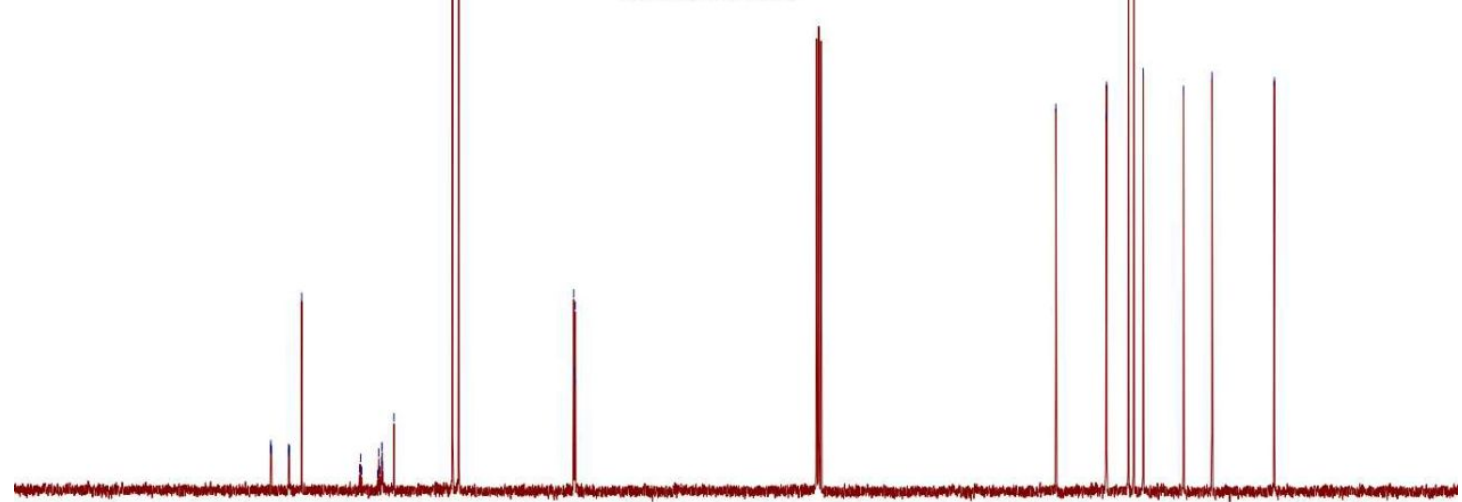

$\begin{array}{llllllllllllllllllllll}180 & 170 & 160 & 150 & 140 & 130 & 120 & 110 & 100 & 90 & 80 & 70 & 60 & 50 & 40 & 30 & 20 & 10 & 0 & -1\end{array}$

${ }^{19} \mathrm{~F}$ NMR $\left(\mathrm{CDCl}_{3}\right)$ spectrum of compound $\mathbf{5 t}$
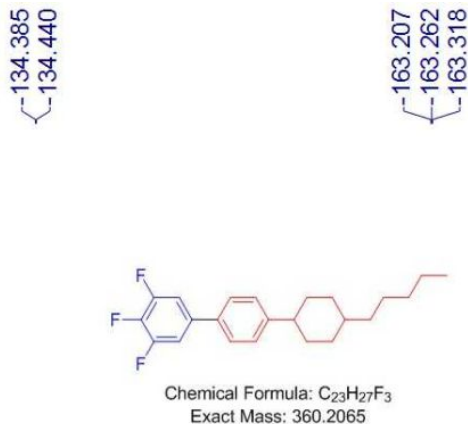

$\begin{array}{llllllllllllllllllllllllllll}-95 & -100 & -105 & -110 & -115 & -120 & -125 & -130 & -135 & -140 & -145 & -150 & -155 & -160 & -165 & -170 & -175 & -180 & -185 & -190 & -195 \\ \mathrm{f} 1(\mathrm{ppm}) & \end{array}$ 
${ }^{1} \mathrm{H}$ NMR $\left(\mathrm{CDCl}_{3}\right)$ spectrum of compound $\mathbf{5 u}$
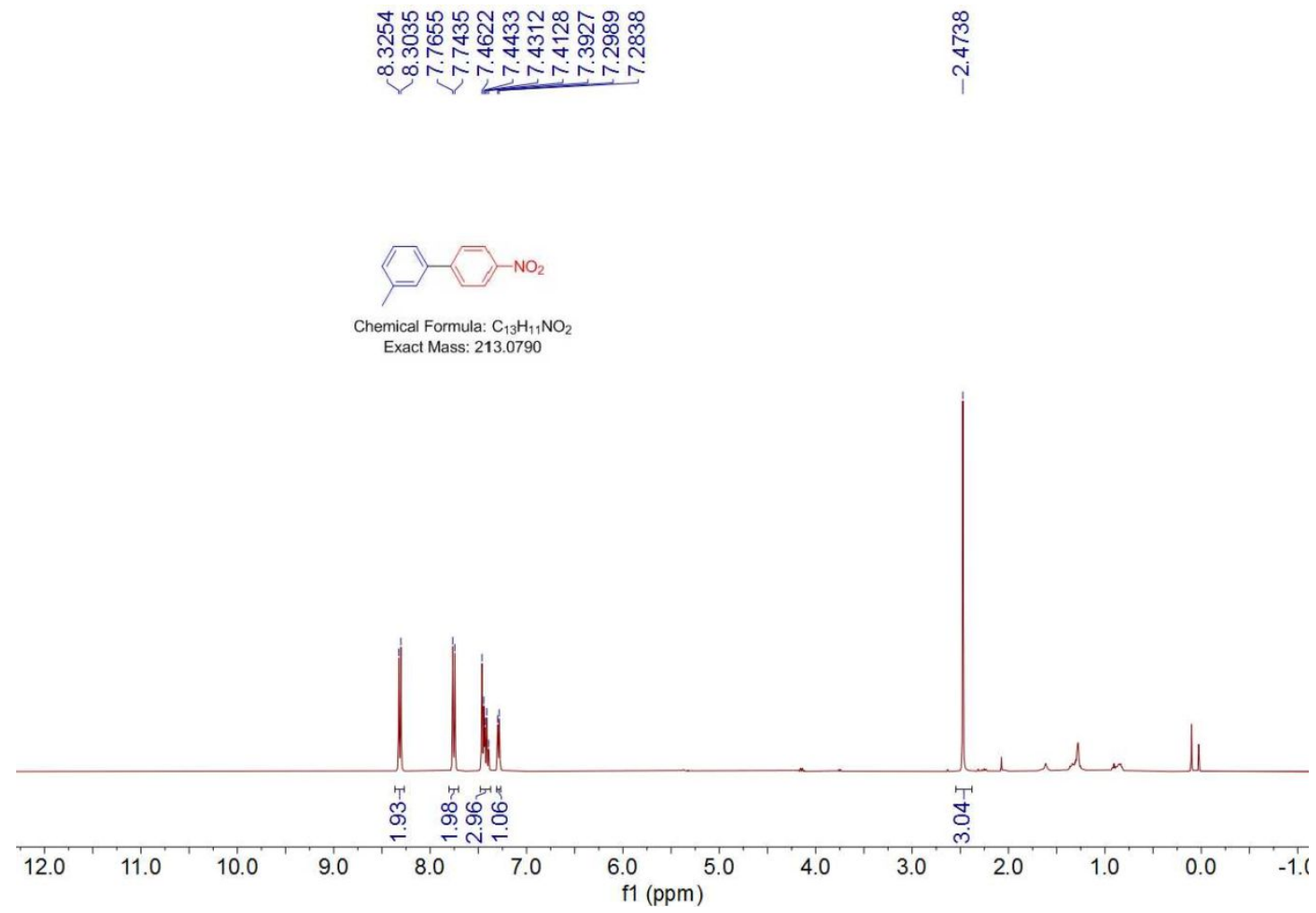

${ }^{13} \mathrm{C}$ NMR $\left(\mathrm{CDCl}_{3}\right)$ spectrum of compound $\mathbf{5 u}$

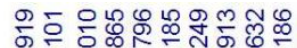

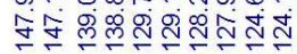

윰

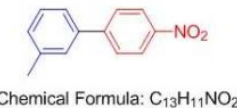

Exact Mass: 213.0790

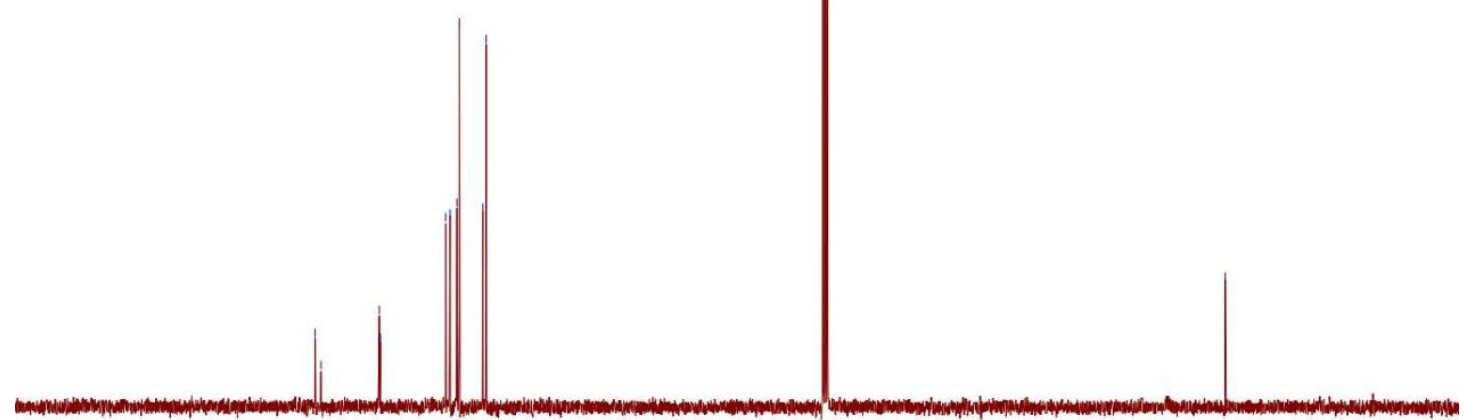

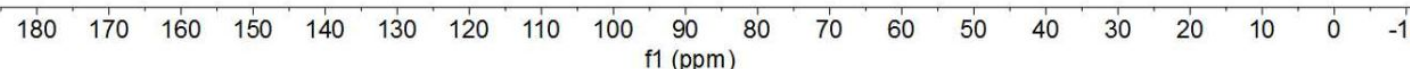


${ }^{1} \mathrm{H}$ NMR $\left(\mathrm{CDCl}_{3}\right)$ spectrum of compound $\mathbf{5 v}$
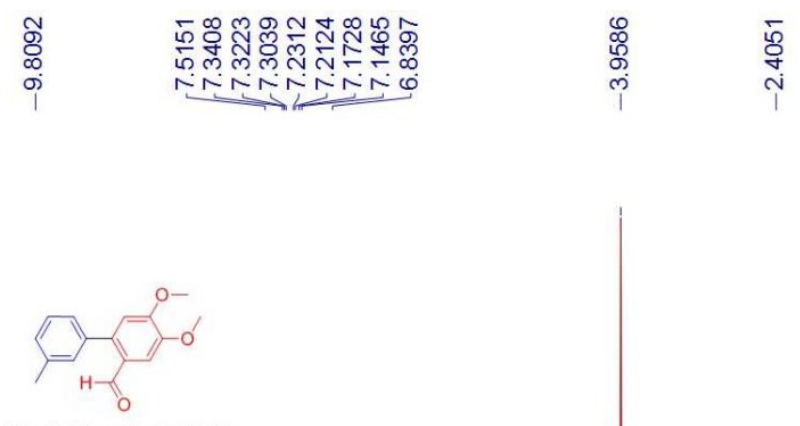

Chemical Formula: $\mathrm{C}_{16} \mathrm{H}_{16} \mathrm{O}_{3}$

Exact Mass: 256.1099
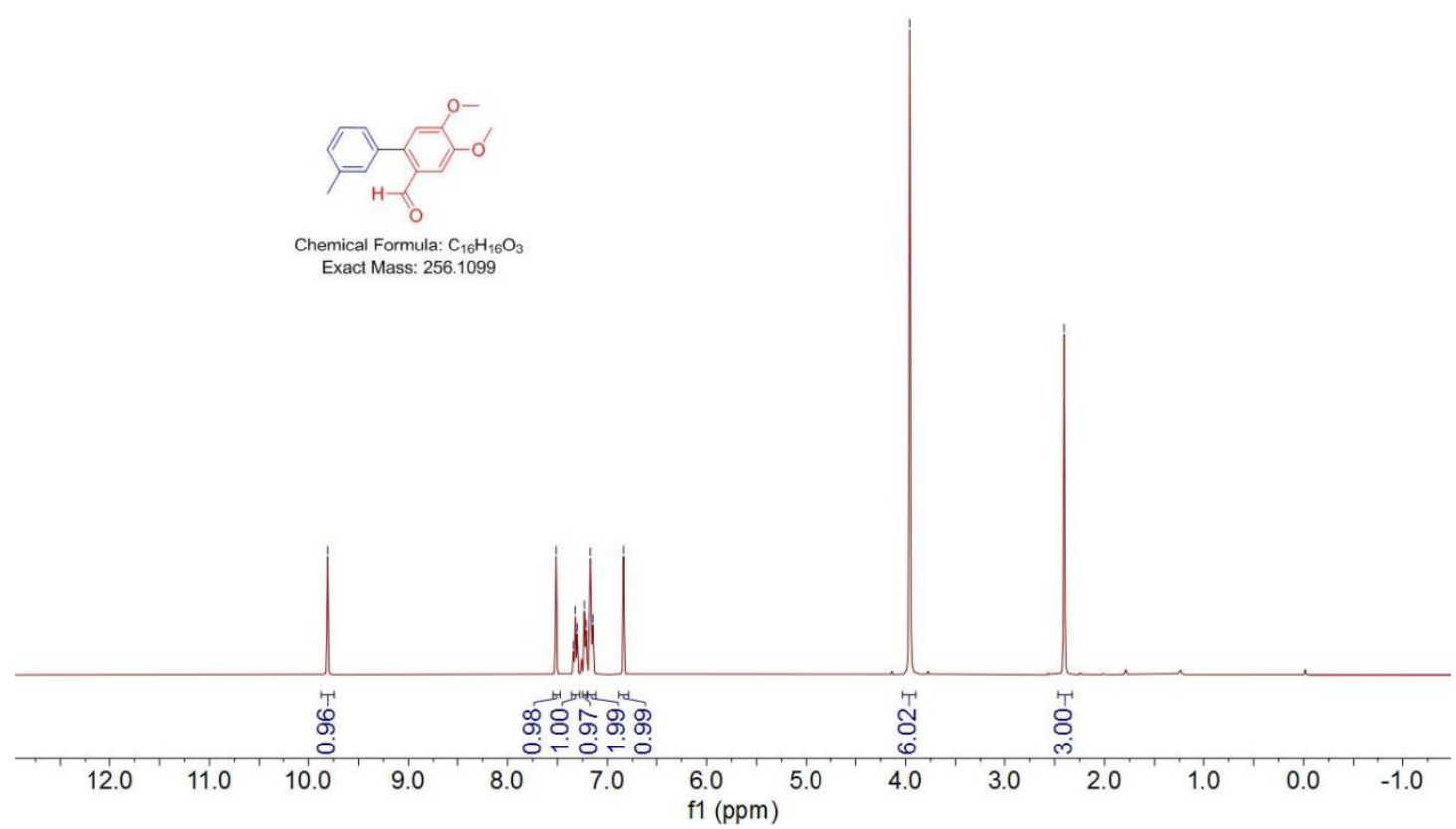

${ }^{13} \mathrm{C}$ NMR $\left(\mathrm{CDCl}_{3}\right)$ spectrum of compound $\mathbf{5 v}$
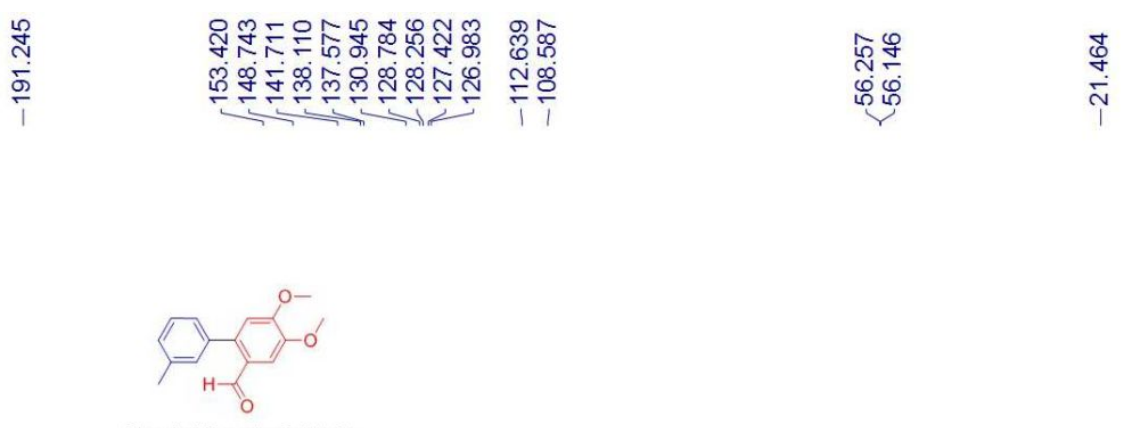

Chemical Formula: $\mathrm{C}_{10} \mathrm{H}_{10} \mathrm{O}_{3}$

Exact Mass: 256.1099

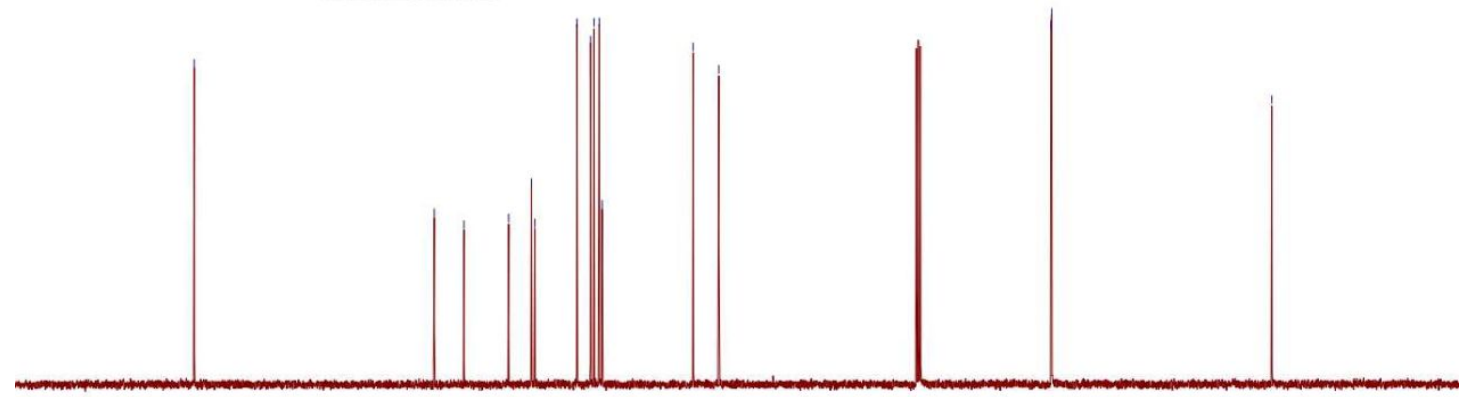

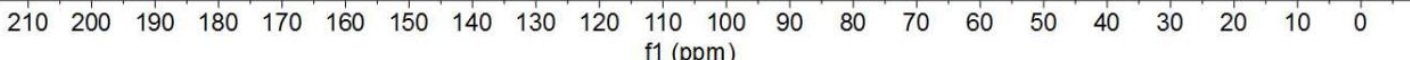


${ }^{1} \mathrm{H} \mathrm{NMR}\left(\mathrm{CDCl}_{3}\right)$ spectrum of compound $\mathbf{5 w}$
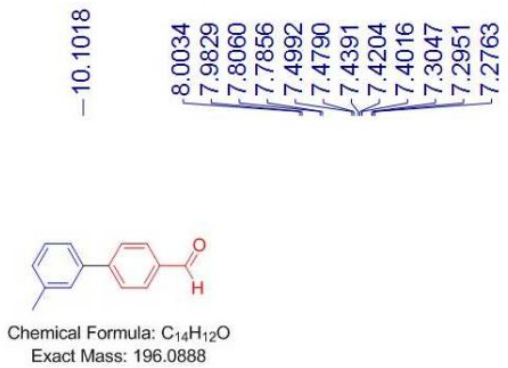

Exact Mass: 196.0888
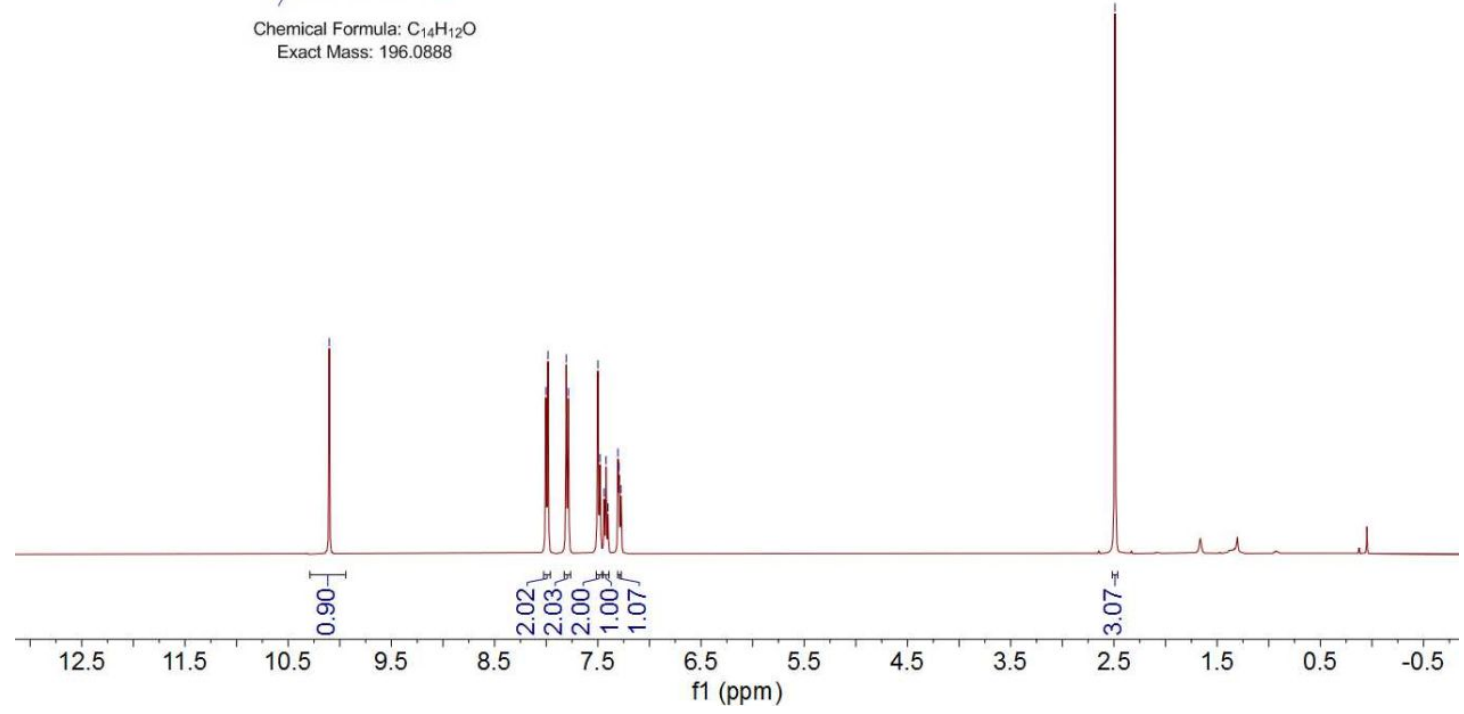

${ }^{13} \mathrm{C}$ NMR $\left(\mathrm{CDCl}_{3}\right)$ spectrum of compound $\mathbf{5 w}$

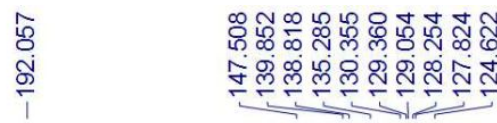

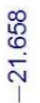

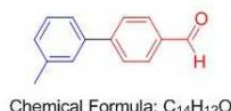

Exact Mass: 196.0888

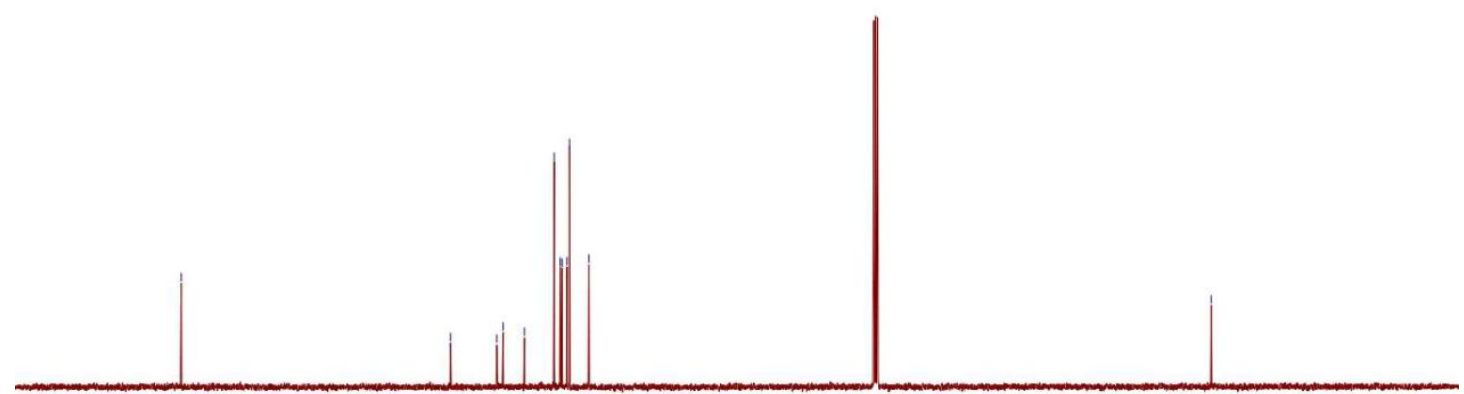

$\begin{array}{llllllllllllllllllllllllllll}210 & 200 & 190 & 180 & 170 & 160 & 150 & 140 & 130 & 120 & 110 & 100 & 90 & 80 & 70 & 60 & 50 & 40 & 30 & 20 & 10 & 0 & -10\end{array}$ f1 (ppm) 
${ }^{1} \mathrm{H}$ NMR $\left(\mathrm{CDCl}_{3}\right)$ spectrum of compound $\mathbf{5 x}$
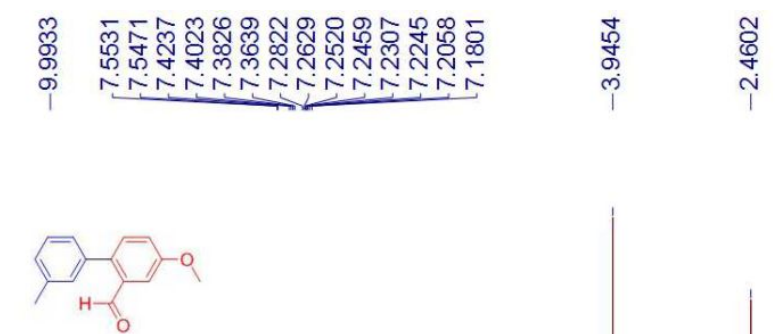

Chemical Formula: $\mathrm{C}_{15} \mathrm{H}_{14} \mathrm{O}_{2}$ Exact Mass: 226.0994

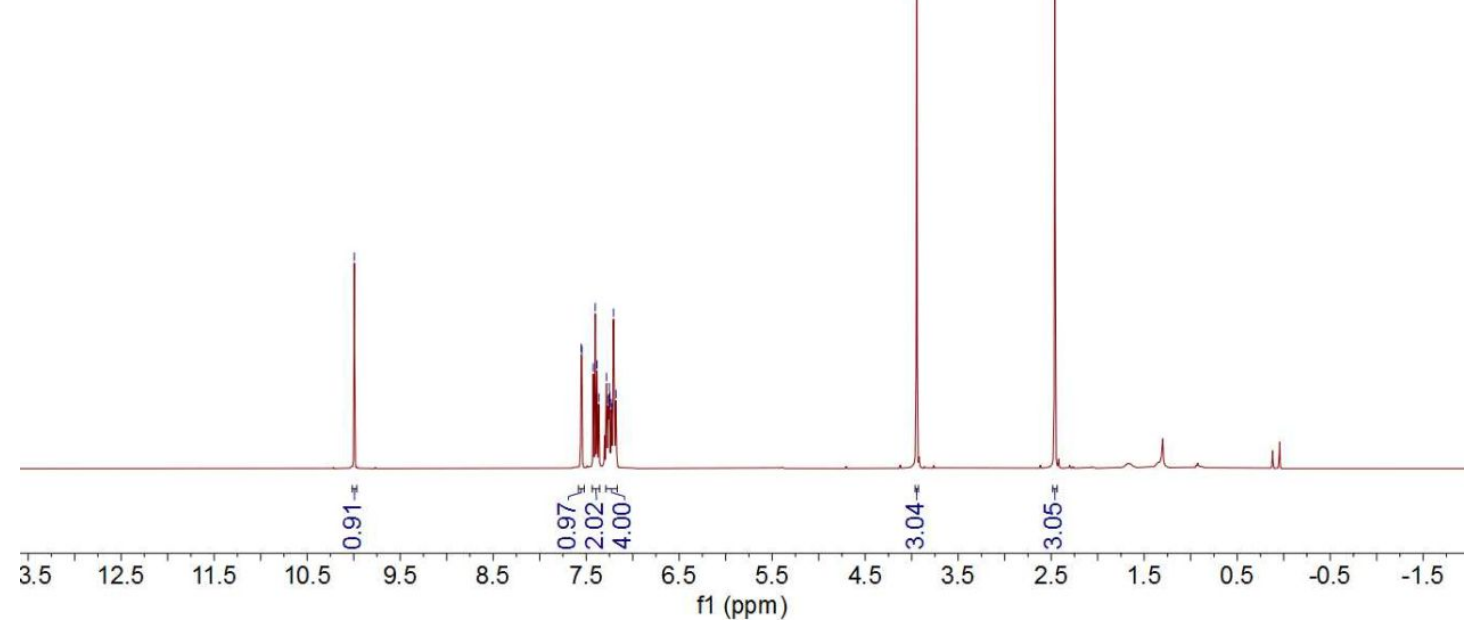

${ }^{13} \mathrm{C}$ NMR $\left(\mathrm{CDCl}_{3}\right)$ spectrum of compound $\mathbf{5 x}$
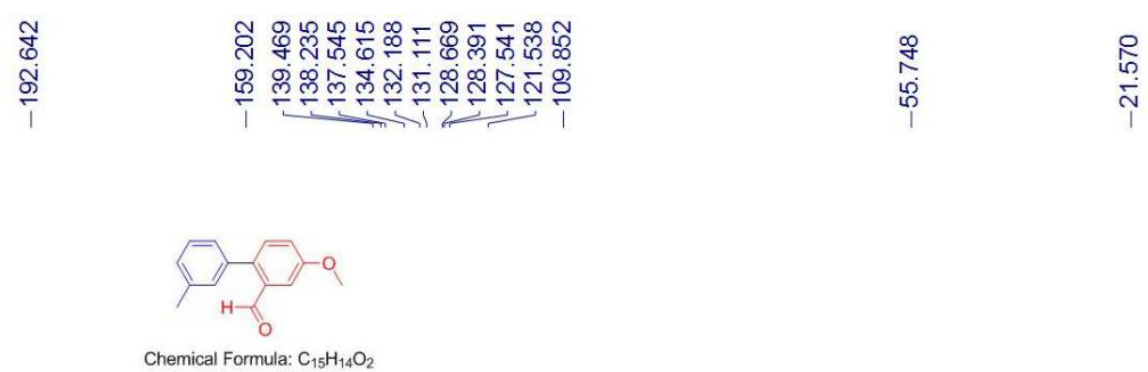

Chemical Formula: $\mathrm{C}_{15} \mathrm{H}_{14} \mathrm{O}_{2}$

Exact Mass: 226.0994

$\begin{array}{lllllllllllllllllllllll}210 & 200 & 190 & 180 & 170 & 160 & 150 & 140 & 130 & 120 & \begin{array}{c}110 \\ \mathrm{f} 1(\mathrm{ppm})\end{array} & 100 & 90 & 80 & 70 & 60 & 50 & 40 & 30 & 20 & 10 & 0\end{array}$ 
${ }^{1} \mathrm{H}$ NMR $\left(\mathrm{CDCl}_{3}\right)$ spectrum of compound $\mathbf{5 y}$

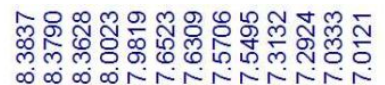

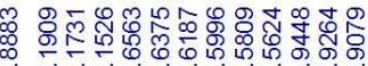

$\infty 0^{\circ} \infty \pi N-N N N$

ले mं m

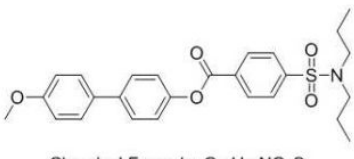

mical Formula: $\mathrm{C}_{26} \mathrm{H}_{29} \mathrm{NO}$

Exact Mass: 467.1766
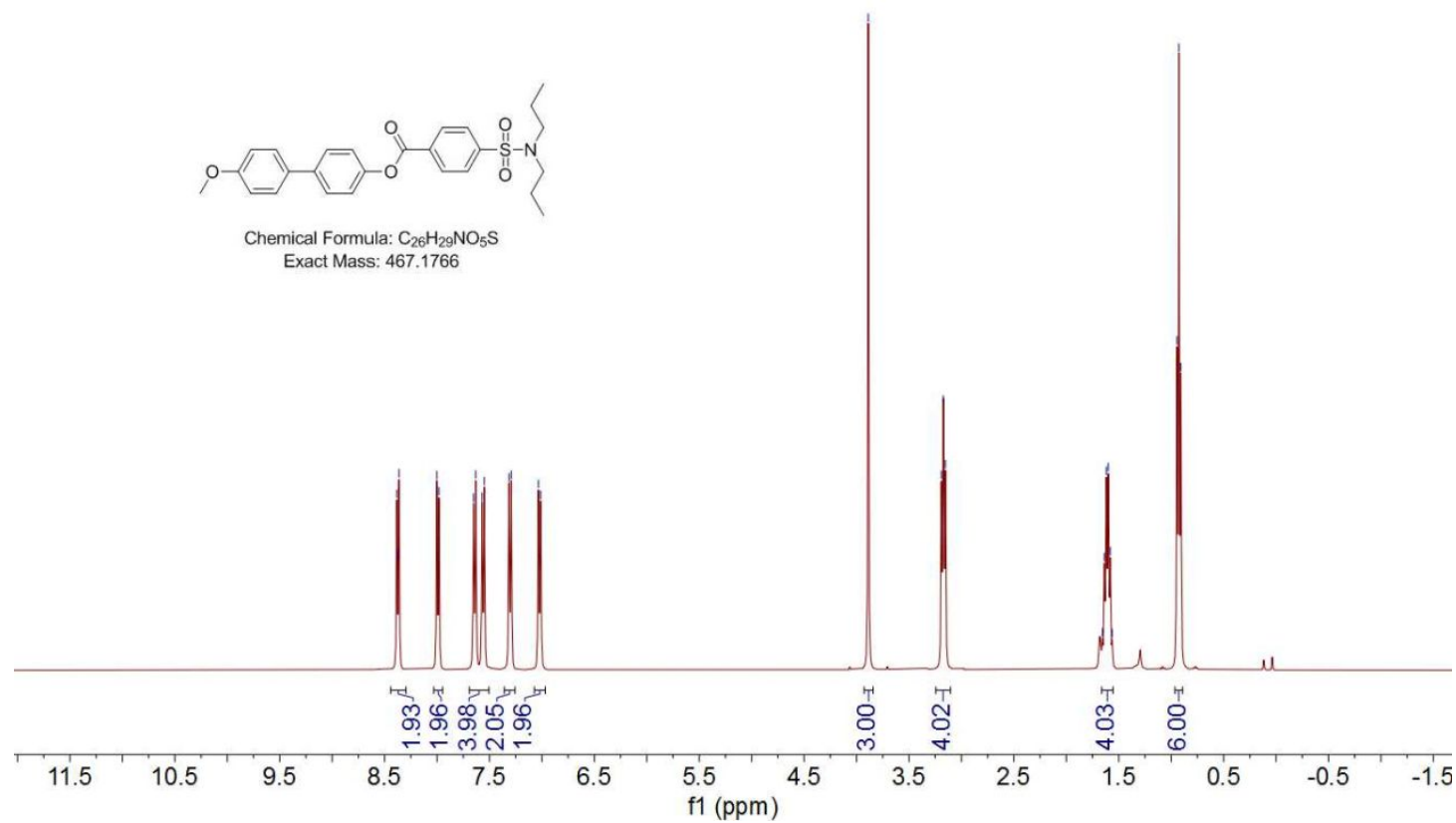

${ }^{13} \mathrm{C}$ NMR $\left(\mathrm{CDCl}_{3}\right)$ spectrum of compound $\mathbf{5 y}$

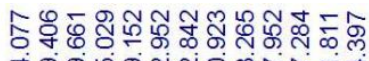

宅官守守色

웅

它

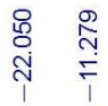

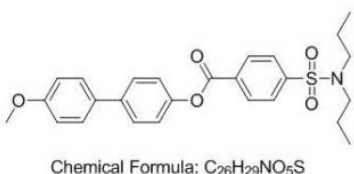

Exact Mass: 467.1766

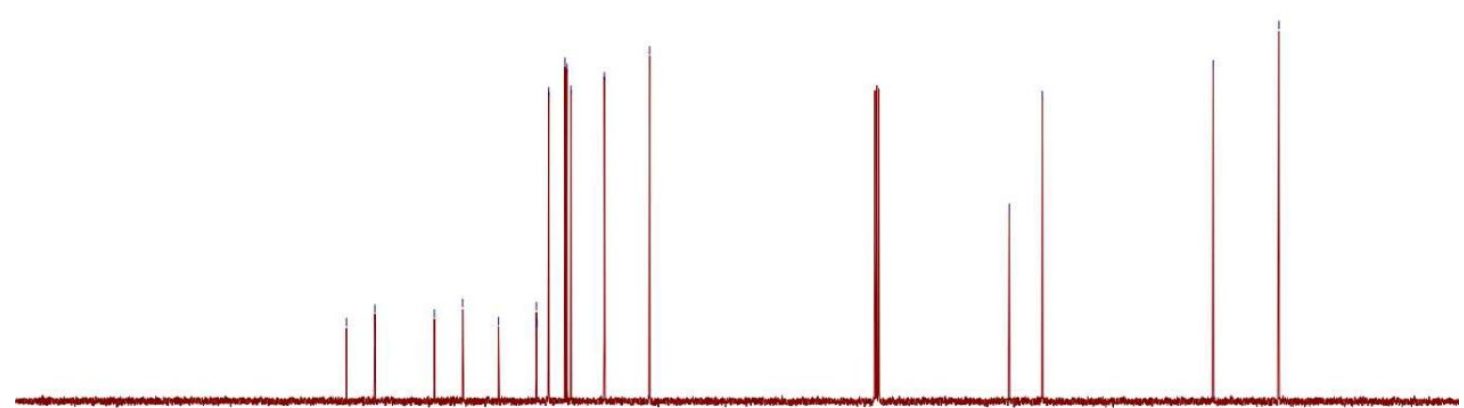

$\begin{array}{lllllllllllllllllllllllllll}210 & 200 & 190 & 180 & 170 & 160 & 150 & 140 & 130 & 120 & 110 & 100 & 90 & 80 & 70 & 60 & 50 & 40 & 30 & 20 & 10 & 0 & -10\end{array}$ 
${ }^{1} \mathrm{H}$ NMR $\left(\mathrm{CDCl}_{3}\right)$ spectrum of compound $\mathbf{5 z}$
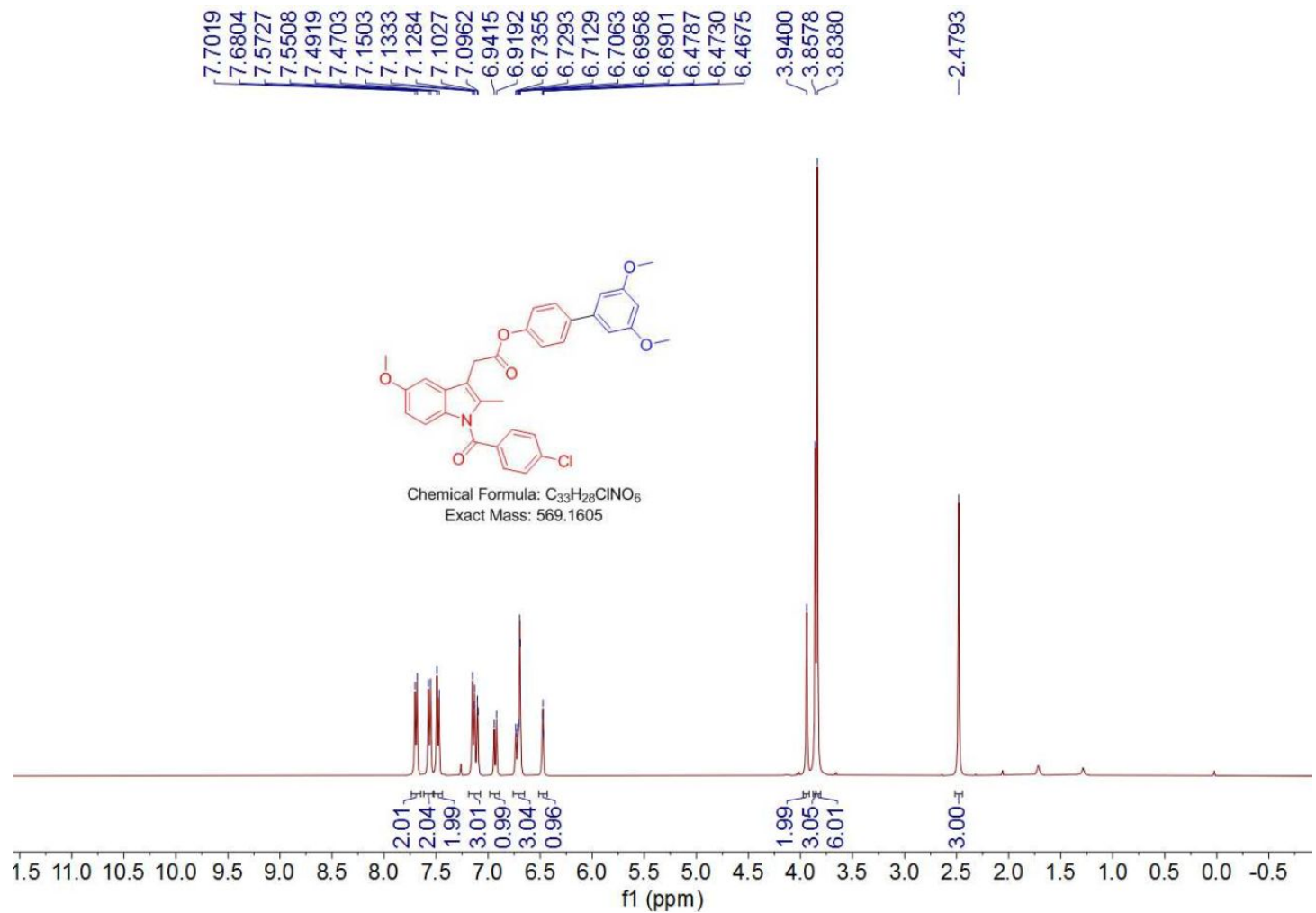

${ }^{13} \mathrm{C}$ NMR $\left(\mathrm{CDCl}_{3}\right)$ spectrum of compound $\mathbf{5 z}$

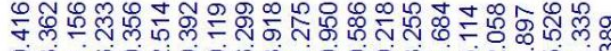

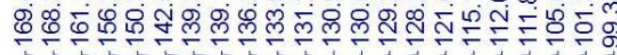
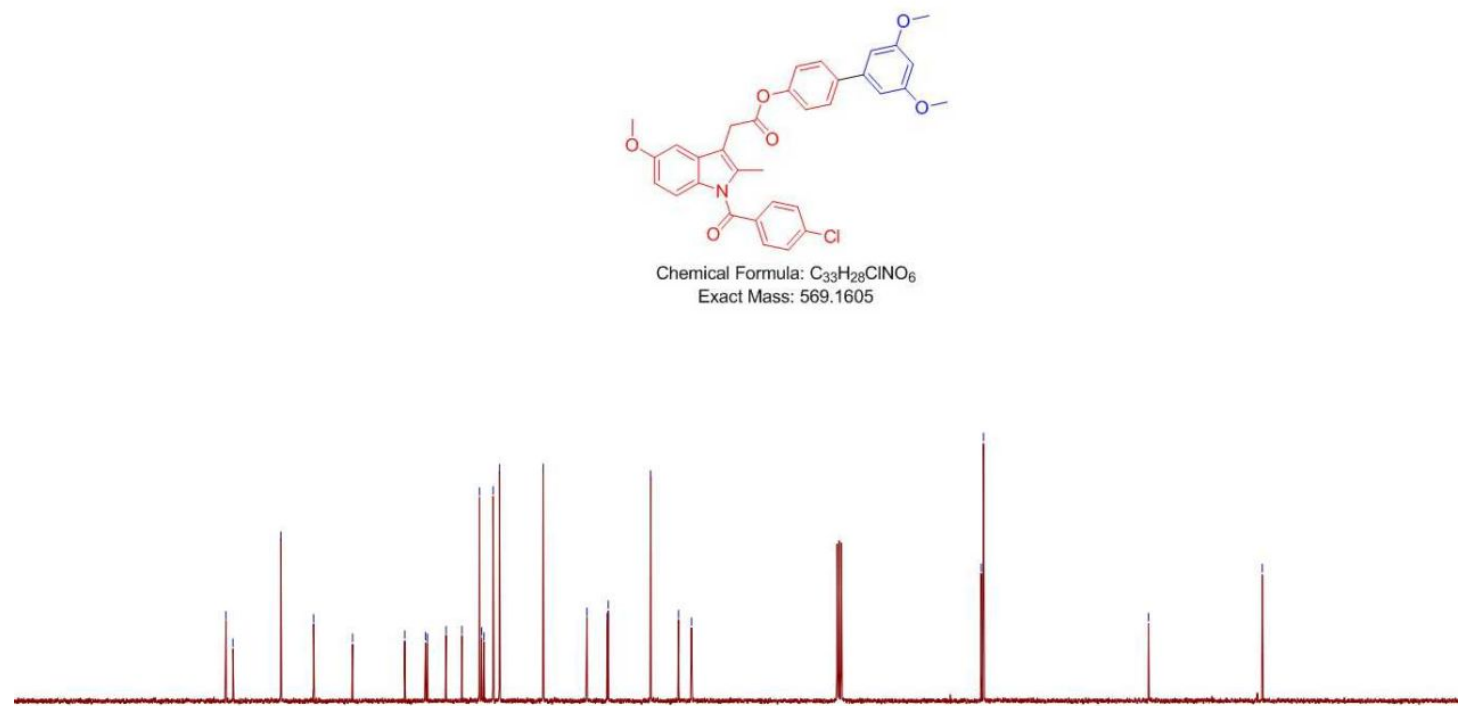

\begin{tabular}{lllllllllllllllllllllll}
\hline 00 & 190 & 180 & 170 & 160 & 150 & 140 & 130 & 120 & 110 & $\begin{array}{c}100 \\
\mathrm{f} 1(\mathrm{ppm})\end{array}$ & 90 & 70 & 60 & 50 & 40 & 30 & 20 & 10 & 0 & -10
\end{tabular} 
${ }^{1} \mathrm{H}$ NMR $\left(\mathrm{CDCl}_{3}\right)$ spectrum of compound $\mathbf{5 a a}$
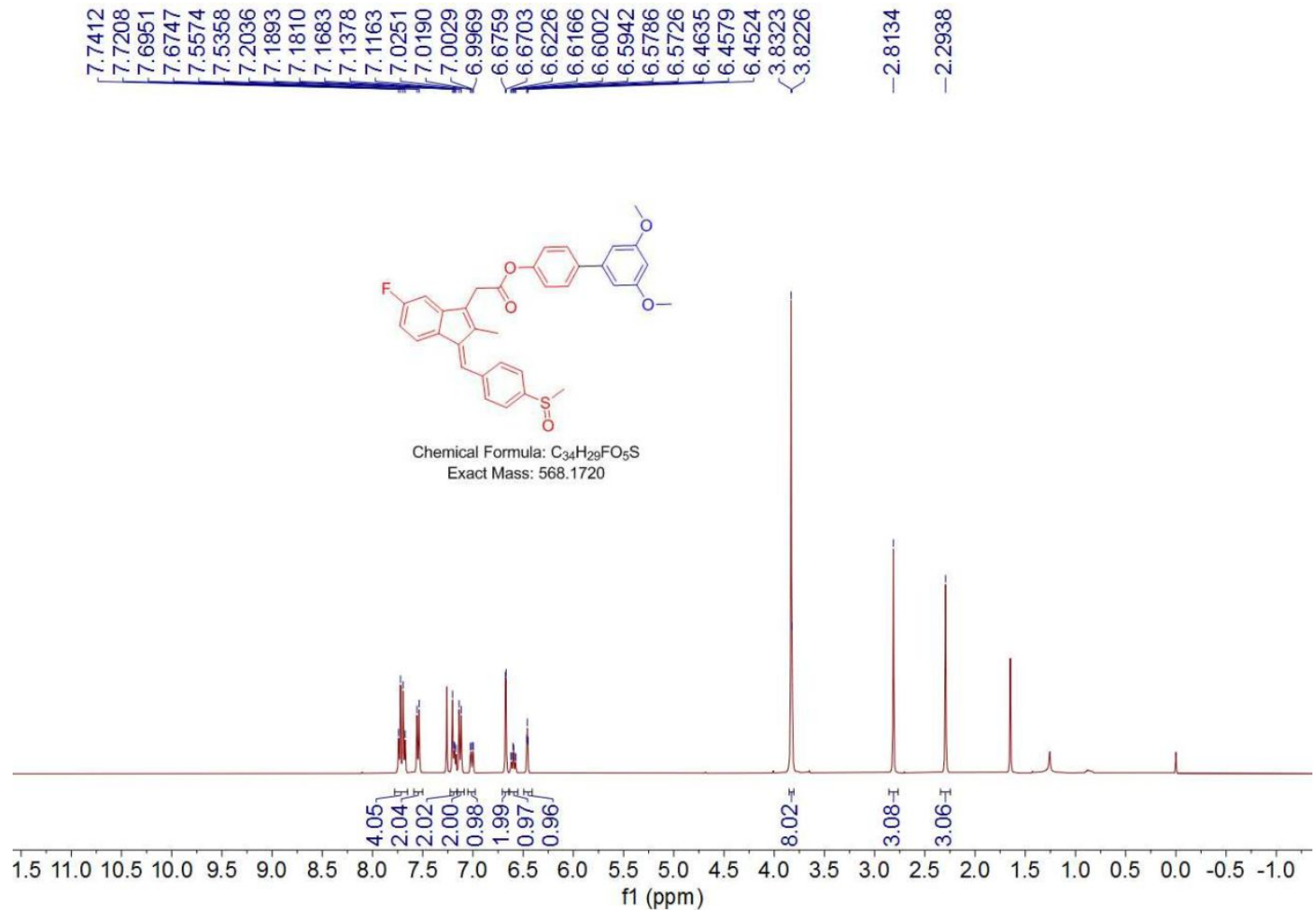

${ }^{13} \mathrm{C} \mathrm{NMR}\left(\mathrm{CDCl}_{3}\right)$ spectrum of compound $\mathbf{5 a a}$

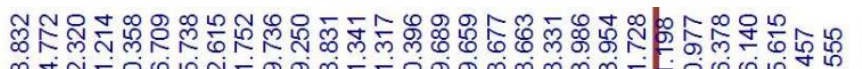

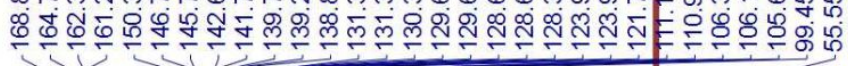

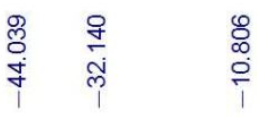

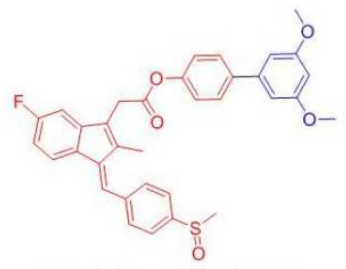

Chemical Formula: $\mathrm{C}_{34} \mathrm{H}_{29} \mathrm{FO}_{5} \mathrm{~S}$

Exact Mass: 568.1720

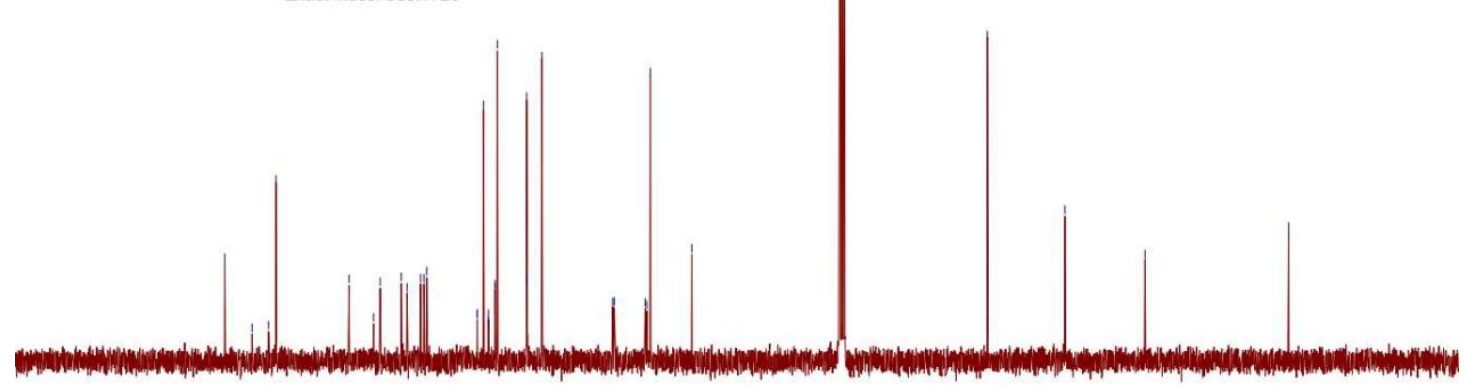

$\begin{array}{llllllllllllllllllllll}190 & 180 & 170 & 160 & 150 & 140 & 130 & 120 & 110 & \underset{\mathrm{f} 1}{100} \mathbf{( \mathrm { ppm } )} & 90 & 80 & 70 & 60 & 50 & 40 & 30 & 20 & 10 & 0 & -10\end{array}$ 
${ }^{19} \mathrm{~F} \mathrm{NMR}\left(\mathrm{CDCl}_{3}\right)$ spectrum of compound $\mathbf{5 a a}$

$\stackrel{N}{6}$
$\stackrel{i}{i}$

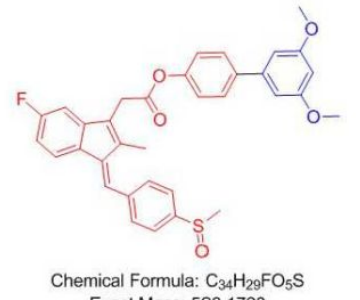

Exact Mass: 568.1720

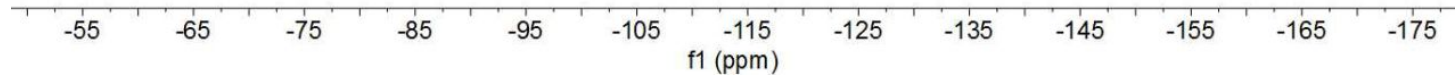

${ }^{1} \mathrm{H}$ NMR $\left(\mathrm{CDCl}_{3}\right)$ spectrum of compound $\mathbf{5 a b}$

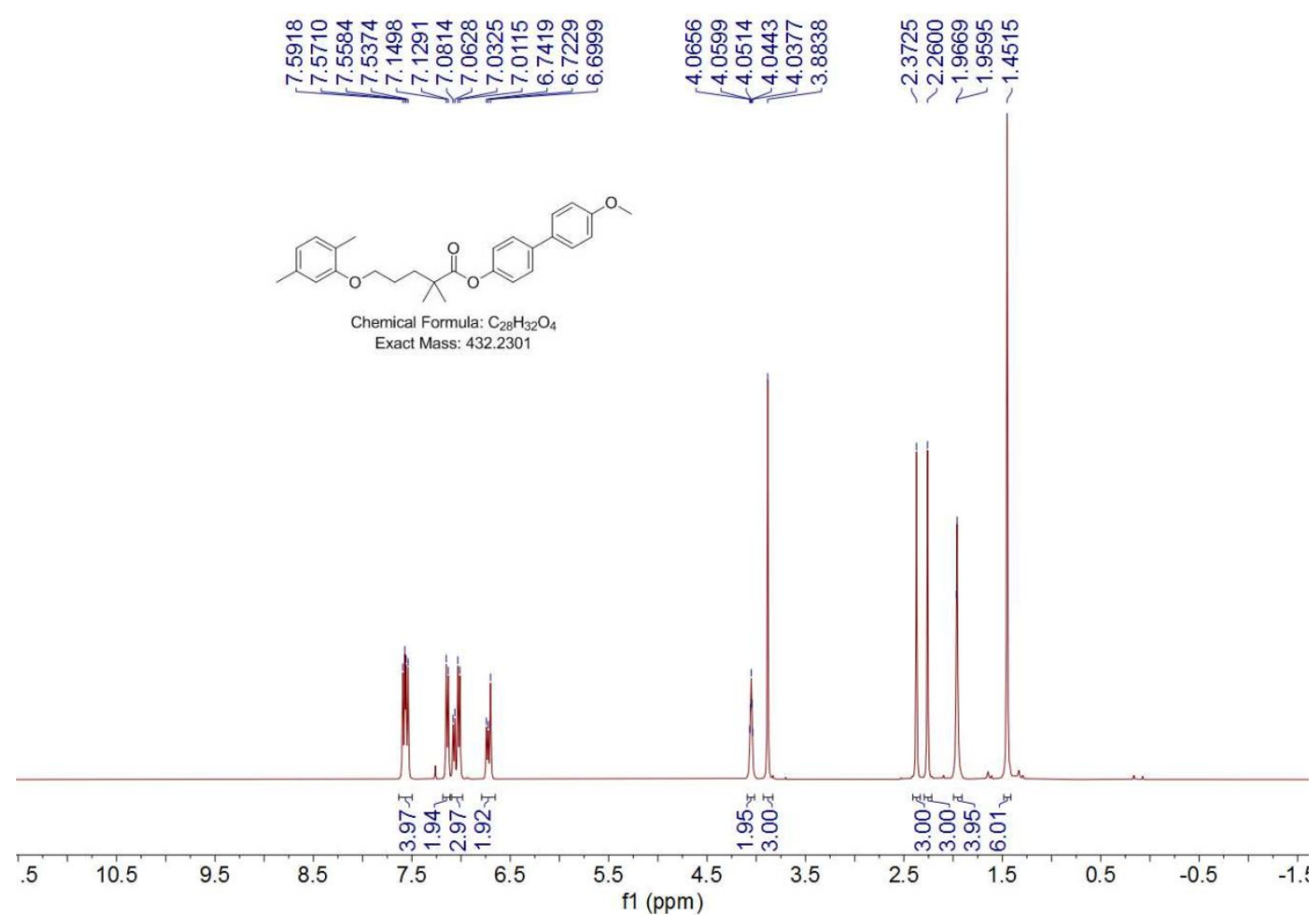


${ }^{13} \mathrm{C}$ NMR $\left(\mathrm{CDCl}_{3}\right)$ spectrum of compound $\mathbf{5 a b}$

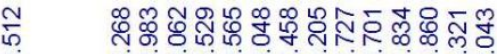

ڤ

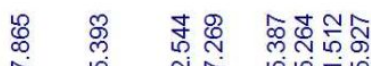

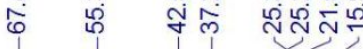

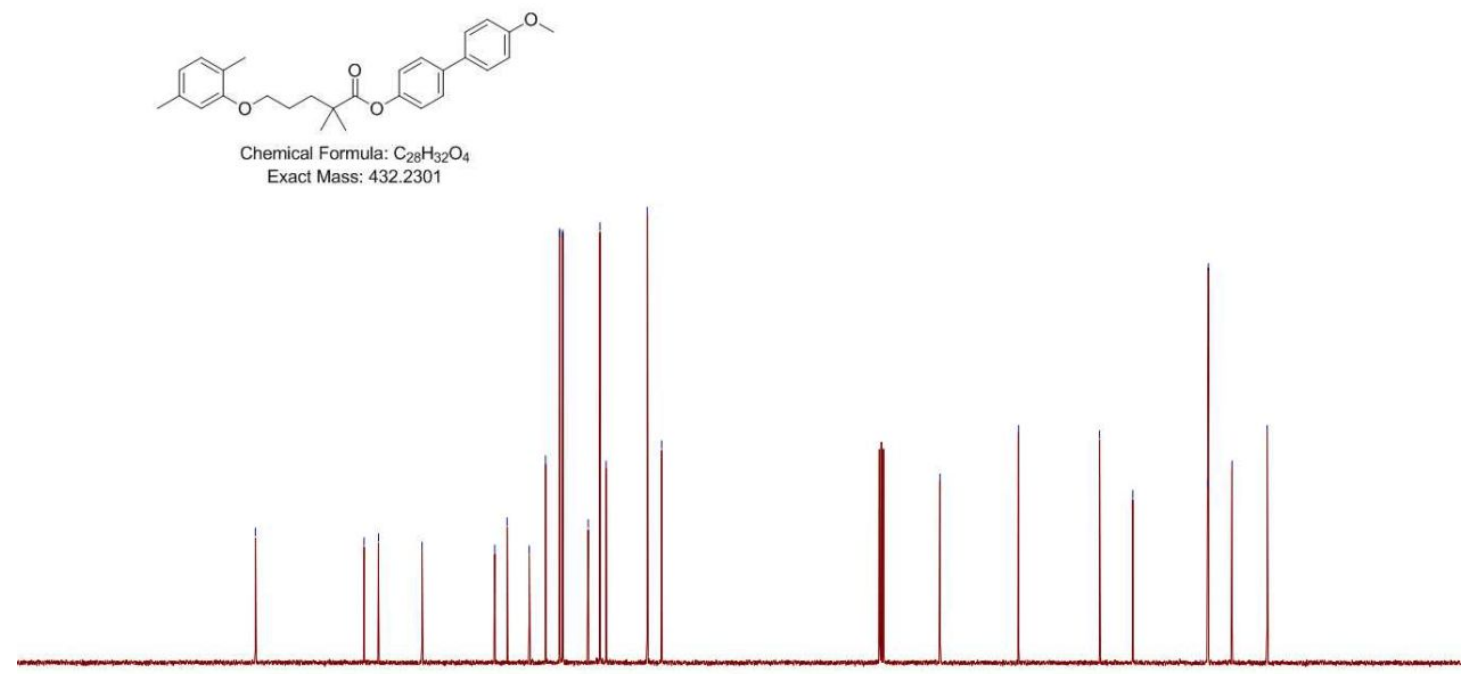

$\begin{array}{lllllllllllllllllllllllll}210 & 200 & 190 & 180 & 170 & 160 & 150 & 140 & 130 & 120 & 110 & 100 & 90 & 80 & 70 & 60 & 50 & 40 & 30 & 20 & 10 & 0 & -10\end{array}$

${ }^{1} \mathrm{H}$ NMR $\left(\mathrm{CDCl}_{3}\right)$ spectrum of compound $\mathbf{5 a c}$

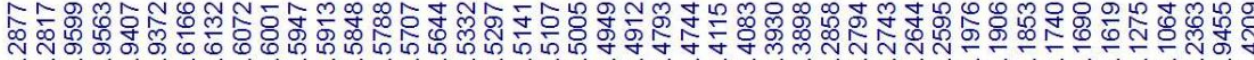

$\infty$

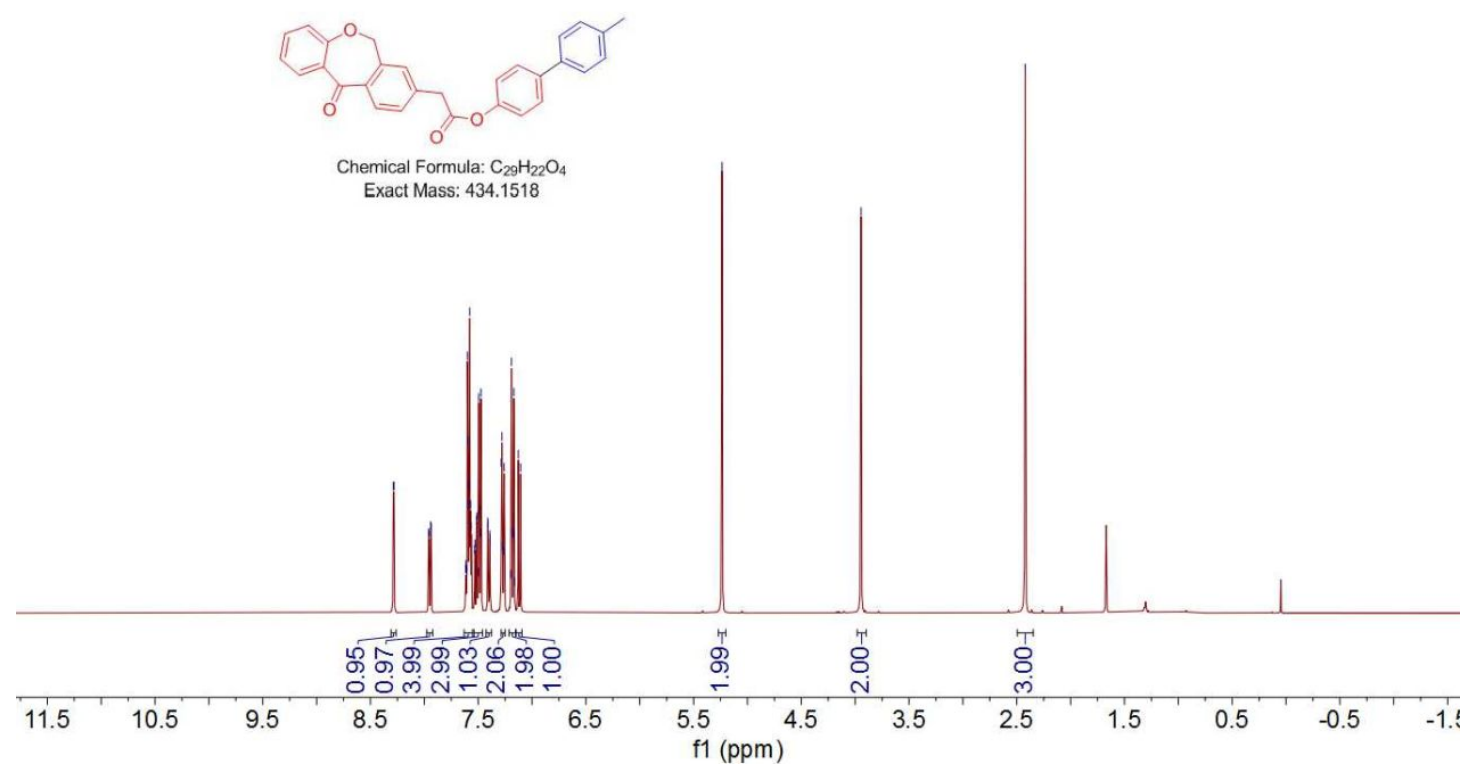


${ }^{13} \mathrm{C} \mathrm{NMR}\left(\mathrm{CDCl}_{3}\right)$ spectrum of compound $\mathbf{5 a c}$

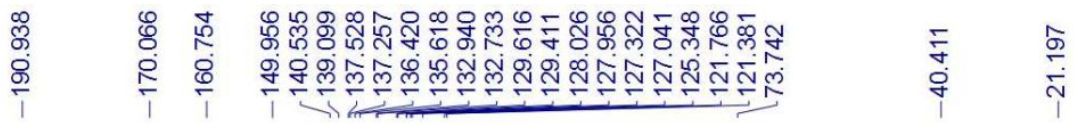

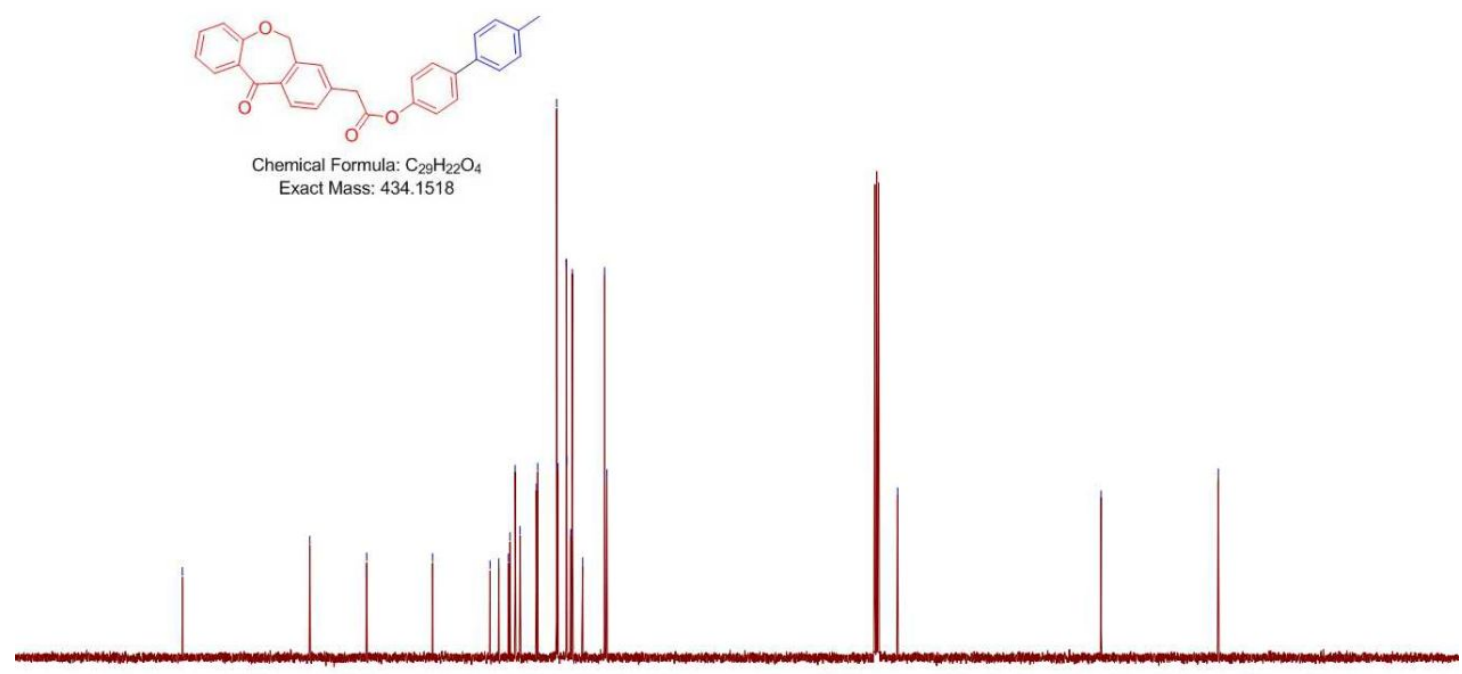

$\begin{array}{llllllllllllllllllllllllll}210 & 200 & 190 & 180 & 170 & 160 & 150 & 140 & 130 & 120 & 110 & 100 & 90 & 80 & 70 & 60 & 50 & 40 & 30 & 20 & 10 & 0 & -10\end{array}$

${ }^{1} \mathrm{H}$ NMR $\left(\mathrm{CDCl}_{3}\right)$ spectrum of compound $\mathbf{5 a d}$

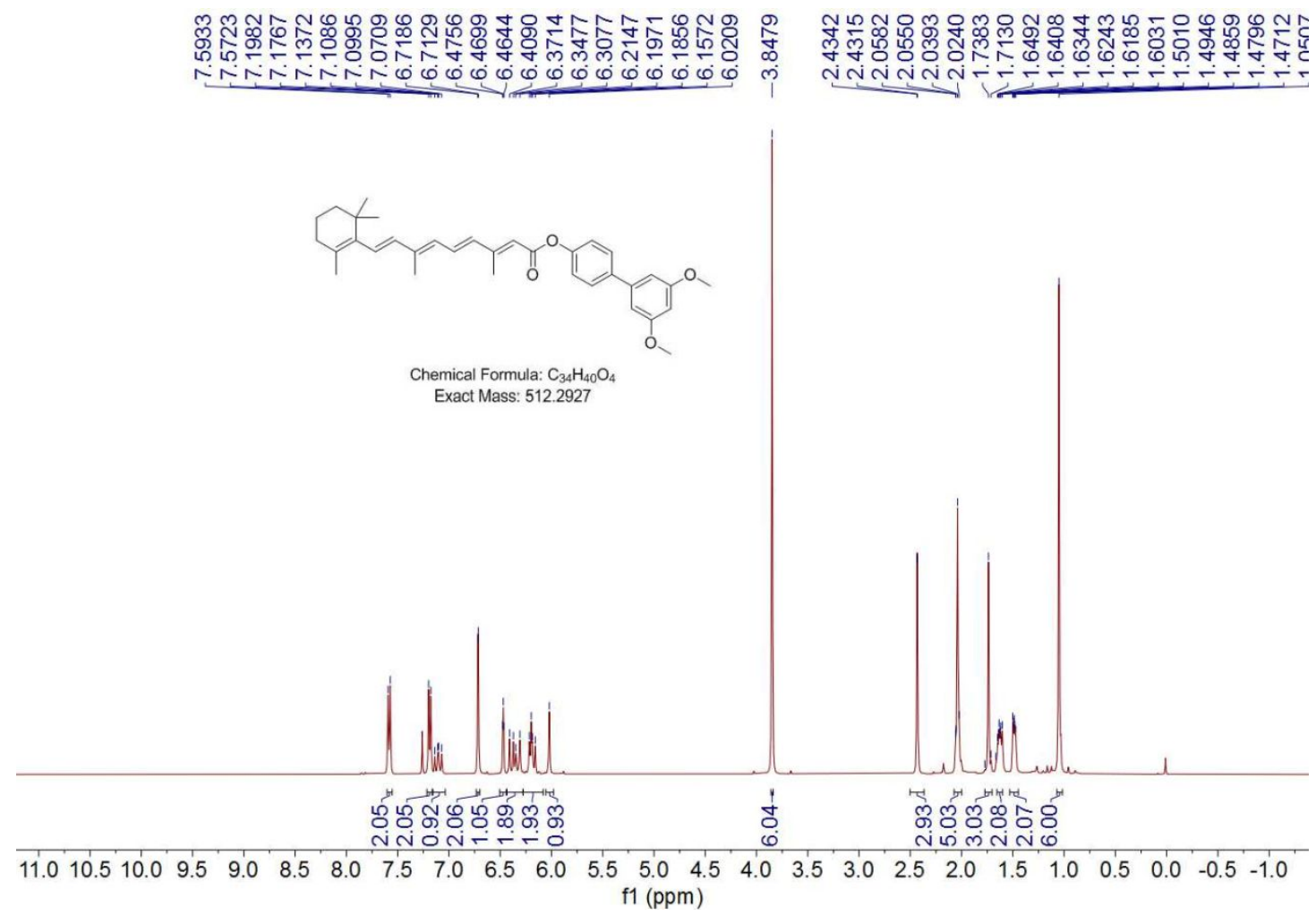


${ }^{13} \mathrm{C}$ NMR $\left(\mathrm{CDCl}_{3}\right)$ spectrum of compound $\mathbf{5 a d}$

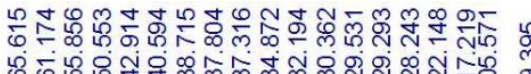

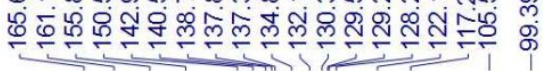

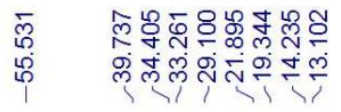

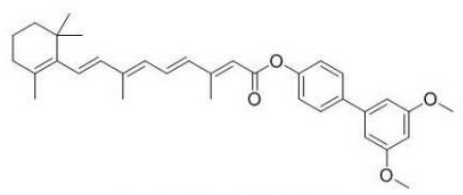

Chemical Formula: $\mathrm{C}_{34} \mathrm{H}_{40} \mathrm{O}$ Exact Mass: 512.2927

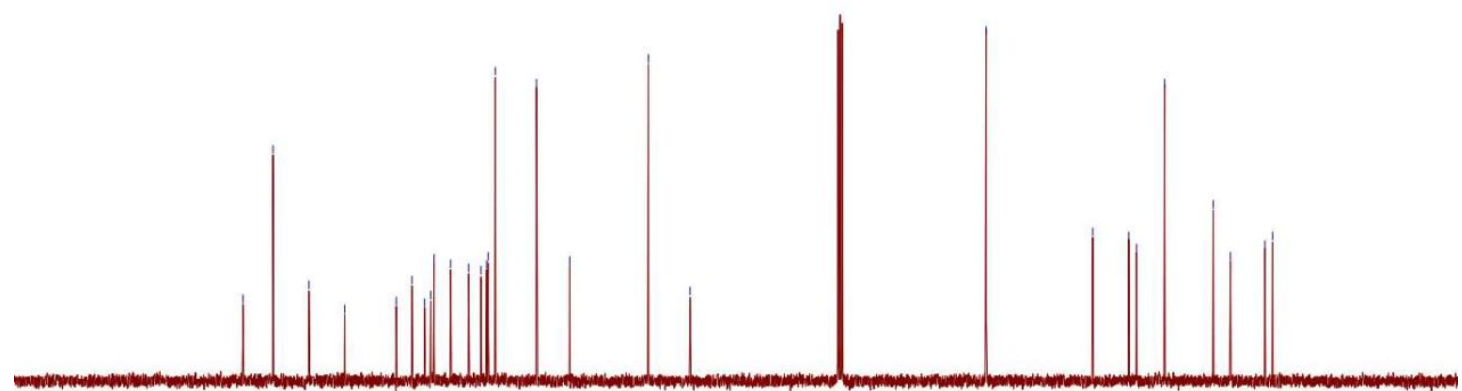

$\begin{array}{lllllllllllllllllllll}190 & 180 & 170 & 160 & 150 & 140 & 130 & 120 & 110 & 100 & 90 & 80 & 70 & 60 & 50 & 40 & 30 & 20 & 10 & 0 & -10\end{array}$

${ }^{1} \mathrm{H}$ NMR $\left(\mathrm{CDCl}_{3}\right)$ spectrum of compound 5ae

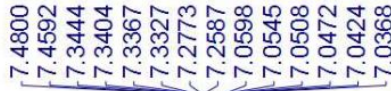

đo
$\infty$

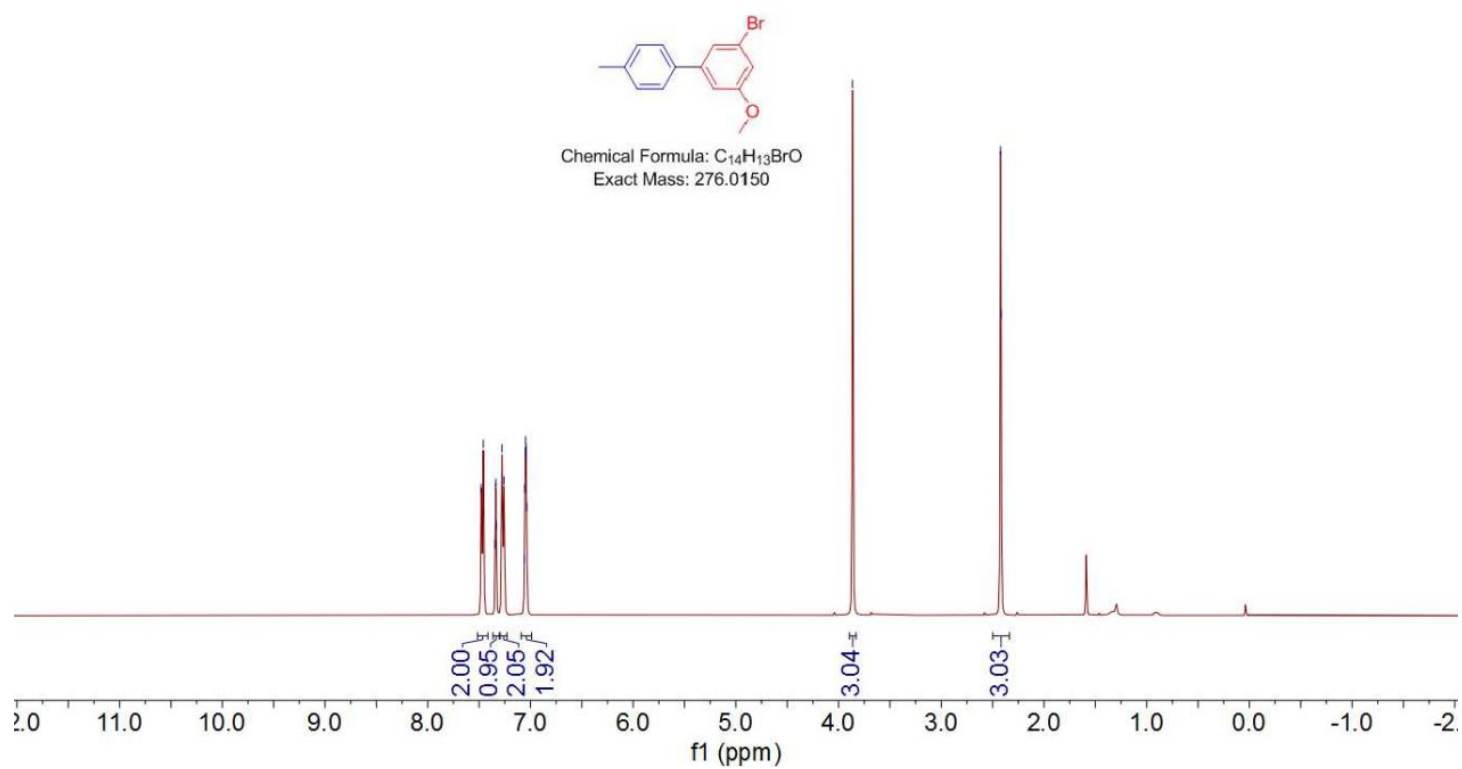


${ }^{13} \mathrm{C} \mathrm{NMR}\left(\mathrm{CDCl}_{3}\right)$ spectrum of compound $5 \mathbf{a e}$

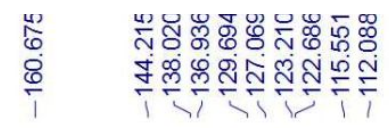

胥

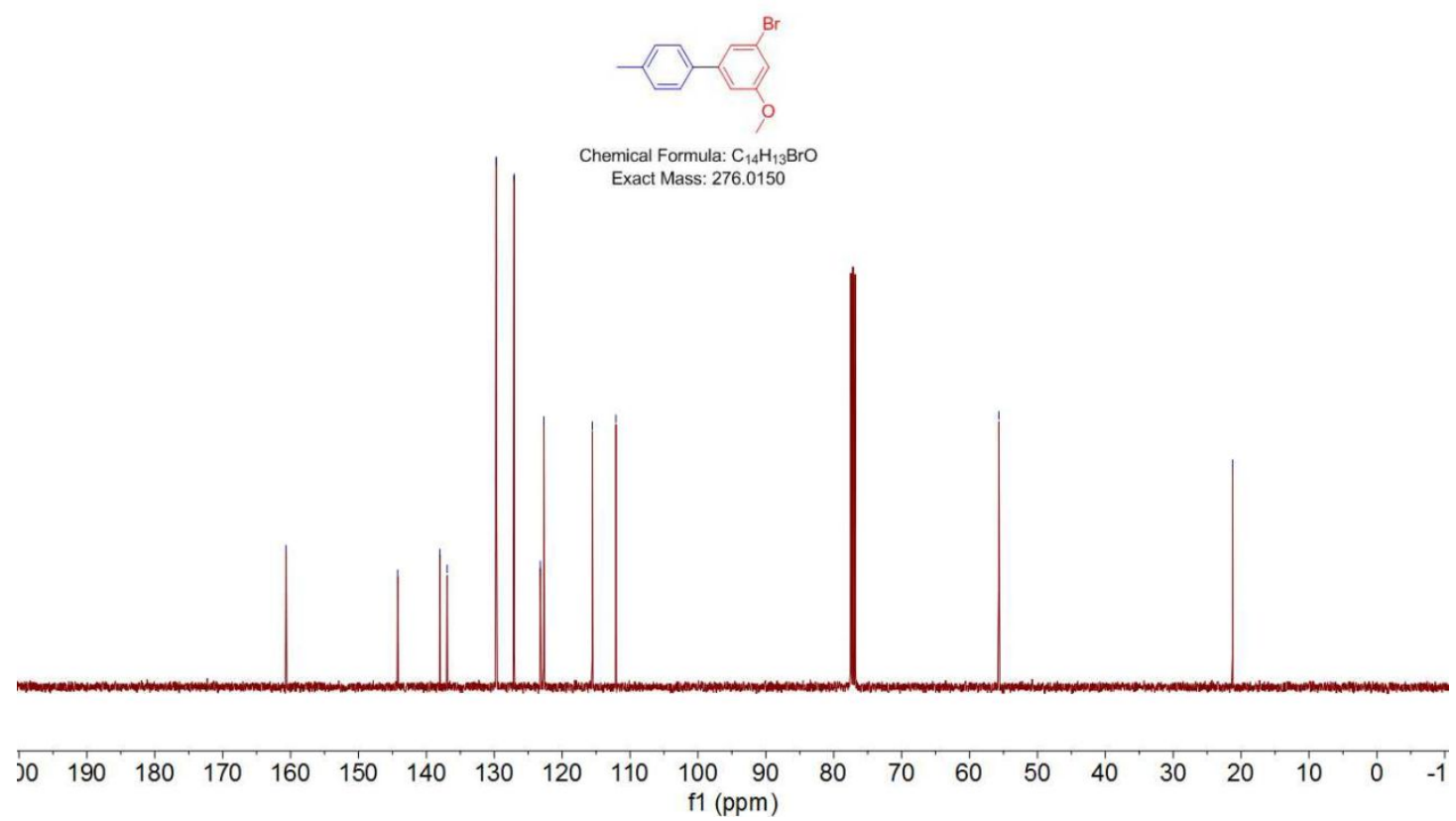

${ }^{1} \mathrm{H}$ NMR $\left(\mathrm{CDCl}_{3}\right)$ spectrum of compound $\mathbf{5 a f}$

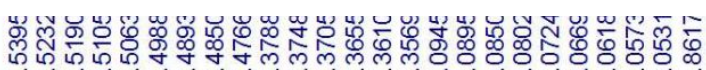

NNNNNNNNNNNNNNNNNNNNNm

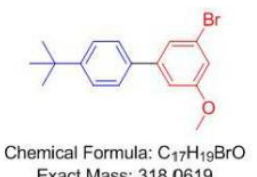

Exact Mass: 318.0619

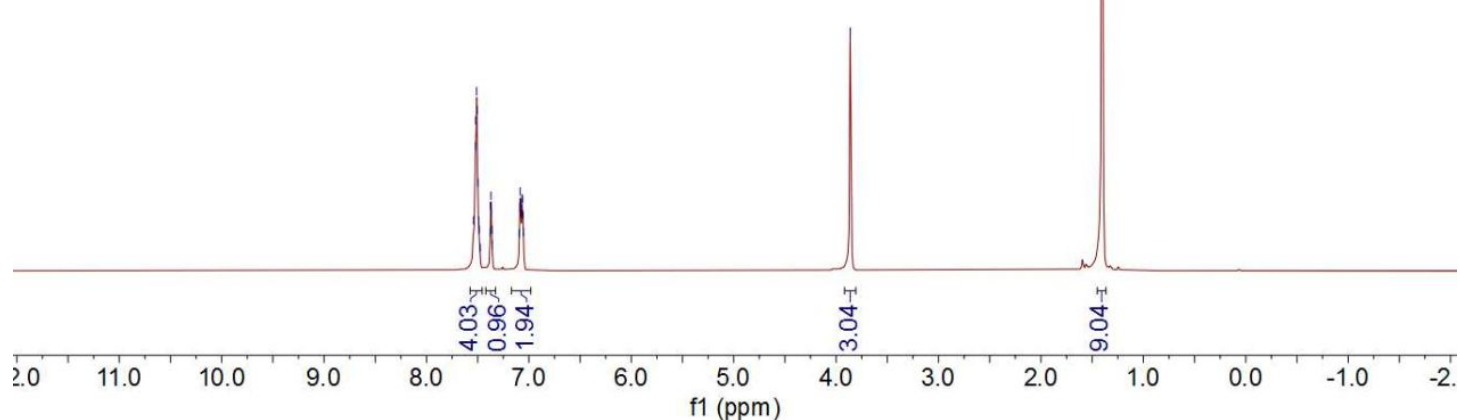


${ }^{13} \mathrm{C}$ NMR $\left(\mathrm{CDCl}_{3}\right)$ spectrum of compound $\mathbf{5 a f}$

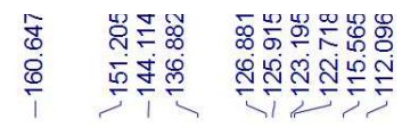

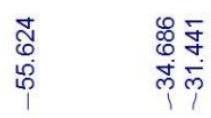

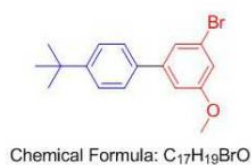

Exact Mass: 318.0619

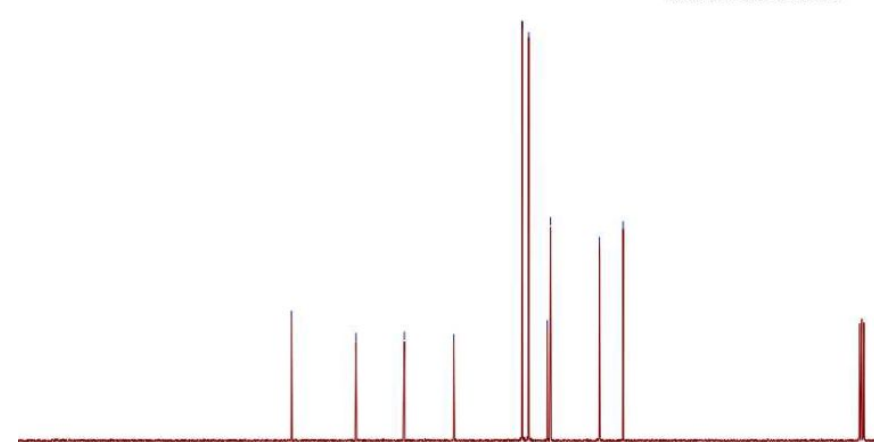

\begin{tabular}{rlllllllllllllllllllll}
\hline 0 & 190 & 180 & 170 & 160 & 150 & 140 & 130 & 120 & 110 & $\begin{array}{r}100 \\
\mathrm{f} 1(\mathrm{ppm})\end{array}$ & 90 & 80 & 70 & 60 & 50 & 40 & 30 & 20 & 10 & 0 & -1
\end{tabular}

${ }^{1} \mathrm{H}$ NMR $\left(\mathrm{CDCl}_{3}\right)$ spectrum of compound $\mathbf{5 a g}$

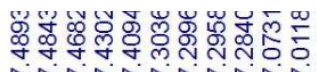

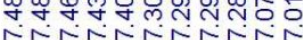

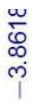

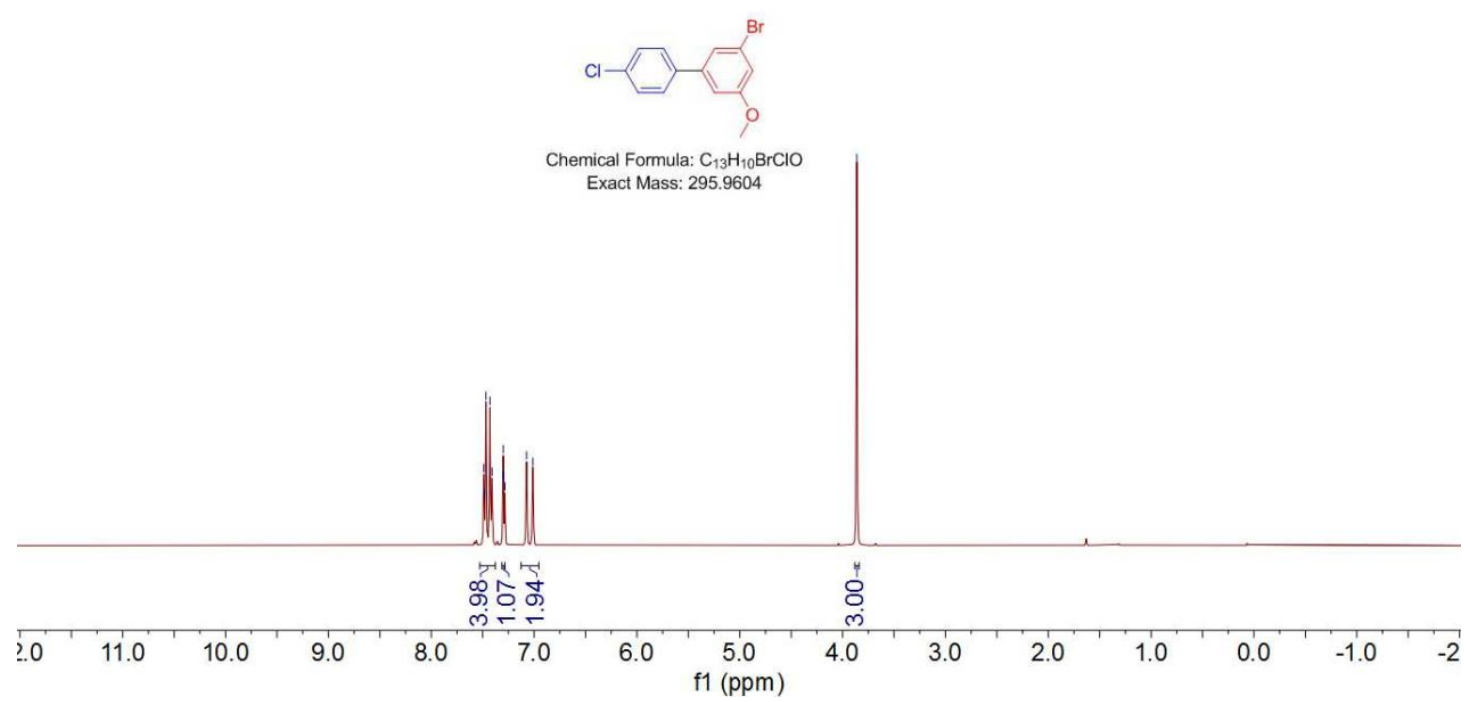


${ }^{13} \mathrm{C}$ NMR $\left(\mathrm{CDCl}_{3}\right)$ spectrum of compound $\mathbf{5 a g}$

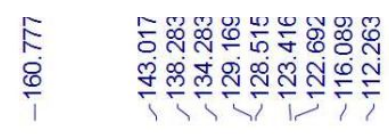

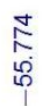

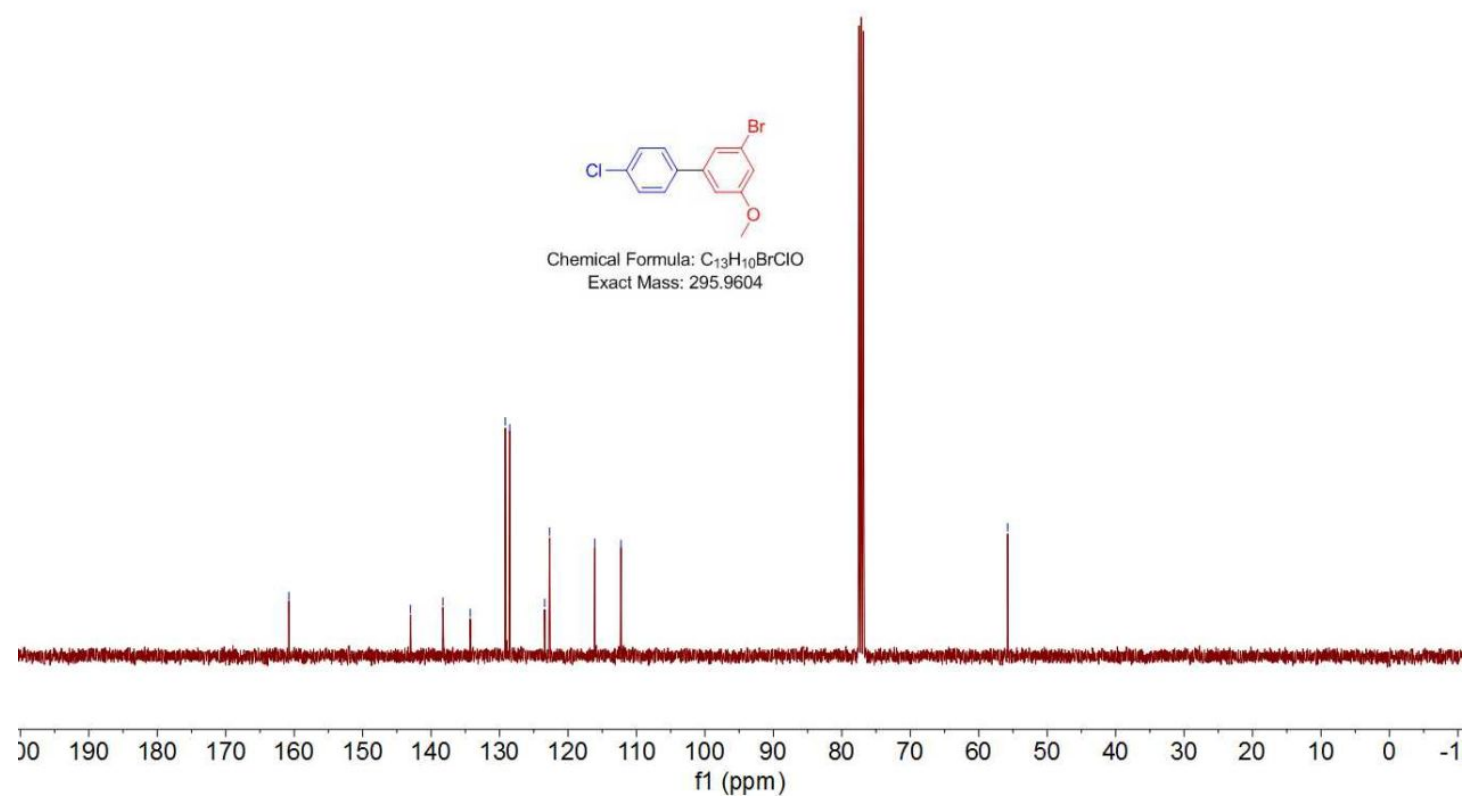

${ }^{1} \mathrm{H}$ NMR $\left(\mathrm{CDCl}_{3}\right)$ spectrum of compound $\mathbf{5 a h}$

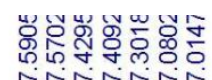

NNNNN

:

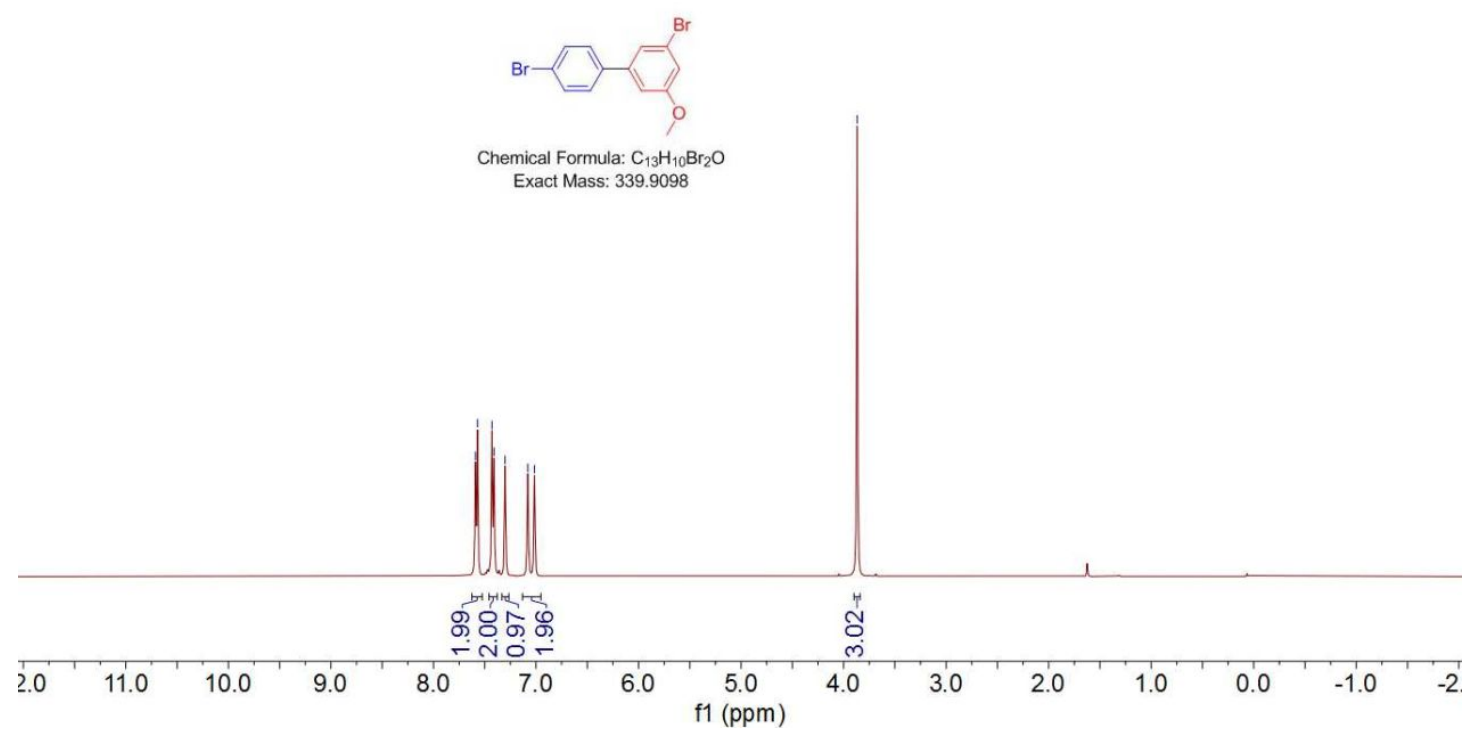


${ }^{13} \mathrm{C} \mathrm{NMR}\left(\mathrm{CDCl}_{3}\right)$ spectrum of compound $\mathbf{5 a h}$
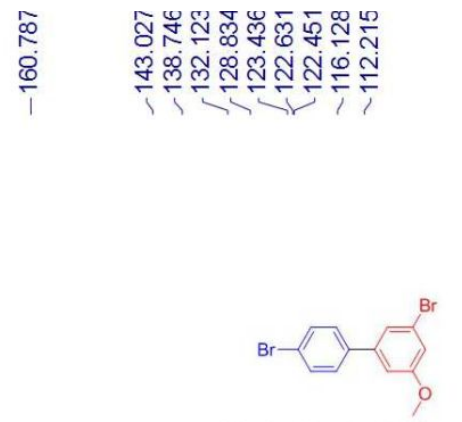

Chemical Formula: $\mathrm{C}_{13} \mathrm{H}_{10} \mathrm{Br}_{2} \mathrm{O}$ Exact Mass: 339.9098

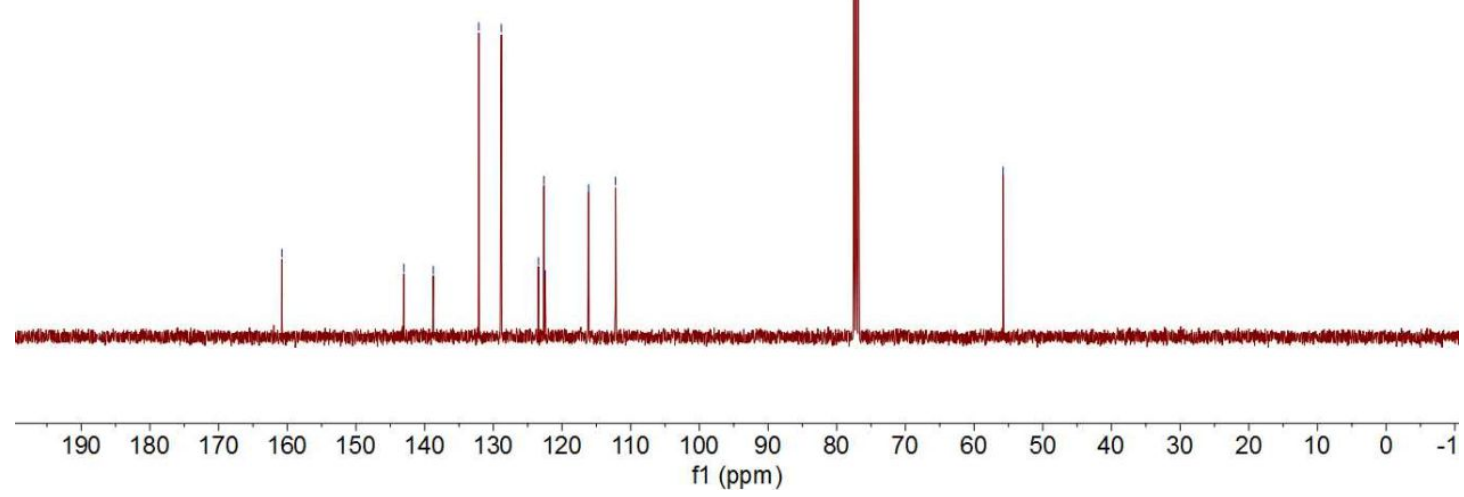

${ }^{1} \mathrm{H} \mathrm{NMR}\left(\mathrm{CDCl}_{3}\right)$ spectrum of compound 5ai

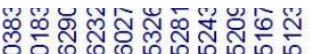

$\infty$

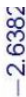
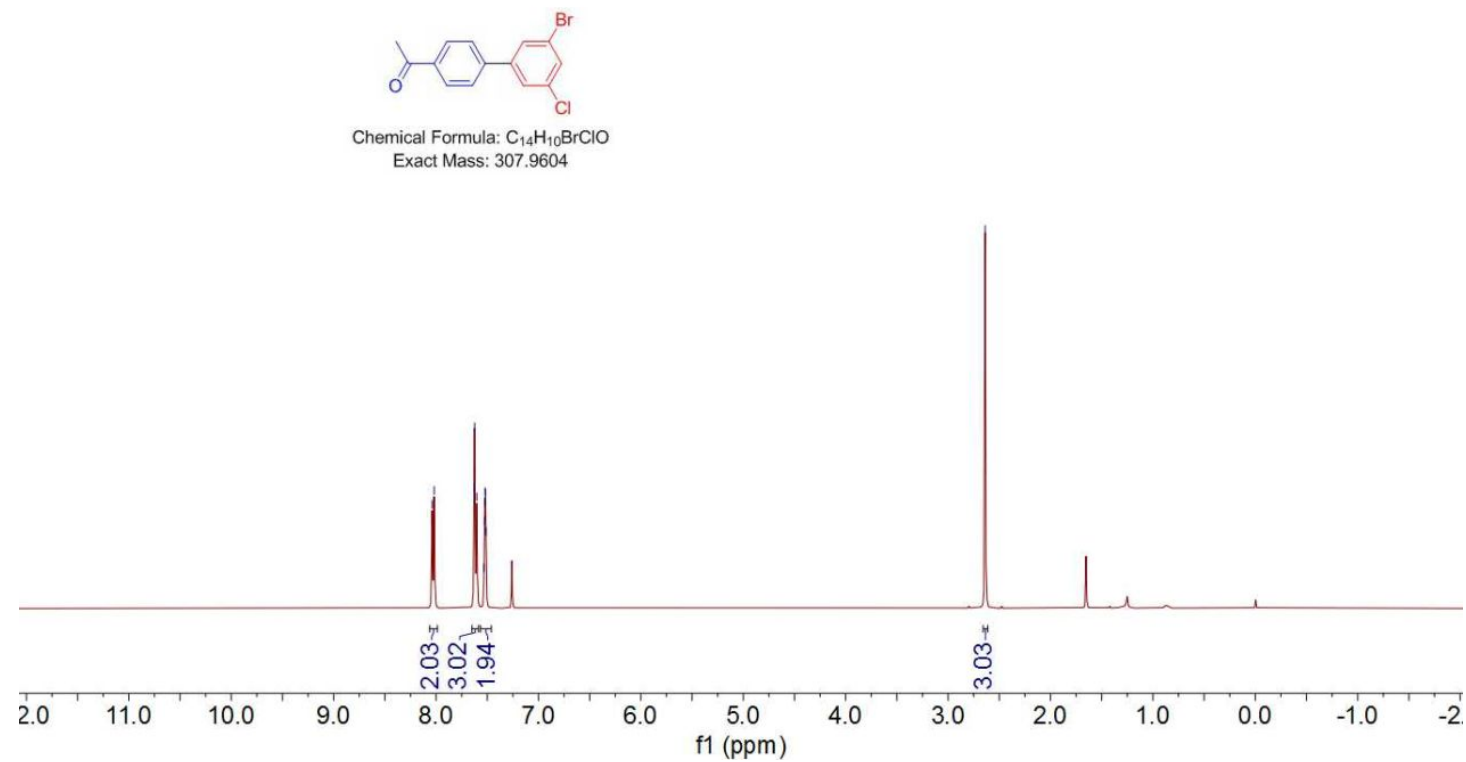
${ }^{13} \mathrm{C} \mathrm{NMR}\left(\mathrm{CDCl}_{3}\right)$ spectrum of compound 5ai
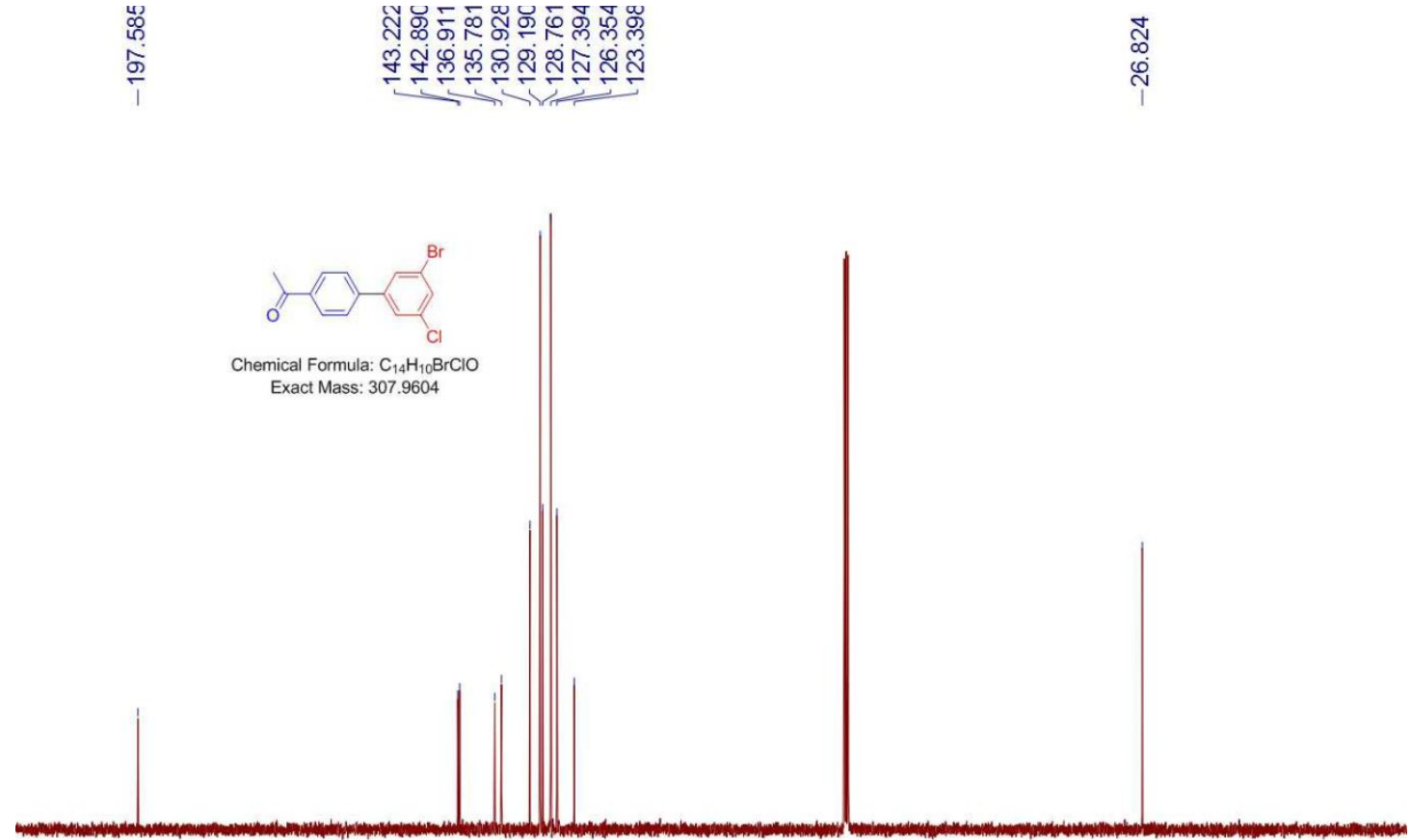

$\begin{array}{lllllllllllllllllllllll}210 & 200 & 190 & 180 & 170 & 160 & 150 & 140 & 130 & 120 & \begin{array}{c}110 \\ \mathrm{f} 1\end{array}(\mathrm{ppm}) & 100 & 90 & 70 & 60 & 50 & 40 & 30 & 20 & 10 & 0 & -10\end{array}$

${ }^{1} \mathrm{H} \mathrm{NMR}\left(\mathrm{CDCl}_{3}\right)$ spectrum of compound 5aj
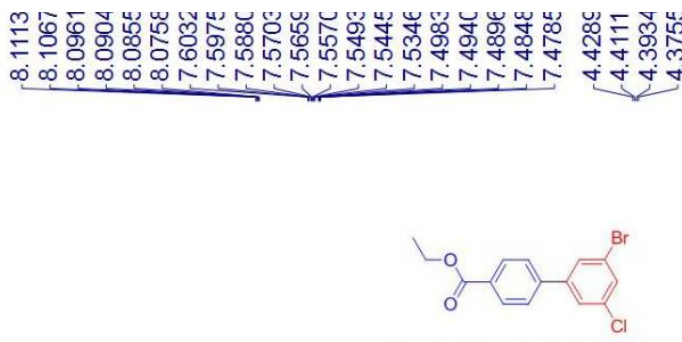

Chemical Formula: $\mathrm{C}_{15} \mathrm{H}_{12} \mathrm{BrClO}$ Exact Mass: 337.9709

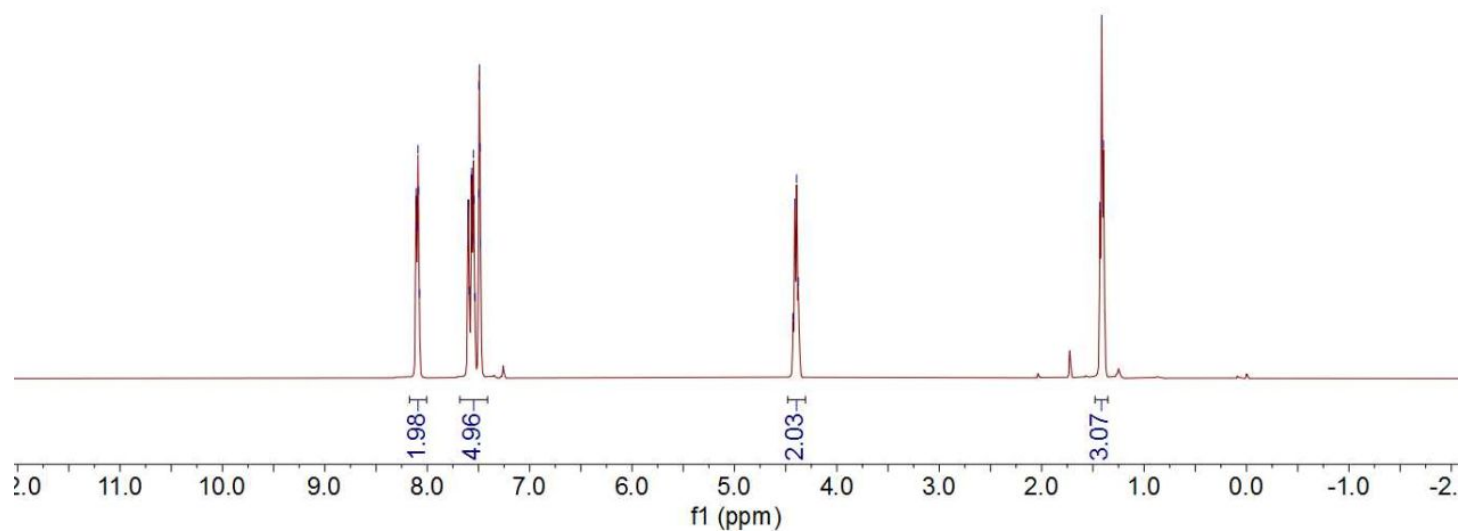


${ }^{13} \mathrm{C} \mathrm{NMR}\left(\mathrm{CDCl}_{3}\right)$ spectrum of compound 5aj

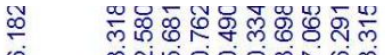

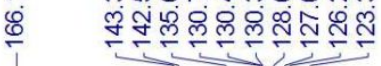

$\stackrel{\substack{n \\ i}}{i}$

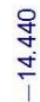

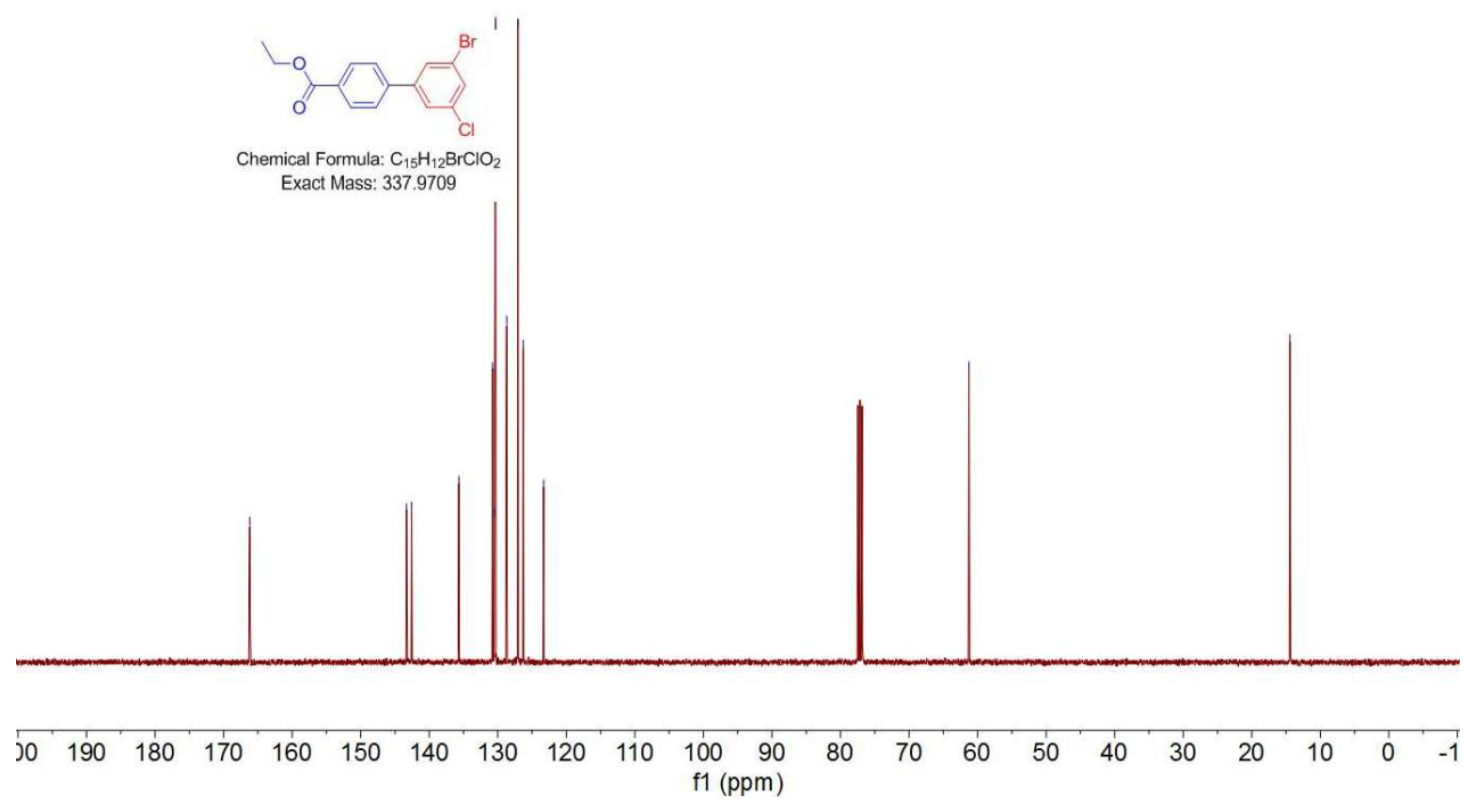

${ }^{1} \mathrm{H}$ NMR $\left(\mathrm{CDCl}_{3}\right)$ spectrum of compound $\mathbf{5 a k}$

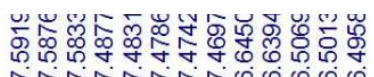

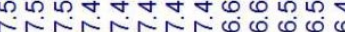

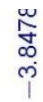

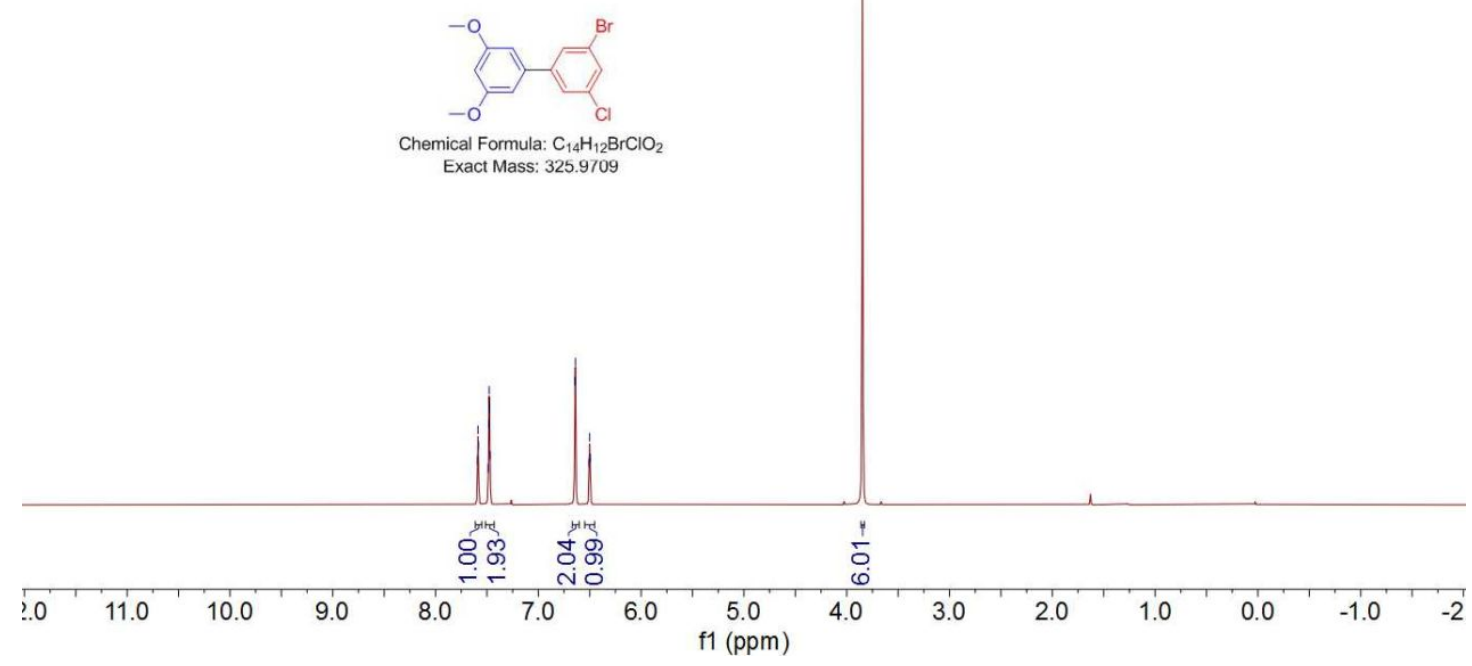


${ }^{13} \mathrm{C}$ NMR $\left(\mathrm{CDCl}_{3}\right)$ spectrum of compound $\mathbf{5 a k}$

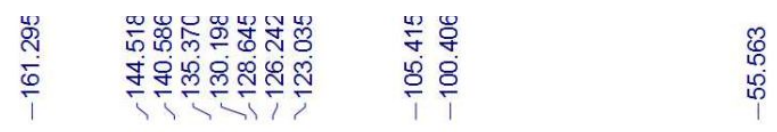

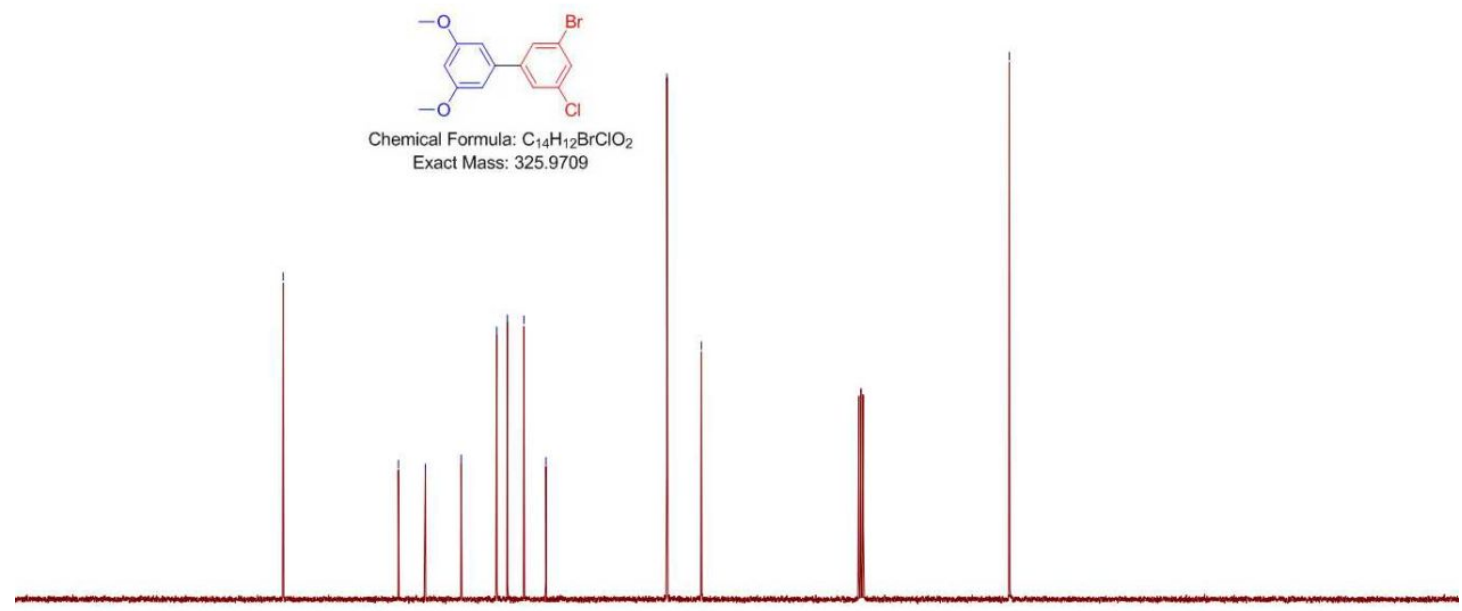

\begin{tabular}{lllllllllllllllllllllll}
\hline 0 & 190 & 180 & 170 & 160 & 150 & 140 & 130 & 120 & 110 & $\begin{array}{l}100 \\
\mathrm{f} 1(\mathrm{pmm})\end{array}$ & 80 & 70 & 60 & 50 & 40 & 30 & 20 & 10 & 0
\end{tabular}

${ }^{1} \mathrm{H} \mathrm{NMR}\left(\mathrm{CDCl}_{3}\right)$ spectrum of compound 5al

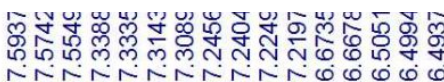

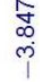

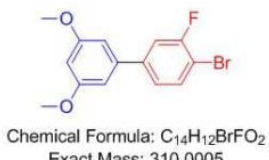

Exact Mass: 310.0005

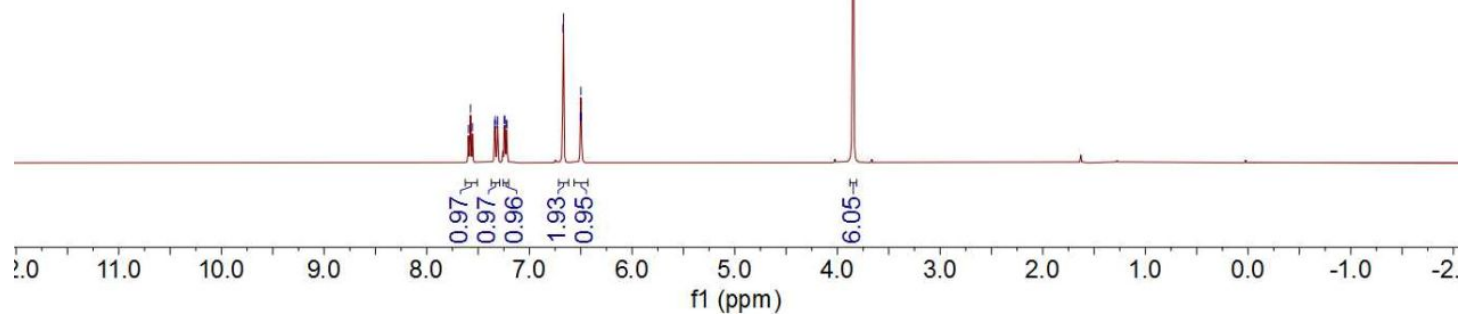


${ }^{13} \mathrm{C} \mathrm{NMR}\left(\mathrm{CDCl}_{3}\right)$ spectrum of compound 5al

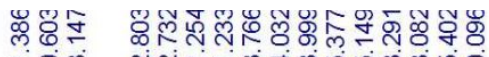

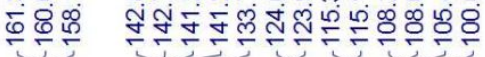

$\frac{1}{6}$

究

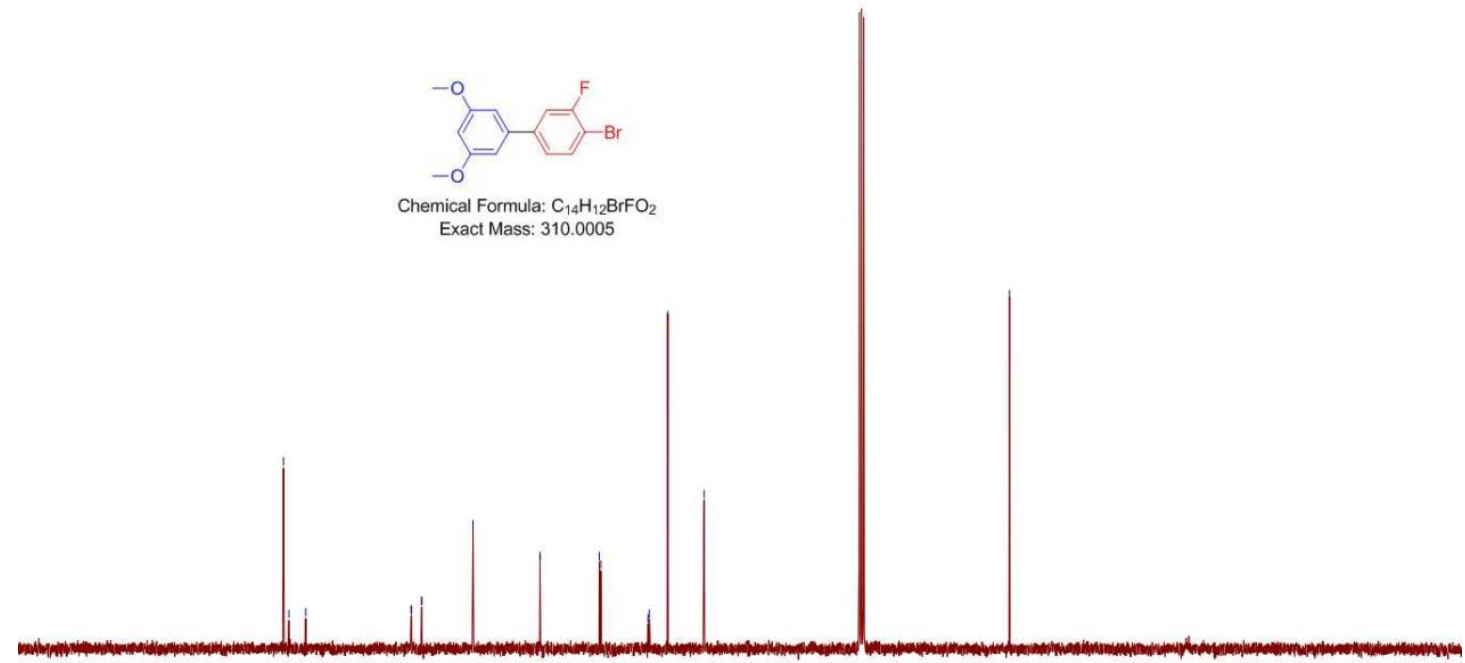

\begin{tabular}{llllllllllllllllllllllll}
\hline 0 & 190 & 180 & 170 & 160 & 150 & 140 & 130 & 120 & 110 & $\begin{array}{r}100 \\
\mathrm{f} 1(\mathrm{ppm})\end{array}$ & 90 & 80 & 70 & 60 & 50 & 40 & 30 & 20 & 10 & 0 & -1
\end{tabular}

${ }^{19} \mathrm{~F}$ NMR $\left(\mathrm{CDCl}_{3}\right)$ spectrum of compound 5al

ํํำ

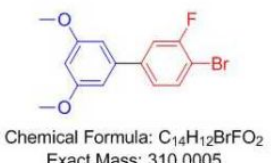

Exact Mass: 310.0005

$\begin{array}{lllllllllllllllllllll}-20 & -30 & -40 & -50 & -60 & -70 & -80 & -90 & -100 & -110 & -120 & -130 & -140 & -150 & -160 & -170 & -180 & -190 & -20( \\ \mathrm{f} 1(\mathrm{ppm})\end{array}$ 
${ }^{1} \mathrm{H}$ NMR $\left(\mathrm{CDCl}_{3}\right)$ spectrum of compound $5 \mathbf{a m}$

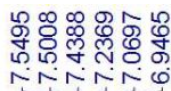

ñNing

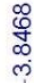

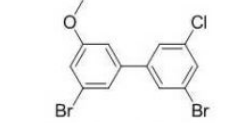

Chemical Formula: $\mathrm{C}_{13} \mathrm{H}_{9} \mathrm{Br}_{2} \mathrm{ClO}$

Exact Mass: 373.8709

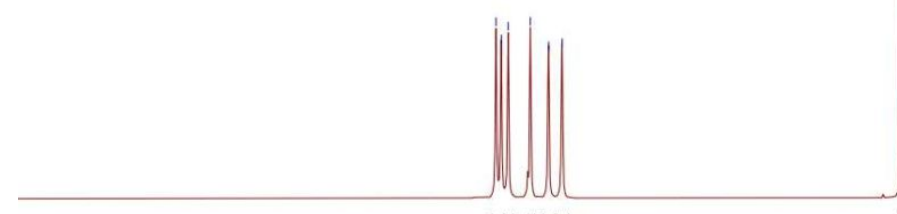

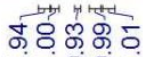

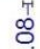

$\begin{array}{llllllllllllllllllllllllllllll}11.5 & 11.0 & 10.5 & 10.0 & 9.5 & 9.0 & 8.5 & 8.0 & 7.5 & 7.0 & 6.5 & 6.0 & 5.5 & 5.0 & 4.5 & 4.0 & 3.5 & 3.0 & 2.5 & 2.0 & 1.5 & 1.0 & 0.5 & 0.0 & -0.5 & -1.0\end{array}$ f1 (ppm)

${ }^{13} \mathrm{C} \mathrm{NMR}\left(\mathrm{CDCl}_{3}\right)$ spectrum of compound $\mathbf{5 a m}$

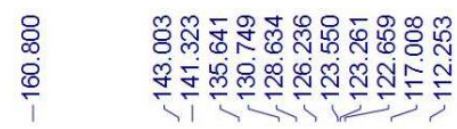

$\infty$
$\infty$
$\infty$
$\infty$
$\infty$

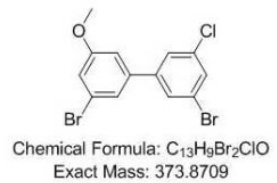

Exact Mass: 373.8709

$\begin{array}{llllllllllllllllll}190 & 180 & 170 & 160 & 150 & 140 & 130 & 120 & \begin{array}{c}1 \\ \mathrm{f} 1\end{array}(\mathrm{ppm}) & 100 & 90 & 80 & 70 & 60 & 50 & 40 & 30 & 2\end{array}$ 
${ }^{1} \mathrm{H}$ NMR $\left(\mathrm{CDCl}_{3}\right)$ spectrum of compound 5 an

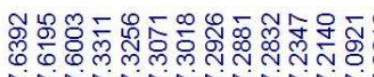

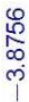

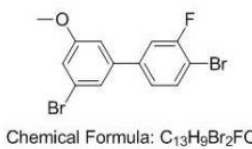

Exact Mass: 357.9004

${ }^{13} \mathrm{C}$ NMR $\left(\mathrm{CDCl}_{3}\right)$ spectrum of compound 5 an

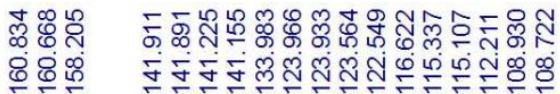

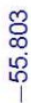

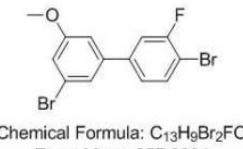

Exact Mass: 357.9004

$\begin{array}{lllllllllllllllll}180 & 170 & 160 & 150 & 140 & 130 & 120 & \begin{array}{c}110 \\ \mathrm{f} 1(\mathrm{ppm})\end{array} & 90 & 80 & 70 & 60 & 50 & 40 & 30 & 20\end{array}$ 
${ }^{19} \mathrm{~F}$ NMR $\left(\mathrm{CDCl}_{3}\right)$ spectrum of compound 5 an

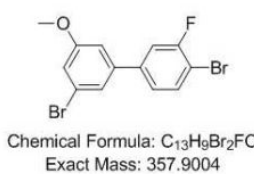

Exact Mass: 357.9004

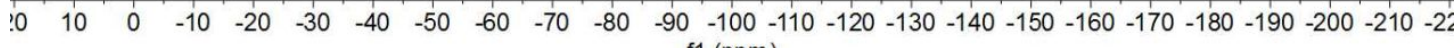
f1 (ppm)

${ }^{1} \mathrm{H}$ NMR $\left(\mathrm{CDCl}_{3}\right)$ spectrum of compound $\mathbf{5 a o}$
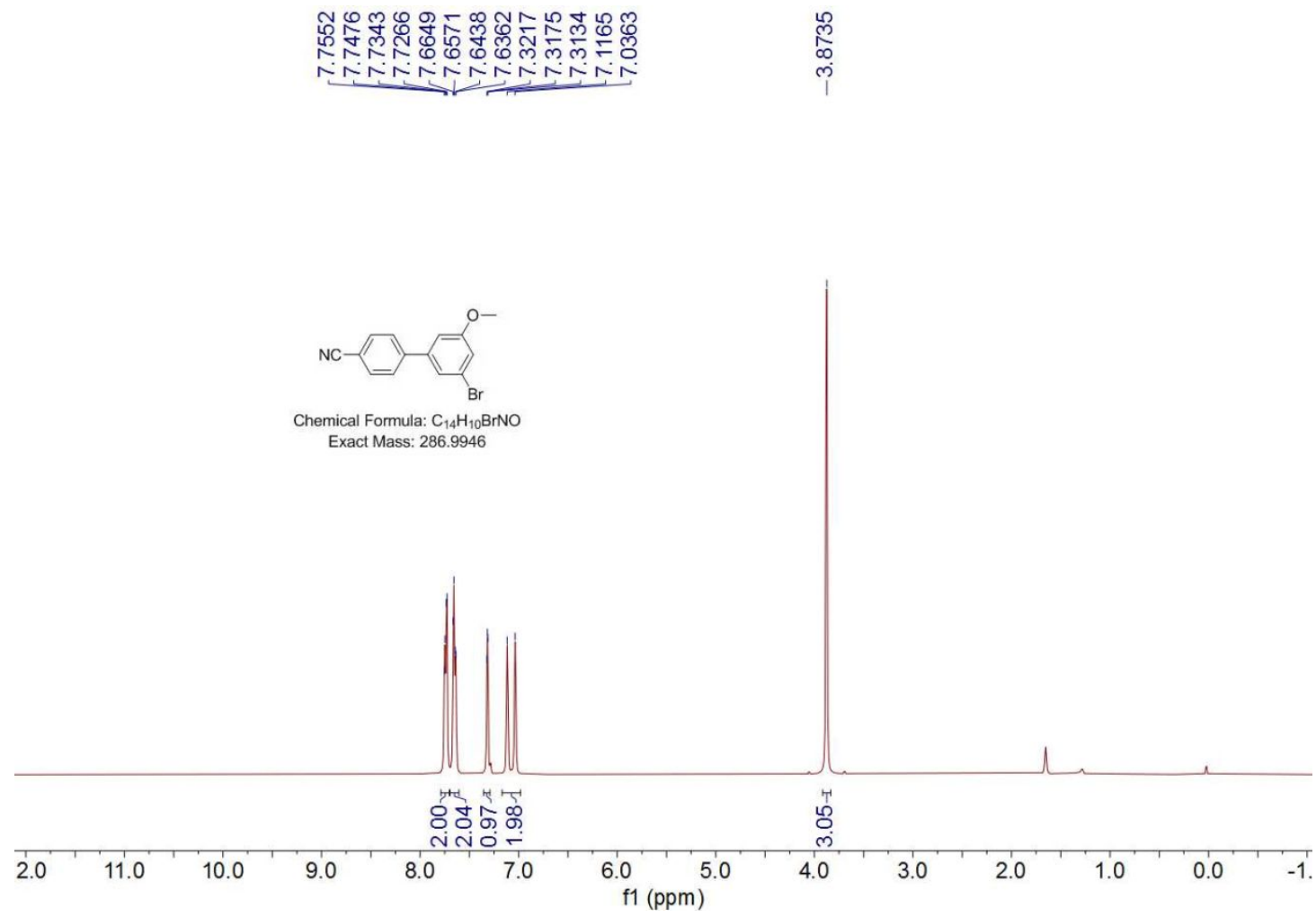

Exact Mass: 286.9946 
${ }^{13} \mathrm{C}$ NMR $\left(\mathrm{CDCl}_{3}\right)$ spectrum of compound $\mathbf{5 a o}$
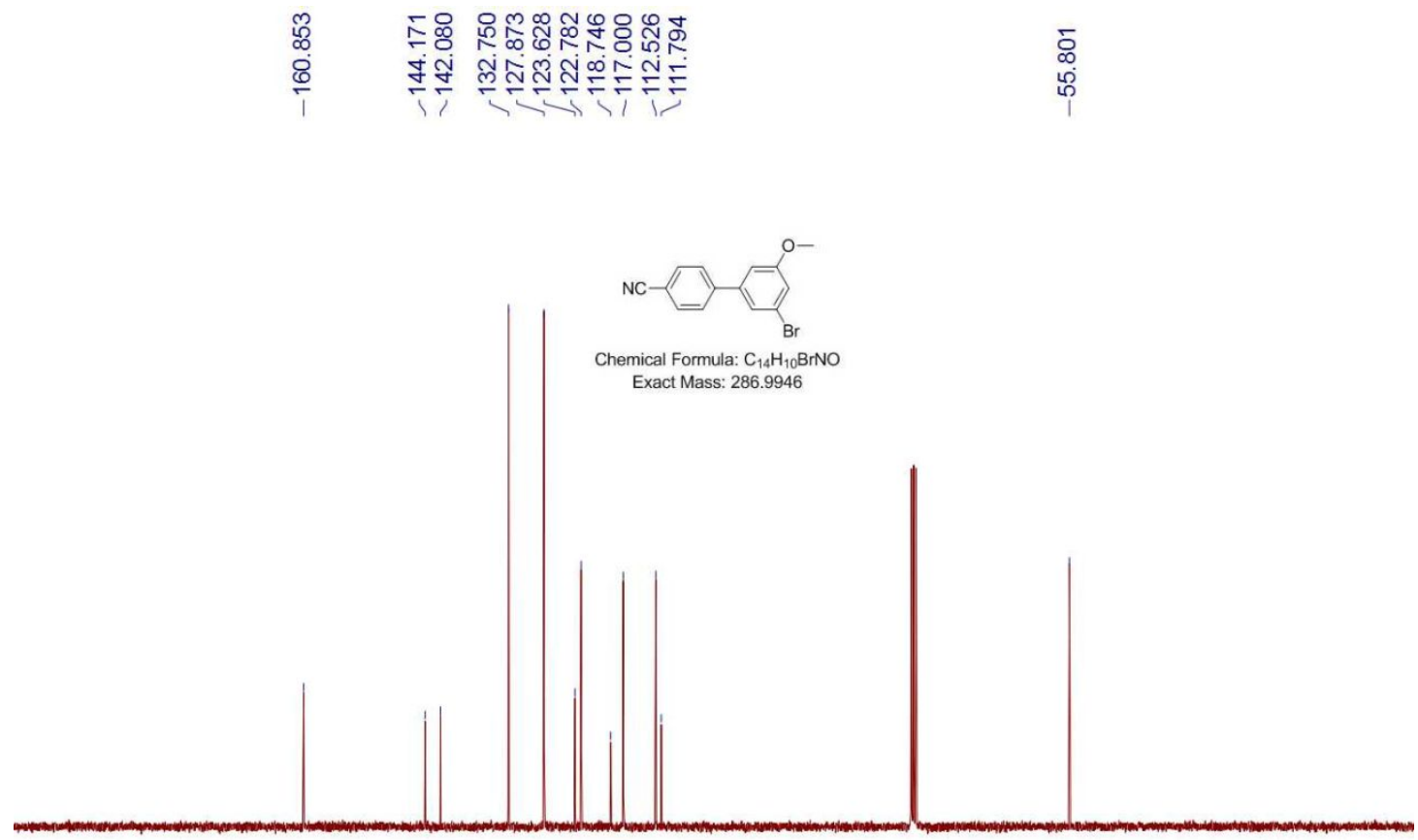

\begin{tabular}{llllllllllllllllllllll}
\hline 00 & 190 & 180 & 170 & 160 & 150 & 140 & 130 & 120 & $\begin{array}{c}110 \\
\mathrm{f} 1(\mathrm{ppm})\end{array}$ & 90 & 80 & 70 & 60 & 50 & 40 & 30 & 20 & 10
\end{tabular}

${ }^{1} \mathrm{H}$ NMR $\left(\mathrm{CDCl}_{3}\right)$ spectrum of compound 5ap

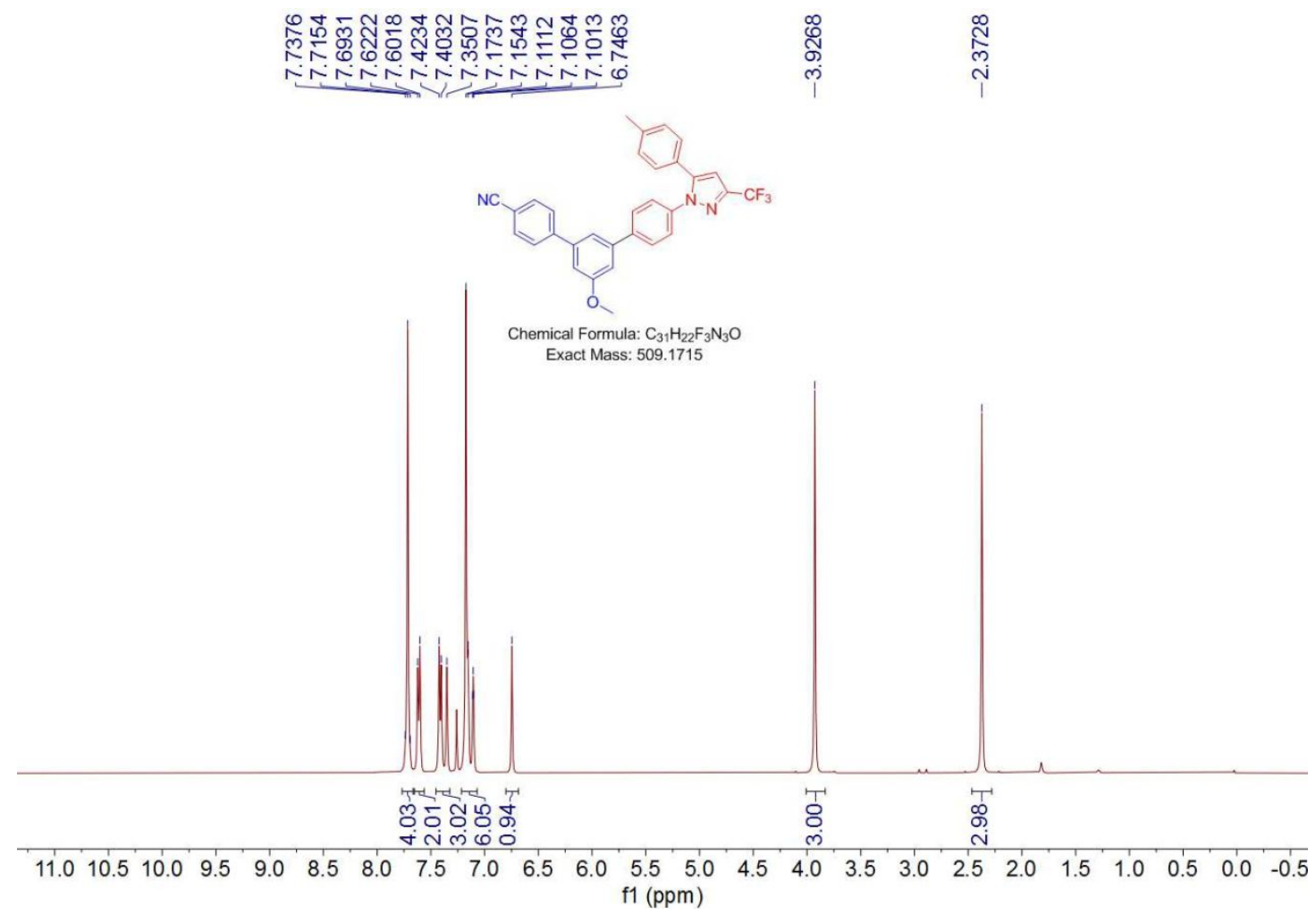


${ }^{13} \mathrm{C} \mathrm{NMR}\left(\mathrm{CDCl}_{3}\right)$ spectrum of compound 5ap

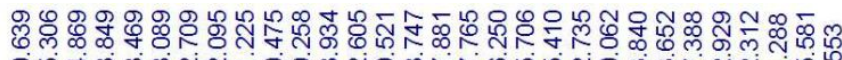

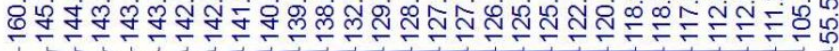

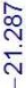

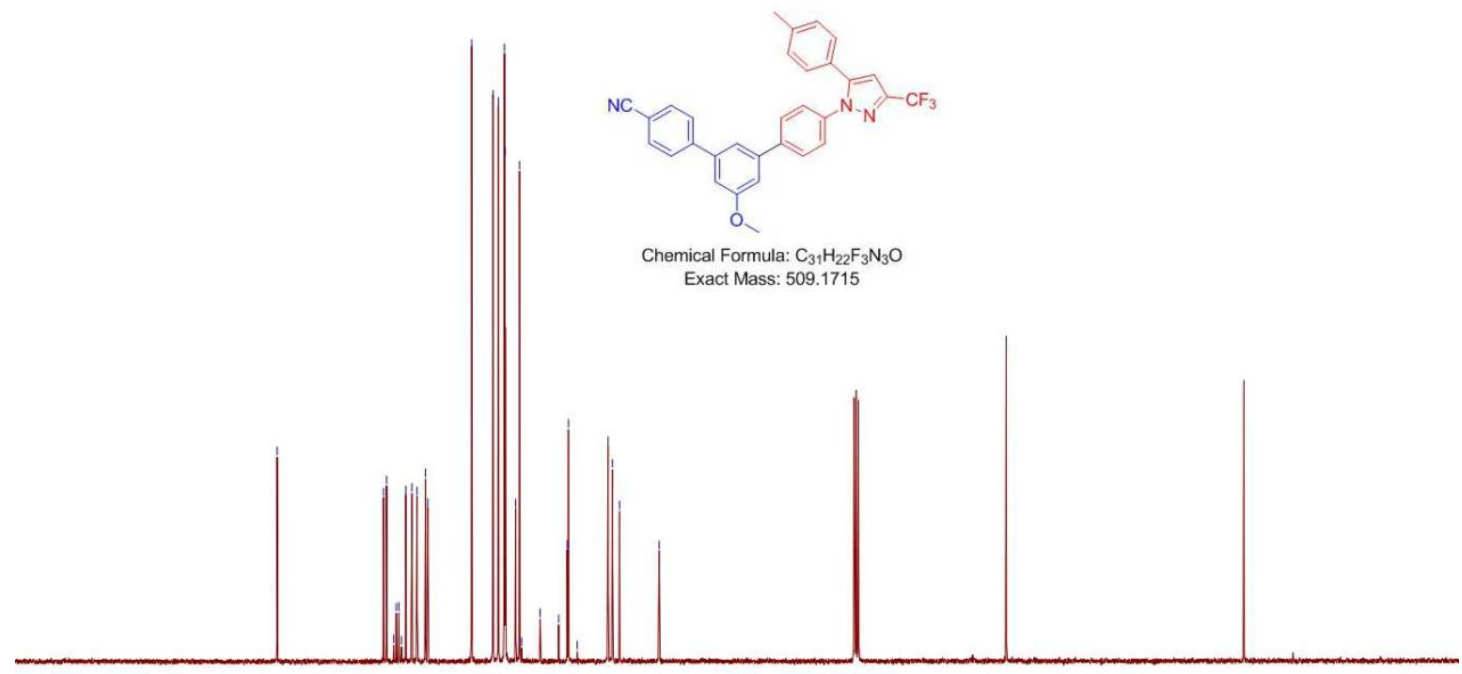

$\begin{array}{llllllllllllllllllll}190 & 180 & 170 & 160 & 150 & 140 & 130 & 120 & 110 & 100 & 90 & 80 & 70 & 60 & 50 & 40 & 30 & 20 & 10 & 0\end{array}$

\section{${ }^{19} \mathrm{~F} \mathrm{NMR}\left(\mathrm{CDCl}_{3}\right)$ spectrum of compound $\mathbf{5 a p}$}

홍

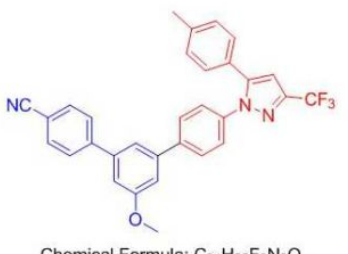

Chemical Formula: $\mathrm{C}_{31} \mathrm{H}_{22} \mathrm{~F}_{3} \mathrm{~N}_{3} \mathrm{O}$ Exact Mass: 509.1715

$-10 \quad-15$

$5 \quad-20$ 
${ }^{1} \mathrm{H}$ NMR $\left(\mathrm{CDCl}_{3}\right)$ spectrum of compound $\mathbf{5 a q}$

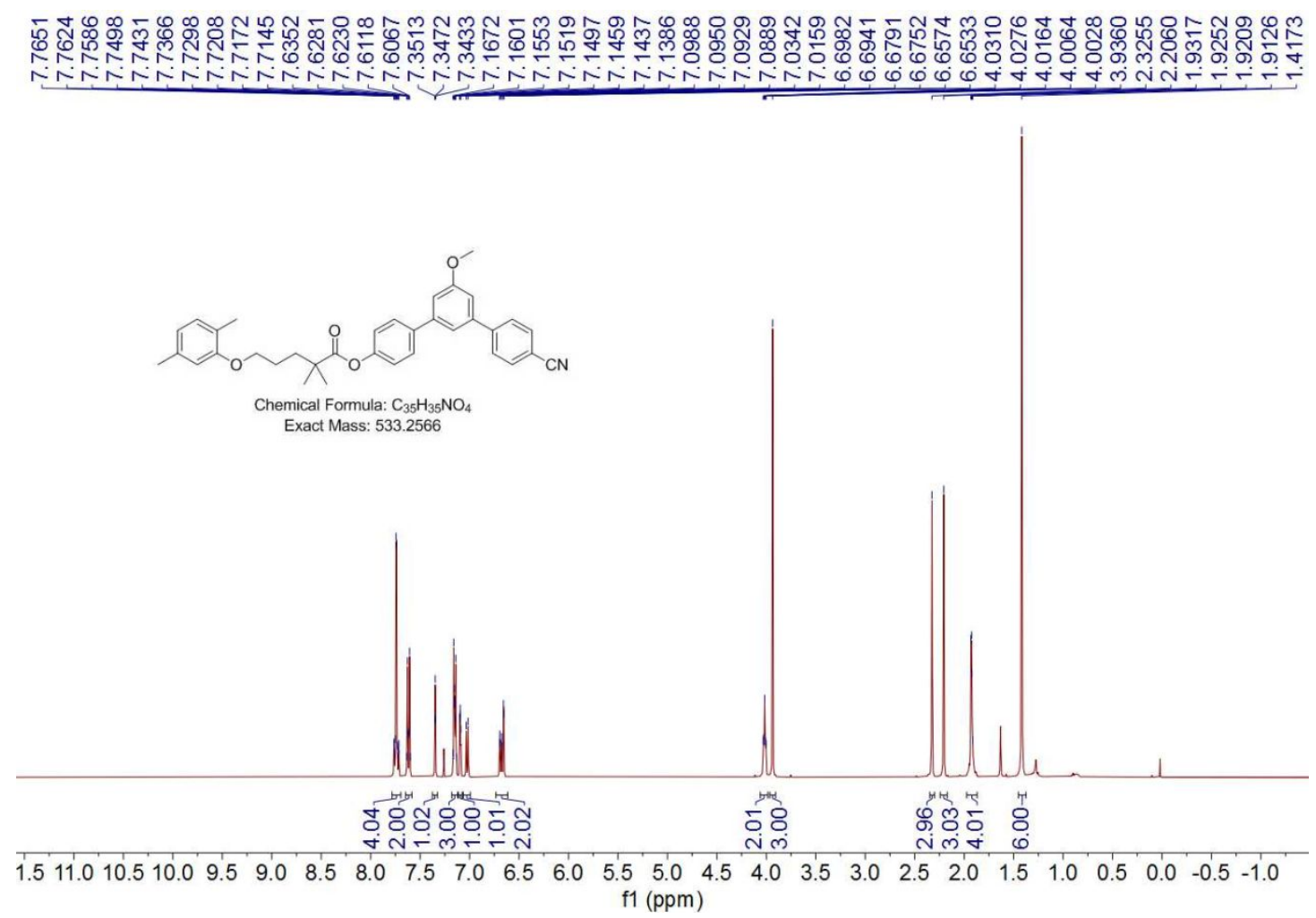

${ }^{13} \mathrm{C}$ NMR $\left(\mathrm{CDCl}_{3}\right)$ spectrum of compound $\mathbf{5 a q}$
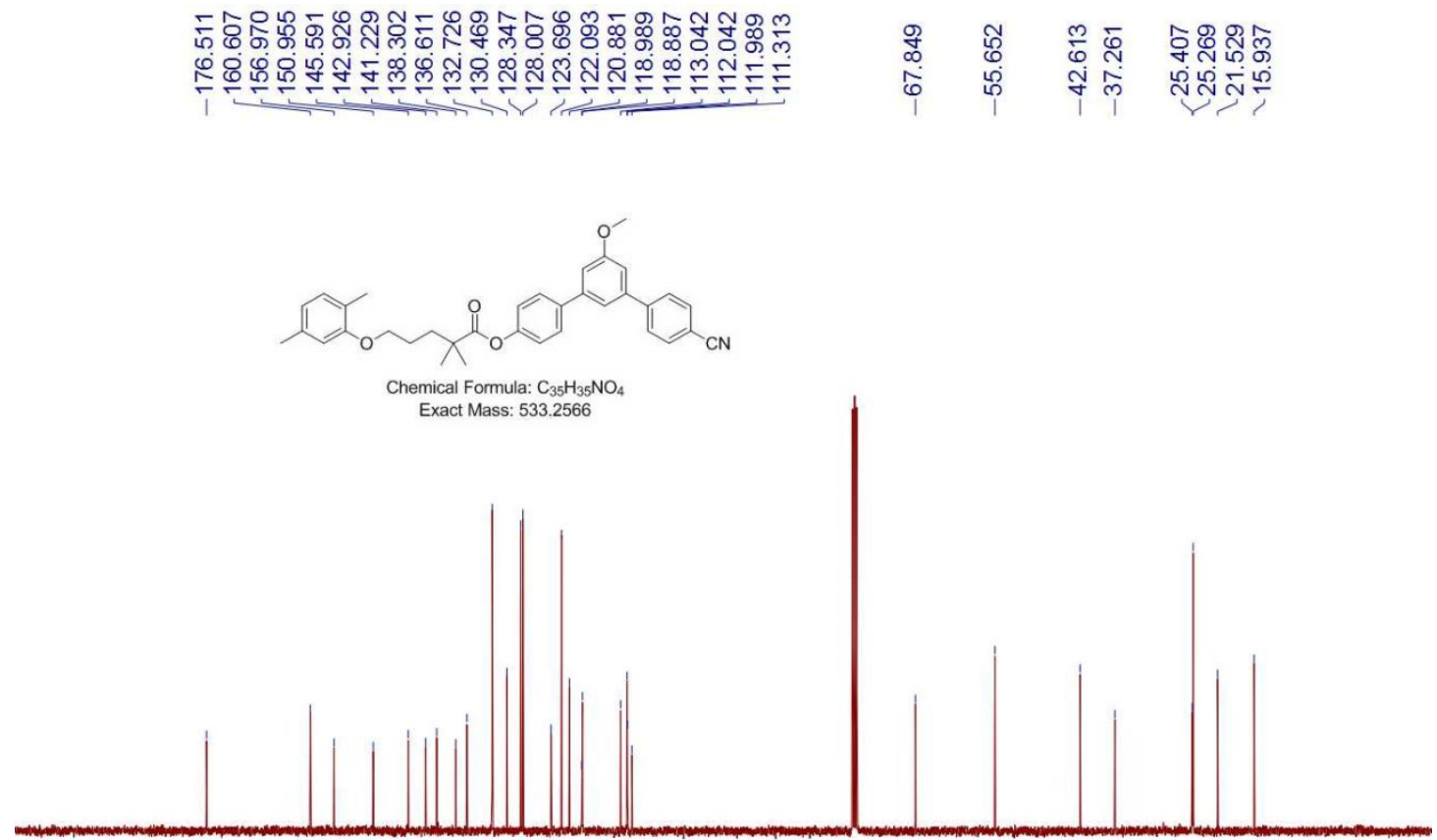

$\begin{array}{lllllllllllllllllllllllll}200 & 190 & 180 & 170 & 160 & 150 & 140 & 130 & 120 & 110 & 100 & 90 & 80 & 70 & 60 & 50 & 40 & 30 & 20 & 10 & 0 & -1(\end{array}$ 
${ }^{1} \mathrm{H}$ NMR $\left(\mathrm{CDCl}_{3}\right)$ spectrum of compound $\mathbf{5 a r}$
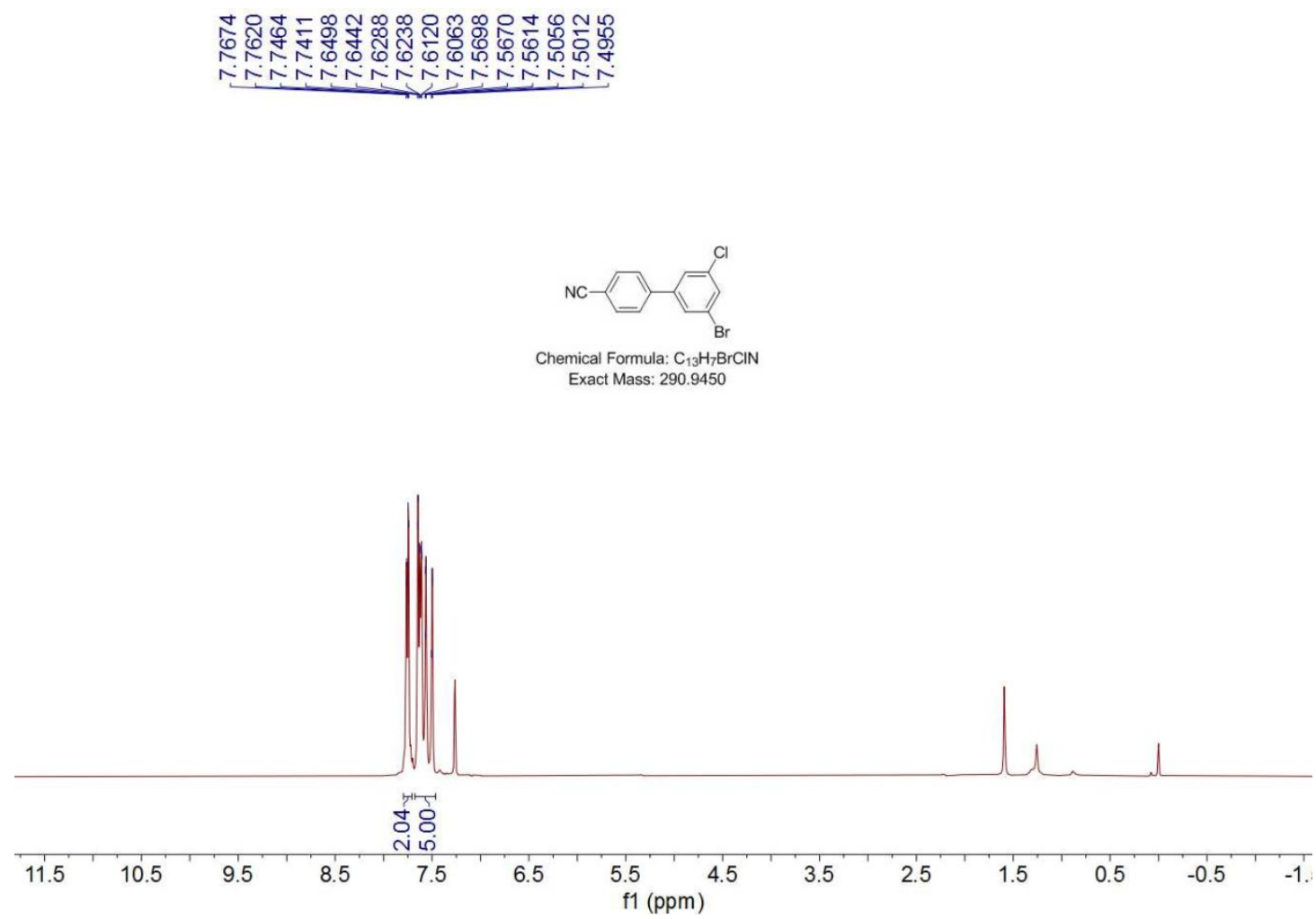

${ }^{13} \mathrm{C}$ NMR $\left(\mathrm{CDCl}_{3}\right)$ spectrum of compound $\mathbf{5 a r}$

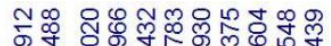

守娄

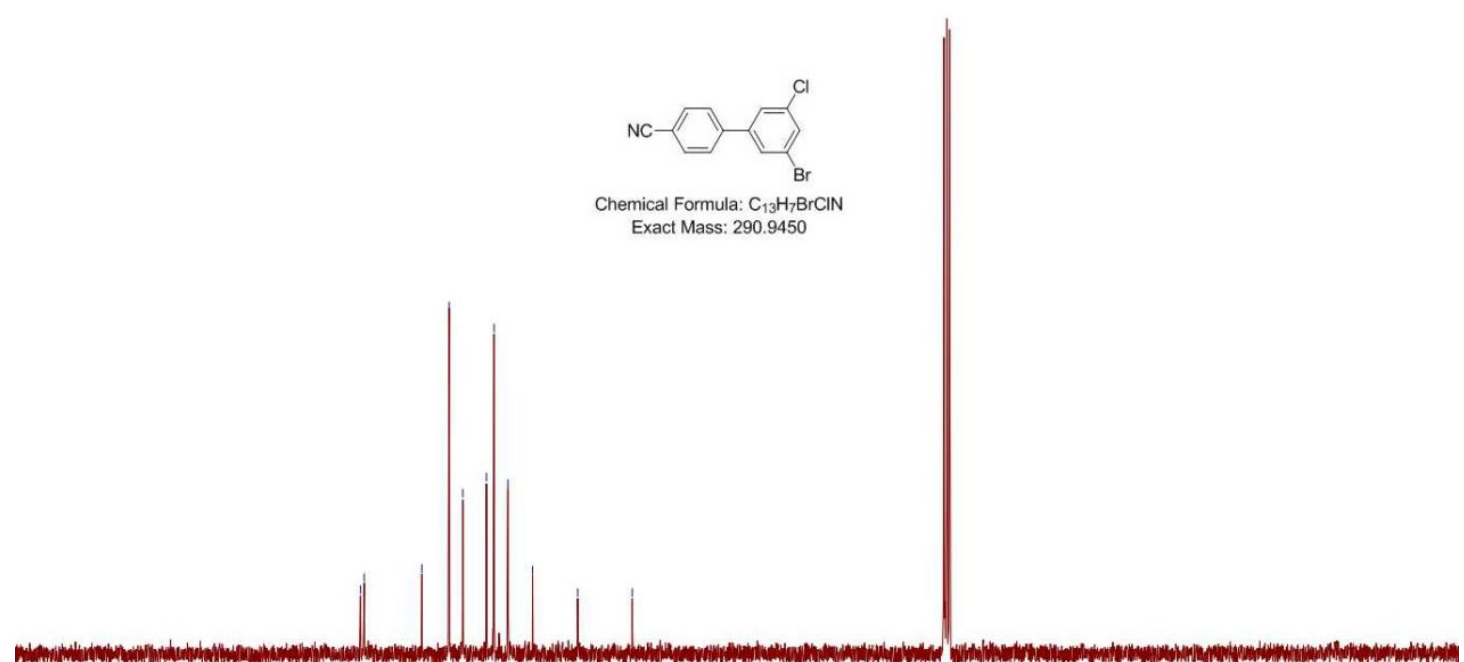

$\begin{array}{lllllllllllllllll}180 & 170 & 160 & 150 & 140 & 130 & 120 & 110 & \begin{array}{c}100 \\ \mathrm{f} 1(\mathrm{ppm})\end{array} & 90 & 80 & 70 & 60 & 50 & 40 & 30 & 21\end{array}$ 
${ }^{1} \mathrm{H} \mathrm{NMR}\left(\mathrm{CDCl}_{3}\right)$ spectrum of compound $\mathbf{5 a s}$
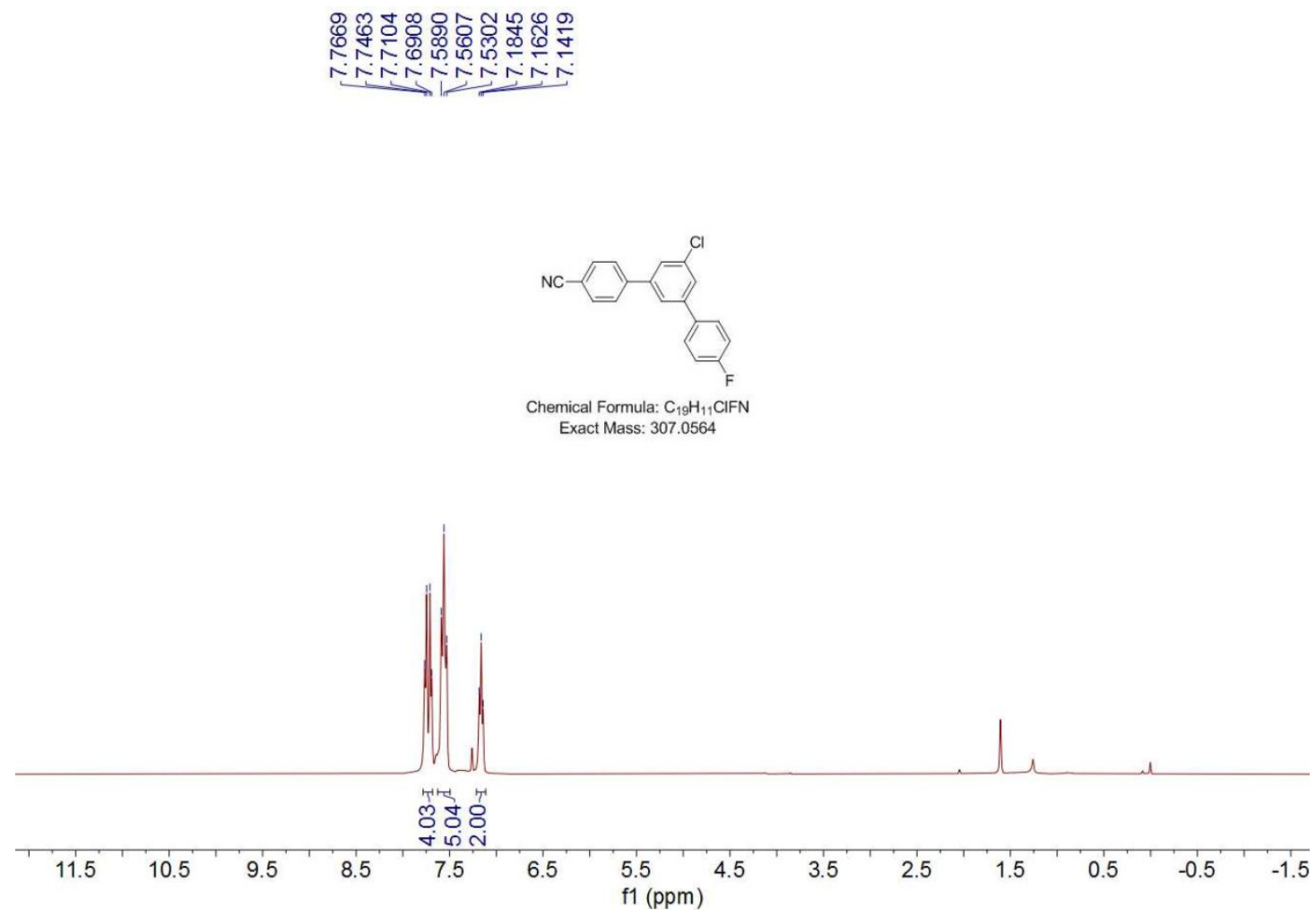

${ }^{13} \mathrm{C} \mathrm{NMR}\left(\mathrm{CDCl}_{3}\right)$ spectrum of compound 5 as

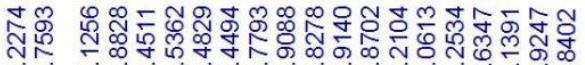

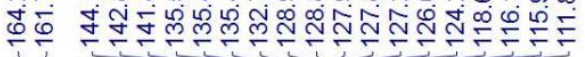

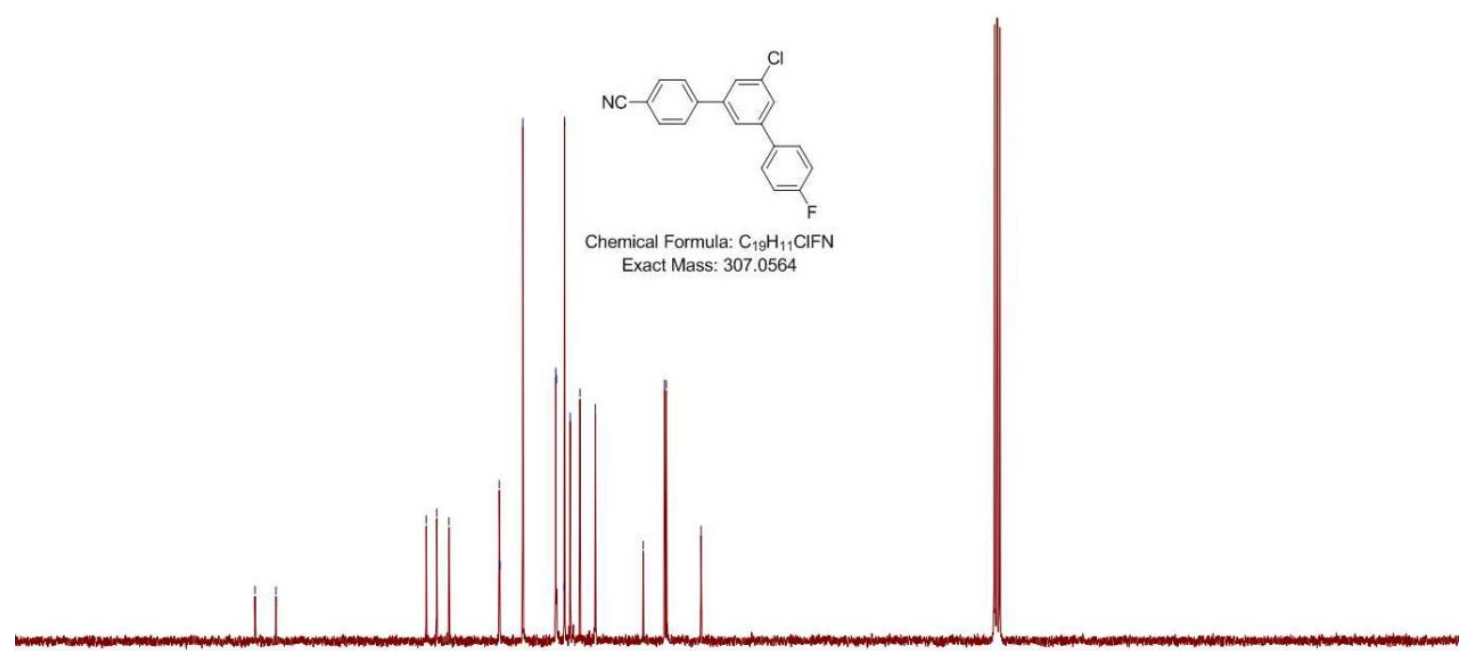

190

$180 \quad 170 \quad 160$

$150 \quad 140$

$130 \quad 120$

110100 
${ }^{19} \mathrm{~F}$ NMR $\left(\mathrm{CDCl}_{3}\right)$ spectrum of compound $\mathbf{5 a s}$

के
$\frac{\text { ल }}{5}$

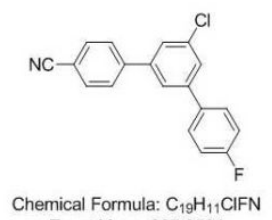

Exact Mass: 307.0564

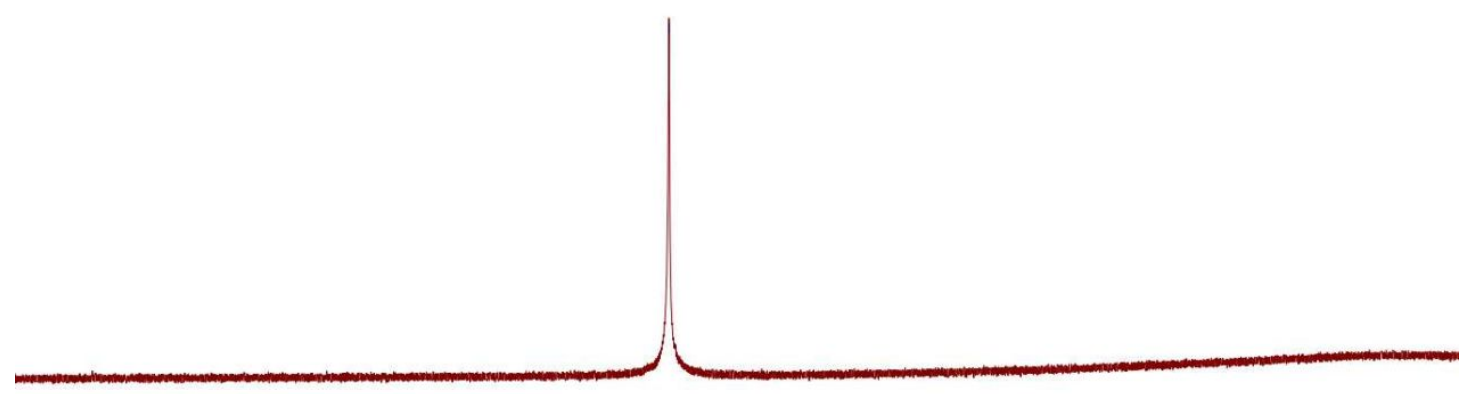

$\begin{array}{rrrrrrrrrrrrrrrrrrrrr}-70 & -75 & -80 & -85 & -90 & -95 & -100 & -105 & -110 & -115 & -120 & -125 & -130 & -135 & -140 & -145 & -150 & -155 & -160 & -165 & -17\end{array}$

${ }^{1} \mathrm{H}$ NMR $\left(\mathrm{CDCl}_{3}\right)$ spectrum of compound 5at

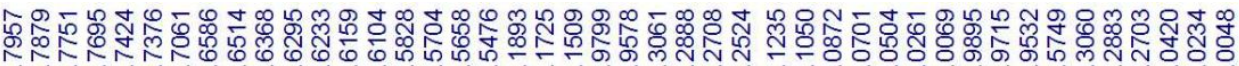

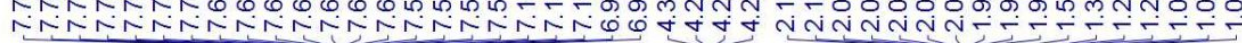

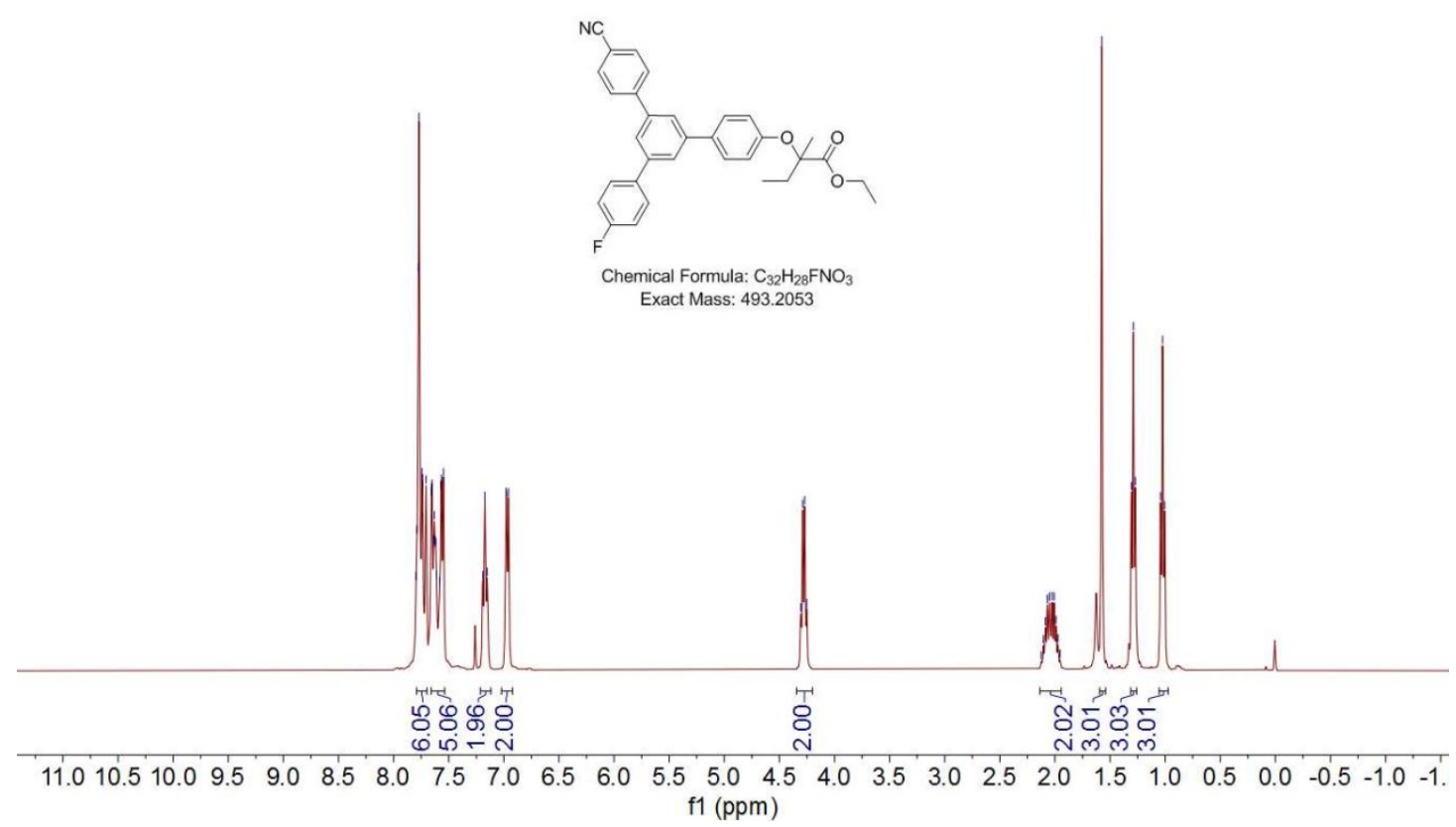


${ }^{13} \mathrm{C}$ NMR $\left(\mathrm{CDCl}_{3}\right)$ spectrum of compound 5at

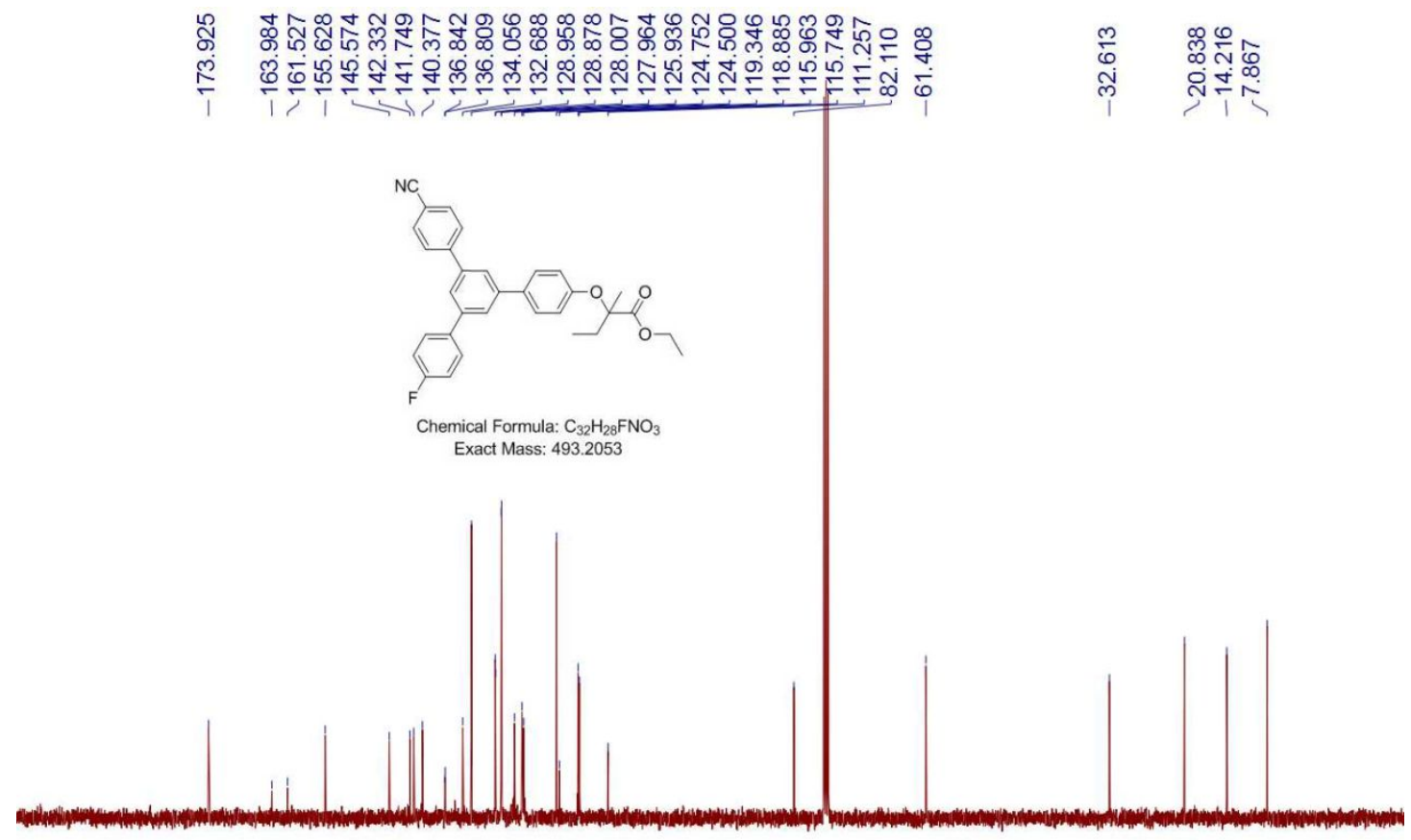

$\begin{array}{lllllllllllllllllllllll}200 & 190 & 180 & 170 & 160 & 150 & 140 & 130 & 120 & 110 & \begin{array}{c}100 \\ \mathrm{f} 1(\mathrm{ppm})\end{array} & 90 & 70 & 60 & 50 & 40 & 30 & 20 & 10 & 0 & -10\end{array}$

${ }^{19} \mathrm{~F}$ NMR $\left(\mathrm{CDCl}_{3}\right)$ spectrum of compound 5at

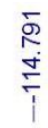

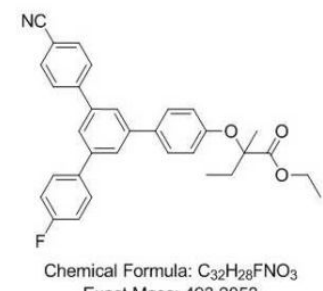

Exact Mass: 493.2053

\begin{tabular}{lllllllllllllllll}
\hline 5 & -80 & -85 & -90 & -95 & -100 & -105 & -110 & -115 & -120 & -125 & -130 & -135 & -140 & -145 & -150
\end{tabular} 
${ }^{1} \mathrm{H}$ NMR $\left(\mathrm{CDCl}_{3}\right)$ spectrum of compound 5au
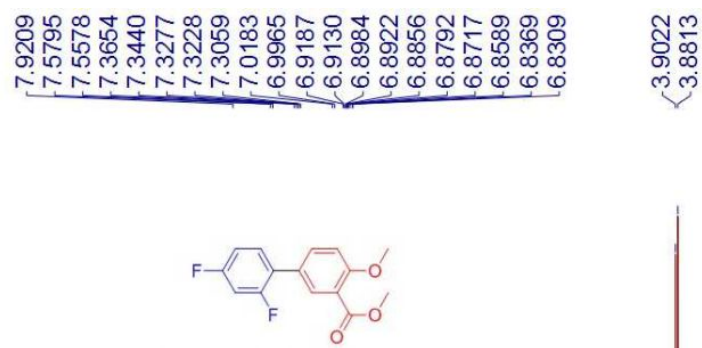

Chemical Formula: $\mathrm{C}_{15} \mathrm{H}_{12} \mathrm{~F}_{2} \mathrm{O}_{3}$ Exact Mass: 278.0755

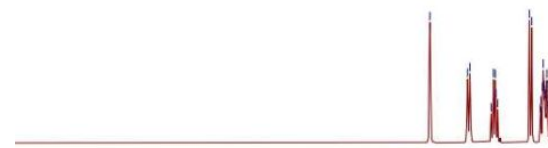

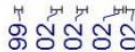

के

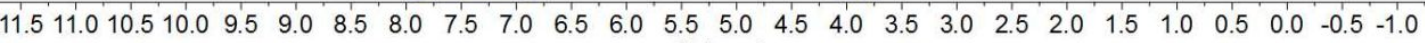
f1 (ppm)

${ }^{13} \mathrm{C}$ NMR $\left(\mathrm{CDCl}_{3}\right)$ spectrum of compound 5au

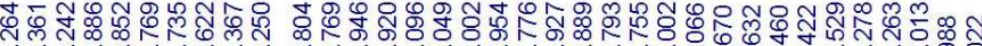

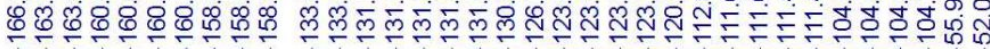

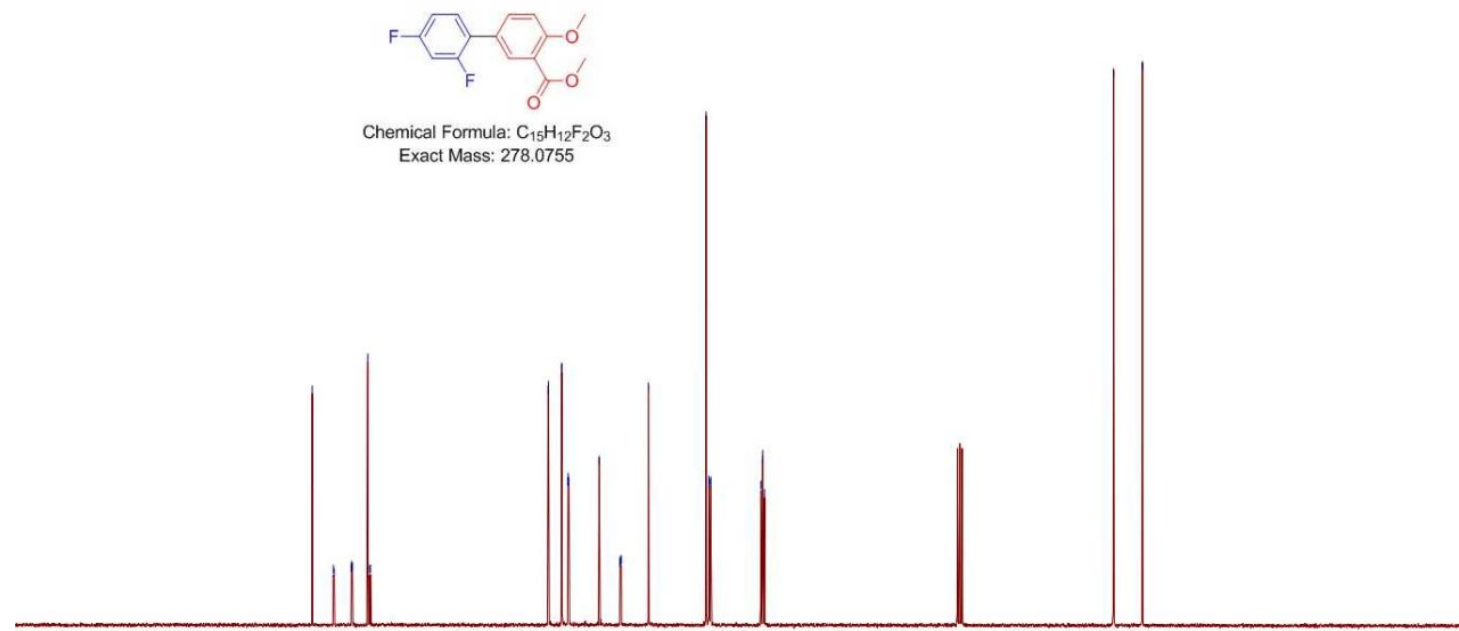

$\begin{array}{lllllllllllllllllllllllllllll}200 & 190 & 180 & 170 & 160 & 150 & 140 & 130 & 120 & 110 & 100 & 90 & 80 & 70 & 60 & 50 & 40 & 30 & 20 & 10\end{array}$ 
${ }^{19} \mathrm{~F} \mathrm{NMR}\left(\mathrm{CDCl}_{3}\right)$ spectrum of compound 5au
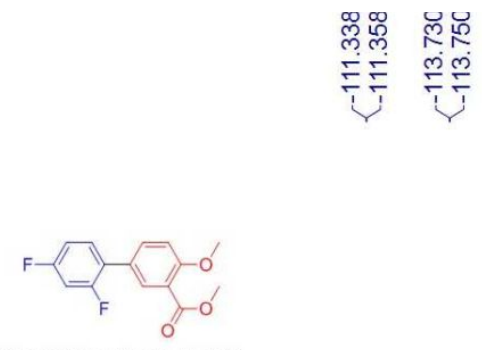

Chemical Formula: $\mathrm{C}_{15} \mathrm{H}_{12} \mathrm{~F}_{2} \mathrm{O}_{3}$

Exact Mass: 278.0755

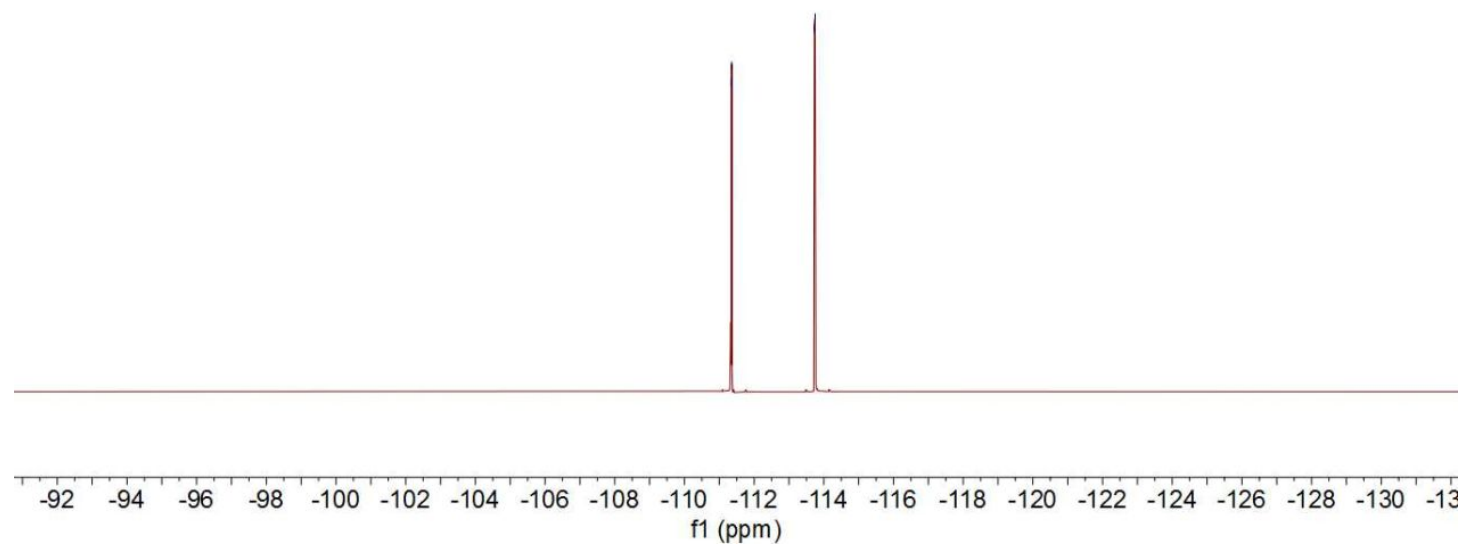

${ }^{1} \mathrm{H}$ NMR (DMSO- $d_{6}$ ) spectrum of compound $\mathbf{5 a v}$

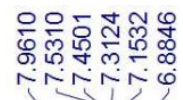

iNiNio

$\mathrm{f1}(\mathrm{ppm}$

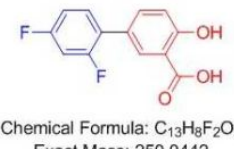

Exact Mass: 250.0442

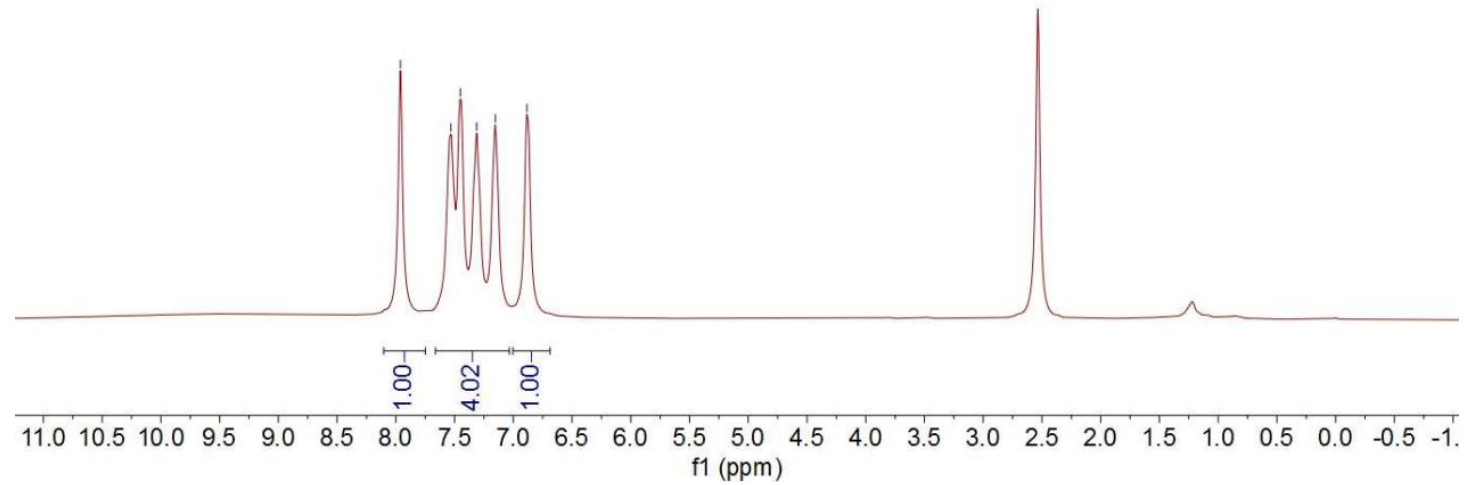


${ }^{13} \mathrm{C}$ NMR (DMSO- $\left.d_{6}\right)$ spectrum of compound 5av

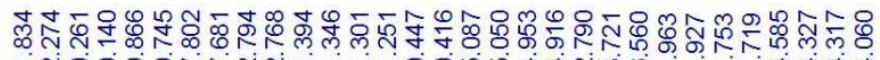

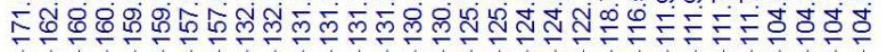

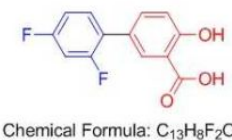

Chemical Formula: $\mathrm{C}_{13} \mathrm{H}_{8} \mathrm{~F}_{2} \mathrm{O}_{3}$ Exact Mass: 250.0442

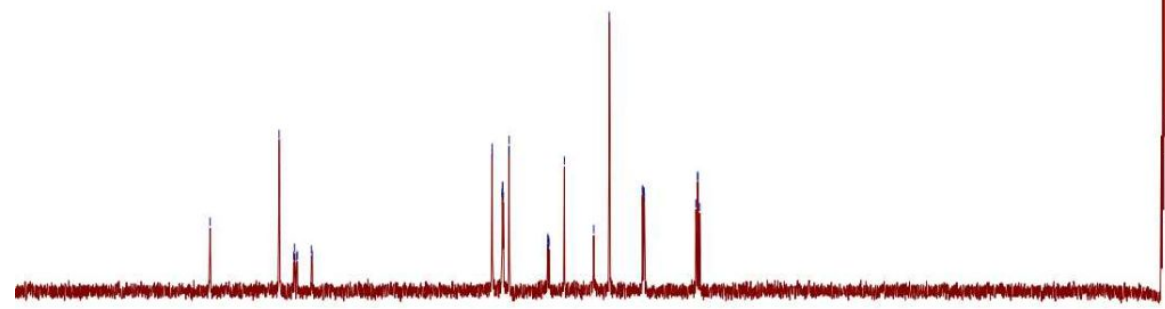

$\begin{array}{lllllllllllllllllllll}190 & 180 & 170 & 160 & 150 & 140 & 130 & 120 & 110 & \begin{array}{c}100 \\ \mathrm{f} 1(\mathrm{ppm})\end{array} & 90 & 80 & 70 & 60 & 50 & 40 & 30 & 20 & 10 & 0\end{array}$

${ }^{19}$ F NMR (DMSO- $\left.d_{6}\right)$ spectrum of compound $\mathbf{5 a v}$

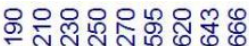

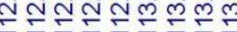

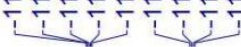

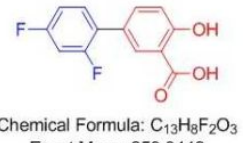

Chemical Formula: $\mathrm{C}_{13} \mathrm{H}_{8} \mathrm{~F}_{2} \mathrm{O}_{3}$

\begin{tabular}{lllllllllllllllllllllllll}
-104 & -105 & -106 & -107 & -108 & -109 & -110 & -111 & -112 & -113 & -114 & -115 & -116 & -117 & -118 & -119 & -120 & -121 & -122 \\
\hline
\end{tabular} 
${ }^{1} \mathrm{H}$ NMR $\left(\mathrm{CDCl}_{3}\right)$ spectrum of compound 5aw

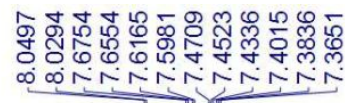

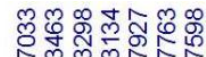

लूलूखूंतुल

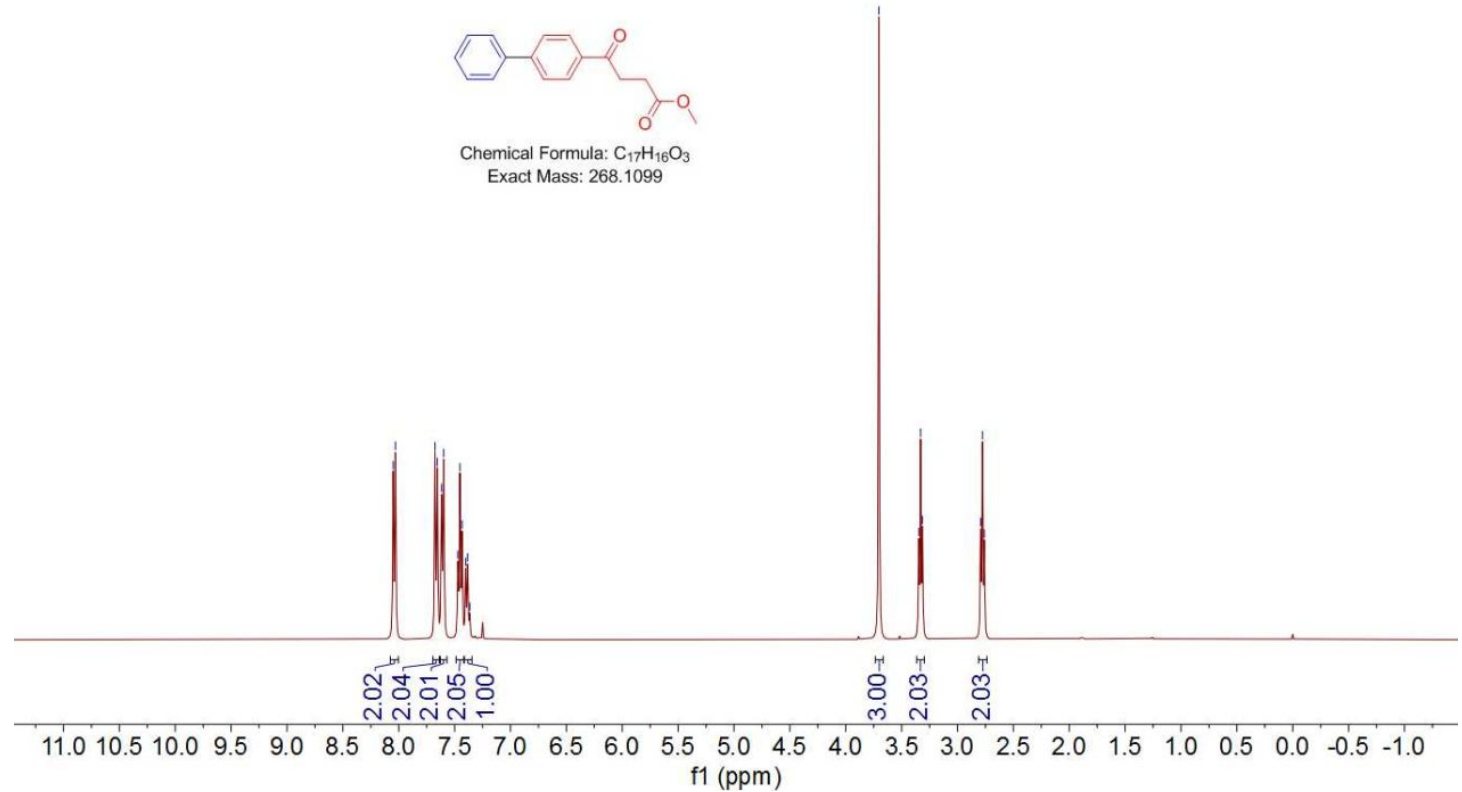

Exact Mass: 268.1099

${ }^{13} \mathrm{C}$ NMR $\left(\mathrm{CDCl}_{3}\right)$ spectrum of compound $\mathbf{5 a w}$

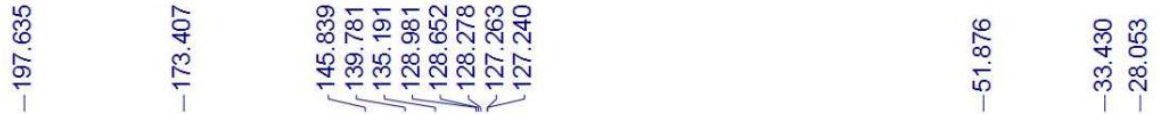

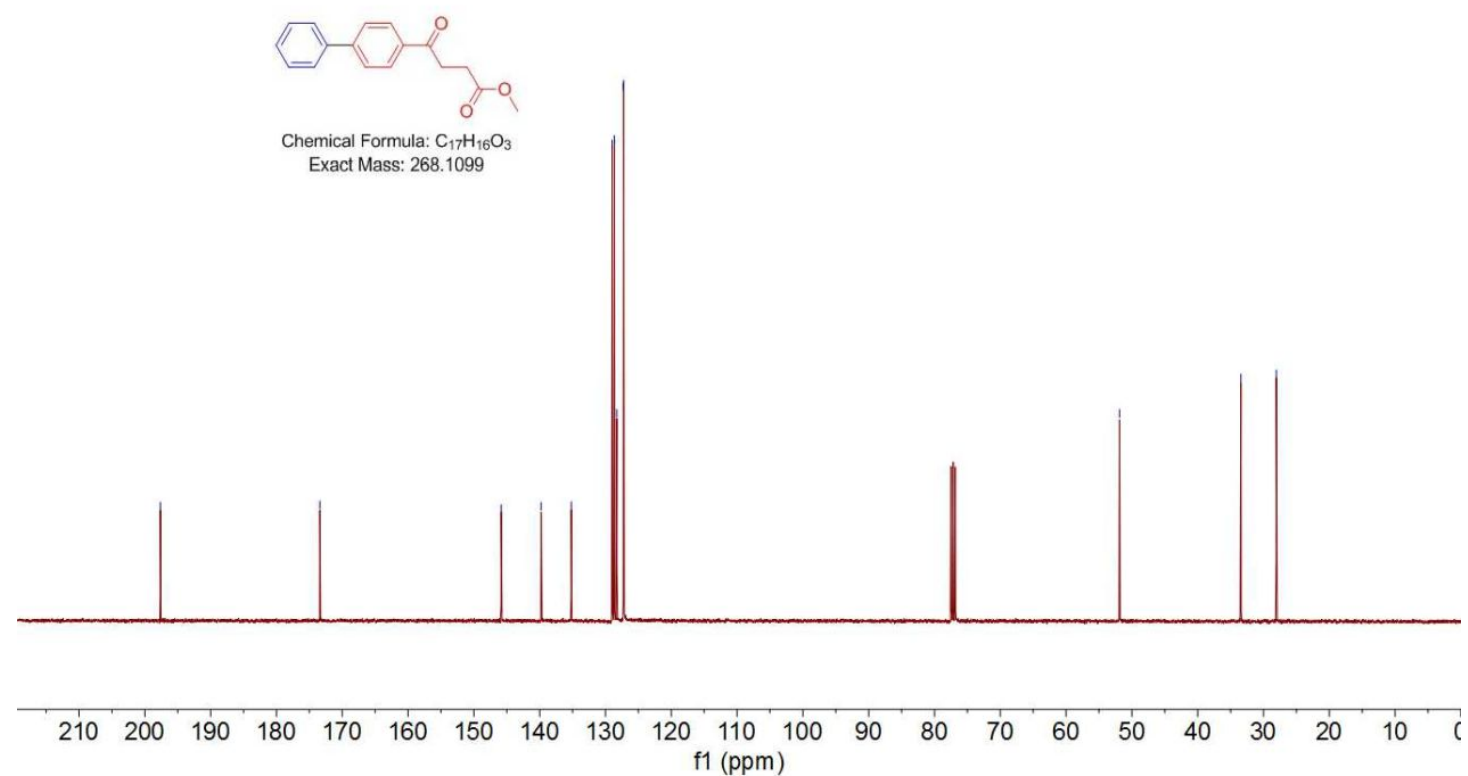


${ }^{1} \mathrm{H}$ NMR (DMSO- $d_{6}$ ) spectrum of compound $\mathbf{5 a x}$
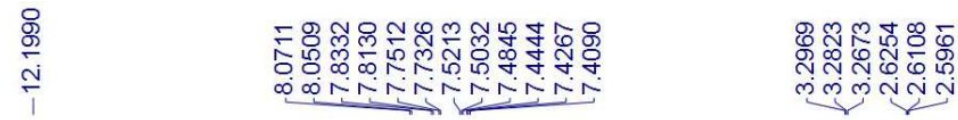

กัก กู่

min $\operatorname{rin}$

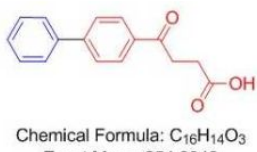

Chemical Formula: $\mathrm{C}_{16} \mathrm{H}_{14} \mathrm{O}_{3}$ Exact Mass: 254.0943

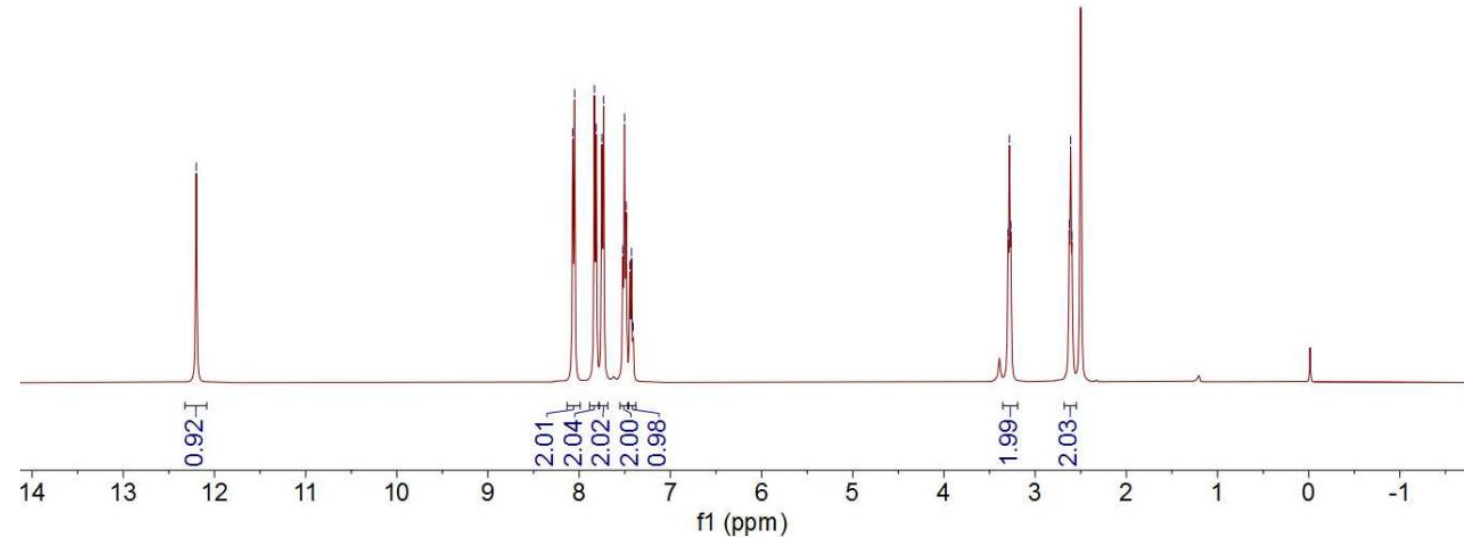

${ }^{13} \mathrm{C}$ NMR (DMSO- $d_{6}$ ) spectrum of compound $\mathbf{5 a x}$

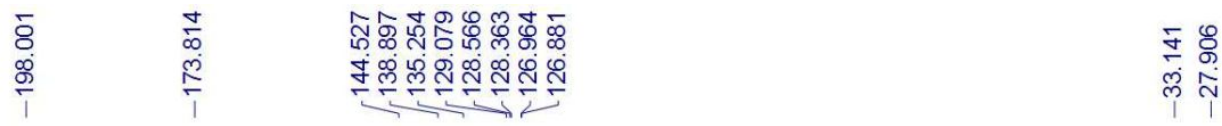

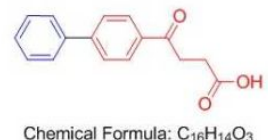

Exact Mass: 254.0943

קָ

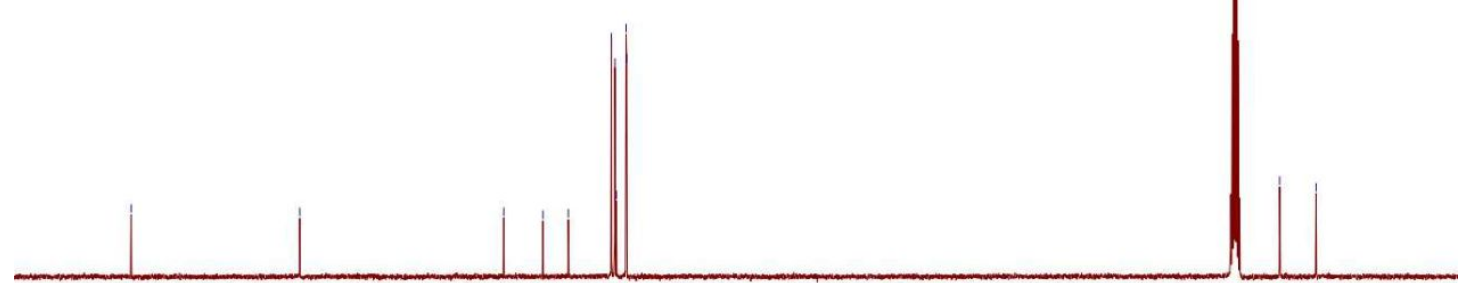

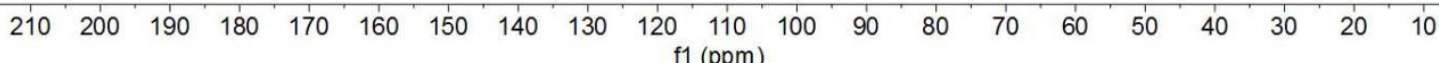


${ }^{1} \mathrm{H}$ NMR $\left(\mathrm{CDCl}_{3}\right)$ spectrum of compound 5ay

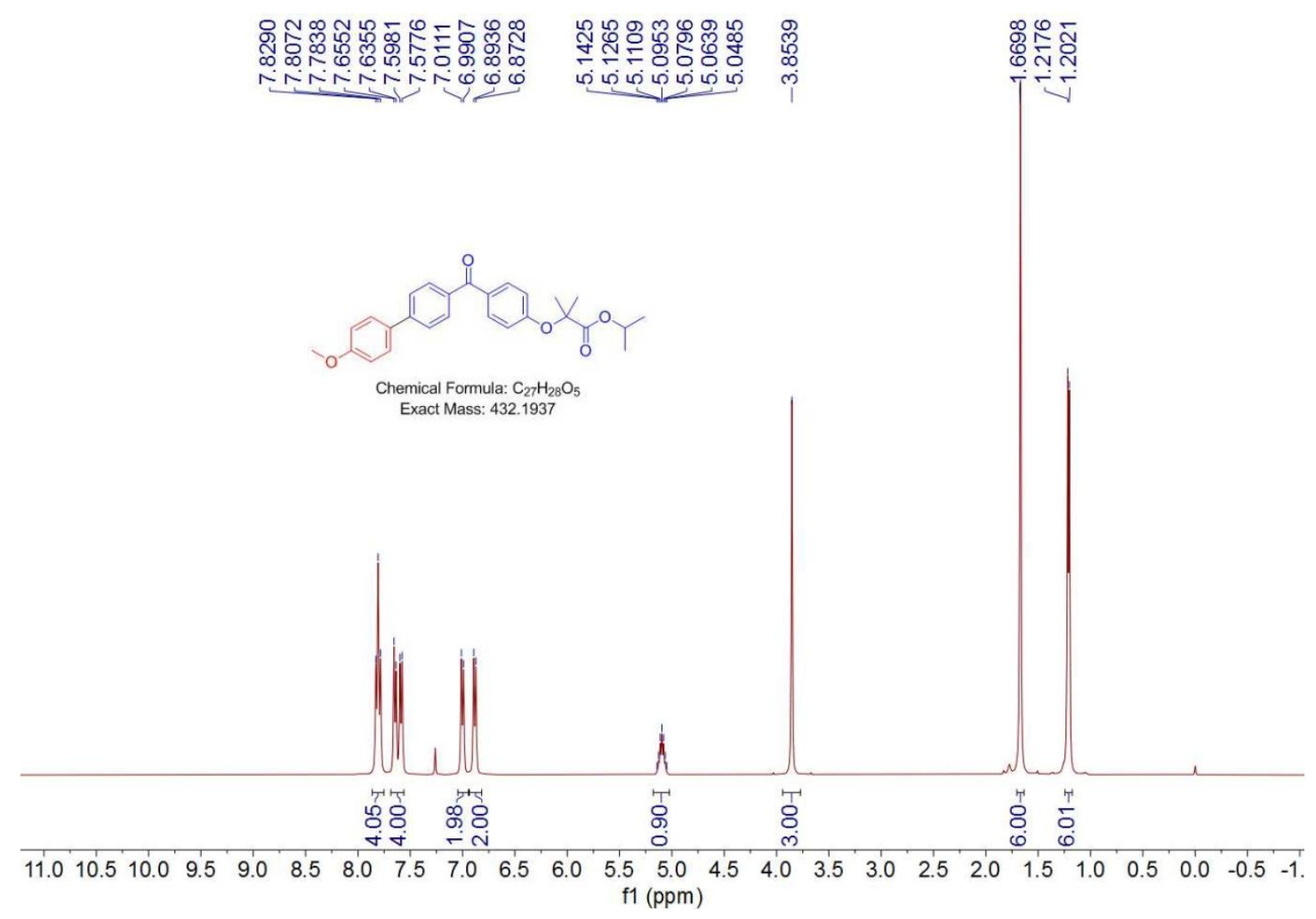

${ }^{13} \mathrm{C}$ NMR $\left(\mathrm{CDCl}_{3}\right)$ spectrum of compound 5ay
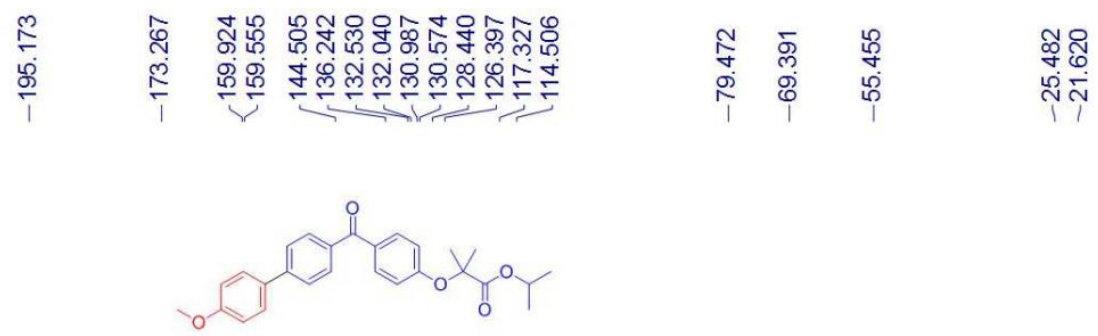

Chemical Formula: $\mathrm{C}_{27} \mathrm{H}_{28} \mathrm{O}_{5}$

Exact Mass: 432.1937

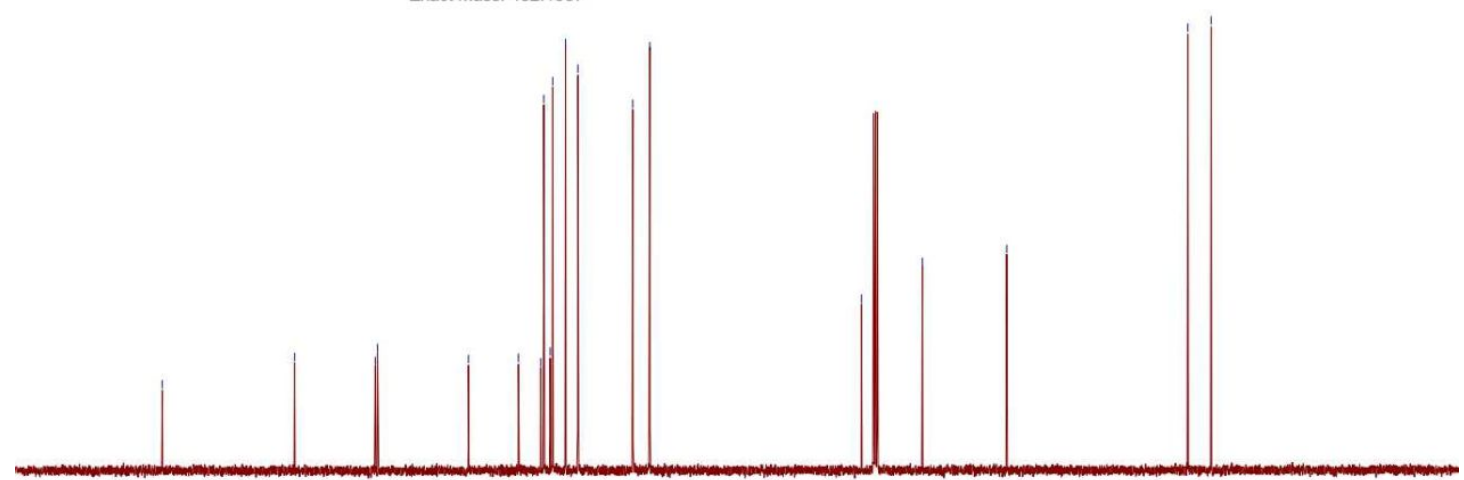

$\begin{array}{llllllllllllllllllllllll}210 & 200 & 190 & 180 & 170 & 160 & 150 & 140 & 130 & 120 & 110 & 100 & 90 & 80 & 70 & 60 & 50 & 40 & 30 & 20 & 10 & 0 & -10\end{array}$ 